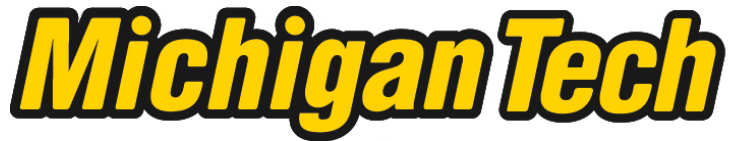 \\ Michigan Technological University Create the Future Digital Commons @ Michigan Tech
}

Dissertations, Master's Theses and Master's Reports - Open

Dissertations, Master's Theses and Master's

Reports

2013

Reverse Engineering the Structural and Acoustic Behavior of a Stradivari Violin

Michael A. Pyrkosz

Michigan Technological University

Follow this and additional works at: https://digitalcommons.mtu.edu/etds

Part of the Mechanical Engineering Commons

Copyright 2013 Michael A. Pyrkosz

\section{Recommended Citation}

Pyrkosz, Michael A., "Reverse Engineering the Structural and Acoustic Behavior of a Stradivari Violin", Dissertation, Michigan Technological University, 2013.

https://doi.org/10.37099/mtu.dc.etds/634

Follow this and additional works at: https://digitalcommons.mtu.edu/etds

Part of the Mechanical Engineering Commons 


\title{
REVERSE ENGINEERING THE STRUCTURAL AND ACOUSTIC BEHAVIOR OF A STRADIVARI VIOLIN
}

\author{
By \\ Michael A. Pyrkosz \\ A DISSERTATION \\ Submitted in partial fulfillment of the requirements for the degree of \\ DOCTOR OF PHILOSOPHY \\ In Mechanical Engineering-Engineering Mechanics \\ MICHIGAN TECHNOLOGICAL UNIVERSITY \\ 2013
}

copyright @ 2013 Michael A. Pyrkosz 

This dissertation has been approved in partial fulfillment of the requirements for the Degree of DOCTOR OF PHILOSOPHY in Mechanical Engineering - Engineering Mechanics.

\section{Department of Mechanical Engineering - Engineering Mechanics}

Dissertation Advisor: Charles D. Van Karsen

Committee Member: Jason R. Blough

Committee Member: Gregory M. Odegard

Committee Member: $\quad$ Chris E. Passerello

Committee Member: Milton L. Olsson

Department Chair: $\quad$ William W. Predebon 

To my wife, Alexis 



\section{Table of Contents}

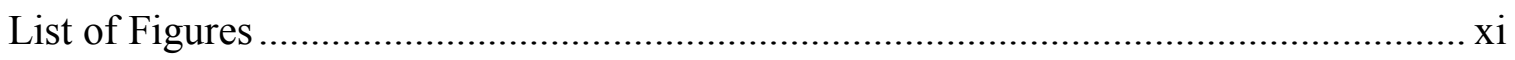

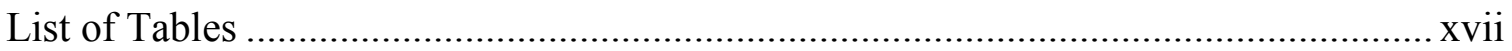

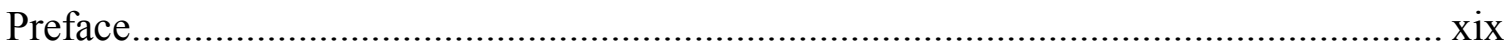

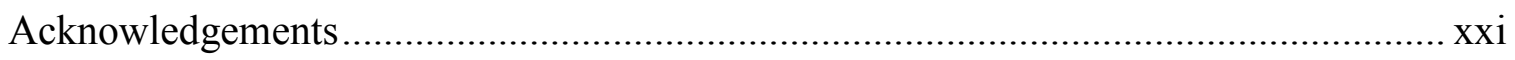

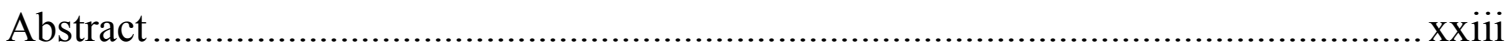

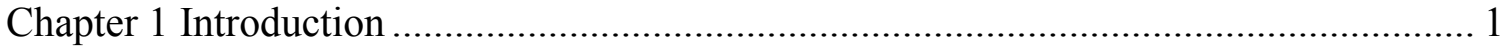

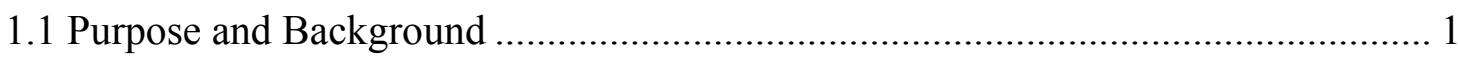

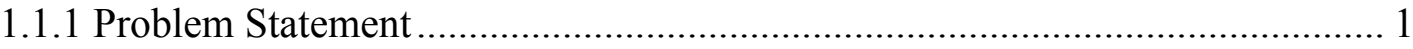

1.1.2 Background and Context...................................................................... 2

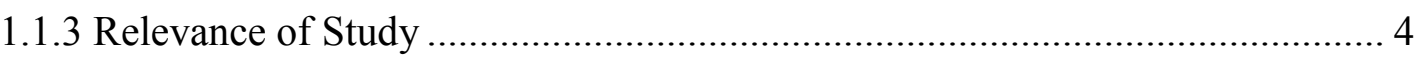

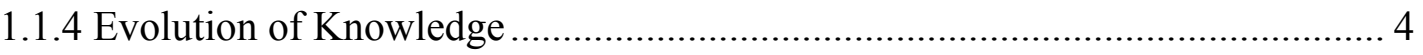

1.1.5 Contributions to Practice................................................................................ 4

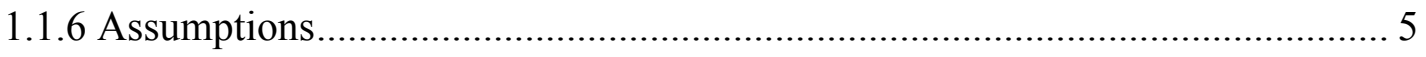

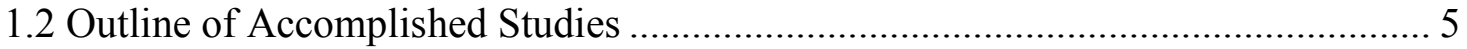

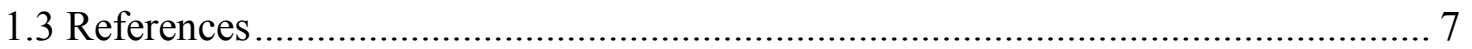

Chapter 2 Converting CT Scans of a Stradivari Violin to a Finite Element Model .......... 9

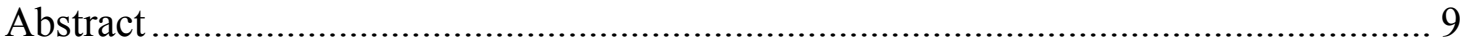

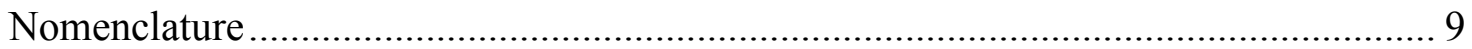

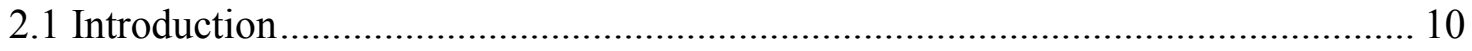

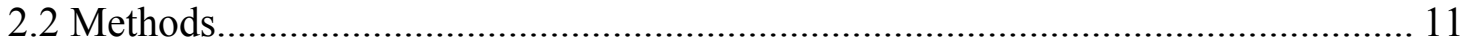

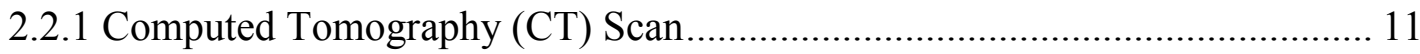

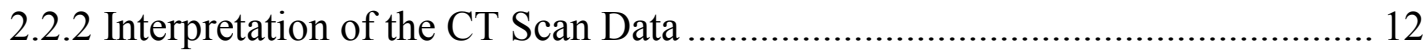

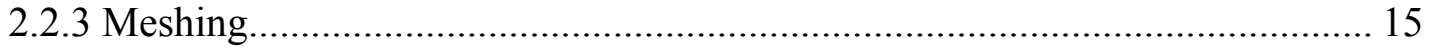

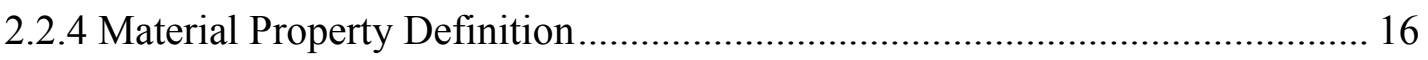

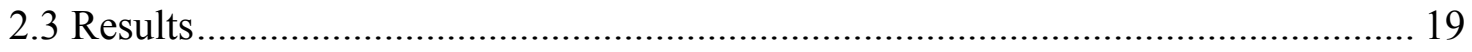

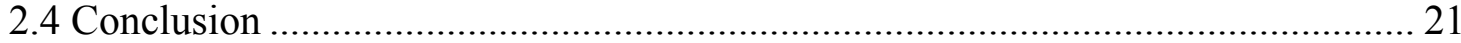

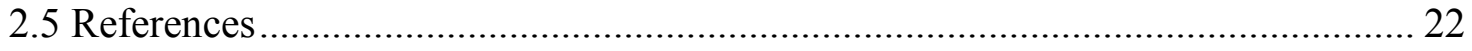

Michael Pyrkosz vii Michigan Technological University 
Chapter 3 Comparative Modal Tests of a Violin ....................................................... 23

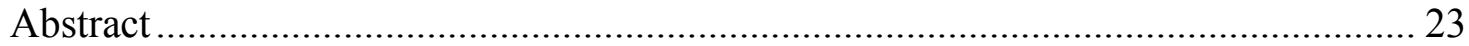

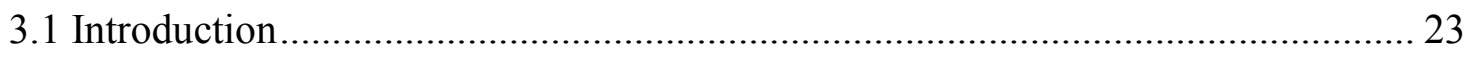

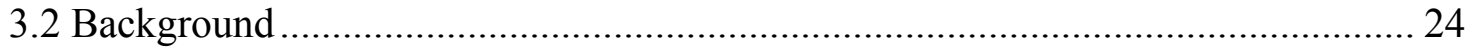

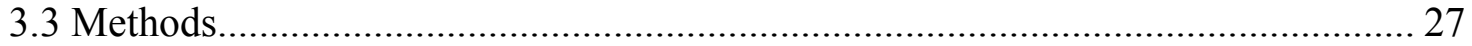

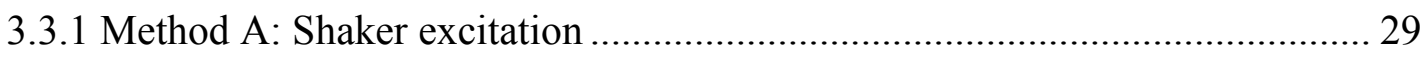

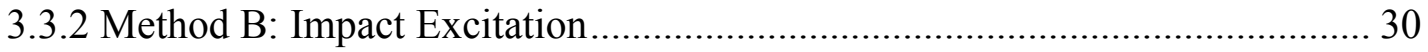

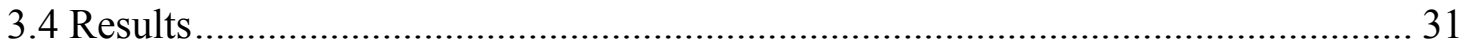

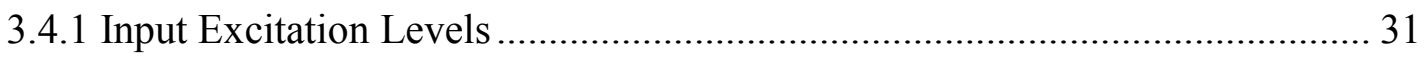

3.4.2 Frequency Response Functions and Coherence......................................... 32

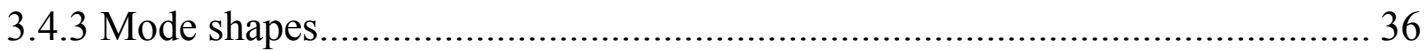

3.4.4 Modal Assurance Criterion before and after post processing ....................... 40

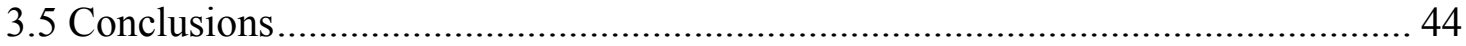

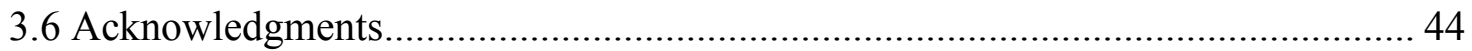

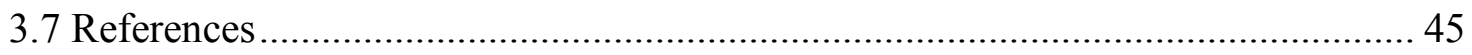

Chapter 4 Determining the Material Properties of a Stradivari Violin through Modal

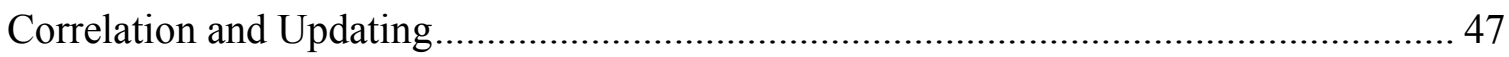

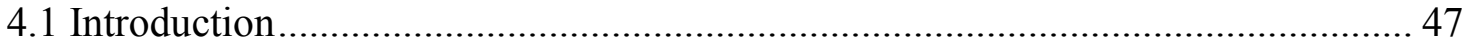

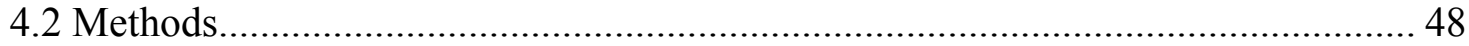

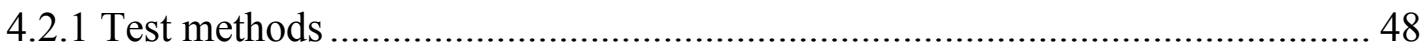

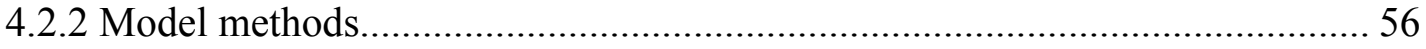

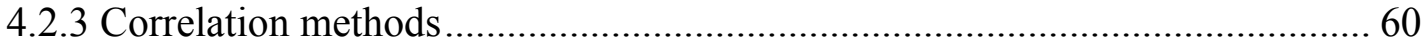

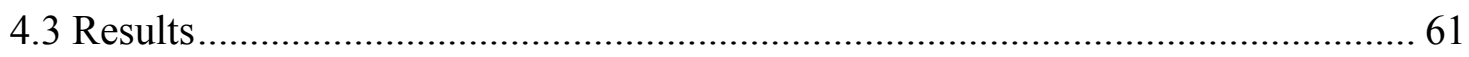

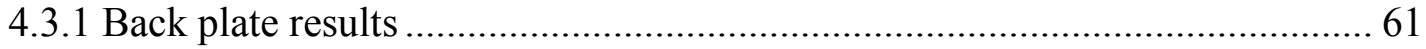

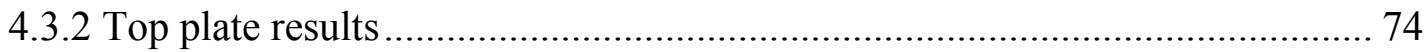

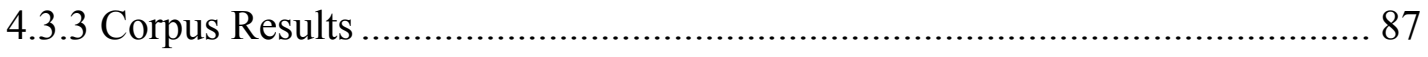

4.3.4 Titian Stradivari Violin Structural Results ................................................ 98

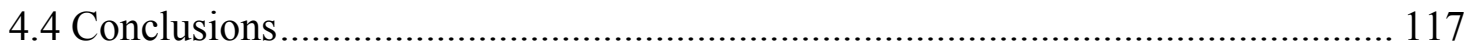

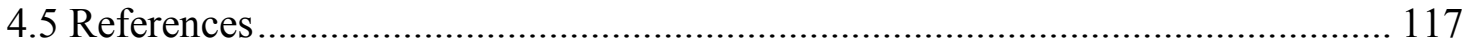

Chapter 5 Coupled Vibro-Acoustic Model of the Titian Stradivari Violin .................... 119

Michael Pyrkosz viii Michigan Technological University 


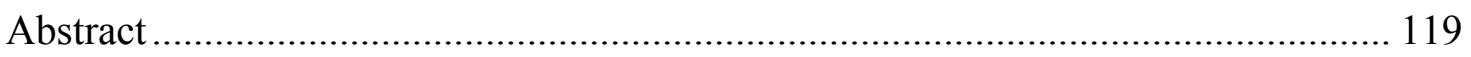

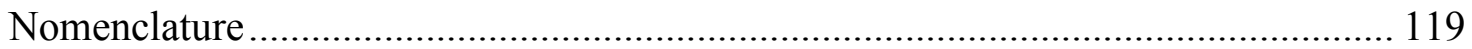

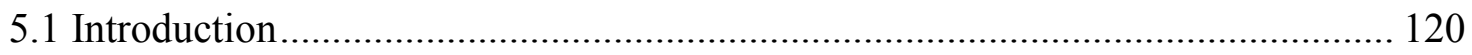

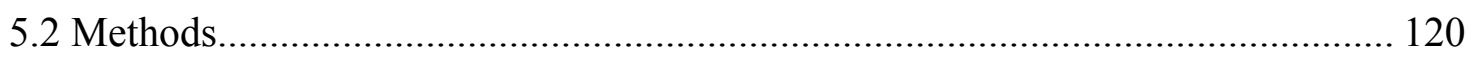

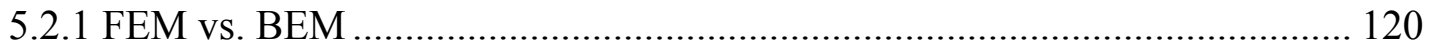

5.2.2 Sommerfeld radiation condition in FEM ....................................................... 121

5.2.3 Acoustic Mesh for FEM with AML.............................................................. 122

5.2.4 Acoustic mesh preparation......................................................................... 123

5.2.5 Coupled Vibro-Acoustic Set-up............................................................... 130

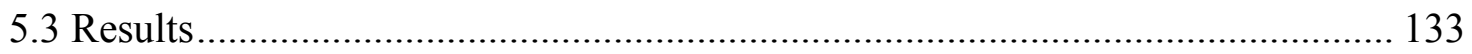

5.3.1 Projected Structural Modes........................................................................ 133

5.3.2 FEM Vibro-Acoustic Noise Transfer Function Analysis .............................. 135

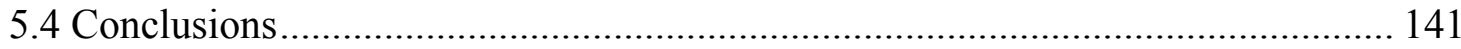

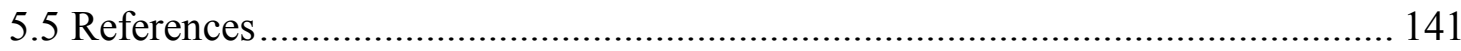

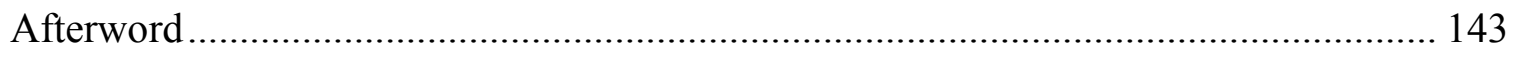

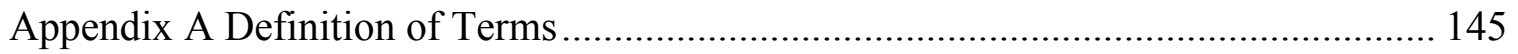

A.1 Engineering Terminology ....................................................................... 145

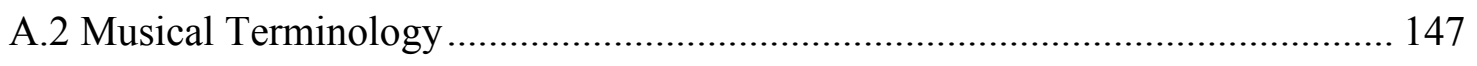

A.3 Parts of the Violin ...................................................................................... 148

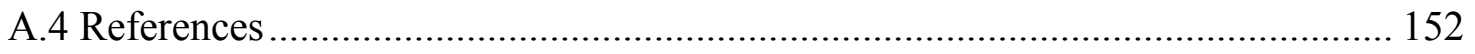

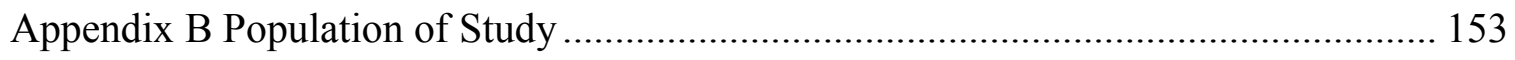

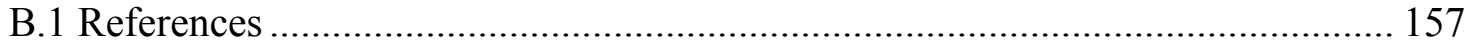

Appendix C Supporting Studies …………………………................................... 159

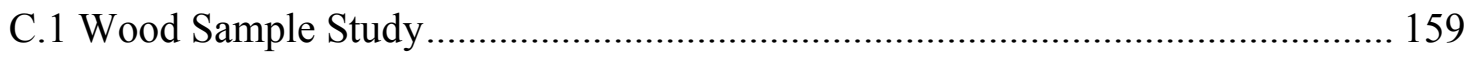

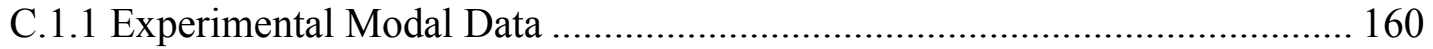

C.1.2 Finite Element Analysis ........................................................................... 164

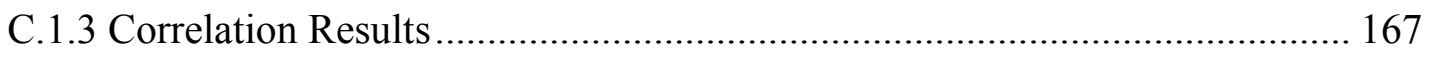

C.2 Cantilever Beam Study................................................................................. 170

C.2.1 Methods ......................................................................................... 170 


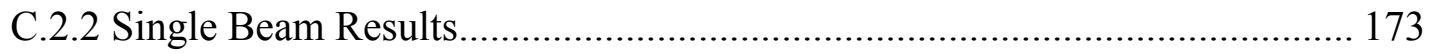

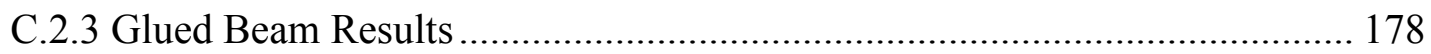

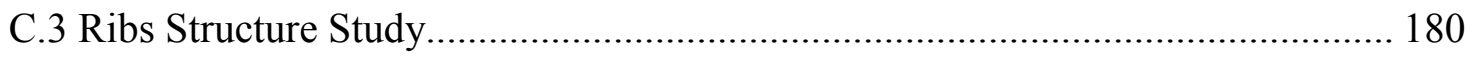

C.3.1 Experimental Modal Analysis ................................................................... 181

C.3.2 Finite Element Model............................................................................ 183

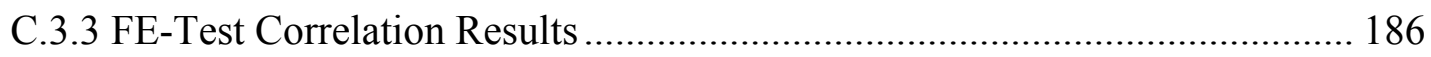

C.4 Reassembled Factory Violin Measurements ......................................................... 187

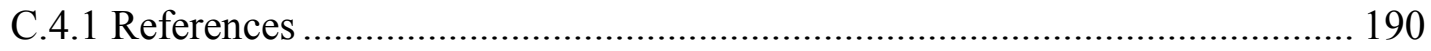

Appendix D Matlab Scripts ……………………………………………………..... 191

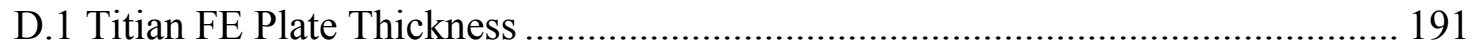

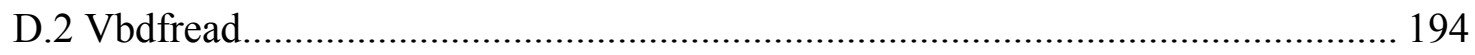

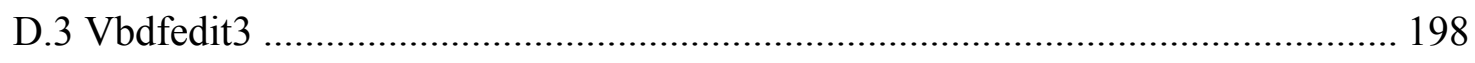

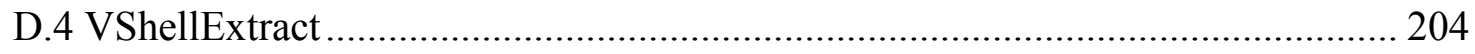

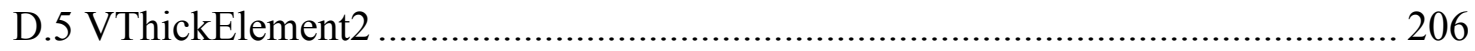

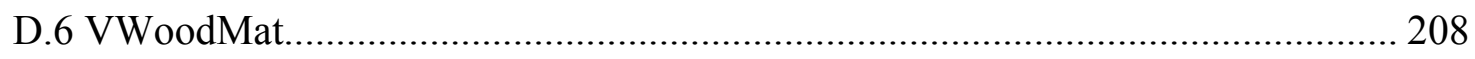

Appendix E Test Support Drawings …………………....................................... 217 


\section{List of Figures}

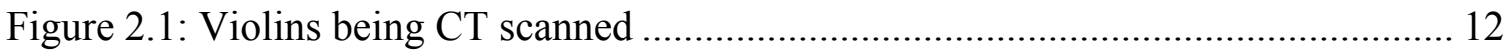

Figure 2.2: Example of mask editing to remove artifacts.............................................. 14

Figure 2.3: Screen capture from Mimics software depicting separation of parts and 3D

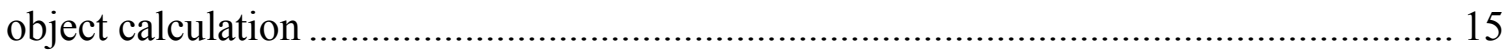

Figure 2.4: Screen capture of tetrahedron mesh in Virtual.Lab..................................... 16

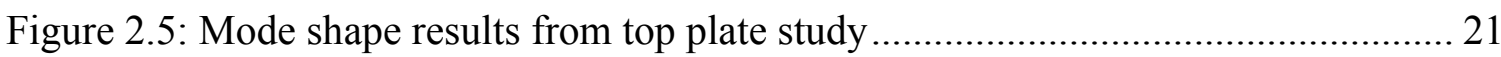

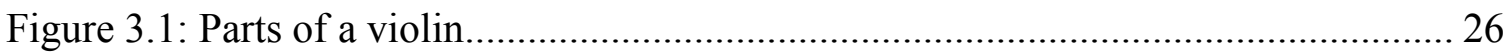

Figure 3.2: Diagram showing exciter input and accelerometer response locations for

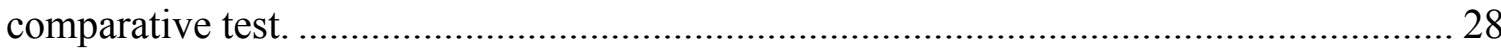

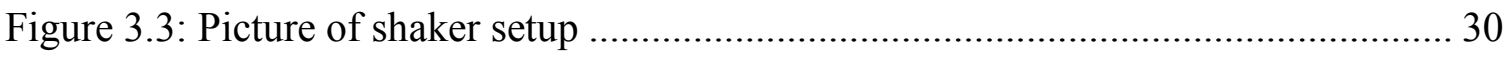

Figure 3.4: Picture of impact hammer setup ................................................................... 31

Figure 3.5: PCB 086E80 impact hammer with actual tip used (US dime shown for scale).

Figure 3.6: Input Autopower spectra for each type of excitation. Burst random excitation (red), stepped-sine excitation (green), impact excitation (blue).

Figure 3.7: Frequency response functions for the driving point at the bass-side corner of the bridge. Burst random excitation (red), stepped-sine excitation (green), impact excitation (blue).

Figure 3.8: Coherence functions for the driving point at the bass-side corner of the bridge. Burst random excitation (red), stepped-sine excitation (green), impact excitation (blue).33 Figure 3.9: Frequency response functions for the bass-side foot of the bridge. Burst random excitation (red), stepped-sine excitation (green), impact excitation (blue)......... 34 Figure 3.10: Coherence functions for the bass-side foot of the bridge. Burst random excitation (red), stepped-sine excitation (green), impact excitation (blue). ...................... 35

Figure 3.11: Frequency response functions for the treble-side foot of the bridge. Burst random excitation (red), stepped-sine excitation (green), impact excitation (blue)......... 35 Figure 3.12: Coherence functions for the treble-side foot of the bridge. Burst random excitation (red), stepped-sine excitation (green), impact excitation (blue). ..................... 36

Figure 3.13: Mode 1 - First bending of violin between neck and corpus (B0) ................ 37

Figure 3.14: Mode 2 - First bending of violin in lateral direction ................................... 37

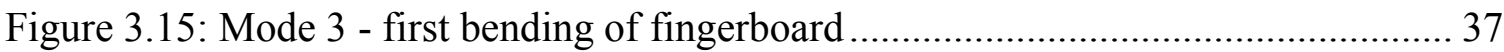

Figure 3.16: Mode 4 - first cavity mode (A0) ............................................................ 37

Figure 3.17: Mode 5 - scroll twist out-of phase with center......................................... 38

Figure 3.18: Mode 6 - scroll twist in-phase with center ................................................ 38

Figure 3.19: Mode 7 Center Bout Rhomboid (CBR) scroll in phase with center............. 38

Figure 3.20: Mode 8 - Center Bout Rhomboid (CBR) scroll out-of-phase with center ... 38

Figure 3.21: Mode 9 - first torsion of fingerboard ...................................................... 38 
Figure 3.22: Mode 10 - Cavity mode (A1) most breathing in lower bout (strong couple to

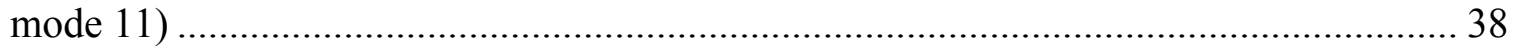

Figure 3.23: Mode 11 - first lateral bending of fingerboard (strong couple to A1)......... 39 Figure 3.24: Mode 12 - First bending of corpus (B1-) top plate transverse, back plate longitudinal 39

Figure 3.25: Mode 13 - First bending of corpus (B1+) top plate longitudinal, back plate transverse 39

Figure 3.26: Mode 14 - A2 ........................................................................................... 39

Figure 3.27: Modal Assurance Criterion between Method A before correction to Method

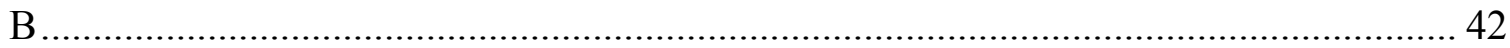

Figure 3.28: Modal Assurance Criterion between Method A after correction to Method B

.

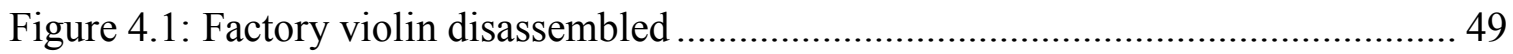

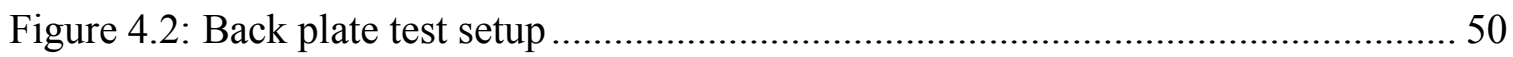

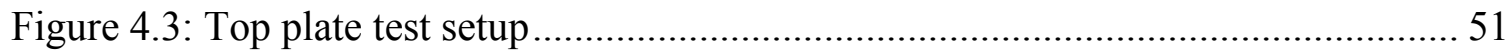

Figure 4.4: Applying hide glue to the back edges of the rib structure ............................. 52

Figure 4.5: Clamping the back plate and rib structure together...................................... 52

Figure 4.6: Appplying hide glue to the top edges of the rib structure ............................ 53

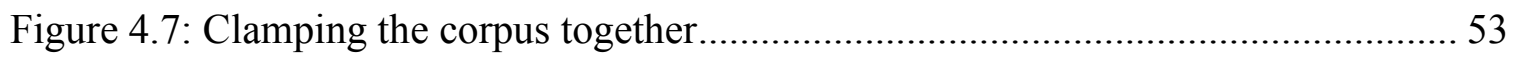

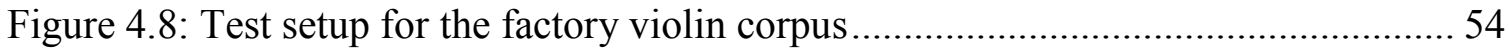

Figure 4.9: Comparison of the mid-treble region of the corpus (a) before and (b) after the

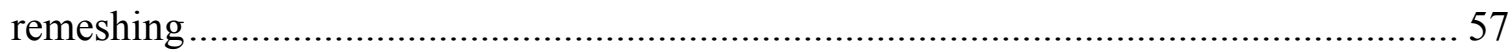

Figure 4.10: Comparison of the corpus-neck joint (a) before and (b) after remeshing .... 58

Figure 4.11: Comparison of the neck-fingerboard-nut interfaces (a) before and (b) after

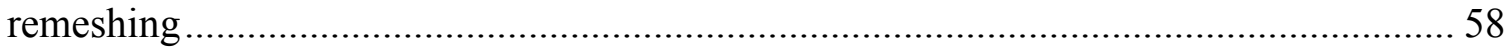

Figure 4.12: Screen capture of Titian Stradivari violin model remesh in ANSA 13.2.2.. 59

Figure 4.13: Driving Point FRFs of the factory violin back plate ................................. 62

Figure 4.14: Driving point coherence functions of the factory violin back plate ............ 63

Figure 4.15: Mode indicator functions for curve fitting the factory violin back plate

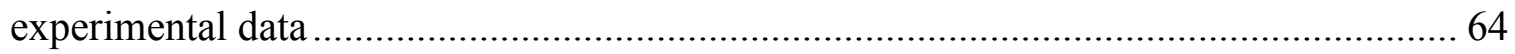

Figure 4.16: First experimental mode of the factory violin back plate ........................... 65

Figure 4.17: Second experimental mode of the factory violin back plate ...................... 65

Figure 4.18: Third experimental mode of the factory violin back plate .......................... 66

Figure 4.19: Fourth experimental mode of the factory violin back plate ........................ 66

Figure 4.20: Fifth experimental mode of the factory violin back plate ............................ 67

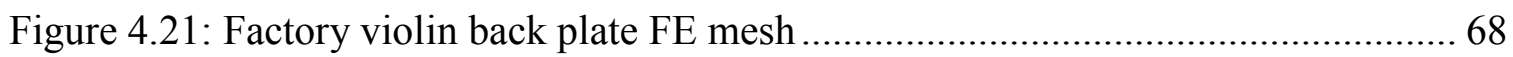

Figure 4.22: Element thickness of the factory violin back plate in $\mathrm{mm}$.......................... 69

Figure 4.23: Mode 1 of the factory violin back plate (first torsion) ............................. 70

Figure 4.24: Mode 2 of the factory violin back plate (longitudinal bending).................. 70

Michael Pyrkosz xii Michigan Technological University 
Figure 4.25: Mode 3 of the factory violin back plate (triad) ...................................... 70

Figure 4.26: Mode 4 of the factory violin back plate (second torsion)........................... 70

Figure 4.27: Mode 5 of the factory violin back plate (ring) ...................................... 71

Figure 4.28: Mode 6 of the factory violin back plate (upper torsion-lower 2nd bending) 71

Figure 4.29: MAC results of factory violin back plate EMA modes with distributed thickness FE model .................................................................................................. 72

Figure 4.30: MAC results of factory violin back plate EMA modes with uniform

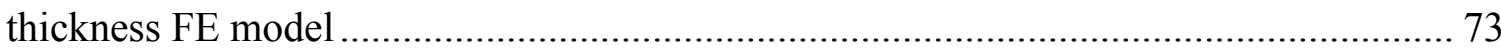

Figure 4.31: Driving point FRFs of the factory violin top plate ................................. 75

Figure 4.32: Driving point coherence functions of the factory violin top plate .............. 76

Figure 4.33: Mode indicator functions for curve fitting the factory violin top plate

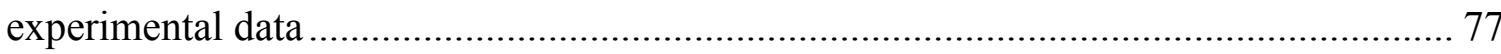

Figure 4.34: First experimental mode of the factory violin top plate ........................... 78

Figure 4.35: Second experimental mode of the factory violin top plate........................ 78

Figure 4.36: Third experimental mode of the factory violin top plate........................... 79

Figure 4.37: Fourth experimental mode of the factory violin top plate......................... 79

Figure 4.38: Fifth experimental mode of the factory violin top plate............................... 80

Figure 4.39: Factory violin top plate FE mesh (left view from top, right view from back)

Figure 4.40: Element thickness of the factory violin top plate in $\mathrm{mm}$............................ 82

Figure 4.41: Mode 1 of the factory violin top plate (first torsion) ............................... 83

Figure 4.42: Mode 2 of the factory violin top plate (first longitudinal bending)............. 83

Figure 4.43: Mode 3 of the factory violin top plate (second torsion) ............................ 83

Figure 4.44: Mode 4 of the factory violin top plate (ring) ........................................... 83

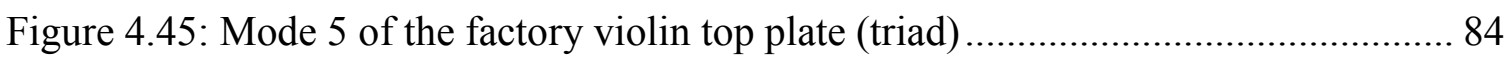

Figure 4.46: Mode 6 of the factory violin top plate (upper torsion-lower 2nd bending).. 84

Figure 4.47: MAC results of factory violin top plate EMA modes with distributed

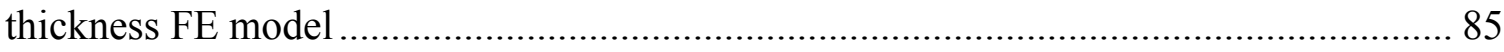

Figure 4.48: MAC results of factory violin top plate EMA modes with uniform thickness

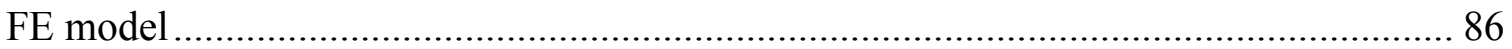

Figure 4.49: Driving point FRFs for factory violin corpus....................................... 88

Figure 4.50: Driving point coherence functions for factory violin corpus ..................... 89

Figure 4.51: Mode indicator functions for curve fitting the factory violin corpus

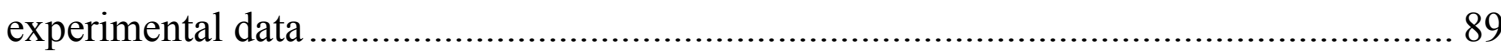

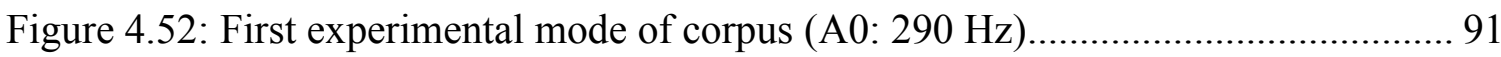

Figure 4.53: Second experimental mode of corpus (CBR: $464 \mathrm{~Hz}$ ) .............................. 91

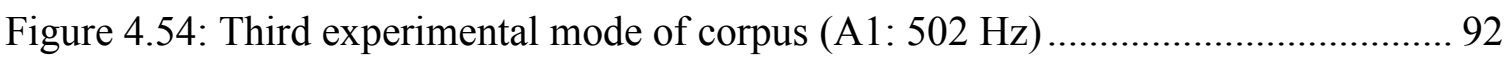

Figure 4.55: Fourth experimental mode of corpus (B1-: $638 \mathrm{~Hz})$................................. 92

Figure 4.56: Fifth experimental mode of corpus $(\mathrm{B} 1+: 657 \mathrm{~Hz})$................................ 93

Michael Pyrkosz xiii Michigan Technological University 


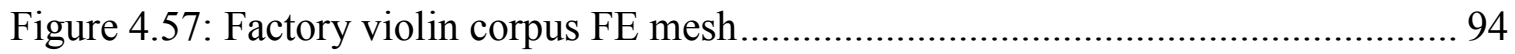

Figure 4.58: First analytical mode of corpus (CBR) .................................................... 95

Figure 4.59: Second analytical mode of corpus (B1-) .................................................... 95

Figure 4.60: Third analytical mode of corpus (B1+) ................................................ 95

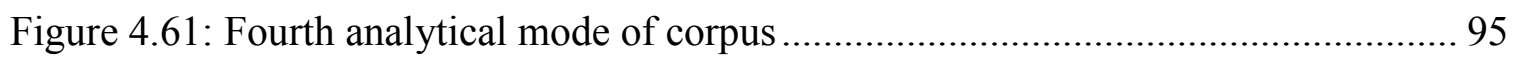

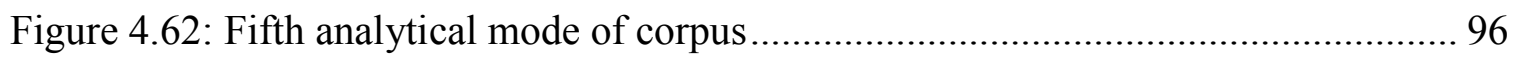

Figure 4.63: Sixth analytical mode of corpus ............................................................ 96

Figure 4.64: MAC results of factory violin corpus EMA modes with structural FE model

Figure 4.65: FRFs of the bass-side bridge foot responses of the Titian Stradivari violin 98 Figure 4.66: Coherence spectra of the bass-side bridge foot responses of the Titian

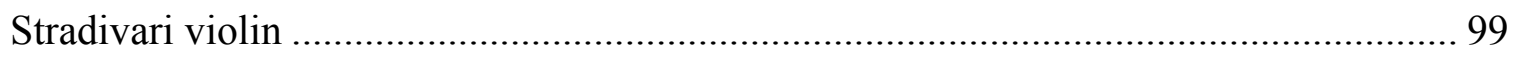

Figure 4.67: FRF sum and Multivariant MIF for the Titian Stradivari violin curve-fitting

Figure 4.68: Final Titian Stradivari violin structural FE model with Pre-loaded strings 103

Figure 4.69: Element thickness of the Titian Stradivari violin back plate in mm .......... 104

Figure 4.70: Element thickness of the Titian Stradivari violin top plate in mm............ 104

Figure 4.71: First mode of Titian FE model: Global bending, B0 (Tail IP) $158.9 \mathrm{~Hz} \ldots 106$

Figure 4.72: Second mode of Titian FE model: Global bending, B0 (Tail OP) $181.1 \mathrm{~Hz}$

Figure 4.73: Third mode of Titian FE model: Global bending, B0 (FB bend) $227.2 \mathrm{~Hz} 106$

Figure 4.74: Lateral bending mode of the Titian FE model (Tail IP) $223.5 \mathrm{~Hz}$............ 107

Figure 4.75: Lateral bending mode of the Titian FE model, (Tail OP) 245.8 Hz........... 107

Figure 4.76: First G-string modes on Titian Stradivari violin FE ................................ 108

Figure 4.77: First D-string modes on Titian Stradivari violin FE ................................ 108

Figure 4.78: First A-string modes on Titian Stradivari violin FE .................................. 109

Figure 4.79: First E-string modes on Titian Stradivari violin FE ................................. 109

Figure 4.80: Second G-string modes on Titian Stradivari violin FE ............................ 110

Figure 4.81: Second D-string modes on Titian Stradivari violin FE ............................ 110

Figure 4.82: Third G-string modes on Titian Stradivari violin FE ............................... 111

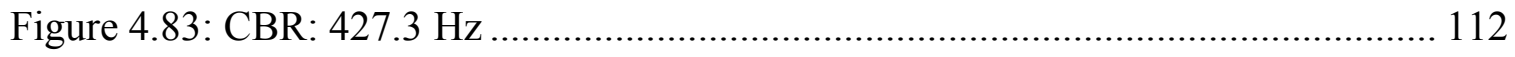

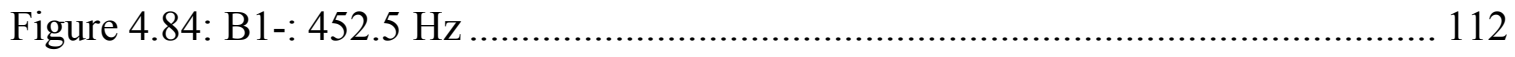

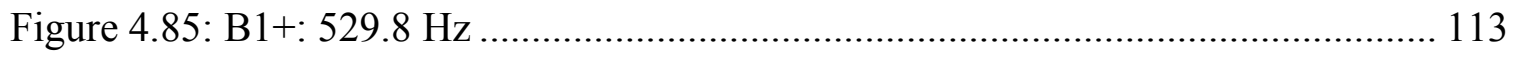

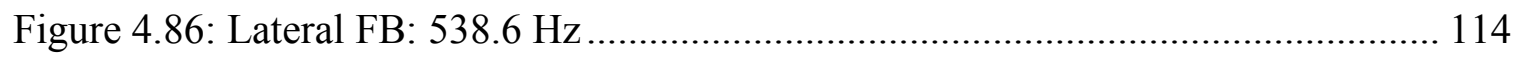

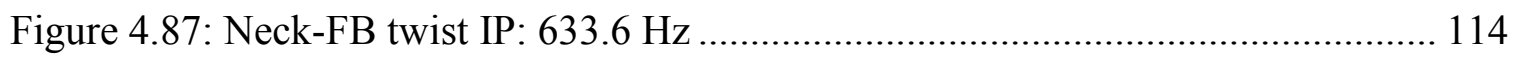

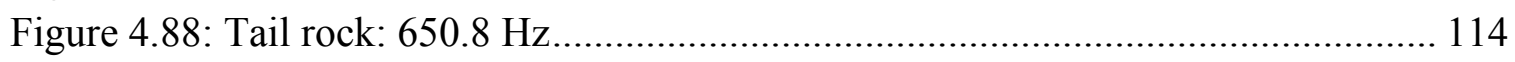

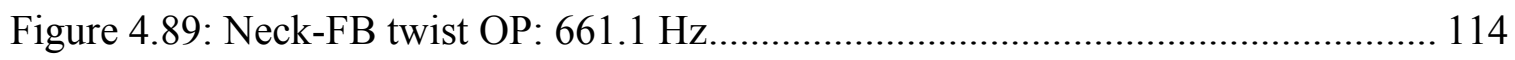

Figure 4.90: Initial Correlation Results for the Titian violin...................................... 115

Figure 4.91: Updated Correlation Results for the Titian violin .................................... 116

Michael Pyrkosz $\quad$ xiv Michigan Technological University 
Figure 5.1: Setting up FE meshes for AML property on an opened structural mesh of a

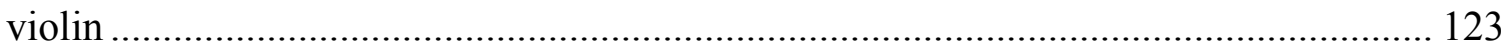

Figure 5.2: Filling in holes: (a) peg box, (b) tail piece, and (c) end pin ....................... 124 Figure 5.3: The edges around the f-holes are selected to be maintained ........................ 125 Figure 5.4: Cross-sectional view of the first pass of the volumize mesh ....................... 126 Figure 5.5: Final volumize mesh of the Titian Stradivari violin and cut-plane at bridge

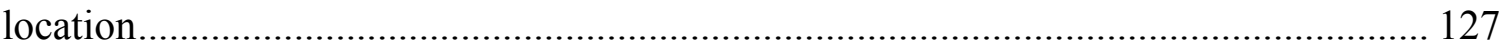

Figure 5.6: Convex mesh around Titian Stradivari violin FE model.............................. 128

Figure 5.7: Sagittal cross-section of the tetra-fill volume mesh ................................... 128

Figure 5.8: Coronal cross-section of the tetra-fill volume mesh .................................. 129

Figure 5.9: Axial cross-section of the tetra-fill volume mesh ....................................... 129

Figure 5.10: Titian Stradivari structural FE mesh inside a cut-away (coronal) of the acoustic volume mesh

Figure 5.11: Cut-away (sagittal) of the acoustic mesh showing the AML property on the

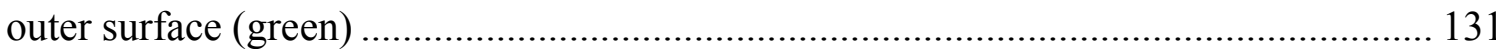

Figure 5.12: Field point mesh around the Titian Stradivari FE model ......................... 132 Figure 5.13: Input locations for the vibro-acoustic transfer function analysis of the Titian Stradivari violin

Figure 5.14: Projection of the CBR mode from the structural mesh (left) to the acoustic envelope (right)

Figure 5.15: Projection of the B1- mode from the structural mesh (left) to the acoustic envelope (right)

Figure 5.16: Projection of the B1+ mode from the structural mesh (left) to the acoustic envelope (right)

Figure 5.17: Radiativity results comparison for point directly in front of violin top plate; Real normal modes on structural FE; Air properties constant real: $\mathrm{c}=340 \mathrm{~m} / \mathrm{s}, \rho=1.225$ $\mathrm{kg} / \mathrm{m} 3$

Figure 5.18: Radiativity results comparison for point directly in front of violin top plate; Modal damping added to all structural FE modes; Air properties constant real: $\mathrm{c}=340$ $\mathrm{m} / \mathrm{s}, \rho=1.225 \mathrm{~kg} / \mathrm{m} 3$ 138

Figure 5.19: Radiativity results comparison for point directly in front of violin top plate; Modal damping added to structural modes (except string modes); Air properties complex: $\mathrm{c}=(340+\mathrm{j} 4) \mathrm{m} / \mathrm{s}, \rho=1.225 \mathrm{~kg} / \mathrm{m} 3$ 139

Figure 5.20: Radiativity results comparison for point directly in front of violin top plate; Modal damping added to structural modes (except string modes); Air properties complex: $\mathrm{c}=(340+\mathrm{j} 8) \mathrm{m} / \mathrm{s}, \rho=1.225 \mathrm{~kg} / \mathrm{m} 3$ 140

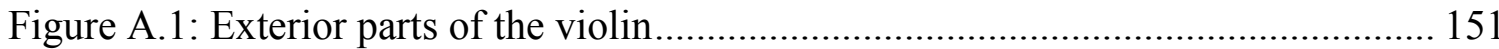

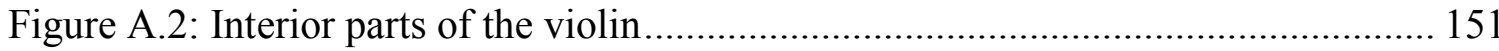

Figure B.1: Comparison of slices taken across middle section of each violin ................ 156 
Figure B.2: Detail sagittal and coronal views of Factory violin A and the Titian Stradivari

Figure C.1: Wood samples to be used in finding material properties of wood species, . 160

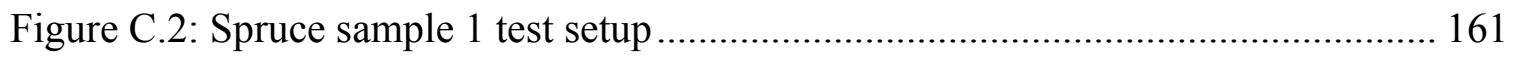

Figure C.3: Driving point FRF measurements for spruce sample 1 ............................ 162

Figure C.4: Driving point coherence measurements for spruce sample 1 .................... 162

Figure C.5: Driving point FRF measurements for maple sample 1 ............................ 163

Figure C.6: Driving point coherence measurements for maple sample 1 ...................... 163

Figure C.7: Wood sample FE meshes, (a) spruce 1, (b) maple 1 ................................. 165

Figure C.8: Mode shapes of the spruce 1 FE model .................................................. 166

Figure C.9: Mode shapes of the maple 1 FE model................................................... 167

Figure C.10: MAC matrix for the Spruce 1 FE-Test correlation .................................... 168

Figure C.11: MAC matrix for the maple 1 FE-Test correlation .................................. 169

Figure C.12: Three beams cut from plywood sheet ............................................... 170

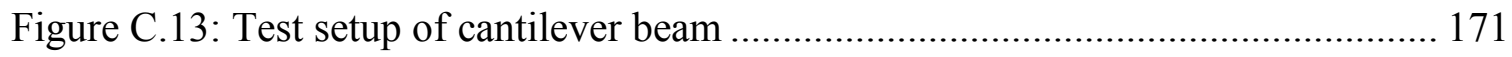

Figure C.14: Applying liquid hide glue to the surface of one beam............................ 172

Figure C.15: Spring clamps on laminated beam ................................................... 172

Figure C.16: Driving point FRFs for single beam ................................................. 173

Figure C.17: FE mesh of the cantilever plywood beam............................................. 174

Figure C.18: Mode shapes of the single plywood beam ........................................... 176

Figure C.19: MAC results for single beam after updating......................................... 177

Figure C.20: Driving point FRFs for laminated beam .............................................. 178

Figure C.21: MAC results for laminated beam after updating .................................... 179

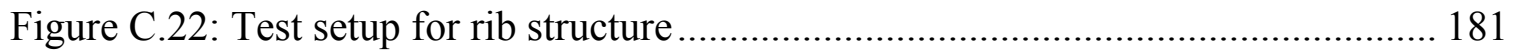

Figure C.23: Driving point FRFs of factory violin rib structure ................................. 182

Figure C.24: Driving point coherence of factory violin rib structure .......................... 182

Figure C.25: Groups for defining shell properties, (a) ribs and lining groups, (b) material

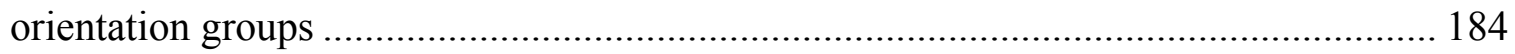

Figure C.26: Mode 1 of the factory violin rib structure (lower bass-side bow) ............. 185

Figure C.27: Mode 2 of the factory violin rib structure (lower treble-side bow) ........... 185

Figure C.28: Mode 3 of the factory violin rib structure (lower treble-side twist) .......... 185

Figure C.29: Mode 4 of the factory violin rib structure (upper bass-side twist) ............ 185

Figure C.30: Mode 5 of the factory violin rib structure (lower treble-side twist) .......... 185

Figure C.31: Mode 6 of the factory violin rib structure (upper bass-side bow) ............. 185

Figure C.32: MAC results for rib structure.......................................................... 186

Figure C.33: Gluing the neck and scroll to the corpus .............................................. 188

Figure C.34: Comparison of driving point FRF before and after factory violin was rebuilt 
Figure C.35: Comparison of bass-side bridge foot FRF before and after factory violin was rebuilt 189

Figure C.36: Comparison of treble-side bridge foot FRF before and after factory violin was rebuilt 190

\section{List of Tables}

Table 2.1: Top plate study results

Table 3.1: Mode merging table, indicating the pole migrations for each acquisition session due to mass loading effect (Color indicates high/low values in each column with red $=$ high and green $=$ low .

Table 3.2: Comparison of mode frequencies and MAC values of Method A before and

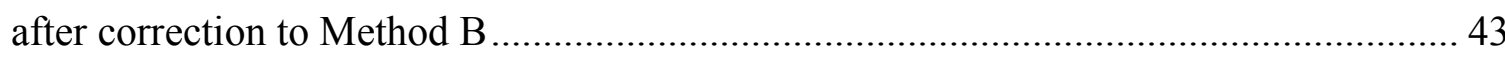

Table 4.1: DSP parameters for factory violin back plate EMA .................................... 62

Table 4.2: Curve-fitting results from the factory violin back plate EMA ....................... 64

Table 4.3: Summary of final factory violin back plate normal modes analysis 69

Table 4.4: Mode pairs of the factory violin back plate correlation with distributed thickness. 72

Table 4.5: Mode pairs of the factory violin back plate correlation with uniform thickness

Table 4.6: Summary of final material properties for factory violin back plate FE model 74

Table 4.7: DSP parameters for factory violin top plate EMA .................................... 75

Table 4.8: Curve-fitting results from the factory violin top plate EMA .......................... 77

Table 4.9: Summary of final factory violin top plate normal modes analysis .................. 82

Table 4.10: Mode pairs of the factory violin top plate correlation with distributed

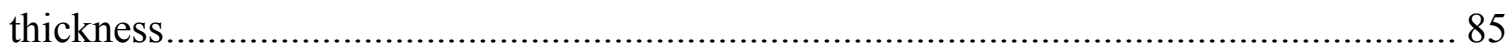

Table 4.11: Mode pairs of the factory violin top plate correlation with uniform thickness

Table 4.12: Summary of final material properties for factory violin top plate FE model 87

Table 4.13: DSP parameters for factory violin corpus EMA ......................................... 88

Table 4.14: Curve-fitting results from the factory violin corpus EMA .......................... 90

Table 4.15: Factory violin corpus FE mesh composition .......................................... 93

Table 4.16: Summary of final factory violin corpus normal modes analysis .................. 94

Table 4.17: Mode pairs of the factory violin corpus correlation .................................. 96

Table 4.18: Summary of final material properties for factory violin corpus FE model ... 97

Table 4.19: Curve-fitting results from the Titian Stradivari violin EMA....................... 101

Table 4.20: Titian Stradivari violin structural FE mesh composition........................... 102

Table 4.21: Summary of updated Titian Stradivari violin normal modes analysis ........ 105

Michael Pyrkosz xvii Michigan Technological University 
Table 4.22: Updated Mode Pair Results for the Titian violin..................................... 116

Table 4.23: Summary of final material properties for Titian Stradivari FE model ........ 117

Table 5.1: Mesh compositions for Vibro-Acoustic FEM Analysis of the Titian Stradivari

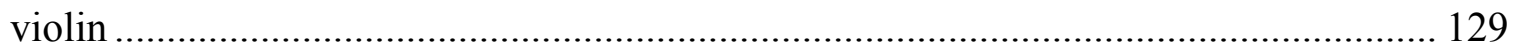

Table 5.2: AML parameters for Vibro-Acoustic Analysis of the Titian Stradivari........ 131

Table 5.3: Analysis system properties .......................................................................... 136

Table 5.4: Vibro-Acoustic FEM Analysis run summary .......................................... 136

Table A.1: Engineering Terms and Definitions .................................................... 145

Table A.2: Musical Terms and Definitions................................................................. 147

Table A.3: Descriptions of various Parts of the Violin............................................... 148

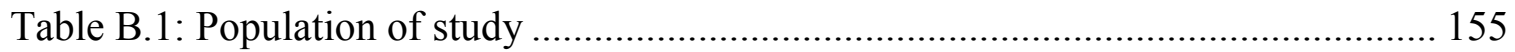

Table C.1: Measured mass of all wood samples........................................................... 160

Table C.2: DSP parameters for wood sample measurements ..................................... 161

Table C.3: Curve-fitting results from the Spruce 1 EMA............................................ 164

Table C.4: Curve-fitting results from the Maple 1 EMA.............................................. 164

Table C.5: Wood sample FE mesh compositions ...................................................... 165

Table C.6: Summary of material properties for wood sample FE models...................... 165

Table C.7: Summary of spruce 1 FE normal modes analysis ..................................... 166

Table C.8: Summary of maple 1 FE normal modes analysis....................................... 167

Table C.9: Mode pairs of the spruce 1 FE-Test correlation.......................................... 168

Table C.10: Mode pairs of the maple 1 FE-Test correlation ........................................ 169

Table C.11: DSP parameters for the plywood beams .............................................. 171

Table C.12: Curve-fitting results from the single beam EMA...................................... 174

Table C.13: Orthotropic materials for the plywood beam.......................................... 175

Table C.14: Summary of single plywood beam normal modes analysis ....................... 175

Table C.15: Mode pairs of the single beam FE-test correlation after updating .............. 177

Table C.16: Curve fit results for the laminated beam EMA ........................................ 178

Table C.17: Initial properties used for the glue layer ............................................... 179

Table C.18: Mode pairs of the laminated beam correlation......................................... 180

Table C.19: Final properties used for the glue layer................................................. 180

Table C.20: DSP parameters for rib structure measurements ................................... 181

Table C.21: Curve-fitting results from the rib structure EMA .................................... 183

Table C.22: Mesh composition of factory violin rib structure..................................... 183

Table C.23: Summary of factory violin rib structure normal modes analysis ............... 184

Table C.24: Mode pairs of the rib structure correlation............................................ 186

Michael Pyrkosz xviii Michigan Technological University 


\section{Preface}

The chapters of this dissertation have either already been published, or are intended for publication in the near future. Due to the collaborative nature of the work, clarification is provided as to the origin of the material for each chapter. A brief summary is given, as well as publication status, and author contributions.

Chapter 1 was written by MAP. It is an overview of the dissertation project as a whole, outlining the goals of the project and describing how each chapter relates to those objectives. The introduction chapter is not intended for separate publication; excerpts were adapted from the original dissertation proposal.

The material contained in Chapter 2 was previously published as "Converting CT Scans of a Stradivari Violin to a FEM" by Michael Pyrkosz, Charles Van Karsen, and George Bissinger in the Proceedings of the 28th International Modal Analysis Conference, Jacksonville, FL (February 2010). MAP was the primary author of this paper. The CT data used was part of GB's earlier work [1]. MAP imported the data into the CT scan interpretation software and developed the finite element mesh and density data. The five analytical case studies included in the project were also performed by MAP. CVK and GB advised the project and helped edit the paper.

The material contained in Chapter 3 was published as "Comparative Modal Tests of a Violin" by M. Pyrkosz and C. Van Karsen in Experimental Techniques (2012), Society for Experimental Mechanics. All data used in this project was collected by MAP with assistance from Kingsley Iduma and Dr. Alexis Black Pyrkosz. CVK was advisor for the project. The paper was primarily written by MAP with editing by CVK and ABP.

Chapter 4 is currently unpublished, but selected portions are planned for future submission. MAP arranged for the CT scan of the factory violin at Portage Health in Hancock, MI. The disassembly and reassembly of the factory violin and was done by MAP with instruction from William "Butch" Gariepy, a carpenter and luthier of Mohawk, MI. All experimental testing of the factory violin sub-components was performed by MAP with assistance from Alexis Black Pyrkosz. Experimental data for the Titian Stradivari violin was previously taken by George Bissinger, et al [2]. All FE models were developed by MAP with instruction from Numair Alani, an engineer at LMS. All correlation and updating was performed by MAP. The chapter was written by MAP with editing by CVK and ABP.

Chapter 5 has currently unpublished, but is planned for submission in the near future. The vibro-acoustic model was developed and run by MAP. Radiativity measurements of the Titian Stradivari violin were provided by GB [3]. Updating to the model was done by 
MAP based on input from GB. The chapter was written by MAP with editing by CVK and $\mathrm{ABP}$.

[1] Bissinger, George, “A Unified Materials-Normal Mode Approach to Violin Acoustics,” ACTA Acustica united with Acustica Vol. 91, pp. 214-228 (2005)

[2] Bissinger, George, Oliver, D., "3-D Laser Vibrometry Focuses on Legendary Old Italian Violins," Sound and Vibration magazine Vol. 41/Num. 7, pp. 10-15 (July 2007)

[3] Bissinger, George, "Structural acoustics model of the violin radiativity profile", Journal Acoustical Society of America, vol. 124, issue 6, pages 4013-4023 (2008) 


\section{Acknowledgements}

The author would like to thank the following people and organizations:

Dr. George Bissinger for initiating this project, and for his continual insight into the physics of violins; he has been instrumental in making this project possible.

Professor Chuck Van Karsen who was willing to take me on as a graduate assistant when I started, and has always succeeded in finding support for me.

The advisory committee for all their help along the way and for their guidance whenever I reached a road block.

The Violin Society of Acoustics and everyone at the Violin Acoustics workshops at Oberlin College; their cumulative knowledge of violins both from a technical and practical standpoint has been essential to my understanding of how violins are constructed and how they work. I only wish that I could have attended the workshops more frequently. A special thank you to Sam Zygmuntowicz who was vital in rounding up the old, Italian instruments for Bissinger's original research, and for convincing their owners to have their priceless violins irradiated in a CT scanner, then tested with something called an impact hammer and laser vibrometer.

All of my colleagues in the Dynamic Systems Laboratory at Michigan Technological University who have taught me a great deal and supported me along the way.

The LMS team in North American has been a great asset to me. The customer support group is one of the best that I have had the pleasure of working with. And my colleagues in the Engineering Services group have been truly supportive; I learn something new from them every day.

The representatives at Materialise Software who came out to Houghton, assisted me with learning MIMICS, and helped me develop a methodology for converting the CT scan data into a usable finite element mesh.

The support group at BETA CAE Systems S.A. helped me figure out how to refine the FE mesh of the Titian Stradivari and make it as outstanding as possible.

PolyTec, Inc. of course was part of the team that collected the original 3D laser vibrometry data of the old, Italian instruments. But I do need to make a special acknowledgement to Mike Stone who happened to be at the LMS open house five years ago, and was able to help me convert the Bissinger data into a format that could be read by LMS software. 
PCB Piezotronics, Inc. and The Modal Shop, Inc. for loaning various test equipment and support, including accelerometers, a micro-impact hammer, and upgrading the DSL's mini shakers to SmartShakers.

Keweenaw Research Center also loaned several of their miniature accelerometers as well as a micro-BNC repair kit.

Radiology Director John Jokela at Portage Health hospital who allowed the factory violins to be CT scanned, as well as technician Jason Griffin who took the scans.

William "Butch" Gariepy of Mohawk, MI, a carpenter and luthier who taught me how to disassemble, repair, and reassemble a violin. (And for sharing many great stories).

For my parents whose love and encouragement throughout my life has facilitated my becoming an engineer and finding success.

And for my wife, Alexis, whose patience, support, and love has helped me get through. She has been my inspiration, my copy editor, and one of the best lab assistants that I've worked with. 


\section{Abstract}

There is a tremendous amount of mystery that surrounds the instruments of Antonio Stradivari. There have been many studies done in the past, but no one completely understands exactly how he made his instruments, or why they are still considered the best in the world. This project is designed to develop an engineering model of one of Stradivari's violins that will accurately simulate the structural and acoustic behavior of the instrument. It also hopes to shine some light on what makes the instruments of Stradivari unique when compared to other violins. It will focus on geometry and material properties, utilizing several modern engineering tools, including CT scanning, experimental modal analysis, finite element analysis, correlation techniques, and acoustic synthesis. 


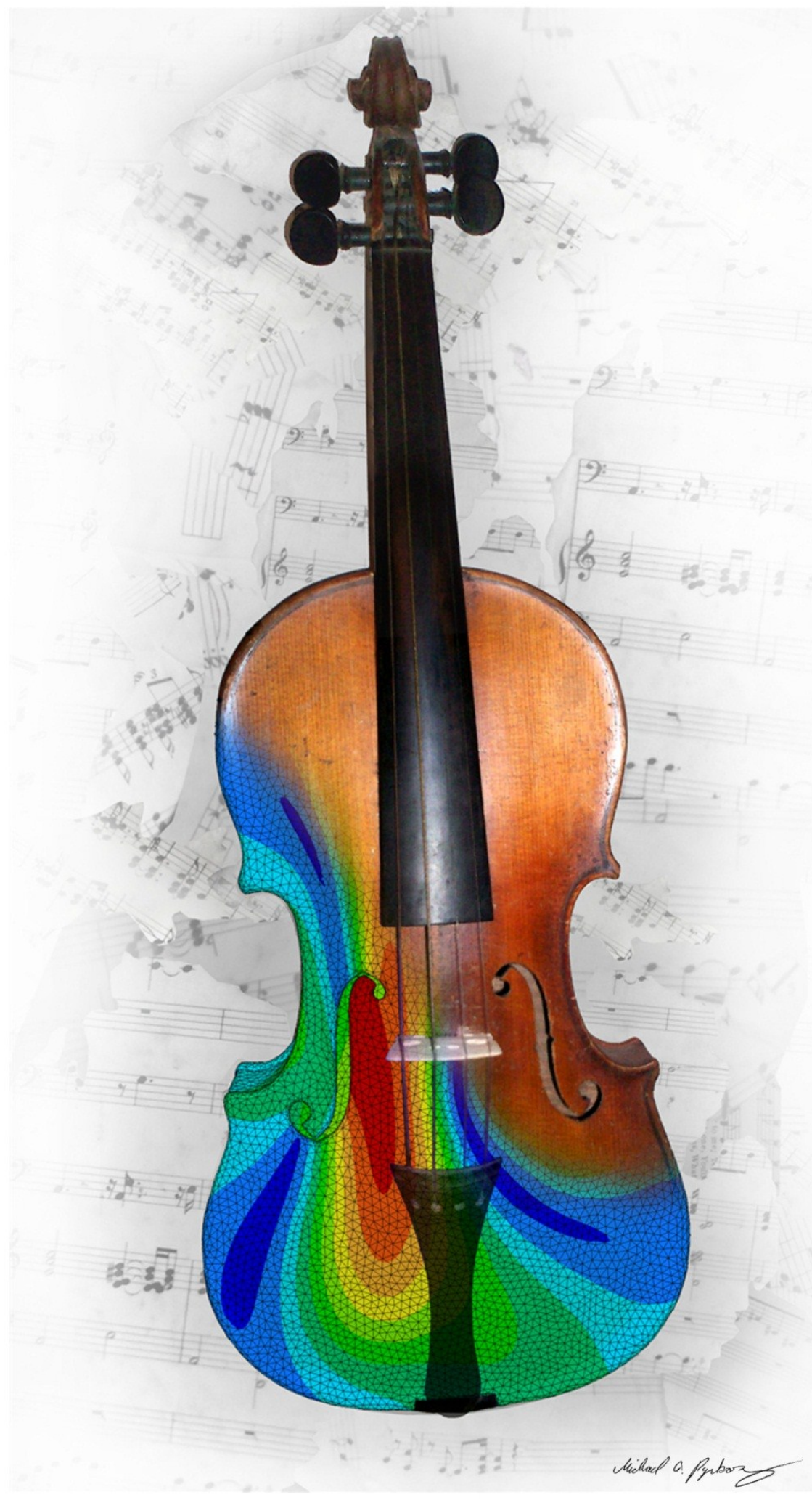




\section{Chapter 1 Introduction ${ }^{1}$}

Approximately 300 years ago in Northern Italy, luthiers, makers of fine violins, were crafting stringed instruments with unsurpassed sound quality. Antonio Stradivari (1644 1737) was one of these luthiers, and is considered by modern violin makers, violinists, and music critics to be the greatest violin maker of all time.

Unfortunately, the trade secrets of these master craftsmen have been lost to the ages. There was a period of about two centuries where the majority of violin makers simply made copies of other violins, based on patterns and moulds, without understanding the underlying principles that had been used to craft the originals.

Today, there are a number of modern violin makers who are striving to rediscover these principles. Some of the modern luthiers have come close, and are capable of producing some truly excellent quality instruments. However, they have all "bumped" their heads on what has been often called the "Stradivari ceiling", and no one has been able to reproduce an instrument that matches a Stradivari.

What is it that makes the instruments of Antonio Stradivari unique? Many people have attempted to answer this question. Yet each study has only yielded more questions rather than finding a conclusive answer.

This project aims to answer some of these questions by developing a computer model that accurately simulates the dynamic behavior of a Stradivari violin. This model was correlated to an actual instrument, namely the 1715 Titian Stradivari.

\subsection{Purpose and Background}

The purpose of this project was to develop a correlated model of the dynamic behavior of a Stradivari violin. This section discusses why this purpose is important. It will contain a brief history and background of what research has been done previously on the violin, what limitations have been apparent in past research, and how this project has overcome them.

\subsubsection{Problem Statement}

Today, the instruments made by Antonio Stradivari in the early $18^{\text {th }}$ century are considered to be the best in the world. Unfortunately, no one in our generation fully

\footnotetext{
${ }^{1}$ The material in this chapter is not intended for separate publication; some excerpts were adapted from the original dissertation proposal.
} 
understands how Stradivari crafted his instruments, and no one has ever produced an instrument that has quite the same musical quality of one of his instruments. There are many theories about how Stradivari made his instruments. The list ranges from the wood he used being unique to his century due to climate changes, to various chemical treatments to stave off wood worm, such as borax, or even apricot brandy.

Most of these theories point to the material properties of the wood as the vital piece to solving the Stradivari puzzle. After all, master luthiers have developed great skills at duplicating the geometric characteristics of any violin they wish to copy. Some have even produced a number of extraordinary pieces that come quite close to matching the quality of a Stradivari. However, someone with a trained ear, such as a professional violinist or another master luthier, can always distinguish between the old, Italians and the modern instruments. So, it stands to reason that the key piece is a difference in material properties.

But what material properties are different? Regardless of what causes Stradivari's wood to be different from modern violin makers' wood, the differences in material properties have to be characterized first. Properties that are critical to the structural vibrations of a material are density, elasticity, and internal damping. The most important acoustic properties are those of the acoustic impedance of the system. These include the properties of the air surrounding the instrument. The density, damping, and speed of sound of the air have direct influence on the acoustical inertance, resistance, and capacitance respectively.

This study aims to answer some of the questions surrounding Stradivari's instruments using the tools of modern engineering. By using experimental modal analysis, CT scans of the old, Italian instruments to build a finite element model, and correlation and updating techniques, realistic material properties can be determined. Once the model was correlated, a coupled vibro-acoustic model was constructed and the damping properties updated to build the most accurate model of violin dynamics. This model can now be used to predict what design modifications of a violin have on the acoustic response.

\subsubsection{Background and Context}

Many years of study have already gone into solving the mystery of Stradivari's instruments. Dr. George Bissinger, of East Carolina University, has done extensive research regarding the violin, especially those of Antonio Stradivari. This project is an extension of much of the work done by Bissinger to reverse engineer a violin, (labeled VIOlin Computer Aided Design Engineering Analysis System, or VIOCADEAS) [1.1], [1.2]. VIOCADEAS is an approach to studying the violin that involves combining 
simulation and experimental modal analysis to determine the material properties of a violin that govern its dynamic behavior [1.1], [1.2].

One of the important aspects of Bissinger's approach is developing Finite Element (FE) models with violin specific geometry and density information through the use of medical CT (Commuted Tomography) X-ray scans. This is a unique approach to developing FE models for violins, as all previous models used generic material properties and geometries [1.6], [1.7]; thus the product is limited to making generalized analyses of the basic violin design, and cannot be used to distinguish one violin from another. Bissinger had CT scans performed on two Stradivari instruments, (Titian 1715, and Willemotte 1734), as well as a Guarneri del Gesù (Plowden, 1735), [1.1]-[1.3]. This data was utilized to develop FE models for the first of these in the current study. By keeping the density and geometry parameters of the model constant, the task of determining elastic moduli for the different types of wood was much easier, through correlation and updating with experimental data [1.2].

The other major advantage to using geometry from CT scan data was that the modeled system could easily be disassembled into its major substructures, unlike the physical instrument, which comes with a price tag of over a million USD [1.2]. This made it possible to understand the behavior of the substructures and develop accurate models to predict their behavior. This was an important step before attempting to model a complete violin.

The experimental modal analysis of the violins being studied is another important aspect to Bissinger's approach. Having an accurate set of test data, under "proper" conditions, (i.e. "free-free" boundary conditions, strings in tension, non-contact measurement sensors, minimal room reflectivity, etc.), is absolutely imperative to correlate the FE model, and determine the actual material properties of the violins, [1.1], [1.2]. A large part of the data in VIOCADEAS is a database of normal modes of several violins ranging in quality from "bad", "good", and "excellent" [1.2], as well as several experimental violins known as the Hutchins-Schelleng violin octet, [1.11]. These modes were measured using a mini-impact hammer, and laser vibro-meter scanner [1.1]-[1.3]. Acoustic data is also included in the VIOCADEAS database; room-averaged acoustic measurements for the octet, and acoustic radiativity over a sphere around the other violins, [1.1], [1.2].

The most important instruments in the VIOCADEAS database are the old, Italian violins. For these, a full 3D laser vibrometry from PolyTec, Inc. was employed to record both the in-plane and out-of-plane motion of the violin, [1.3]. Acoustic radiativity measurements were also included. 


\subsubsection{Relevance of Study}

It may seem that a violin is an unlikely candidate for a mechanical engineering study. This is untrue, as many studies have already been done, [1.1]-[1.7]. And yet it still remains that no one seems to have a definitive answer to the Stradivari mystery; many of the previous studies were performed on the violin in general. It is reasonable to argue then, that this study is relevant in both the evolution of knowledge, and the contributions to practice.

\subsubsection{Evolution of Knowledge}

Previous studies have established methodology and base knowledge about the violin. There is a baseline for the dynamic behavior of a violin. There are a number of established, recognizable "signature" modes that all violins seem to possess, in the lower frequency range; although, there is still some debate as to the importance of each of these [1.1], [1.2]. There are even some measurable quantities that have been identified as statistical markers for distinguishing a "good" violin from a "bad" violin [1.1], [1.2], \& [1.4].

\subsubsection{Contributions to Practice}

Some standards of testing have also come about in the studies of the violin. This is of great value because it provides some direction for the methodology of this study. Simple test practices such as setting up "free-free" boundary conditions, excitation through the bridge, and non-contact measurement devices, have already been established [1.2], which enabled this study to focus on a Stradivari violin specifically.

This study offers a number of contributions to the study of violins. First, there is the CT scan to FE conversion process. Using CT scans to develop analytical engineering models is a relatively new technology. And although a number of FE Analyses have been done on the violin, to date no one has done an in-depth, high fidelity FE Analysis on a Stradivari using geometry and density data collected from a CT scanner. Second, there is the method of bringing simulation and experiment together in a correlation and updating process for determining material properties of a particular violin. This has become a fairly common practice in the engineering world, but so far, Bissinger is the only one to propose applying the technique to the violin, [1.1], [1.2].

Finally, a vibro-acoustic finite element model was developed for the 1715 Titian Stradivari violin. This model takes advantage of recent developments in acoustic analysis such as the Perfectly Matched Layer (PML), which reduces the size required for the model over the older Infinite Element Method (IFEM), and still meets the 
Sommerfeld radiation condition. Additionally, faster solvers are now available that take advantage of the symmetric sparse matrices of FEM models.

\subsubsection{Assumptions}

A number of important assumptions exist in this study. First, there is the assumption of linearity. The assumption that a structure behaves linearly is an approximation that is limited to the materials and level of vibration the structure undergoes. Second, there are a number of assumptions being made about material properties. Wood is not a simple engineering material. Density is not uniform; it was approximated with a distribution. The elastic properties of wood are generally approximated with an orthotropic model. One fault of this model is that it is defined in rectangular coordinates, even though the growth pattern in wood is predominantly cylindrical. There is an additional complication of the seeming randomness of anything produced by nature. Fortunately, the majority of the pieces of the violin are fairly thin, and approximating the orthotropic materials in rectangular coordinates was sufficient. Finally, boundary conditions always contain several assumptions that have to be considered when analyzing data. Everything is measured in "free-free" boundary conditions, because the interest is in the uniqueness of the violin, and not its support structure, (the violinist) [1.2]. But, it is technically impossible to achieve truly "free-free" boundary conditions in a laboratory environment on Earth. Fortunately, the lowest elastic modes tend to be no less than $200 \mathrm{~Hz}$, and pushing the rigid-body modes of the structure below this threshold is relatively simple; making the assumption valid.

\subsection{Outline of Accomplished Studies}

The following chapters will describe the details of this project. These chapters were either published or are planned to be submitted for publishing in future, and each represents a milestone in the project.

In Chapter 2 the process used for converting the CT scan data into a Finite Element (FE) model is discussed. This study was important to determine which details were needed in the model to accurately predict the dynamic behavior of a spruce violin top plate. An initial solid FE mesh was created from the CT scans of the Titian Stradivari violin. Five case studies were performed varying between isotropic and anisotropic elasticity, uniform and distributed density, and utilizing first order versus second order tetrahedral elements within the mesh. It was determined that, in the case of the Titian Stradivari top plate, a uniform average density was acceptable, but that the elasticity must include the orthotropic nature of the wood to accurately predict its behavior. It was also determined 
that for a solid model using tetrahedral elements, the elements must be a minimum of second order.

To develop the methodology for correlation and updating the violin model, initial experimental modal analyses were performed on a factory violin that could be disassembled. This is discussed in detail in Chapter 3. Comparative modal tests were performed on the same violin using two of the most common types of exciters: a stationary shaker and a roving impact hammer. The results showed that both methods have their benefits and limitations. Overall the shaker method resulted in less noise than the impact hammer. However, the shaker method also contained errors due to mass loading issues of the response sensors, as well as issues with the boundary conditions at the input location. Based on these results, for the purposes of this study the impact hammer is shown to be the preferred method of excitation for the modal analysis of a violin.

In Chapter 4 the use of modal correlation and updating techniques to estimate the material properties of a violin is discussed. Experimental modal data was collected on various sub-components of a factory violin as well as the 1715 Titian Stradivari. Structural finite element models were constructed based on CT scans of the actual instruments. Correlation between the experimental data and the initial FE models was used to compare the modal behavior of each system. The sensitivity of the correlation to the different properties was determined and the material properties were updated to bring the model dynamic behavior in greater agreement with the real test structure. This process is iterated until the model is a reasonable representation of reality. It was found that this method was viable for determining the orthotropic material properties of the various wood components of a violin. This was a critical step toward developing a reliable structural model of the 1715 Titian Stradivari violin.

The development of the vibro-acoustic finite element model of the 1715 Titian Stradivari violin is discussed in Chapter 5. This was the final step in the process of reverse engineering the Titian Stradivari violin. An acoustic mesh was created first by volumizing the existing structural mesh, wrapping this with a convex mesh to form the outer boundary, and finally filling the space in between with tetrahedral elements to represent the air. The correlated structural modes were then projected onto the acoustic envelope. The vibro-acoustic transfer function between force input at the violin bridge and acoustic pressure response $1.2 \mathrm{~m}$ away was predicted. These results were compared to experimental radiativity measurements made on the actual instrument. The damping properties of both the structure and the air were updated to bring the vibro-acoustic model into greater alignment with the experimental data. This represents the first 
comprehensive analytical vibro-acoustic model of a Stradivari instrument and can be used to determine how structural modifications effect violin sound quality.

\subsection{References}

[1.1] Bissinger, George, "Structural Acoustics of Good and Bad Violins" J. Acoust. Soc. Am. Vol.124 (3), (2008) pp.1764-1769

[1.2] Bissinger, George, "A Unified Materials-Normal Mode Approach To Violin Acoustics" Acustica Vol.91, (2005) pp.214-228

[1.3] Bissinger, George, "3-D Laser Vibrometry Focuses On Legendary Old Italian Violins" Sound and Vibration magazine Vol.41/ Num.7 (July 2007) $10-15$

[1.4] Bissinger, George, "Contemporary Generalized Normal Mode Violin Acoustics" Acustica Vol.90 (2004) pp.590-599

[1.5] Bissinger, George, "The violin Bridge as Filter" J. Acoust. Soc. Am. Vol.120/Num.1 (2006) pp.482-491

[1.6] Marshall, Kenneth D., "Modal Analysis of a Violin" J. Acoust. Soc. Am. Vol.77/Num.2 (February 1985) 695-709

[1.7] Knott, George A., "A Modal Analysis of the Violin Using MSC/NASTRAN and PATRAN" M.S. Thesis, Naval Postgraduate School. Available through NTIS website. (March 1987)

[1.8] Lord, Harold W., Gately, William S., Evensen, Harold A., "Noise Control for Engineers" Original Edition: Robert E. Krieger Pub. Co., Inc., Malabar, FL (1980). Reprint Edition: McGraw Hill Book Co., New York, NY (1987)

[1.9] Rao, Singiresu S., "Mechanical Vibrations" 3rd edition, Addison-Wesley Pub. Co., Inc., Reading, MA, (1995)

[1.10] Olson, Harry F., "Music, Physics and Engineering" 2nd edition, Dover Publications, Inc., New York, NY (1967). Originally published as "Musical Engineering” McGraw Hill Book Co., Inc., New York, NY (1952)

[1.11] Fletcher, Neville H., Rossing, Thomas D., "The Physics of Musical Instruments" 2nd edition, Springer Science+Business Media, LLC. New York, NY (1998)

[1.12] Thompson, William T., Dahleh, Marie D., "Theory of Vibration with Application" 5th edition, Prentice Hall, Inc. Upper Saddle River, NJ (1998)

[1.13] Heron-Allen, Edward, "Violin Making: A Historical and Practical Guide" Dover Publications, Inc., Mineola, NY (2005). Originally published as "Violin Making, as it was and is" 2nd edition, Ward, Lock, and Co., London, UK (1885) 
[1.14] U. S. Department of Agriculture, "The Encyclopedia of Wood", Skyhorse Pub., Inc., New York, NY (2007). Originally published by U.S.D.A. Washington, D.C. (1999) 


\title{
Chapter 2 Converting CT Scans of a Stradivari Violin to a Finite Element Model $^{2}$
}

\begin{abstract}
This study is part of an ongoing project to reverse engineer the structural and acoustic behavior of the Titian Stradivari violin. Several violins, ranging from some low quality factory fiddles, to those from Stradivari's "golden era" have been measured using a medical Computed Tomography (CT) scanner. This paper discusses the process used for converting the CT scan data into a Finite Element (FE) model. With comprehensive density data from the CT scans, accurate frequencies and mode shapes from a true threedimensional (3D) modal analysis and a complete acoustic scan over a sphere, the Titian FE model has the unique potential to allow extraction of the elastic moduli of this violin without the disassembly needed to test the various substructures. There will also be a discussion of determining material properties to be used in the model that will be correlated with experimental data and updated in Chapter 4.
\end{abstract}

\section{Nomenclature}

$L \quad$ Orthotropic axis directed longitudinally along the grain of the wood

$R \quad$ Orthotropic axis directed radially across the grain in the wood

$T \quad$ Orthotropic axis directed tangentially across the grain in the wood

$H U \quad$ Hounsfield Unit, on a scale from -1024 to 3072

$\rho_{i} \quad$ Density of element or material i

$A, B \quad$ Linear coefficients

$x_{i} \quad$ Average value of the voxels that are contained in element $\mathrm{i}$, in HU

$E_{i i} \quad$ Elastic modulus along axis i

$G_{i j} \quad$ Shear modulus in ij plane

$\varepsilon_{i i} \quad$ Strain along axis i

$\gamma_{i j} \quad$ Shear strain in ij plane

$\sigma_{i i} \quad$ Stress along axis i

$\tau_{i j} \quad$ Shear stress in ij plane

\footnotetext{
${ }^{2}$ Reprinted with permission from "Converting CT Scans of a Stradivari Violin to a FEM", M. Pyrkosz, C. Van Karsen, and G. Bissinger in the Proceedings of the 28th International Modal Analysis Conference, Jacksonville, FL (February 2010), copyright 2010 Society for Experimental Mechanics, Inc., copyright 2010 Michigan Technological University
}

Michael Pyrkosz 


\subsection{Introduction}

There are many theories about how Stradivari made his instruments, and why they are superior to other violins. Most of these theories point to the material properties of the wood. Properties that are critical to the structural vibrations of a material are density, elasticity, and internal damping. The most important acoustic properties of the material are the acoustic impedance or admittance, including the radiation efficiency.

High fidelity solid finite element models of several different violins, including a Stradivari violin, were constructed using geometry information gathered from a medical CT (Computed Tomography) scan. CT scan data provides accurate geometry and density information. This information is then interpreted to build the finite element (FE) model. This paper focuses on the process of interpreting the CT scan data to build a usable FE model of a violin.

Dr. George Bissinger, of East Carolina University, has done extensive research on violins, especially those of Antonio Stradivari. This project is an extension of his work to reverse engineer a violin (labeled VIOlin Computer Aided Design Engineering Analysis System, or VIOCADEAS) [2.1], [2.2]. VIOCADEAS is an approach to studying the violin that involves combining simulation and experimental modal analysis to determine the material properties that govern its dynamic behavior [2.1], [2.2].

One of the important aspects of Bissinger's approach is developing Finite Element (FE) models with violin-specific geometry and density information through the use of medical CT X-ray scans. Bissinger had CT scans performed on two Stradivari instruments, (Titian 1715, and Willemotte 1734), as well as a Guarneri del Gesù, a contemporary of Stradivari, (Plowden, 1735), [2.1]-[2.3]. This data was utilized to develop FE models for these particular violins. Keeping the density and geometry parameters of the model constant simplifies the task of determining elastic moduli for the different types of wood, through correlation and updating with experimental data [2.2].

The experimental modal analysis of the violins being studied is another important aspect to Bissinger's approach. A significant portion of the data in VIOCADEAS is a database of normal modes of several violins ranging in quality from "bad", "good", and "excellent" [2.2], as well as several experimental violins known as the HutchinsSchelleng violin octet, [2.4].

The total population of the current study includes a large number of violins for comparative analyses. In addition to the old, Italian instruments, CT scans have been performed on three factory violins, as well as several intermediate quality violins. The overall goal is to eventually have correlated models for each violin to compare. 
However, since this paper is primarily concerned with the process used for converting the CT data to a FE model, the discussion will mostly be limited to one violin in particular, the Titian Stradivari.

The Titian is unique in that not only is it a considered to be one of Stradivari's finest violins, it is the only one of his instruments to have measurements of 3D mobility, radiativity around a sphere, and a high quality $\mathrm{CT}$ scan [2.1]. This means that updating the solid model can be extended beyond just surface normal motion. Also, although there have been a few vacuum FE models of violins constructed over the years, none have included accurate anisotropic density data, vibroacoustic analysis, or correlation with experimental data.

\subsection{Methods}

\subsubsection{Computed Tomography (CT) Scan}

One of the difficulties in modeling a violin is that it is not an easily definable engineering structure. Its shape is a complex arrangement of curves that are hand-carved from various species of woods, whose material properties are distinctly heterogeneous. For these reasons the $\mathrm{CT}$ scanner presents itself as a useful tool for reverse-engineering a violin. CT is a medical imaging method similar to an X-ray, where individual crosssection slices of density information of a patient are taken. By taking a multitude of these single slice images along the length of the body, three-dimensional density information is collected and digitized. This data can then be used to reconstruct various aspects of the patient (organs, skeletal structure, etc.) to be used in diagnosis and treatment planning.

A violin is not an easily definable engineering structure. Its shape is a complex arrangement of curves that are carved from various pieces of wood, whose material properties are anything but homogeneous. For these reasons the CT scanner presents itself as a useful tool for reverse-engineering a violin. First, accurate geometry information can be determined through edge detection. Secondly, accurate density data is available at any location within the structure.

A CT scanner digitizes density information on a scale of 4096 values (i.e. 12 bits), which are commonly referred to as Hounsfield Units (HU). The scale used by the CT scanner for the violin data ranges from -1024 to 3071, where -1024 represents the density of air and 0 represents that of water. The digitized data is sampled in three dimensions. The resolution in the XY directions is defined by the pixels per unit length of the individual slices. The resolution in the $\mathrm{Z}$ direction is defined by the number of slices per unit length. The old, Italian instruments were scanned with an XY resolution of 1.462 pixels $/ \mathrm{mm}$ ( or $0.684 \mathrm{~mm} / \mathrm{pixel}$ ). The $\mathrm{Z}$ resolution of all the violin scans was $1 \mathrm{slice} / \mathrm{mm}$. 
Where the smallest unit of 2D image data (one slice) is commonly referred to as a pixel, the smallest unit of 3D volume data is generally referred to as a voxel (Volumetric pixel). The voxel size of the violin data therefore ranges from $0.343 \mathrm{~mm}^{3}$ to $0.604 \mathrm{~mm}^{3}$.

It has been shown that the typical behavior of a CT scanner is that the calibration curve consists of two linear relationships, one for values less dense than water, and another for values more dense than water [2.5]. Fortunately, most wood is less dense than water and therefore only one relationship will be required for the violin data. Figure 2.1 shows a picture of two violins being CT scanned. Note that the violins are supported by foam, which can be easily separated out later due to its lack of density.

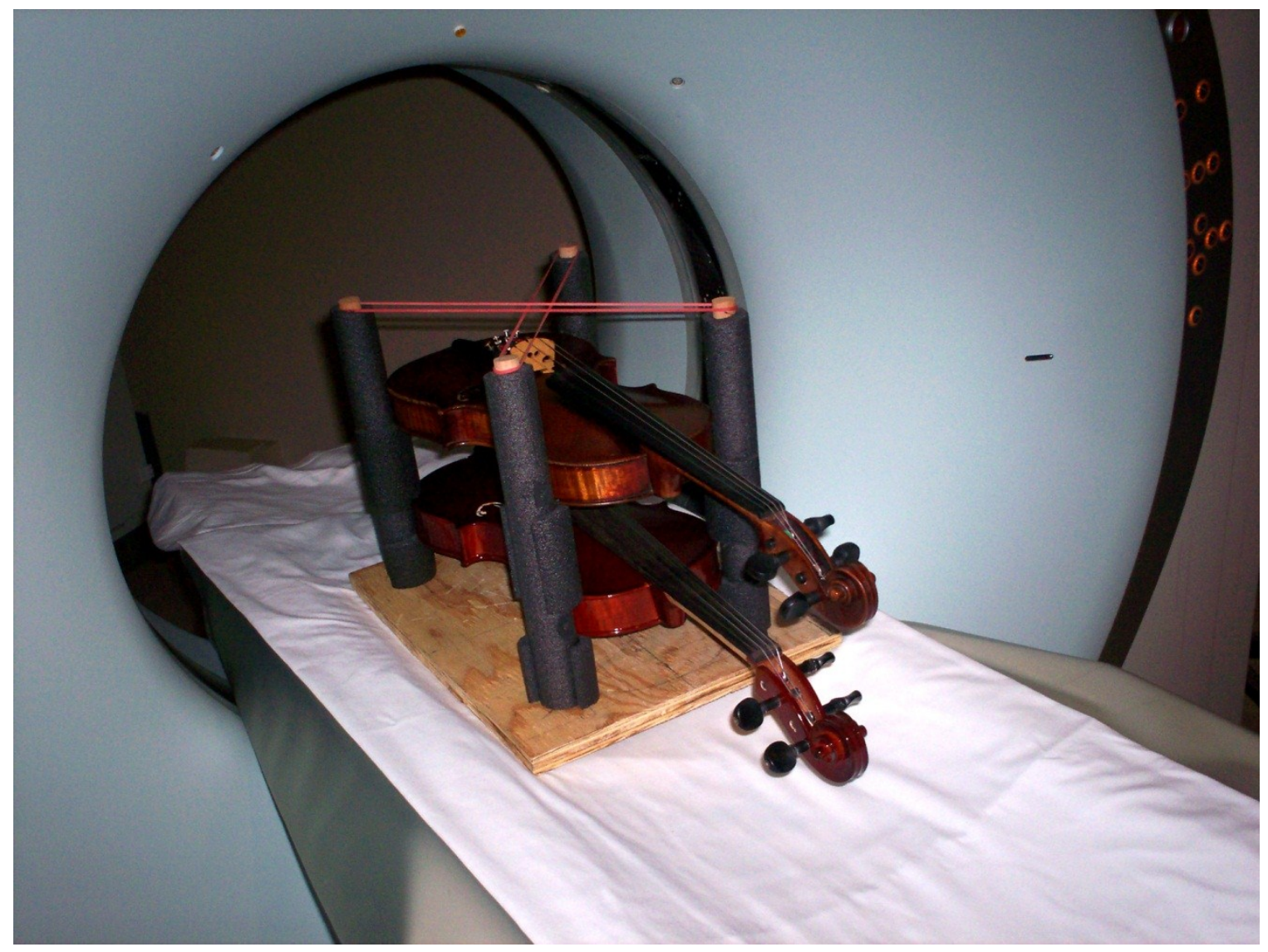

Figure 2.1: Violins being CT scanned

\subsubsection{Interpretation of the CT Scan Data}

The first step in processing the CT scan data is to import it into a CT interpretation program. This type of program allows the user to segment the images and render them as three-dimensional objects. The program used here was Mimics by Materialise. 
Segmentation is a process of separating specific structures of interest based on density information. This is accomplished by creating a mask using a threshold of HU. For processing the violin data, thresholding is primarily used to separate the violins from the foam supports. An upper threshold can also be used for removing various metal components. It may be tempting to use thresholding to separate out different species of wood, since spruce is a softwood, and maple is a hardwood, and ebony is considerably more dense than both. However, there is enough overlap in density between summer and winter growth that this proves impractical.

Once the thresholding step is complete, the violins can be separated into different masks. Since the threshold effectively removed the foam supports from the mask each violin was floating freely, and separating them was trivial.

Unfortunately, the scans contain artifacts that interfere with this process. Line-artifacts can be caused by abrupt changes in density from one material to another that exceeds the dynamic range of the processing electronics. The result is "scatter" noise, which appears as bright and dark lines that radiate away the offending area in the scan. Metallic objects are particularly troublesome since iron and steel can completely extinguish or reflect the $\mathrm{X}$-ray. Mask editing tools within the $\mathrm{CT}$ interpretation software can be used to remove the effects of scatter on the calculated mask. Figure 2.2 demonstrates the process of correcting the mask to remove scatter caused by a fine tuner. It should be noted that this method only effectively changes the shape of the calculated object; the HU values within the mask, and therefore the calculated density, will still be affected by the noise. One possible correction may be to edit the images directly, but the values that are affected will have to be interpolated based on the nearby slices. 


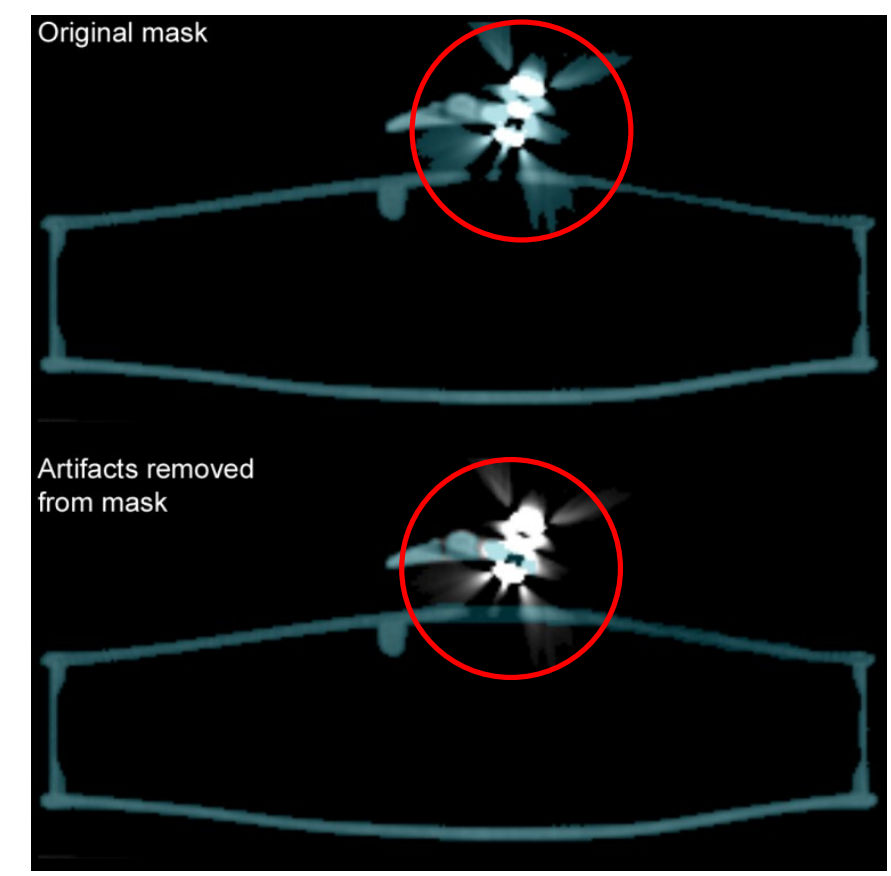

Figure 2.2: Example of mask editing to remove artifacts

It is also necessary to separate out the different parts of each violin. The reason for this is to simplify material property assignment of each wood part based on its species and grain orientation. Again, the mask editing tools in the CT interpretation software can be used for separating out the different parts.

Once the masks have been cleaned of artifacts and the parts separated, each mask can be used to create $3 \mathrm{D}$ objects. In calculating the $3 \mathrm{D}$ object, the surfaces of the object are triangulated based on the edges of the mask. This effectively creates a basic triangular surface mesh of the object. Different interpolation methods are available to account for voxels that are only partially filled by the volume of the object.

By separating all the parts into separate masks, the best set of parameters can be applied when calculating each 3D object. For example, the thicknesses of the top and bottom plates of the violin are critical since they will act as the primary resonators of the instrument. These were compared to measurements of the Titian top and back plates to ensure accuracy. However, obtaining accurate rib thickness is not as straight forward. Due to the ribs being so thin (approximately $1.0 \mathrm{~mm}$ ), the majority of the voxels that contain information on the ribs also contain a fair amount of air. This leads to the average density within a voxel to be estimated considerably lower, and using the same threshold on the ribs as the back plate, (since they are both maple) creates holes in the ribs. 
Therefore the rib dimensions are estimated from contours around the rib voxels rather than on the specific density data.

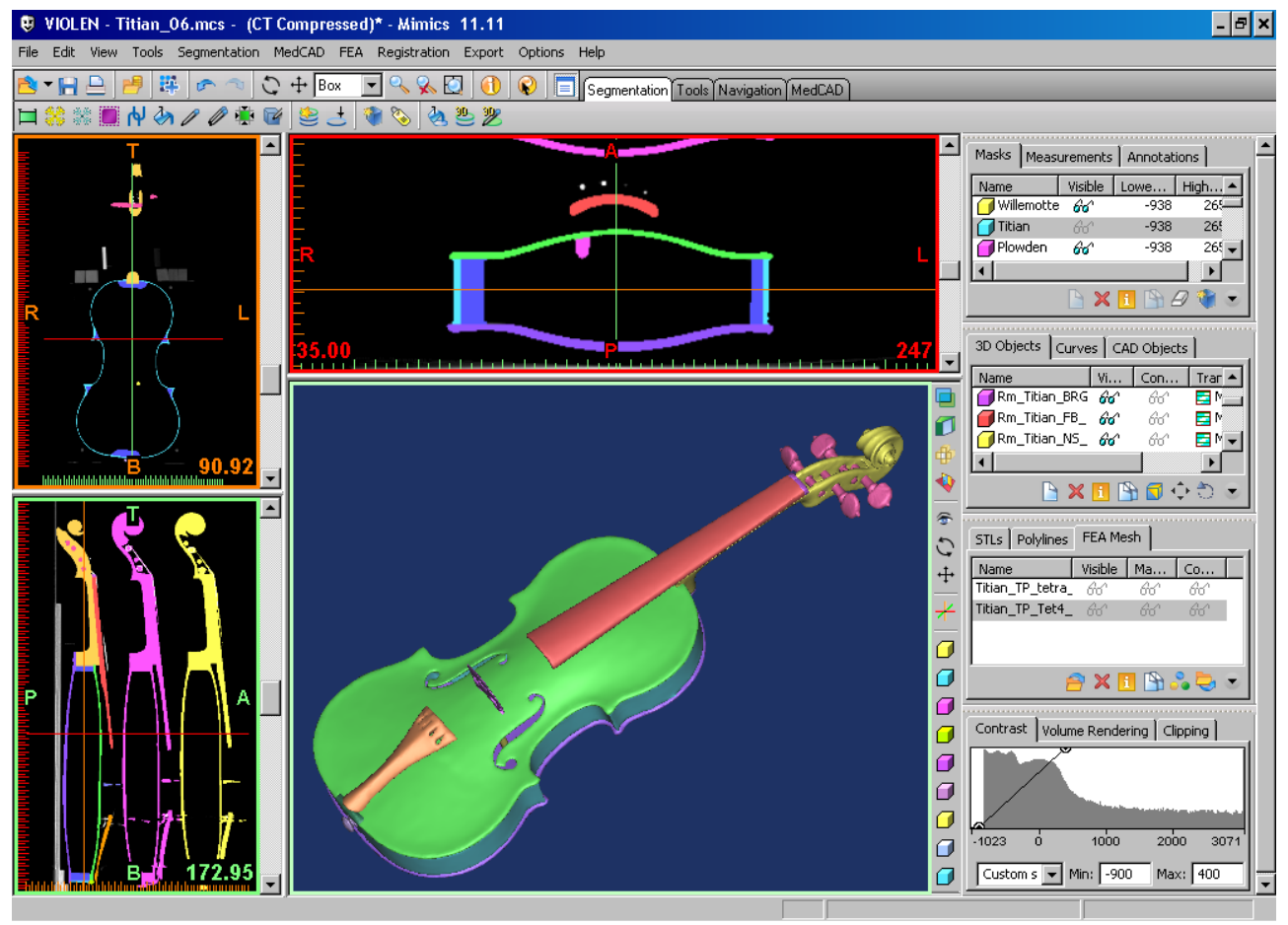

Figure 2.3: Screen capture from Mimics software depicting separation of parts and 3D object calculation

\subsubsection{Meshing}

At this point the $3 \mathrm{D}$ objects exist as triangular surface mesh over the boundary between the air and solid material. The next step is to remesh this surface to improve the mesh quality. This optimizes the mesh for FEA. This includes reducing the amount of detail, reducing the number of triangles, and improving the quality of the triangles. For structural vibration simulation fine detail in the mesh is not generally necessary and can lead to slow computation time and numerical instability. Reducing the amount of detail increases efficiency and stability without sacrificing significant accuracy. Reducing the number of triangles also improves computation time, but can lead to lower quality triangles. Therefore it is necessary to remesh using a quality threshold based on various shape parameters. Finally, it is necessary to ensure that none of the triangles intersect.

Once the 3D object has been remeshed, it is exported to the FEA preprocessor, which is used to generate a volumetric mesh within the surface mesh. The preprocessor used on the violins is LMS Virtual.Lab since it is has tools for performing both structural and 
acoustic studies. The volume enclosed by the surface mesh is filled in with tetrahedrons. It should be apparent that the quality of the created solid mesh is largely dependent on that of the surface mesh. One important consideration here is the type of tetrahedron elements to use. Linear elements contain only four nodes that are connected by straight edges. These are commonly called TETRA4 elements. Parabolic elements contain ten nodes, four on the vertices of the tetrahedron and six intermediate nodes on the edges. These intermediate nodes define curved edges between the vertices, and increase the overall number of degrees of freedom without having to decrease the elements size. These are called TETRA10 elements. TETRA4 elements are easier to implement, but TETRA10 elements are preferred as they improve accuracy. Figure 2.4 depicts the result of computing a solid tetrahedron mesh for the top plate of the Titian Stradivari.

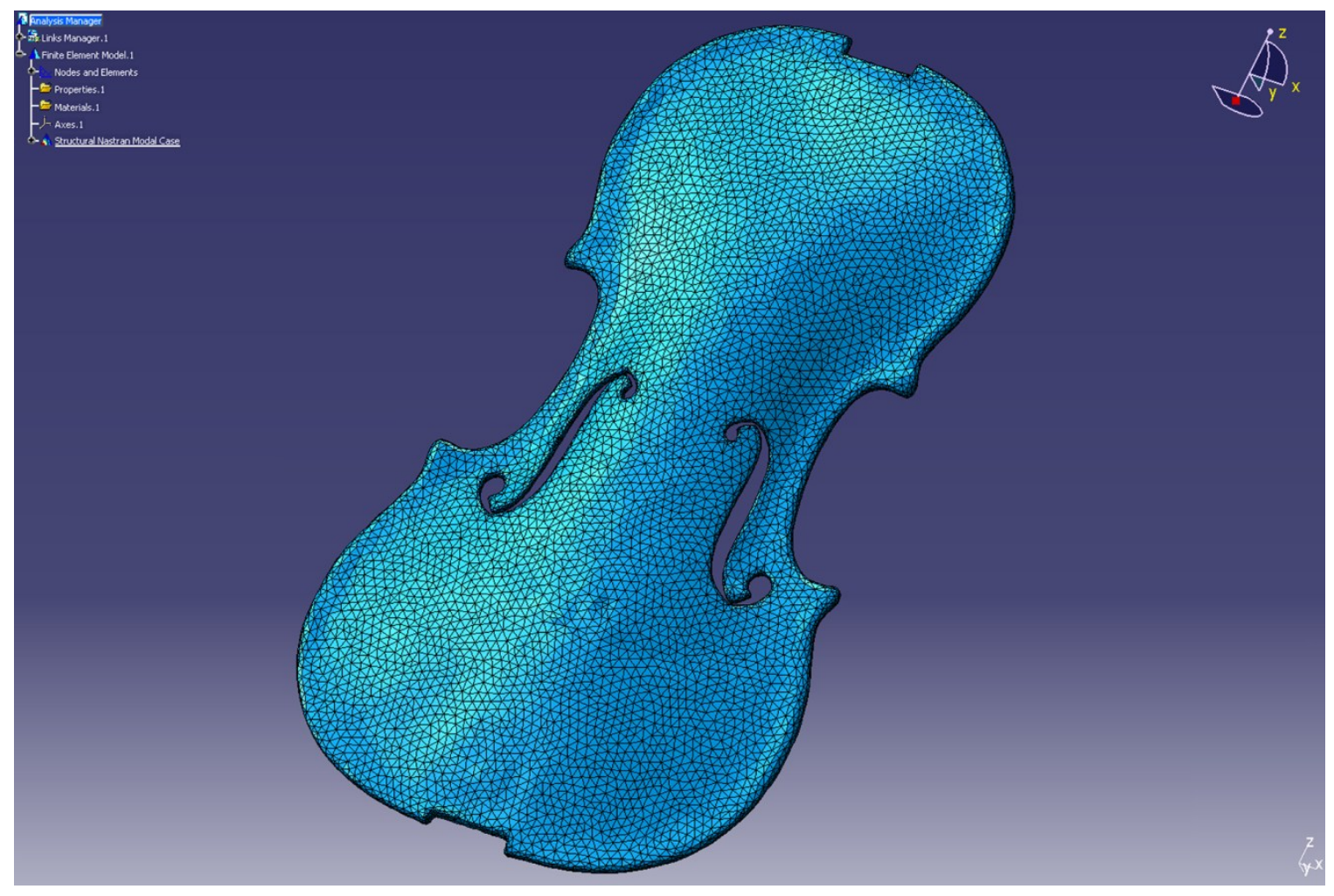

Figure 2.4: Screen capture of tetrahedron mesh in Virtual.Lab

\subsubsection{Material Property Definition}

Before finite element analysis can be performed several material properties need to be defined. Among these are density, elasticity, and structural damping. Most of these properties will eventually need to be updated during the correlation process so that the final model will reflect the structural characteristics of each particular violin. It is also 
noted here that the different material properties can be defined for each of the individual parts to account for each species of wood and grain orientation.

The CT scan data provides highly detailed density information. This data can be extracted and used in the model. After the volumetric mesh has been created in the FEA preprocessor, it is imported back into the CT interpretation software for extracting the density information and assigning it to the material properties. The range of gray values or Hounsfield Units in the CT scan data is divided into groups that are each assigned a specific density value. To do this, the relationship between HU and density must be defined within the software. This relationship has been shown to be linear [2.5]; from $1024 \mathrm{HU}=\rho_{\text {air }}$ to $0 \mathrm{HU}=\rho_{\text {water }}$. (see Equation 1).

$$
\rho_{i}=A \cdot x_{i}+B
$$

Where $\rho_{i}$ is the density of the element in $\frac{g m}{(\mathrm{~mm})^{3}}, x_{i}$ is the average value of the voxels, in $H U$, that define the element, $A \approx 975.4 \times 10^{-9} \frac{\mathrm{gm}}{(\mathrm{mm})^{3} \cdot \mathrm{HU}}$, and $B \approx 1.00 \times 10^{-3} \frac{\mathrm{gm}}{(\mathrm{mm})^{3}}$.

This is a fairly straightforward calculation and is easily compared to the specific weight of some known wood species (specific weight is density normalized with respect to water). Note that the range of gray values (i.e. Hounsfield units) contained within the mask is divided into a smaller number of approximate values. This decreases the total number of material groups generated, simplifying data management.

Mechanically, wood is typically treated as an orthotropic material; that is, mechanical properties are unique along orthogonal axes [2.6]. These axes consist of the longitudinal (L) axis along the grain, the radial (R) axis across the grain normal to the growth rings, and the tangential (T) axis across the grain but tangent to the growth rings. Due to the structure of the wood, the properties such as elastic and shear moduli, and Poisson's ratios are different on each of these axes. Most studies measuring the properties of wood are done by bending beams; the results are only given in one direction, usually the longitudinal. There is data on a few species of wood for properties along the other axes, but this is limited. This issue is complicated further by elasticity of the wood being affected by moisture content.

Other properties of interest are the speed of sound traveling within the wood and the internal damping. This is primarily a function of the elasticity and density, and as a result also dependent on the grain direction and moisture content. In general, since the cross- 
gain elastic moduli are significantly smaller than the longitudinal elastic modulus, the speed of sound across the grain is usually smaller than it is along the grain. Also, the speed of sound decreases proportionally with an increase in temperature or in moisture content, depending on species [2.6]. The internal friction or damping of wood depends greatly on temperature and moisture content [2.7]. Internal damping is also particularly troublesome because there are no exact numbers available for most species of wood. Bissinger has measured the total damping of the Titian Stardivari violins as a sum of radiation damping and internal damping [2.1]. By computing radiation efficiency and radiation damping a semi-empirical internal damping value can be assigned to each violin. However, this means that the internal damping property may not be fully updated until an acoustic simulation is performed to ensure that the total damping is realistic.

A few studies have been done to show a linear regression between the longitudinal modulus of elasticity and density for certain samples of softwoods, (primarily Norway spruce) [2.7]. These are simply average properties of the samples used, however, it may be possible to determine a linear relationship for the density and elasticity changes within a sample. During material assignment within the CT interpretation software such a relationship between density and elasticity may be included, such that the mesh groups created for different density ranges would also have a proportional elastic modulus assigned. However, this elasticity assignment does not account for the orthotropic nature of the wood; that is, elasticity is different for each density group created, but equal along each orthogonal axis.

To account for the orthotropic properties, elasticity and Poisson's ratio must be adjusted for the materials used in the model. It can be shown that an orthotropic material is a specific case of an anisotropic material. This is shown Equation 2, which gives the compliance tensor of an orthotropic material. 


$$
\left\{\begin{array}{l}
\varepsilon_{L L} \\
\varepsilon_{R R} \\
\varepsilon_{T T} \\
\gamma_{L R} \\
\gamma_{R T} \\
\gamma_{T L}
\end{array}\right\}=\left[\begin{array}{ccccc}
\frac{1}{E_{L L}} & \frac{-v_{R L}}{E_{R R}} & \frac{-v_{T L}}{E_{T T}} & & \\
\frac{-v_{L R}}{E_{L L}} & \frac{1}{E_{R R}} & \frac{-v_{T R}}{E_{T T}} & & \text { zeros } \\
\frac{-v_{L T}}{E_{L L}} & \frac{-v_{R T}}{E_{R R}} & \frac{1}{E_{T T}} & & \\
& & & \frac{1}{G_{L R}} & \\
& & & & \frac{1}{G_{R T}} \\
\\
z e r o s & & & & \frac{1}{G_{T R}}
\end{array}\right]\left\{\begin{array}{c}
\sigma_{L L} \\
\sigma_{R R} \\
\sigma_{T T} \\
\tau_{L R} \\
\tau_{R T} \\
\tau_{T L}
\end{array}\right\}
$$

The compliance matrix can be inverted to determine the stiffness matrix to be used in the material definition. Matlab is used to solve transverse terms based on average ratios from similar species listed in "The Encyclopedia of Wood" [2.6]. The result is then exported into a format compatible with a Nastran MAT9 material definition for anisotropic materials.

\subsection{Results}

One question that arises is how complex does the model need to be in order to accurately simulate each violin in question. It is a fairly straightforward task to create an anisotropic material in the model, and using CT interpretation software makes it possible to account for the non-uniform density distribution in the wood. However, including both of these details in the model is more complicated since each density group needs to be assigned a different anisotropic elasticity matrix, each containing 9 independent values. This long list of properties increases the complexity of the model making bookkeeping cumbersome. Also, for correlation and updating to be effective, influences of all properties need to be observable in the modal data [2.8].

To gain deeper understanding of the sensitivity of the model to different modeling complexities, an initial study was done using the same mesh of the top plate from the Titian Stradivari changing the features one at a time. Five case studies were performed varying between isotropic and anisotropic elasticity, uniform and distributed density, and utilizing TETRA4 and TETRA10 elements within the mesh. 
The results of the first five modes and mode shapes for each of these cases are shown in Table 2.1 and Figure 2.5. Blue indicates the nodal lines, and red indicates areas of maximum deflection. The modes vary tremendously, but this is not a surprise since the different elastic moduli and densities vary considerably. What is interesting is the effect on the different mode shapes. The first mode shape is mostly insensitive to these modeling changes. The model also performs similarly with uniform density or distributed based on the CT scan data. The reason for this is unknown. It is possible that the density groups created are too low-resolution to see a noticeable difference, which is plausible since the groups were created by dividing the total range of $\mathrm{HU}$ values contained within the mask, which includes points affected by the scatter from fine tuner.

Table 2.1: Top plate study results

\begin{tabular}{|c|c|c|c|c|c|}
\hline & Case 1 & Case 2 & Case 3 & Case 4 & Case 5 \\
\hline Element Type & TETRA4 & TETRA4 & TETRA4 & TETRA10 & TETRA10 \\
\hline Density & Uniform & Distributed & Uniform & Uniform & Uniform \\
\hline Elasticity & Isotropic & Isotropic & Anisotropic & Isotropic & Anisotropic \\
\hline Mode 1 & 332.3 & 273.3 & 219.2 & 178.7 & 131.1 \\
\hline Mode 2 & 494.9 & 394.5 & 330.6 & 321.5 & 196.1 \\
\hline Mode 3 & 821.4 & 669.1 & 535.4 & 487.2 & 328.9 \\
\hline Mode 4 & 922.1 & 753.4 & 556.7 & 522.7 & 365.8 \\
\hline Mode 3 & 1131.0 & 901.0 & 622.5 & 654.5 & 408.9 \\
\hline
\end{tabular}

However, the change from isotropic to anisotropic elasticity has a dramatic effect. With the softer cross-grain moduli the structure bends more easily about the longitudinal axis than it does against the longitudinal axis, which can be seen by comparing mode shapes. The effect is more dramatic with TETRA4 elements than it is with TETRA10 elements. In fact, the TETRA4 elements with anisotropic elastic moduli model (Case 3) is the only case where the mode shapes change order. The fifth mode shape actually drops below both the $3^{\text {rd }}$ and $4^{\text {th }}$ mode shapes. 


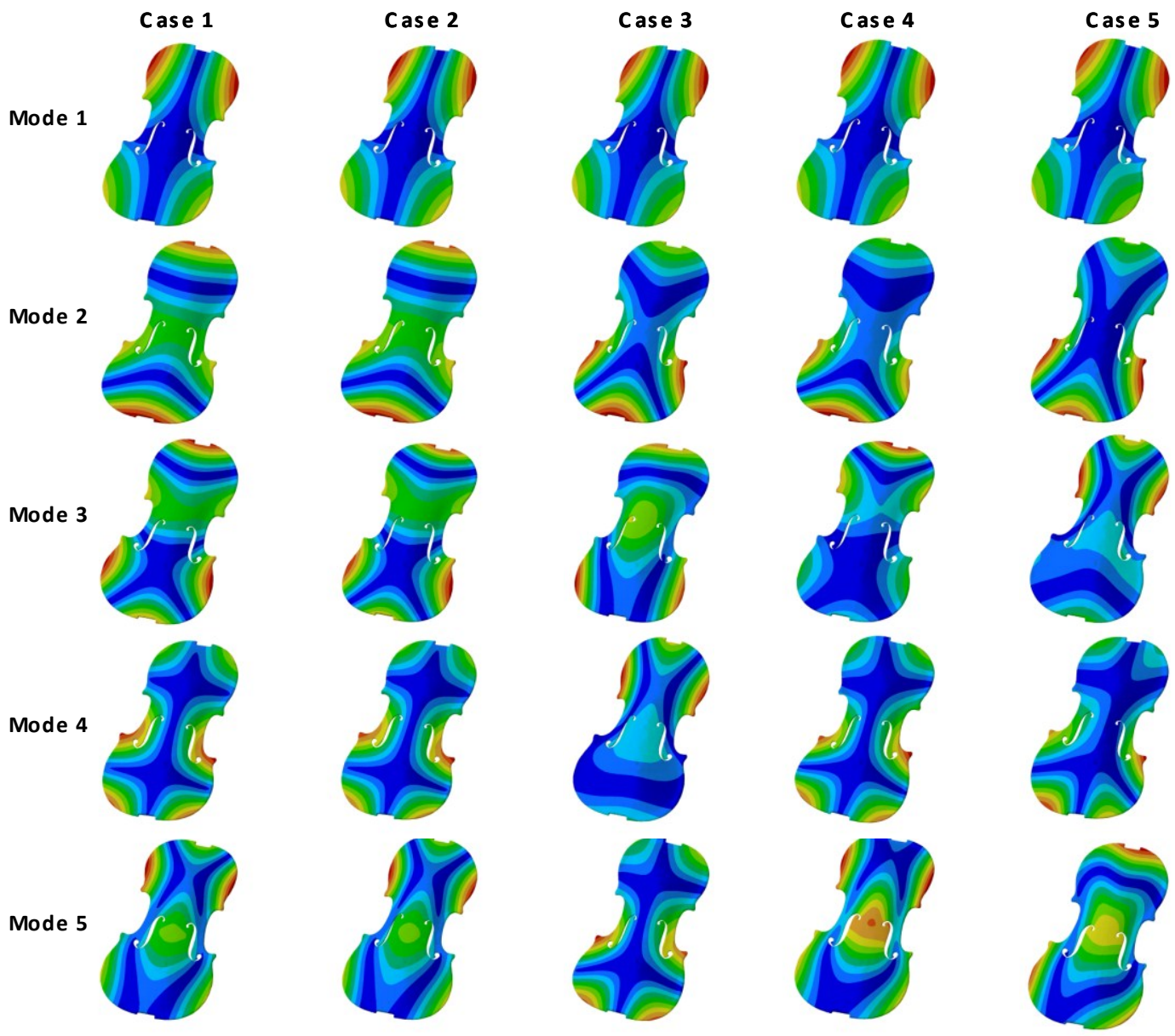

Figure 2.5: Mode shape results from top plate study

The next question becomes which one of these models more closely represents the actual top plate of the Titian Stradivari. This question is not so easy to answer, given the pricetag associated with the instrument. Based on experiments that have been done on violin top plates case 5 does appear to yield the most reasonable results both in mode frequency and shape. To determine how to best model the wood, and to identify the correct material properties, a simpler structure must be modeled that can be readily tested, and a correlation analysis performed.

\subsection{Conclusion}

This project aims to determine the structural and acoustic properties of priceless, old, Italian violins, and compare them to that of cheap factory fiddles, as well as some middle class instruments. This is done by developing high-fidelity FE models specific to each violin based on a CT scan of the instrument. It has been shown that this is possible with 
the aid of modern software like Mimics and Virtual.Lab. What remains is correlating the models to experimental data and updating the material properties. Several iterations of this correlation and updating process may have to be performed to get values representative of the actual instruments. Furthermore, to start with values that are close to the real values some testing, modeling, and correlation work will be done on some samples of the wood species of interest. This will simplify the process and reduce the number of iterations needed to match the model with the data.

\subsection{References}

[2.1] Bissinger, George, "Structural acoustics of good and bad violins," J. Acoust. Soc. Am. Vol. 124 (3), pp. 1764-1773 (September 2008)

[2.2] Bissinger, George, "A Unified Materials-Normal Mode Approach To Violin Acoustics," ACTA Acustica united with Acustica Vol. 91, pp. 214$228(2005)$

[2.3] Bissinger, G., Oliver, D., "3-D Laser Vibrometry Focuses On Legendary Old Italian Violins," Souns and Vibration magazine Vol. 41/Num. 7, pp. 10-15 (July 2007)

[2.4] Fletcher, Neville H., Rossing, Thomas D., "The Physics of Musical Instruments" $2^{\text {nd }}$ edition, Springer Science+Business Media, LLC, New York, NY (1998)

[2.5] Saw, C. B., Loper, A., Komanduri, K., Combine, T., Huq, S., Scicutella, C., "Determination of CT-to-Density Conversion Relationship for ImageBased Treatment Planning Systems" Medical Dosimetry, Vol. 30, No. 3, pp. 145-148 (2005)

[2.6] U. S. Department of Agriculture, "The Encyclopedia of Wood," Skyhorse Pub., Inc., New York, NY (2007). Originally published by U.S.D.A. Washington, D.C. (1999)

[2.7] Bucur, Voichita, “Acoustics of Wood," CRC Press, Inc., Boca Raton, FL (1995)

[2.8] Carne, T. G., and Stasiunas, E. C., "Identification of a Full Orthotropic Constitutive Model Using Measured Modal Data", Proceedings of the $11^{\text {th }}$ International Congress and Exposition, Society for Experimental Mechanics, Inc., (June 2008) 


\title{
Chapter 3 Comparative Modal Tests of a Violin ${ }^{3}$
}

\begin{abstract}
To determine the properties that make the violins of Antonio Stradivari among the best in the world, a project is underway to reverse engineer one of the great master's instruments. To develop the methodology, a factory violin that can be disassembled is used as the initial test case. The focus of this paper is to determine the best modal test methods to use on the violin. Comparative modal tests were performed on the same violin using two of the most common types of exciters: a shaker and an impact hammer. The results show that both methods have their benefits and limitations. Overall the shaker method resulted in less noise than the impact hammer. However, the shaker method also contained error due to mass loading issues of the response sensors, as well as issues with the boundary conditions at the input location. Based on these results, for the purposes of this study the impact hammer is shown to be the preferred method of excitation for the modal analysis of a violin.
\end{abstract}

\subsection{Introduction}

Violins crafted by eighteenth century Italian violin makers are still considered the finest in the world today. The greatest of these are the instruments of Antonio Stradivari (1644 - 1737). Unfortunately the trade secrets of these masters have been lost to the ages.

Even the best violinmakers of today, though they have produced some excellent quality instruments, have not been able to match those of Stradivari and his contemporaries. The testing discussed in this article is part of a larger project to reverse engineer a Stradivari violin. The purpose of this project is to develop an analytical model of the dynamic behavior of a Stradivari violin and to understand which measurable properties are unique, if any, to Stradivari's instruments. The key to this is to successfully identify the material properties of the wood used to construct these instruments [3.1]. The assumption being made here is that even if a modern violin were to precisely match the geometric shape and setup of one of the great masters', it would still not produce the same sound, indicating that the variables of interest must lie in the material properties of the wood.

To gain insight into the material properties of a violin, it is useful to disassemble one and modally test the individual pieces. Disassembling a Stradivari is out of the question; they

\footnotetext{
${ }^{3}$ Reprinted with permission from "Comparative Modal Tests of a Violin”, M. Pyrkosz and C. Van Karsen, Experimental Techniques 37 (2013) 47-62 (C) 2012, Society for Experimental Mechanics, (C) 2012, Michigan Technological University
} 
usually come with a price tag of over a million USD [3.3]. For this reason a factory violin costing less than 100 USD was obtained to be used as a test case. This can be tested both intact and disassembled to identify a starting point for material properties to use in the analytical model. The objective of this study is to compare different test methods and determine which methods best provide insight into the structure and material properties of the violin.

Material properties in the analytical model are adjusted to match experimental results using correlation and updating. The primary method for correlation is the Modal Assurance Criterion (MAC), which compares the mode shape vectors of the models with those of the experimental modal analysis. In addition to mode shape vectors, the frequencies of the modes predicted by the model will be compared to those of the experiment. The experimental modal analysis requires frequency response functions (FRF). These are measured by giving the test structure a known excitation force and measuring the response over the frequency range of interest. The ratio of the response to the force input and the phase delay between them at each frequency is the FRF. Natural frequencies, or modes, occur at local peaks in the magnitude of the FRF, or when the phase passes through $90^{\circ}$. Each mode will have a unique mode shape representing structural motion at the mode frequency. Violins also have a number of acoustic modes due to the volume cavity inside the body of the violin and the f-holes. Because the cavity walls are thin these acoustic modes feedback on the structure and cause it to move as well. Both the motion of the structure and air through the f-holes are important to the sound of the instrument. Once the structural model is correlated, an acoustic model is to follow, and a similar approach can be used to correlate the acoustic modes. Suitable excitation is needed across the entire frequency range of interest to ensure that all modes within the frequency range are measured.

Two types of exciters will be discussed in this article: an electrodynamic shaker producing either a burst random input force or a stepped-sine input force, and an impact hammer. The factory violin was tested with both methods. The results are compared and evaluated for their merits in terms of model correlation and material identification. Since the correlation is based on modal frequencies and mode shapes of the test results with those of the analytical model, it is imperative to obtain clear, accurate results in the experiment with the structure in "free-free" boundary conditions.

\subsection{Background}

This project is an extension of the work done by Bissinger to reverse engineer a violin (labeled VIOlin Computer Aided Design Engineering Analysis System, or VIOCADEAS)[3.2],[3.3]. VIOCADEAS is an approach to studying the violin that 
involves combining simulation and experimental modal analysis to determine the material properties that govern its dynamic behavior [3.2],[3.3].

One of the important aspects of Bissinger's approach is developing Finite Element (FE) models with violin specific geometry and density information through the use of medical Computed Tomography (CT) X-ray scans. This is a unique approach to developing FE models for violins, as all previous models that have been made used generic material properties and violin geometries [3.5],[3.6]. Thus the product was limited to making generalized analyses of the basic violin design, and could not be used to distinguish one violin from another. Bissinger had CT scans performed on two Stradivari instruments ("Titian" 1715, and "Willemotte" 1734), as well as a violin by Guarneri del Gesù ("Plowden", 1735) [3.2]-[3.4]. In the scope of the larger project this data will be utilized to develop FE models for these specific violins. By keeping the density and geometry parameters of the model constant, the task of determining elastic moduli for the different types of wood becomes much easier through correlation and updating with experimental data [3.3].

Another important aspect to Bissinger's approach is the experimental modal analysis of the old, Italian violins mentioned above. Part of the data in VIOCADEAS is a database of normal modes of the old, Italian instruments. These modes were measured using a mini-impact hammer excitation; and full 3D laser vibrometry was employed to record both the in-plane and out-of-plane motion of each violin [3.4]. Unfortunately, a laser vibrometer was not available for use on the factory violin, hence accelerometers were used for measuring the response. These are contact transducers that will introduce mass loading issues, which will be discussed in more detail in the following sections.

A detailed diagram of the parts of the violin is shown in Figure 3.1. The violin is essentially a wooden box formed by two plates (top and back) sandwiching a rib structure comprised of six blocks (two end blocks and four corners), the ribs themselves, and twelve lining strips. The violin has four strings; starting from the lowest string and going up by major fifths, they are tuned to G $(196.00 \mathrm{~Hz}), \mathrm{D}(293.66 \mathrm{~Hz}), \mathrm{A}(440.00 \mathrm{~Hz})$, and E $(659.26 \mathrm{~Hz})$, respectively. These are tuned primarily by pegs supported by a peg box between the neck and scroll. Often fine tuners are added to the tail piece for more precise adjustment (usually on the E string). Different notes are achieved by pressing the strings to different lengths between the player's fingers and the fingerboard. 


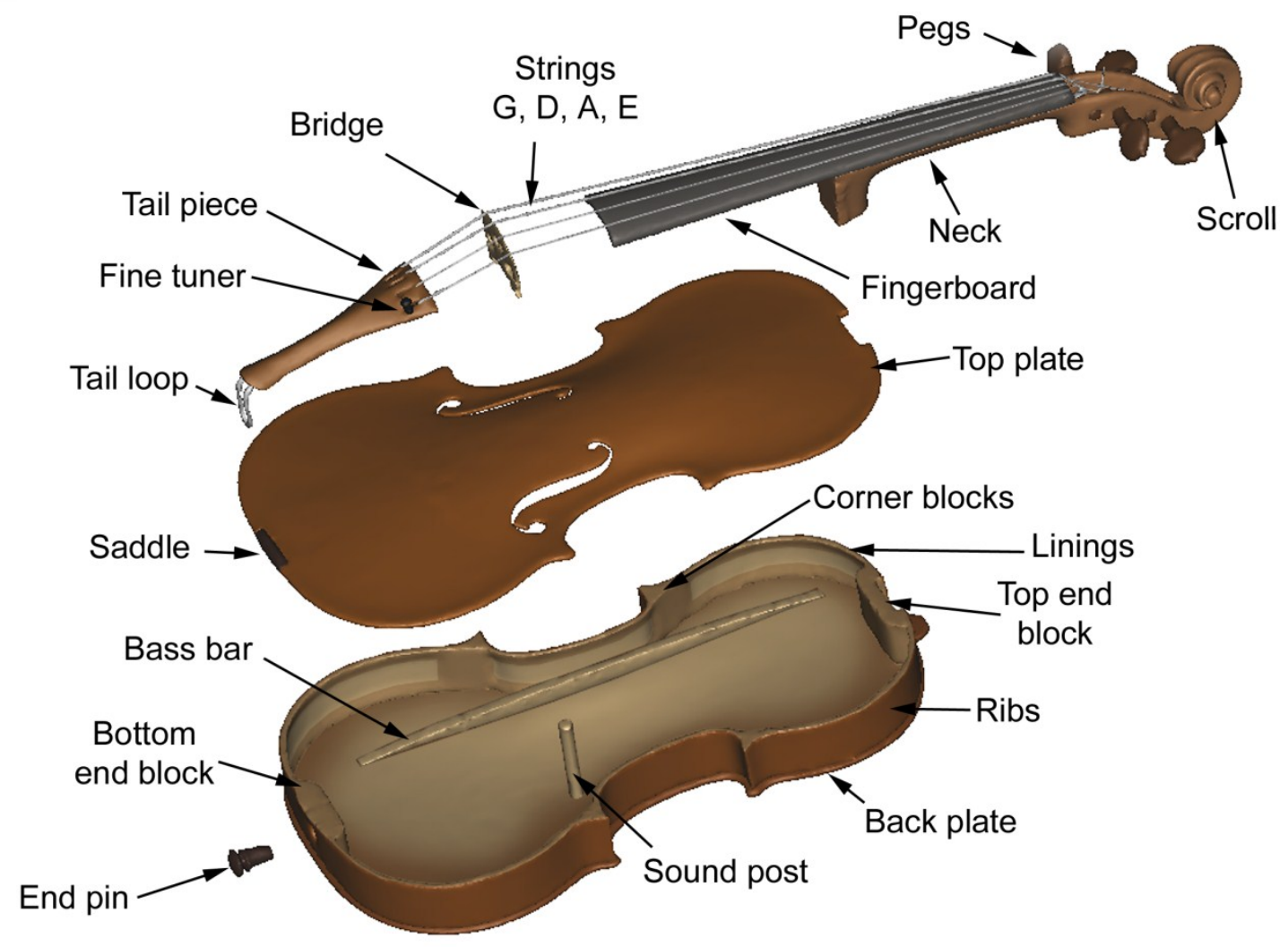

Figure 3.1: Parts of a violin

Under normal operating conditions energy is imparted to the strings by a bow. This energy is then transmitted through the bridge to the top plate. This step has been long considered one of the most critical in the performance quality of the violin [3.7], [3.8]. Heron-Allen also notes that the bridge's "important functions, its proportions, and position on the belly must be very nicely adjusted to the quality of the violin to which it is affixed... Its height must also be most carefully adjusted to the quality of the instrument..." [3.9]. The bass bar (located under the bass side bridge foot) acts as a structural member to transmit energy throughout the entire length of the top plate. The top plate is coupled to the back plate through the rib structure as well as the sound post (located under the treble side bridge foot).

The plates are generally regarded as the most important part of the structure when it comes to violin sound. The Bissinger data contains measurements of 3-dimensional mobility of the top and back plates for the Titian Stradivari and the Plowden Guarneri del Gesù, and top plate measurements for the Willemotte Stradivari [3.4]. This data was the 
first time 3D measurements were made on a Stradivari and revealed in-plane motion of the plates. The relative in-plane motion between the top and back plates reveals that flexture of the rib structure is involved in all of the corpus modes, and thus the ribs play an important role in how the plates are coupled.

As mentioned previously, a 3D laser vibrometry system was unavailable for the factory violin and accelerometers were used for measuring the responses. To capture the full motion of the violin, response locations for the modal test included measurements primarily on the plates and ribs, with some additional measurements on the fingerboard and other smaller substructures. This will be discussed in greater detail in the Results section.

\subsection{Methods}

The purpose of this article is to determine the best method for testing violins to find modes and mode shapes that will be correlated with the analytical model. These are found by measuring frequency response functions and developing a system response matrix, $[\mathrm{H}(\mathrm{j} \omega)]$, which is defined by the following equation.

$$
\{X(j \omega)\}=[H(j \omega)]\{F(j \omega)\}
$$

Where $\{X(j \omega)\}$ is the response vector and $\{F(j \omega)\}$ is the force input vector.

Each element of the system response matrix represents the FRF of one response with respect to a given input force. These FRFs are measured by applying a known force excitation into the system and measuring the response at several locations. Alternatively, assuming that the system response matrix is symmetric, a known force can be applied at each of the measurement locations while measuring the response at one or more reference locations. This is referred to as reciprocity and relies on the assumption that the system is linear.

Two types of exciters were explored for the factory violin: an electrodynamic shaker exciter (Method A), and an impact hammer exciter (Method B). The exciter in Method A remains stationary while a number of response transducers (accelerometers, in this case) are roved over all measurement locations. The advantage to this method is that it takes less time to collect data. Since multiple points are measured in each acquisition session, it requires fewer sessions total. The disadvantage is that the accelerometers add mass to the system. This mass loading condition changes between acquisition sessions, and may cause the system poles to shift, leading to an inconsistent data set.

In Method B, one or more response transducers are left stationary, while an impact hammer is roved over all measurement locations. This measures the same system 
response matrix as Method A, assuming that the system is linear and that reciprocity holds. The advantage is that the mass loading of any response transducers is consistent for all measurements. The disadvantage is that each point must be measured in a separate acquisition session, which may take more time.

To determine if these methods would have similar results, a set of driving point and bridge feet accelerance frequency response functions were measured using both exciters. The driving point was located on the bass side of the bridge, as shown in Figure 3.2. The accelerometers for this test were PCB model 352A73 (nominal sensitivity: $5 \mathrm{mV} / \mathrm{g}$, mass: $0.3 \mathrm{gm}$ ) and were located as shown in Figure 3.2. This strategy is derived from the primary energy path of the violin when it is being played. That is, normally energy from the strings is transmitted through the bridge into the top plate and then to the rest of the instrument. It is noted that at $\sim 0.3$ gm each the accelerometers may not seem like a significant mass compared to a violin weighing $\sim 0.4 \mathrm{~kg}(<0.1 \%)$, however the bridge (a critical substructure is a mere $2 \mathrm{gm}$ and the driving point accelerometer presents a considerable mass loading problem locally $(\sim 15 \%)$. An attempt is made therefore to remove the mass effects of all accelerometers using modal modification prediction [10], as will be discussed later. In both methods the violin is suspended in "free-free" boundary conditions using rubber bands around the lower corners of the violin and the peg box. Having "free-free" boundary conditions is preferred for many reasons, primarily because of the difficulty in measuring and modeling difficulties that would be presented by a violin support such as a player [3.11].

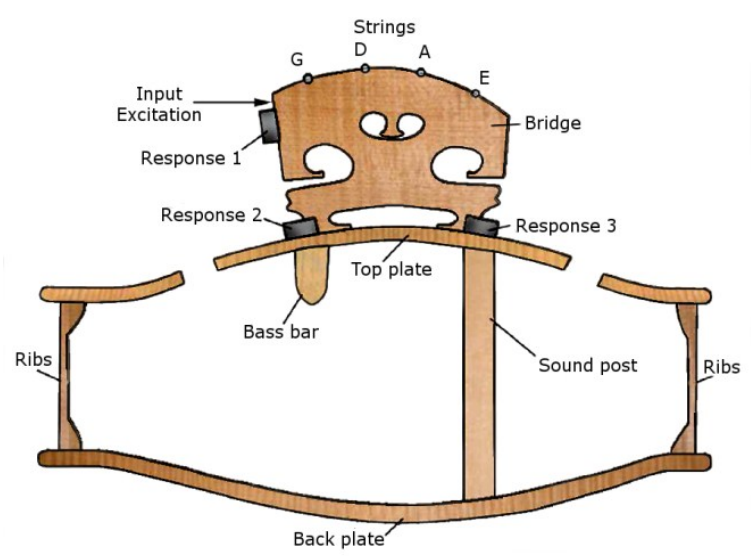

Figure 3.2: Diagram showing exciter input and accelerometer response locations for comparative test. 


\subsubsection{Method A: Shaker excitation}

For the electrodynamic shaker exciter two different signals were used: a burst random signal and a stepped-sine signal. The burst random signal was set to have a burst length $75 \%$ of the $1.0 \mathrm{~s}$ time block, so that a fully observed transient would be measured in each of the 10 linear averages. This type of signal has the advantage of being quick $(10 \mathrm{~s}$ per acquisition session). The disadvantage to this type of signal is that the actual force input across the frequency range depends on the impedance characteristics of the shakeramplifier system.

In the stepped-sine test each spectral line of the frequency response function is measured in series. The amplitude of the electrical signal to the shaker-amplifier is adjusted automatically to ensure that the same force level is used across the frequency range of interest. The main advantage to using a stepped-sine force is that at each frequency the level of the shaker is automatically adjusted to guarantee the same input level, compensating for the impedance mismatch. The one disadvantage to this method is that it takes a considerable amount of time (approximately 2 hours for 2000 spectral lines and 5 averages).

The shaker used for this test was a TMS mini "SmartShaker" with an integrated power amplifier model K2007E01. This shaker was selected for its small size and was easy to suspend at the same level as the bridge. The stinger was a nylon threaded rod with an aluminum tube stiffener. The force input to the violin was measured using a PCB dynamic force sensor model 209C11 (nominal sensitivity: $494.604 \mathrm{mV} / \mathrm{N}$ ). Finally, to attach the force sensor to the violin bridge, an adapter was made using a wooden dowel of diameter $1 / 8$ inch $(3.2 \mathrm{~mm})$ and length $1 / 2$ inch $(12.7 \mathrm{~mm})$, which was glued to the bridge of the violin. The presence of the wooden dowel was accounted for by recalibrating the force sensor with the dowel attached to a known mass. The setup for Method A is shown in Figure 3.3.

It should be noted that the presence of the stinger is difficult to back out of the modal data in this case due to the sensitive nature of the bridge [3.12]. The dowel added an additional $0.5 \mathrm{gm}$ to the already mass loaded bridge. This was included in the modal modification prediction used to adjust the shaker data. 


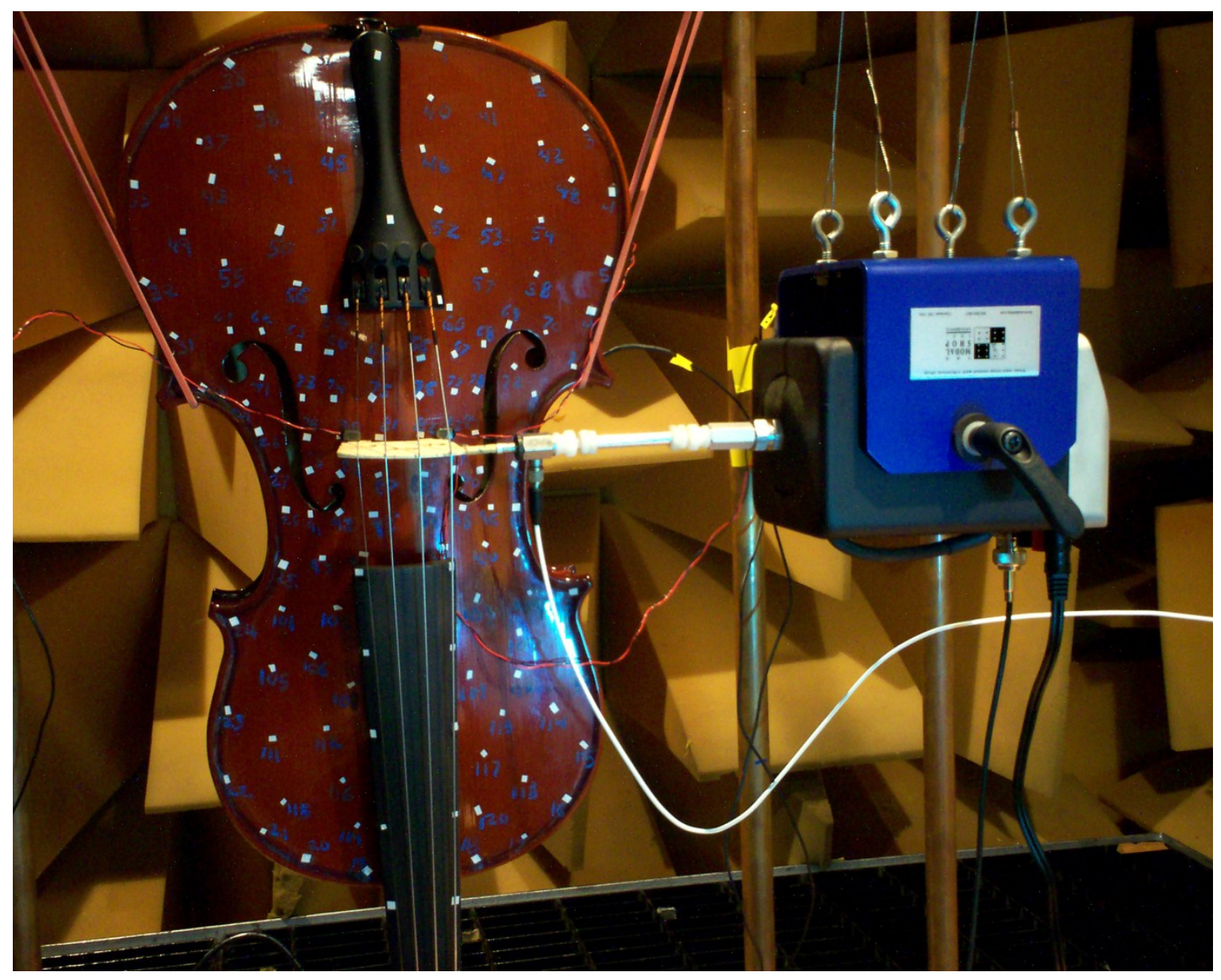

Figure 3.3: Picture of shaker setup

\subsubsection{Method B: Impact Excitation}

The impact exciter used was a PCB model 086E80 (nominal sensitivity: $22.5 \mathrm{mV} / \mathrm{N}$ ). This hammer has an integral conical impact tip made of steel. This is effective for exciting high frequencies (above $2000 \mathrm{~Hz}$ ), but detrimental to the finish of the violin. The hammer has an optional vinyl impact cap to "soften" the impact, which successfully protected the violin finish, but softened the impact to the point that the hammer did not suitably excite the higher end of the frequency range of interest $(196-3520 \mathrm{~Hz}$; the typical playing range for the violin). For this reason a custom tip was made for the hammer using acetal plastic. The setup for Method B is shown in Figure 3.4. A detailed view of the impact hammer is shown in Figure 3.5 with the actual tip used. The speed of this test is greatly dependent on the skill of the hammer operator to strike the bridge consistently for all averages (average time for each acquisition session was $\sim 66 \mathrm{~s}$ for 5 averages per session). The other major advantage to the impact excitation method is that 
vibration energy is imparted to the violin bridge without any additional mass loading effects from a stinger [3.12].

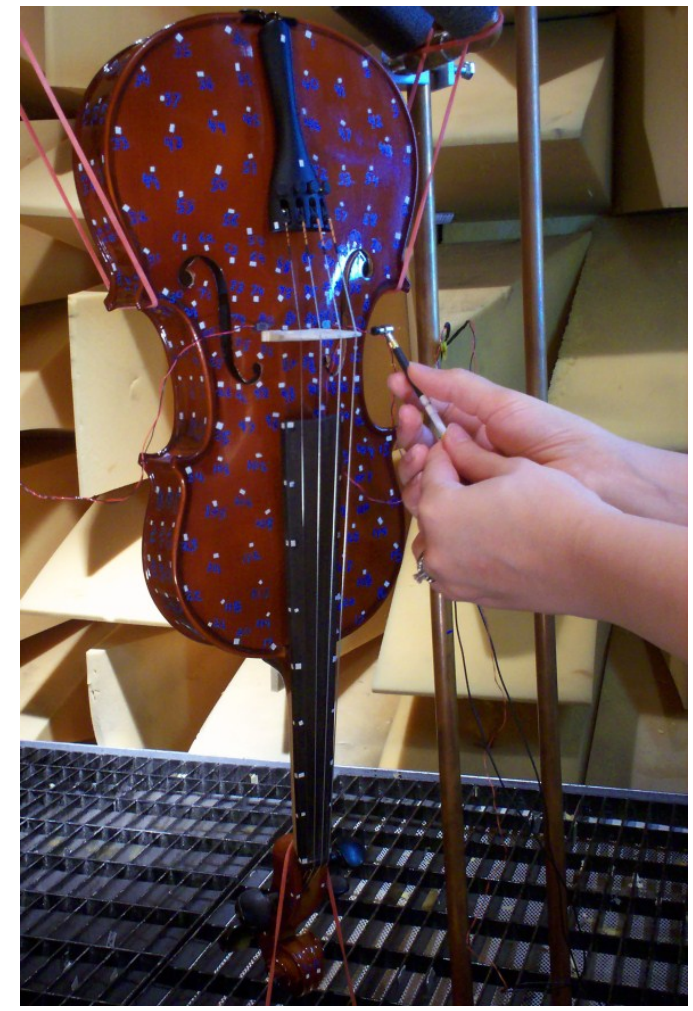

Figure 3.4: Picture of impact hammer setup

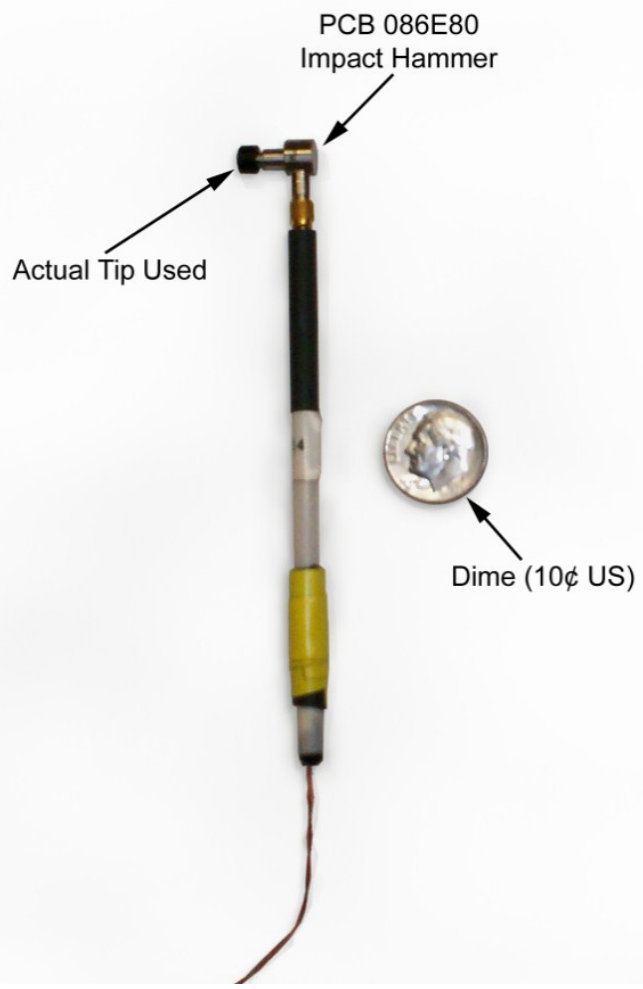

Figure 3.5: PCB 086E80 impact hammer with actual tip used (US dime shown for scale).

\subsection{Results}

Once data was collected using both methods, it was analyzed and compared to determine which method would yield results best suited for updating material properties in the analytical model. This section will discuss the different means of comparison and how they relate to the objective.

\subsubsection{Input Excitation Levels}

The input autopower spectra for the different methods are shown in Figure 3.6. These are real valued functions equivalent to the magnitude of the linear spectrum squared, and are used to discern the actual level of force each excitation input imparted to the system across the frequency range. There are significant differences between the amounts of energy that are being input to the system for each method. The stepped-sine excitation is a full three orders of magnitude greater than the impact excitation. The burst random excitation begins two orders of magnitude greater than the impact excitation at the start of 
the frequency range, and ends nearly a full order of magnitude below it. This difference is due to the impedance mismatch between the shaker and the structure. The fact that these excitations span nearly four orders of magnitude may push the limits of the assumption that the structure is linear.

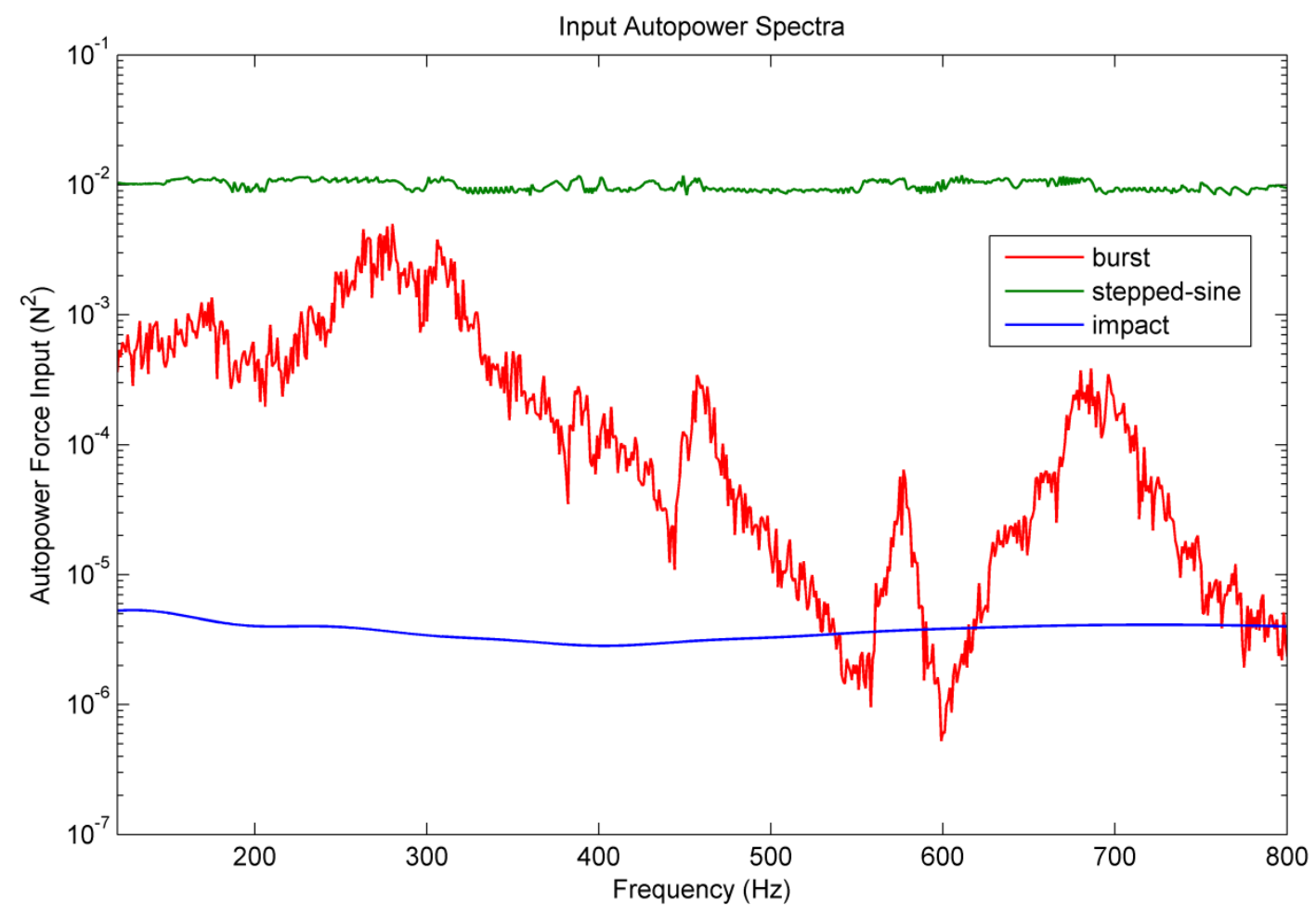

Figure 3.6: Input Autopower spectra for each type of excitation. Burst random excitation (red), stepped-sine excitation (green), impact excitation (blue).

\subsubsection{Frequency Response Functions and Coherence}

The driving point frequency response functions (FRF) and coherence functions are shown in Figure 3.7 and Figure 3.8 respectively. Figure 3.9 through Figure 3.12 show the frequency response and coherence functions of the bridge feet acceleration. The FRFs of Method A (burst random and stepped-sine excitation) are in good agreement. The only significant difference between these two is near the G string fundamental $(196 \mathrm{~Hz})$ where the stepped-sine result is consistently $5 \mathrm{~dB}$ higher than the burst random result for all three locations. There are additional discrepancies around $362 \mathrm{~Hz}$ and $420 \mathrm{~Hz}$ (both less than $3 \mathrm{~dB}$ ), but these do not appear to correspond to any string frequencies or mode frequencies. The results match within $1 \mathrm{~dB}$ at all other frequencies within the range of interest, suggesting good linear behavior. 


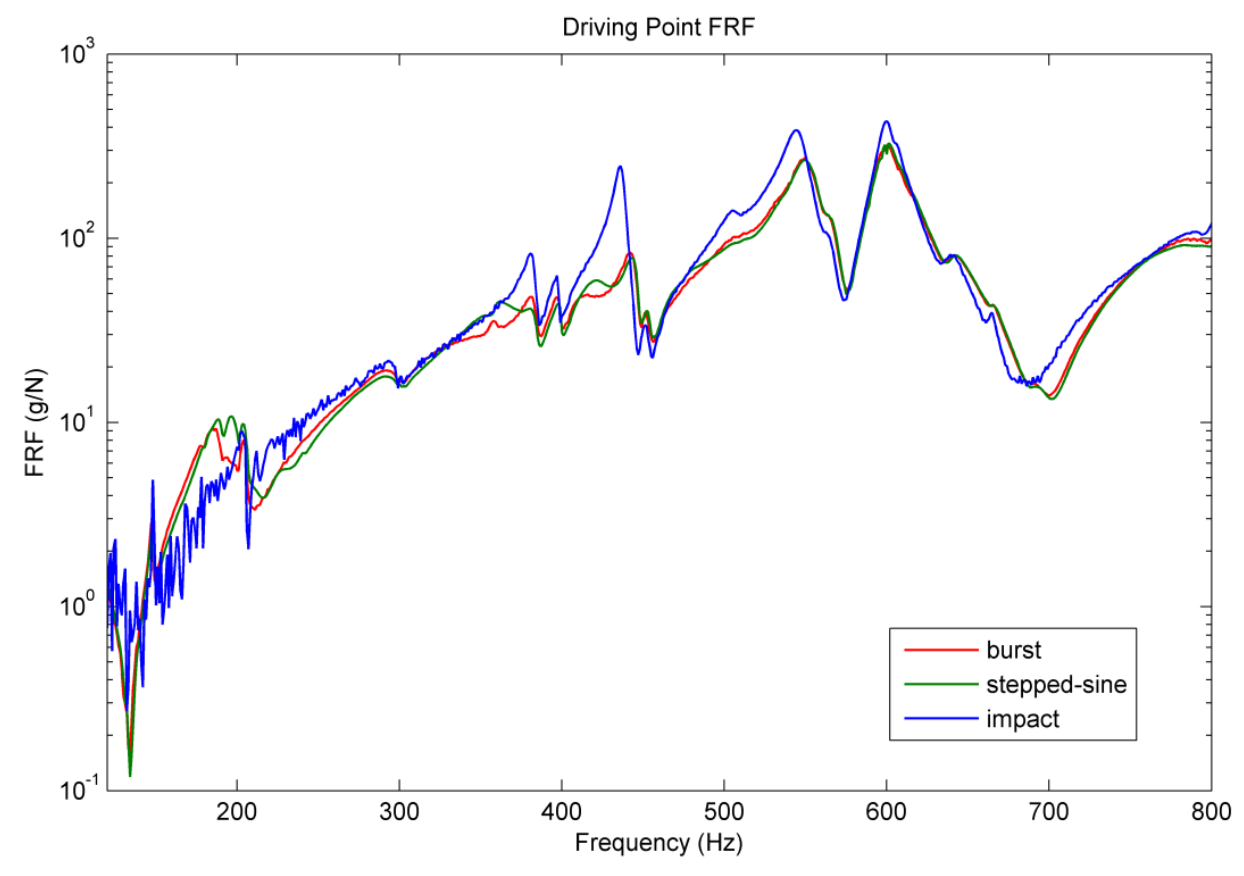

Figure 3.7: Frequency response functions for the driving point at the bass-side corner of the bridge. Burst random excitation (red), stepped-sine excitation (green), impact excitation (blue).

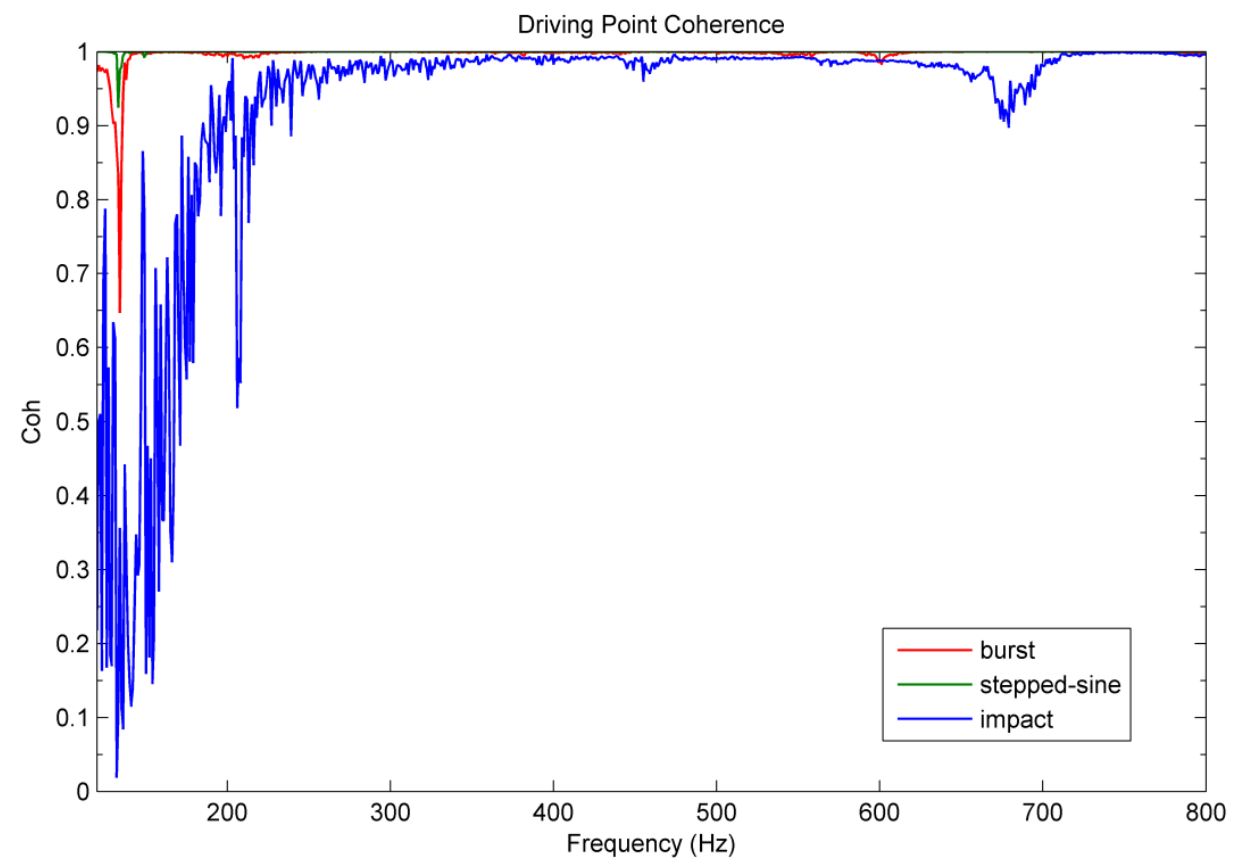

Figure 3.8: Coherence functions for the driving point at the bass-side corner of the bridge. Burst random excitation (red), stepped-sine excitation (green), impact excitation (blue). 
The FRFs of Method B however are strikingly dissimilar to those of Method A in a few key areas; namely around the CBR (Center-Bout-Rhomboid) mode near $433 \mathrm{~Hz}$ to 435 Hz. This is a mode that involves a twisting motion of the middle portion of the corpus (center bouts), out of phase with the upper and lower bouts, forming a double-cross nodal line pattern on the plates [3.2]. Here the CBR mode of Method A occurs about $2 \mathrm{~Hz}$ higher than that of Method B. Even more significant is the difference in the magnitude of the peaks associated with this mode; Method B is consistently $10 \mathrm{~dB}$ higher than Method A. The most reasonable explanation is that the presence of the shaker system (including the stinger, force sensor, and wooden dowel) has affected the system. The CBR mode involves significant rocking motion of the bridge. When the shaker system is glued to the corner of the bridge, it constrains the rotational component of that motion. This effect is seen in other modes that involve a rotational component to the bridge, such as mode 5 (around $383 \mathrm{~Hz}$ ), which is similar to the CBR mode.

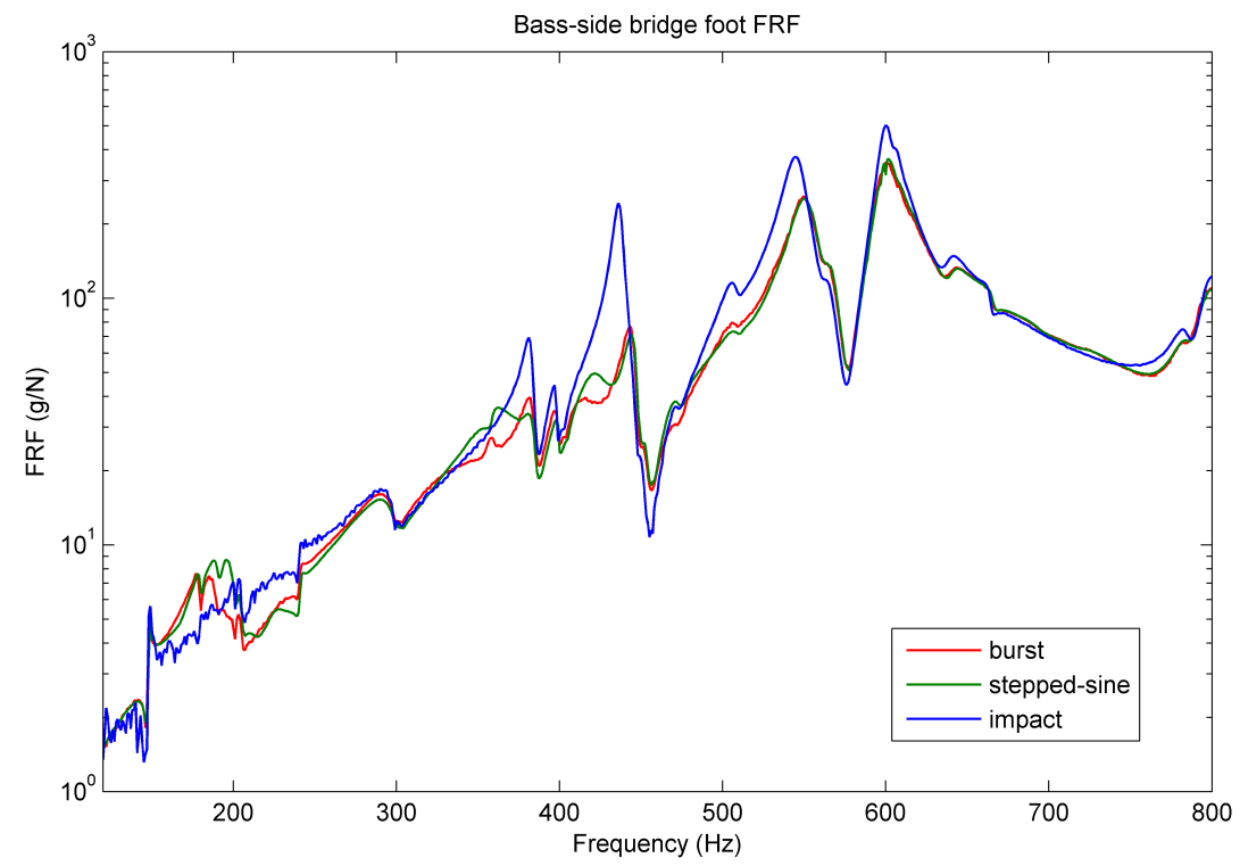

Figure 3.9: Frequency response functions for the bass-side foot of the bridge. Burst random excitation (red), stepped-sine excitation (green), impact excitation (blue). 


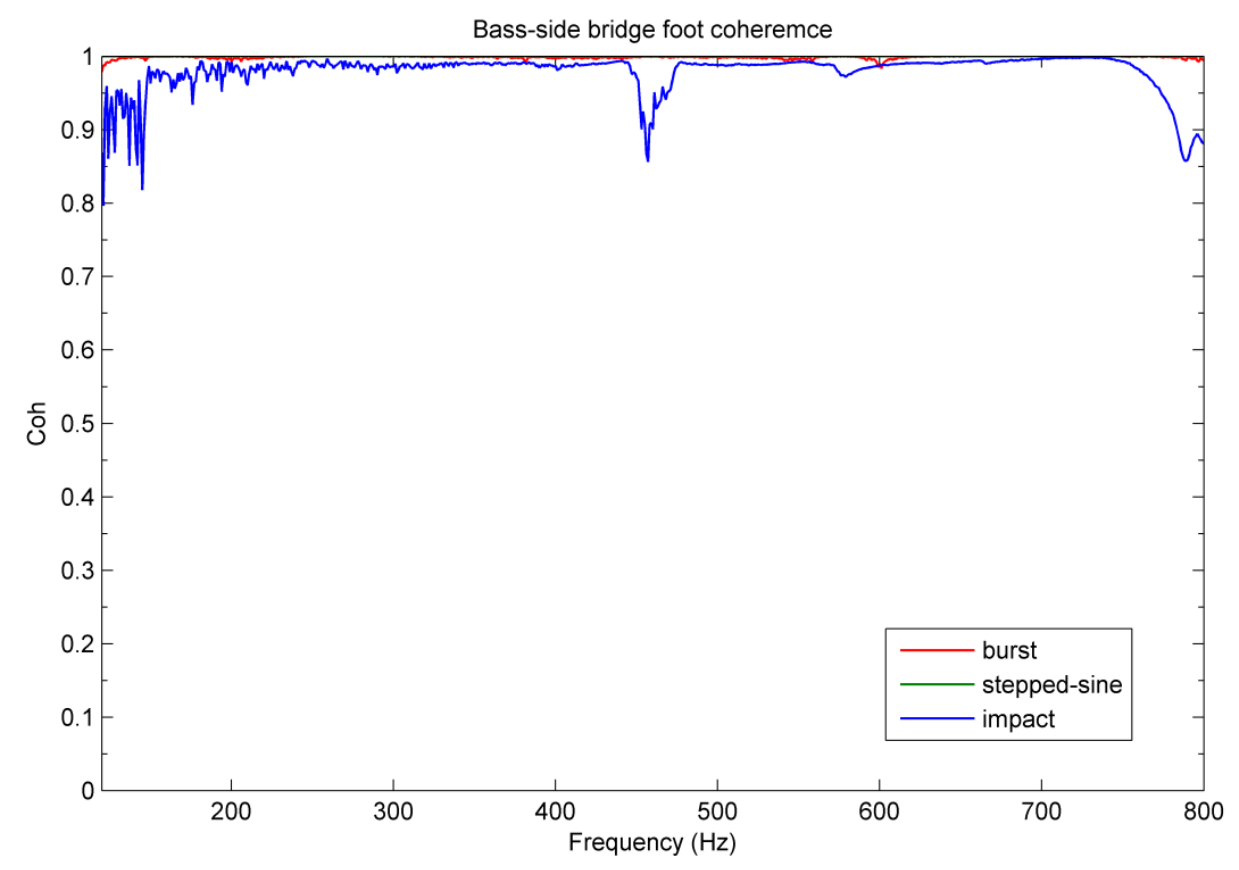

Figure 3.10: Coherence functions for the bass-side foot of the bridge. Burst random excitation (red), stepped-sine excitation (green), impact excitation (blue).

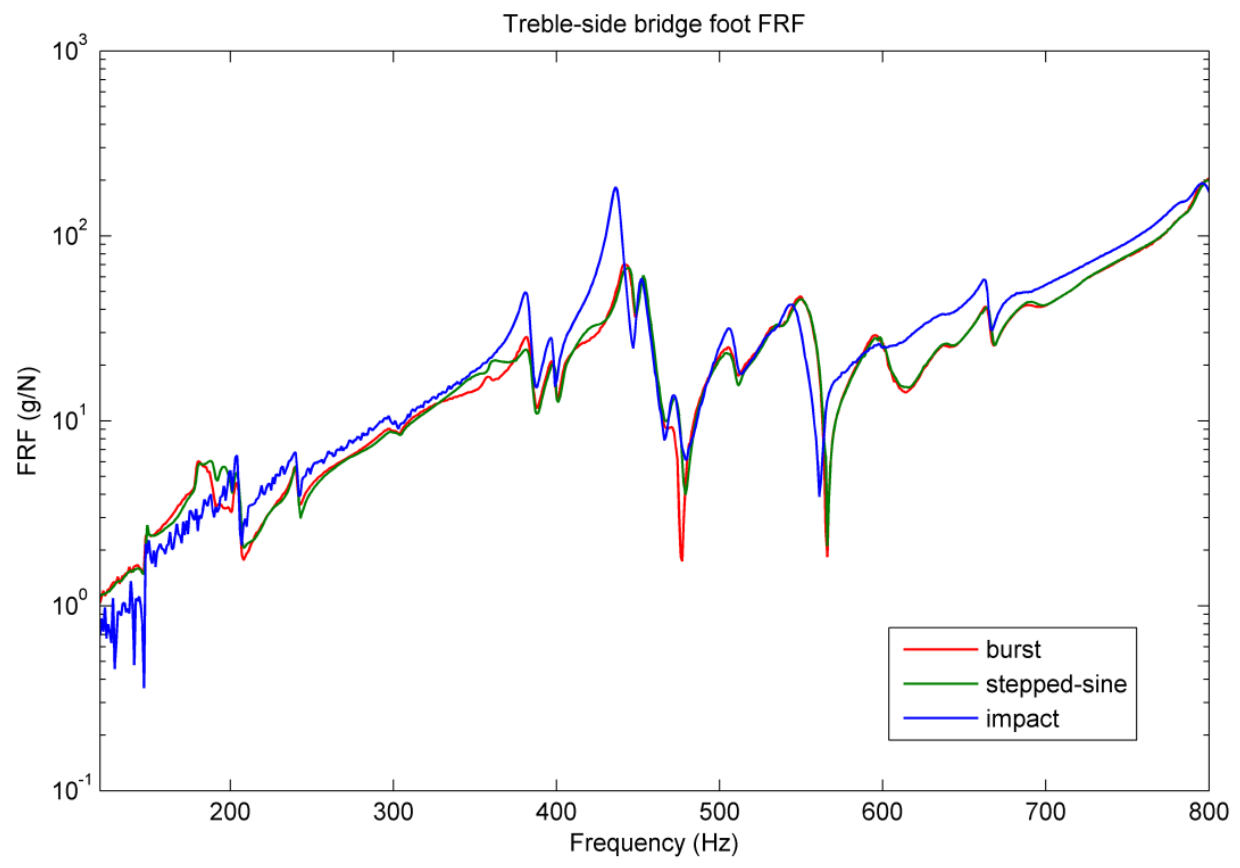

Figure 3.11: Frequency response functions for the treble-side foot of the bridge. Burst random excitation (red), stepped-sine excitation (green), impact excitation (blue). 


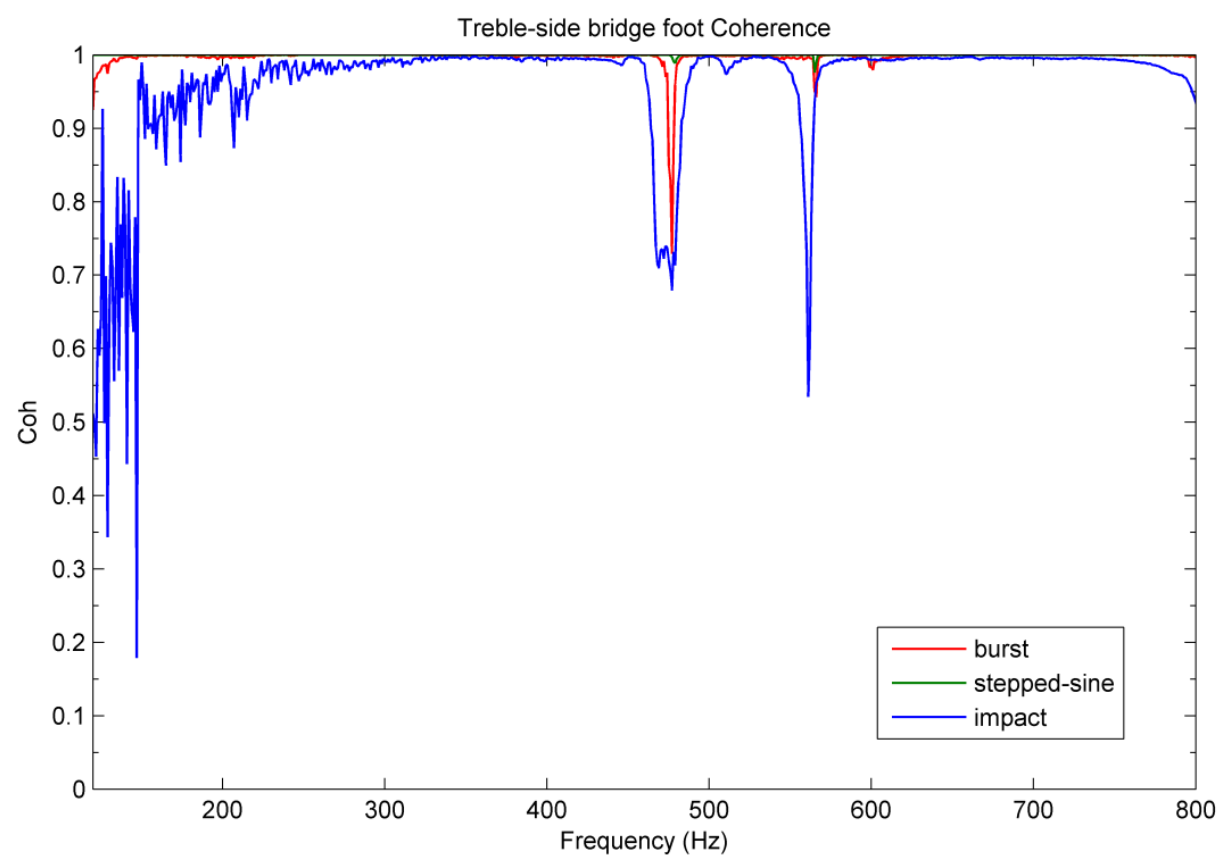

Figure 3.12: Coherence functions for the treble-side foot of the bridge. Burst random excitation (red), stepped-sine excitation (green), impact excitation (blue).

The other important comparison between these methods is the quality of the measurements. Both the FRFs and the coherence functions indicate that Method A has results with less noise than Method B, particularly in the lower frequency range. The stepped-sine force input results in the least amount of noise, consistently having the highest coherence throughout the frequency range. The impact force results in the lowest coherence, with a significant drop in the low frequency range. This is due to the smaller input excitation provided by the hammer. Less excitation results in less acceleration response, and a poorer signal to noise ratio. Data quality does not appear to be an issue for the modes above the $\mathrm{G}$ string $(196 \mathrm{~Hz})$ as the coherence values of these modes are all above 0.95 for all three excitations.

\subsubsection{Mode shapes}

Ultimately, the objective of this testing will be to obtain suitable experimental mode shapes for correlating to the analytical model of the factory violin. The final comparison of the test methods considers the results from curve fitting the different results. As mentioned previously, measuring all locations with the stepped-sine excitation would have taken far longer than is practical. Therefore only the burst excitation and impact excitation were used for a full modal test. 
For Method A the shaker input is kept stationary, one accelerometer is kept on the driving point and six others are roved over the remaining measurement points. Modal parameter estimation of this data is extremely difficult since the movement of the accelerometers leads to changes in the mass loading condition, which may cause the system poles to shift in frequency. For Method B the response transducers were stationary while the impact hammer input was roved over all locations. For this testing the mass loading conditions do not change and the system poles should remain constant. Overall, Method B resulted in more real normal modes than Method A in the range of interest. The mode shapes found using Method B are shown in Figure 3.13 through Figure 3.26. The color scale shown is for the absolute displacement, i.e. red is large motion, while blue is little to no motion (nodal lines).

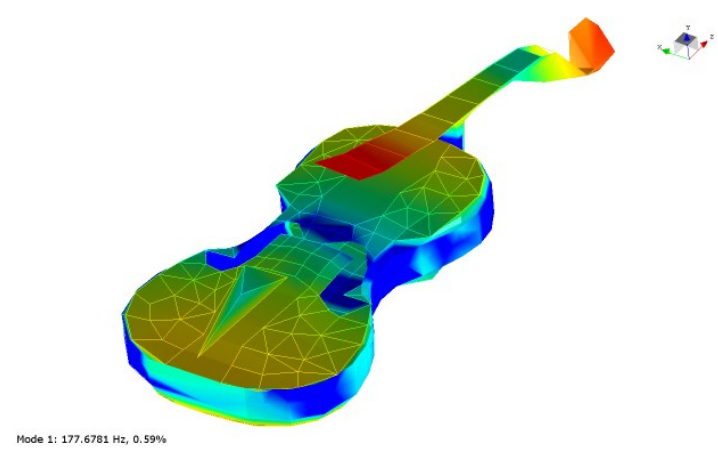

Figure 3.13: Mode 1 - First bending of violin between neck and corpus (BO)

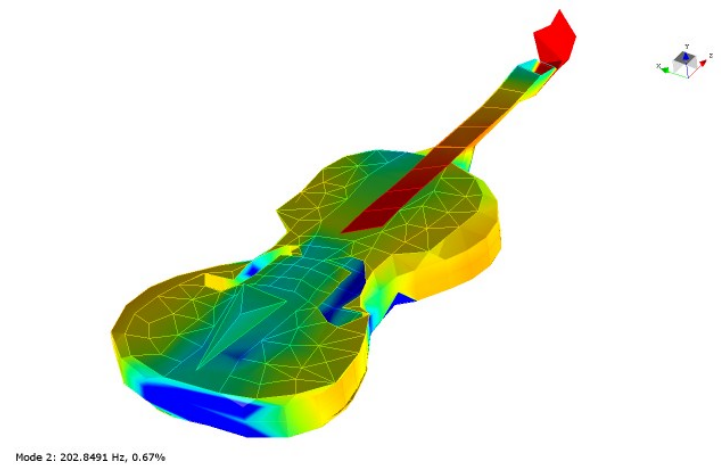

Figure 3.14: Mode 2 - First bending of violin in lateral direction

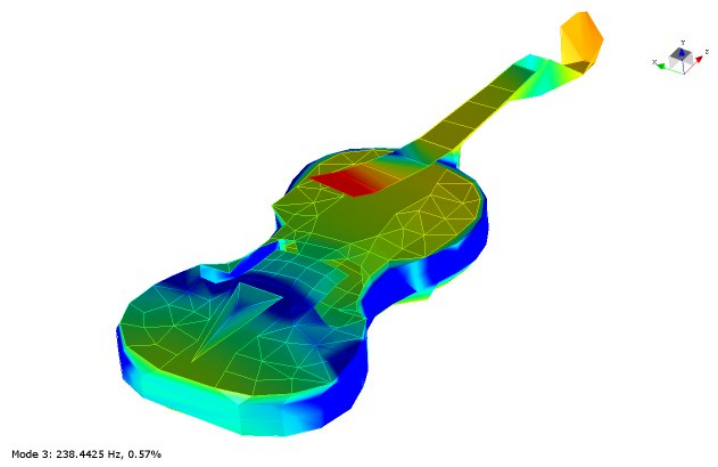

Figure 3.15: Mode 3 - first bending of fingerboard

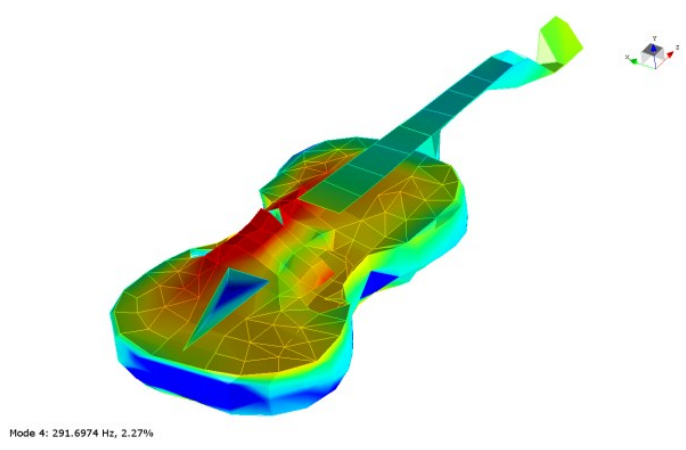

Figure 3.16: Mode 4 - first cavity mode (A0) 


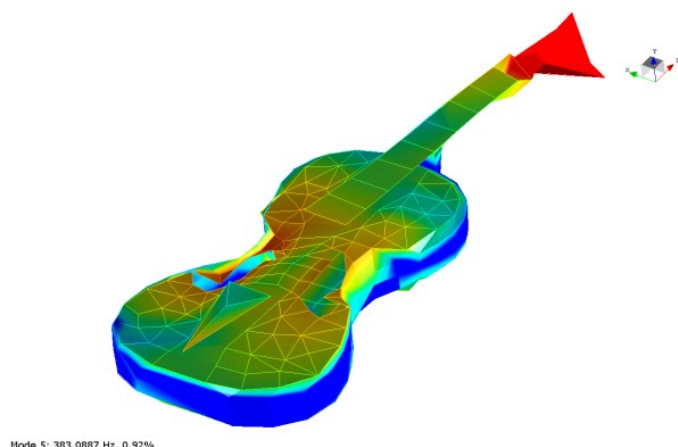

Figure 3.17: Mode 5 - scroll twist out-of phase with center

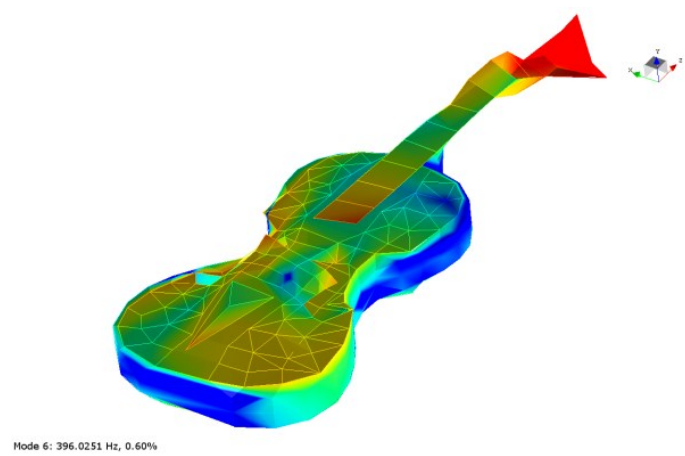

Figure 3.18: Mode 6 - scroll twist in-phase with center

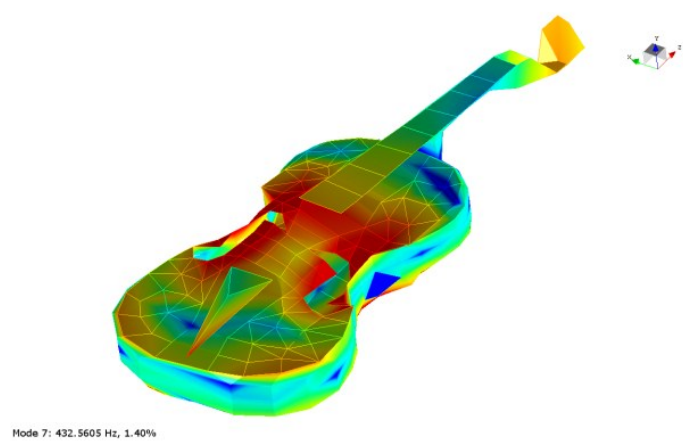

Figure 3.19: Mode 7 Center Bout Rhomboid (CBR) scroll in phase with center

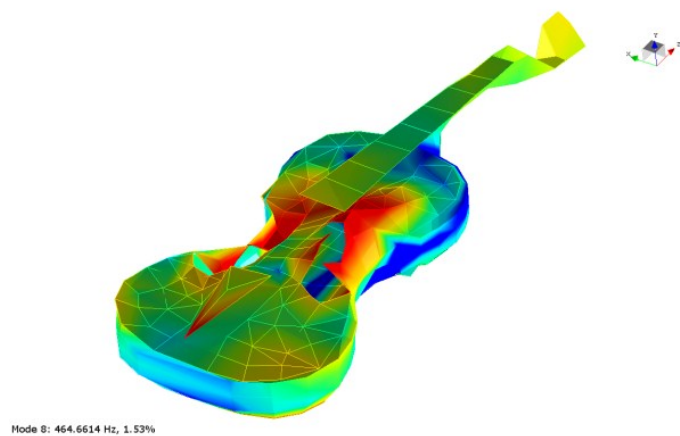

Figure 3.20: Mode 8 - Center Bout Rhomboid (CBR) scroll out-of-phase with center

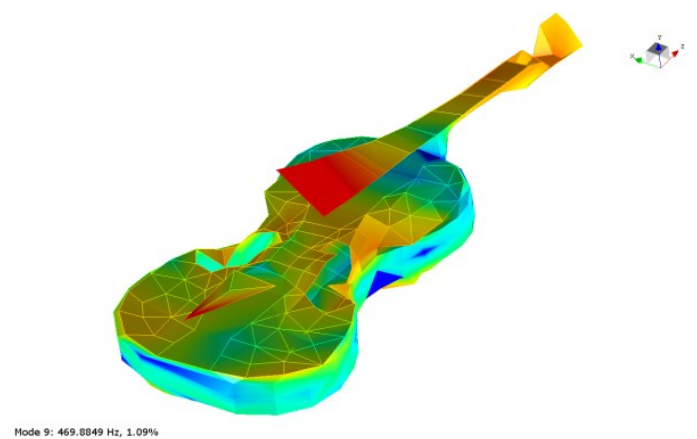

Figure 3.21: Mode 9 - first torsion of fingerboard

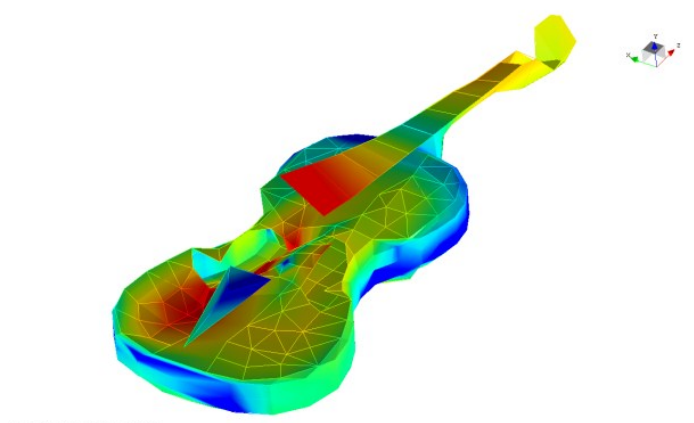

Figure 3.22: Mode 10 - Cavity mode (A1) most breathing in lower bout (strong couple to mode 11) 


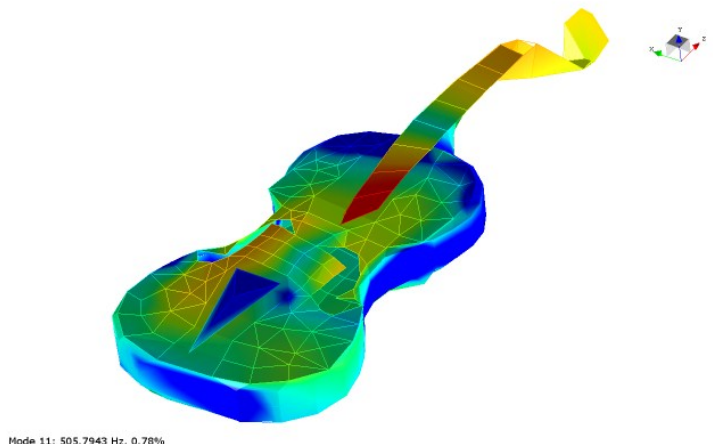

Figure 3.23: Mode 11 - first lateral bending of fingerboard (strong couple to A1)

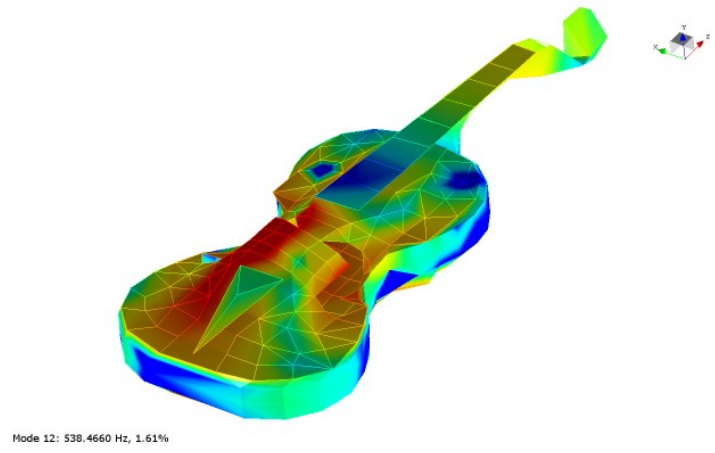

Figure 3.24: Mode 12 - First bending of corpus (B1-) top plate transverse, back plate longitudinal

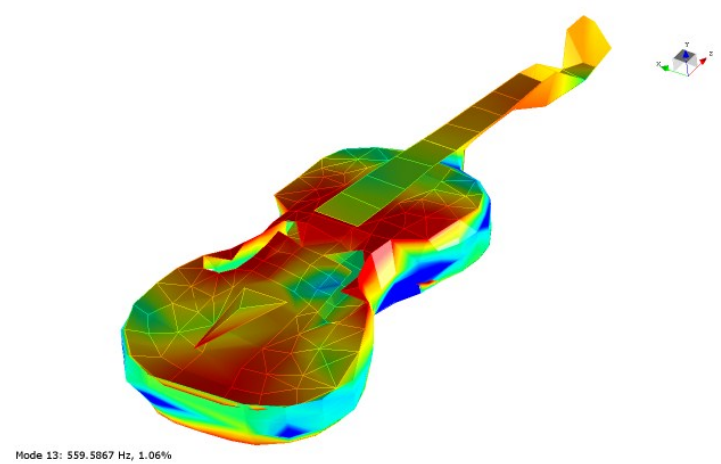

Figure 3.25: Mode 13 - First bending of corpus (B1+) top plate longitudinal, back plate transverse
Figure 3.26: Mode 14 - A2 


\subsubsection{Modal Assurance Criterion before and after post processing}

To correct for the shifting poles in Method A, an additional processing technique was applied. Modal parameter estimation was performed on data from each individual acquisition session. (In theory, since each acquisition session included the driving point, the same modes should have been measured every time, even if the poles shifted.) Modal modification is then used to remove the mass loading effects. It should be noted that this only accounts for translational mass loading; the rotational inertia loading cannot be accounted for in this data, which can lead to additional differences in both the mode frequencies as well as the mode shapes [3.7]. The results from all acquisition sessions are then merged into a final set of modal parameters. As previously mentioned, the driving point and bridge feet measurements used only three accelerometers $(0.3$ grams each), while the roving accelerometer testing of Method A used an additional four accelerometers ( 0.7 grams each). For this reason the FRFs from the driving point and bridge feet measurements were used to determine target poles for the modal merge since this set had the least amount of mass loading and therefore contains the smallest amount of error.

Table 3.1 shows a list of poles found for each individual acquisition session. Note that some modes vary more than others due to the changing mass load condition of the accelerometer placement for each acquisition. For example, the first bending mode of the fingerboard (mode 3, 242.5 Hz for corrected Method A) is particularly sensitive to accelerometers placed on the end of the fingerboard since this part of the structure moves the most for this particular mode. Note that in Table 3.1 mode 3 is measured the lowest for acquisition session \#15 at around $233.6 \mathrm{~Hz}$. Two of the larger accelerometers are mounted on the fingerboard itself during acquisition session \#15; one near the midway point on the treble side and one on the bass-side corner close to the bridge. The accelerometer near the midway point of the fingerboard is probably not affecting the mode itself since it also happens to be close to a nodal line. However, the accelerometer on the corner of the fingerboard does affect this mode since it is adding mass (and inertia) to the part of the structure that is under the greatest amount of motion for this mode. This causes the system pole for this mode to go down.

The added processing time to do this correction is relatively short (total processing time increases by no more than a factor of 1.5). It should be remembered however that this does not include time setting up the merge. Caution must be taken to be sure that the modes are aligned correctly as there is potential for them to switch. Fortunately that did not happen here, but it was necessary to adjust the merging table as some of the poles are 
close and the range in which they shift overlap, potentially confusing the automated process.

Table 3.1: Mode merging table, indicating the pole migrations for each acquisition session due to mass loading effect (Color indicates high/low values in each column with red $=$ high and green $=$ low).

\begin{tabular}{|c|c|c|c|c|c|c|c|c|c|c|c|c|c|c|}
\hline $\begin{array}{c}\text { Acq. } \\
\#\end{array}$ & $\begin{array}{c}\text { Mode } \\
1 \\
181.6 \\
\mathrm{~Hz} \\
\end{array}$ & $\begin{array}{c}\text { Mode } \\
2 \\
206.8 \\
\text { Hz } \\
\end{array}$ & $\begin{array}{c}\text { Mode } \\
3 \\
242.5 \\
\mathrm{~Hz} \\
\end{array}$ & $\begin{array}{c}\text { Mode } \\
4 \\
295.1 \\
\mathrm{~Hz} \\
\end{array}$ & $\begin{array}{c}\text { Mode } \\
5 \\
385.0 \\
\text { Hz } \\
\end{array}$ & $\begin{array}{c}\text { Mode } \\
6 \\
392.4 \\
\mathrm{~Hz} \\
\end{array}$ & $\begin{array}{c}\text { Mode } \\
7 \\
433.4 \\
\mathrm{~Hz} \\
\end{array}$ & $\begin{array}{c}\text { Mode } \\
8 \\
465.7 \\
\mathrm{~Hz} \\
\end{array}$ & $\begin{array}{c}\text { Mode } \\
9 \\
485.0 \\
\mathrm{~Hz} \\
\end{array}$ & $\begin{array}{c}\text { Mode } \\
10 \\
509.2 \\
\mathrm{~Hz} \\
\end{array}$ & $\begin{array}{c}\text { Mode } \\
11 \\
515.1 \\
\mathrm{~Hz} \\
\end{array}$ & $\begin{array}{c}\text { Mode } \\
12 \\
566.4 \\
\mathrm{~Hz} \\
\end{array}$ & $\begin{array}{c}\text { Mode } \\
13 \\
581.6 \\
\mathrm{~Hz} \\
\end{array}$ & $\begin{array}{c}\text { Mode } \\
14 \\
599.9 \\
\mathrm{~Hz} \\
\end{array}$ \\
\hline 1 & 182.1 & 205.9 & 241.8 & 295.9 & 384.7 & 392.3 & 434.6 & 463.7 & 490.1 & 505.0 & 513.2 & 550.2 & 571.7 & 597.0 \\
\hline 2 & 180.6 & 205.8 & 241.8 & 295.4 & 384.8 & 392.0 & 434.2 & 463.2 & 484.3 & 508.3 & 514.9 & 552.7 & 572.2 & 596.0 \\
\hline 3 & 180.8 & 205.9 & 241.8 & 296.3 & 384.4 & 391.7 & 435.5 & 462.9 & 485.1 & 504.3 & 509.8 & 550.3 & 571.1 & 596.7 \\
\hline 4 & 180.6 & 205.9 & 241.9 & 297.0 & 384.2 & 391.9 & 434.4 & 462.9 & 493.0 & 504.9 & 514.0 & 546.7 & 569.9 & 595.4 \\
\hline 5 & 180.8 & 205.9 & 241.9 & 296.8 & 384.0 & 392.0 & 433.9 & 462.7 & 485.7 & 507.9 & 514.2 & 547.1 & 567.0 & 596.4 \\
\hline 6 & 180.6 & 206.0 & 241.8 & 295.5 & 384.4 & 391.7 & 435.1 & 462.1 & 482.4 & 508.8 & 514.9 & 550.8 & 569.3 & 596.6 \\
\hline 7 & 180.6 & 205.9 & 241.6 & 297.3 & 383.8 & 391.4 & 434.4 & 461.1 & 481.6 & 508.4 & 515.3 & 548.0 & 567.6 & 594.8 \\
\hline 8 & 180.4 & 205.8 & 241.7 & 297.0 & 384.0 & 391.5 & 434.4 & 461.2 & 481.2 & 508.8 & 516.1 & 549.9 & 567.9 & 596.3 \\
\hline 9 & 180.5 & 205.8 & 241.7 & 297.5 & 383.5 & 391.4 & 433.8 & 461.5 & 480.9 & 508.6 & 513.9 & 546.8 & 569.7 & 594.5 \\
\hline 10 & 180.4 & 205.8 & 241.6 & 297.9 & 383.8 & 391.2 & 434.5 & 461.3 & 481.0 & 509.6 & 514.7 & 549.4 & 568.5 & 595.8 \\
\hline 11 & 180.5 & 205.6 & 241.5 & 297.7 & 384.1 & 391.4 & 435.2 & 461.5 & 481.8 & 510.4 & 513.8 & 550.8 & 571.1 & 595.3 \\
\hline 12 & 180.6 & 205.6 & 241.5 & 297.9 & 383.9 & .0 & 435.0 & 461.7 & 481.6 & 510.0 & 513.5 & 547.8 & 570.2 & 594.6 \\
\hline 13 & 180.6 & 206.5 & 241.9 & 298.9 & 385.0 & 393.4 & 437.7 & 464.2 & 485.2 & 510.5 & 513.7 & 543.9 & 568.6 & 611.1 \\
\hline 14 & 179.8 & 206.1 & 240.9 & 299.5 & 384.5 & 391.3 & 437.3 & 462.8 & 485.1 & 509.1 & 511.9 & 553.9 & 579.4 & 611.6 \\
\hline 15 & 176.1 & 205.7 & 233.6 & 298.4 & 384.6 & 391.3 & 437.3 & 462.7 & 482.7 & 506.5 & 513.0 & 561.2 & 579.5 & 612.0 \\
\hline 16 & 179.9 & 206.3 & 241.6 & 299.7 & 384.3 & 391.5 & 437.3 & 462.9 & 485.4 & 510.1 & 511.9 & 560.7 & 579.3 & 611.4 \\
\hline 17 & 177.7 & 206.4 & 237.3 & 295.6 & 384.5 & 391.9 & 436.3 & 463.9 & 483.9 & 509.2 & 513.3 & 557.7 & 577.0 & 605.1 \\
\hline 18 & 179.2 & 205.4 & 240.0 & 296.8 & 384.3 & 391.4 & 436.4 & 463.0 & 485.8 & 509.5 & 512.1 & 557.0 & 577.2 & 605.4 \\
\hline 19 & 180.2 & 206.1 & 241.8 & 297.6 & 384.5 & 391.7 & 437.0 & 462.4 & 486.1 & 511.6 & 514.5 & 559.6 & 580.3 & 608.1 \\
\hline 20 & 180.5 & 206.0 & 241.8 & 299.2 & 384.4 & 391.4 & 436.0 & 462.8 & 187.6 & 508.9 & 515.1 & 556.7 & 574.4 & 601.4 \\
\hline 21 & 179.0 & 205.8 & 239.4 & 300.3 & 384.7 & 391.6 & 436.3 & 463.2 & 487.1 & 512.2 & 517.2 & 557.9 & 576.1 & 603.8 \\
\hline 22 & 180.0 & 205.7 & 241.4 & 297.6 & 384.4 & 391.4 & 435.9 & 463.0 & 487.1 & 509.0 & 514.6 & 555.9 & 575.3 & 600.5 \\
\hline 23 & 180.2 & 206.0 & 241.6 & 299.7 & 384.6 & 391.5 & 437.0 & 464.3 & 487.2 & 502.0 & 514.3 & 558.3 & 579.8 & 613.6 \\
\hline 24 & 180.4 & 206.3 & 241.6 & 299.4 & 384.3 & 392.1 & 437.0 & 462.3 & 487.3 & 510.7 & 513.6 & 557.0 & 580.2 & 615.4 \\
\hline 25 & 180.3 & 206.0 & 241.6 & 298.1 & 384.5 & 391.8 & 436.4 & 463.6 & 486.8 & 510.0 & 515.7 & 556.7 & 576.6 & 605.9 \\
\hline
\end{tabular}

To visualize the effectiveness of this technique, modal parameter results from Method A both with and without the additional processing were correlated to the results from Method B using the Modal Assurance Criterion [3.13]. Figure 3.27 shows the MAC of Method A without any correction to Method B, while Figure 3.28 shows the MAC of

Michael Pyrkosz 
Method A with correction to Method B. The mode frequencies and MAC mode pair values are reported in Table 3.2. The correction technique improves the results significantly, particularly for modes 1, 3, 4, 10 and 14. Although this is positive, the results fall short of showing a good correlation between the two methods. Modes 1 through 5, 7, and 14 are reasonable matches, having MAC values over 0.80 (after correction). However, modes 6 , and 8 through 13 are all less than 0.80 , indicating that the two methods do not consistently yield the same results.

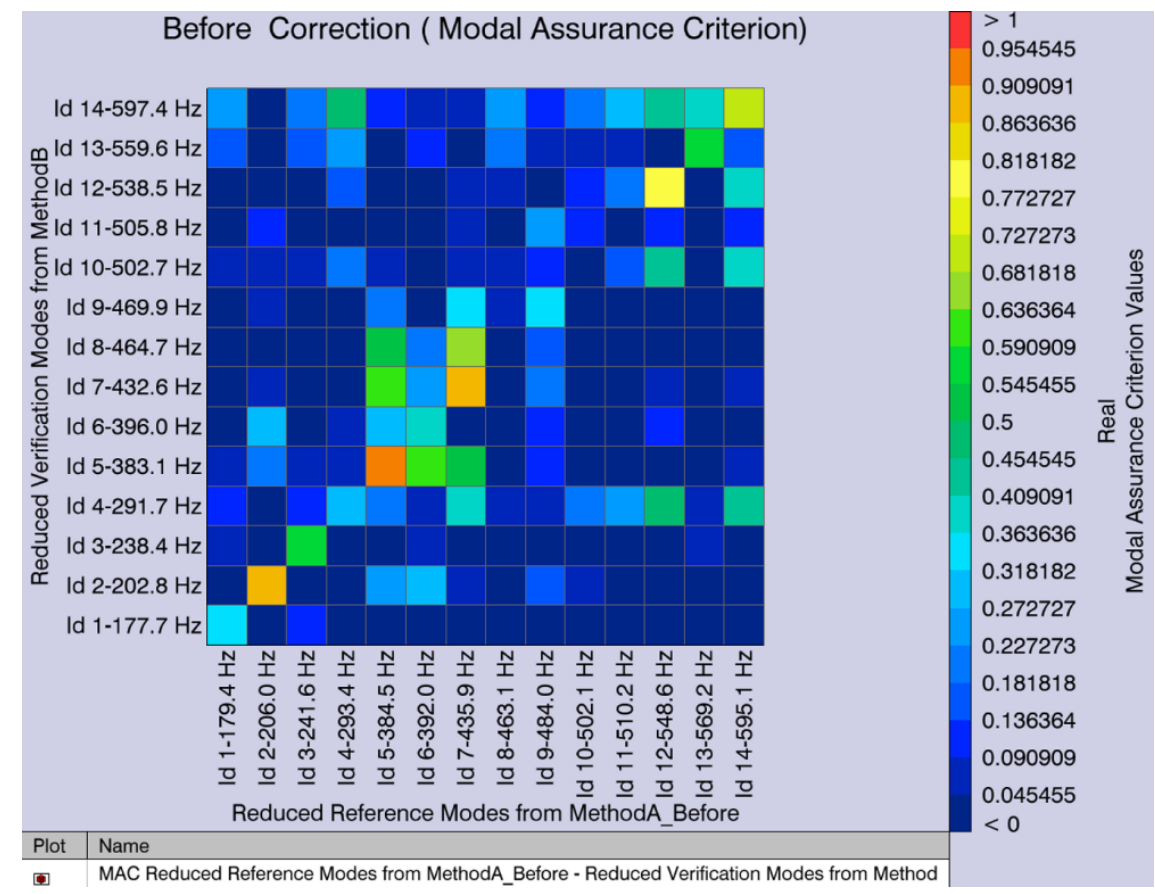

Figure 3.27: Modal Assurance Criterion between Method A before correction to Method B 


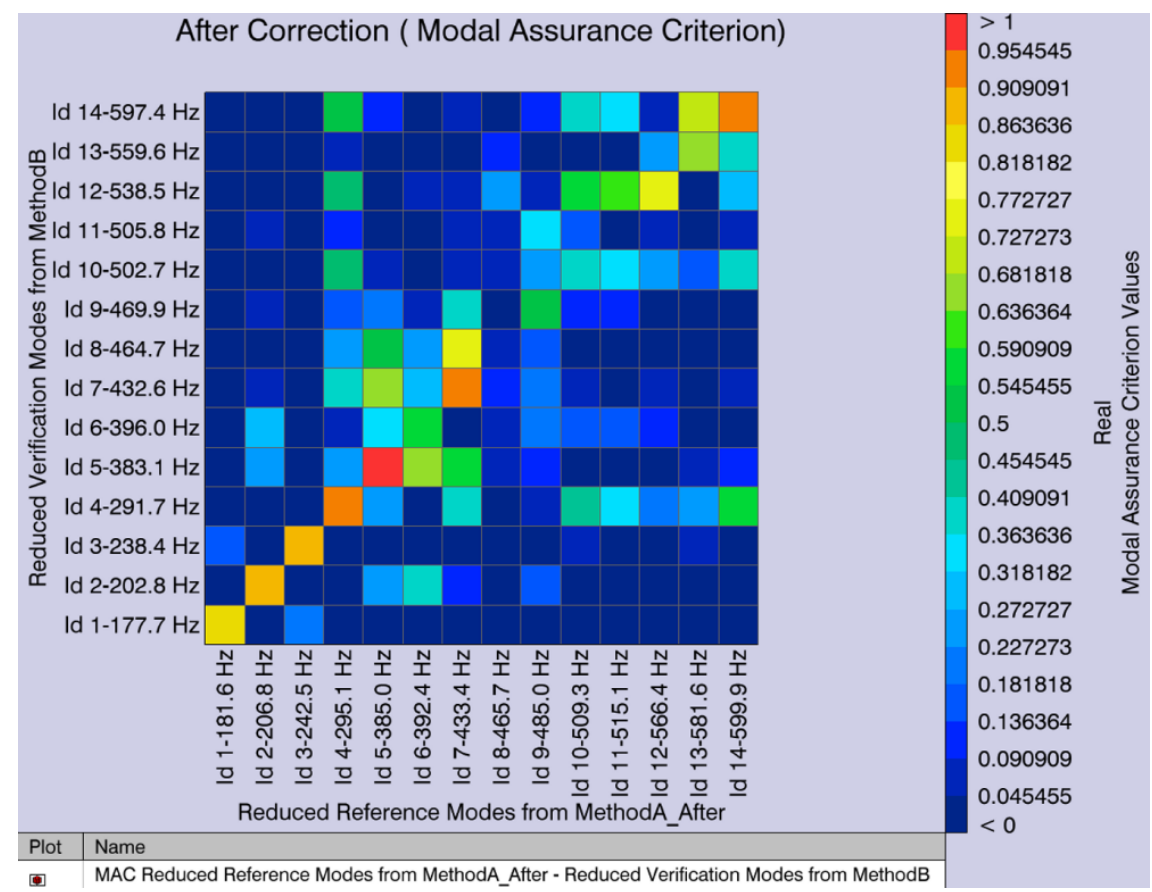

Figure 3.28: Modal Assurance Criterion between Method A after correction to Method B

It should also be noted that the mode results from Method A are typically higher in frequency than the results from Method $\mathrm{B}$, in most cases even before corrections are made to Method A. This is indicative of the stiffening effect due to the presence of the shaker. By constraining the rotational motion of the bridge, modes in which the bridge would rotate under true "free-free" conditions are shifted higher in frequency.

Table 3.2: Comparison of mode frequencies and MAC values of Method $A$ before and after correction to Method B

\begin{tabular}{|c|c|c|c|c|c|c|c|c|c|}
\hline \multirow[b]{2}{*}{ Mode } & \multirow[b]{2}{*}{ Description } & \multicolumn{2}{|c|}{$\begin{array}{l}\text { Method A } \\
\text { before } \\
\text { correction }\end{array}$} & \multicolumn{2}{|c|}{$\begin{array}{l}\text { Method A } \\
\text { after } \\
\text { correction }\end{array}$} & \multicolumn{2}{|c|}{ Method B } & \multirow{2}{*}{$\begin{array}{c}\text { MAC } \\
\text { before } \\
\text { correction }\end{array}$} & \multirow{2}{*}{$\begin{array}{c}\text { MAC } \\
\text { after } \\
\text { correction }\end{array}$} \\
\hline & & $\begin{array}{l}\text { freq } \\
(\mathrm{Hz})\end{array}$ & $\begin{array}{c}\text { damp } \\
\%\end{array}$ & $\begin{array}{l}\text { freq } \\
(\mathrm{Hz})\end{array}$ & $\begin{array}{c}\text { damp } \\
\%\end{array}$ & $\begin{array}{l}\text { freq } \\
(\mathrm{Hz})\end{array}$ & $\begin{array}{c}\text { damp } \\
\%\end{array}$ & & \\
\hline 1 & B0 : first bending & 179.4 & 0.4933 & 181.6 & 0.5224 & 177.7 & 0.5858 & 0.321 & 0.837 \\
\hline 2 & Lateral bending & 206.0 & 0.6325 & 206.8 & 0.5179 & 202.8 & 0.6681 & 0.883 & 0.877 \\
\hline 3 & Fingerboard bending & 241.6 & 0.4296 & 242.5 & 0.4891 & 238.4 & 0.566 & 0.590 & 0.878 \\
\hline 4 & A0 : first cavity & 293.4 & 4.1676 & 295.1 & 3.2678 & 291.7 & 2.2661 & 0.318 & 0.933 \\
\hline 5 & Scroll twist out-of-phase with center & 384.5 & 0.6694 & 385.0 & 0.5911 & 383.1 & 0.9248 & 0.932 & 0.964 \\
\hline 6 & Scroll twist in-phase with center & 392.0 & 0.1661 & 392.4 & 0.1186 & 396.0 & 0.6023 & 0.393 & 0.563 \\
\hline 7 & CBR scroll in-phase with center & 435.9 & 0.4945 & 433.4 & 0.807 & 432.6 & 1.3954 & 0.880 & 0.931 \\
\hline 8 & CBR scroll out-of-phase with center & 463.1 & 0.5443 & 465.7 & 0.3984 & 464.7 & 1.3954 & 0.034 & 0.084 \\
\hline 9 & Fingerboard torsion & 484.0 & 0.7042 & 485.0 & 0.7721 & 469.9 & 1.0912 & 0.341 & 0.508 \\
\hline 10 & A1 : second cavity (upper-lower) & 502.1 & 0.4384 & 509.3 & 1.1446 & 502.7 & 1.3538 & 0.045 & 0.403 \\
\hline 11 & First lateral bending of fingerboard & 510.2 & 0.9074 & 515.1 & 0.7659 & 505.8 & 1.0912 & 0.039 & 0.039 \\
\hline 12 & B1- : Corpus bend & 548.6 & 2.1902 & 566.4 & 1.0403 & 538.5 & 1.6112 & 0.775 & 0.743 \\
\hline 13 & $\mathrm{~B} 1+:$ Corpus bend & 569.2 & 0.6700 & 581.6 & 0.5681 & 559.6 & 1.0574 & 0.580 & 0.682 \\
\hline 14 & A2 : second cavity (bass-treble) & 595.1 & 0.5389 & 599.9 & 0.0125 & 597.4 & 0.9432 & 0.686 & 0.948 \\
\hline
\end{tabular}

Michael Pyrkosz 


\subsection{Conclusions}

The primary motivation for the testing in this article was to determine which experimental methods are best for finding accurate modal parameters of the factory violin. These methods will be implemented in the future to gain insight into system properties of the violin and later applied to an analytical model. Two experimental methods were used and their results compared.

Using excitation from a shaker, as in Method A (either stepped-sine or burst random), was a novel technique and produced frequency response curves with less variance throughout the frequency range. However, for a structure as light as the violin, the mass loading from the accelerometers roving over different response locations between acquisition sessions leads to inconsistencies in system poles. These were corrected by performing modal parameter estimation on individual acquisition session, removing the added translational mass of the accelerometers through structural dynamic modification, and merging the modified modal results together to form new modal parameters. Additionally, the presence of the shaker-stinger-force sensor system constrained the motion of the modes that involved rotational motion of the bridge.

The impact hammer excitation in Method B is the preferred method for testing the violin. The results are more consistent from one acquisition session to the next since the mass loading condition does not change. It also does not constrain the rotation motion of the bridge. The frequency response curves of Method B contain more variance in the lower frequency range than those of Method A. This may be improved by increasing the signal to noise ratio for the impact hammer. However, this is not a problem because most of the noise in this data is below the frequency range of interest; that is, below the lowest string on the violin. It was determined that it is legitimate to impact test the violin since the system behaved relatively linear within the frequency range of interest. It would also have been beneficial to have made these measurements with a non-contact transducer, such as a laser vibrometer. An attempt was made to remove the mass loading effects of the accelerometers using modal modification, however this did not account for the rotational inertia effects. Both methods had their benefits and limitations, but the impact test proved to be the best method for testing the violin.

\subsection{Acknowledgments}

The authors would like to acknowledge Dr. George Bissinger for initiating this project; PolyTec, Inc. for the 3D laser vibrometry data collection; PCB Piezotronics, Inc., The Modal Shop, Inc., and the Keweenaw Research Center for equipment support; LMS

Michael Pyrkosz

Michigan Technological University 
International for software support; and Kingsley Iduma and Dr. Alexis Black Pyrkosz for technical assistance.

\subsection{References}

[3.1] Pyrkosz, Michael, Van Karsen, Charles, Bissinger, George, "Converting CT Scans of a Stradivari Violin to a FEM", 28th International Modal Analysis Conference, Jacksonville, FL, (February 2010)

[3.2] Bissinger, George, "Structural Acoustics of Good and Bad Violins" J. Acoust. Soc. Am. Volume 124, Issue 3, pp. 1764-1773 (September 2008)

[3.3] Bissinger, George, "A Unified Materials-Normal Mode Approach To Violin Acoustics” Acustica Volume 91, Number 2, pp. 214-228 (March/April 2005)

[3.4] Bissinger, George, "3-D Laser Vibrometry Focuses On Legendary Old Italian Violins" Sound and Vibration magazine Vol.41/ Num.7, pp. 10-15 (July 2007)

[3.5] Marshall, Kenneth D., "Modal Analysis of a Violin" J. Acoust. Soc. Am. Vol.77/Num.2, pp. 695-709 (February 1985)

[3.6] Knott, George A., "A Modal Analysis of the Violin Using MSC/NASTRAN and PATRAN" M.S. Thesis, Naval Postgraduate School. Available through NTIS website. (March 1987)

[3.7] Bissinger, George, "The violin bridge as filter" J. Acoust. Soc. Am. Volume 120, Issue 1, pp. 482-491 (July 2006)

[3.8] Bailey, M., Bissinger, G., "Modal analysis study of mode frequency and damping changes due to chemical treatments of the violin bridge", 13th International Modal Analysis Conference, Bethel, CT, (1995) pp.828-833

[3.9] Heron-Allen, Edward, "Violin-Making, As It Was And Is" (Ward, Lock \& Co., London, 1885) p. 161

[3.10] Avitable, P., O'Callahan, J., Chou, C-M., Kalkunte,V., "Expansion of Rotational Degrees of Freedom for Structural Dynamic Modification," 5th International Modal Analysis Conference, London, England, pp. 950-955 (April 1987)

[3.11] Ye, K., Bissinger, G., “Attaining 'Free-Free' Normal Mode Frequency and Damping conditions for the Violin" 18th International Modal Analysis Conference, Bethel, CT, 2000, pp. 398-403

[3.12] Bissinger, George, "Modern Vibration Measurement Techniques for Bowed String Instruments", Experimental Techniques (July/August 2001) pp. 43-46

[3.13] Allemang, Randall J., "Investigation of some Multiple Input/Output Frequency Response Function Experimental Modal Analysis Techniques" Ph.D. Dissertation, University of Cincinnati. Available through University Microfilms International. pp. 150 - 214 (September 1980) 


\section{Chapter 4 Determining the Material Properties of a Stradivari Violin through Modal Correlation and Updating $^{4}$}

\subsection{Introduction}

This chapter discusses the use of modal correlation and updating techniques to estimate the material properties of a violin. In particular, the objective is to determine the properties of the 1715 Titian Stradivari. This technique uses correlation of experimental modal data with the modal results of an approximate FE model with initial material properties. The sensitivity of the correlation to the different properties is determined and the material properties are updated to bring the model dynamic behavior in greater agreement with the real test structure. This process is iterated until the model is a reasonable representation of reality.

The violin parts are made of various types of wood. For example the top plate is made of spruce while the back plate is typically constructed from maple. The exact species of each wood, the growing conditions during the original tree's life, the drying process, possible wood treatments, and environmental exposure over time can all affect the material properties of the wood. It is logical that no two pieces of wood are identical, and the precise material values that give the best modal correlation may be different from piece to piece. Each type of wood has heterogeneous density and orthotropic elasticity. A previous study of the violin top plate model showed that the distribution of the density did not have a significant effect on the modal parameters of the top plate [4.1]. Based on those results it is assumed that a model of uniform density is a reasonable approximation of the structure within the frequency range of interest $(196-3520 \mathrm{~Hz}$; the typical playing range for the violin).

The orthotropic elasticity conversely was far more significant. The compliance tensor of a solid orthotropic material is known to have twelve independent properties (3 elastic moduli, 3 shear moduli, and 6 Poisson ratios). The assumption of a symmetric elastic matrix creates constraint equations between the pairs of Poisson ratios reducing the effective number of independent terms to nine. For shell structures (such as the plates and ribs) only a 2D property definition is required. This removes the need for the out-ofplane elastic modulus and the compliance tensor is reduced by removing the associated

\footnotetext{
${ }^{4}$ The material contained in this chapter is currently unpublished; selected portions are planned for future submission, copyright (C) 2013 Michael A. Pyrkosz
}

Michael Pyrkosz 
row and column, thereby also removing the associated Poisson ratios. The final number of independent terms for a 2D orthotropic property is six (2 in-plane elastic moduli, 3 shear moduli, and the in-plane Poisson ratio).

Trying to perform the updating process on all parts of the violin simultaneously is impractical. To reliably identify all the material properties involved in the structure there must be at least as many modal parameters measured; these modal parameters need to be sensitive to the properties to be identified; and every property must have at least one modal parameter sensitive to it. This means that for a property to be identified it must have an observable effect in the data [4.2]. Attempting to identify all the properties of the entire structure at once requires a huge amount of modal data and there is no guarantee that the measured modes will be sensitive to all the parameters. By splitting the violin structure into its major substructures (primarily the top and back plates) a smaller number of properties can be identified at a time. This does however require that a set of test data be available for each substructure; something that is not the case for most Stradivari instruments. For this reason a factory violin was used as a case study. This violin cost less than 100 USD, (compared to a Stradivari worth more than one million USD). A modal analysis was performed on the factory violin as a whole, then it was disassembled and the major substructures (back plate, top plate, and rib structure) were also tested. The corpus of the violin was then reassembled and another modal analysis was performed. Finally, the neck and scroll were reattached (the fingerboard remained glued to the neck) and the instrument restrung. Final driving point measurements were taken to compare the violin before and after disassembly/reassembly.

Model correlation and updating is performed first on the plates and ribs structure, then on the corpus, followed by the fully assembled factory violin. This provides a good estimate of which material properties are reasonable for this violin, which provides a starting point for the model of the Titian Stradivari. Having good starting values will be especially important in this case since there is limited modal data available on the Titian. The updating process will indicate how the material properties of the Titian Stradivari differ from those of a commonplace instrument: a significant step in understanding Stradivari's work.

\subsection{Methods}

\subsubsection{Test methods}

The test methods employed for the full factory violin were discussed in detail in the previous chapter. The experimental data for the Titian Stradivari was part of Bissinger's 
original work [4.3]. The testing discussed in this chapter primarily focuses on the factory violin substructures.

First the factory violin was carefully disassembled. To do this, the violin was exposed to steam for approximately 20 minutes, the purpose of which was to soften the hide glue. A small X-acto blade (\#11) was used to separate the top plate from the rib structure. It became necessary to apply water directly to the joint between the blocks and top plate and let it soak into the glue for approximately 10 minutes to thoroughly soften the glue in these areas. Once the top plate had been removed a temporary support frame was made to hold the blocks in place relative to one another. A small spot of hide glue was used to secure each block to the frame. This was to ensure that the rib structure would retain its shape after the back plate was removed. The neck and scroll of the factory violin was found to have been poorly glued to the upper end block by the manufacturer. After another twenty minutes of exposure to steam the neck was easily removed. The joints between the blocks and the back plate were then wetted and allowed to soak for 10 minutes. The X-acto blade was again used to separate the back plate from the rib structure. Figure 4.1 shows the fully disassembled factory violin.

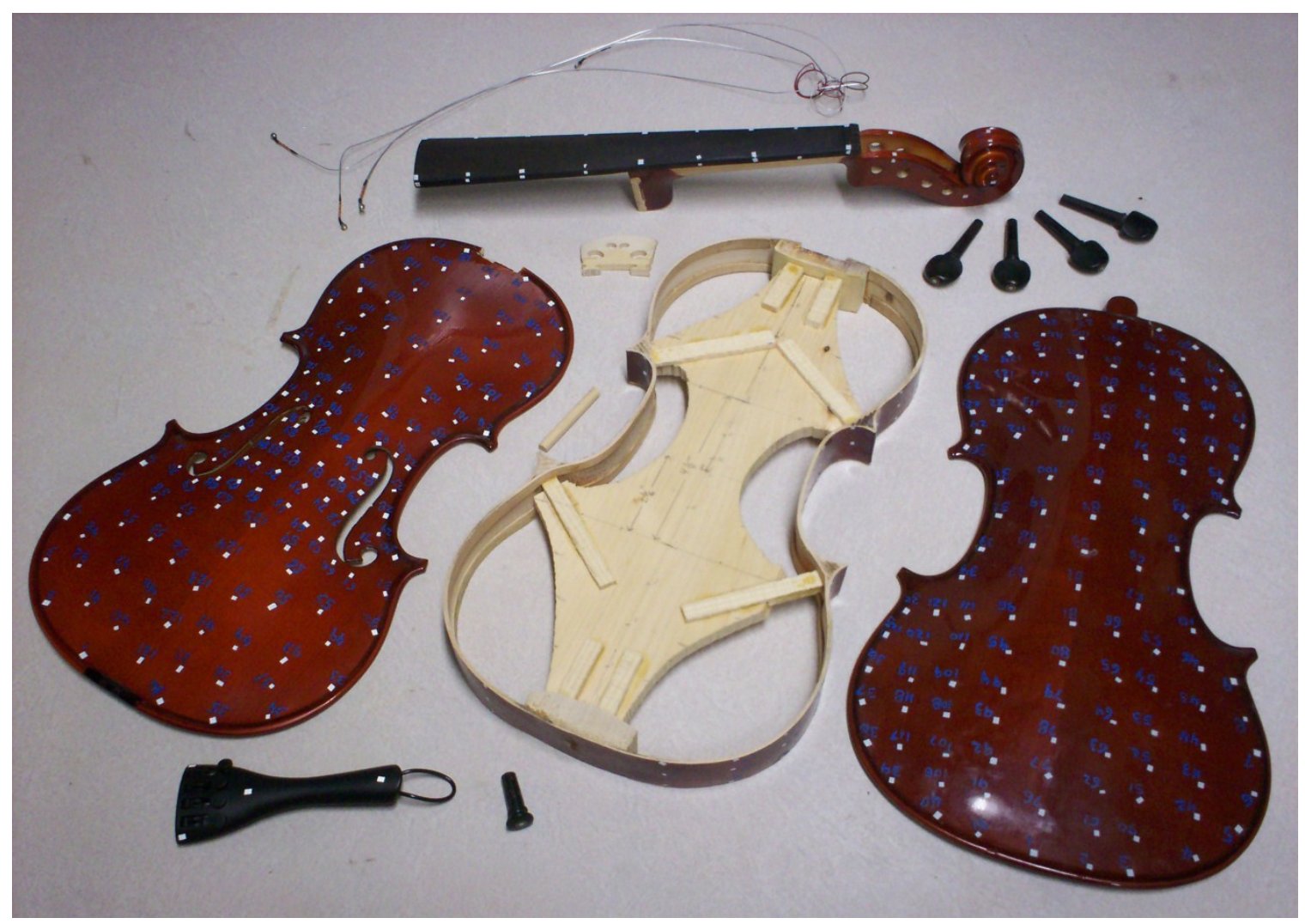

Figure 4.1: Factory violin disassembled

Michael Pyrkosz 
The parts were allowed to dry to bring the wood moisture content back to equilibrium with the lab environment. Testing then proceeded with multi-reference roving impact testing of the plates and rib structure. Both plates were tested using "free-free" boundary conditions accomplished by placing each test piece on crisscrossing rubber bands. The placement of the plate was selected so that the contact points between the structure and the rubber bands would be near the nodal lines of the first mode, which for a typical violin plate is torsion. Figures 4.2 and 4.3 show the actual test setup used for the back and top plates respectively.

While the plates were separated the thickness was also measured at every test location. This was done by setting up a round headed bolt on a steel table. A dial gauge was set up on the bolt and zeroed. Each plate was then placed between the bolt and the gauge and the thickness recorded. These data were later used for mapping element thickness to the shell elements of the FE model. Similar data for the Titian Stradivari is available, which was measured with a Hacklinger gauge [4.4].

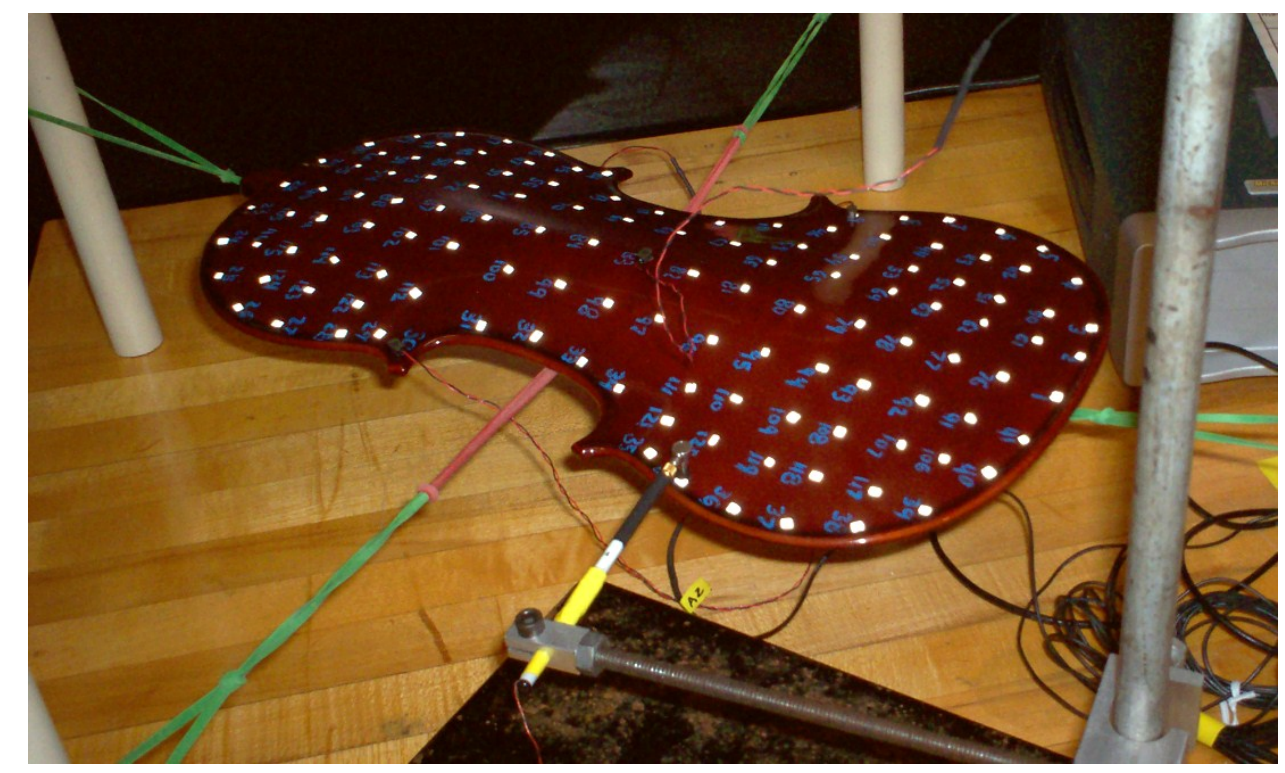

Figure 4.2: Back plate test setup 


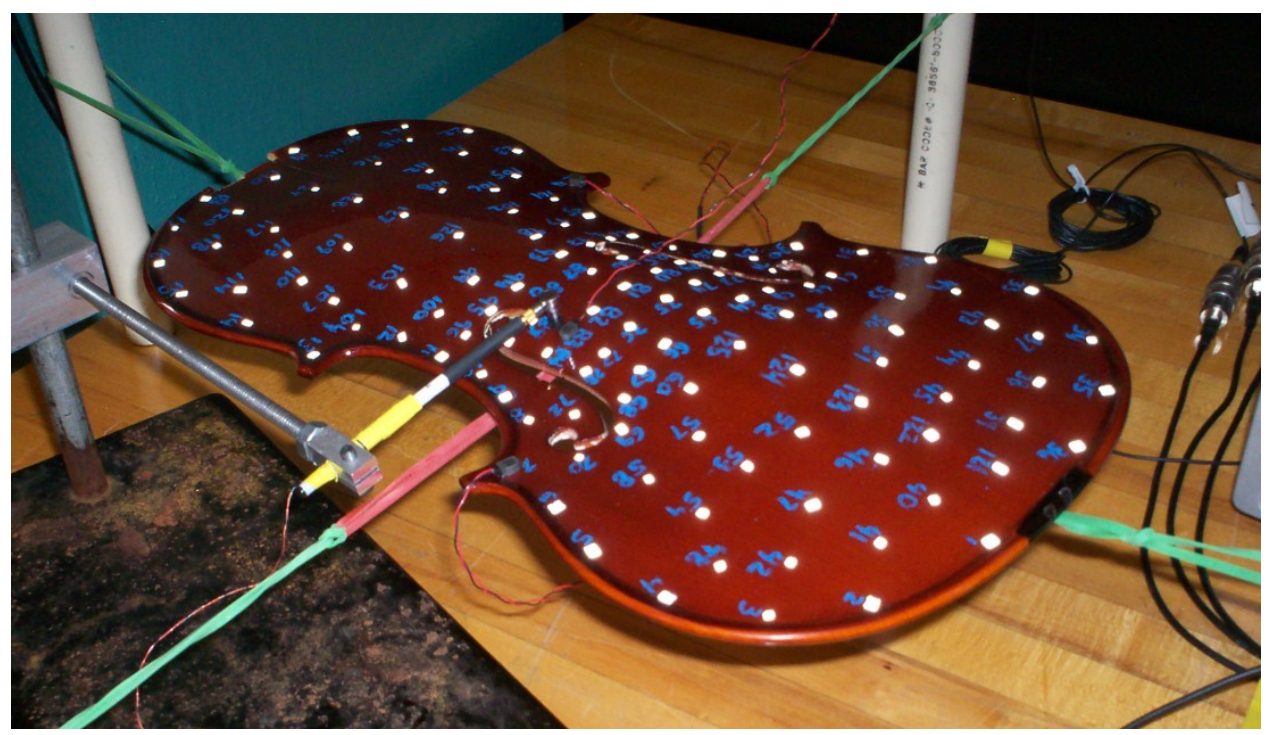

Figure 4.3: Top plate test setup

Once the plates and rib structure modal tests were completed, the corpus of the factory violin was reassembled. A total of 26 spool clamps were used. These were constructed from circular spools cut from pine, $4.5 "(114.3 \mathrm{~mm})$ long $1 / 4-20$ thread carriage bolts, and a thin cork backing. First, a layer of hide glue was lightly brushed onto the back edges of the rib structure, shown in Figure 4.4. The back plate was set in place and the spool clamps distributed around the edge as shown in Figure 4.5. Once the glue had set, the support frame was separated from the blocks and removed. The top plate was then glued and clamped in a similar fashion: shown in Figures 6 and 7. 


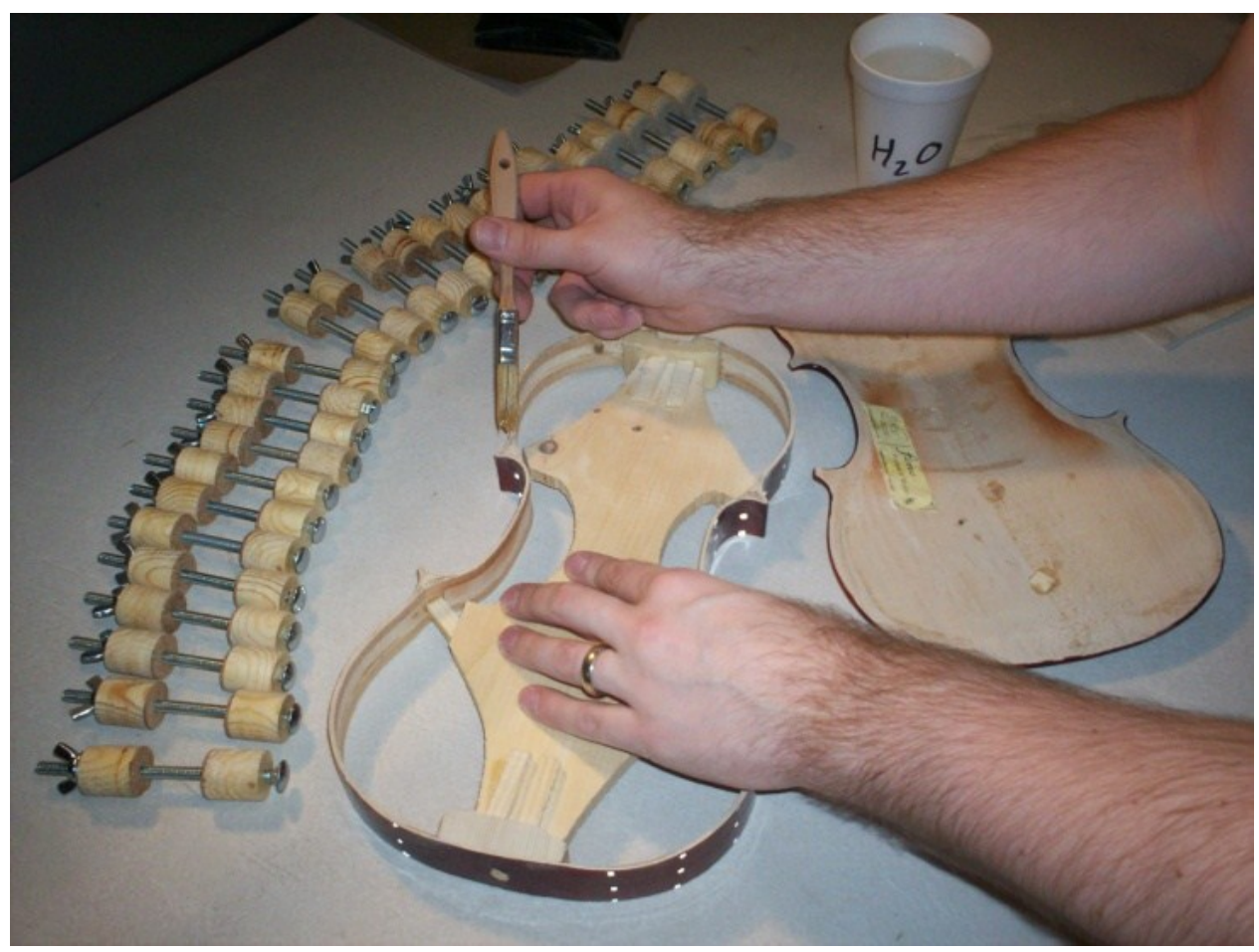

Figure 4.4: Applying hide glue to the back edges of the rib structure

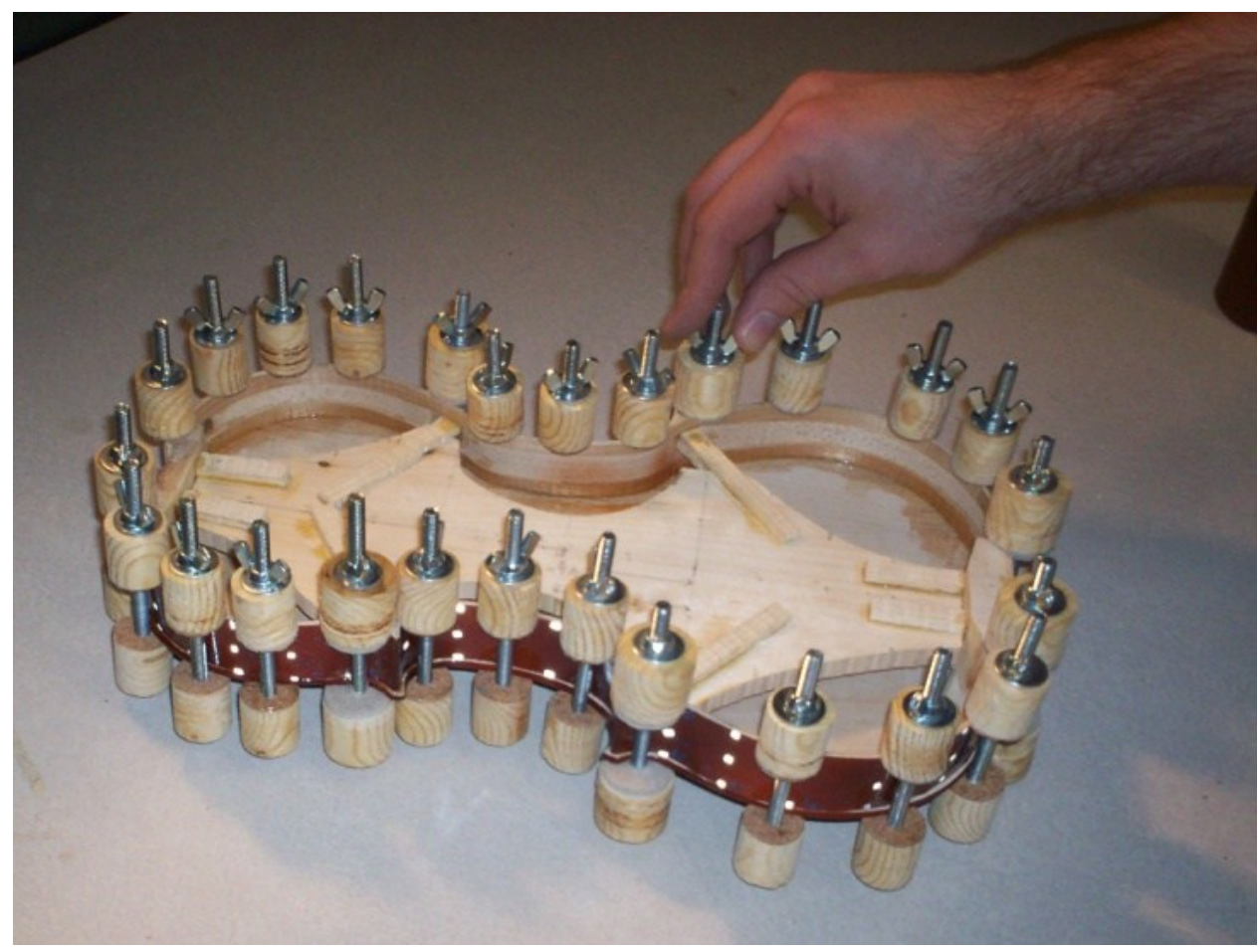

Figure 4.5: Clamping the back plate and rib structure together 


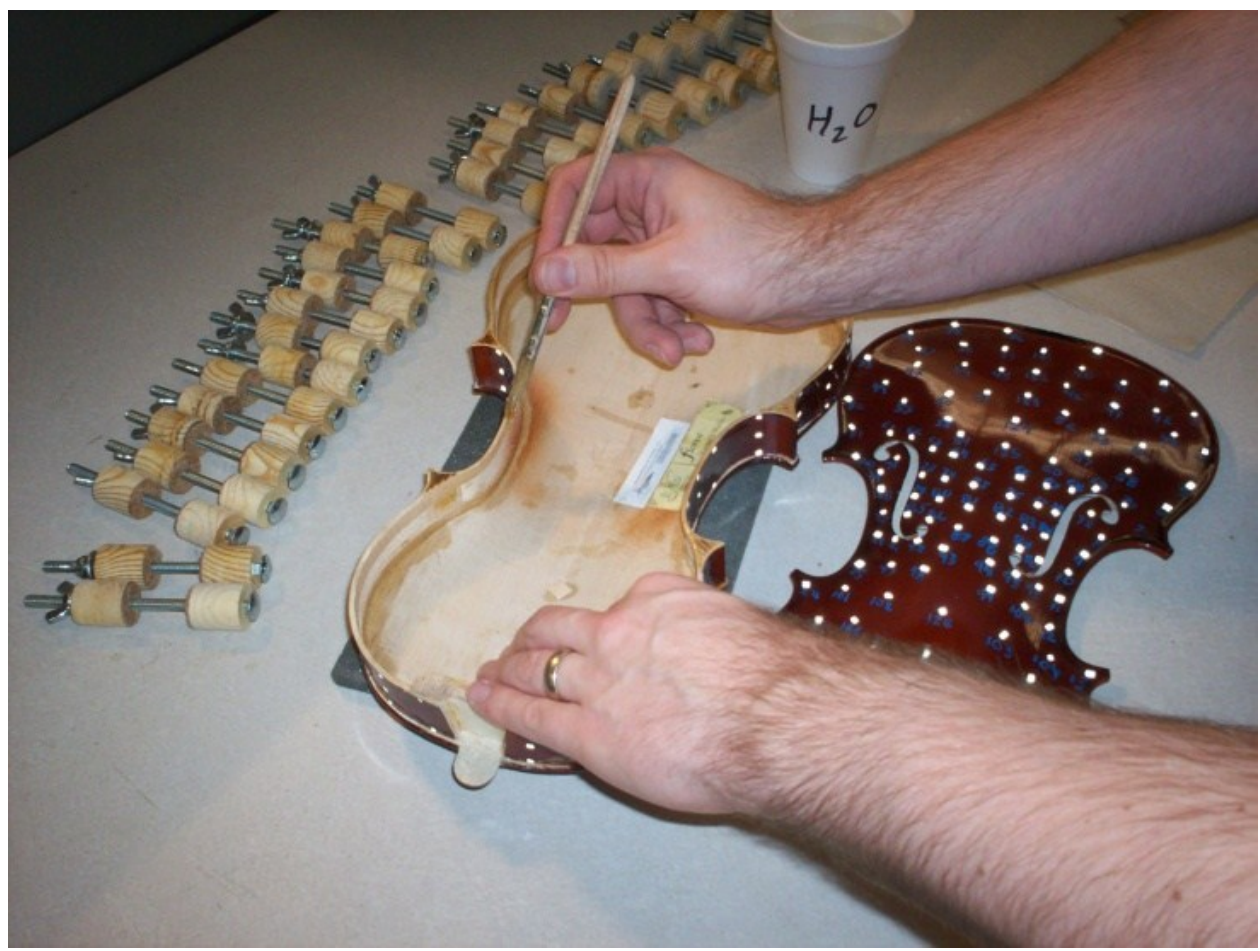

Figure 4.6: Appplying hide glue to the top edges of the rib structure

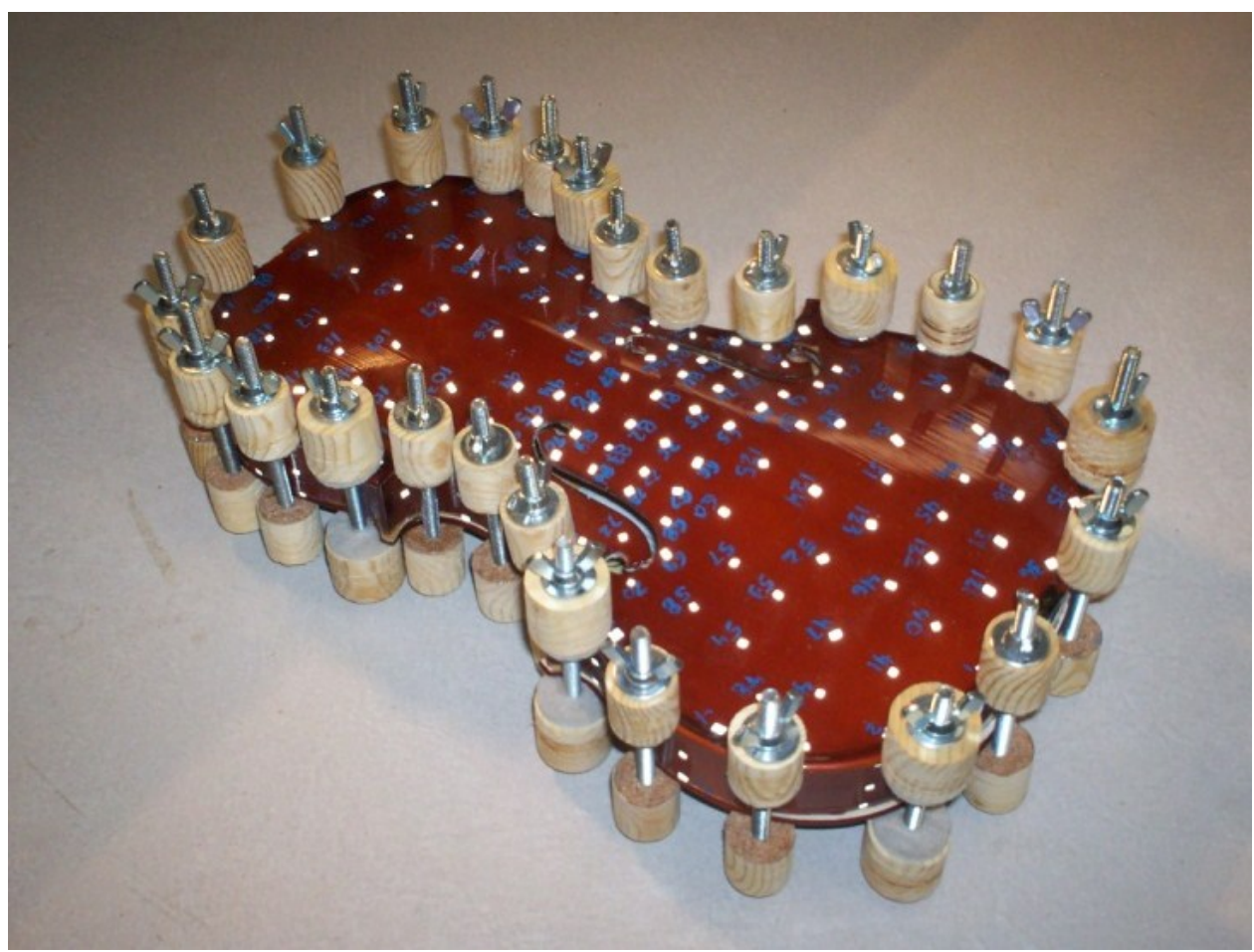

Figure 4.7: Clamping the corpus together

Michael Pyrkosz 
As soon as the top plate was set, the clamps were all removed and the sound post was inserted. To place the sound post into the same place as it was previously, the CT scans were used to measure the original sound post location relative to the nearest edges of the treble side f-hole in the X (lateral) and Z (longitudinal) directions. Once the sound post was set its position was checked with a depth probe on a dial caliper and adjusted according to these measurements.

Following the reassembly of the corpus a roving impact modal test was performed. For this test the structure was suspended in "free-free" boundary conditions using rubber bands. These rubber bands were connected to all four corners of the structure as shown in Figure 4.8.

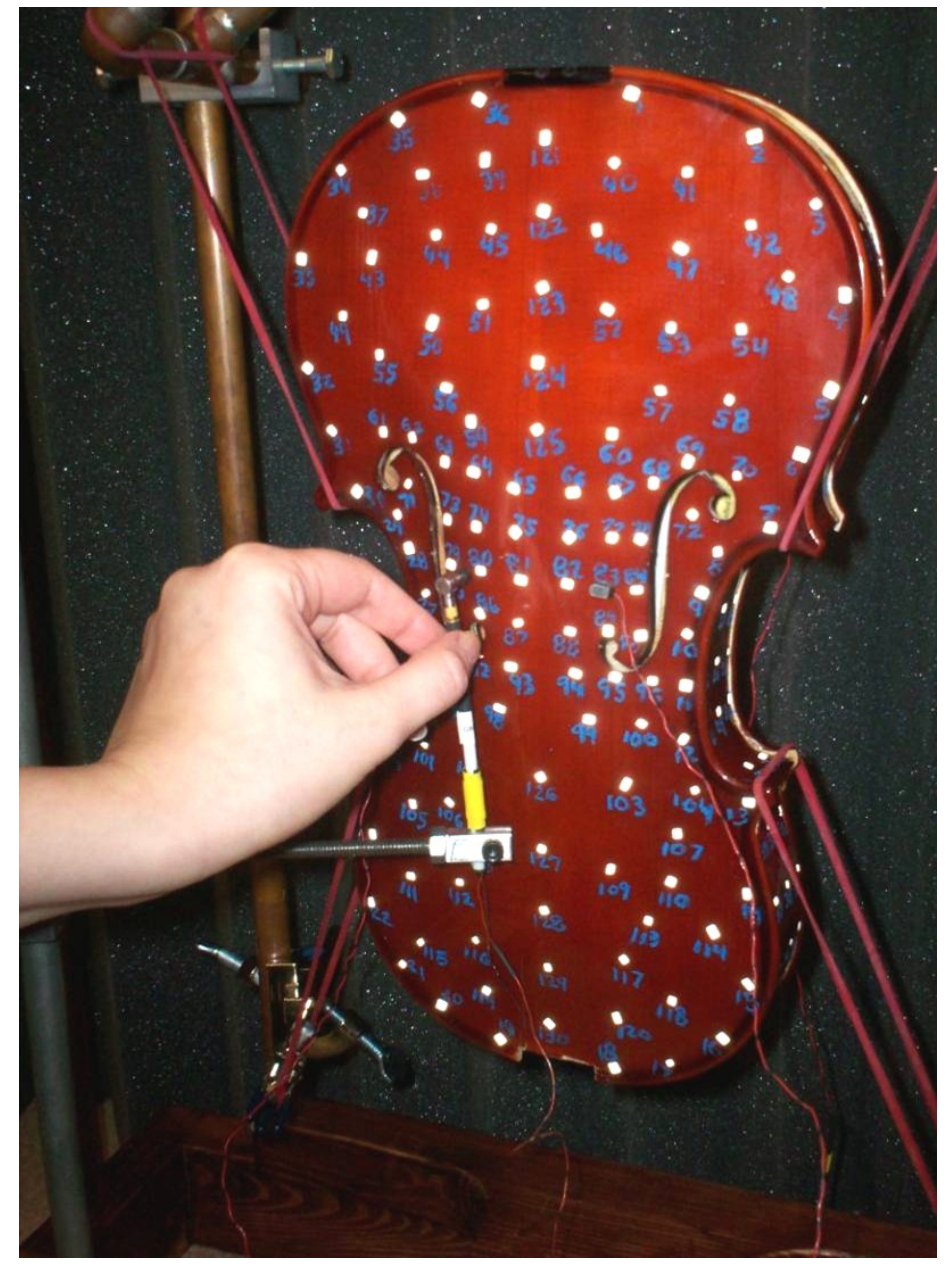

Figure 4.8: Test setup for the factory violin corpus 
After the corpus testing was completed the neck and scroll (still with the fingerboard attached) was reattached. An additional clamp block was made to fit over the shoulder of the neck and clamped to the corners of the corpus.

Once the neck was set, the violin was restrung and tuned. The instrument was allowed to "settle", meaning that the structure required time to reach static equilibrium as the strain introduced by the strings was distributed to the various joints and wood fibers. As this occurred, the strings relaxed slightly and the violin sound went flat. The violin was therefore retuned once a day for three days before it was close enough to static equilibrium that it would reliably remain in tune during the final tests. The driving point measurements from the original full violin model tests [4.5] were repeated to measure how much the factory violin changed from being rebuilt. As noted previously, the factory violin had been manufactured with a number of poorly glued joints. Attempts were made in the reassembly process to fit the pieces more tightly together; as such, it was expected that some of the structural modes would increase in frequency due to these joints being effectively stiffer.

\subsubsection{Curve fitting}

Development of an analytical model that reliably represents the dynamics of a physical test structure requires experimental data sets to validate the model parameters that define the analytical model.

A modal analysis provides a set of modal parameters that characterize the dynamic behavior of a structure. The modal measurement process, also referred to as Experimental Modal Analysis (EMA), is implemented to obtain Frequency Response Function (FRF) measurements of the structure from which the modal properties of the structure are determined. The modal properties include modal frequencies, corresponding damping, and corresponding mode shape information [4.6]. The structure's modal frequencies are defined as the natural decaying vibration of the structure due to a transient excitation force (free from external influences). Frequency damping is a term related to the decay time of a specific vibration frequency, and the mode shape is the structure's deflection profile for a specific frequency.

The fundamental problem of parameter estimation consists of estimating the parameters in the model, such that the data predicted by the model approximate the measured data. Modal parameters can be estimated using a number of techniques, both in the time domain and the frequency domain. The starting point is usually a set of measured data either Impulse Response (IR) in the time domain, or frequency response functions (FRFs) in the frequency domain. 
The data in this study was curve-fit using a variant of the Least Squares Frequency Domain method (LSFD).

\subsubsection{Model methods}

The basic modeling methods employed in this project were thoroughly discussed in an earlier chapter [4.1]. The following is a summary. Geometry and density information is taken from CT scans of the actual instrument. The CT images are taken through a CT interpretation program where the various parts are converted into $3 \mathrm{D}$ objects and surface meshed. The meshes are exported to a Finite Element (FE) format and edited in a FE preprocessor. These surface meshes envelope the original 3D objects computed by the CT interpretation software. For thin parts, such as the plates and ribs, the surface mesh is reduced to a single layer on the outer surface. For solid parts, such as the blocks, and the neck and scroll, the original surface mesh is filled with solid tetrahedral elements.

For the 2D shell meshes, the removal of one side of the surface leaves the edges somewhat jagged, since the original meshing algorithm was only constrained to the surface and not to a specific edge.

For the factory violin, the original mesh was used to select a sample of edge points that were then used to define a spline curve, which was used to define the edge of the new mesh. These spline curves were used to create flat CAD representations of the factory violin plates and were initially meshed. A set of sample nodes of the original mesh was used to describe the arching of each plate. Each point of the flat plate meshes were then interpolated on the 3-dimensional surface described by that sample data.

During the assembly process of the Titian Stradivari violin model some modification of the existing meshes was required, due to overlap between the substructure meshes. The smoothing operations that were performed on the individual component surface meshes (which were done to remove the voxel-ation of the volumes from the CT scans) had shifted the surface into one another. It was necessary to create common planes and surfaces to align meshes at interfaces between components. 


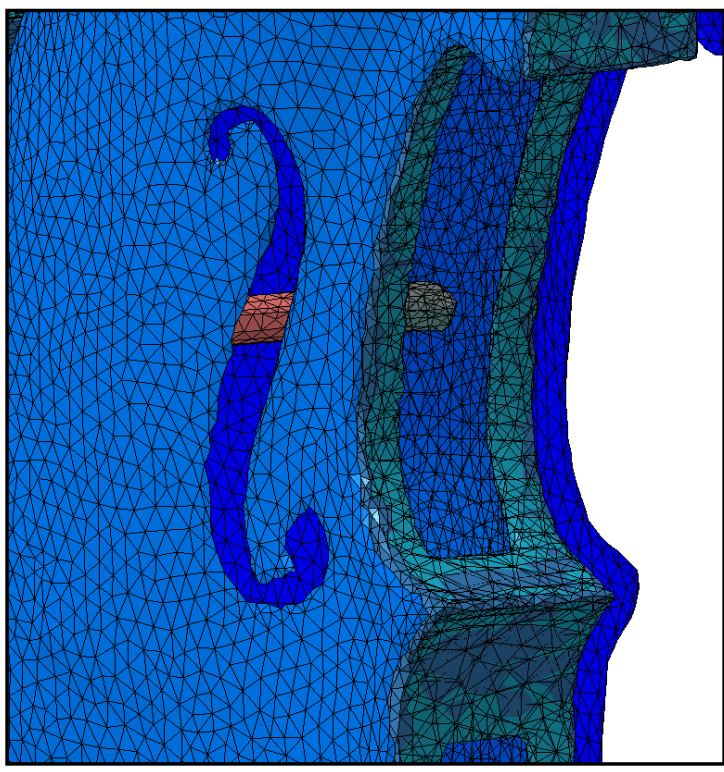

(a)

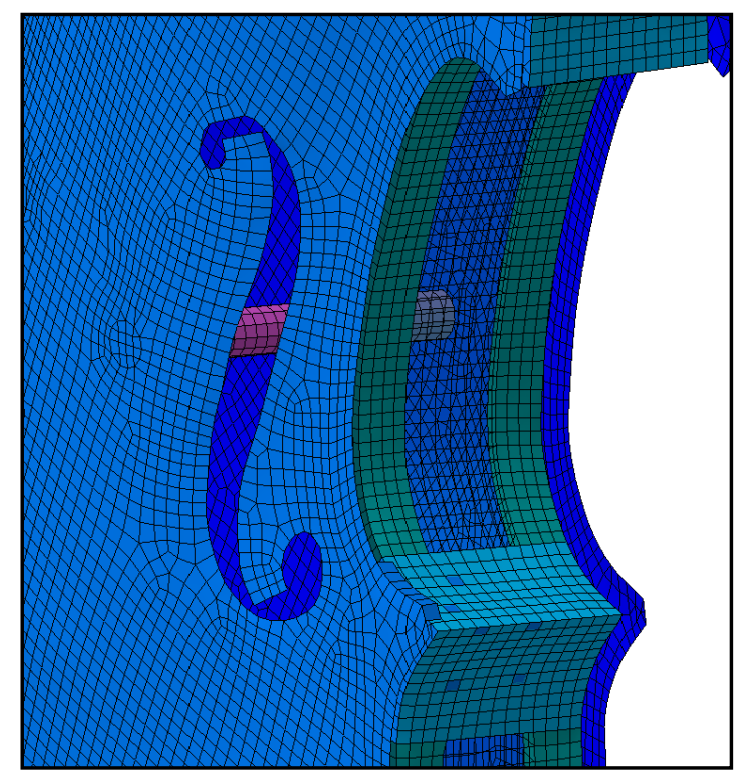

(b)

Figure 4.9: Comparison of the mid-treble region of the corpus (a) before and (b) after the remeshing

During this process the surfaces were re-meshed. The triangular elements were upgraded to quadrangular elements for improved performance. Tetrahedral elements used in the solid components (blocks, sound post, bass bar, neck and scroll) were replaced with hexdominant meshes. Figure 4.9 shows a side-by-side comparison of the mid-treble region of the corpus before and after the re-meshing process. Note that the edges around plates and f-holes are now smooth.

The issue with interface surfaces overlapping was handled in the following way: common planes between the parts were created using three existing nodes near the intersections; the nodes of each surface were aligned to these planes and remeshed. Again, triangular elements were replaced by quadrangular, and the elements at the interfaces were mapped such that each part would have coincident nodes. Figure 4.10 shows a comparison of the joint between the top end block and the base of the neck before and after this process.

Similarly, the interface surfaces between the neck, fingerboard, and nut are shown in Figure 4.11. Note the overlap that exists in the original mesh versus the corrected one. 


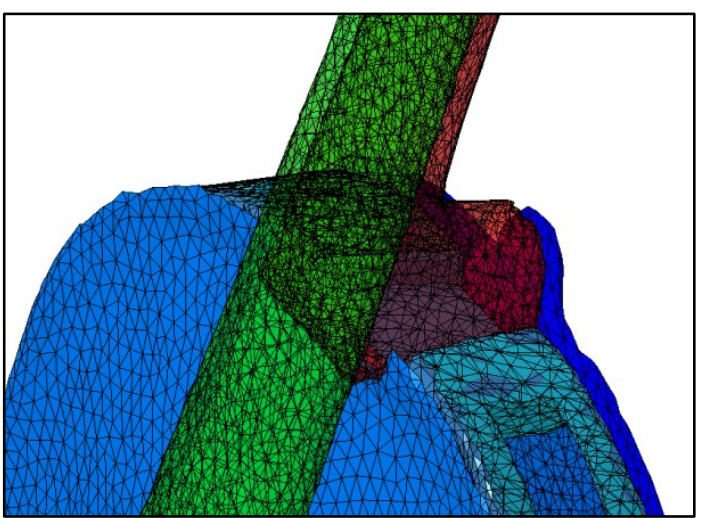

(a)

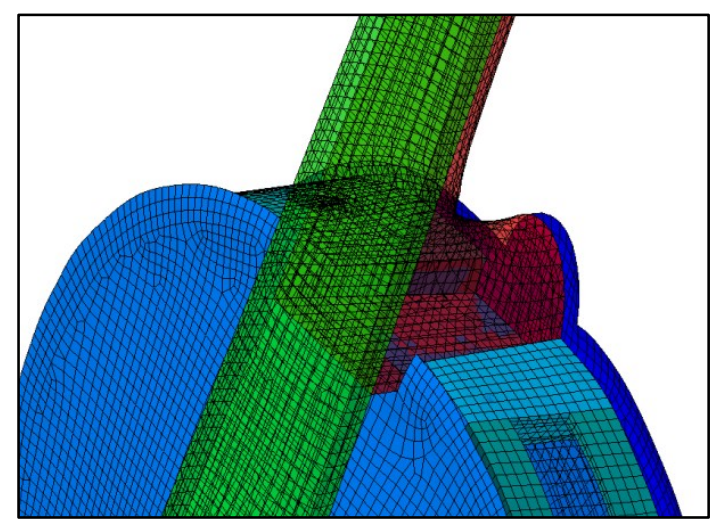

(b)

Figure 4.10: Comparison of the corpus-neck joint (a) before and (b) after remeshing

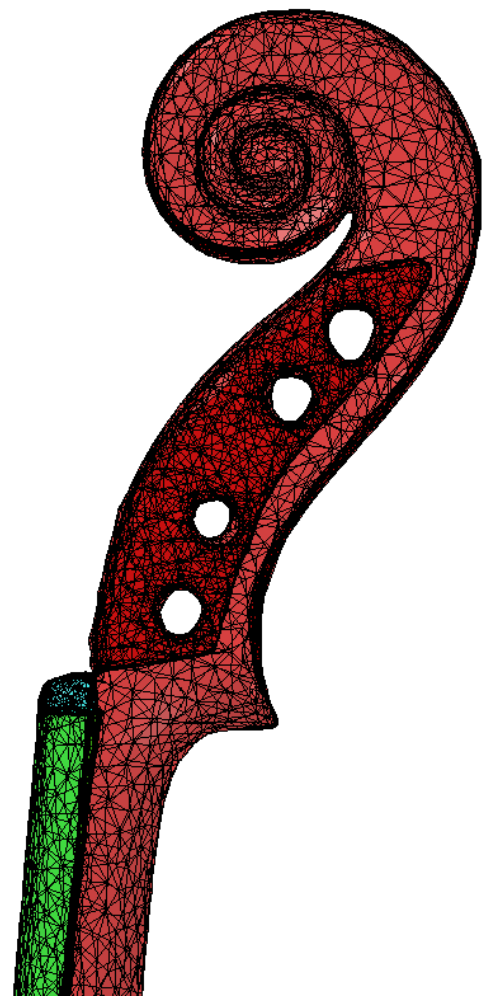

(a)

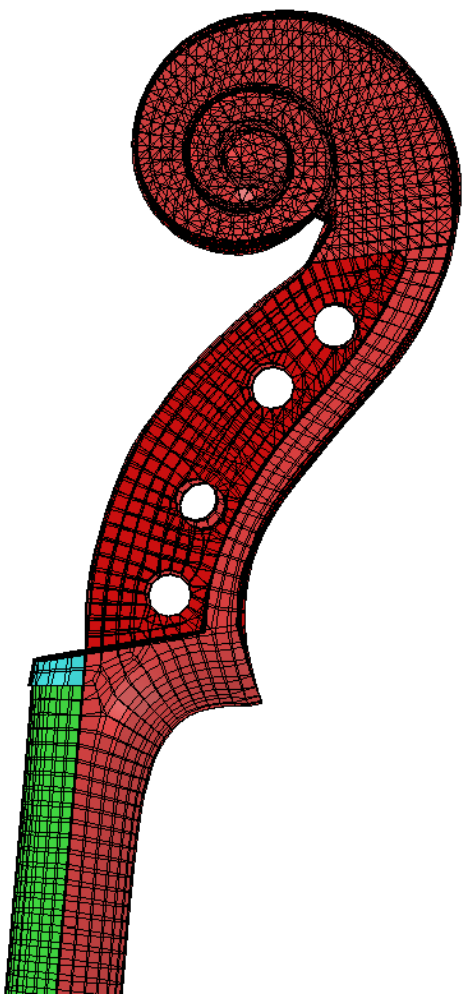

(b)

Figure 4.11: Comparison of the neck-fingerboard-nut interfaces (a) before and (b) after remeshing

Most of these issues were resolved using ANSA by BETA CAE Systems S.A. ANSA is an advanced preprocessing tool that is used for preparing meshes for Finite Element Analysis [4.7]. The original surface envelopes from MIMICS were imported into ANSA 
and re-meshed as "skins" for the various sub-volumes of the violin structure. In this way, shells could be upgraded to mostly quadrangular elements, and the skins could be used to map hexahedral dominant meshes for the solid volumes. A screen capture of the ANSA interface is shown in Figure 4.12.

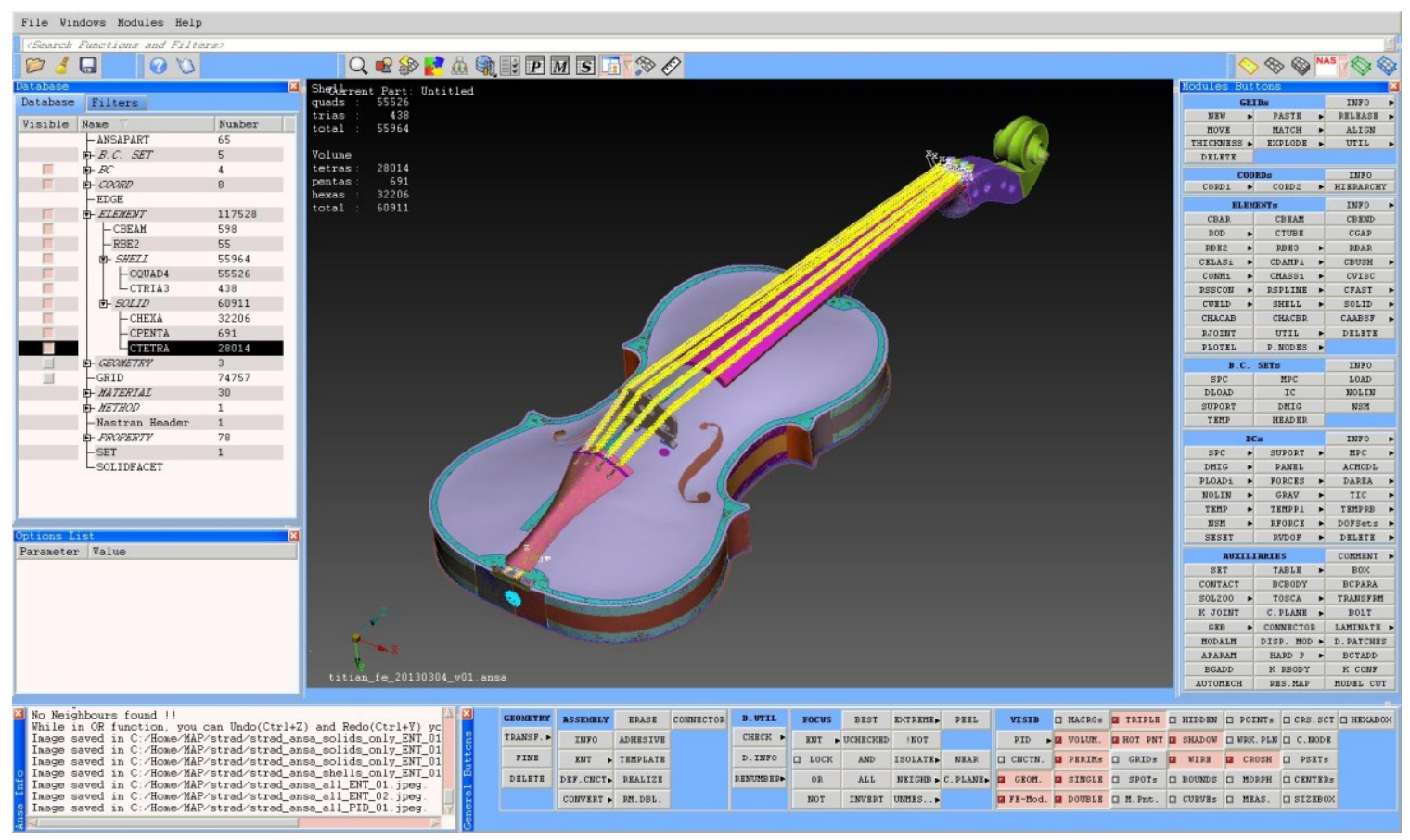

Figure 4.12: Screen capture of Titian Stradivari violin model remesh in ANSA 13.2.2

\subsubsection{Titian FE model with Pre-loaded strings}

Strings were modeled with a series of beam elements between the bridge and the nut (128 elements each) and between the bridge and the tail piece (20 elements each). The windings on the G, D, and A strings were modeled as non-structural mass. Properties for the beam elements were taken from those used in Knott's model [4.8]. Similarly, the tail loop is modeled as two lines of beam elements between the bottom of the tail piece and the saddle (3 elements each).

To apply tension in the strings a static preload subcase was included. At the top of each string a coincident node was added at the string-nut interface and connected with a zerolength rigid bar element (RBE). In this way a local axis system could be created for each string to orient the DOF of the RBE with that of the beams. The constraint in the axial direction was then excluded for each RBE. Initial preloads were applied in the axial direction at each of the beams, based on input from Fan Tao at D'Addario Strings [Ref: 
personal communication with Fan Tao, 3/4/2011]. After an initial run, these tension preloads were adjusted to "tune" the stings.

\subsubsection{Correlation methods}

\subsubsection{Modal Assurance Criterion}

Ensuring reliable validity of an FE model is essential for realistic noise and vibration simulations of penetrator systems. This requires that component, subsystem, and fullsystem models be compared to experimental data or to validated models of similar structures. One common tool for comparing and validating an FE model with experimental data is the Modal Assurance Criterion (MAC).

The modal assurance criterion is a statistical indicator that measures the degree of relationship between a pair of vectors [4.9]. These vectors can be the Modal vectors (normal or complex mode shapes) or Response vectors from a Forced Response Case for instance. The MAC values are normalized by the magnitude of the vectors, and thus always lie between 0 and 1, where values near 1 indicate consistency between the vectors, while values near 0 indicate the vectors are not consistent [4.9]. Generally, reasonable values of MAC are 0.7 whereas good correlation is above 0.9 [4.6].

\subsubsection{Modification prediction}

A common problem when comparing experimental modal data with an analytical model is differences in mass-loading effects of accelerometers in the test data. For this reason, modal-based modification prediction (also known as structural dynamic modification) is used to estimate the effects of the accelerometer masses if they were applied to the FE model.

Modal-based Modification Prediction makes it possible to estimate the impact of a small structural change to the modal behavior of a structure. Starting from a mode set (mode shapes and frequencies) of a system, a new mode set can be computed for a modified system, consisting of the original system and a few small structural changes. It should be noted that the two most significant sources of errors associated with this method are often the lack of rotational degrees-of-freedom (DOF) and the truncation of the modal database [4.10]. Although the FE model does contain rotational DOF, the mass-modifications are treated as point masses, and rotational inertia effects of the accelerometers are considered negligible. To reduce truncation errors the maximum frequency in the modification prediction is increased by a factor of two. 


\subsection{Results}

The experimental, analytical, and correlation results for the major substructures of the factory violin are presented here. This is followed by those of the Titian Stradivari violin.

\subsubsection{Back plate results}

\subsubsection{Experimental Results for the back plate}

For the back plate a roving hammer modal test was performed using three reference accelerometers. These were miniature uniaxial ICP accelerometers (PCB Model 352A73, $\sim 0.3$ grams each). Driving point locations for the reference accelerometers were selected with the foreknowledge of typical violin plate modes. For many of the mode shapes the most active areas of the plate are around the corners. The first mode is always torsion, in which all four corners are fairly active. The next few modes alternate between the upper and lower corners being active or near nodal lines. It is important to have at least one reference active for each mode shape, otherwise a mode may be missed entirely. For this reason the first two accelerometers were placed on opposite corners of the back plate, one on the lower-bass corner (back:9), and one on the upper-treble corner (back:30). The third accelerometer was placed near the center (back:83), to account for the "ring" mode (usually mode 5 for back plate) the nodal lines of which are often extremely close to the corners. The modal impact hammer (PCB Model 086E80) was roved over 125 points on the back plate.

The driving point FRF measurements are shown in Figure 4.13. The first peak $(126 \mathrm{~Hz})$ occurs in both the upper (green) and lower (red) corner measurements while the center (blue) measurement shows no motion; this is expected for the torsion mode. The next few peaks alternate between the upper and lower corner measurements with the exception of the fifth peak in which the center is the most active, indicating the ring mode.

The coherence measurements for the driving points are shown in Figure 4.14. The only significant drops in coherence occur near anti-resonances. For the rest of the frequency range the coherence remains well above 0.95 , indicating good consistency.

The Digital Signal Processing (DSP) parameters for the back plate experimental modal analysis are summarized in Table 4.1 . 
Table 4.1: DSP parameters for factory violin back plate EMA

\begin{tabular}{|l|l|}
\hline Averaging & 5 Averages, Linear \\
\hline Bandwidth & $4096 \mathrm{~Hz}$ \\
\hline Frequency Resolution & $1.0 \mathrm{~Hz}$ \\
\hline Reference Window & Force-Exponential, 100\% \\
\hline Response Window & Exponential, 100\% \\
\hline
\end{tabular}

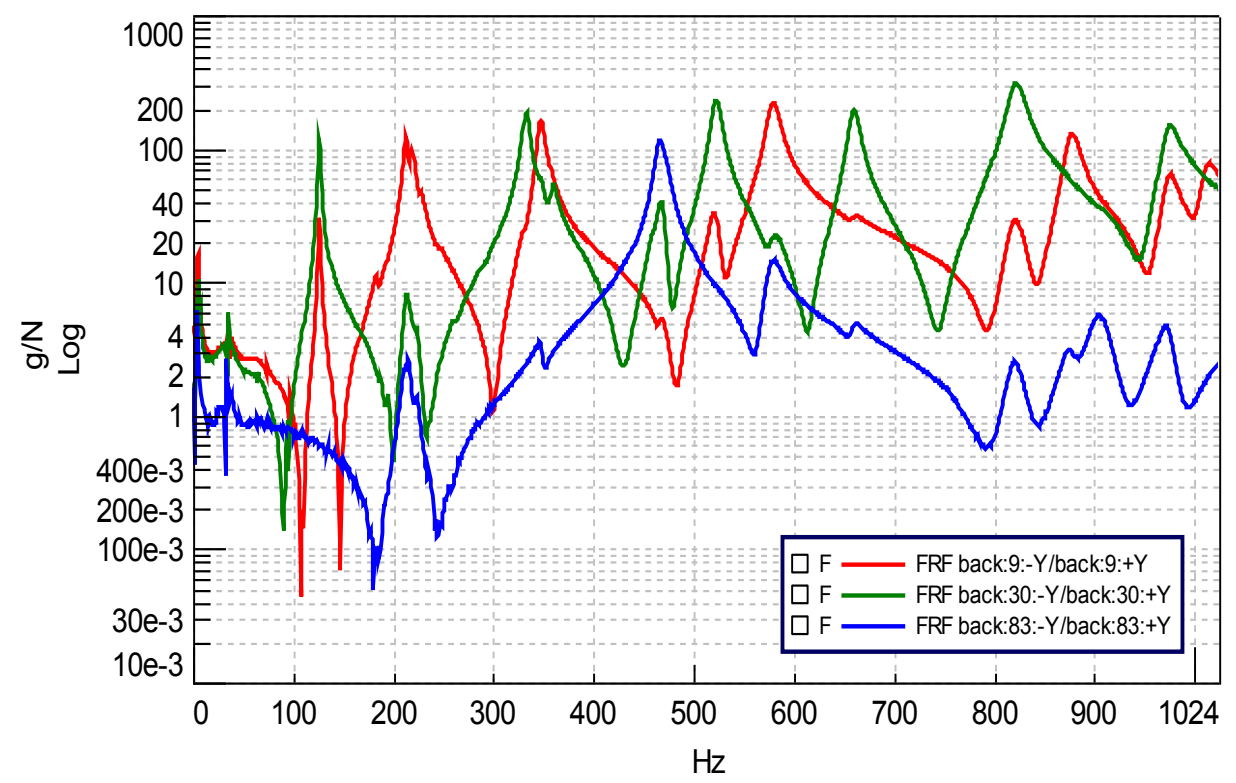

Figure 4.13: Driving Point FRFs of the factory violin back plate 


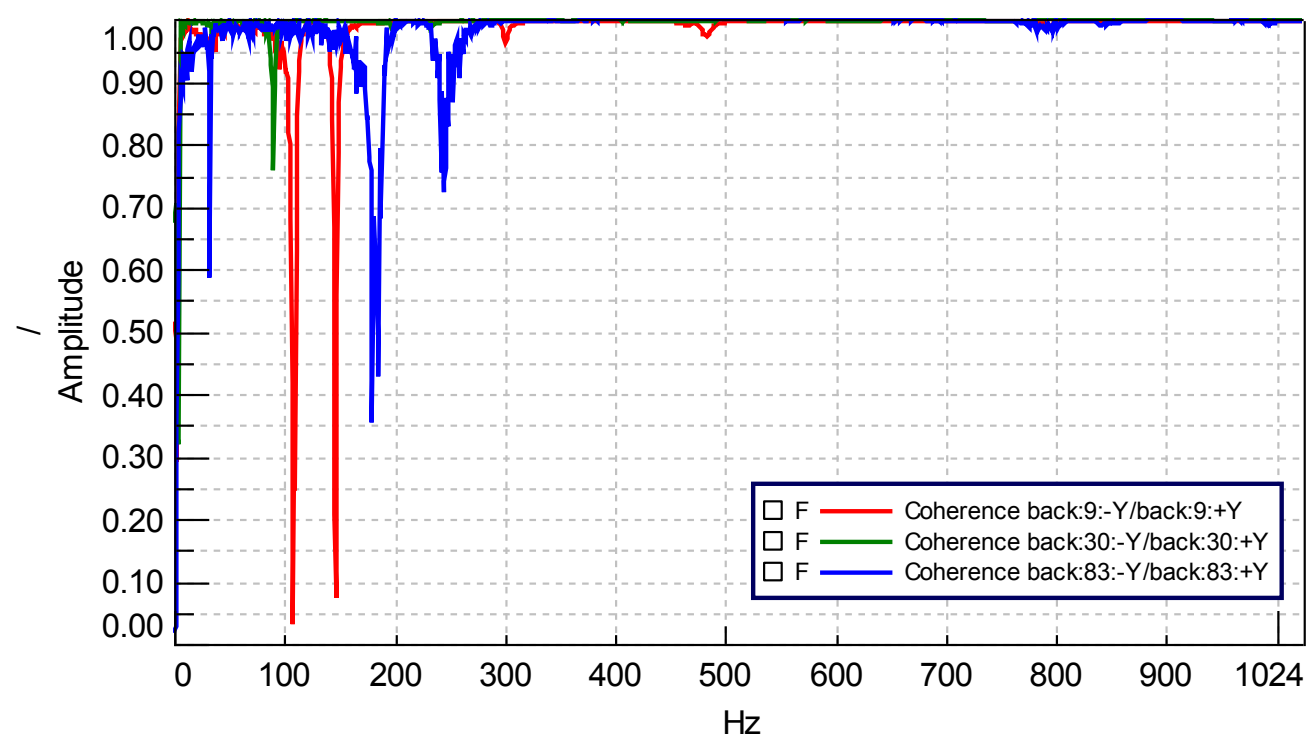

Figure 4.14: Driving point coherence functions of the factory violin back plate

Curve fitting of the back plate modal data was performed using LMS Test.Lab PolyMAX. Several consecutive frequency bands from $72.3 \mathrm{~Hz}$ to $1120 \mathrm{~Hz}$ were selected for stabilization. The Mode Indicator Functions (MIFs) used for modal parameter selection are shown in Figure 4.15. The red curve is the FRF sum, while the blue, magenta, and cyan curves are the Multivariant MIFs. The Automatic Modal Parameter Selection (AMPS) feature was used. 


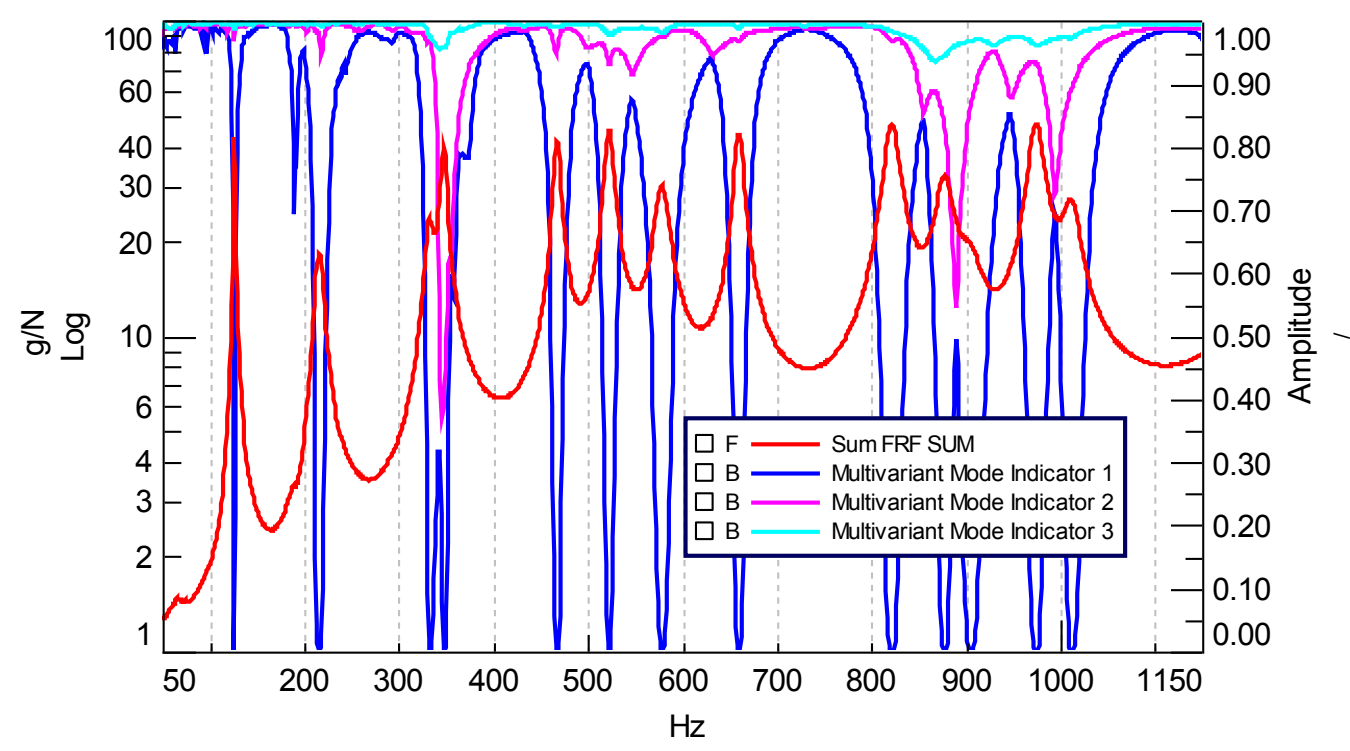

Figure 4.15: Mode indicator functions for curve fitting the factory violin back plate experimental data

Table 4.2: Curve-fitting results from the factory violin back plate EMA

\begin{tabular}{|c|c|c|l|}
\hline Mode \# & $\begin{array}{c}\text { Frequency } \\
(\mathbf{H z})\end{array}$ & $\begin{array}{c}\text { Damping } \\
(\mathbf{\%})\end{array}$ & Description \\
\hline 1 & 126.1 & 0.66 & $1^{\text {st }}$ Torsion \\
\hline 2 & 215.5 & 1.76 & Longitudinal bending (X-mode) \\
\hline 3 & 334.5 & 1.21 & Triad \\
\hline 4 & 347.8 & 0.92 & $2^{\text {nd }}$ Torsion \\
\hline 5 & 467.2 & 0.87 & Ring (O-mode) \\
\hline 6 & 522.5 & 0.92 & \\
\hline 7 & 578.2 & 1.24 & \\
\hline 8 & 659.7 & 0.80 & \\
\hline 9 & 821.4 & 1.06 & \\
\hline 10 & 877.0 & 0.84 & \\
\hline 11 & 904.6 & 1.18 & \\
\hline 12 & 973.8 & 0.87 & \\
\hline 13 & 1011.7 & 0.87 & \\
\hline
\end{tabular}

The modal frequency and damping results are given in Table 4.2. These are followed by the first five experimental mode shapes in Figures 4.16 through 4.20. The color scale indicates the absolute displacement (red indicating large displacement and blue indicating no displacement).

Michael Pyrkosz 


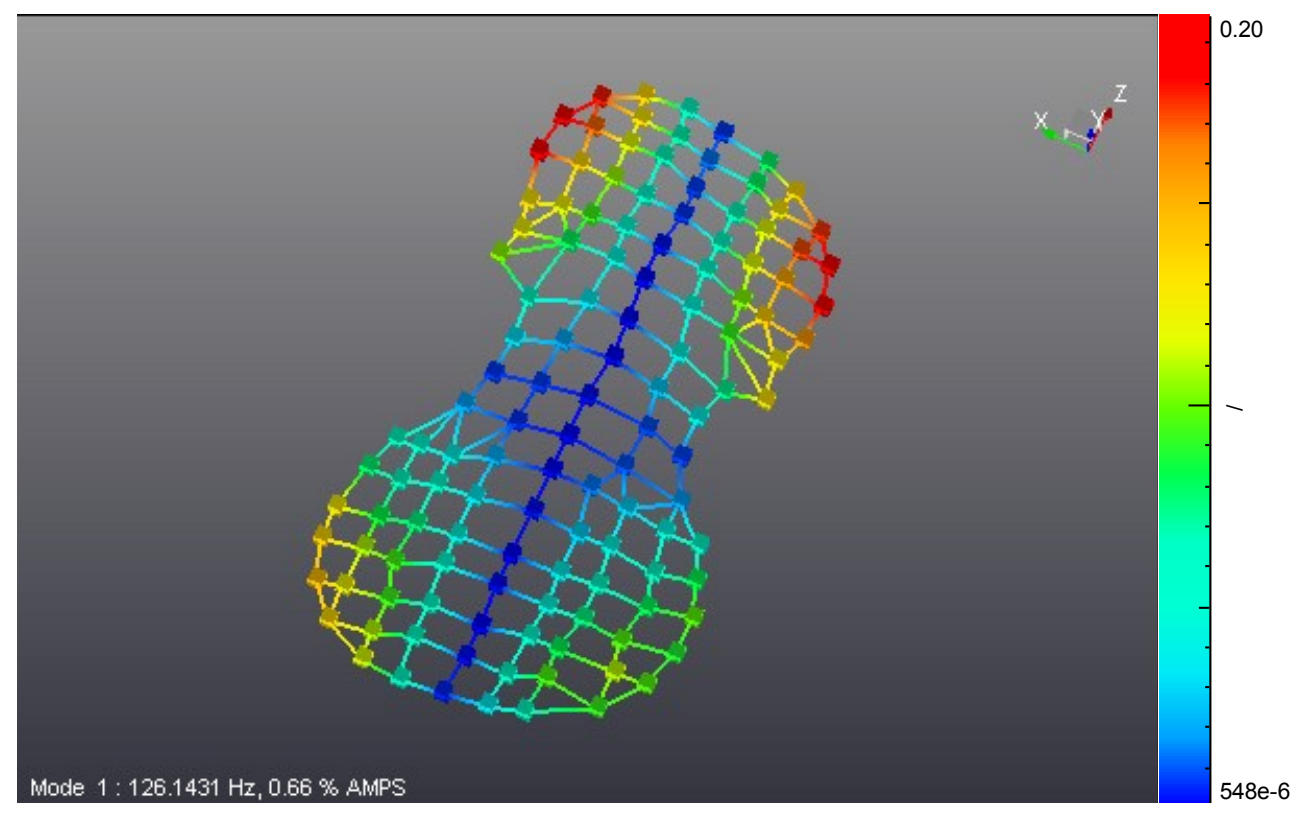

Figure 4.16: First experimental mode of the factory violin back plate

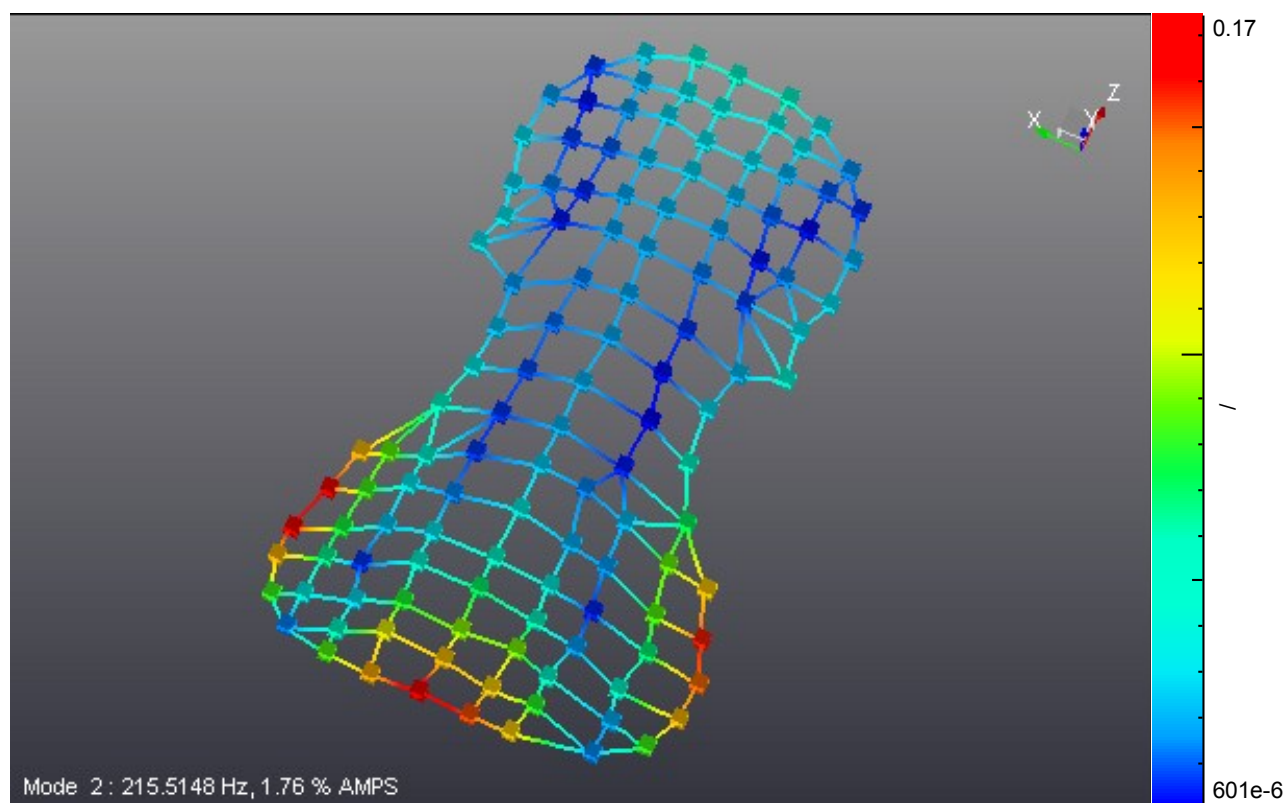

Figure 4.17: Second experimental mode of the factory violin back plate 


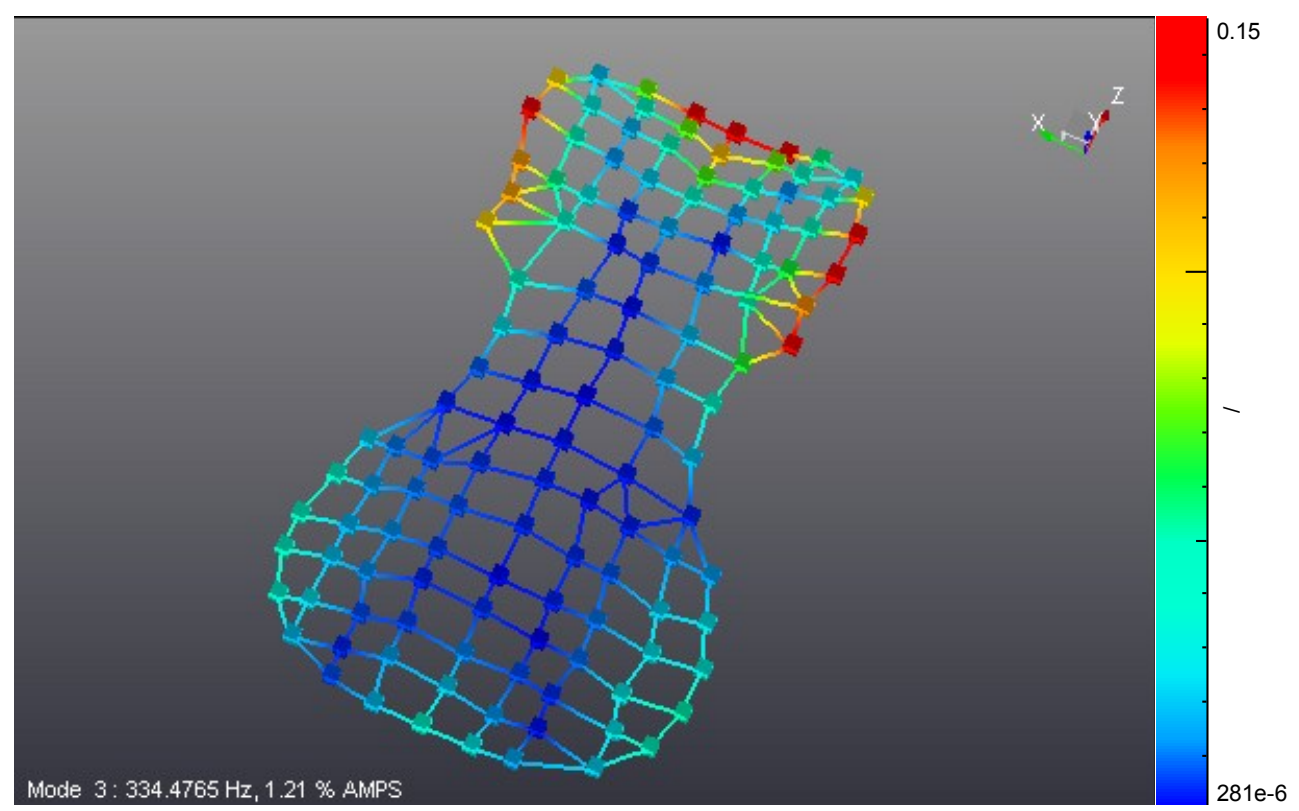

Figure 4.18: Third experimental mode of the factory violin back plate

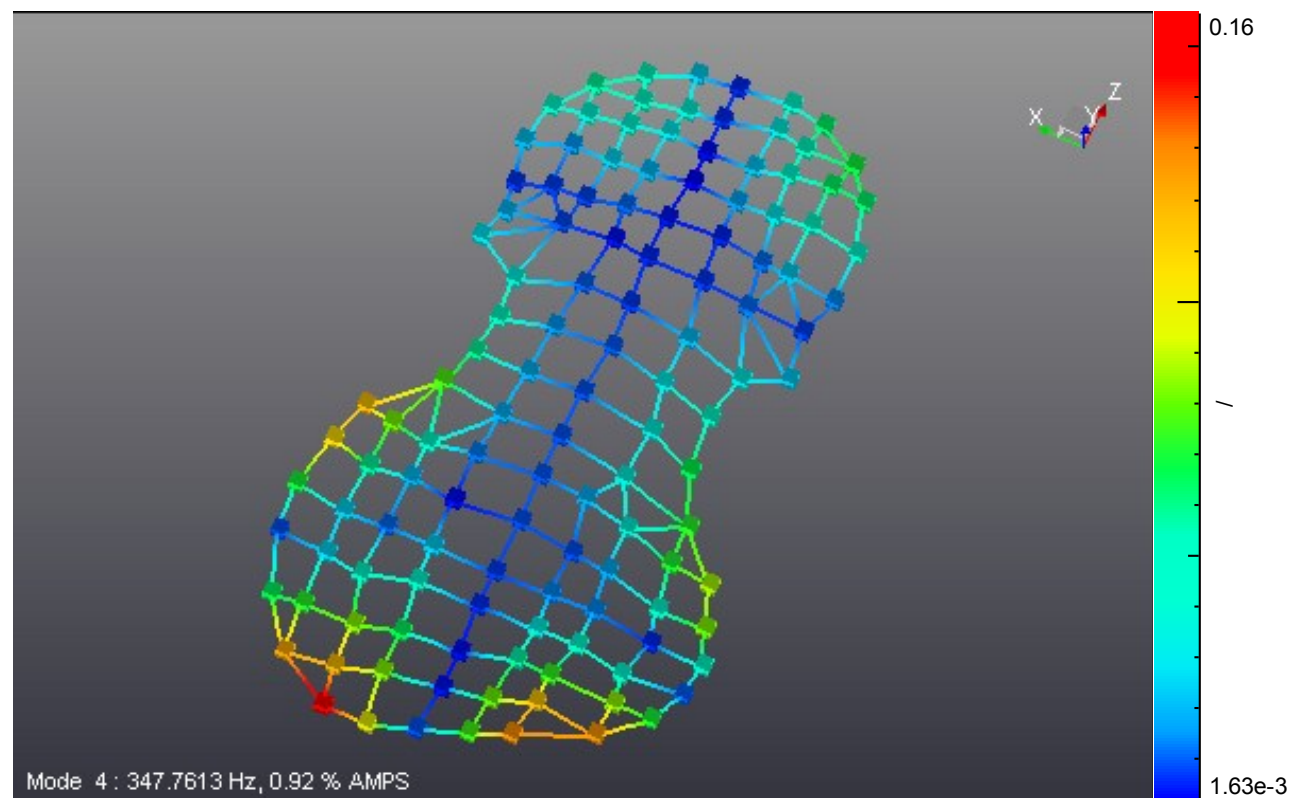

Figure 4.19: Fourth experimental mode of the factory violin back plate 


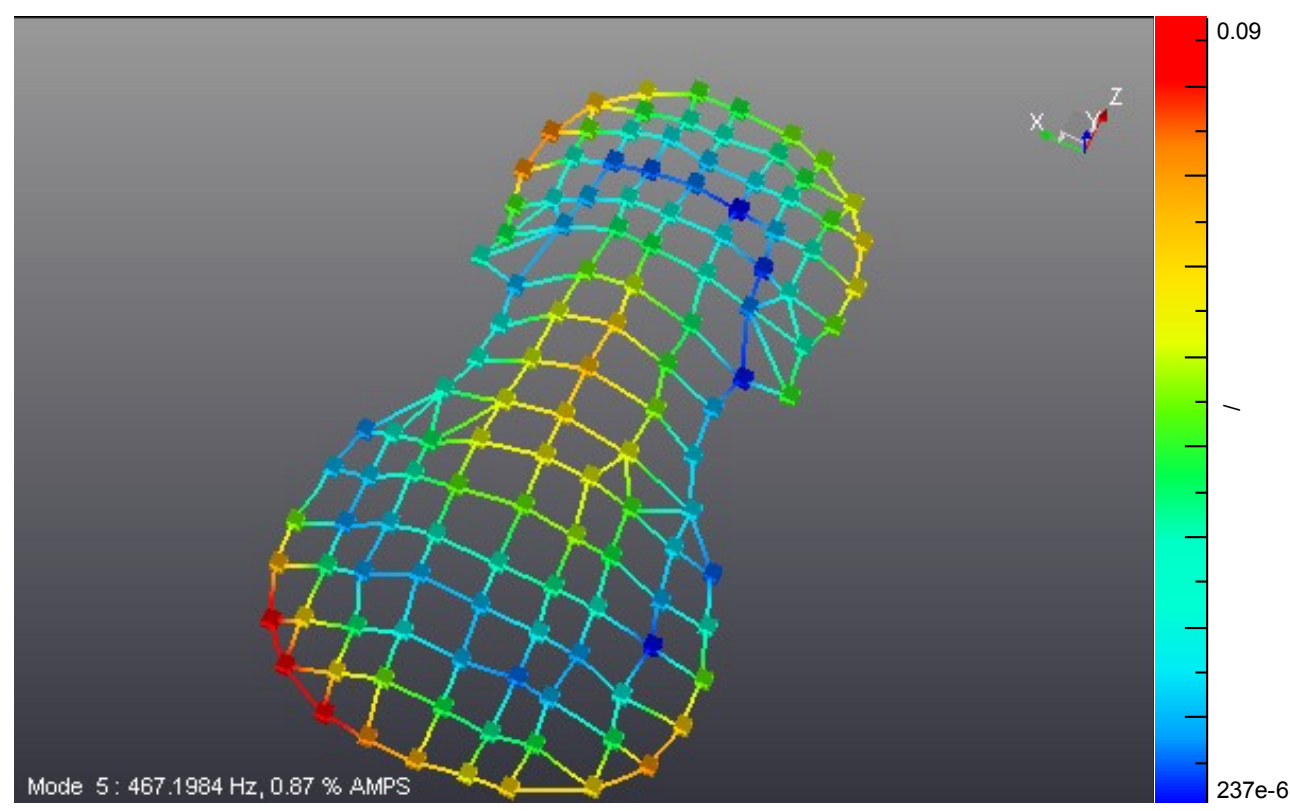

Figure 4.20: Fifth experimental mode of the factory violin back plate

Modification prediction was used to remove the effect of the 0.90 grams of the three accelerometers. It should be noted that the modification prediction method only accounts for the translational mass, while rotational inertias are neglected. This had the effect of raising some of the modal frequencies as much as $1.5 \%$ depending on the shape.

\subsubsection{Analytical results for back plate}

The factory violin back plate structural FE mesh contains 2810 nodes and 5404 linear triangular elements. Figure 4.21 shows the final factory violin back plate FE mesh. 


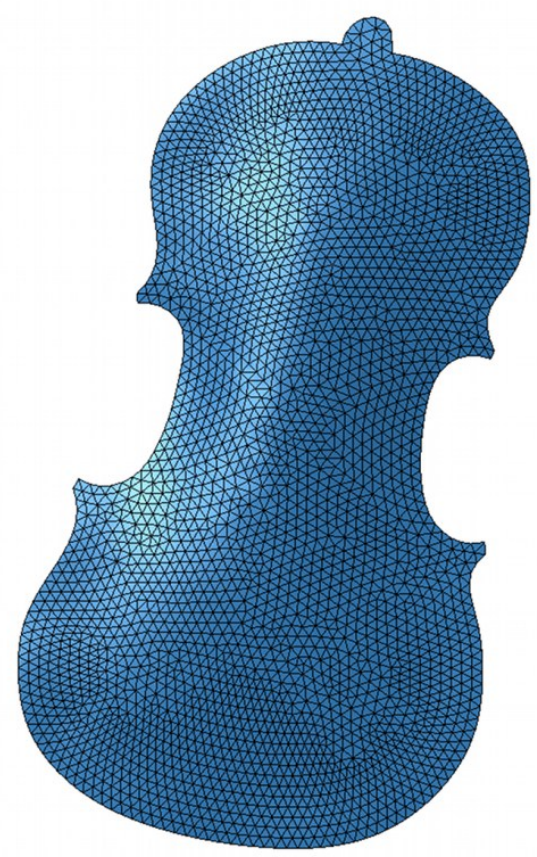

Figure 4.21: Factory violin back plate FE mesh

The initial model assumed distributed thicknesses based on the gauge measurements. The thickness was interpolated at every node location. The results of the thickness mapping are shown in Figure 4.22. During the updating process, it was determined that for the back plate, a uniform thickness mesh (using the average of the measurements) yielded better results. Several iterations were performed on both versions of the model to "tune" the material properties to better match the modal frequencies. 

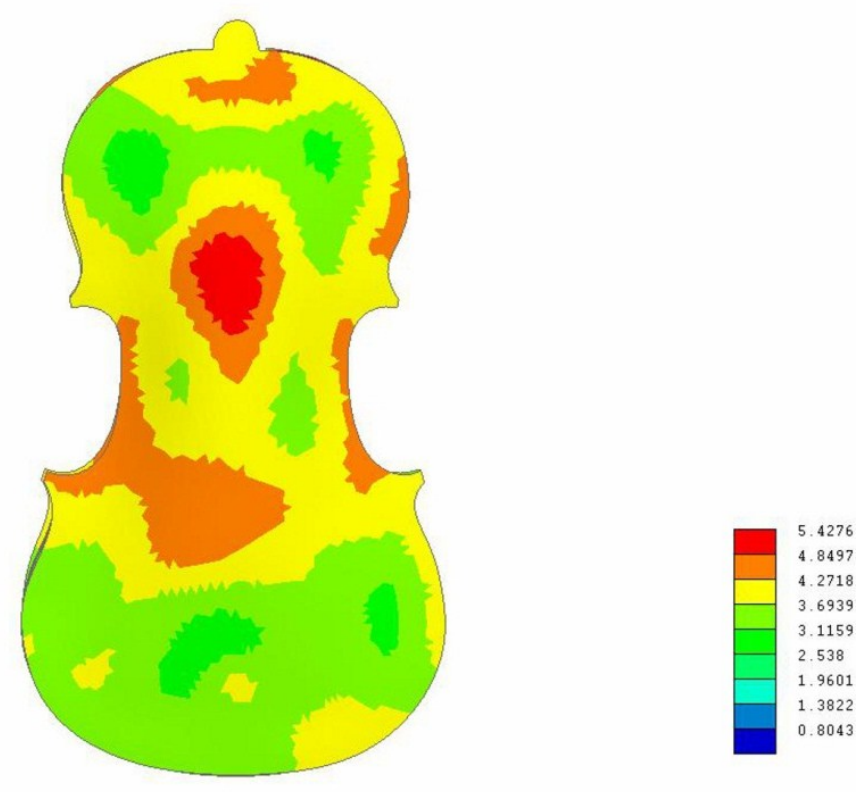

Figure 4.22: Element thickness of the factory violin back plate in $\mathrm{mm}$

The final FE model normal modes analysis results are summarized in Table 4.3. Examples of the first six FE mode shapes are shown in Figures 4.23 through 4.28.

Table 4.3: Summary of final factory violin back plate normal modes analysis

\begin{tabular}{|c|c|l|}
\hline Mode \# & Freq $(\mathrm{Hz})$ & Description \\
\hline 1 & 120.8 & $1^{\text {st }}$ Torsion \\
\hline 2 & 214.5 & Longitudinal Bending (X-mode) \\
\hline 3 & 350.6 & Triad \\
\hline 4 & 351.6 & $2^{\text {nd }}$ torsion \\
\hline 5 & 441.7 & Ring (O-Mode) \\
\hline 6 & 535.2 & Upper bout torsion with Lower bout 2nd bending \\
\hline 7 & 611.0 & \\
\hline 8 & 672.1 & \\
\hline 9 & 769.7 & \\
\hline 10 & 877.2 & \\
\hline 11 & 905.6 & \\
\hline 12 & 1036.8 & \\
\hline
\end{tabular}




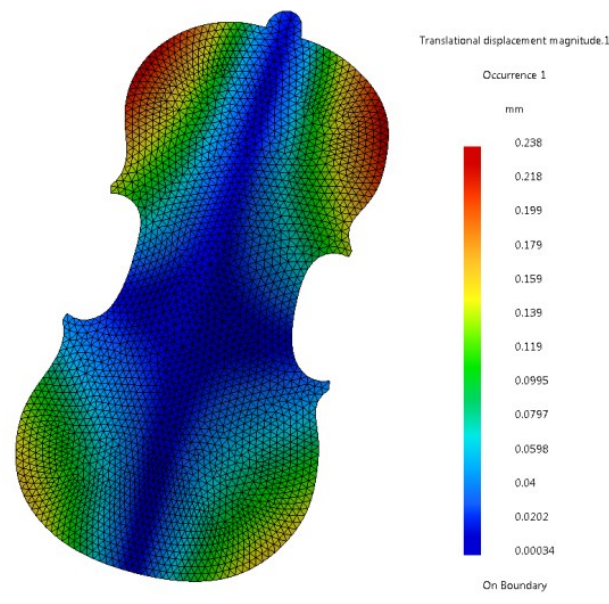

Figure 4.23: Mode 1 of the factory violin back plate (first torsion)

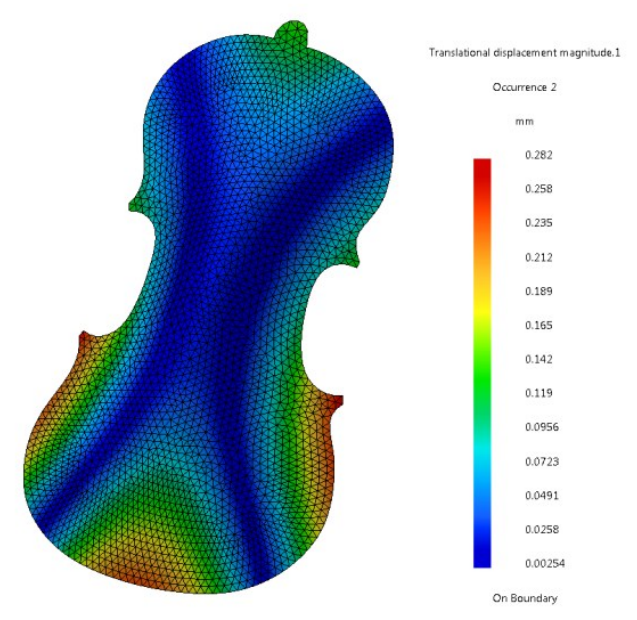

Figure 4.24: Mode 2 of the factory violin back plate (longitudinal bending)

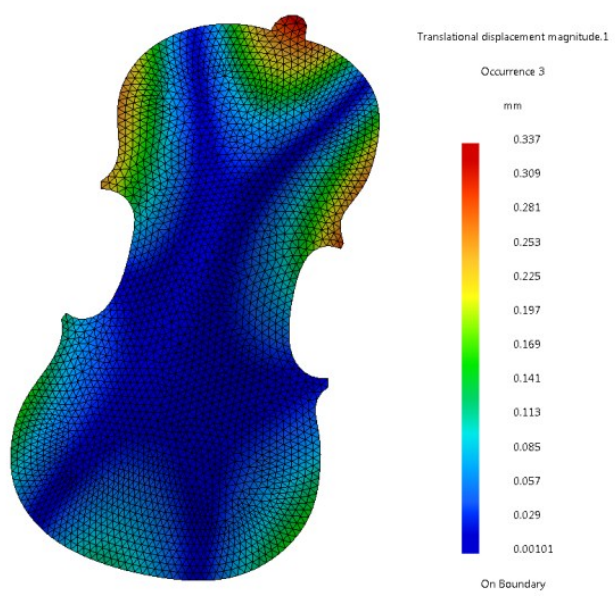

Figure 4.25: Mode 3 of the factory violin back plate (triad)

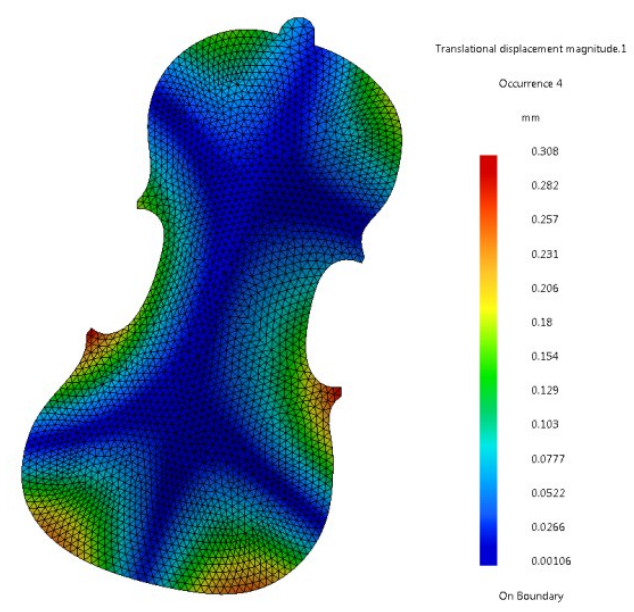

Figure 4.26: Mode 4 of the factory violin back plate (second torsion) 


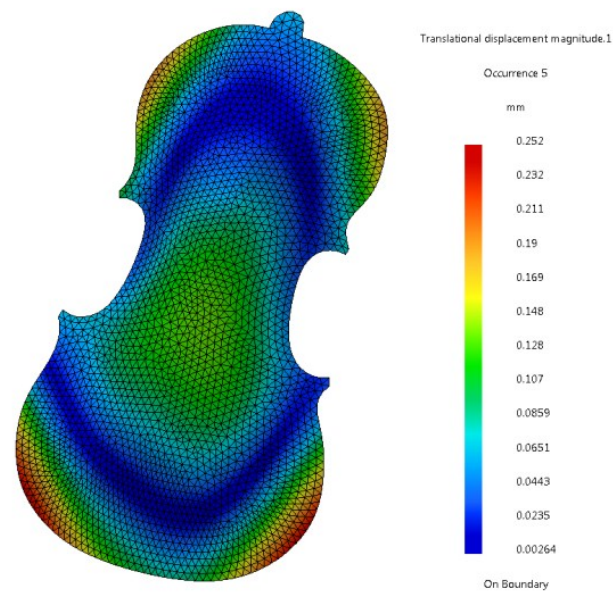

Figure 4.27: Mode 5 of the factory violin back plate (ring)

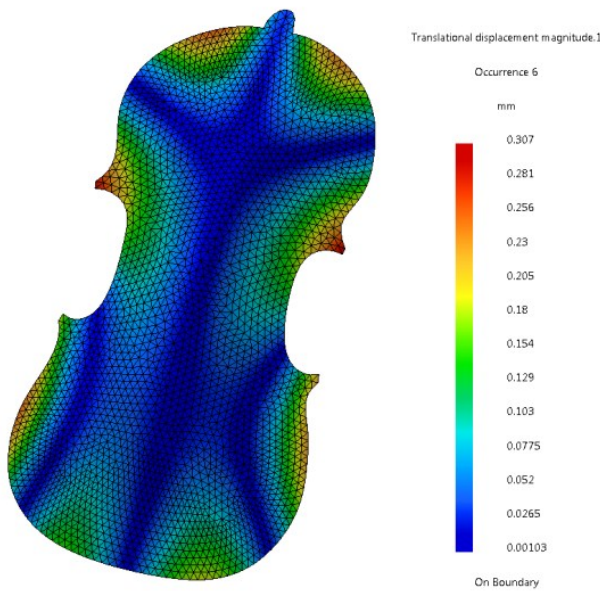

Figure 4.28: Mode 6 of the factory violin back plate (upper torsion-lower 2nd bending)

\subsubsection{Correlation results for back plate}

Interestingly, a uniform thickness back plate model correlated more closely with the experimental data. Correlation results for both versions of the factory violin back plate model are presented here. Figure 4.29 shows a plot of the final MAC matrix results for the correlation between the test and the original distributed thickness model, while Figure 4.30 shows that of the correlation with the uniform thickness model. The corresponding Mode pair tables are given in Tables 4.4 and 4.5. 


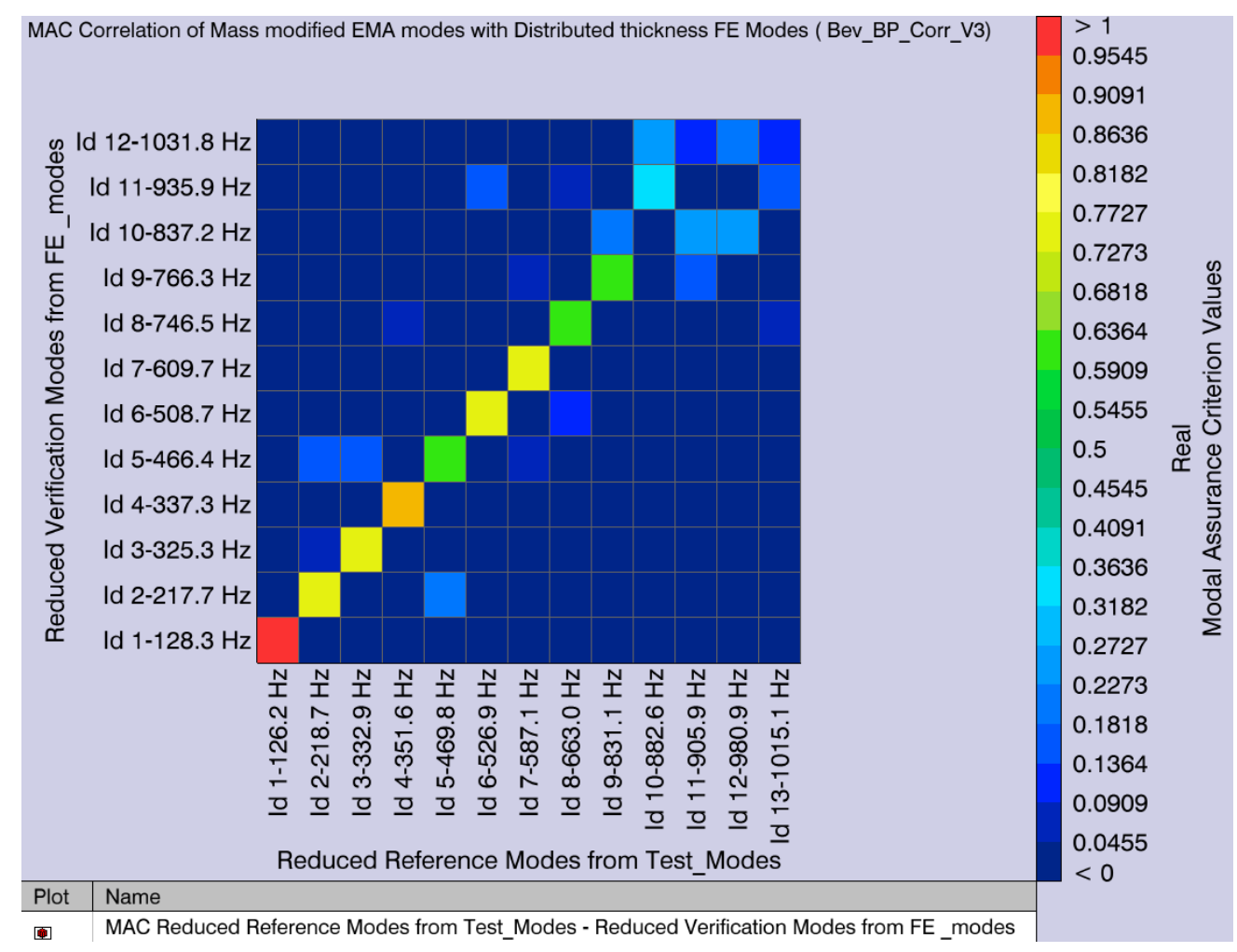

Figure 4.29: MAC results of factory violin back plate EMA modes with distributed thickness FE model

Table 4.4: Mode pairs of the factory violin back plate correlation with distributed thickness

\begin{tabular}{|c|c|c|c|c|c|c|}
\hline $\begin{array}{c}\text { Test } \\
\text { Id }\end{array}$ & $\begin{array}{c}\text { Test Freq } \\
(\mathrm{Hz})\end{array}$ & $\begin{array}{c}\text { FE } \\
\text { Id }\end{array}$ & $\begin{array}{c}\text { FE Freq } \\
(\mathrm{Hz})\end{array}$ & $\begin{array}{c}\text { MAC } \\
\text { Value }\end{array}$ & $\begin{array}{c}\text { Freq Diff } \\
(\mathrm{Hz})\end{array}$ & $\begin{array}{c}\text { Freq Diff } \\
(\% \text { of Test })\end{array}$ \\
\hline 1 & 126.2 & 1 & 128.3 & 0.967 & 2.1 & 1.7 \\
\hline 2 & 218.7 & 2 & 217.7 & 0.763 & 1.04 & 0.5 \\
\hline 3 & 332.9 & 3 & 325.3 & 0.764 & 7.54 & 2.3 \\
\hline 4 & 351.6 & 4 & 337.3 & 0.887 & 14.29 & 4.1 \\
\hline 5 & 469.8 & 5 & 466.4 & 0.607 & 3.39 & 0.7 \\
\hline 6 & 526.9 & 6 & 508.7 & 0.755 & 18.22 & 3.5 \\
\hline 7 & 587.1 & 7 & 609.7 & 0.752 & 22.59 & 3.8 \\
\hline 8 & 663 & 8 & 746.5 & 0.62 & 83.49 & 12.6 \\
\hline 9 & 831.1 & 9 & 766.3 & 0.605 & 64.77 & 7.8 \\
\hline 10 & 882.6 & 11 & 935.9 & 0.319 & 53.35 & 6.0 \\
\hline 11 & 905.9 & 10 & 837.2 & 0.271 & 68.72 & 7.6 \\
\hline 12 & 980.9 & 10 & 837.2 & 0.243 & 143.73 & 14.7 \\
\hline 13 & 1015.1 & 11 & 935.9 & 0.156 & 79.21 & 7.8 \\
\hline
\end{tabular}




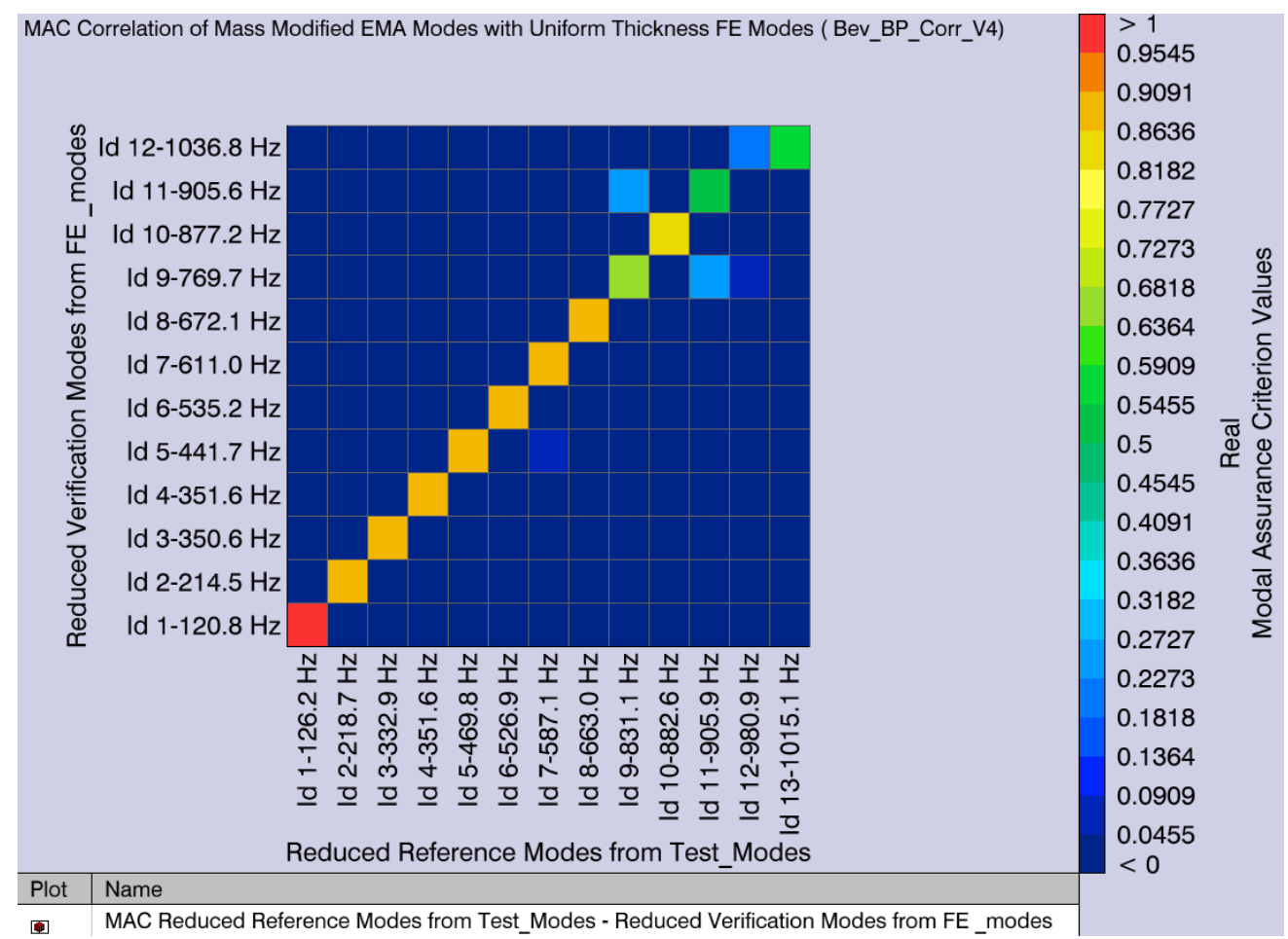

Figure 4.30: MAC results of factory violin back plate EMA modes with uniform thickness FE model

Table 4.5: Mode pairs of the factory violin back plate correlation with uniform thickness

\begin{tabular}{|c|c|c|c|c|c|c|}
\hline $\begin{array}{c}\text { Test } \\
\text { Id }\end{array}$ & $\begin{array}{c}\text { Test Freq } \\
(\mathrm{Hz})\end{array}$ & $\begin{array}{c}\text { FE } \\
\text { Id }\end{array}$ & $\begin{array}{c}\text { FE Freq } \\
(\mathrm{Hz})\end{array}$ & $\begin{array}{c}\text { MAC } \\
\text { Value }\end{array}$ & $\begin{array}{c}\text { Freq Diff } \\
(\mathrm{Hz})\end{array}$ & $\begin{array}{c}\text { Freq Diff } \\
(\% \text { of Test })\end{array}$ \\
\hline 1 & 126.2 & 1 & 120.8 & 0.966 & 5.39 & 4.3 \\
\hline 2 & 218.7 & 2 & 214.5 & 0.864 & 4.27 & 2.0 \\
\hline 3 & 332.9 & 3 & 350.6 & 0.879 & 17.72 & 5.3 \\
\hline 4 & 351.6 & 4 & 351.6 & 0.879 & 0.06 & 0.0 \\
\hline 5 & 469.8 & 5 & 441.7 & 0.903 & 28.1 & 6.0 \\
\hline 6 & 526.9 & 6 & 535.2 & 0.89 & 8.33 & 1.6 \\
\hline 7 & 587.1 & 7 & 611 & 0.865 & 23.94 & 4.1 \\
\hline 8 & 663 & 8 & 672.1 & 0.894 & 9.08 & 1.4 \\
\hline 9 & 831.1 & 9 & 769.7 & 0.658 & 61.4 & 7.4 \\
\hline 10 & 882.6 & 10 & 877.2 & 0.859 & 5.37 & 0.6 \\
\hline 11 & 905.9 & 11 & 905.6 & 0.504 & 0.37 & 0.0 \\
\hline 12 & 980.9 & 12 & 1036.8 & 0.219 & 55.81 & 5.7 \\
\hline 13 & 1015.1 & 12 & 1036.8 & 0.561 & 21.63 & 2.1 \\
\hline
\end{tabular}


Overall the uniform thickness model correlates more closely with the test data. The average MAC for the first 10 mode pairs goes from $\sim 0.704$ for the distributed thickness model to $\sim 0.866$ for the uniform thickness model. This is particularly true for mode 5 (the "ring" mode, $469 \mathrm{~Hz}$ ), which after updating had a MAC value of 0.607 for the distributed thickness model versus a MAC of 0.903 for the uniform thickness model. This indicates that although thickness is important, this particular plate is relatively uniform in thickness such that the simpler model yields more correct results in this case, as the mode shapes may be sensitive to small errors in the thickness measurements.

It was noted that the material properties of both versions of the model were updated to "tune" the modal frequencies. In both cases, updating was performed until the first five mode frequencies were within $6 \%$ of the test frequency and the average percent difference for the first 9 modes was below 5\%. A summary of the final material properties is given in Table 4.6.

Table 4.6: Summary of final material properties for factory violin back plate FE model

\begin{tabular}{|c|c|c|c|c|c|c|c|}
\hline $\begin{array}{c}\text { Thickness } \\
\text { method }\end{array}$ & $\begin{array}{c}\text { E1 } \\
(\mathrm{MPa})\end{array}$ & $\begin{array}{c}\mathrm{E} 2 \\
(\mathrm{MPa})\end{array}$ & NU12 & $\begin{array}{c}\mathrm{G} 12 \\
(\mathrm{MPa})\end{array}$ & $\begin{array}{c}\mathrm{G} 1 \mathrm{Z} \\
(\mathrm{MPa})\end{array}$ & $\begin{array}{c}\mathrm{G} 2 \mathrm{Z} \\
(\mathrm{MPa})\end{array}$ & $\begin{array}{c}\mathrm{RHO} \\
\left(\mathrm{kg} / \mathrm{m}^{3}\right)\end{array}$ \\
\hline Distributed & 9049.6 & 1600.0 & 0.490 & 979.3 & 399.4 & 296.4 & 587.0 \\
\hline Uniform & 14727.0 & 1573.8 & 0.467 & 1114.4 & 771.2 & 405.3 & 571.1 \\
\hline
\end{tabular}

The mass of the factory violin back plate was measured experimentally on an electronic scale to be 123.3 grams. The mass of the final FE model of the factory violin back plate was $\sim 124.9$ grams, a difference of $\sim 1.3 \%$.

\subsubsection{Top plate results}

\subsubsection{Experimental results Top plate}

The top plate was tested in a similar fashion to the back plate. A roving impact hammer test was performed with three reference accelerometers; two on opposite corners, one on the lower-bass corner (topp:7), one on the upper-treble corner (topp:24), and one near the middle just above the bass bar, (topp:83). The driving point FRFs are shown in Figure 4.31. The behavior is similar to that of the back plate, although the mode order is slightly different. This is due to the presence of the bass bar, which significantly stiffens the plate in the longitudinal direction. 
The driving point coherences are shown in Figure 4.32. Again, coherence is well above 0.95 for all frequencies of interest with the exception of the anti-resonances.

The Digital Signal Processing (DSP) parameters for the top plate experimental modal analysis are summarized in Table 4.7.

Table 4.7: DSP parameters for factory violin top plate EMA

\begin{tabular}{|l|l|}
\hline Averaging & 5 Averages, Linear \\
\hline Bandwidth & $4096 \mathrm{~Hz}$ \\
\hline Frequency Resolution & $1.0 \mathrm{~Hz}$ \\
\hline Reference Window & Force-Exponential, 100\% \\
\hline Response Window & Exponential, 100\% \\
\hline
\end{tabular}

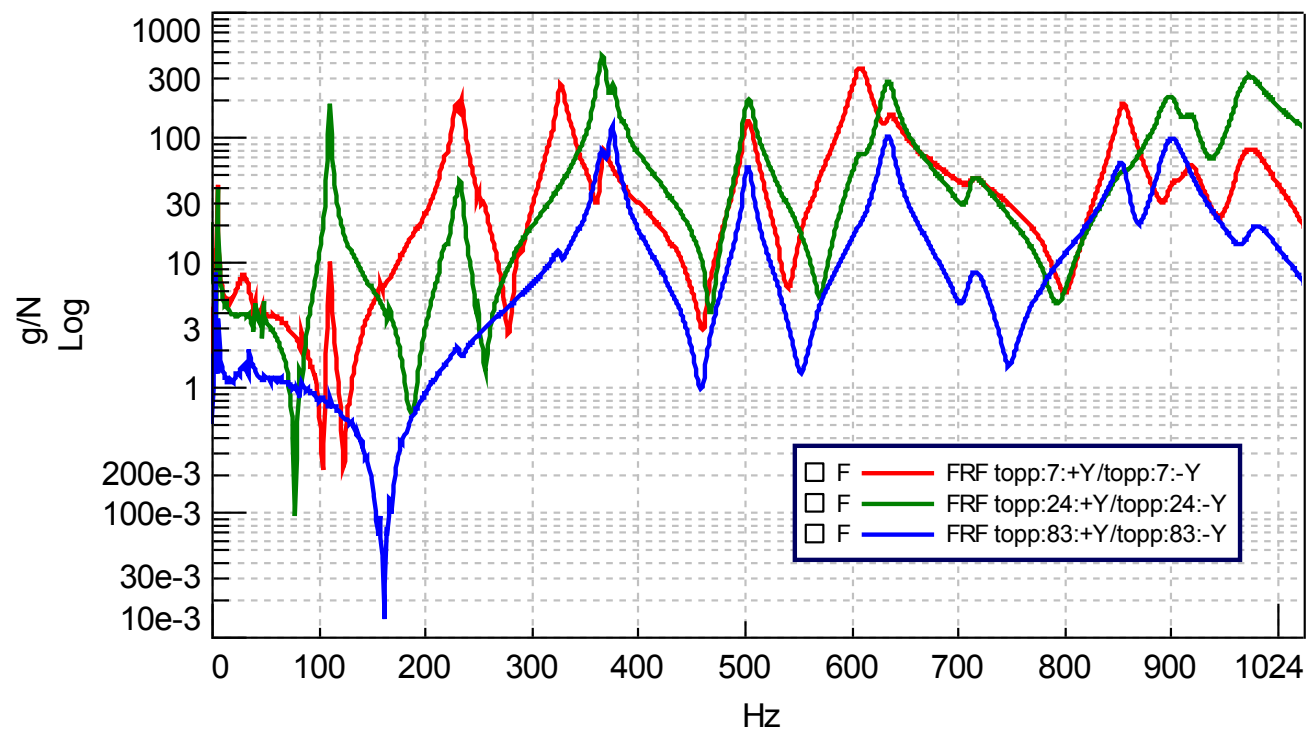

Figure 4.31: Driving point FRFs of the factory violin top plate 


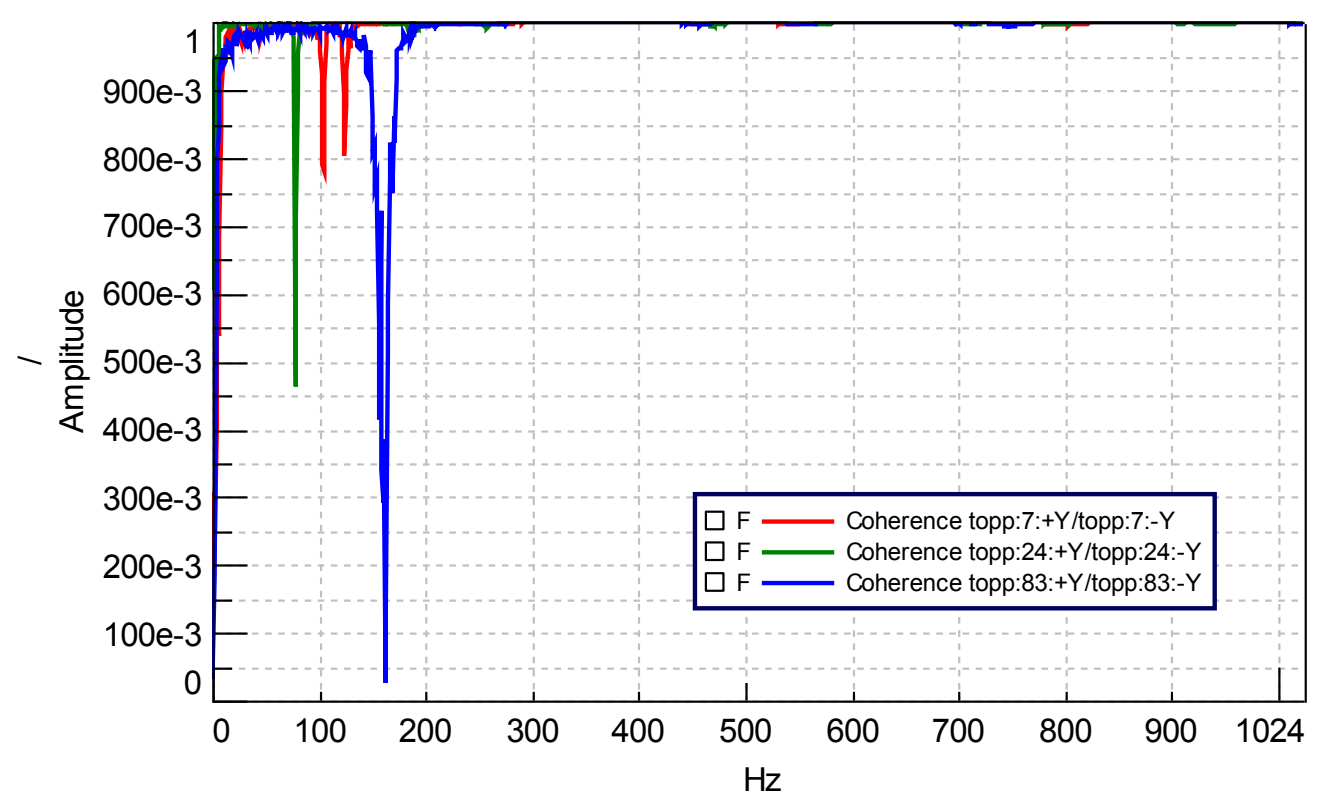

Figure 4.32: Driving point coherence functions of the factory violin top plate

Curve fitting of the top plate modal data was performed using LMS Test.Lab PolyMAX. Several consecutive frequency bands from $73.1 \mathrm{~Hz}$ to $797 \mathrm{~Hz}$ were selected for stabilization. The Mode Indicator Functions (MIFs) used for modal parameter selection are shown in Figure 4.33. The red curve is the FRF sum, while the blue, magenta, and cyan curves are the Multivariant MIFs. The Automatic Modal Parameter Selection (AMPS) feature was used. 


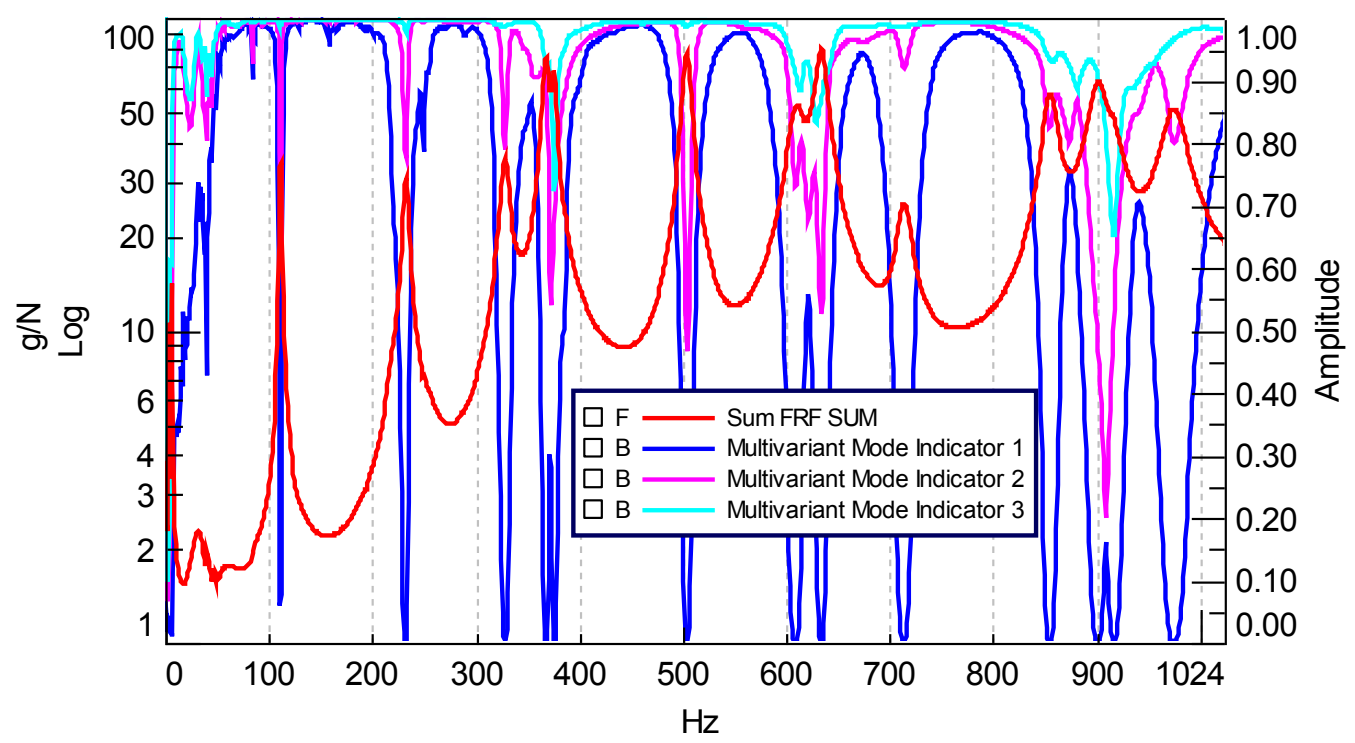

Figure 4.33: Mode indicator functions for curve fitting the factory violin top plate experimental data

The modal frequency and damping results for the factory violin top plate are given in

Table 4.8. These are followed by the first five mode shapes in Figures 4.34 through 4.38.

Table 4.8: Curve-fitting results from the factory violin top plate EMA

\begin{tabular}{|c|c|c|l|}
\hline $\begin{array}{c}\text { Mode } \\
\#\end{array}$ & $\begin{array}{c}\text { Frequency } \\
(\mathbf{H z})\end{array}$ & $\begin{array}{c}\text { Damping } \\
\mathbf{( \% )}\end{array}$ & Description \\
\hline 1 & 111.7 & 0.73 & $1^{\text {st }}$ Torsion \\
\hline 2 & 232.4 & 1.40 & $\begin{array}{l}\text { Longitudinal Bending } \\
\text { (X-mode) }\end{array}$ \\
\hline 3 & 328.5 & 1.15 & $2^{\text {nd }}$ Torsion \\
\hline 4 & 367.3 & 0.91 & Ring (O-mode) \\
\hline 5 & 375.9 & 0.74 & Triad \\
\hline 6 & 503.9 & 0.87 & \\
\hline 7 & 609.3 & 1.14 & \\
\hline 8 & 634.8 & 0.95 & \\
\hline 9 & 713.9 & 1.02 & \\
\hline
\end{tabular}

Michael Pyrkosz 


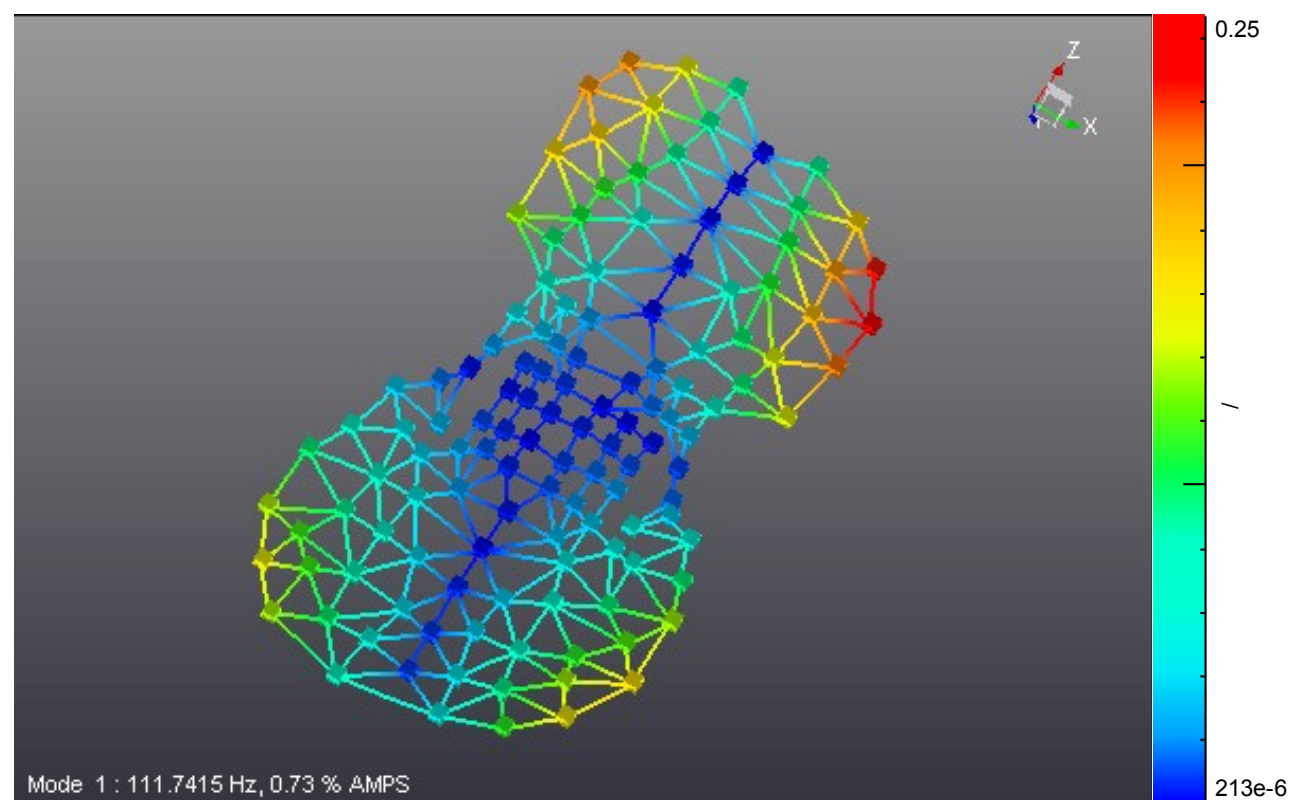

Figure 4.34: First experimental mode of the factory violin top plate

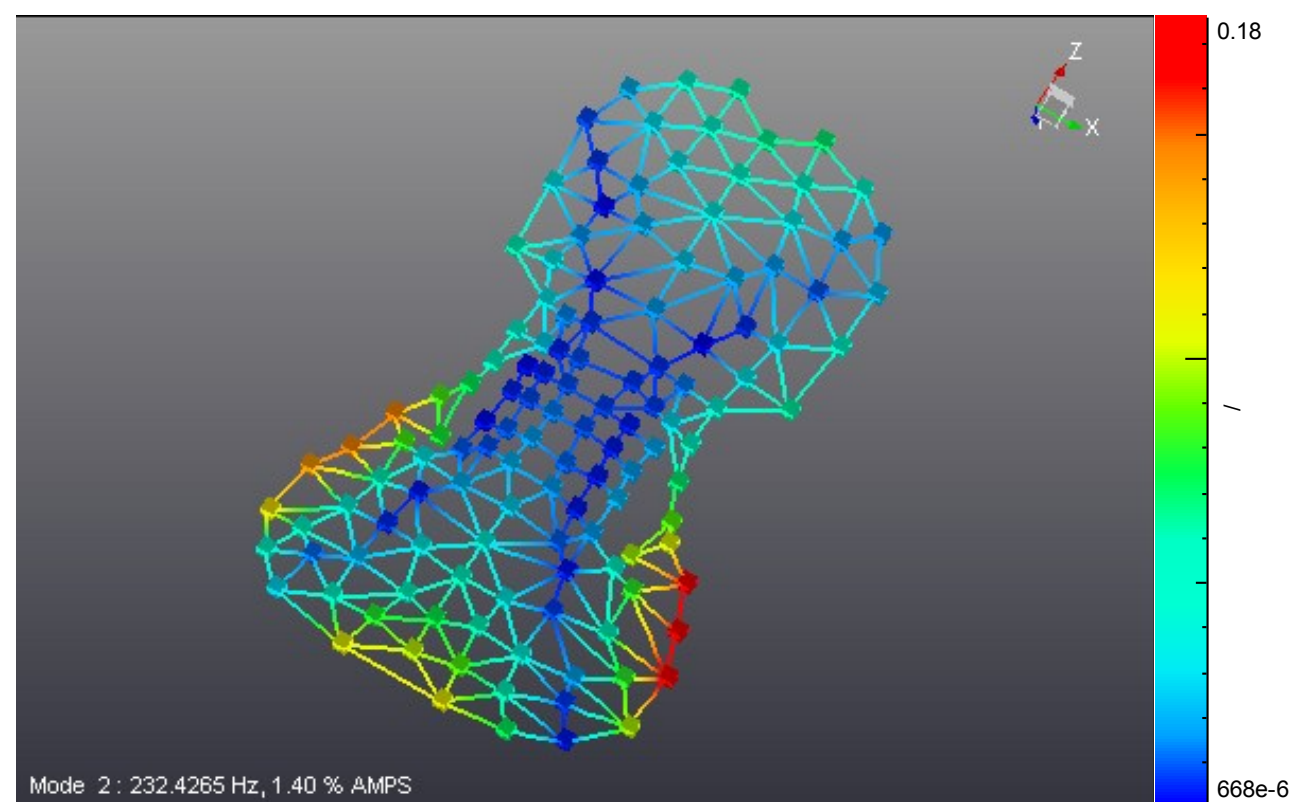

Figure 4.35: Second experimental mode of the factory violin top plate 


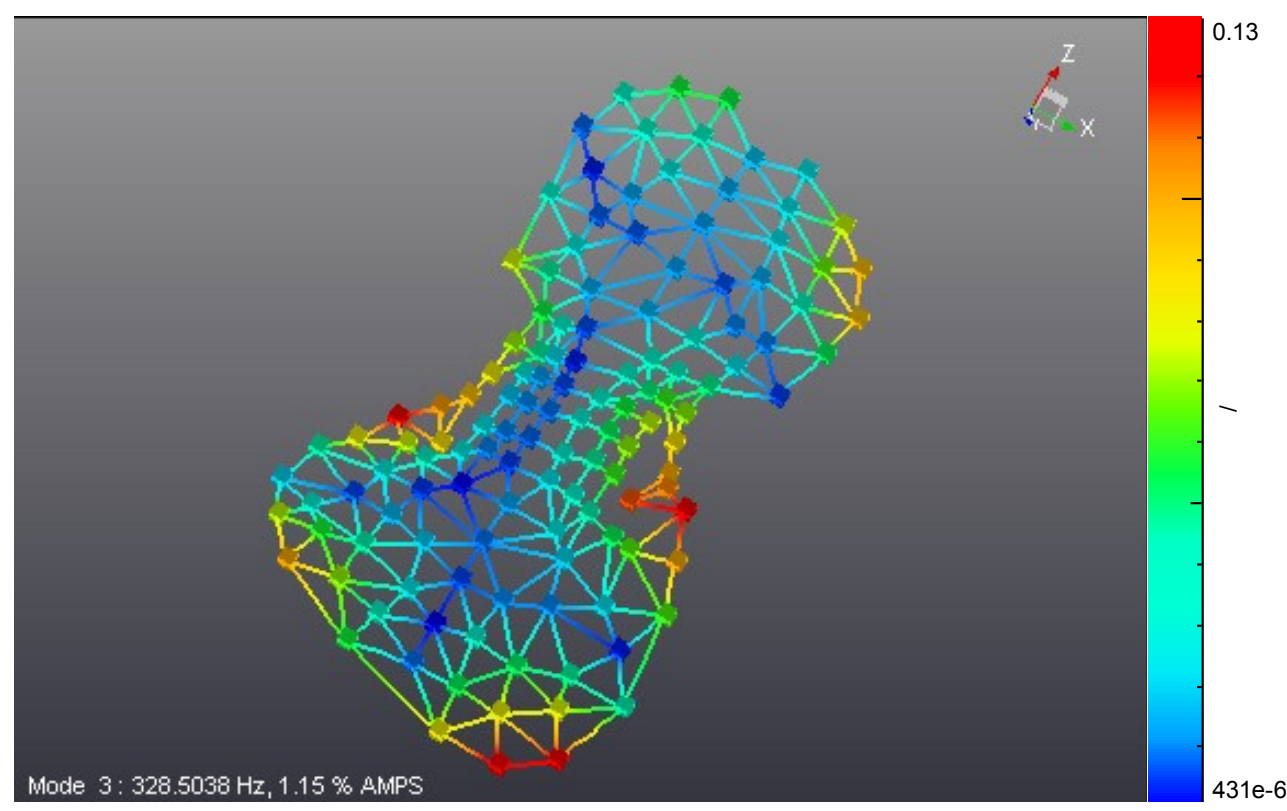

Figure 4.36: Third experimental mode of the factory violin top plate

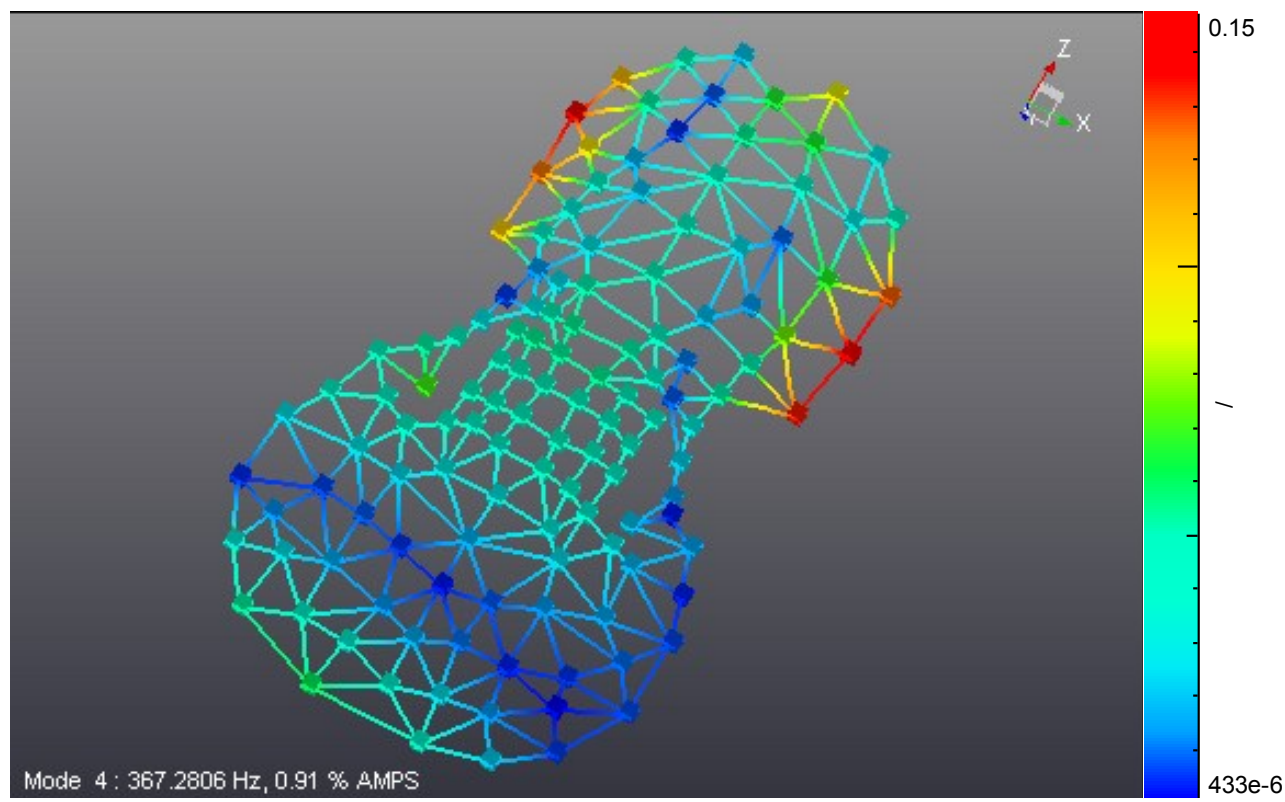

Figure 4.37: Fourth experimental mode of the factory violin top plate 


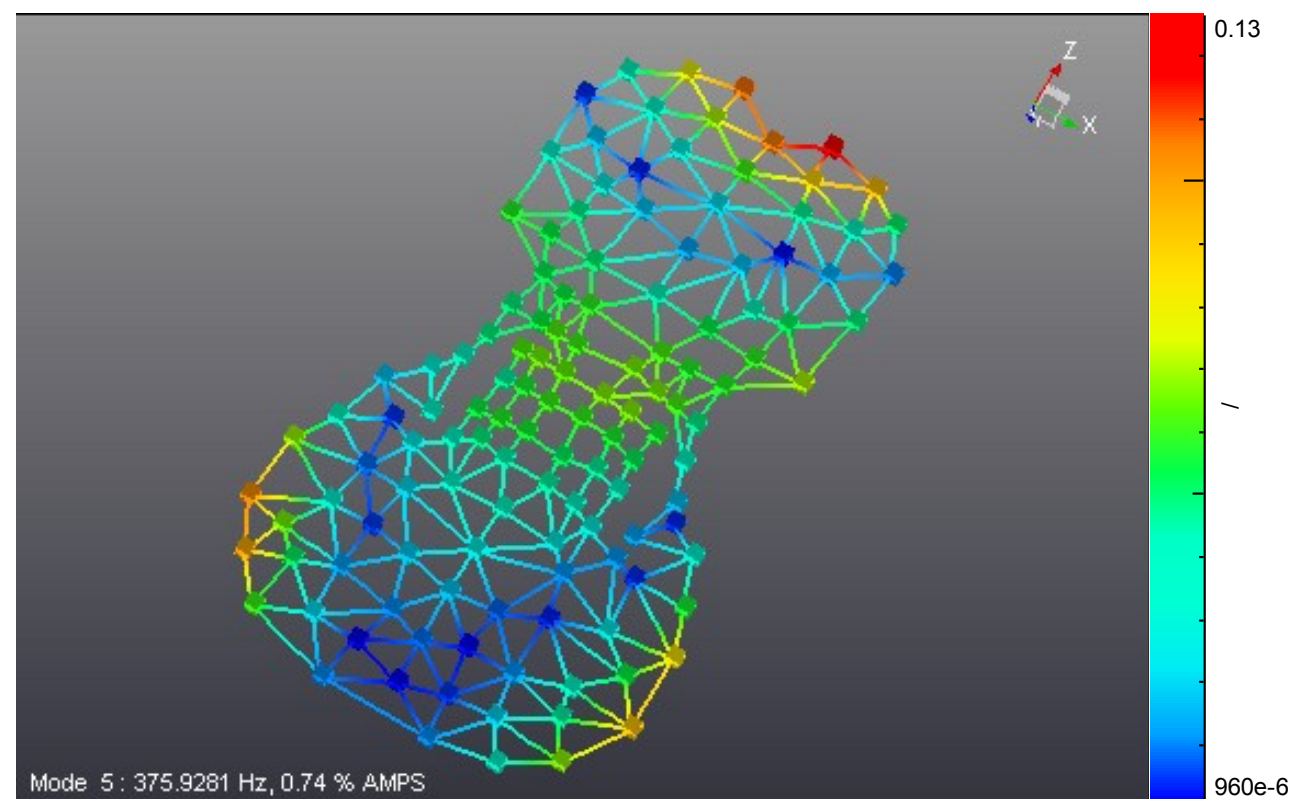

Figure 4.38: Fifth experimental mode of the factory violin top plate

Modification prediction was used to remove the effect of the 0.90 grams of the three accelerometers. Again, this had the effect of raising some of the mode frequencies to varying degrees depending on the location of the accelerometers with respect to the mode shape. The largest change observed was $\sim 1.6 \%$.

\subsubsection{Analytical results for the top plate}

The mesh of the top plate itself contains 2773 nodes and 5244 linear triangular elements. The bass bar is modeled as a solid volume with 4366 nodes and 2193 elements. These are connected via 140 RBEs. Figure 4.39 shows the final factory violin top plate FE mesh. 

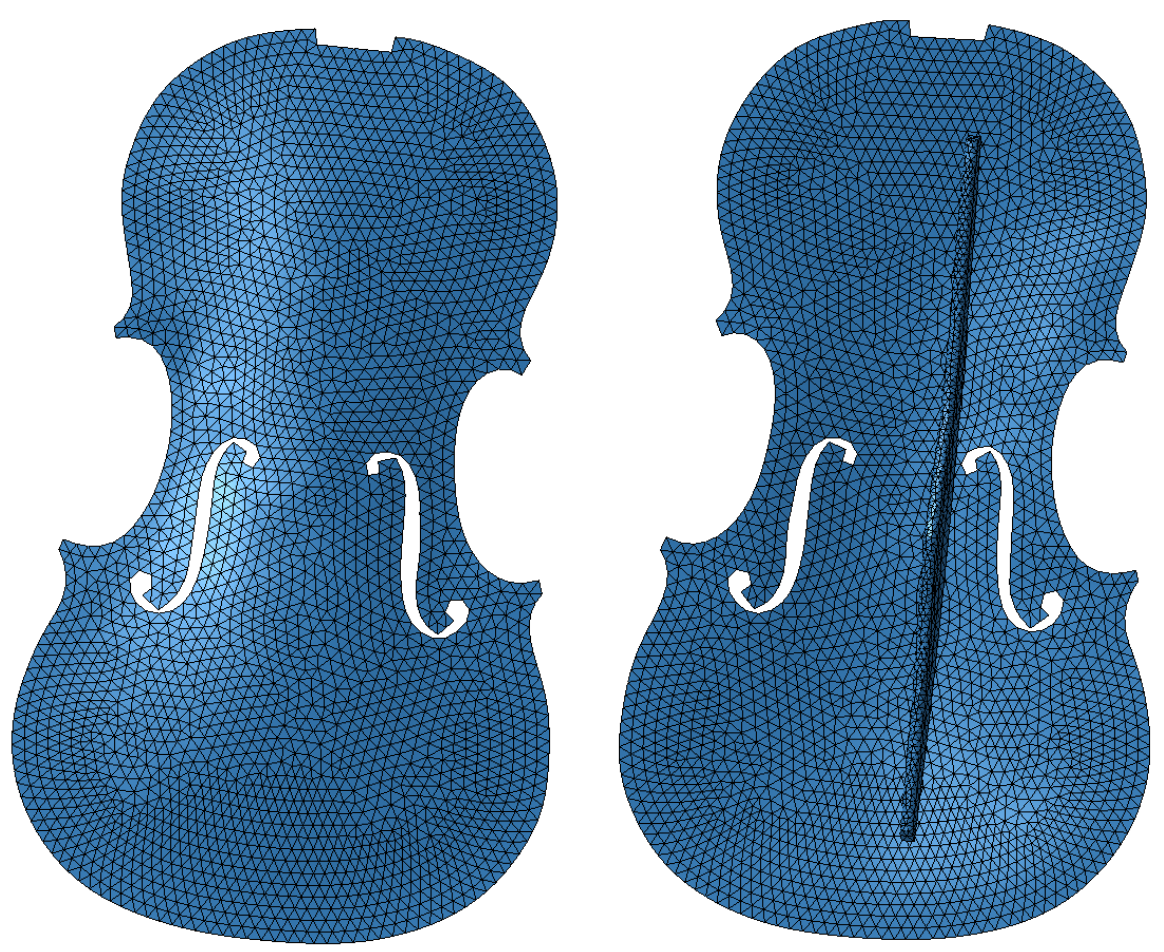

Figure 4.39: Factory violin top plate FE mesh (left view from top, right view from back)

The top plate was also initially modeled with distributed thickness. Figure 4.40 shows the result of the thickness mapping for the factory violin top plate. A version of the model with a uniform thickness was also created for comparison purposes. Based on the correlation results the distributed thickness model was more accurate in predicting the top plate behavior. Again, the material properties of both versions of the model were adjusted to "tune" the modal frequencies with those of the test. 

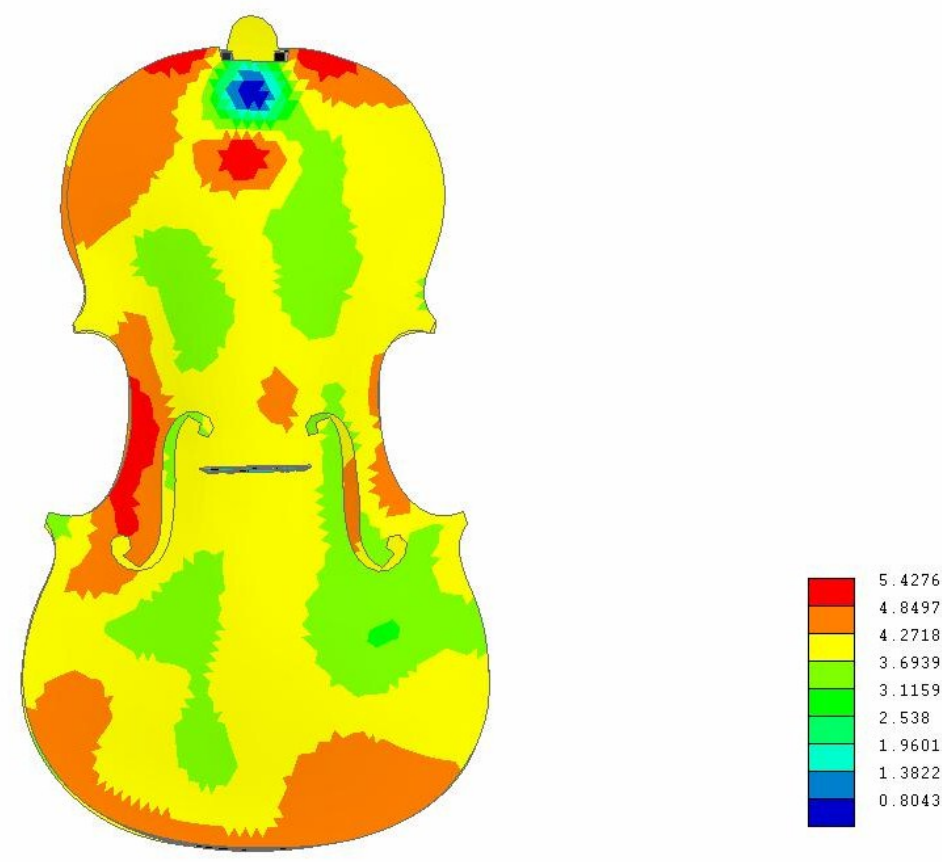

Figure 4.40: Element thickness of the factory violin top plate in $\mathrm{mm}$

The final FE model normal modes analysis results are summarized in Table 4.9.

Examples of the first six FE mode shapes are shown in Figures 4.41 through 4.46.

Table 4.9: Summary of final factory violin top plate normal modes analysis

\begin{tabular}{|c|c|l|}
\hline Mode \# & Freq (Hz) & Description \\
\hline 1 & 116.5 & $1^{\text {st }}$ Torsion \\
\hline 2 & 236.0 & Longitudinal Bending (X-mode) \\
\hline 3 & 331.4 & $2^{\text {nd }}$ Torsion \\
\hline 4 & 374.1 & Ring (O-mode) \\
\hline 5 & 385.3 & Triad (3 nodal lines) \\
\hline 6 & 513.2 & upper torsion-lower 2nd bending \\
\hline 7 & 589.7 & \\
\hline 8 & 639.5 & \\
\hline 9 & 731.0 & \\
\hline 10 & 847.8 & \\
\hline 11 & 891.7 & \\
\hline 12 & 944.1 & \\
\hline
\end{tabular}

Michael Pyrkosz 


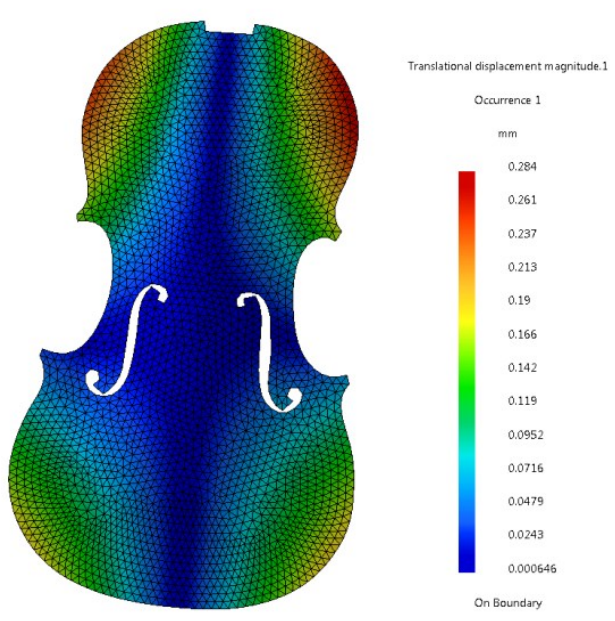

Figure 4.41: Mode 1 of the factory violin top plate (first torsion)

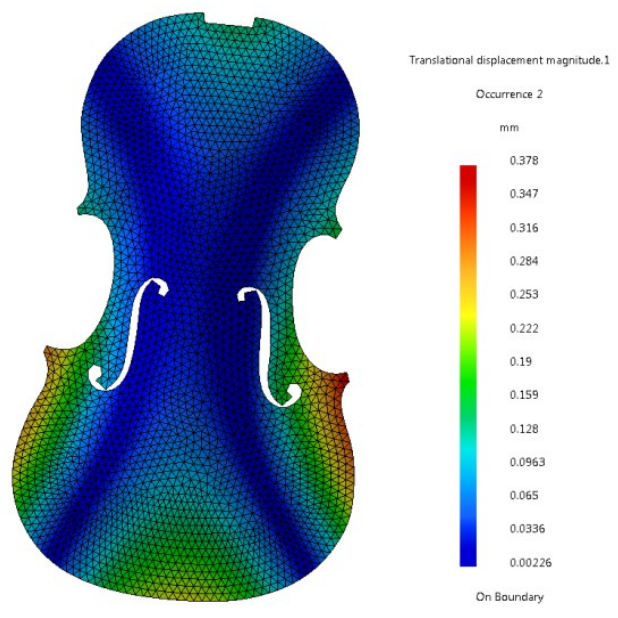

Figure 4.42: Mode 2 of the factory violin top plate (first longitudinal bending)

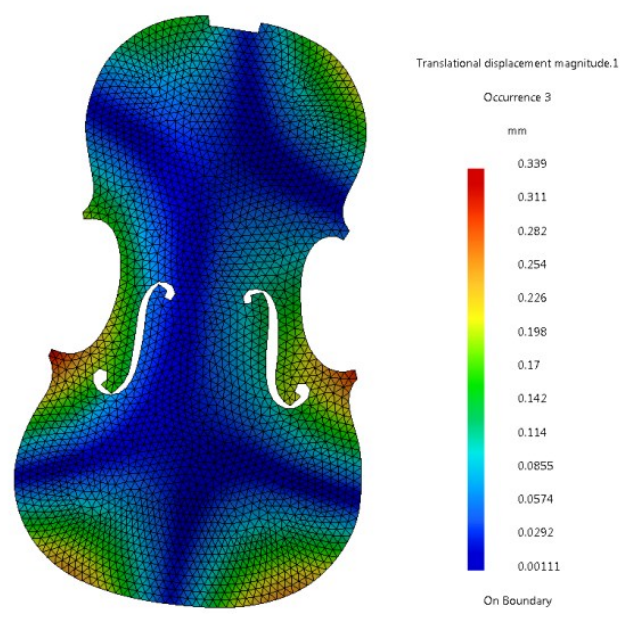

Figure 4.43: Mode 3 of the factory violin top plate (second torsion)

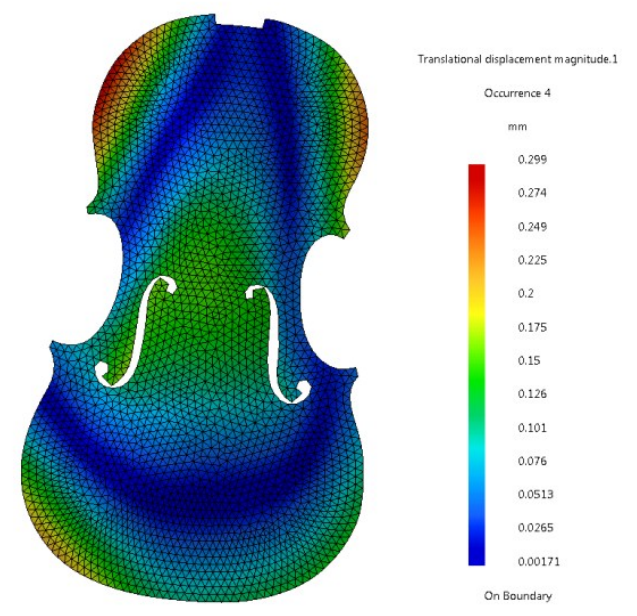

Figure 4.44: Mode 4 of the factory violin top plate (ring) 


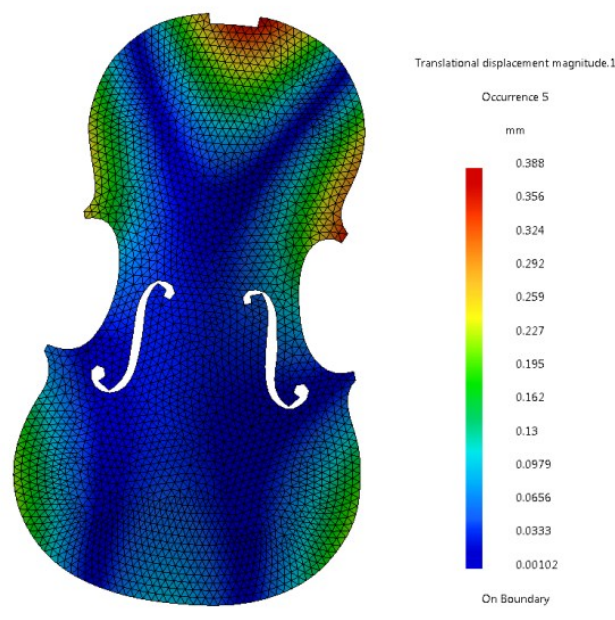

Figure 4.45: Mode 5 of the factory violin top plate (triad)

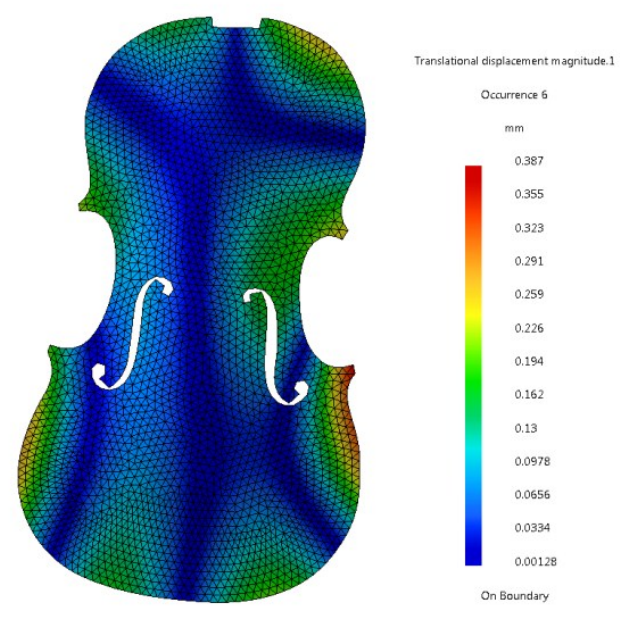

Figure 4.46: Mode 6 of the factory violin top plate (upper torsion-lower 2 nd bending)

It is noted that for this factory violin plate the "ring" mode (mode 4) exhibits nodal lines extending to the upper edge rather than forming a closed ring. This is a behavior that Hutchins mentions in an example of a "poorly tuned plate" [4.11].

\subsubsection{Correlation results for top plate}

Correlation results for both versions of the factory violin top plate model are presented here. Figure 4.47 shows a plot of the final MAC matrix results for the correlation between the test and the original distributed thickness model, while Figure 4.48 shows that of the correlation with the uniform thickness model. The corresponding mode pair tables are given in Tables 4.10 and 4.11. 


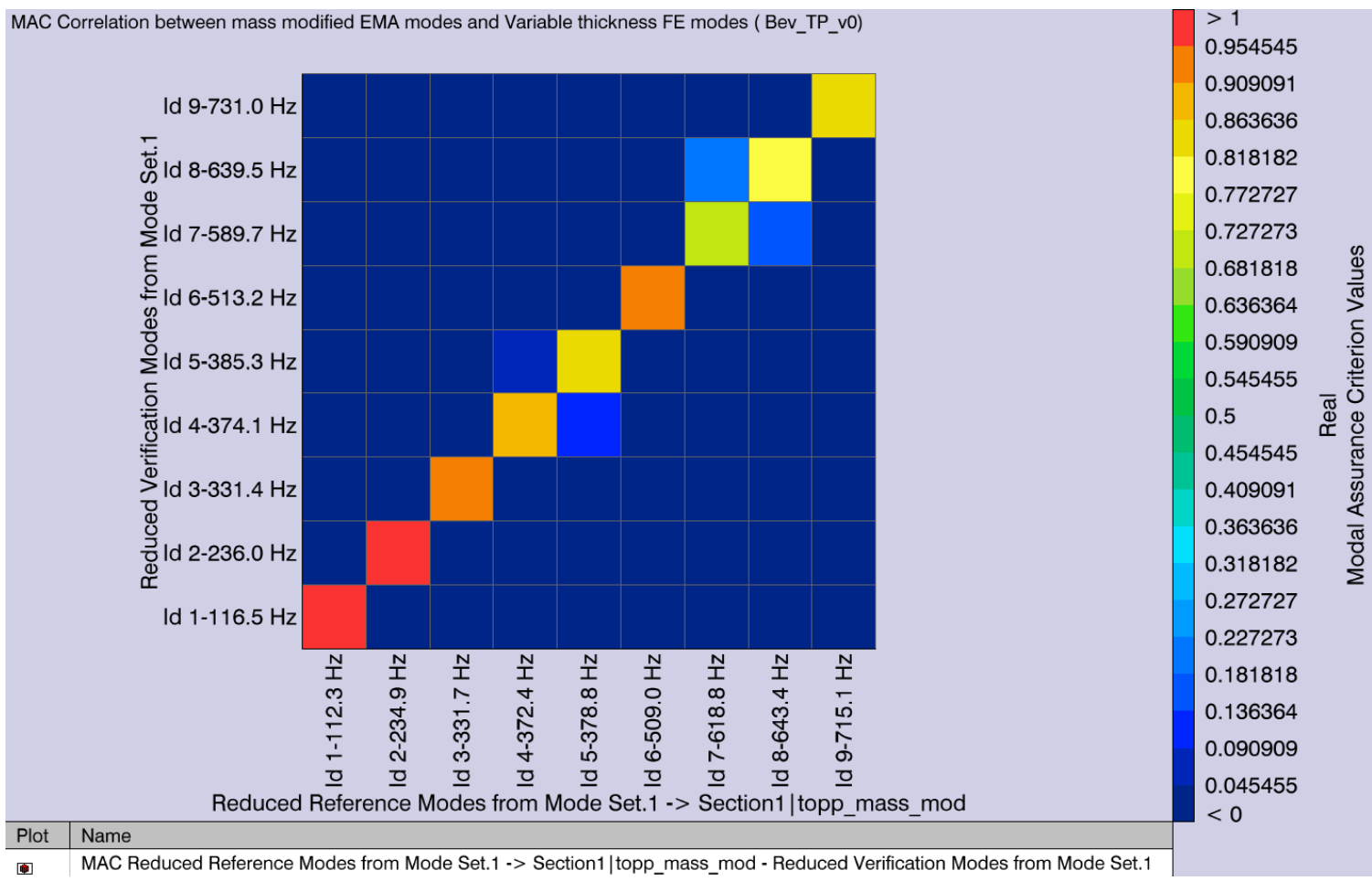

Figure 4.47: MAC results of factory violin top plate EMA modes with distributed thickness FE model

Table 4.10: Mode pairs of the factory violin top plate correlation with distributed thickness

\begin{tabular}{|c|c|c|c|c|c|c|}
\hline $\begin{array}{c}\text { Test } \\
\text { Id }\end{array}$ & $\begin{array}{c}\text { Test Freq } \\
(\mathrm{Hz})\end{array}$ & $\begin{array}{c}\text { FE } \\
\mathrm{Id}\end{array}$ & $\begin{array}{c}\text { FE Freq } \\
(\mathrm{Hz})\end{array}$ & $\begin{array}{c}\text { MAC } \\
\text { Value }\end{array}$ & $\begin{array}{c}\text { Freq Diff } \\
(\mathrm{Hz})\end{array}$ & $\begin{array}{c}\text { Freq Diff } \\
(\% \text { of Test })\end{array}$ \\
\hline 1 & 112.3 & 1 & 116.5 & 0.98 & 4.19 & 3.7 \\
\hline 2 & 234.9 & 2 & 236 & 0.957 & 1.04 & 0.4 \\
\hline 3 & 331.7 & 3 & 331.4 & 0.946 & 0.33 & 0.1 \\
\hline 4 & 372.4 & 4 & 374.1 & 0.876 & 1.66 & 0.4 \\
\hline 5 & 378.8 & 5 & 385.3 & 0.861 & 6.48 & 1.7 \\
\hline 6 & 509 & 6 & 513.2 & 0.945 & 4.14 & 0.8 \\
\hline 7 & 618.8 & 7 & 589.7 & 0.713 & 29.1 & 4.7 \\
\hline 8 & 643.4 & 8 & 639.5 & 0.808 & 3.84 & 0.6 \\
\hline 9 & 715.1 & 9 & 731 & 0.848 & 15.94 & 2.2 \\
\hline
\end{tabular}




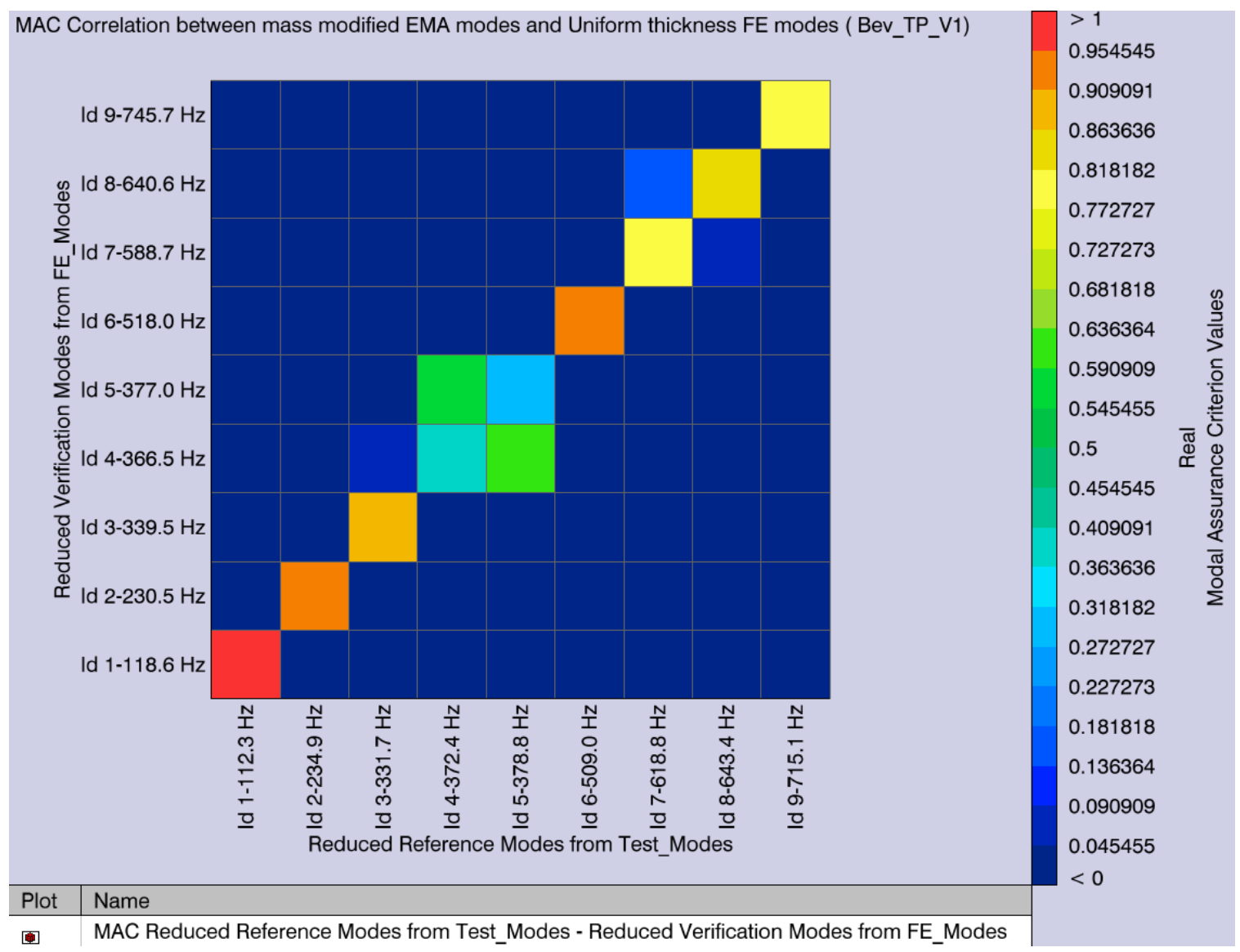

Figure 4.48: MAC results of factory violin top plate EMA modes with uniform thickness FE model

Table 4.11: Mode pairs of the factory violin top plate correlation with uniform thickness

\begin{tabular}{|c|c|c|c|c|c|c|}
\hline $\begin{array}{c}\text { Test } \\
\text { Id }\end{array}$ & $\begin{array}{c}\text { Test Freq } \\
(\mathrm{Hz})\end{array}$ & $\begin{array}{c}\text { FE } \\
\text { Id }\end{array}$ & $\begin{array}{c}\text { FE Freq } \\
(\mathrm{Hz})\end{array}$ & $\begin{array}{c}\text { MAC } \\
\text { Value }\end{array}$ & $\begin{array}{c}\text { Freq Diff } \\
(\mathrm{Hz})\end{array}$ & $\begin{array}{c}\text { Freq Diff } \\
(\% \text { of Test })\end{array}$ \\
\hline 1 & 112.3 & 1 & 118.6 & 0.978 & 6.37 & 5.7 \\
\hline 2 & 234.9 & 2 & 230.5 & 0.952 & 4.44 & 1.9 \\
\hline 3 & 331.7 & 3 & 339.5 & 0.909 & 7.75 & 2.3 \\
\hline 4 & 372.4 & 5 & 377 & 0.575 & 4.58 & 1.2 \\
\hline 5 & 378.8 & 4 & 366.5 & 0.617 & 12.34 & 3.3 \\
\hline 6 & 509 & 6 & 518 & 0.931 & 8.96 & 1.8 \\
\hline 7 & 618.8 & 7 & 588.7 & 0.78 & 30.1 & 4.9 \\
\hline 8 & 643.4 & 8 & 640.6 & 0.828 & 2.82 & 0.4 \\
\hline 9 & 715.1 & 9 & 745.7 & 0.814 & 30.64 & 4.3 \\
\hline
\end{tabular}


In the case of the top plate the distributed thickness model yielded better correlation results than the uniform one. For instance after updating mode 4 (again the "ring" mode, $372 \mathrm{~Hz}$ ) had a MAC value of 0.904 for the distributed thickness model versus 0.575 for the uniform model. This is most likely due to inconsistent thickness distribution across the plate. The bass side edge was measured at $5.4 \mathrm{~mm}(0.2125 \mathrm{in})$, whereas the treble side was measured as thin as $3.5 \mathrm{~mm}(0.1375 \mathrm{in})$. This bass-treble asymmetry leads to the need for a more sophisticated model. Moreover, the upper bout region of the plate contains a point that drops less than $0.8 \mathrm{~mm}$ with another point above $5.4 \mathrm{~mm}$.

It is noted that the material properties of both versions of the model were updated to "tune" the modal frequencies. In both cases, updating was performed until the first five mode frequencies were within $6 \%$ of the test modes and the average percent difference for the first 9 modes was below 5\%. A summary of the final material properties is given in Table 4.12.

Table 4.12: Summary of final material properties for factory violin top plate FE model

\begin{tabular}{|c|c|c|c|c|c|c|c|}
\hline $\begin{array}{c}\text { Thickness } \\
\text { method }\end{array}$ & $\begin{array}{c}\text { E1 } \\
(\mathrm{MPa})\end{array}$ & $\begin{array}{c}\mathrm{E} 2 \\
(\mathrm{MPa})\end{array}$ & NU12 & $\begin{array}{c}\text { G12 } \\
(\mathrm{MPa})\end{array}$ & $\begin{array}{c}\mathrm{G} 1 \mathrm{Z} \\
(\mathrm{MPa})\end{array}$ & $\begin{array}{c}\mathrm{G} 2 Z \\
(\mathrm{MPa})\end{array}$ & $\begin{array}{c}\mathrm{RHO} \\
\left(\mathrm{kg} / \mathrm{m}^{3}\right)\end{array}$ \\
\hline Distributed & 12396 & 1468.1 & 0.4473 & 969.2 & 813.7 & 61.85 & 448.0 \\
\hline Uniform & 10706 & 1288.5 & 0.3946 & 1076.1 & 846.9 & 60.02 & 440.7 \\
\hline
\end{tabular}

The mass of the factory violin top plate and bass bar was experimentally measured on an electronic scale to be 99.3 grams. The mass of the final FE model of the factory violin top plate and bass bar was $\sim 99.27$ grams, a difference of $\sim 0.03 \%$.

\subsubsection{Corpus Results}

\subsubsection{Corpus Test Results}

The experimental setup for the factory violin corpus modal analysis was as follows. "Free-free" boundary conditions were obtained using rubber bands. Three driving point locations were selected at the upper treble-side corner (topp:24), approximately under the bass-side bridge foot (topp:83), and near the lower-bass corner of the back plate (back:9). These were instrumented with miniature uniaxial ICP accelerometers (PCB Model 352A73, 0.3gm each), which were mounted using petro-wax (PCB Model 080A24). The modal impact hammer (PCB Model 086E80) was roved over 364 points on the top plate, back plate, and ribs. FRFs between the impact force inputs and accelerometer responses were measured. The driving point FRFs are shown in Figure 4.49, while the 
corresponding coherence functions are shown in Figure 4.50. The Digital Signal Processing (DSP) parameters for the test are summarized in Table 4.13.

Table 4.13: DSP parameters for factory violin corpus EMA

\begin{tabular}{|l|l|}
\hline Averaging & 5 Averages, Linear \\
\hline Bandwidth & $4096 \mathrm{~Hz}$ \\
\hline Frequency Resolution & $1.0 \mathrm{~Hz}$ \\
\hline Reference Window & Force-Exponential, 100\% \\
\hline Response Window & Exponential, 100\% \\
\hline
\end{tabular}

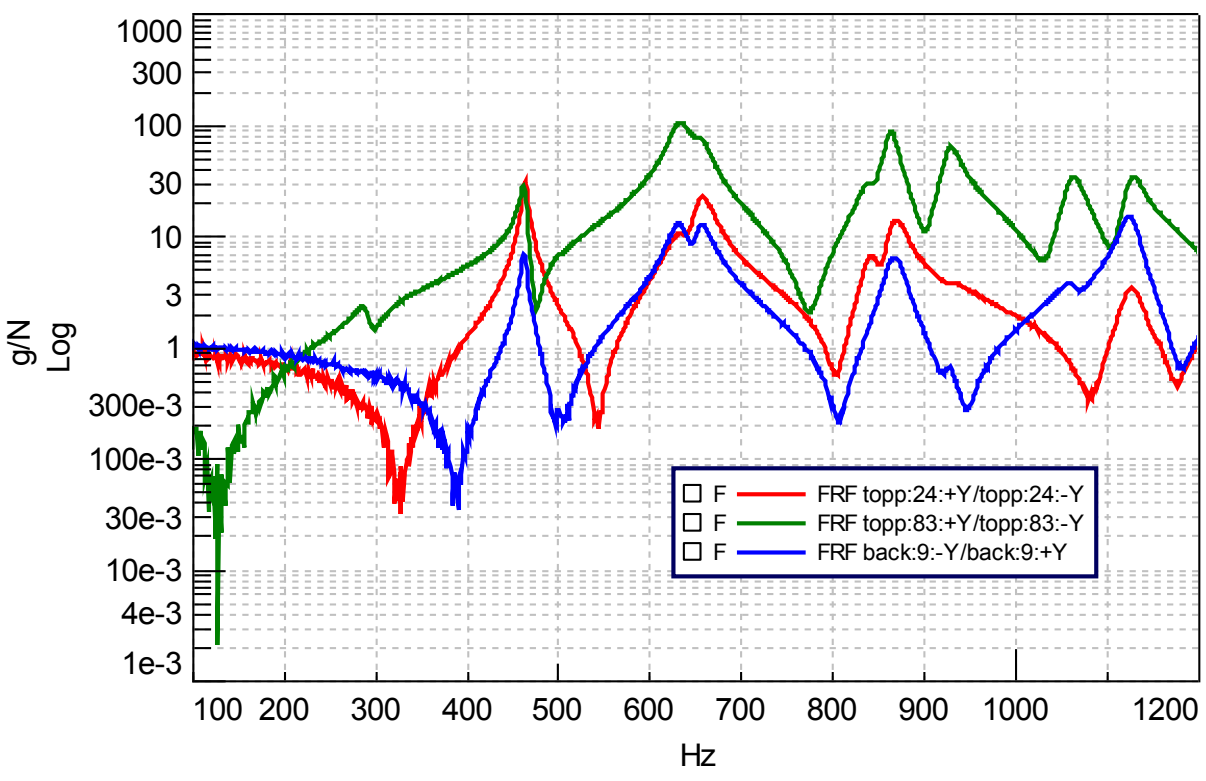

Figure 4.49: Driving point FRFs for factory violin corpus 


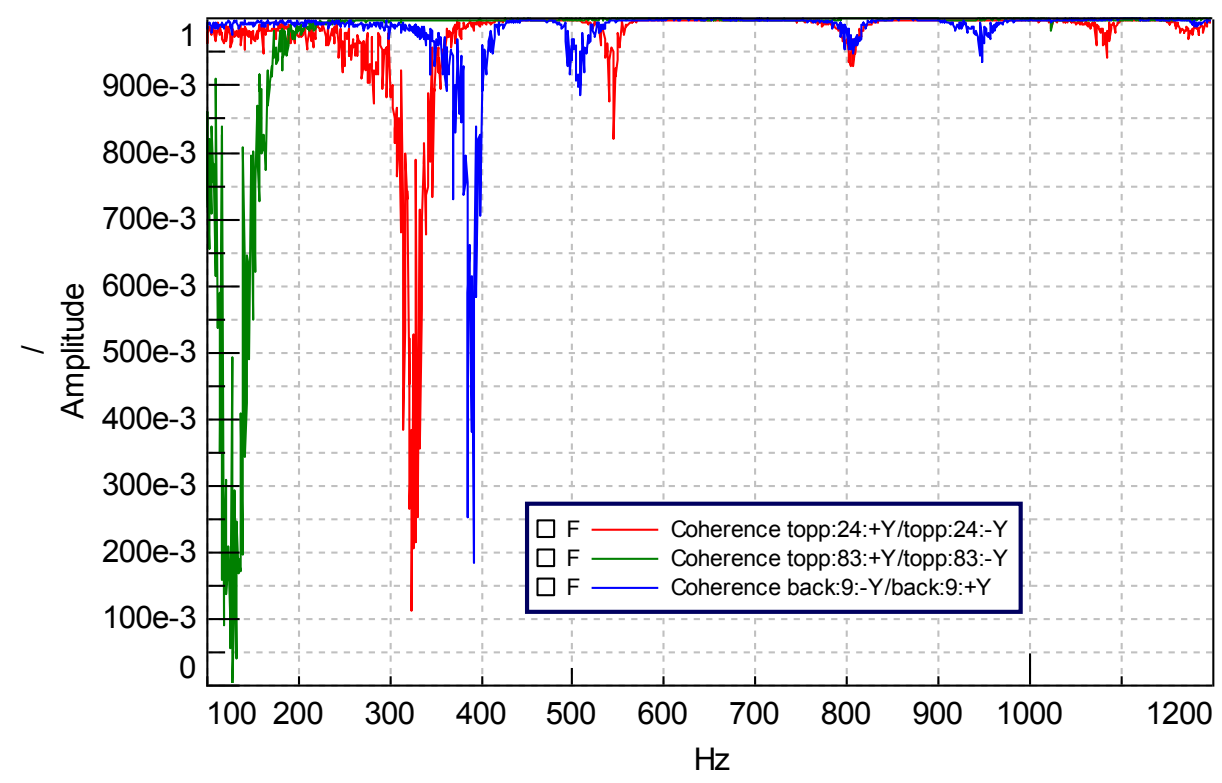

Figure 4.50: Driving point coherence functions for factory violin corpus

Curve fitting of the modal data was performed using LMS Test.Lab PolyMAX. Several consecutive frequency bands from $213 \mathrm{~Hz}$ to $1230 \mathrm{~Hz}$ were selected for stabilization. The Mode Indicator Functions (MIFs) used for modal parameter selection are shown in Figure 4.51. The red curve is the FRF sum, while the blue, magenta, and cyan curves are the Multivariant MIFs. Once again AMPS was used for selecting poles.

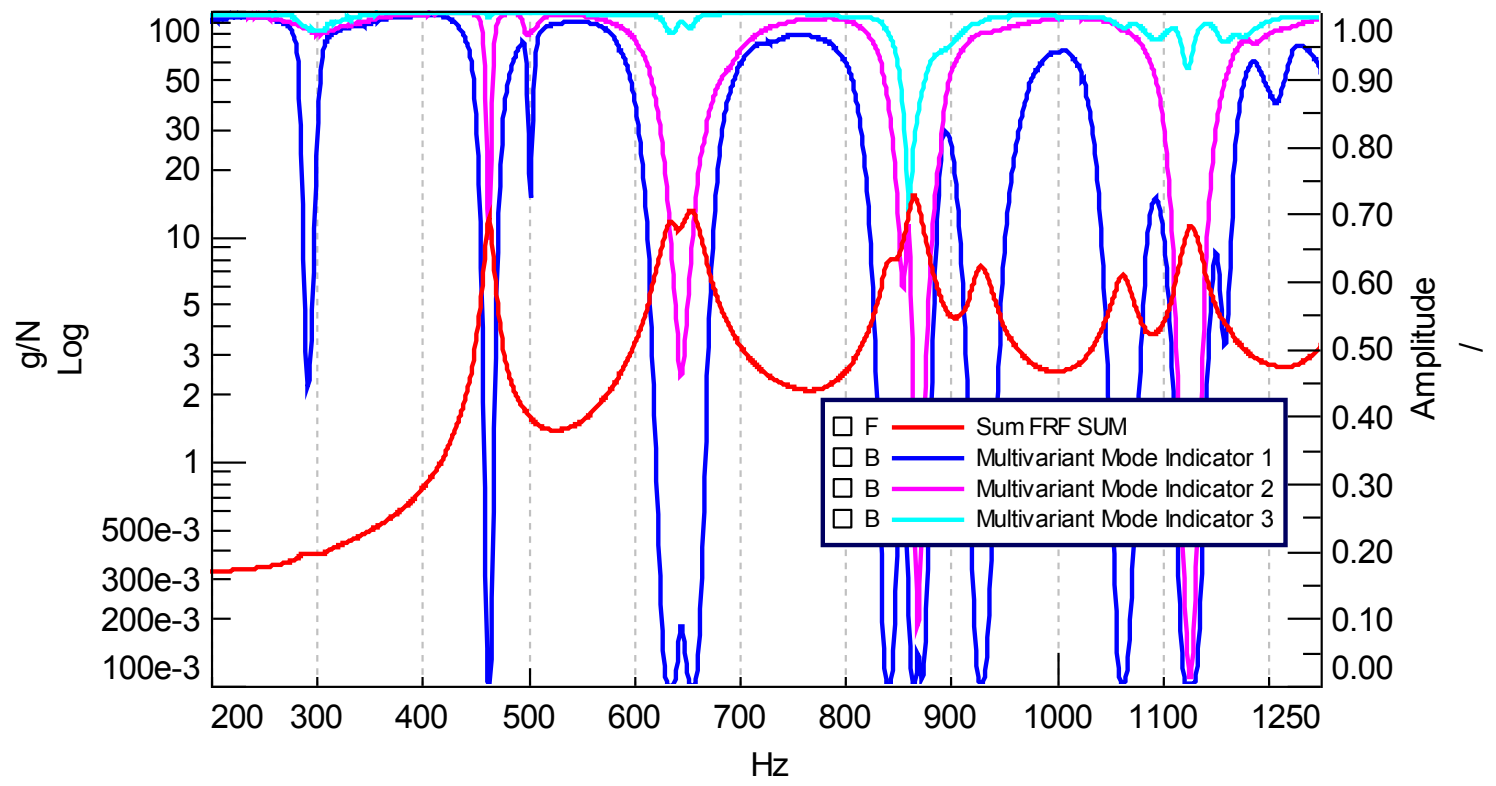

Figure 4.51: Mode indicator functions for curve fitting the factory violin corpus experimental data

Michael Pyrkosz

Michigan Technological University 
The modal frequency and damping results are given in Table 4.14. These are followed by the first five mode shapes in Figures 4.52 through 4.56. Once again the color scale indicates absolute displacement (blue $=0$, red $=$ maximum). These are the signature modes of the violin corpus without the participation of the other sub-components (such as the neck, fingerboard, and tail piece). Note that the first and third modes are acoustic (A0 and $\mathrm{A} 1$ respectively) and as such, are not expected to be in the structural FE model, which is effectively in a vacuum.

Table 4.14: Curve-fitting results from the factory violin corpus EMA

\begin{tabular}{|c|c|c|c|}
\hline Mode \# & Frequency (Hz) & Damping (\%) & Description \\
\hline 1 & 290.5 & 1.62 & A0 \\
\hline 2 & 463.3 & 0.72 & CBR \\
\hline 3 & 501.8 & 0.77 & A1 \\
\hline 4 & 635.2 & 1.53 & B1- \\
\hline 5 & 655.2 & 1.42 & B1+ \\
\hline 6 & 841.8 & 0.92 & \\
\hline 7 & 865.3 & 0.82 & \\
\hline 8 & 872.8 & 0.84 & \\
\hline 9 & 929.2 & 1.10 & \\
\hline 10 & 1064.4 & 0.97 & \\
\hline 11 & 1123.4 & 0.85 & \\
\hline 12 & 1127.8 & 0.85 & \\
\hline 13 & 1158.8 & 0.67 & \\
\hline
\end{tabular}




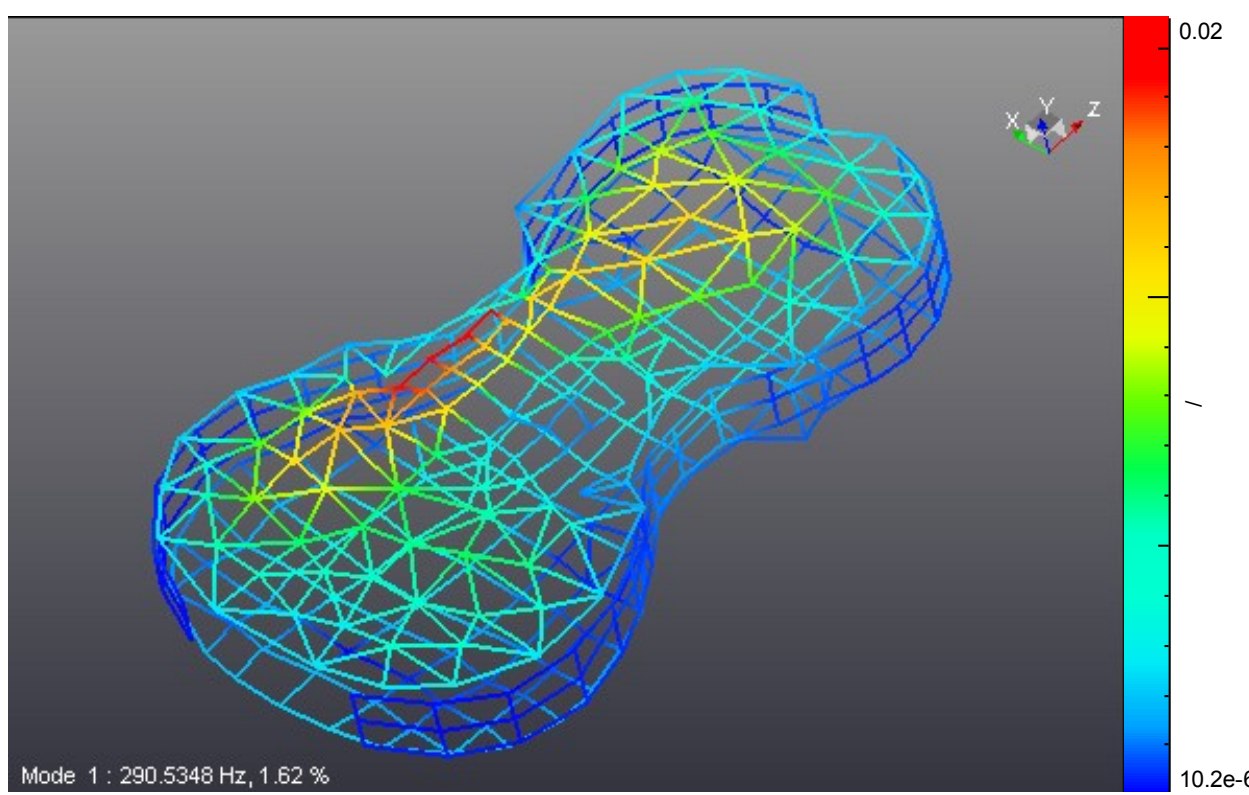

Figure 4.52: First experimental mode of corpus (A0: $290 \mathrm{~Hz}$ )

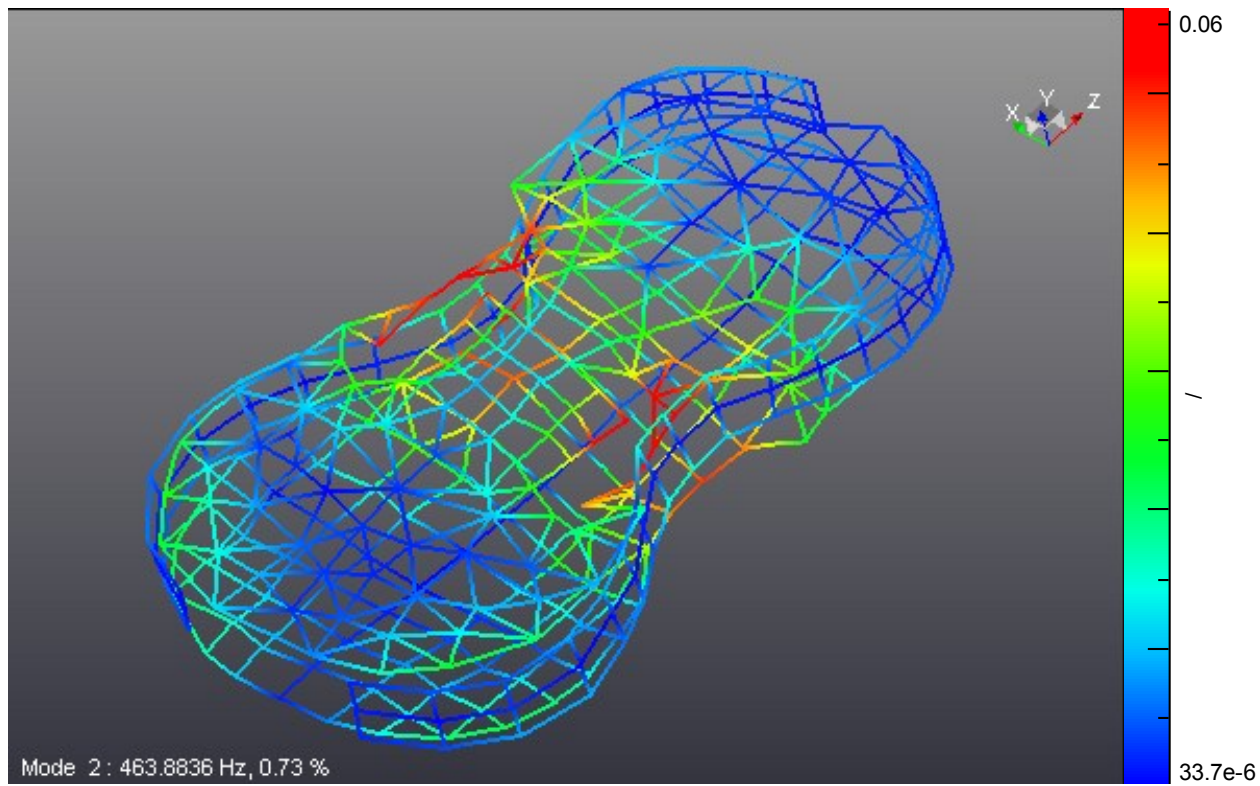

Figure 4.53: Second experimental mode of corpus (CBR: $464 \mathrm{~Hz}$ )

Michael Pyrkosz 


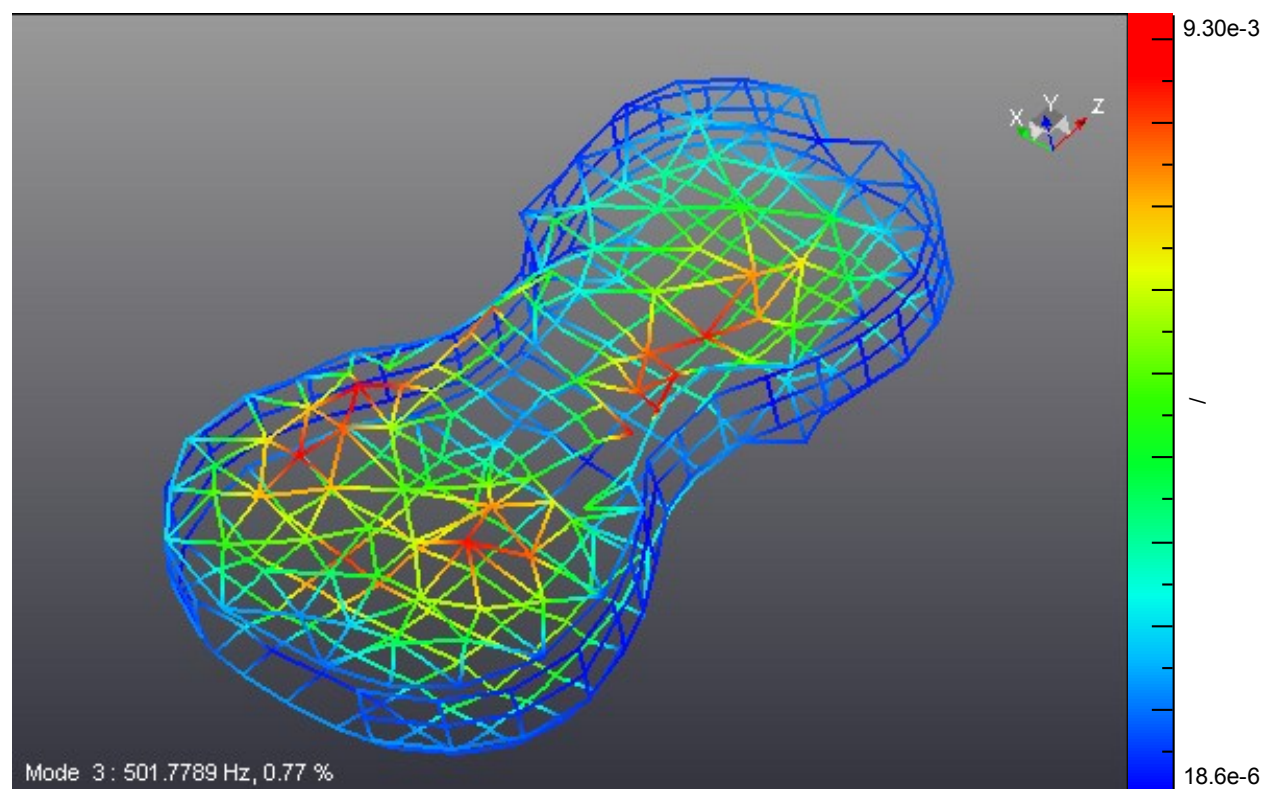

Figure 4.54: Third experimental mode of corpus (A1: $502 \mathrm{~Hz}$ )

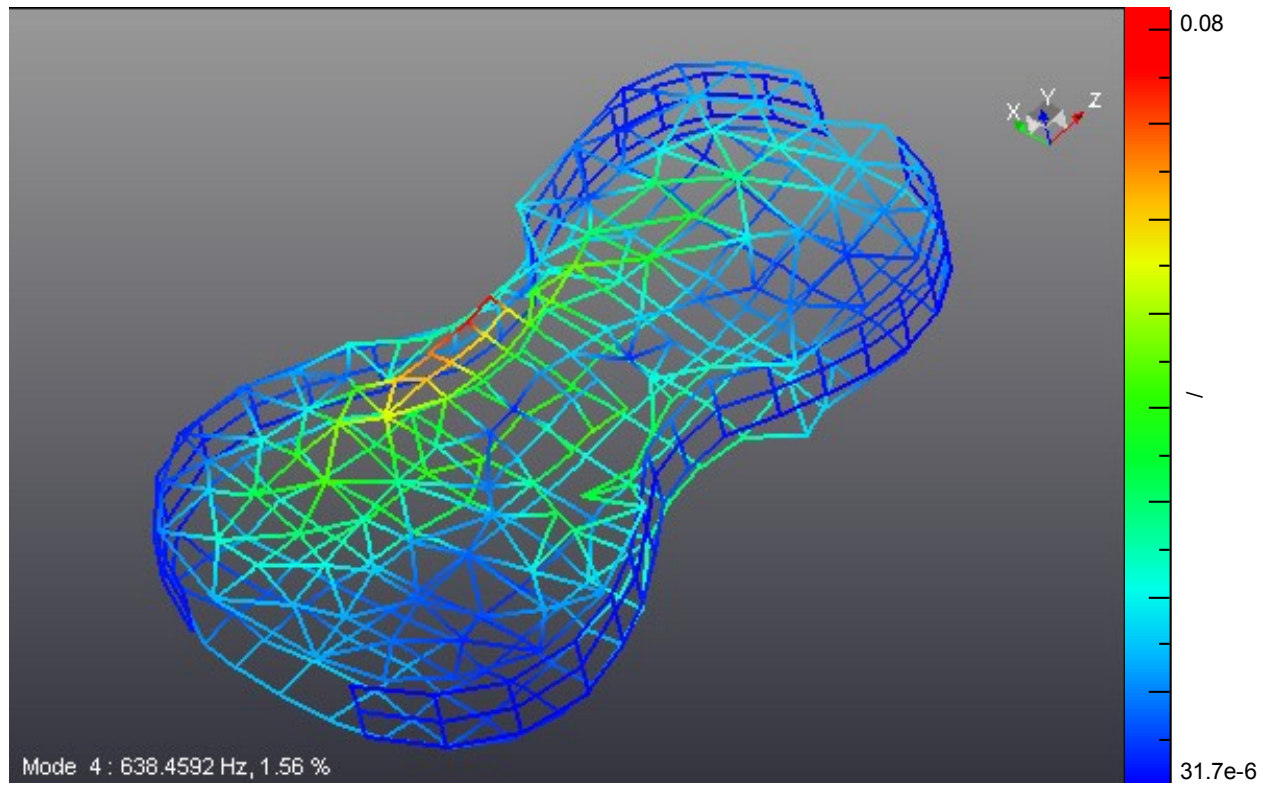

Figure 4.55: Fourth experimental mode of corpus (B1-: $638 \mathrm{~Hz})$ 


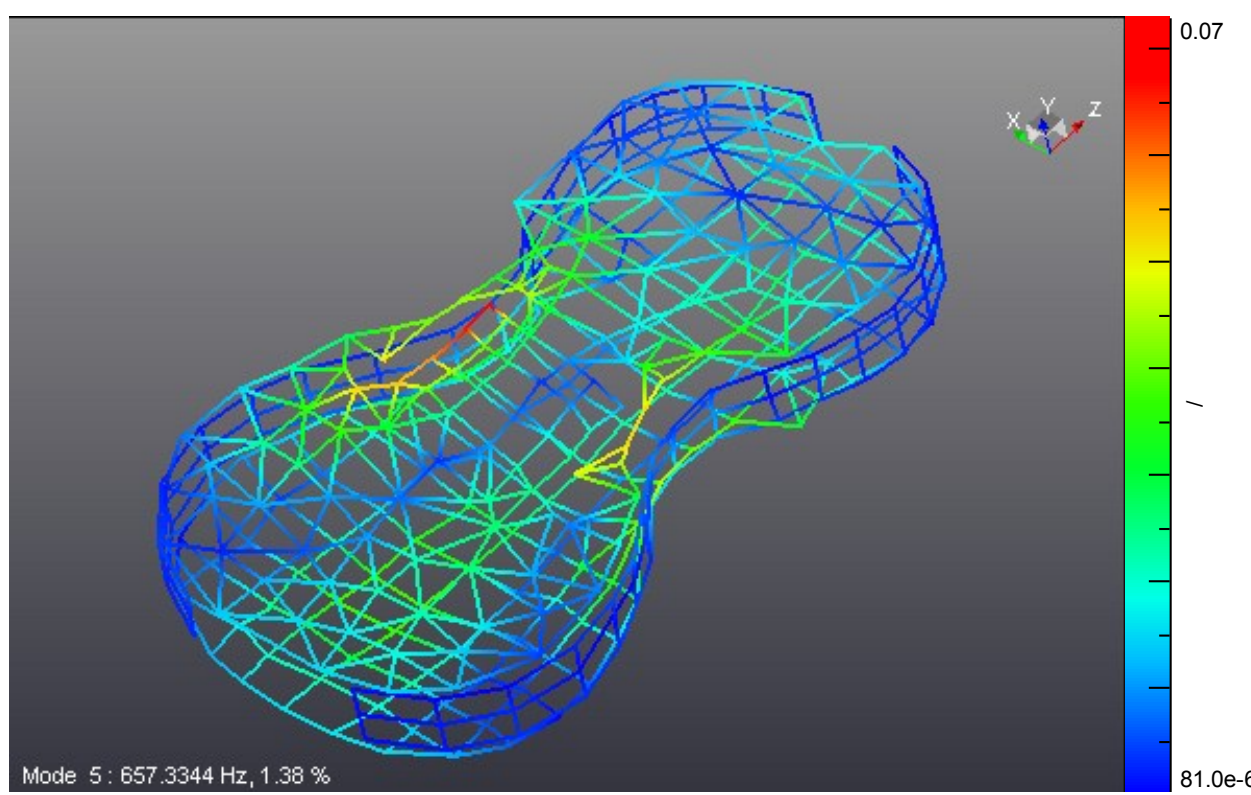

Figure 4.56: Fifth experimental mode of corpus (B1+: $657 \mathrm{~Hz})$

Modification prediction was used to remove the effect of the 0.90 grams of the three accelerometers.

\subsubsection{Corpus FE Results}

The final factory violin corpus structural FE mesh contained 21782 nodes and 28312 elements. A full description of the corpus mesh composition is given in Table 4.15. Figure 4.57 shows the final factory violin corpus FE mesh.

Table 4.15: Factory violin corpus FE mesh composition

\begin{tabular}{|l|c|c|c|c|c|c|c|}
\hline Component & Nodes & TRIA3 & TRIA6 & TETRA4 & TETRA10 & RBE2 & $\begin{array}{c}\text { Total } \\
\text { Elements }\end{array}$ \\
\hline \hline Rib Structure & 9626 & $\sim$ & 2791 & 9559 & $\sim$ & 1081 & 13431 \\
\hline Back plate & 2810 & 5404 & $\sim$ & $\sim$ & $\sim$ & $\sim$ & 5404 \\
\hline Top plate + BB & 7139 & 5244 & $\sim$ & $\sim$ & 2193 & 140 & 7577 \\
\hline Sound post & 2207 & $\sim$ & $\sim$ & $\sim$ & 1178 & $\sim$ & 1178 \\
\hline Full mesh & $\mathbf{2 1 7 8 2}$ & $\mathbf{1 0 6 4 8}$ & $\mathbf{2 7 9 1}$ & $\mathbf{9 5 5 9}$ & $\mathbf{3 3 7 1}$ & $\mathbf{1 9 4 3}$ & $\mathbf{2 8 3 1 2}$ \\
\hline
\end{tabular}




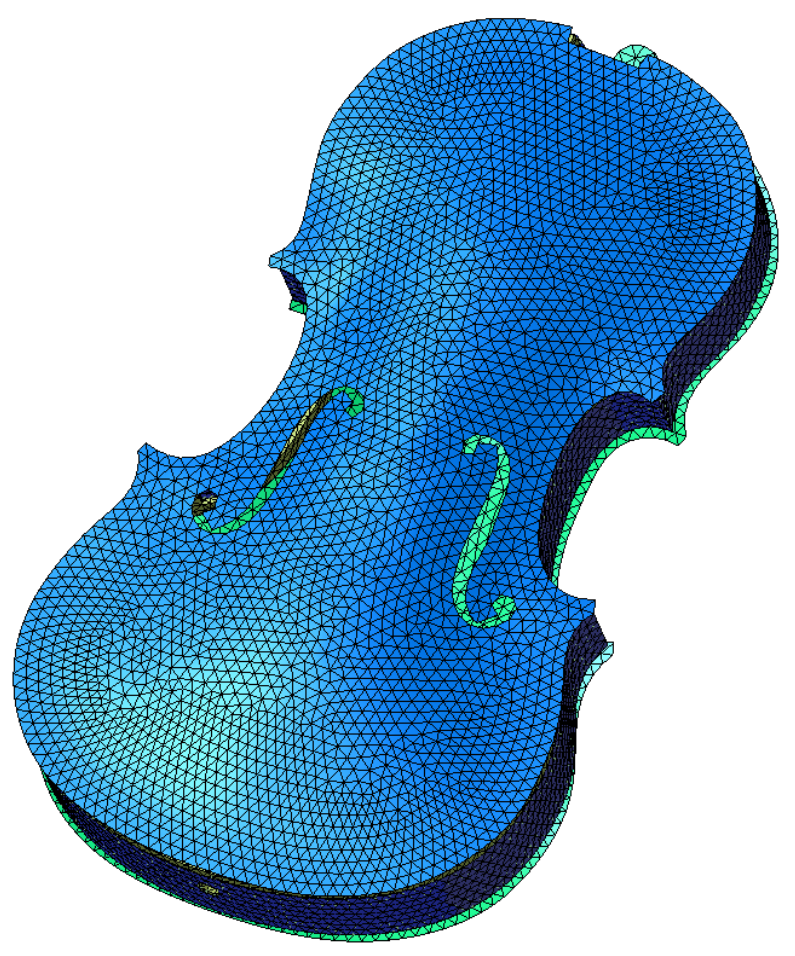

Figure 4.57: Factory violin corpus FE mesh

Results of the final FE model normal modes analysis are summarized in Table 4.16. Examples of the first six FE mode shapes are shown in Figures 4.58 through 4.63.

Table 4.16: Summary of final factory violin corpus normal modes analysis

\begin{tabular}{|c|c|l|}
\hline Mode \# & Freq (Hz) & Description \\
\hline 1 & 503.9 & Center Bout Rhomboid, CBR \\
\hline 2 & 598.9 & First Bending, B1- \\
\hline 3 & 637.7 & First Bending, B1+ \\
\hline 4 & 824.7 & $1^{\text {st }}$ Torsion \\
\hline 5 & 841.9 & $2^{\text {nd }}$ Bending \\
\hline 6 & 851.7 & \\
\hline 7 & 998.2 & \\
\hline 8 & 1103.2 & \\
\hline 9 & 1114.6 & \\
\hline 10 & 1123.1 & \\
\hline
\end{tabular}




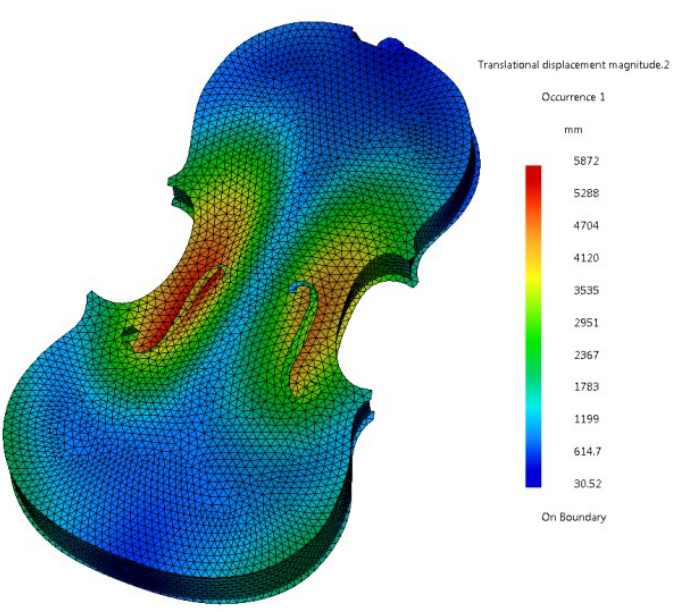

Figure 4.58: First analytical mode of corpus (CBR)

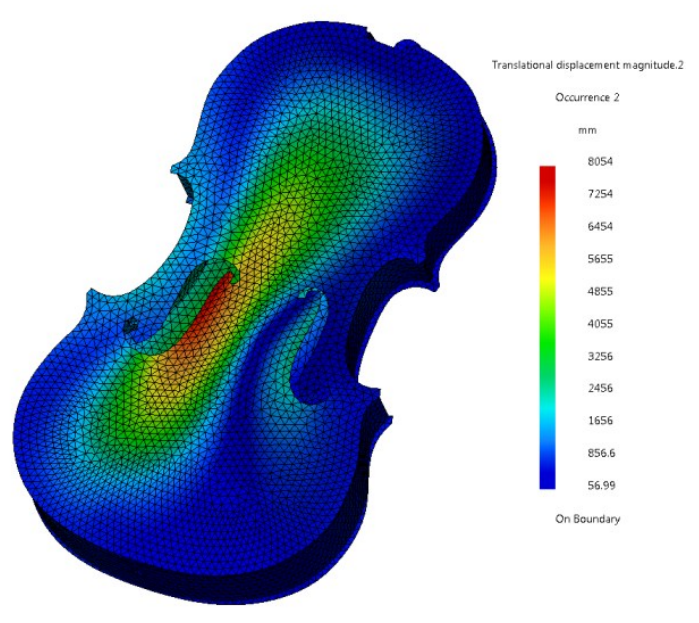

Figure 4.59: Second analytical mode of corpus (B1-)

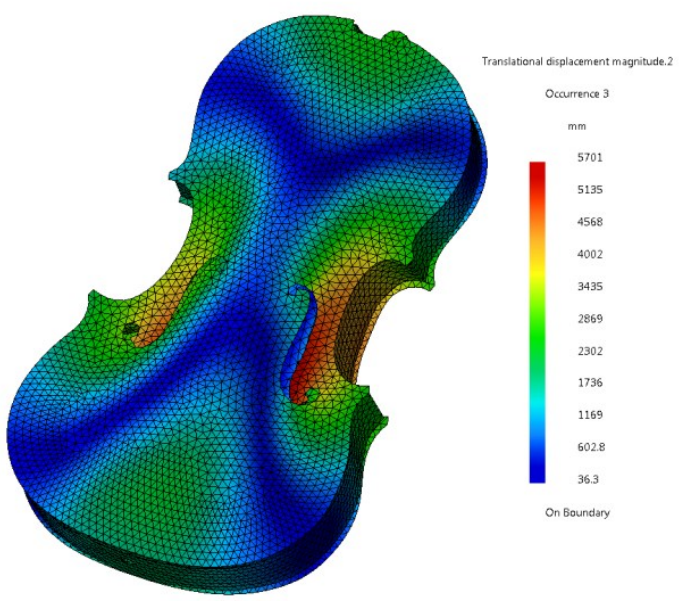

Figure 4.60: Third analytical mode of corpus (B1+)

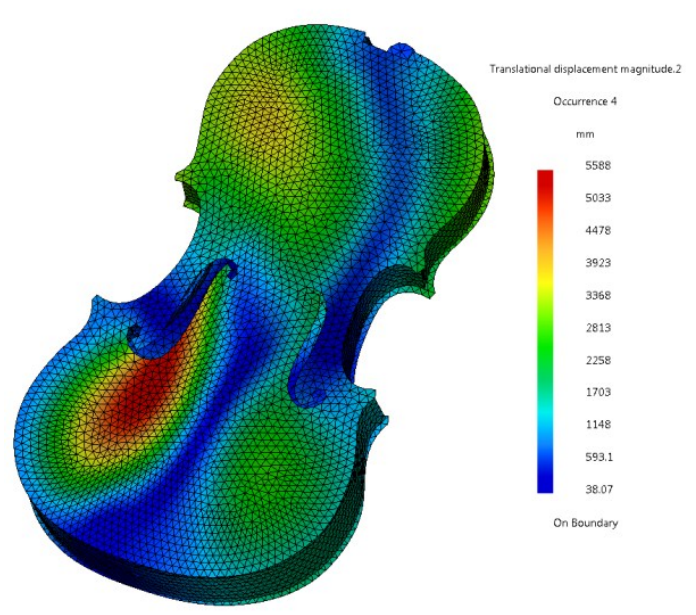

Figure 4.61: Fourth analytical mode of corpus 


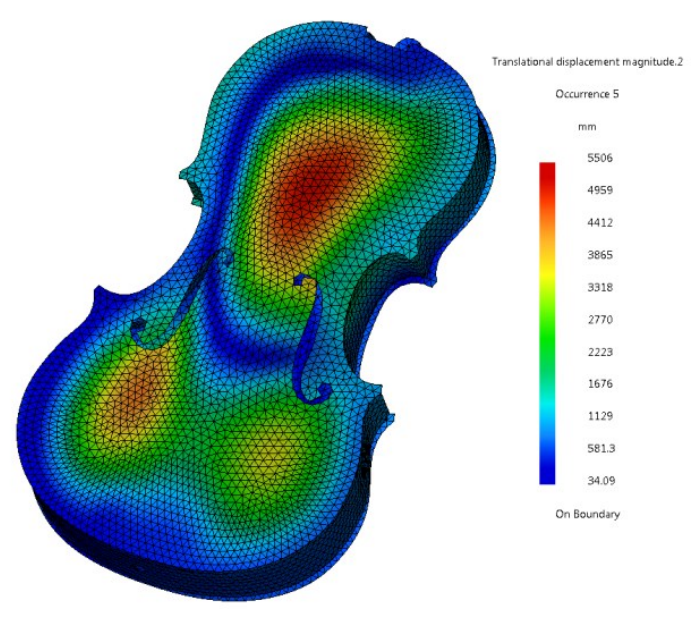

Figure 4.62: Fifth analytical mode of corpus

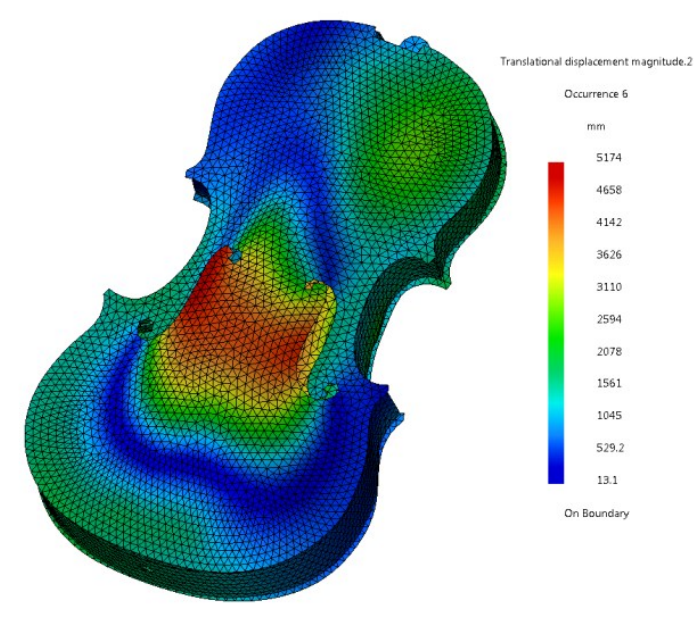

Figure 4.63: Sixth analytical mode of corpus

\subsubsection{Corpus Correlation and Updating}

Results of the factory violin corpus FE model were correlated with those from experimental data using the Modal Assurance Criterion (MAC). Figure 4.64 shows a plot of the final MAC matrix results for the correlation between the test and the final factory violin corpus normal modes analysis. The corresponding mode pairs are given in Table 4.17 .

Table 4.17: Mode pairs of the factory violin corpus correlation

\begin{tabular}{|c|c|c|c|c|c|c|}
\hline $\begin{array}{c}\text { Test } \\
\text { Id }\end{array}$ & $\begin{array}{c}\text { Test Freq } \\
(\mathrm{Hz})\end{array}$ & $\begin{array}{c}\text { FE } \\
\text { Id }\end{array}$ & $\begin{array}{c}\text { FE Freq } \\
(\mathrm{Hz})\end{array}$ & $\begin{array}{c}\text { MAC } \\
\text { Value }\end{array}$ & $\begin{array}{c}\text { Freq Diff } \\
(\mathrm{Hz})\end{array}$ & $\begin{array}{c}\text { Freq Diff } \\
(\% \text { of Test })\end{array}$ \\
\hline 2 & 463.9 & 1 & 503.9 & 0.936 & 40.02 & 8.6 \\
\hline 4 & 638.5 & 2 & 598.9 & 0.768 & 39.61 & 6.2 \\
\hline 5 & 657.3 & 3 & 637.7 & 0.758 & 19.63 & 3 \\
\hline 6 & 842.2 & 4 & 824.7 & 0.63 & 17.50 & 2.1 \\
\hline 7 & 867.3 & 6 & 851.7 & 0.625 & 15.57 & 1.8 \\
\hline 8 & 873.1 & 5 & 841.9 & 0.488 & 31.20 & 3.6 \\
\hline 9 & 931.2 & 7 & 998.2 & 0.48 & 67.06 & 7.2 \\
\hline 10 & 1065.4 & 8 & 1103.2 & 0.714 & 37.80 & 3.5 \\
\hline 11 & 1123.7 & 9 & 1114.6 & 0.369 & 9.07 & 0.8 \\
\hline 12 & 1129 & 10 & 1123.1 & 0.628 & 5.83 & 0.5 \\
\hline
\end{tabular}




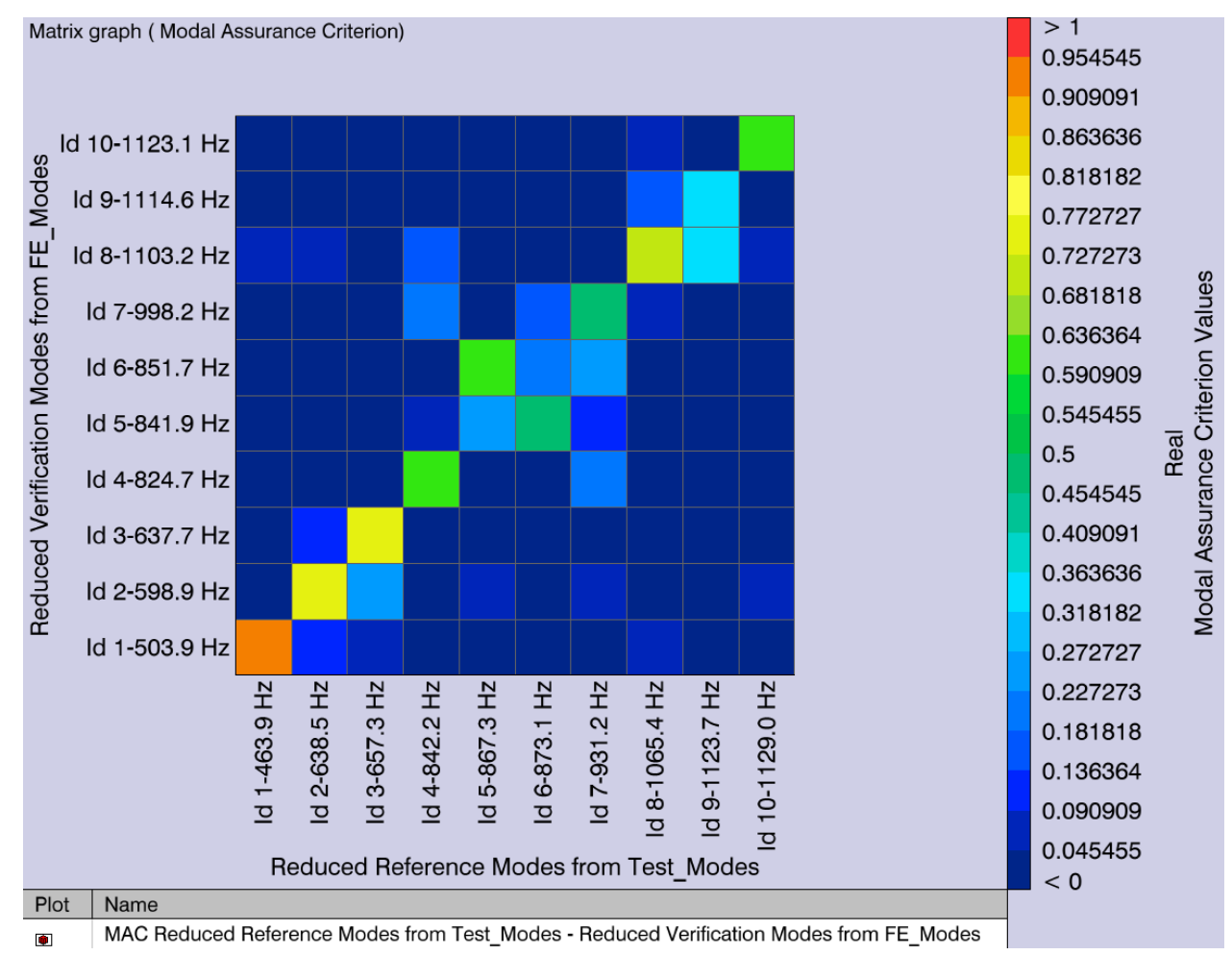

Figure 4.64: MAC results of factory violin corpus EMA modes with structural FE model

It is noted that the material properties of the factory violin corpus FE model were updated to "tune" the modal frequencies. Updating was performed until the average percent difference for the first 12 modes was below 5\%. A summary of the final material properties is given in Table 4.18 .

Table 4.18: Summary of final material properties for factory violin corpus FE model

\begin{tabular}{|c|c|c|c|c|c|c|c|c|}
\hline Component & $\begin{array}{c}\text { Thickness } \\
(\mathrm{mm})\end{array}$ & $\begin{array}{c}\mathrm{E} 1 \\
(\mathrm{MPa})\end{array}$ & $\begin{array}{c}\mathrm{E} 2 \\
(\mathrm{MPa})\end{array}$ & $\mathrm{NU12}$ & $\begin{array}{c}\text { G12 } \\
(\mathrm{MPa})\end{array}$ & $\begin{array}{c}\text { G1Z } \\
(\mathrm{MPa})\end{array}$ & $\begin{array}{c}\mathrm{G} 2 Z \\
(\mathrm{MPa})\end{array}$ & $\begin{array}{c}\mathrm{RHO} \\
\left(\mathrm{kg} / \mathrm{m}^{3}\right)\end{array}$ \\
\hline Back plate & 3.98 & 13965 & 1735.9 & 0.434 & 1294.6 & 778.7 & 407.7 & 571.1 \\
\hline Top plate & Variable & 11074 & 1280.5 & 0.391 & 1029.8 & 823.7 & 59.38 & 448.0 \\
\hline Ribs & 1.0 & 10594 & 2024.4 & 0.495 & 2200.0 & 540.2 & 325.3 & 587.0 \\
\hline
\end{tabular}

The overall weight of the factory violin corpus model was $\sim 276.1$ grams.

Michael Pyrkosz 


\subsubsection{Titian Stradivari Violin Structural Results}

\subsubsection{Review of test data}

The experimental modal analysis of the Titian Stradivari violin was performed by Bissinger, et al, at East Carolina University, and is described more fully in the references [4.12], [4.13]. A brief review is provided here in the context of the current research.

The Titian Stradivari experimental data contains FRF measurements taken at 295 response locations on the top and back plates using a Polytec 3-axis laser system, for a total of 885 response DOFs. Input excitation was supplied by a micro impact hammer at the upper bass corner of bridge in the bowing direction (X-axis). No responses were measured for other substructures such as the neck, fingerboard, or tail piece.

Technically there is no driving point available in the data since the laser could not be placed on the corner of the bridge in the same direction as the hammer. The closest measurement to the driving point is actually at the bass-side bridge foot on the top plate (titian:48). The FRFs of all three bass-side bridge foot responses with respect to the input are given in Figure 4.65 The out-of-plane direction (Y-axis) was measured directly by one laser while the two remaining in-plane directions (X-and $\mathrm{Z}$ - axes) are computed from two other off-angle lasers. As a result the signal-to- noise ratio of the Y-DOF for any point is much greater than the others. It is observed that the phase of the $\mathrm{X}$ and $\mathrm{Y}$ responses both roll past zero $\sim 409 \mathrm{~Hz}$ (where the CBR mode occurs).

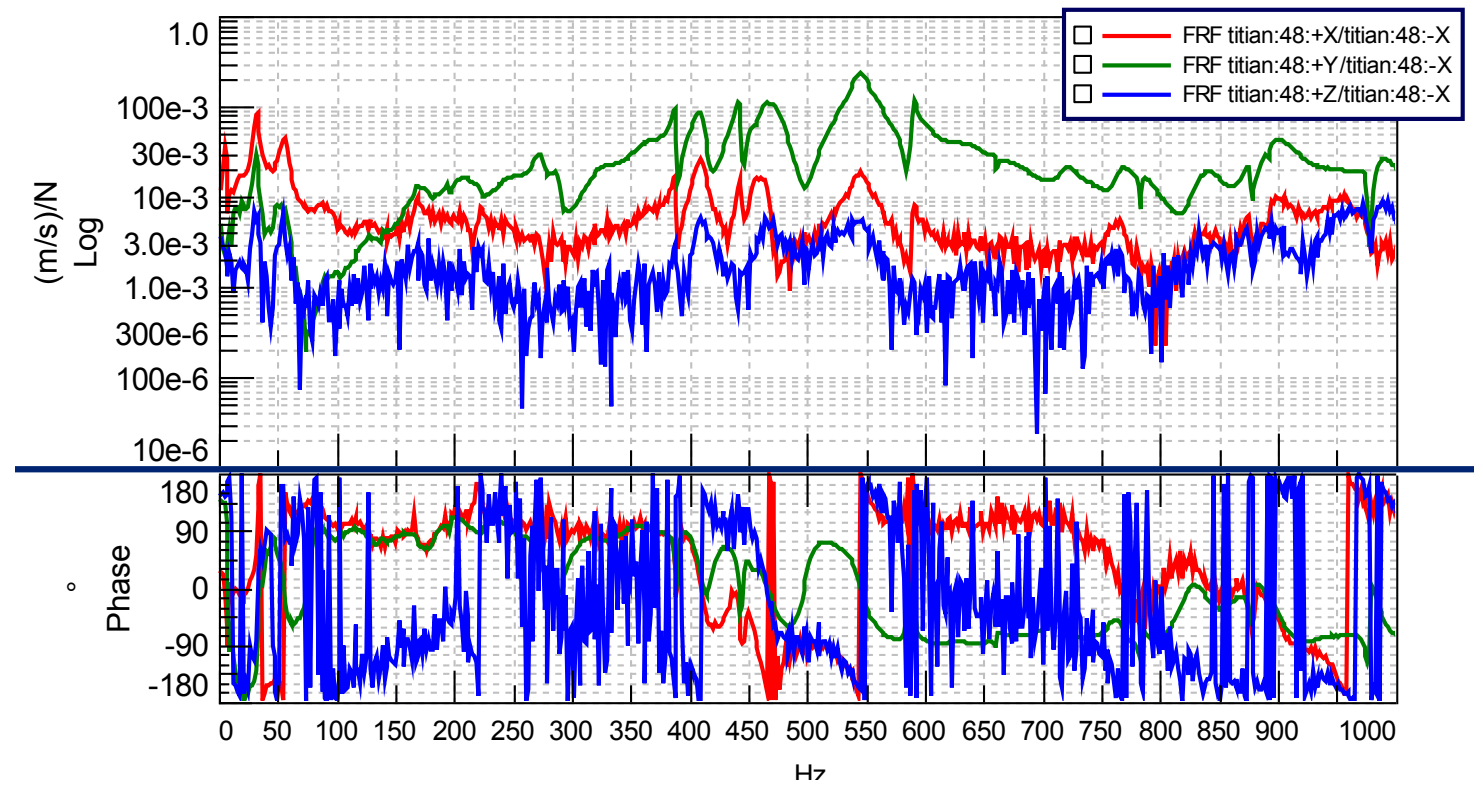

Figure 4.65: FRFs of the bass-side bridge foot responses of the Titian Stradivari violin 
The coherence of all three bass-side bridge foot responses with respect to the input are given in Figure 4.66 The Y-direction response is excellent, having coherence values greater than 0.95 throughout the frequency range. The coherence in the $\mathrm{X}$ and $\mathrm{Z}$ directions indicate greater variance, but result in values above 0.90 at each of the structural signature modes (CBR $\sim 409 \mathrm{~Hz}, \mathrm{~B} 1-\sim 471 \mathrm{~Hz}, \mathrm{~B} 1+\sim 545 \mathrm{~Hz}$ ).

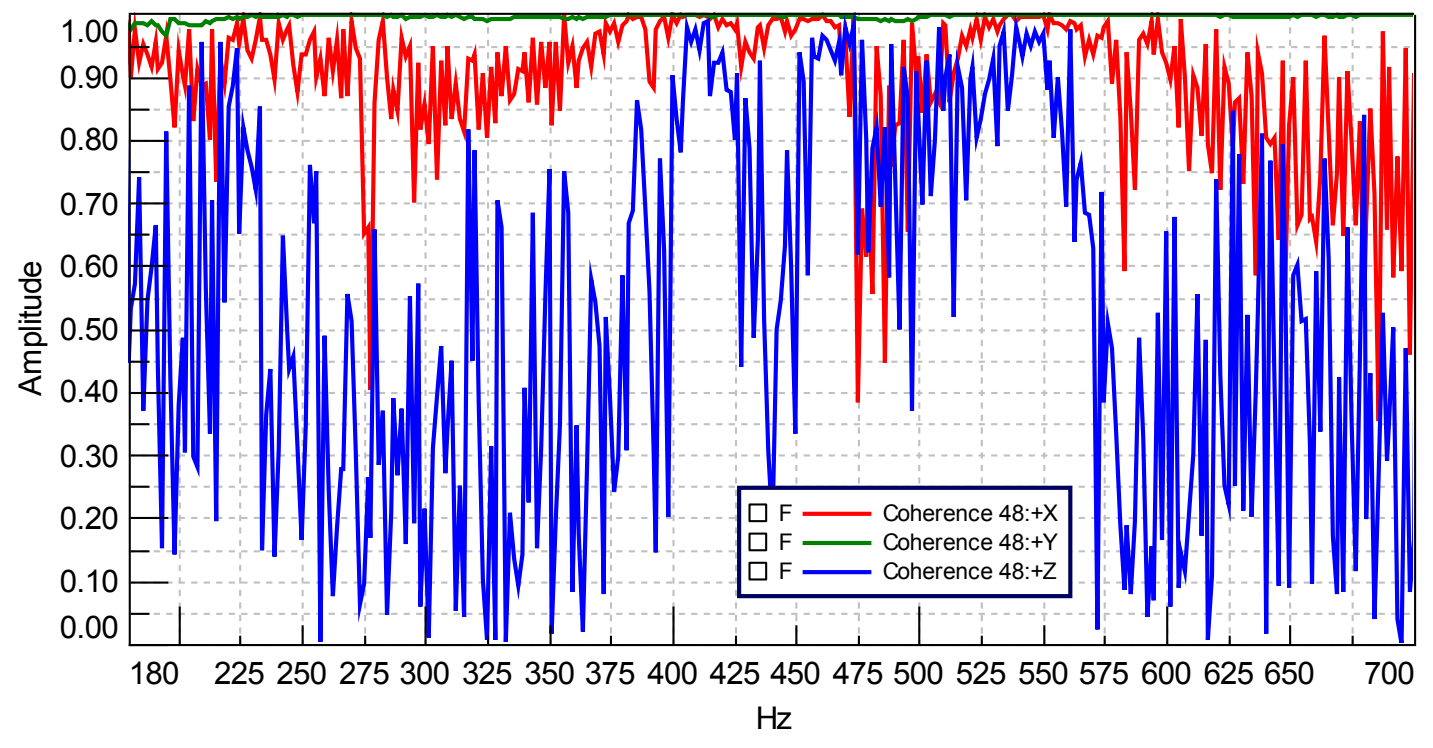

Figure 4.66: Coherence spectra of the bass-side bridge foot responses of the Titian Stradivari violin

A number of points, such as 141, had poor coherence in X, Z, or both, and it was found that the results were improved by removing these DOFs from the curve fitting process. Some points were apparently distorted due to the hammer fixture (such as 118) and were excluded from the curve-fit. The DOFs of these points were slaved to neighboring points.

The PolyTec data was imported into LMS Test.Lab for the final curve fitting. Several bands from $150 \mathrm{~Hz}$ to $700 \mathrm{~Hz}$ were selected for stabilization. The Mode Indicator Functions (MIFs) used for modal parameter selection are shown in Figure 4.67. The red curve is the FRF sum, while the blue curve is the multivariant MIF. The Automatic Modal Parameter Selection (AMPS) feature was used. 


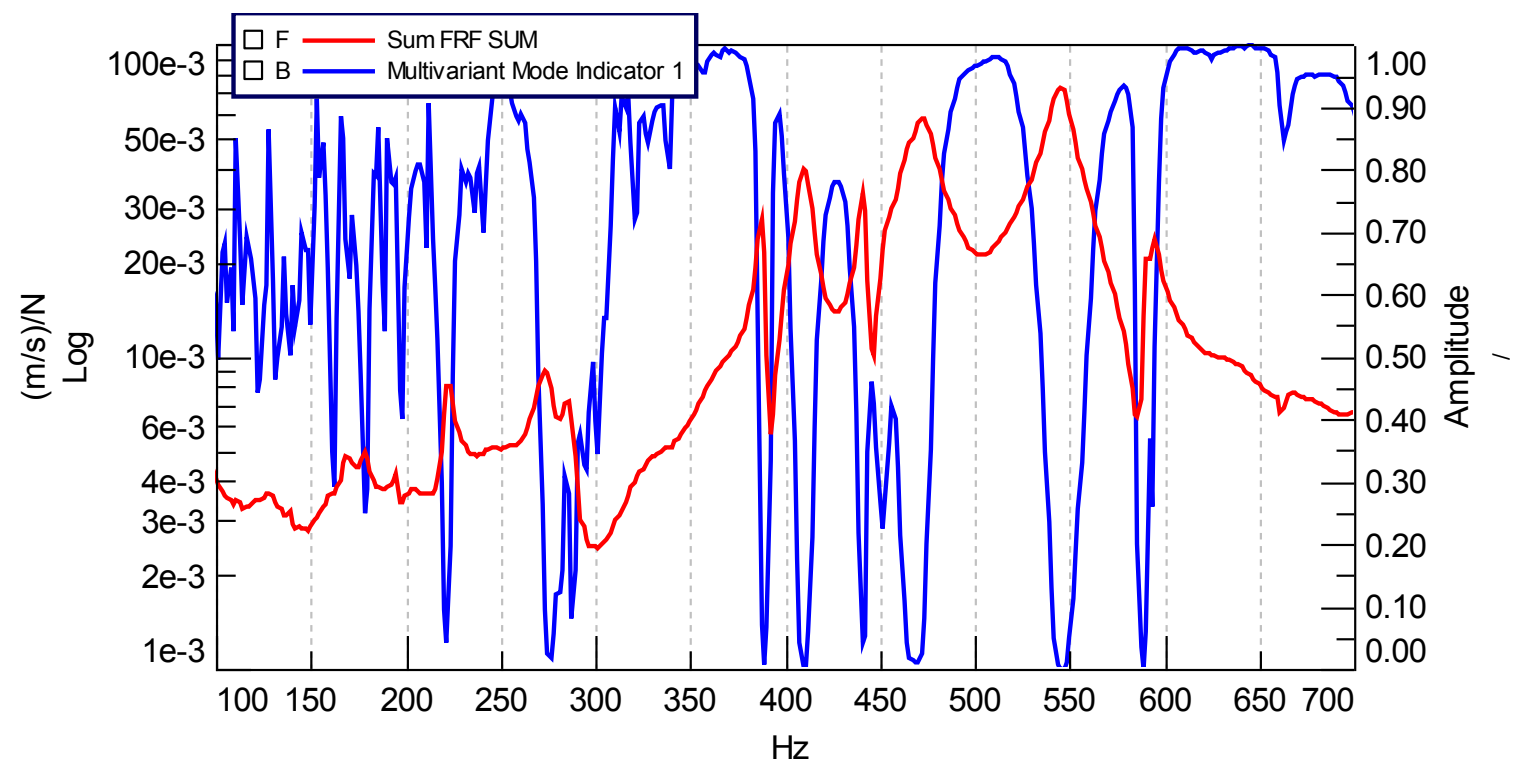

Figure 4.67: FRF sum and Multivariant MIF for the Titian Stradivari violin curve-fitting

The resulting modal frequencies and damping are given in Table 4.19. Notes have been included to identify string frequencies as well as signature modes. 
Table 4.19: Curve-fitting results from the Titian Stradivari violin EMA

\begin{tabular}{|c|c|c|c|}
\hline Mode \# & Freq (Hz) & Damping (\%) & Notes \\
\hline 1 & 168.9 & 0.55 & \\
\hline 2 & 179.7 & 0.72 & \\
\hline 3 & 197.4 & 0.47 & $1^{\text {st }}$ G-string \\
\hline 4 & 222.0 & 0.96 & \\
\hline 5 & 253.6 & 0.22 & \\
\hline 6 & 274.0 & 1.68 & \\
\hline 7 & 286.0 & 0.68 & $1^{\text {st }} \mathrm{D}-$ string \\
\hline 8 & 387.1 & 0.25 & $2^{\text {nd }}$ G-string \\
\hline 9 & 389.2 & 0.36 & $2^{\text {nd }}$ G-string \\
\hline 10 & 409.3 & 1.02 & CBR \\
\hline 11 & 441.6 & 0.44 & $1^{\text {st }} \mathrm{A}-$ string \\
\hline 12 & 452.2 & 1.06 & $1^{\text {st }}$ A-string \\
\hline 13 & 465.3 & 1.07 & \\
\hline 14 & 471.8 & 1.29 & B1- \\
\hline 15 & 517.8 & 0.38 & \\
\hline 16 & 519.3 & 0.50 & \\
\hline 17 & 544.3 & 0.25 & \\
\hline 18 & 545.9 & 1.38 & B1+ \\
\hline 19 & 556.7 & 0.45 & \\
\hline 20 & 557.6 & 0.67 & \\
\hline 21 & 590.2 & 0.35 & $2^{\text {nd }} \mathrm{D}-$ string \\
\hline 22 & 593.9 & 0.36 & $2^{\text {nd }} \mathrm{D}-$ String \\
\hline 23 & 661.2 & 0.11 & $1^{\text {st }}$ E-string \\
\hline & & & \\
\hline
\end{tabular}

\subsubsection{FE Results for the Titian Stradivari violin}

The final Titian Stradivari violin structural FE mesh contains 74756 nodes and 117528 elements. A full description of the Titian Stradivari mesh composition is given in Table 4.20. Note that skins are not included in the individual component counts but are included in the full mesh. The plates were attached to the rib structure with a single layer of hexahedral elements. Figure 4.68 shows a screen capture of the final Titian Stradivari FE mesh. 
Table 4.20: Titian Stradivari violin structural FE mesh composition

\begin{tabular}{|c|c|c|c|c|c|c|c|}
\hline Component & Beams & TRIA & QUAD & TETRA & PENTA & HEXA & $\begin{array}{c}\text { Total Elements } \\
\text { (no skins) }\end{array}$ \\
\hline Back plate & $\sim$ & 62 & 11193 & $\sim$ & $\sim$ & $\sim$ & 11255 \\
\hline $\begin{array}{c}\text { Top plate + Bass } \\
\text { Bar }\end{array}$ & $\sim$ & 46 & 10619 & 186 & 18 & 1176 & 12045 \\
\hline Rib Structure & $\sim$ & $\sim$ & 6458 & $\sim$ & 266 & 10724 & 17448 \\
\hline Sound post & $\sim$ & $\sim$ & $\sim$ & $\sim$ & 116 & 348 & 464 \\
\hline Neck \& Scroll & $\sim$ & $\sim$ & $\sim$ & 27828 & 159 & 7502 & 35489 \\
\hline Fingerboard & $\sim$ & $\sim$ & $\sim$ & $\sim$ & $\sim$ & 4032 & 4032 \\
\hline Bridge & $\sim$ & $\sim$ & $\sim$ & $\sim$ & 30 & 1300 & 1330 \\
\hline Tail piece & $\sim$ & $\sim$ & $\sim$ & $\sim$ & 64 & 4776 & 4840 \\
\hline Nut & $\sim$ & $\sim$ & $\sim$ & $\sim$ & $\sim$ & 72 & 72 \\
\hline Saddle & $\sim$ & $\sim$ & $\sim$ & $\sim$ & $\sim$ & 84 & 84 \\
\hline $\begin{array}{c}\text { Strings + Tail } \\
\text { Loop }\end{array}$ & 598 & $\sim$ & $\sim$ & $\sim$ & $\sim$ & $\sim$ & 598 \\
\hline $\begin{array}{c}\text { Full Mesh } \\
\text { (including skins) }\end{array}$ & 598 & 438 & 55526 & 28014 & 691 & 32206 & 117528 \\
\hline
\end{tabular}




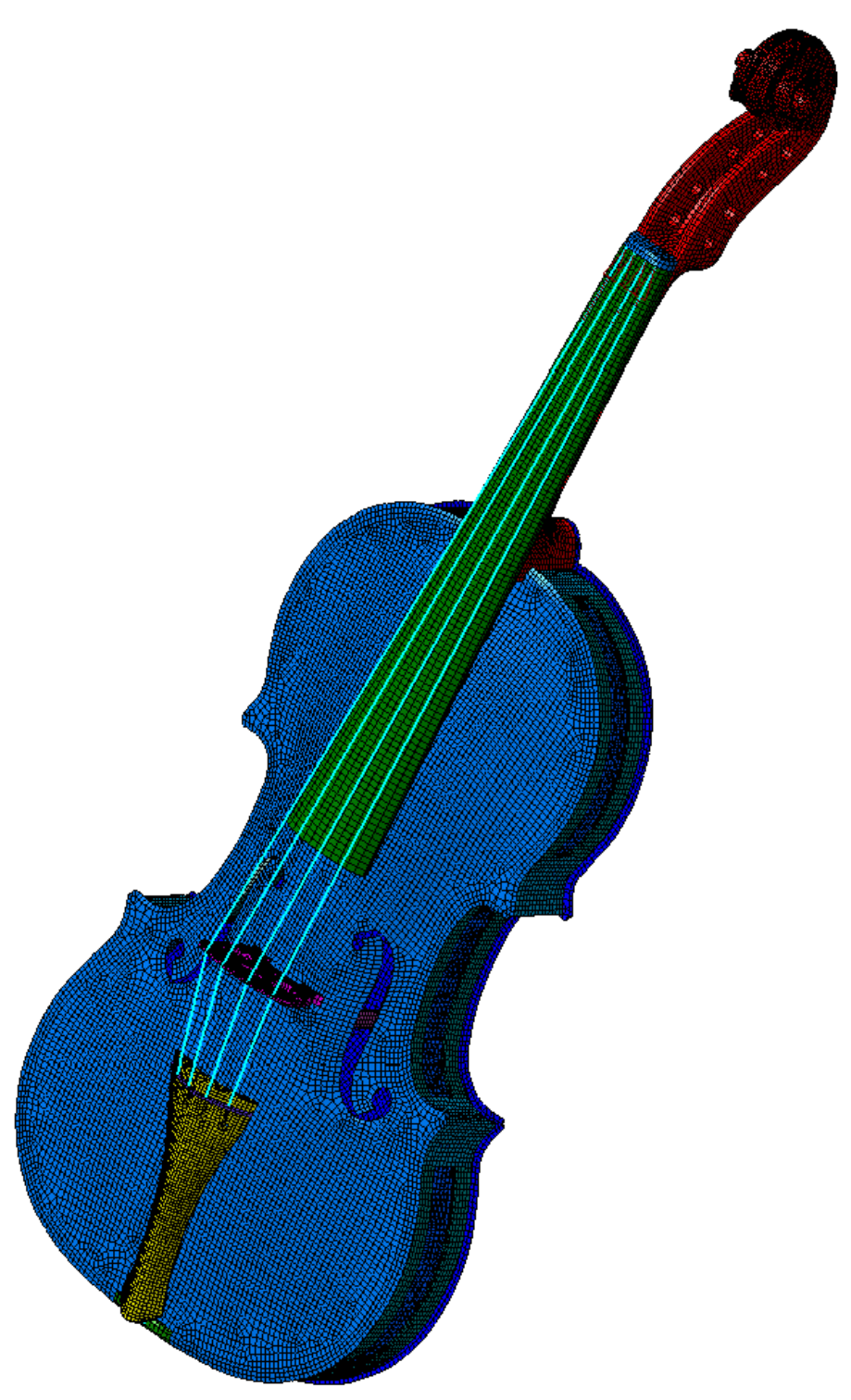

Figure 4.68: Final Titian Stradivari violin structural FE model with Pre-loaded strings

The thickness graduations of both plates were mapped similarly to the factory violin, using the data collected by Zygmuntowicz and Loen [4.4]. The published graduation measurements were transferred to the coordinates of the model through a set of transformation vectors estimated from points taken at the corners. The results of the thickness mapping are shown in Figures 4.69 and 4.70 (color indicates thickness in mm). 

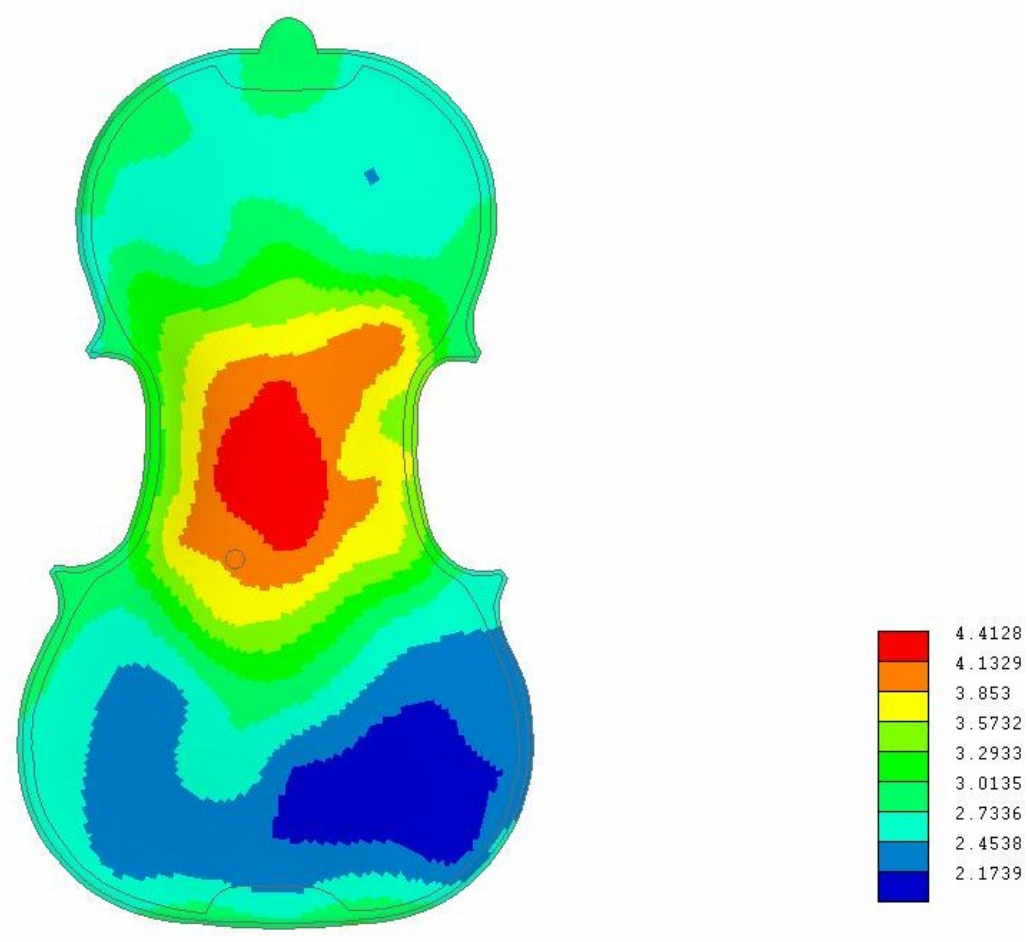

Figure 4.69: Element thickness of the Titian Stradivari violin back plate in $\mathrm{mm}$
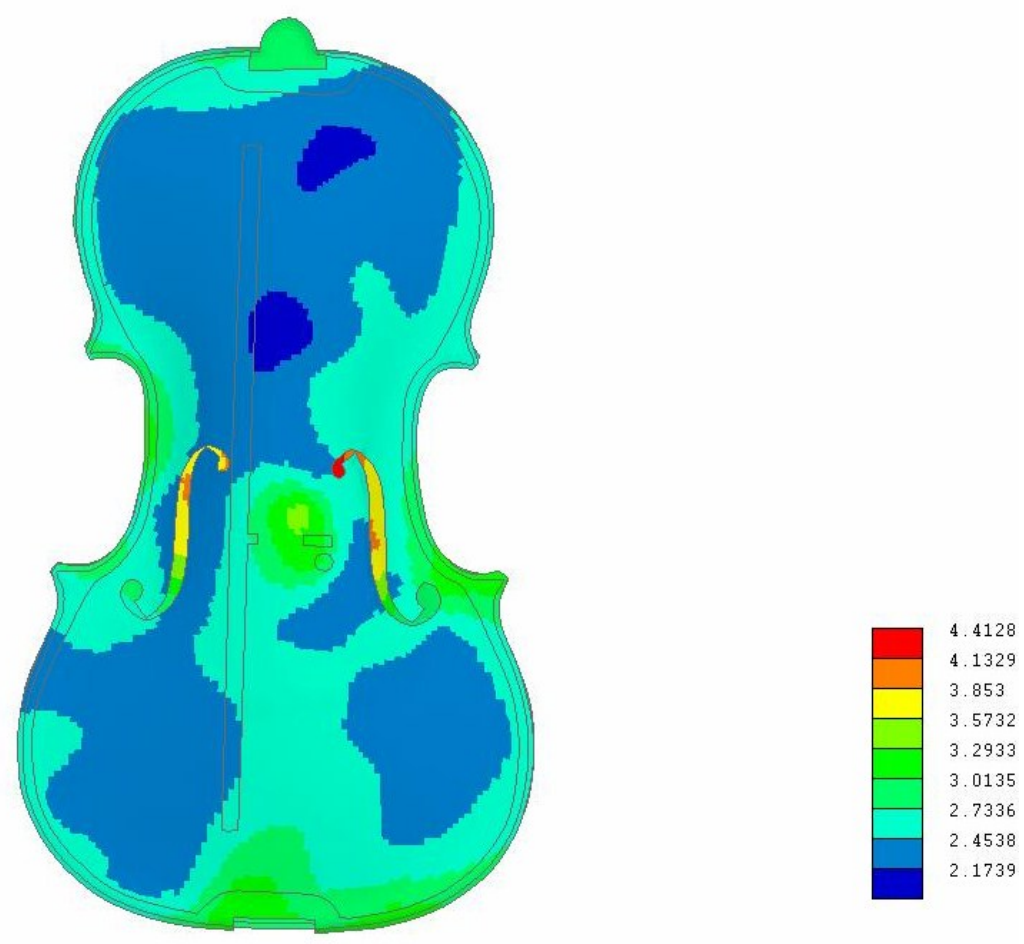

Figure 4.70: Element thickness of the Titian Stradivari violin top plate in $\mathrm{mm}$ 
The final results of the Titian Stradivari violin structural FE normal modes analysis is given in Table 4.21 .

Table 4.21: Summary of updated Titian Stradivari violin normal modes analysis

\begin{tabular}{|c|l|c|}
\hline Mode \# & Description & Frequency (Hz) \\
\hline 1 & B0 (Tail IP) & $158.9 \mathrm{~Hz}$ \\
\hline 2 & B0 (Tail OP) & $181.1 \mathrm{~Hz}$ \\
\hline 3 & Lateral B0 (Tail IP) & $223.5 \mathrm{~Hz}$ \\
\hline 4 & B0 (FB bend) & $227.2 \mathrm{~Hz}$ \\
\hline 5 & Lateral B0 (Tail OP) & $245.8 \mathrm{~Hz}$ \\
\hline 6,7 & $1^{\text {st }}$ G-string & $195.0 \mathrm{~Hz}, 197.0 \mathrm{~Hz}$ \\
\hline 8,9 & $1^{\text {st }}$ D-string & $292.2 \mathrm{~Hz}, 294.8 \mathrm{~Hz}$ \\
\hline 10,11 & $2^{\text {nd }}$ G-string & $387.9 \mathrm{~Hz}, 393.0 \mathrm{~Hz}$ \\
\hline 12 & CBR & $427.3 \mathrm{~Hz}$ \\
\hline 13,14 & $1^{\text {st }}$ A-string & $436.2 \mathrm{~Hz}, 442.5 \mathrm{~Hz}$ \\
\hline 15 & B1- & $452.5 \mathrm{~Hz}$ \\
\hline 16 & B1+ & $529.8 \mathrm{~Hz}$ \\
\hline 17,18 & $2^{\text {nd }}$ D-string & $587.5 \mathrm{~Hz}, 589.0 \mathrm{~Hz}$ \\
\hline 19,20 & $3^{\text {rd }}$ G-string & $590.3 \mathrm{~Hz}, 593.3 \mathrm{~Hz}$ \\
\hline 21,22 & $1^{\text {st }}$ E-string & $654.4 \mathrm{~Hz}, 657.5 \mathrm{~Hz}$ \\
\hline 23 & Lateral FB & $538.6 \mathrm{~Hz}$ \\
\hline 24 & Neck-FB twist IP & $633.6 \mathrm{~Hz}$ \\
\hline 25 & Tail rock & $650.8 \mathrm{~Hz}$ \\
\hline 26 & Neck-FB twist OP & $661.1 \mathrm{~Hz}$ \\
\hline
\end{tabular}

\subsubsection{Low frequency modes}

At the low frequency end (below the $1^{\text {st }} \mathrm{G}$-string mode $\sim 196 \mathrm{~Hz}$ ) there are several violin first order bending (B0) modes. These occur first out of plane with the instrument, and then within the plane of the instrument. The number of bending modes is increased by substructures such as the tail piece or fingerboard moving in-phase (IP) or out-of-phase (OP) with the shape. These mode shapes are of less interest for several reasons, but mainly due to most of the flexure occuring at the corpus-neck joint with little flexure of the corpus itself. As a result, these modes typically do not radiate efficiently [4.14].

The first five mode shapes of the Titian Stradivari violin FE structure are shown in Figures 4.71 through 4.75 (color indicates translational displacement magnitude). The other item to note is that these modes, although present in the test data, are not well captured in the test data. This is because only measurement points on the plates were included, and for some of these modes the bridge is sitting close to a nodal line for the shape, and probably would not have been excited by a drive point on the bridge. 


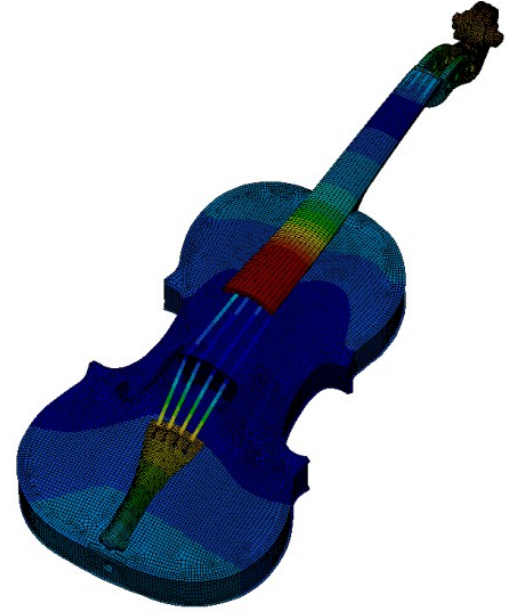

Figure 4.71: First mode of Titian FE model: Global bending, B0 (Tail IP) 158.9 $\mathrm{Hz}$

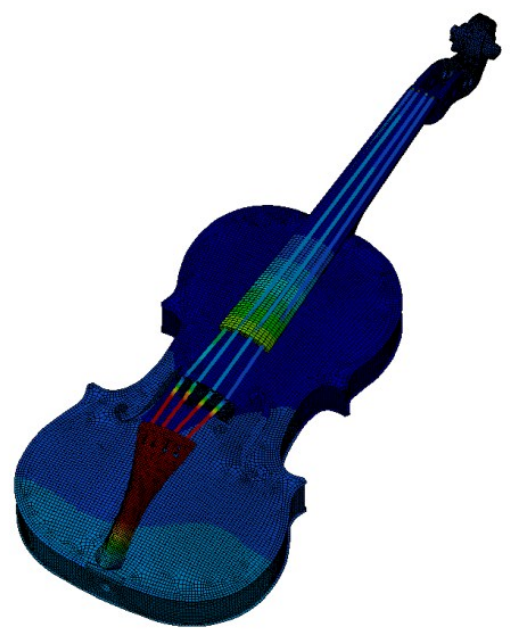

Figure 4.72: Second mode of Titian FE model: Global bending, B0 (Tail OP) 181.1 Hz

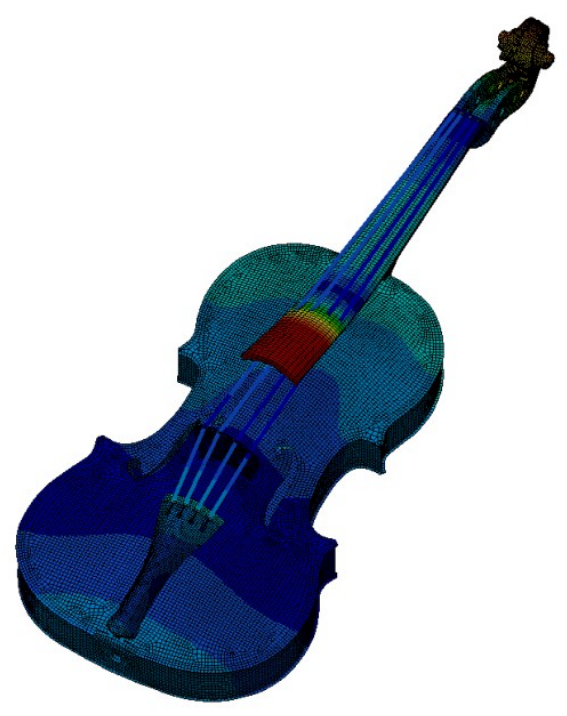

Figure 4.73: Third mode of Titian FE model: Global bending, B0 (FB bend) $227.2 \mathrm{~Hz}$ 


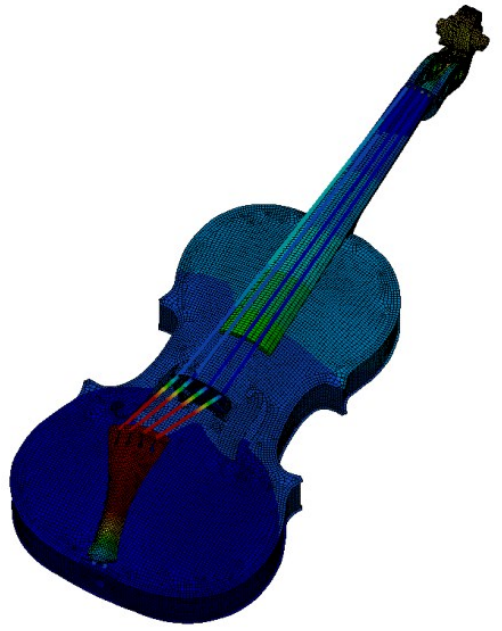

Figure 4.74: Lateral bending mode of the Titian FE model (Tail IP) $223.5 \mathrm{~Hz}$

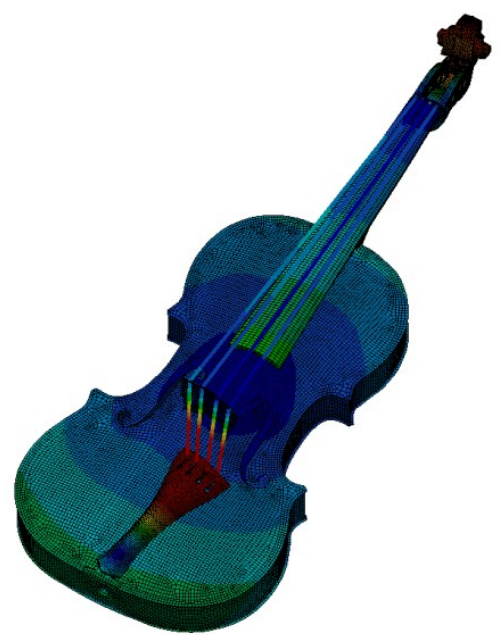

Figure 4.75: Lateral bending mode of the Titian FE model, (Tail OP) $245.8 \mathrm{~Hz}$

\subsubsection{String modes}

Mode shapes for the string modes are shown in Figures 4.76 through 4.82. These are bending modes, and due to the symmetric nature of the cross-section, occur in pairs of repeated roots of the characteristic system equation; that is, bending in the vertical direction occurs at nearly the same frequency as bending in the horizontal direction. The same separation of these mode pairs is due to the relative effective stiffness of the violin structure in bending in and out of the plane of the instrument.

Most of these string modes show minimal relative deflection of the plates, and thus are not well captured in the test data. It should be noted however, that some of the repeated roots are coupled to corpus modes and are expected to be present in the test data. A good example of this is one of the A-string modes $(\sim 440 \mathrm{~Hz})$, which is clearly coupled to the B1- corpus mode $(\sim 452.5 \mathrm{~Hz})$. For this reason, string modes were not included in the correlation and updating process. 


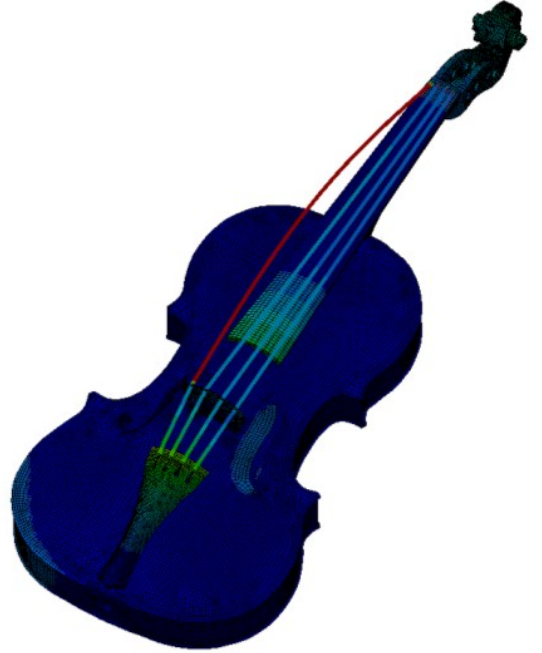

$195.0 \mathrm{~Hz}$

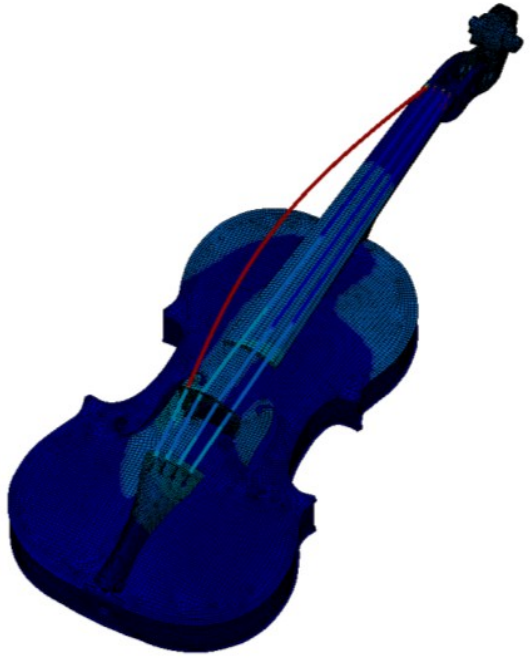

$197.0 \mathrm{~Hz}$

Figure 4.76: First G-string modes on Titian Stradivari violin FE

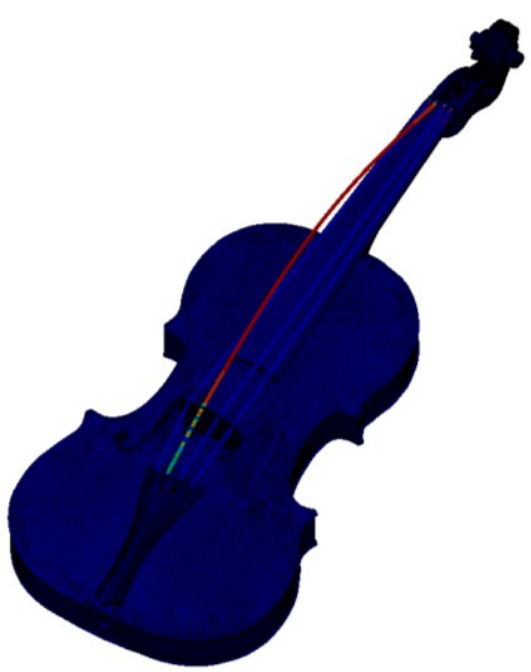

$292.2 \mathrm{~Hz}$

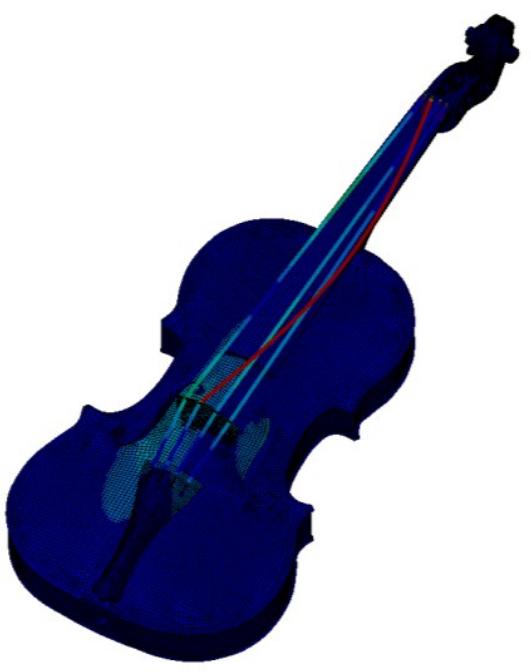

$294.8 \mathrm{~Hz}$

Figure 4.77: First D-string modes on Titian Stradivari violin FE 


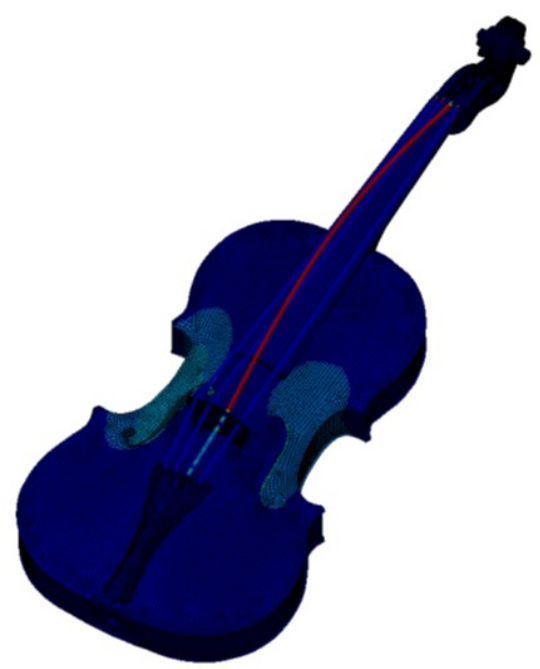

$436.2 \mathrm{~Hz}$

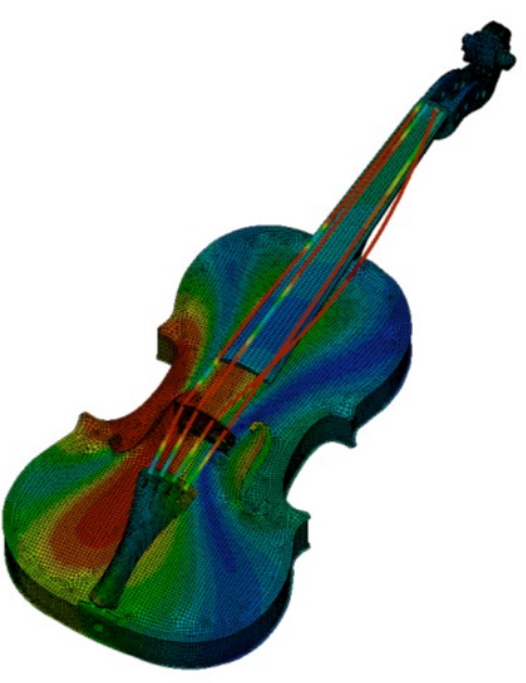

$442.5 \mathrm{~Hz}$

Figure 4.78: First A-string modes on Titian Stradivari violin FE

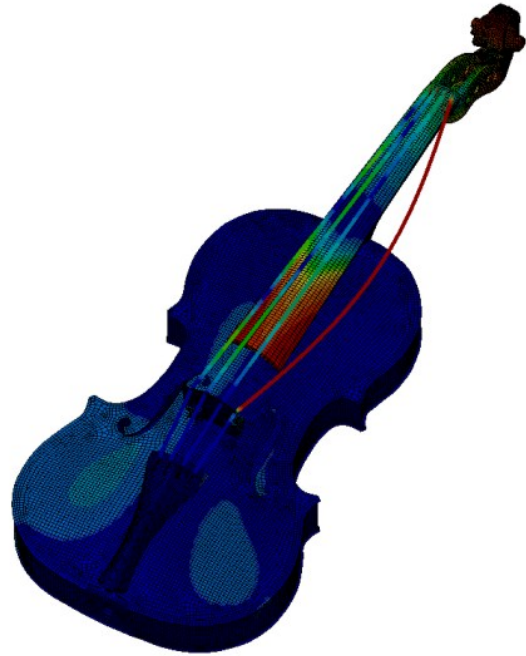

$654.4 \mathrm{~Hz}$

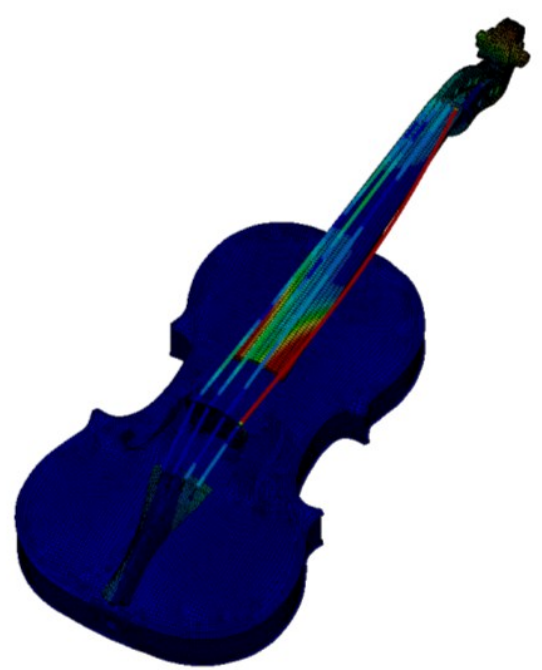

$657.5 \mathrm{~Hz}$

Figure 4.79: First E-string modes on Titian Stradivari violin FE 


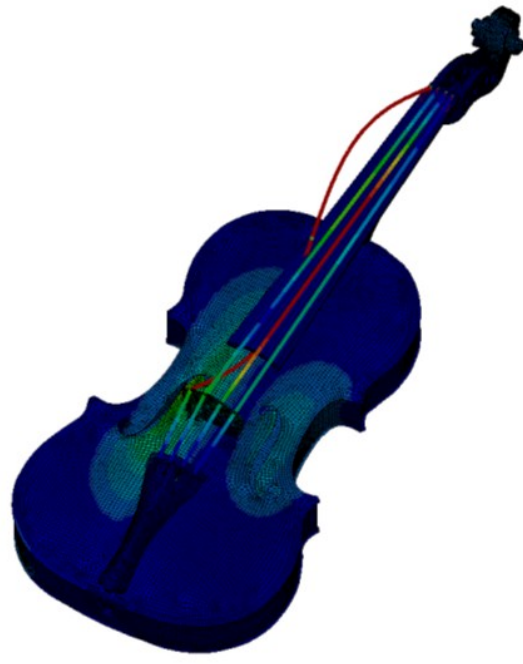

$387.9 \mathrm{~Hz}$

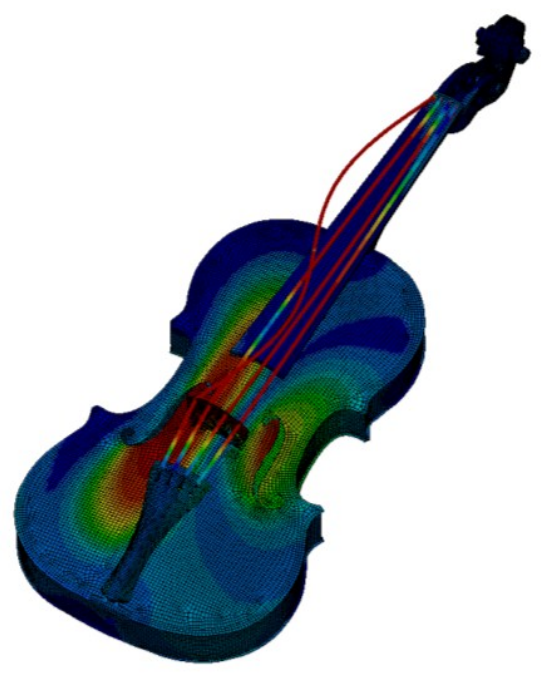

$393.0 \mathrm{~Hz}$

Figure 4.80: Second G-string modes on Titian Stradivari violin FE

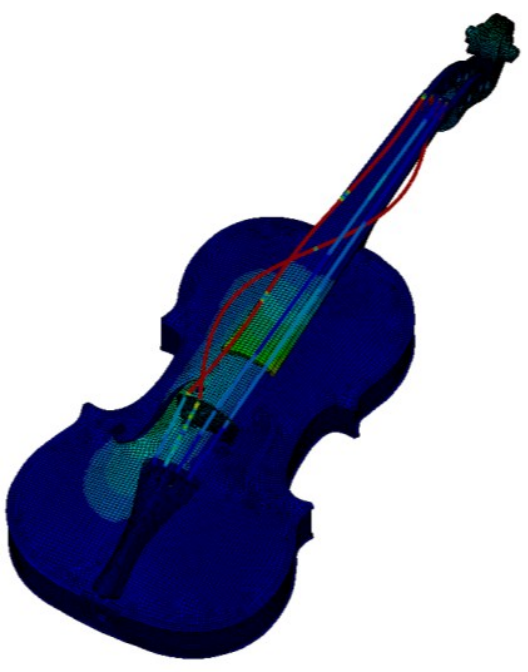

$587.5 \mathrm{~Hz}$

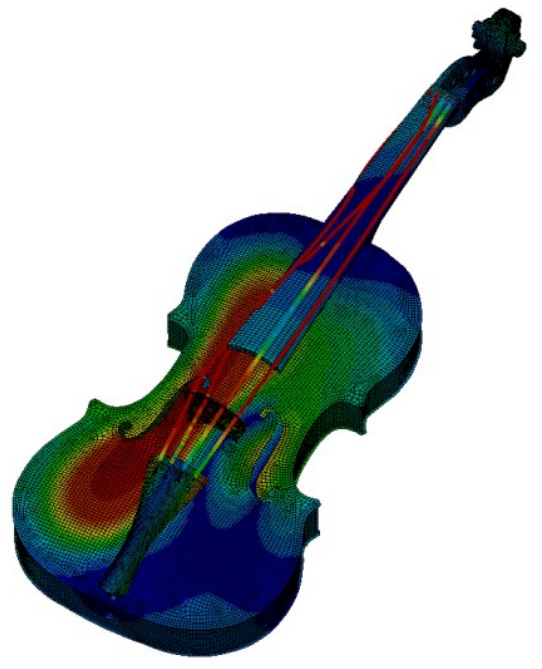

$589.0 \mathrm{~Hz}$

Figure 4.81: Second D-string modes on Titian Stradivari violin FE 


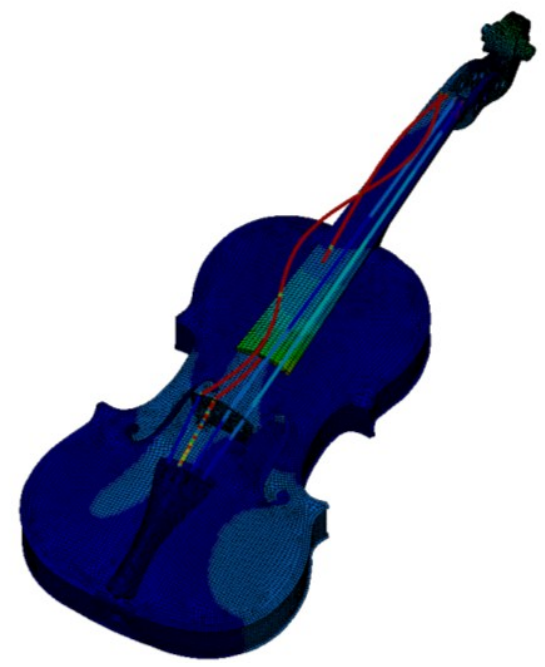

$590.3 \mathrm{~Hz}$

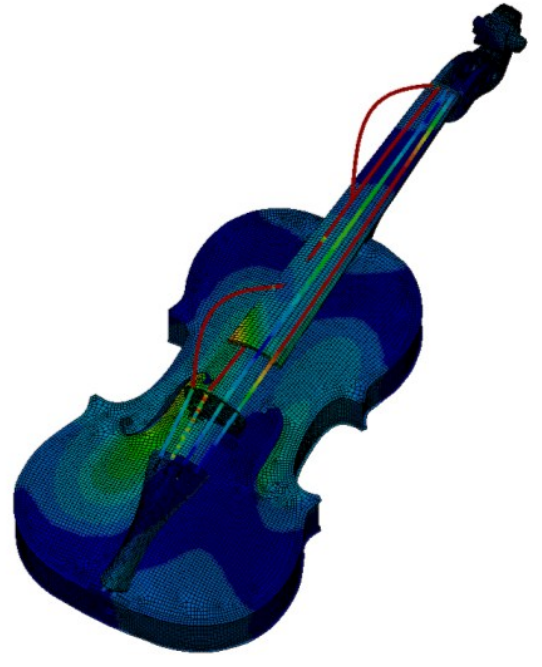

$593.3 \mathrm{~Hz}$

Figure 4.82: Third G-string modes on Titian Stradivari violin FE

\subsubsection{Corpus modes (Signature modes)}

The most important modes of the violin structural FE are the three corpus flexural modes, shown in Figures 4.83 through 4.85. These are the corpus twisting mode known as the CBR (Center-Bout-Rhomboid) mode, and the two corpus bending modes, B1- and B1+. These involve flexure of the plates, and are the efficient radiators of sound in the open string frequency range of the violin $(196 \mathrm{~Hz}-659 \mathrm{~Hz})$, and are the structural signature modes [4.13]. 


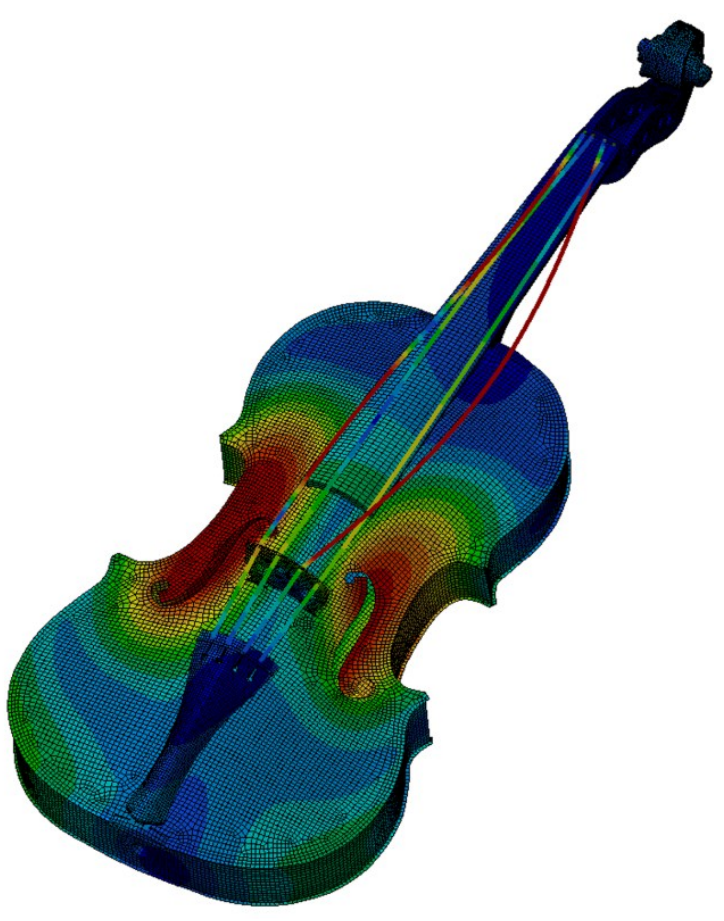

Figure 4.83: CBR: $427.3 \mathrm{~Hz}$

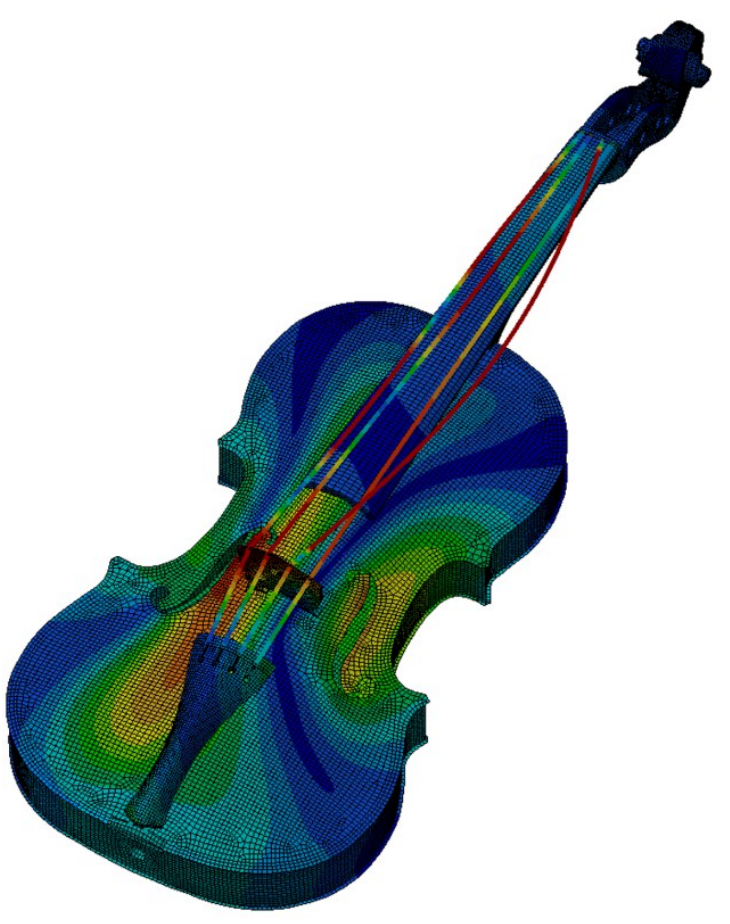

Figure 4.84: B1-: $452.5 \mathrm{~Hz}$ 


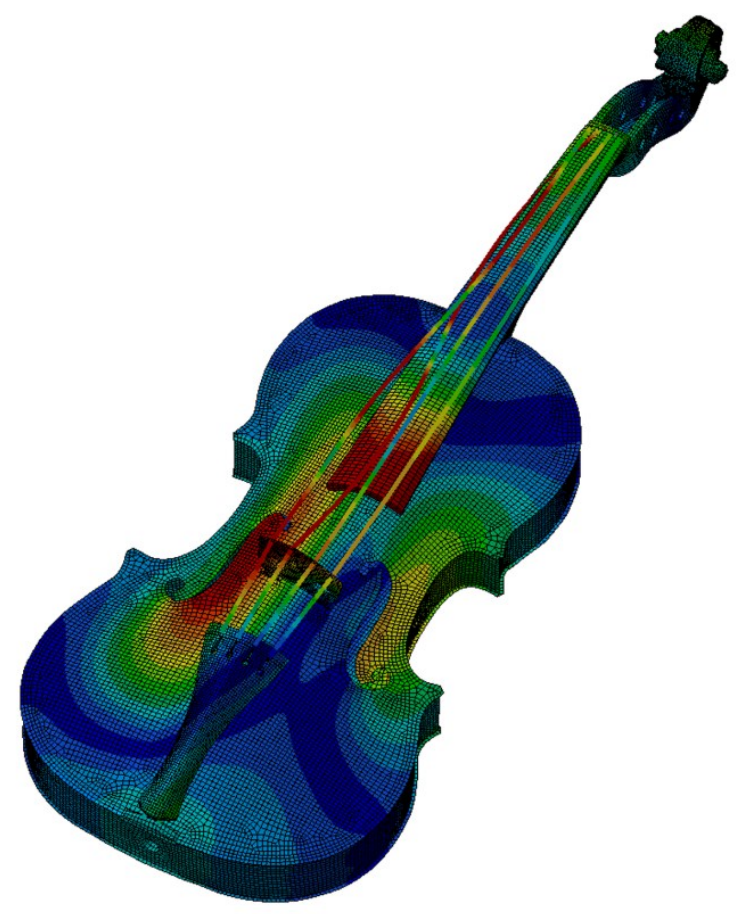

Figure 4.85: B1+: $529.8 \mathrm{~Hz}$

\subsubsection{Additional modes}

Some additional modes that occur in this frequency range are shown in Figures 4.86 through 4.89 .

These include a lateral bending mode of the fingerboard, torsion modes of the neck and fingerboard (both IP and OP), and a tail rock mode. The lateral bending mode of the finger board appears to be partially coupled to the B1+ corpus bending mode. The other modes appear to have minimal plate deflection. 


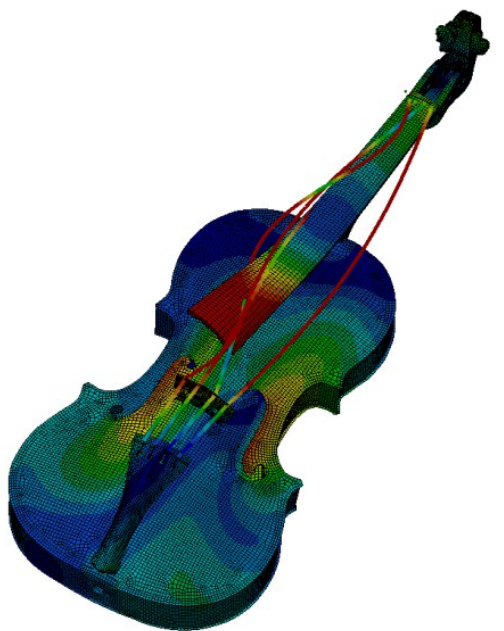

Figure 4.86: Lateral FB: $538.6 \mathrm{~Hz}$

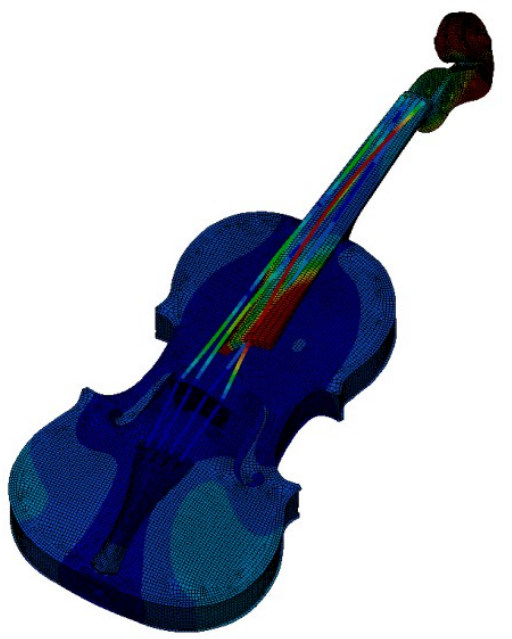

Figure 4.87: Neck-FB twist IP: $633.6 \mathrm{~Hz}$

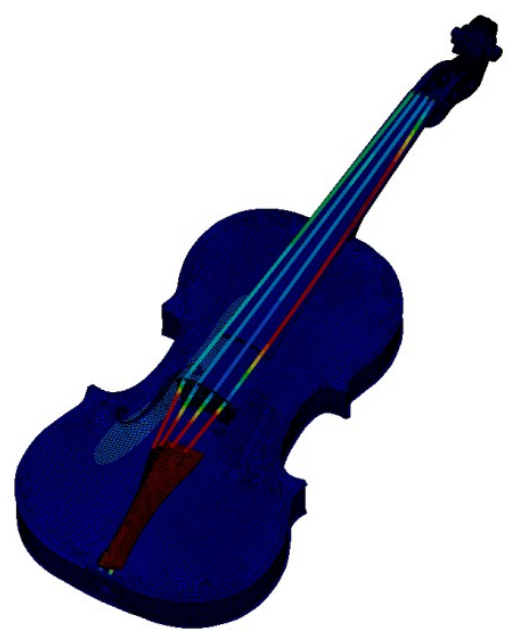

Figure 4.88: Tail rock: $650.8 \mathrm{~Hz}$

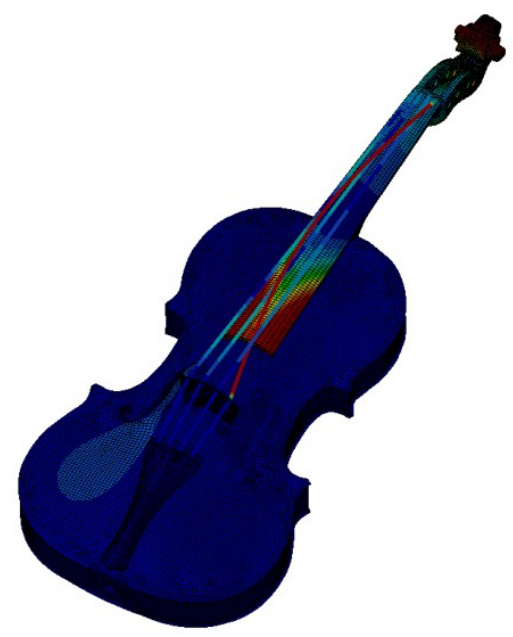

Figure 4.89: Neck-FB twist OP: $661.1 \mathrm{~Hz}$

\subsubsection{Correlation results of the Titian Stradivari structural model}

The initial results of the Titian violin FE model were correlated with those from test data using the Modal Assurance Criterion (MAC). A plot of the MAC matrix is shown in Figure 4.90. Note that string frequencies were removed from both sets. The color scale indicates MAC values from 0 (poor correlation, blue) to 1 (good correlation, red). A magenta box has been added to the figure around the signature modes sub-matrix. These 
are the main modes of interest as they are the strongest radiators of sound, and are expected to be well captured in the experimental data.

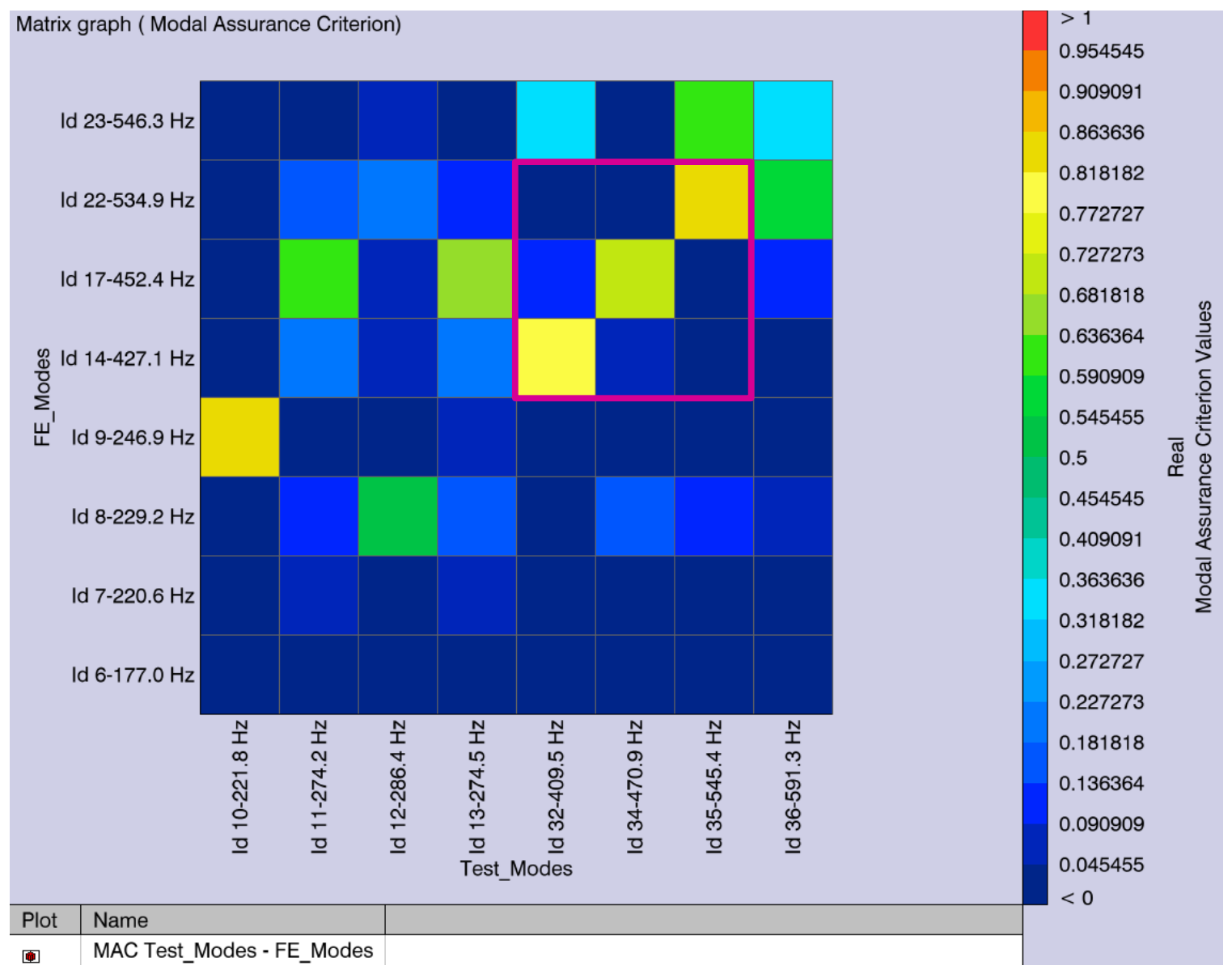

Figure 4.90: Initial Correlation Results for the Titian violin

The correlation results were updated to include all modifications and material updates made to the model. The final MAC matrix results are shown in Figure 4.91. Again, string frequencies have been removed from both sets. The final mode pairs are given in Table 4.22; note that only mode pairs with MAC values greater than 0.6 are included. Overall the final correlation results are excellent. The average absolute percent frequency difference for the signature modes is $\sim 3.8 \%$. The average MAC value for the signature modes is 0.873 . 


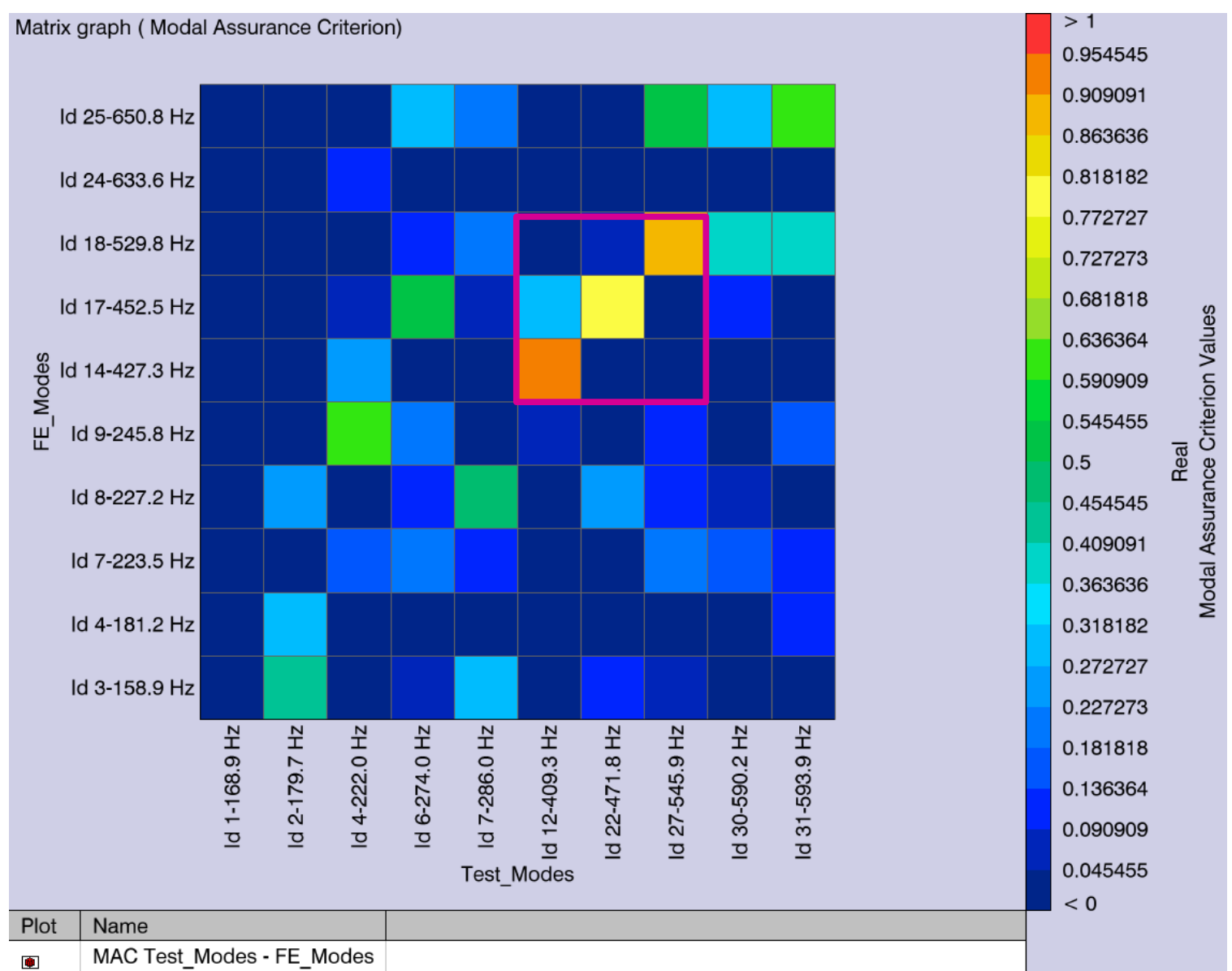

Figure 4.91: Updated Correlation Results for the Titian violin

Table 4.22: Updated Mode Pair Results for the Titian violin

\begin{tabular}{|c|c|c|c|c|c|c|}
\hline $\begin{array}{c}\text { Test } \\
\text { Id }\end{array}$ & $\begin{array}{c}\text { Test Freq } \\
(\mathbf{H z})\end{array}$ & $\begin{array}{c}\text { FE } \\
\text { Id }\end{array}$ & $\begin{array}{c}\text { FE Freq } \\
(\mathbf{H z})\end{array}$ & $\begin{array}{c}\text { MAC } \\
\text { Value }\end{array}$ & $\begin{array}{c}\text { Freq Diff } \\
(\mathbf{H z})\end{array}$ & $\begin{array}{c}\text { Freq Diff } \\
(\% \text { of Test) }\end{array}$ \\
\hline 4 & 222 & 9 & 245.8 & 0.631 & 23.71 & 10.7 \\
\hline 12 & 409.3 & 14 & 427.3 & 0.916 & 18.02 & 4.4 \\
\hline 22 & 471.8 & 17 & 452.5 & 0.808 & -19.25 & -4.1 \\
\hline 27 & 545.9 & 18 & 529.8 & 0.896 & -16.08 & -2.9 \\
\hline 31 & 593.9 & 25 & 650.8 & 0.634 & 56.85 & 9.6 \\
\hline
\end{tabular}

It is noted that the material properties of the Titian Stradivari violin FE model were updated to "tune" the modal frequencies. Updating was performed until the average percent difference for the three signature modes was below 5\%. A summary of the final material properties is given in Table 4.23.

Michael Pyrkosz 
Table 4.23: Summary of final material properties for Titian Stradivari FE model

\begin{tabular}{|c|c|c|c|c|c|c|c|c|}
\hline Component & $\begin{array}{c}\text { Thickness } \\
(\mathrm{mm})\end{array}$ & $\begin{array}{c}\mathrm{E} 1 \\
(\mathrm{MPa})\end{array}$ & $\begin{array}{c}\mathrm{E} 2 \\
(\mathrm{MPa})\end{array}$ & NU12 & $\begin{array}{c}\text { G12 } \\
(\mathrm{MPa})\end{array}$ & $\begin{array}{c}\text { G1Z } \\
(\mathrm{MPa})\end{array}$ & $\begin{array}{c}\mathrm{G} 2 Z \\
(\mathrm{MPa})\end{array}$ & $\begin{array}{c}\text { RHO } \\
\left(\mathrm{kg} / \mathrm{m}^{3}\right)\end{array}$ \\
\hline Back plate & Variable & 11730 & 1560 & 0.424 & 892 & 505 & 265 & 570 \\
\hline Top plate & Variable & 9350 & 1190 & 0.422 & 786 & 760 & 63.7 & 350 \\
\hline
\end{tabular}

Final mass of the full Titian Stradivari violin structural FE model was 323.2 grams. The back plate of the model weighed $\sim 88.8$ grams, while the top plate + bass bar of the model weighed $\sim 48.1$ grams.

\subsection{Conclusions}

Based on the results of these analyses the material properties of a violin may be estimated through the use of modal correlation and updating techniques. Experimental modal data was collected on various sub-components of a factory violin as well as the 1715 Titian Stradivari [4.12]. Structural finite element models were constructed based on CT scans of the actual instruments. Correlation between the experimental data and the initial FE models was used to compare the modal behavior, and the material properties in the models were iterated to determine a set of parameters that reflect the dynamic behavior of the real instruments.

Overall this method is viable for determining the orthotropic material properties of the various wood components of a violin. All models were updated so that the first several modes were within $5 \%$ of the experimental modal frequencies, and all MAC values for mode pairs of interest were above 0.6. Future studies on other violins could be done using these methods to compare the dynamic material properties of these instruments.

Now that a reliable structural model of the Titian Stradivari violin is available, a coupled vibro-acoustic model can be created to simulate the acoustic response of the violin.

\subsection{References}

[4.1] Pyrkosz, M., Van Karsen, C., Bissinger, G., "Converting CT Scans of a Stradivari Violin to a FEM", Proceedings of the 28th International Modal Analysis Conference, Jacksonville, FL, (February 2010)

[4.2] Carne, Thomas G., Neilsen, Michael K., Stasiunas, Eric C., "Experimental and Analytical Validation of a Computationally Developed Orthotropic Constitutive Model", Proceedings of 21st International Modal Analysis Conference, Kissimee, FL, 2003 
[4.3] Bissinger, G., "A Unified Materials-Normal Mode Approach To Violin Acoustics," ACTA Acustica united with Acustica Vol. 91, pp. 214-228 (2005)

[4.4] "Thickness Contour Maps" by Jeffrey Loen with Violin specification and thickness measurements by S. Zygmuntowicz, Strad3D, 2009

[4.5] Pyrkosz, M., Van Karsen, C., "Comparative Modal Tests of a Violin," Experimental Techniques , 2012, Society for Experimental Mechanics

[4.6] Heylen, W., Lammens, S., Sas, P., "Modal Analysis Theory and Testing”, Katholieke Universiteit Leuven, Department of Mechanical Engineering, Division of Production Engineering, Machine Design and Automation, Leuven, Belgium, 1998

[4.7] ANSA version 13.2.1 User's Guide, BETA CAE SYSTEMS S.A., December 2011, Printed in Greece

[4.8] Knott, G. A., "A Modal Analysis of the Violin Using MSC/NASTRAN and PATRAN" M.S. Thesis, Naval Postgraduate School. Available through NTIS website. (March 1987)

[4.9] Allemang, R. J., "The Modal Assurance Criterion (MAC): Twenty Years of Use and Abuse", Proceedings of the International Modal Analysis Conference, Los Angleles, CA, February 2002.

[4.10] Avitabile, P., "Twenty Years of Structural Dynamic Modification - A Review", Proceedings of the 20th International Modal Analysis Conference, Los Angeles, California, Feb 2002

[4.11] Hutchins, C. M., "The Acoustics of Violin Plates," Scientific American Oct 1981, Vol. 245, No.4, page 170

[4.12] Bissinger, G., Oliver, D., "3-D Laser Vibrometry Focuses On Legendary Old Italian Violins," Sound and Vibration magazine Vol. 41/Num. 7, pp. 10-15 (July 2007)

[4.13] Bissinger, G., "Structural acoustics of good and bad violins," J. Acoust. Soc. Am. Vol. 124 (3), pp. 1764-1773 (September 2008)

[4.14] Bissinger, G., "Structural acoustics model of the violin radiativity profile," J. Acoust. Soc. Am. Vol. 124 (6), pp. 4013-4023, (December 2008) 


\title{
Chapter 5 Coupled Vibro-Acoustic Model of the Titian Stradivari Violin ${ }^{5}$
}

\begin{abstract}
The instruments of Antonio Stradivari (1644 - 1737) are still considered the finest in the world and have set the standard for violin quality that has yet to be exceeded by a modern luthier. To determine the relationship between the sound of the instrument and its structure and material properties a vibro-acoustic finite element model was developed for the 1715 Titian Stradivari violin. The structural model was created by measuring the structural geometry and density from CT scans of the actual instrument. The predicted structural modes were correlated with experimental modal data on the Titian and updated accordingly. The correlated structural modes were then projected onto the acoustic envelope. The vibro-acoustic transfer function between force input at the violin bridge and acoustic pressure response $1.2 \mathrm{~m}$ away was predicted. These results were compared to experimental radiativity measurements made on the actual instrument. It was shown that including structural and acoustic damping characteristics are important to accurately predict the vibro-acoustic behavior of the Titian Stradivari violin. Predicted structural modal frequencies were all within $5 \%$ of the experimental data, while the main acoustic mode was within $7 \%$. The predicted radiativity magnitude was within $1.5 \mathrm{~dB}$ for all mode peaks. This is the first comprehensive analytical vibro-acoustic model of a Stradivari instrument and can be used to determine how structural modifications affect violin sound quality.
\end{abstract}

\section{Nomenclature}

C speed of sound

j imaginary, sqrt(-1)

$\mathrm{k}$ wave number

p acoustic pressure

$r \quad$ acoustic field position vector

$t_{P M L}$ thickness of the perfectly matched layer

$\zeta$ damping factor, $\zeta=\mathrm{C} / \mathrm{Cc}$

$\eta \quad$ loss factor, energy dissipation per cycle

$\lambda \quad$ wave length, $\lambda=1 / f=2 \pi / \omega$

$\rho \quad$ mass density

\footnotetext{
${ }^{5}$ The material contained in this chapter is planned for submission in the near future, copyright $(\subset) 2013$ Michael A. Pyrkosz
}

Michael Pyrkosz 


\subsection{Introduction}

The final step in the process of reverse engineering the Titian Stradivari violin was to create an accurate vibro-acoustic model of the instrument. To do this an acoustic finite element mesh was developed based on the existing structural FE model. The predicted structural modes were projected onto this mesh, and a coupled vibro-acoustic analysis was performed to predict the transfer function between acoustic pressure response and force input at the violin bridge. These results were compared to experimental radiativity measurements made on the actual instrument [5.1], [5.2], [5.3]. The model properties were updated accordingly. The structural and acoustic damping properties were both found to play an important role in vibro-acoustic response.

Violin makers are always asking how they can improve their violins:

- "What if thickness is added to the center of the top plate?"

- "What if the arching is made flatter?"

- "What if the density of the top plate is increased?"

So on, and so forth. With a working model to predict the vibro-acoustic behavior of a Stradivari violin, many of these questions can be examined.

Models are a virtual surrogate for a product of interest, and can be used to predict how small design changes will affect overall performance. One advantage of models is that running multiple scenarios with a model is significantly less expensive than building and testing several different prototype structures (in this case, precisely crafted violins). The other advantage is that it is far easier to control design variables during experimentation.

\subsection{Methods}

\subsubsection{FEM vs. BEM}

Traditionally, the only practical way to handle an interior/exterior acoustic problem (such as a violin with an inner cavity connected to the outside air via the f-holes) was using the Boundary Element Method (BEM). However, technology has been developed in recent years that makes the Finite Element Method (FEM) more practical, and indeed better suited for the violin acoustic problem.

One of the previous disadvantages of FEM was that it requires a solid mesh of the air, regardless of whether it was for an interior or exterior problem, which can lead to larger model sizes (which more computationally expensive). The second disadvantage of FEM 
was that for exterior acoustic problems, the Sommerfeld Radiation condition is not implicitly fulfilled as it is in BEM.

Faster solvers are now available that take advantage of the symmetric sparse matrices of FEM models, so that model size is less of an issue. These include the MUlti-frontal Massively Parallel Sparse (MUMPS) Direct solver, and the Iterative Krylov Subspace solver. These are up to 100 times faster than the current BEM solvers [5.4].

For the Titian Stradivari violin vibro-acoustic model the Direct solver was used.

\subsubsection{Sommerfeld radiation condition in FEM}

The Sommerfeld radiation condition requires that the boundary surface of the acoustic FEM mesh allows all acoustic waves to propagate freely towards infinity, and that no reflections occur at this boundary. This is represented by the following equation [5.5].

$$
\lim _{|\vec{r}| \rightarrow \infty}|\vec{r}| \cdot\left(\frac{\partial p(\vec{r})}{\partial|\vec{r}|}+j k p(\vec{r})\right)=0
$$

There are currently two methodologies for handling the Sommerfeld radiation condition in exterior FEM problems: the Infinite Element Method (IFEM), and the Perfectly Matched Layer (PML).

IFEM is the older of these two, which requires that the boundary surface of the air be meshed as a canonical shape (such as a sphere or ellipsoid). Special decay shape functions are used on the boundary elements to minimize reflections. The radius of the boundary surface must be at least a full wavelength for the lowest frequency of interest, $\mathrm{R}$ $>\lambda$, and up to two full wavelengths away from radiating bodies. This leads to extremely large models with many elements to have a useful dynamic range. For example, to create a spherical mesh that is valid for frequencies from $100 \mathrm{~Hz}$ to $1000 \mathrm{~Hz}$, assuming that the speed of sound is $\sim 340 \mathrm{~m} / \mathrm{s}$, the radius of the sphere would need to be $\sim 3.4 \mathrm{~m}$ to meet the wavelength requirement. To fill this with elements small enough to provide at least 6 elements per wavelength at a $1000 \mathrm{~Hz}$, the element edge length would be $\sim 56.7 \mathrm{~mm}$. The model would have $\sim 1.6$ million nodes and $\sim 9.8$ million elements. This is approximately eight times greater that our current model, and would be even larger when the violin is included due to the higher local resolution at the acoustic envelope.

With PML a special perfectly matched layer of elements is added to the outer boundary of a convex mesh that is locally-conformal to the structure mesh. This layer utilizes an anti-reflection function to create a coating around the radiating body to absorb the waves. 
In this case the thickness of the PML only needs to be greater than one fifteenth of the lowest frequency wavelength, $\mathrm{t}_{\mathrm{PML}}>(1 / 15)^{*} \lambda$. Additionally, the distance between the radiating surface and the boundary of the PML only needs to be 1-2 elements thick.

In both IFEM and PML response points may be placed inside or outside of the FEM boundary. For responses outside the FEM domain the solver will use Kirchoff surface integration to compute the solution for these points once the FEM solution is known.

\subsubsection{Acoustic Mesh for FEM with AML}

LMS Virtual.Lab software now includes an Automatically Matched Layer (AML)

property. This feature constructs the absorbing layer on the solver level by extruding the boundary surface elements automatically [5.4]. This is similar to PML except that the layer is created on the solver level. This eliminates the need for the user to create the layer manually within the GUI.

For an opened structural mesh, the acoustic medium is present on both sides of the structure, and a volumize mesher was used to create duplicated meshes projected on both sides of the structural shell elements. This is shown in Figure 5.1. The structure mesh of the violin is shown in green (lines represent shell elements and filled-in areas represent solid elements). The volumize mesh is shown as a black line enveloping the structure mesh. The convex mesh is then created to wrap around the outside of the volumize mesh with room for at least one or two elements in between. The AML property is applied on this surface. A tetrahedron filler mesh is used to fill the space between the volumize mesh and the convex mesh with tetrahedral elements. 


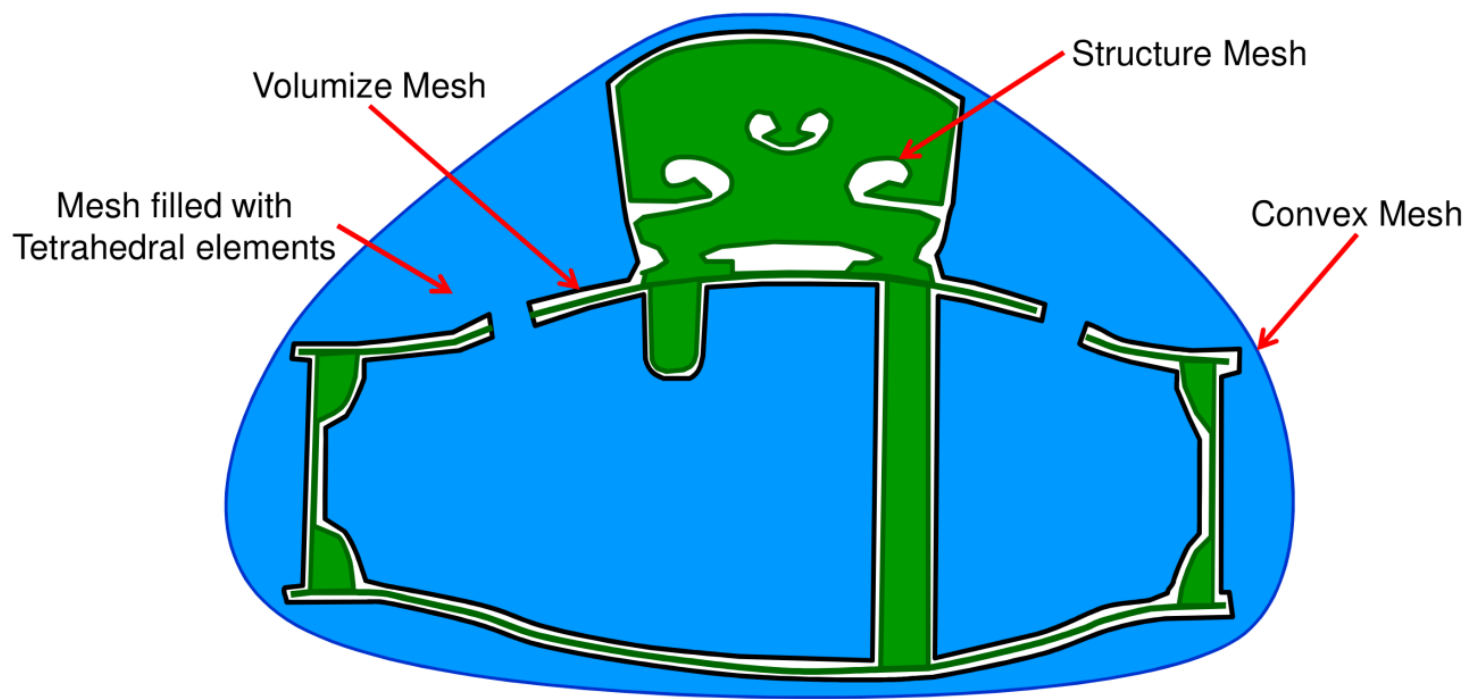

Figure 5.1: Setting up FE meshes for AML property on an opened structural mesh of a violin

\subsubsection{Acoustic mesh preparation}

The acoustic mesh for the Titian Stradivari violin was created using the following steps:

- Starting from the structural mesh the volumize mesh (acoustic envelope) is created

- The convex mesh (AML surface) is then wrapped around the outside of the volumize mesh

- The tetrahedral-filled mesh is created

Most of this work was done using the various modules in LMS Virtual.Lab R11.

Some parts of the structural mesh include small holes and other features that are important structurally, but do not have a significant influence on the surrounding air. These holes were initially filled (for the purposes of creating the acoustic envelope) with shell elements. Examples of this process are shown in Figure 5.2 for the peg box, tailpiece, and end pin hole. 
(a)

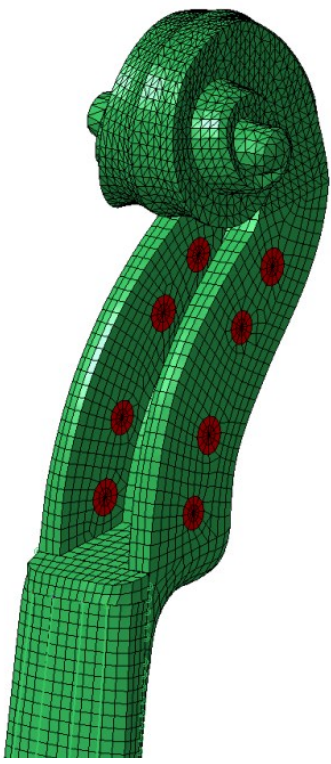

(b)
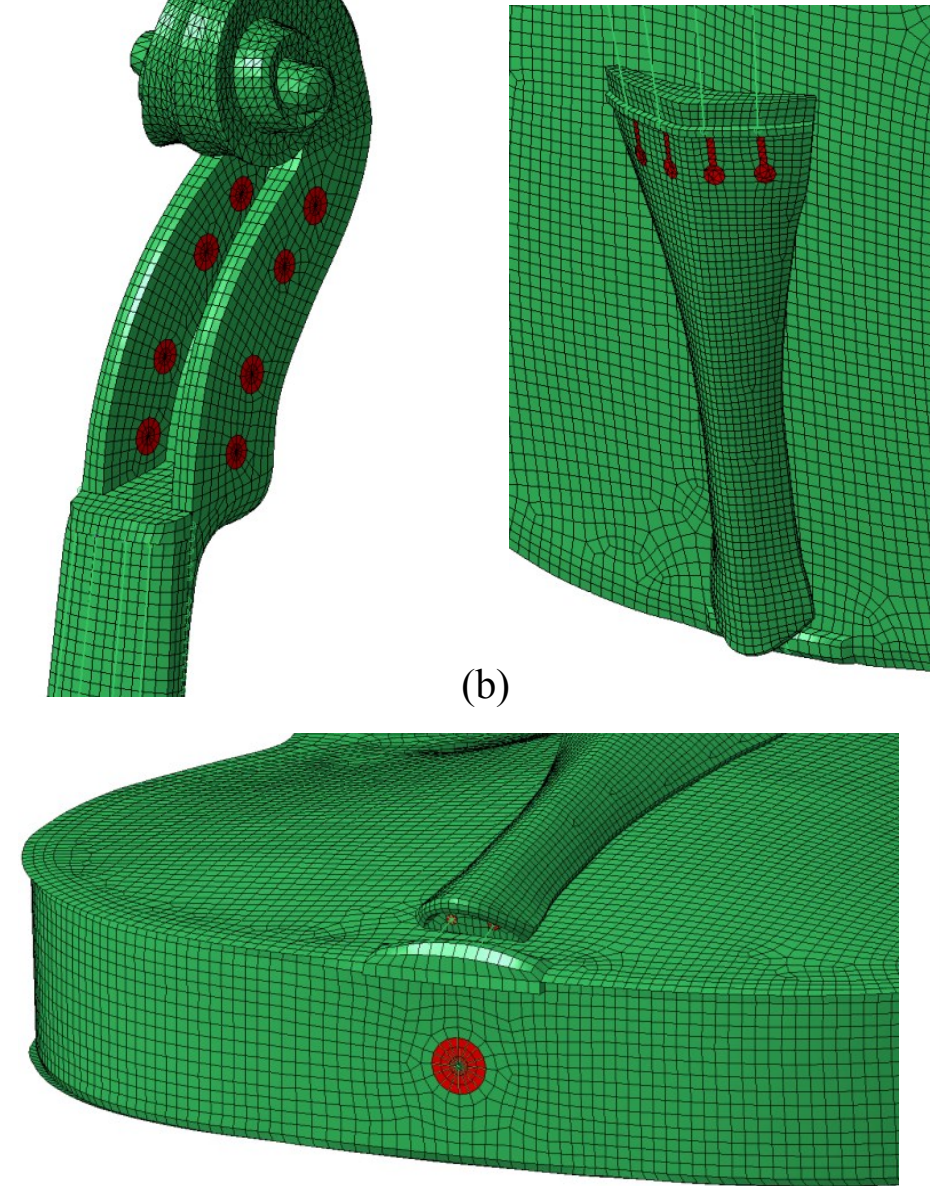

(c)

Figure 5.2: Filling in holes: (a) peg box, (b) tail piece, and (c) end pin

It should be noted that although the structural mesh must be fine to capture the bending waves through the wood materials, the acoustic mesh can be considerably coarser. By coarsening the acoustic mesh, the size of the model was kept small, reducing the solve time. This coarsening is performed before the volumize step. To ensure that the area around the f-holes is not compromised the edges of the f-holes are selected and a mesh group is created to maintain these edges during the coarsening process. This is shown in Figure 5.3. 


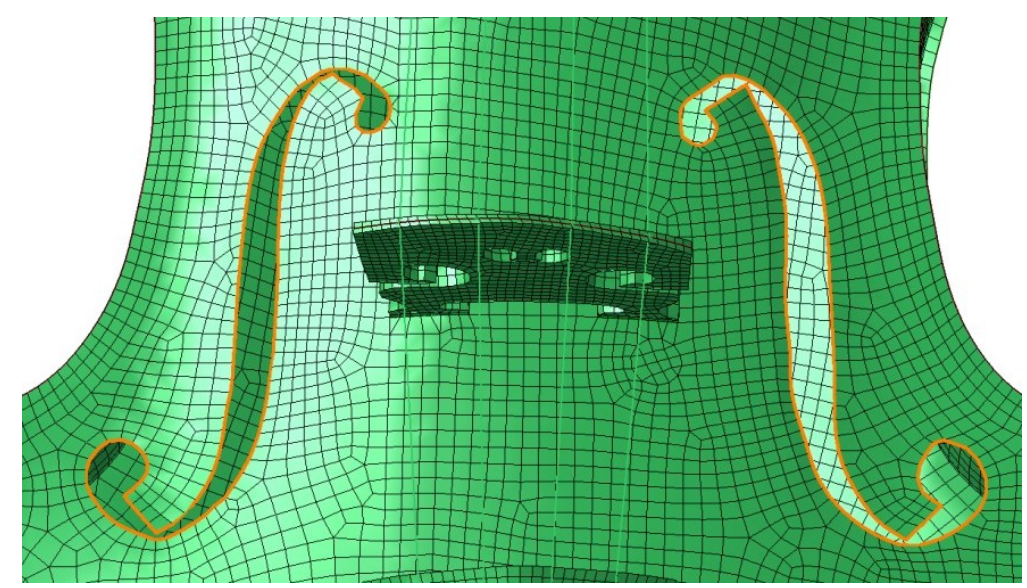

Figure 5.3: The edges around the f-holes are selected to be maintained

All shell elements (including skins of the solid elements) are included in the mesh coarsening step. Several iterations were performed with the "coarser mesher" tool in LMS Virtual.Lab.

The volumize step creates surface meshes on both sides of shell elements, including the interior of solids. A cross-sectional view of the initial volumize mesh is shown in Figure 5.4. The plates and ribs were represented as shell elements, so the volumize step worked correctly. The bass bar, sound post, and other solids already had volume, such that their skins needed to be used for the volumize step to make sure that the interfaces between solid and shell components would remain aligned. This however meant that the solid components all had internal meshes that needed to be removed manually. This was done using ANSA v.13.2.0 by BETA CAE Systems S.A. Additional coarsening was applied at this time, and the entire acoustic envelope was remeshed with a maximum element size of $8 \mathrm{~mm}$, ensuring that the acoustic envelop is valid up to $4000 \mathrm{~Hz}$ at 10 elements per wavelength. The heart and waist areas of the bridge were filled in as well. These areas were important to include in the structural part of the model since the bridge is the main energy path for the structural vibrations from the strings into the body of the violin [5.6]. However these areas are too small to have an impact on the acoustic field surrounding the instrument (compared to the plates). 


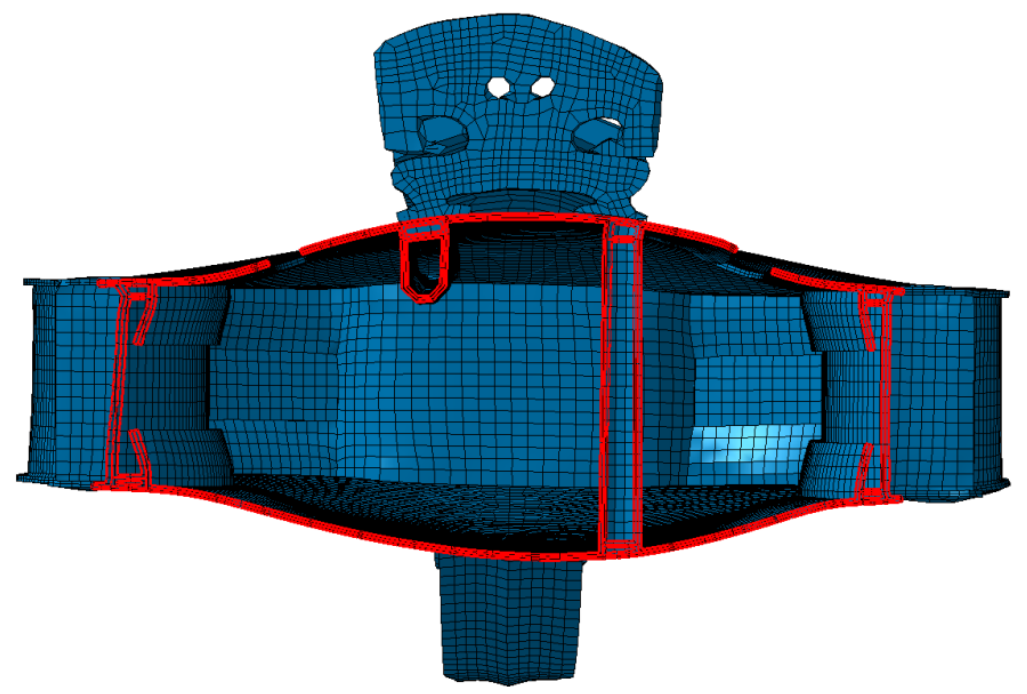

Figure 5.4: Cross-sectional view of the first pass of the volumize mesh

The final volumize mesh is shown in Figure 5.5. This mesh is composed of 56,147 nodes, and 56,234 shell elements (56064 QUAD, and 170 TRIA). A cut-plane view of the final volumize mesh is also shown in Figure 5.5. This mesh will become the "acoustic envelope" where the structural modes will be transferred to the surrounding air. 


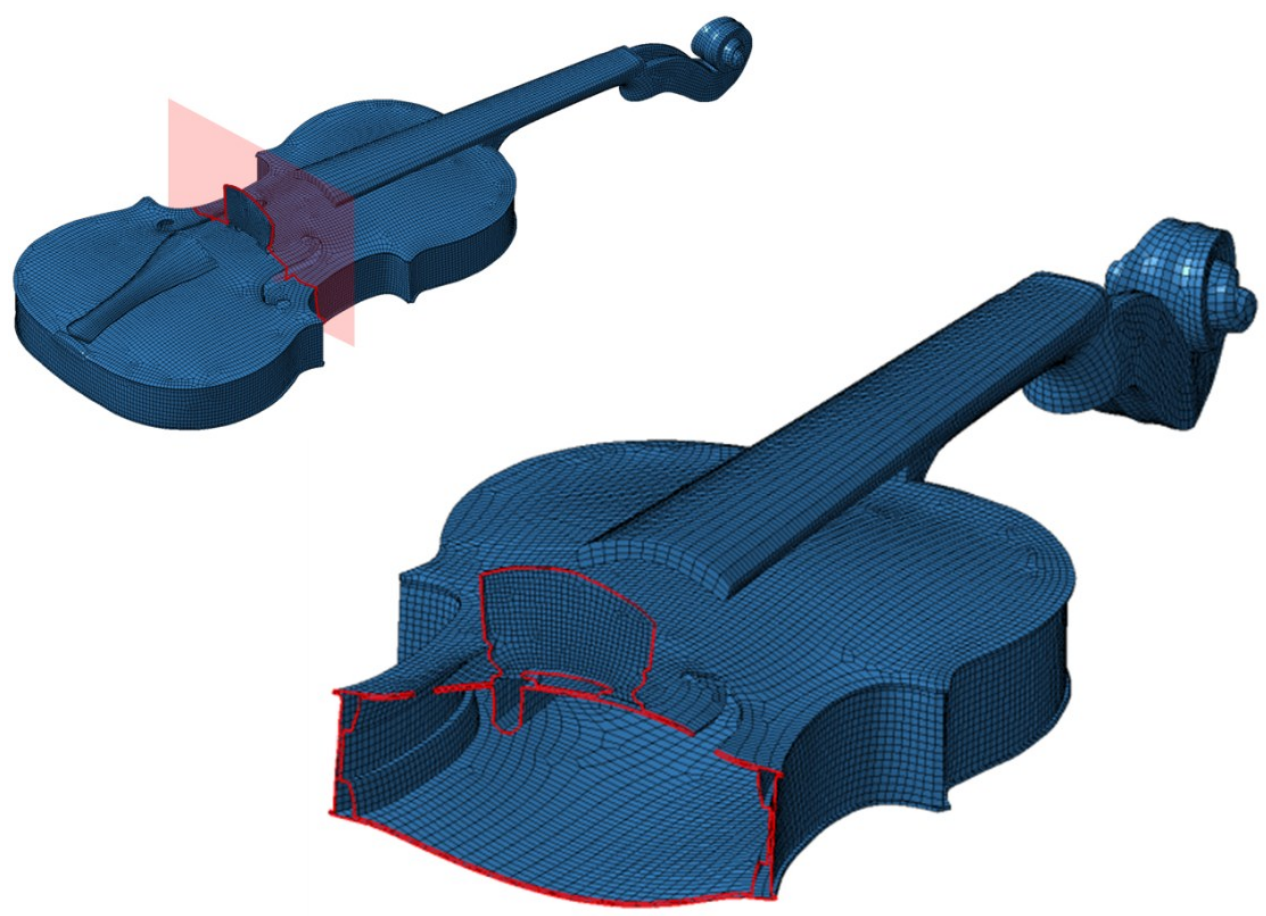

Figure 5.5: Final volumize mesh of the Titian Stradivari violin and cut-plane at bridge location

A convex mesh was created around the volumize mesh. A scaling factor of 1.1 was applied, and the element size was set to $14 \mathrm{~mm}$ (this guarantees at least 6 elements per wavelength up to a frequency of $4000 \mathrm{~Hz}$, assuming speed of sound $\sim 340 \mathrm{~m} / \mathrm{s}$ ). The final convex mesh for the Titian Stradivari violin vibro-acoustic model is shown in Figure 5.6. This mesh defines the point at which the AML property is applied. 


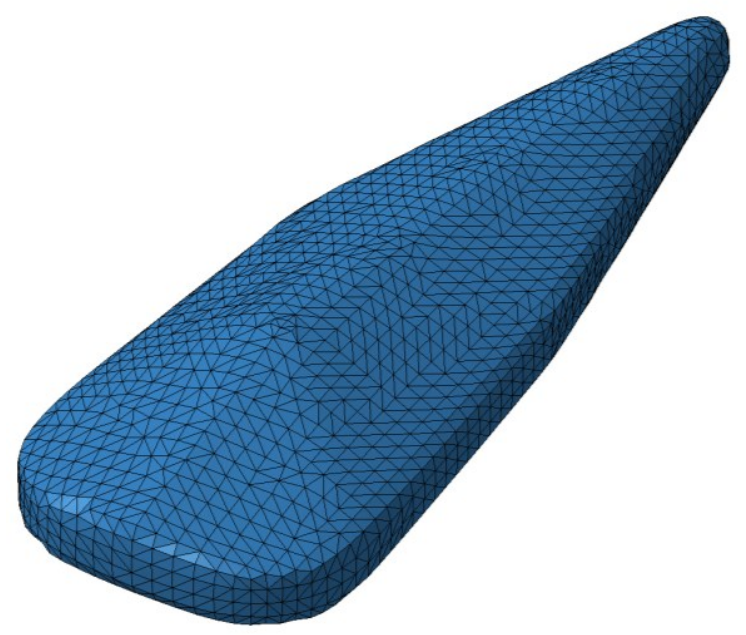

Figure 5.6: Convex mesh around Titian Stradivari violin FE model

Once a convex mesh was wrapped around the outside of volumize mesh the volume between the meshes was filled with tetrahedral elements. Cross-sectional views in the sagittal, coronal, and axial directions are shown in Figures 5.7, 5.8, and 5.9 respectively.

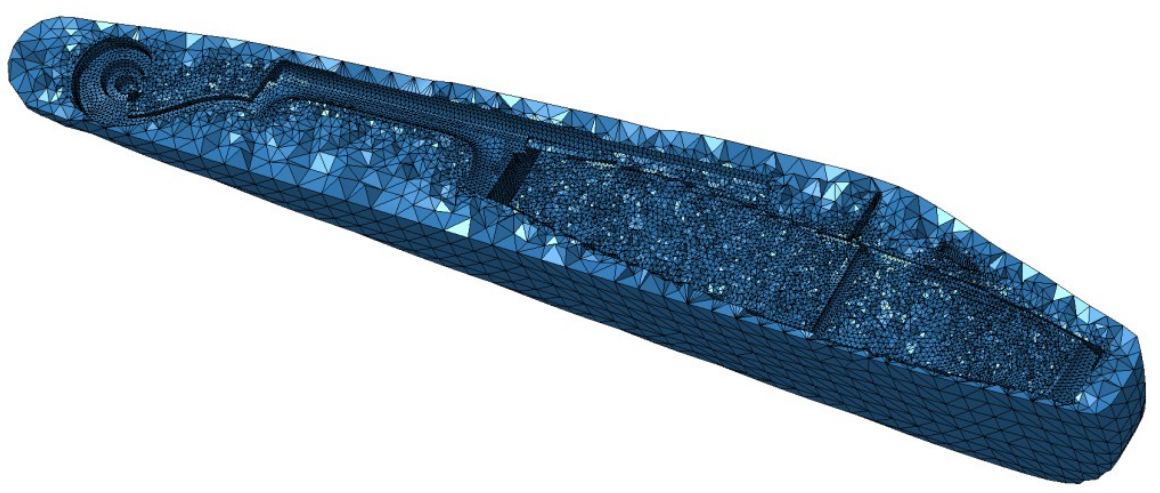

Figure 5.7: Sagittal cross-section of the tetra-fill volume mesh 


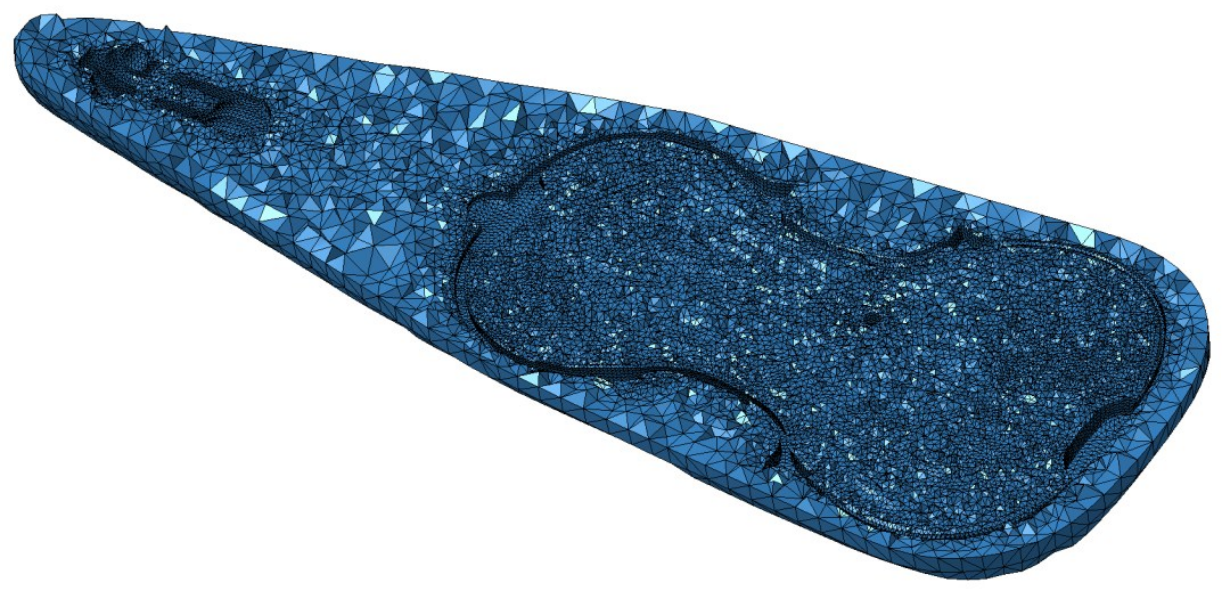

Figure 5.8: Coronal cross-section of the tetra-fill volume mesh

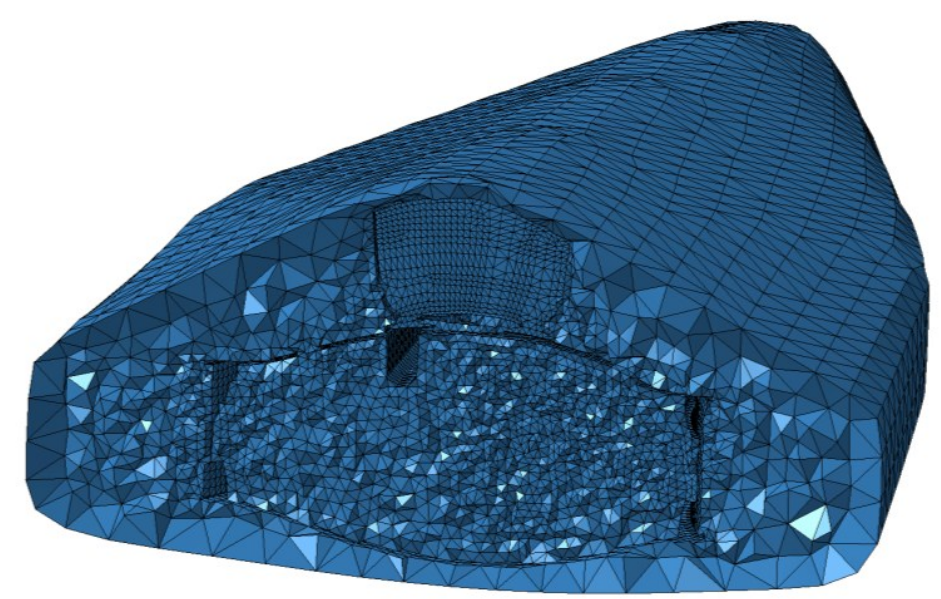

Figure 5.9: Axial cross-section of the tetra-fill volume mesh

Table 5.1: Mesh compositions for Vibro-Acoustic FEM Analysis of the Titian Stradivari violin

\begin{tabular}{|l|l|}
\hline Structure Mesh & Acoustic Mesh \\
\hline 74756 Nodes & 226142 Nodes \\
438 TRIA3 elements & 1191283 TETRA4 elements \\
55526 QUAD4 elements & \\
28014 TETRA4 elements & \\
691 PENTA6 elements & \\
32206 HEXA8 elements & \\
598 Beam elements & \\
55 Rigid spider elements & \\
\hline
\end{tabular}

Michael Pyrkosz 
The final mesh compositions of both the structure mesh and acoustic mesh are given in Table 5.1. Figure 5.10 shows the structural mesh inside a cut-away view of the acoustic mesh.

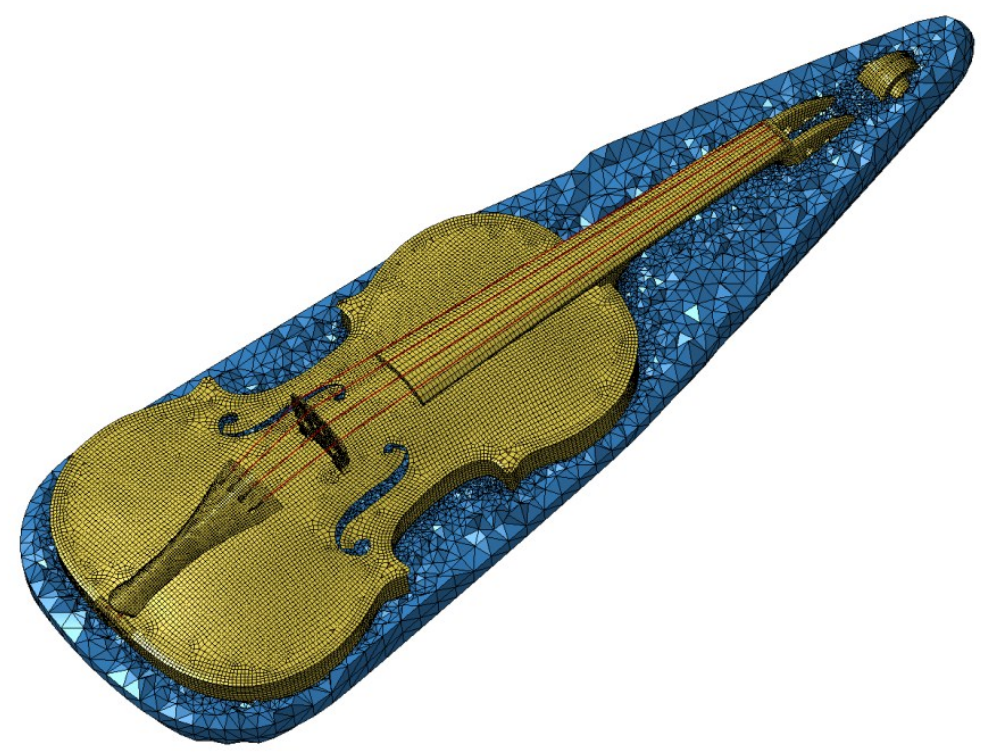

Figure 5.10: Titian Stradivari structural FE mesh inside a cut-away (coronal) of the acoustic volume mesh

\subsubsection{Coupled Vibro-Acoustic Set-up}

Once the acoustic mesh was prepared, several pre-processing steps were performed. These include setting the properties for air, defining the AML property on the convex mesh, transferring the structural modes onto the acoustic envelope, and defining input and output locations for the Vibro-acoustic transfer function analysis case.

Properties of air were initially assumed as follows:

Speed of Sound $=340 \mathrm{~m} / \mathrm{s}$

Mass density $=1.225 \mathrm{~kg} / \mathrm{m} 3$ 


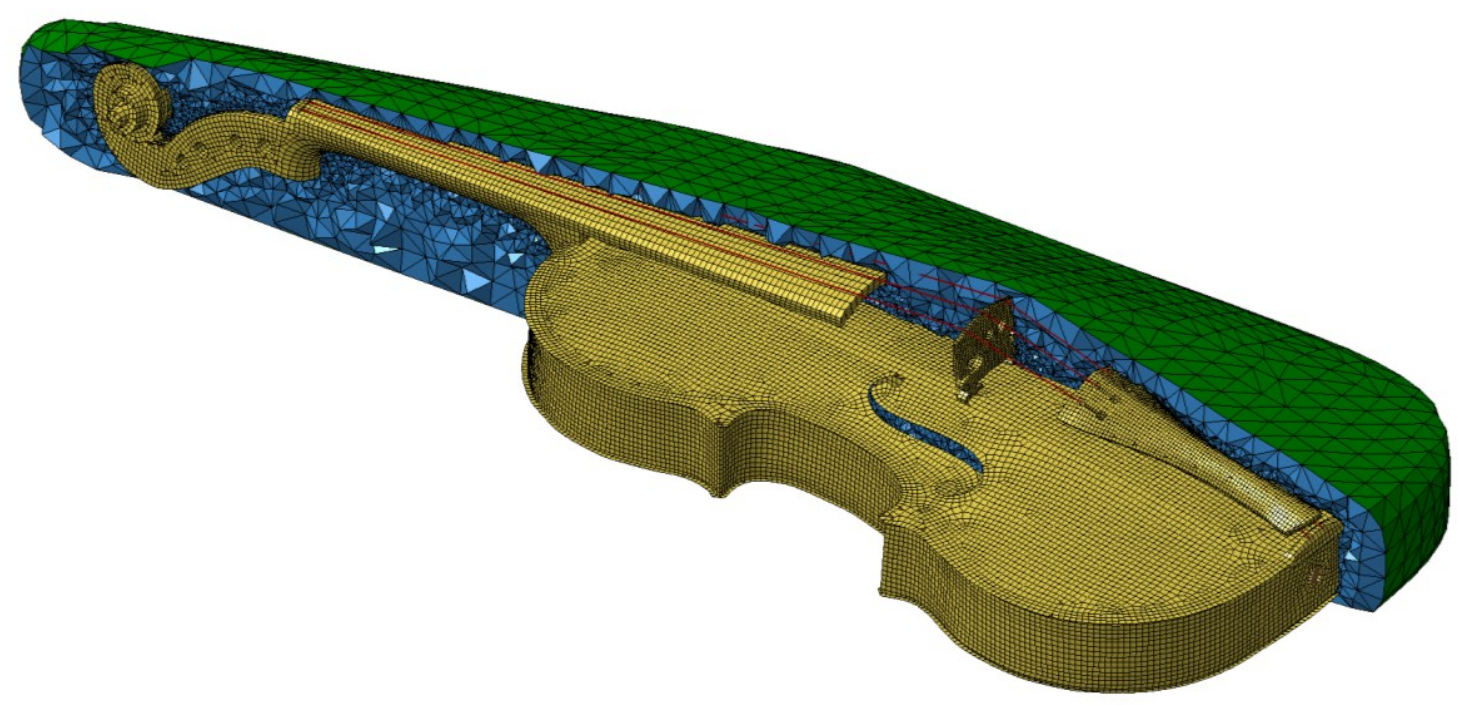

Figure 5.11: Cut-away (sagittal) of the acoustic mesh showing the AML property on the outer surface (green)

The AML property is applied to the outer surface of the acoustic mesh, as shown in

Figure 5.11 in green. The parameters used for the AML property are given in Table 5.2.

Table 5.2: AML parameters for Vibro-Acoustic Analysis of the Titian Stradivari

\begin{tabular}{|l|l|}
\hline Number of layers & 5 \\
\hline $\begin{array}{l}\text { Minimum Thickness } \\
\text { (as a ratio of wavelength) }\end{array}$ & 0.16 \\
\hline Surface element count & 3728 \\
\hline
\end{tabular}

Response locations for the vibro-acoustic transfer function analysis were selected using field point meshes. These virtual microphones can be placed anywhere in the model. To create results comparable to the available experimental data [5.1], a spherical field point mesh was placed around the Titian Stradivari vibro-acoustic mesh. Like the Bissinger experiment its center point was set on the top plate, midway between the two bridge feet. The radius of the sphere was set to $1.2 \mathrm{~m}$, and the number of equatorial elements was set to 24 , creating a response location every $15^{\circ}$ along the equator. The resulting sphere contained 218 nodes and is shown in Figure 5.12. 


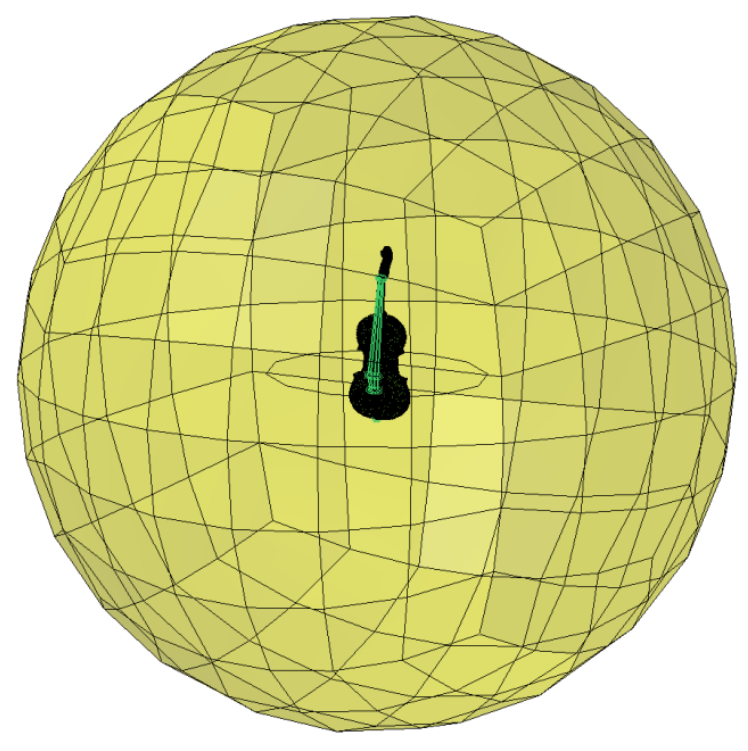

Figure 5.12: Field point mesh around the Titian Stradivari FE model

Five input locations were selected for the vibro-acoustic transfer function analysis case, as shown in Figure 5.13. The first of these was at the upper bass-side corner of the bridge (again to generate results comparable to the Bissinger data [5.1]). The other four were selected at each of the string-bridge interface points. These were selected to be used in future studies that may include measurements of the forces from the strings being transmitted to the bridge during normal operation (i.e. a musician playing the violin). All three degrees-of-freedom were included for each input point, bringing the total number of inputs to fifteen. 


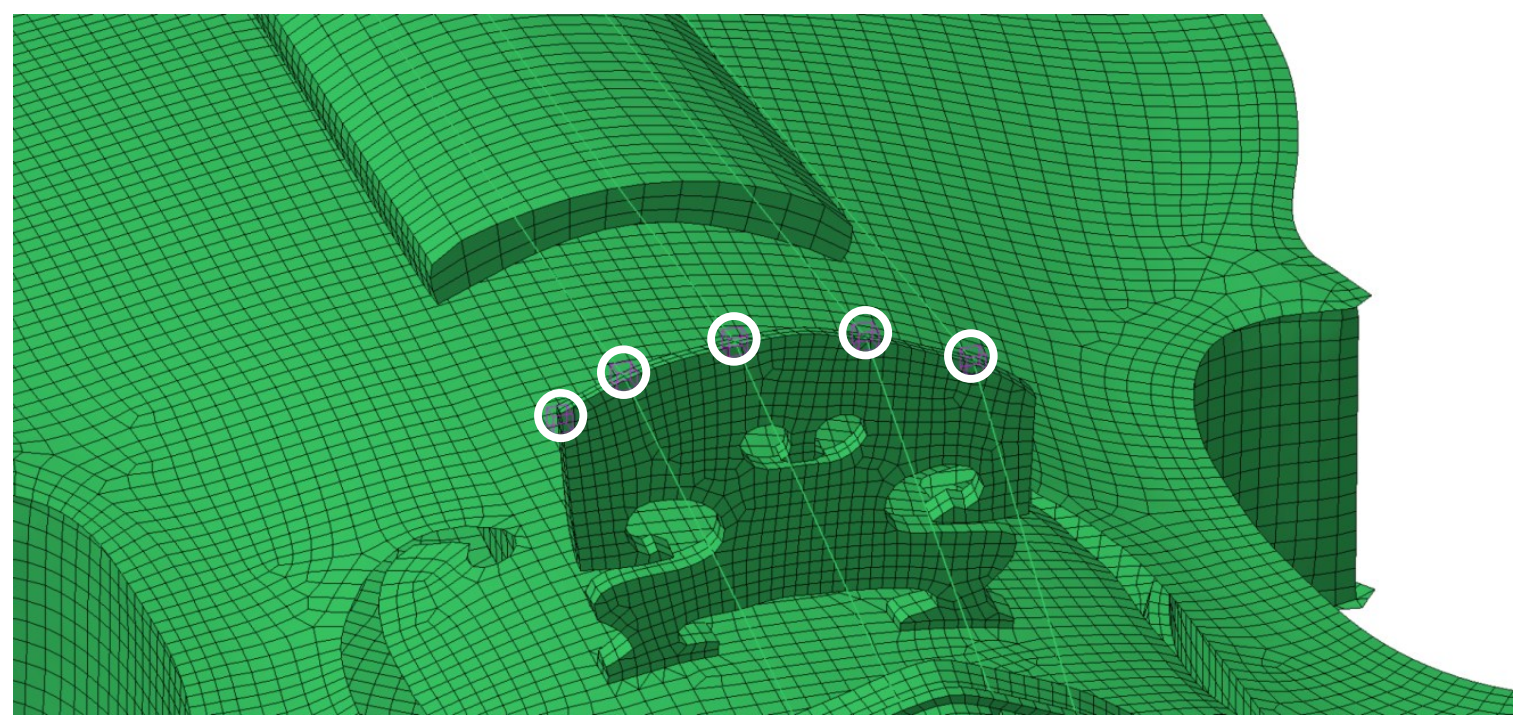

Figure 5.13: Input locations for the vibro-acoustic transfer function analysis of the Titian Stradivari violin

\subsection{Results}

\subsubsection{Projected Structural Modes}

As mentioned earlier the structural modes needed to be mapped onto the acoustic envelope. The most important of these were the three structural signature modes, CBR $(427 \mathrm{~Hz}), \mathrm{B} 1-(451 \mathrm{~Hz})$, and B1+ $(527 \mathrm{~Hz})$. The results of the projected mode mapping for these are shown in Figures 14 through 16. The original structural mode is shown on the left in each figure, while the projected mode on the acoustic envelope is shown on the right. The projected modes are similar, as all the most significant radiating surfaces for each structural mode appear to be the most influential on the acoustic mesh. It should be noted that the strings were excluded from the mode projections, since they have negligible influence on the acoustic field, but some sensitivity of the mapping was observed near the top of the fingerboard when they were included. 

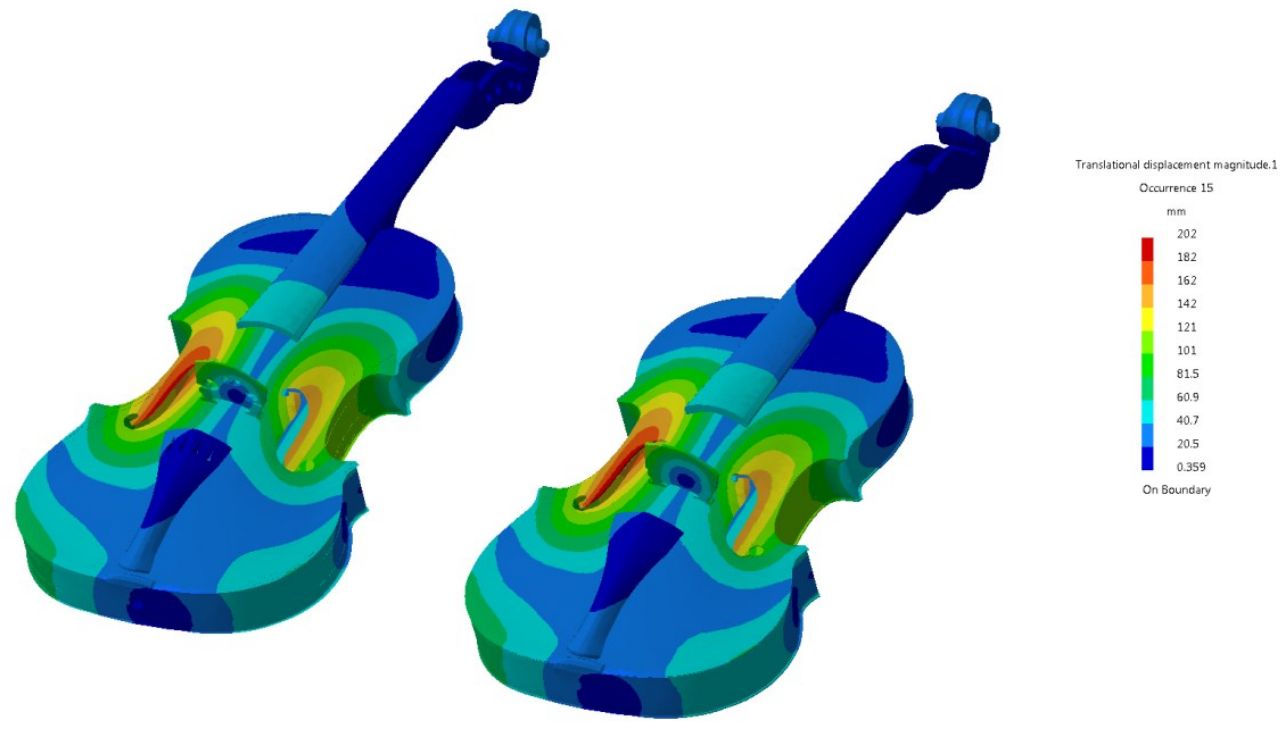

Figure 5.14: Projection of the CBR mode from the structural mesh (left) to the acoustic envelope (right)
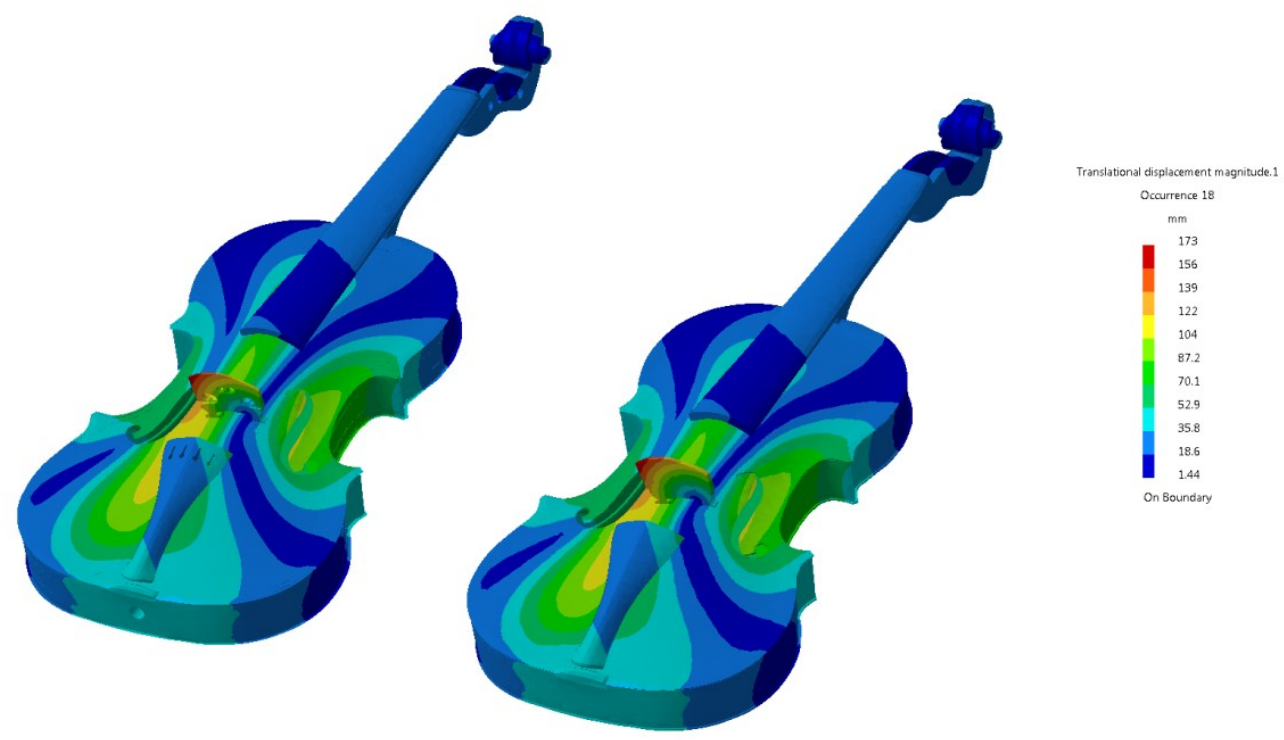

Figure 5.15: Projection of the B1- mode from the structural mesh (left) to the acoustic envelope (right) 


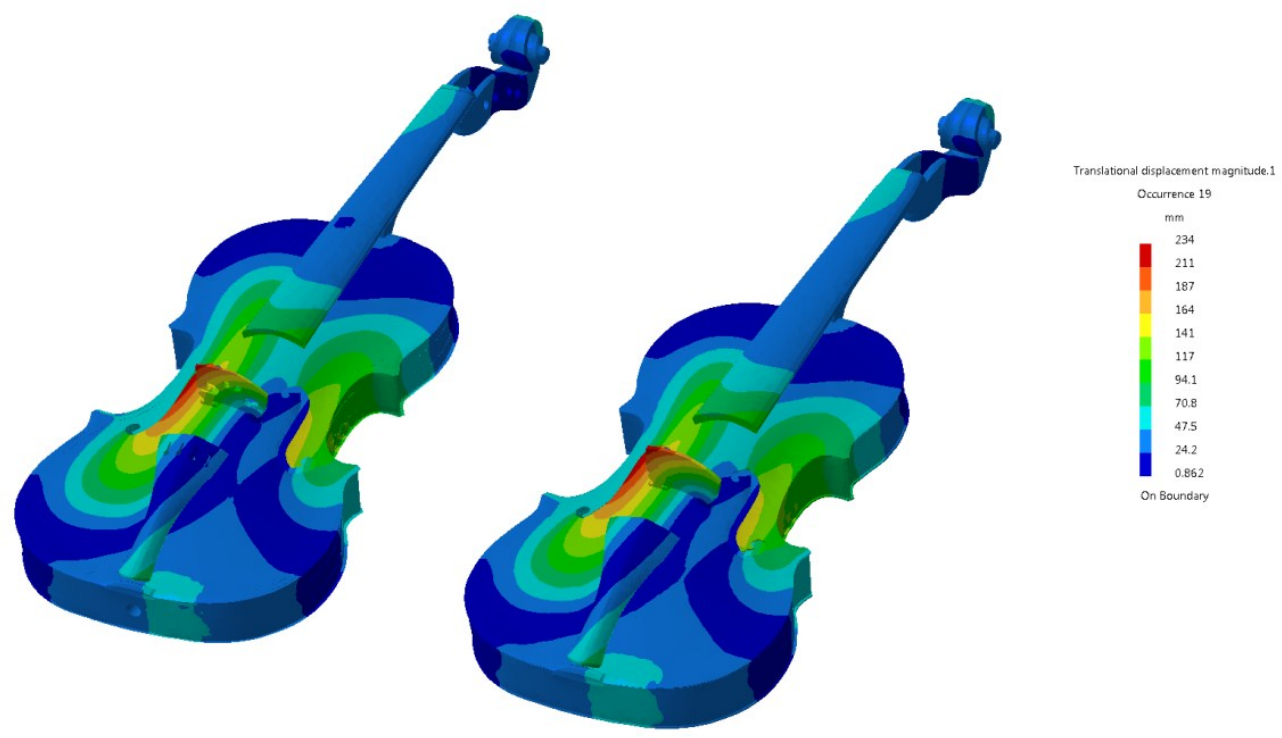

Figure 5.16: Projection of the B1+ mode from the structural mesh (left) to the acoustic envelope (right)

\subsubsection{FEM Vibro-Acoustic Noise Transfer Function Analysis}

The initial modal based vibro-acoustic noise transfer function case was set to calculate the transfer function for frequencies from $100 \mathrm{~Hz}$ to $1000 \mathrm{~Hz}$ with a $2 \mathrm{~Hz}$ increment. The Direct solver was used (rather than the iterative solver) as well as the multi-process combined level option.

The Direct solver was selected for several reasons. First, the total number of nodes is only 226,142 , which for three dimensions yields 678,426 DOFs; this was a relatively small model and memory usage was not an issue, so the iterative solver was not necessary. Second, despite the relatively small size of the model, it is rather complex, involving both interior and exterior domains with a complicated geometry, and a mix of structural elements. For these reasons the more robust Direct solver was preferred. Finally, the Direct solver is recommended for multi-load case scenarios [5.4] (15 in our case). It should also be noted that multi-threading was only supported by the Direct solver.

The combined level multi-processing utilizes both frequency level and matrix level parallelization. This was used to speed up the solve time and to reduce total memory required. The system used for solving the model had two processor cores with two threads per processor. 
The computational system properties used for solving the vibro-acoustic transfer function of the Titian Stradivari violin are listed in Table 5.3. A total of four runs were completed, which varied the modal damping on the structural modes and the complex properties of air, namely the speed of sound. These are summarized in Table 5.4. It was observed that the CPU core temperature generally reached $\sim 90^{\circ} \mathrm{C}$ during each run.

Table 5.3: Analysis system properties

\begin{tabular}{|l|l|}
\hline Processor & Intel ${ }^{\circledR}$ Core ${ }^{\mathrm{TM}}$ i7-2640M CPU @ $2.8 \mathrm{GHz}$ \\
\hline Installed RAM & $8 \mathrm{~GB}$ \\
\hline $\begin{array}{l}\text { Maximum Allocated } \\
\text { memory }\end{array}$ & $85 \%$ of System Physical Memory \\
\hline
\end{tabular}

The response of the point directly in front of the violin top plate was selected for comparison with the Bissinger test data. The transfer function (radiativity) magnitude for this point with respect to the lateral (X-direction) input at the upper bass-side corner of the bridge was extracted from each run and plotted against that of the Bissinger data [5.1]. These are shown in Figures 5.17 through 5.20.

Table 5.4: Vibro-Acoustic FEM Analysis run summary

\begin{tabular}{|c|l|c|c|c|}
\hline Run & Structural Modal Damping & Speed of Sound & A0 peak & CPU Time \\
\hline 1 & $\begin{array}{l}\text { Real Normal Modes (no } \\
\text { damping) }\end{array}$ & $340 \mathrm{~m} / \mathrm{s}$ & $1.742 \mathrm{~Pa} / \mathrm{N}$ & $13: 37: 49$ \\
\hline 2 & $\begin{array}{l}\text { Modal damping applied to all } \\
\text { structural modes }\end{array}$ & $340 \mathrm{~m} / \mathrm{s}$ & $1.1899 \mathrm{~Pa} / \mathrm{N}$ & $13: 38: 56$ \\
\hline 3 & $\begin{array}{l}\text { Modal damping applied to violin } \\
\text { modes (strings undamped) }\end{array}$ & $340+\mathrm{j} 4 \mathrm{~m} / \mathrm{s}$ & $0.8518 \mathrm{~Pa} / \mathrm{N}$ & $13: 45: 12$ \\
\hline 4 & $\begin{array}{l}\text { Modal damping applied to violin } \\
\text { modes (strings undamped) }\end{array}$ & $340+\mathrm{j} 8 \mathrm{~m} / \mathrm{s}$ & $0.5537 \mathrm{~Pa} / \mathrm{N}$ & $13: 48: 12$ \\
\hline
\end{tabular}

Since the structural FE model was correlated with experimental modal data previously, the frequencies of peaks in the radiativity curve that are associated with structural FE modes are all within $5 \%$ of those of the experimental data. The acoustic modes, A0 $(\sim 275 \mathrm{~Hz})$ and A1 $(\sim 464 \mathrm{~Hz})$, are of interest for verification of the vibro-acoustic model. The A1 peak is somewhat obfuscated by the B1- peak making it difficult to use as a reliable verifier. The A0 peak has a split in the experimental data of the Titian Stradivari violin, most likely due to a substructure mode. Despite this, the A0 peak is clearly visible, and can be used for updating the acoustic results. The radiativity magnitude of 
the A0 peak from the FE results are also noted in Table 5.4 for each run. For comparison, the experimental peak in radiativity for A0 was $\sim 0.396 \mathrm{~Pa} / \mathrm{N}$.

In Run 1 the structural modes are left as is; that is, they are real normal modes and have no modal damping. The properties of air were kept as default real constant values, $\mathrm{c}=$ $340 \mathrm{~m} / \mathrm{s}, \rho=1.225 \mathrm{~kg} / \mathrm{m}^{3}$. The comparison of the radiativity result with experiment is given in Figure 5.17. In almost all cases the peaks of the FE modes are sharper than those of the experiment, indicating that damping is important and needs to be included. The A0 mode is also considerably more prominent in the FE results ( $\sim 6.4 \mathrm{~dB}$ higher) than in the test data.

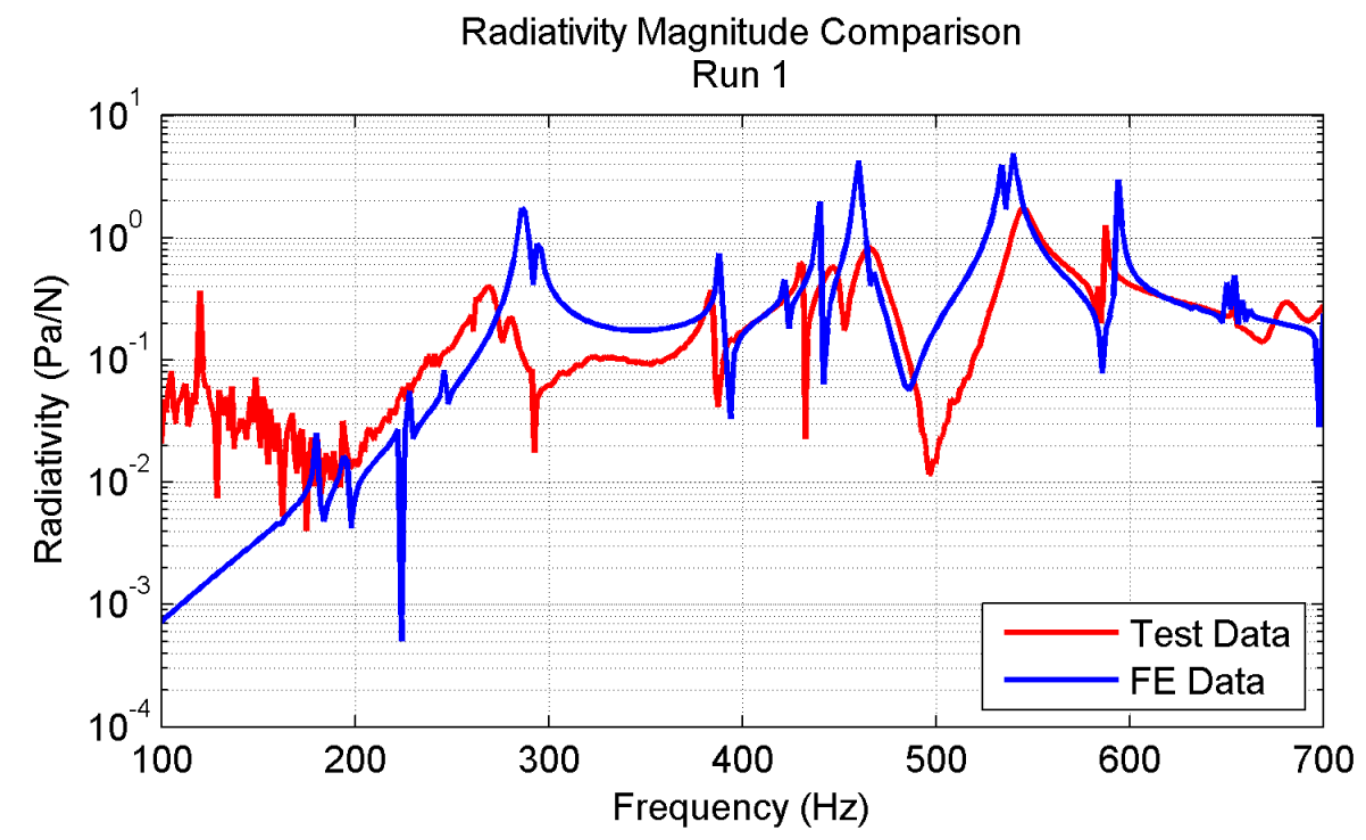

Figure 5.17: Radiativity results comparison for point directly in front of violin top plate; Real normal modes on structural FE; Air properties constant real: $c=340 \mathrm{~m} / \mathrm{s}, \rho=1.225$ $\mathrm{kg} / \mathrm{m} 3$

In Run 2 modal damping is applied to the structural modes across the frequency range, following the estimated trends of the structural damping given by Bissinger based on a set of 14 different violin modal analyses [5.3]. The properties of air are the same constant real values. Figure 5.18 shows the result of the FE predicted radiativity compared with the experimental data. This result shows stronger agreement in the peaks 
related to the structural modes. The exception to this is that the string modes of the test are now sharper than those of the FE. This is due to the strings themselves having less structural damping than the wood of the violin. A0 decreases by $\sim 1.6 \mathrm{~dB}$ and is now $\sim 4.8$ $\mathrm{dB}$ higher in the FE results than the test results, leading to better agreement between the results. The fact that although modal damping was only applied to the structural modes but the acoustic modes were also affected is indicative of the structure-acoustic coupling of the violin system.

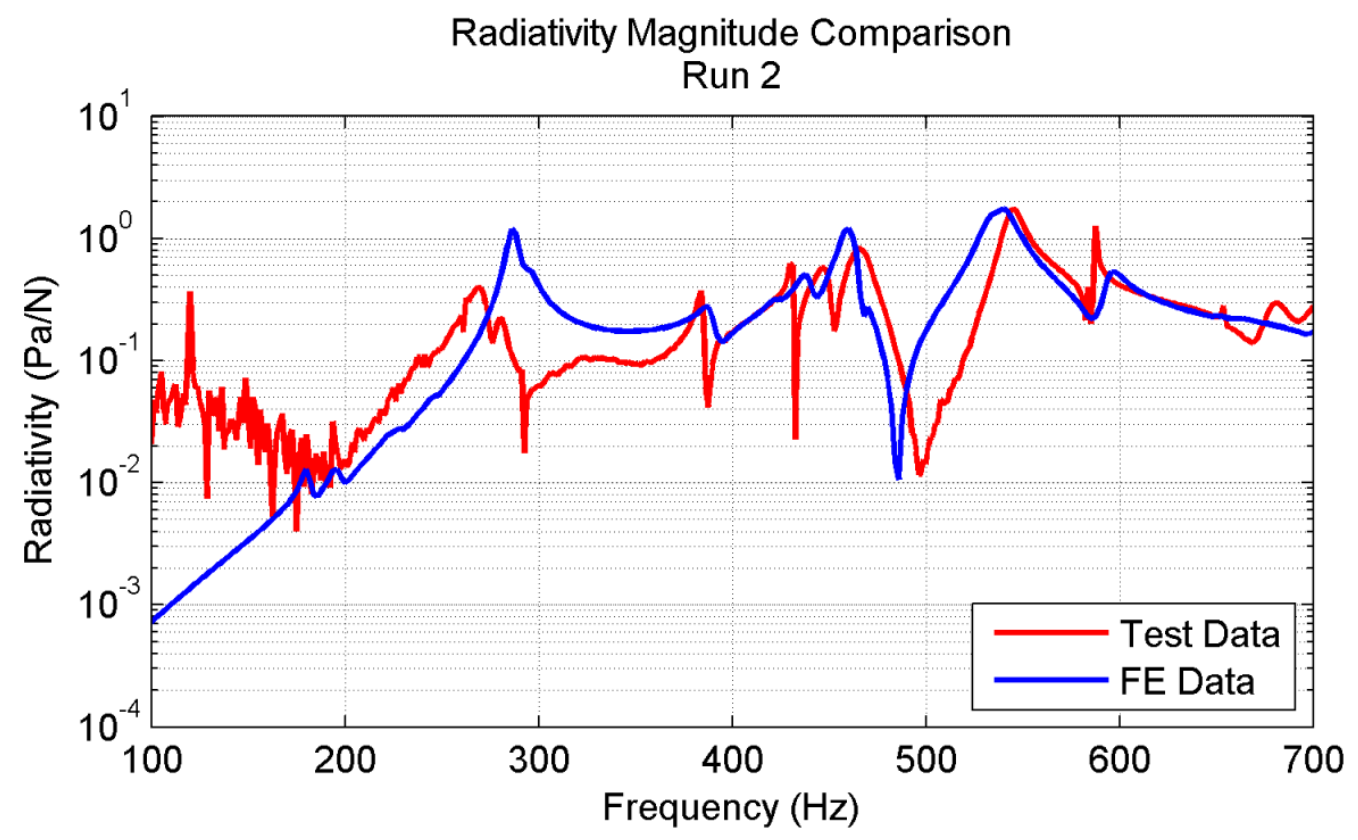

Figure 5.18: Radiativity results comparison for point directly in front of violin top plate; Modal damping added to all structural FE modes; Air properties constant real: $c=340 \mathrm{~m} / \mathrm{s}$, $\rho=1.225 \mathrm{~kg} / \mathrm{m} 3$

In Run 3 modal damping is only applied to modes involving flexure of the violin itself (corpus, neck, fingerboard, etc.) No damping is applied to modes involving the strings. The properties of air are adjusted to include a loss factor in media. Skudrzyk shows that a loss factor, $\eta$, can be added to the speed of sound to make it complex [5.7].

$$
c=c_{0}\left(1+j \frac{\eta}{2}\right)
$$

Lord also discusses this loss factor in terms of bending waves in a plate, and concludes that for small values of damping $(\zeta \leq 0.1)$ this loss factor is approximately proportional to the damping factor, $\zeta[5.8]$. 


$$
\eta \cong 2 \zeta
$$

For the Titian Stradivari violin, the A0 mode has an estimated damping factor of $\sim 1.2 \%$.

This yields a loss factor of 0.024 . The speed of sound is thus made complex, $\mathrm{c}=340+\mathrm{j} 4$ $\mathrm{m} / \mathrm{s}$ (assuming an ambient speed of sound of $340 \mathrm{~m} / \mathrm{s}$ ). The air density is held constant at $\rho=1.225 \mathrm{~kg} / \mathrm{m}^{3}$.

The result of this analysis is shown in Figure 5.19 for the point directly over the top plate compared to the experimental FRF. All FE structural peaks have approximately the same shape as those of the experimental curve, indicating that the modal damping properties being applied are realistic. The acoustic modes are also improved; the peak of the predicted A0 mode is now only $\sim 3.3 \mathrm{~dB}$ higher than that of the test data.

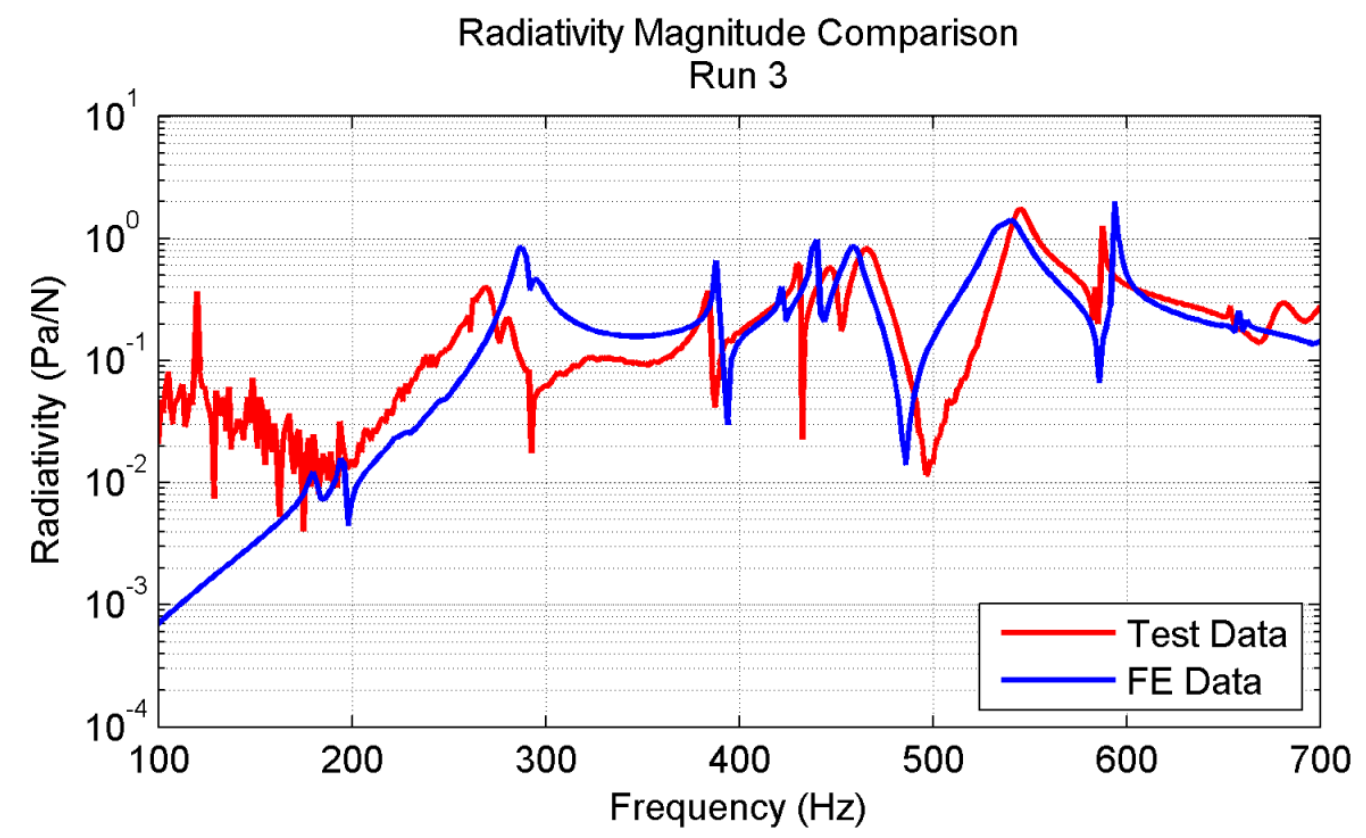

Figure 5.19: Radiativity results comparison for point directly in front of violin top plate; Modal damping added to structural modes (except string modes); Air properties complex: $c=(340+\mathrm{j} 4) \mathrm{m} / \mathrm{s}, \rho=1.225 \mathrm{~kg} / \mathrm{m} 3$

In the experimental data of the Titian Stradivari violin the radiativity has a split in the A0 peak. This is most likely due to a local structure mode of either the tail piece or fingerboard; however, since no response points were measured on either of these substructures, it is difficult to curve fit this area reliably, and the damping factor estimate would be particularly sensitive. 
The average damping factor of the A0 mode for all 14 violins in the VIOCADEAS database [5.3] is $\sim 2.12 \%$, nearly twice that estimated from the Titian experimental modal analysis. The loss factor in the air property is thus doubled in Run 4, such that the speed of sound was $\mathrm{c}=340+\mathrm{j} 8 \mathrm{~m} / \mathrm{s}$. The structural modal damping was the same as in Run 3 , that is, the string modes were undamped, while all others had an estimated modal damping applied.

The comparison of the transfer function of the point over the top with respect to the bassside bridge input for this final analysis run compared to the experimental data is given in Figure 5.20. The FE and test curves follow the same trend. Structural modes all have approximately the same shape (again, structural damping is realistic). The peak of the predicted $\mathrm{A} 0$ mode is now only $1.45 \mathrm{~dB}$ above that of the test.

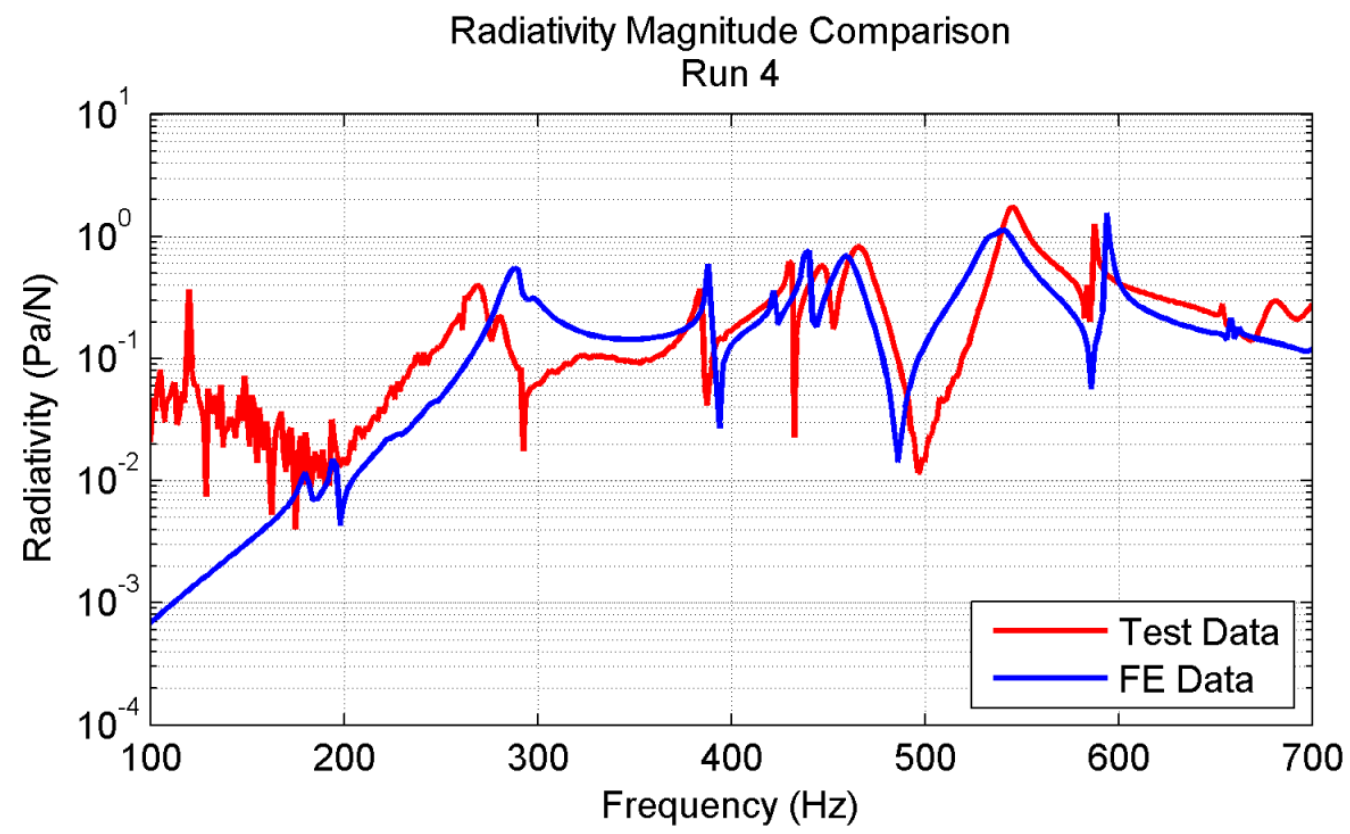

Figure 5.20: Radiativity results comparison for point directly in front of violin top plate; Modal damping added to structural modes (except string modes); Air properties complex: $c=(340+\mathrm{j} 8) \mathrm{m} / \mathrm{s}, \rho=1.225 \mathrm{~kg} / \mathrm{m} 3$

The predicted frequency of the A 0 mode is consistently above that of test by $\sim 6.8 \%$ for all 4 runs; this could be a function of the air density, or related to sound post stress loading [5.9],[5.10]. 


\subsection{Conclusions}

Based on the results of these analyses, the coupled vibro-acoustic FEM model of the Titian Stradivari violin is an excellent representation of the real structure. This model was created by measuring the structural geometry and density from CT scans of the actual instrument, structural elasticity was found through model correlation and updating with experimental measurements, and most importantly structural and acoustic damping characteristics were taken from a 14 violin experimental study including the Titian Stradivari [5.1]. It was shown that by including all these details, the vibro-acoustic behavior of the Titian Stradivari violin is accurately predicted. Predicted structural mode frequencies were all within $5 \%$ of the experimentally measured modes. The main acoustic mode, A0, of the Titian Stradivari was predicted within $7 \%$ of the experimental result, and its associated peak in the radiativity magnitude was within $1.5 \mathrm{~dB}$.

A comprehensive model such as this has never been built for a violin before (let alone a Stradivarius). The real value of this model is that further studies can be done to answer all those "what if..." questions that violin makers all over the world have been asking. By starting from a known system, and making incremental changes to the design variables (plate thickness, arch height, density, stiffness, damping, etc.) the effect of these on acoustic response can now be simulated (rather than needing to build hundreds of violins and testing them all). The Titian Stradivari violin, one of the world's best violins, now has a comprehensive analytical surrogate.

\subsection{References}

[5.1] Bissinger, G., "Structural acoustics of good and bad violins", Journal Acoustical Society of America, vol. 124, issue 3, pages 1764-1773 (2008)

[5.2] Bissinger, G., "A unified materials-normal mode approach to violin acoustics", Acustica 91, pages 214-228 (2005)

[5.3] Bissinger, G., "Structural acoustics model of the violin radiativity profile", Journal Acoustical Society of America, vol. 124, issue 6, pages 4013-4023 (2008)

[5.4] "A Next Generation Solution for Functional Performance Engineering - LMS Virtual.Lab Acoustic Training", LMS International (2008)

[5.5] Desmet, W., et al, "Numerical Acoustics - Theoretical Manual", LMS International (1998)

[5.6] Bissinger, G., "The violin bridge as filter", Journal Acoustical Society of America, vol. 120, pages 482-491 (2006)

[5.7] Skudrzyk, E., "The Foundation of Acoustics: Basic Mathematics and Basic Acoustics", Springer-Verlag, New York, p.319-322 (1972) 
[5.8] Lord, H., Gatley, W., Evensen, H., "Noise Control for Engineers", Krieger Pub. Co., Malabar, FL, p. 258-263, (1980)

[5.9] Meinl, H., "On the frequency curves of violins," Akust. Z. 2, 22-33 (1937), Translated by Fan, C., (2007)

[5.10] Bissinger, G., "Some mechanical and acoustical consequences of the violin soundpost Journal Acoustical Society of America, vol. 97, pages 3154-3164 (1995) 


\section{Afterword}

The purpose of this project was to develop a correlated model of the dynamic behavior of a Stradivari violin. A high fidelity finite element model of a Stradivari violin was constructed using geometry information gathered from a medical CT (Computed Tomography) scan of the Titian Stradivari. The model was then correlated with experimental data taken on the same instrument, and the model was updated with material properties that more closely matched the experimental results. Finally, the correlated structural FE model was used to develop a coupled vibro-acoustic model, which was used to predict the radiativity of the Titian Stradivari violin. This is the first time a fully vibro-acoustic model of a violin has been made, and represents a huge step in understanding violin dynamics. This model represents the first of its kind, and will provide much insight into violin dynamics.

This opens the door to many exciting possibilities. Structural modification prediction can be done virtually with the model, saving a tremendous amount of time, effort, and expense for violin makers, who want to know how design changes will affect the performance of their instruments.

Future studies may offer even further insights. Operating forces from a violin being played could be applied to the model to predict the acoustic response. Acoustic Transfer Vector (ATV) analysis could then be done on the model to show which areas of the violin structure have the greatest contribution to different frequencies at specific locations.

Furthermore, now that this project has developed a methodology for creating a comprehensive vibro-acoustic analytical model of a violin, any number of other violins may be modeled in a similar fashion. The overall process is as follows:

1. Obtain a priceless violin by Stradivari (or Guarneri) and have it CT scanned

2. Convert the CT scan data into a working FE model (see Chapter 2)

3. Perform an Experimental Modal Analysis on the violin (See Chapter 3)

4. Correlate the FE model with the test data and update (see Chapter 4)

5. Create a coupled vibro-acoustic model of the violin (see Chapter 5)

Scientists and engineers have been using computer modeling to understand complex systems and improve product design for years. Presenting these tools to violin makers has the potential to improve the quality of violins in the future. Perhaps one day a modern luthier will be able to break the "Stradivari ceiling" by applying science and engineering to the age old art of violin making, with the ultimate goal of creating superior music.

Michael Pyrkosz 


\section{Appendix A Definition of Terms}

There exists a large gap between the fields of violin making and vibro-acoustics. The following section is intended to provide definitions for readers, whether they come from a musical background or an engineering one. Three subsections have been included: Engineering terms, Musical terms, and Parts of the violin.

\section{A.1 Engineering Terminology}

Table A.1 is a selection of engineering terms that will be useful for readers.

Table A.1: Engineering Terms and Definitions

\section{Engineering Terms Definitions}

Anechoic chamber Room in which the walls are designed to be almost 100 percent absorptive for a specified range of frequencies, and free-field conditions exist nearly to the boundaries of the room. [A.1]

Automatically Similar to the Perfectly Matched Layer (PML), AML constructs Matched Layer (AML) the absorbing layer on the solver level, reducing model preparation time by the user.

Coupling of modes

Often a system may be modified in such a way that the normal modes of the system are perturbed. The new set of normal modes that results are linear combinations of the original system modes. This is synonymous with "mode coupling" [A.2].

Damping, Internal When materials are deformed, energy is absorbed and dissipated by the internal friction within the molecular structure of the material [A.3].

Far field

A region that is far enough away from the sound source that particle velocity and acoustic pressure become simply related, as in a plane wave [A.1].

Finite Element

The Finite element method is a numerical technique used for Method (FEM) solving complex mechanical and structural vibration problems. In this method, the actual structure is approximated by smaller pieces or elements, each of which is assumed to behave as a continuous 
structural member called a finite element. [A.3]

Free field Regions where the sound field is predominately caused by the source [A.1].

Impedance, Acoustic The complex quotient of the alternating pressure applied to the system by the resulting volume current, or velocity [A.4].

Impedance, The complex quotient of the alternating force applied to the Mechanical system by the resulting linear velocity at some point in the structure [A.4].

Infinite Element An older method for handling Sommerfeld radiation condition in Method (IFEM) exterior acoustic FEM problems. Requires that the boundary surface of the air be meshed as a canonical shape of an order similar to the longest wavelength. Special decay shape functions are used on the boundary elements to minimize reflections. [A.5]

Modal analysis The process of describing the dynamic properties of an elastic structure in terms of its normal modes of vibration [A.6].

Modal Assurance A scalar constant relating the degree of consistency (linearity) Criterion (MAC) between one modal and another reference modal vector [A.7].

Near field A region that is so close to the sound source that the acoustic pressure and particle velocity are not in phase, thus the intensity at any point in the region cannot be expressed as a simple relationship involving the mean square pressure and the characteristic impedance of the medium. [A.1]

Orthotropic Having unique and independent properties in three mutually orthogonal (perpendicular) planes of symmetry [A.8].

Perfectly Matched A newer method for handling the Sommerfeld radiation condition Layer (PML) in exterior acoustic FEM problems. A layer of elements is added to the outer boundary of a convex mesh that is locally-conformal to the structure mesh. This layer utilizes an anti-reflection function to create a coating around the radiating body to absorb the waves. [A.5]

Sommerfeld $\quad$ For exterior acoustic problems all acoustic waves must propagate freely towards infinity and no reflections may occur at the 
Radiation Condition boundary surface of the model [A.9]

Transfer Function The ratio of the output over the input with all initial conditions equal to zero [A.10].

\section{A.2 Musical Terminology}

Table A.2 is a list of important terms and definitions when discussing musical instruments.

Table A.2: Musical Terms and Definitions

\section{Music Terms}

Complex tone

Fundamental frequency

Fundamental tone

Harmonic

Note

Overtone

Partial

Simple tone

Sub-harmonic

Michael Pyrkosz

\section{Definitions}

A sound sensation characterized by having more than one pitch [A.4].

The lowest frequency component in a complex sound [A.4].

A component of a complex tone having the lowest pitch [A.4].

A partial or overtone whose frequency is an integer multiple of the fundamental tone or fundamental frequency [A.4].

A conventional sign used to indicate the pitch of the duration or both of a tone sensation, also the sensation itself or the vibration causing the sensation. The word serves when no distinction is desired between the symbol, the sensation, and the physical stimulus. [A.4]

A component of a complex tone having a pitch higher than the fundamental [A.4].

A component of a sound sensation which may be distinguished as a simple sound that cannot be further analyzed by the ear and which contributes to the character of the complex tone. It is a physical component of a complex tone. [A.4]

A sound sensation characterized by its singleness of pitch [A.4].

A tone that is an integer sub-multiple of the fundamental 
frequency of the sound to which it is related [A.4].

Tone

A sound sensation having pitch or a sound wave capable of exciting an auditory sensation having pitch [A.4].

\section{A.3 Parts of the Violin}

Table A. 3 contains a list of some of the most important parts of the violin along with some brief definitions. Figure A.1 and Figure A.2 are also included to give the reader a frame of reference.

Table A.3: Descriptions of various Parts of the Violin

\section{Parts of the violin Description}

Back plate (back) The back face of a violin body, (opposite the top plate), generally made of maple.

Bass bar

A strip of wood glued to the inside of the belly of a violin under the lowest string [A.4]. This serves to distribute vibrational energy from the bridge to the upper and lower regions of the top plate.

Blocks

These six pieces of wood, being fixed at the top, bottom, and corners of a violin, serve to strengthen the whole structure, and thereby give a firm base for the vibrations of the back and belly [A.11].

Bow

A long stick strung with a flat group of horsehair, which is drawn across the strings of a violin, or other string instrument, to actuate the strings. The iterated force is increased by applying rosin to the bow [A.4].

Bridge

Catgut

A small piece of wood, in contact with the body or soundboard, of a string instrument, over which the strings are stretched. The bridge transmits the vibrations of the strings to the body, or soundboard, thus increasing the output of the string [A.4].

Term used for the gut strings of a musical instrument [A.4]. 
End-pin (Tail-pin) A peg of ebony or box-wood, which is firmly fixed into the bottom block, through the center of the join of the lower bouts, to which is fastened the loop of the tail-piece [A.11].

F-Holes

Two holes in the belly of a violin that couple the air chamber of the body to the outside air. The term comes from the f-hole being shaped similarly to a lower case "f" [A.4].

Finger board

A long, narrow piece of wood, (usually ebony), on the front of the neck in a string instrument, over which the strings are stretched. The resonant frequency of the strings is altered by changing the length of the string by pressing it against the finger board with a finger [A.4].

Linings

These are twelve strips of wood that run round the top and bottom of the sides, and connect the blocks with one another, [A.11]. They also serve as a stronger mating surface for gluing the back and top plates to the ribs.

Neck

Generally the same piece of wood as the scroll, the neck serves as a place for the violinist to support the instrument in their hand while the fingers curl around to the strings on the other side of the fingerboard.

Nut

A piece of wood that forms the ridge at the top end of the finger board over which the strings pass to the tuning pegs. The nut and the bridge form the open length of the string [A.4].

Ribs (sides) The vertical strips that join the belly and back of the instruments in the violin family [A.4].

Rosin

A turpentine distillate in solid state, which is applied to bows to increase the friction between the bow and the string of a bowedstring instrument [A.4].

Saddle (rest)

A small piece of ebony, or other hard material, over which the loop passes from the end-pin to the tail-piece [A.11].

Scroll

A term used to designate the head of the instruments in the violin family [A.4]. 
Sound post

Strings

Tail piece

Top plate (belly)

Tuning peg
A short wooden prop between the belly and back of the instruments in the violin family [A.4]. This piece is highly important and provides energy transmission from the top plate into the back. It is held in place by pressure only, and if set improperly has a deleterious effect of the sound quality of the instrument.

The tightly stretched threads of steel, gut, or other material which is induced to vibrate by some actuating means in order to produce a musical tone [A.4].

A triangular piece of wood, (usually ebony, rosewood, or box wood), to which the lower ends of the strings are attached in the violin family [A.4].

The face of the body upon which the strings are stretched in a violin [A.4]. This is the primary sounding board for the violin.

Thumb pins around which one end of the strings of a violin are wound. The tension in the string and the resultant pitch is changed by turning the peg. [A.4] 


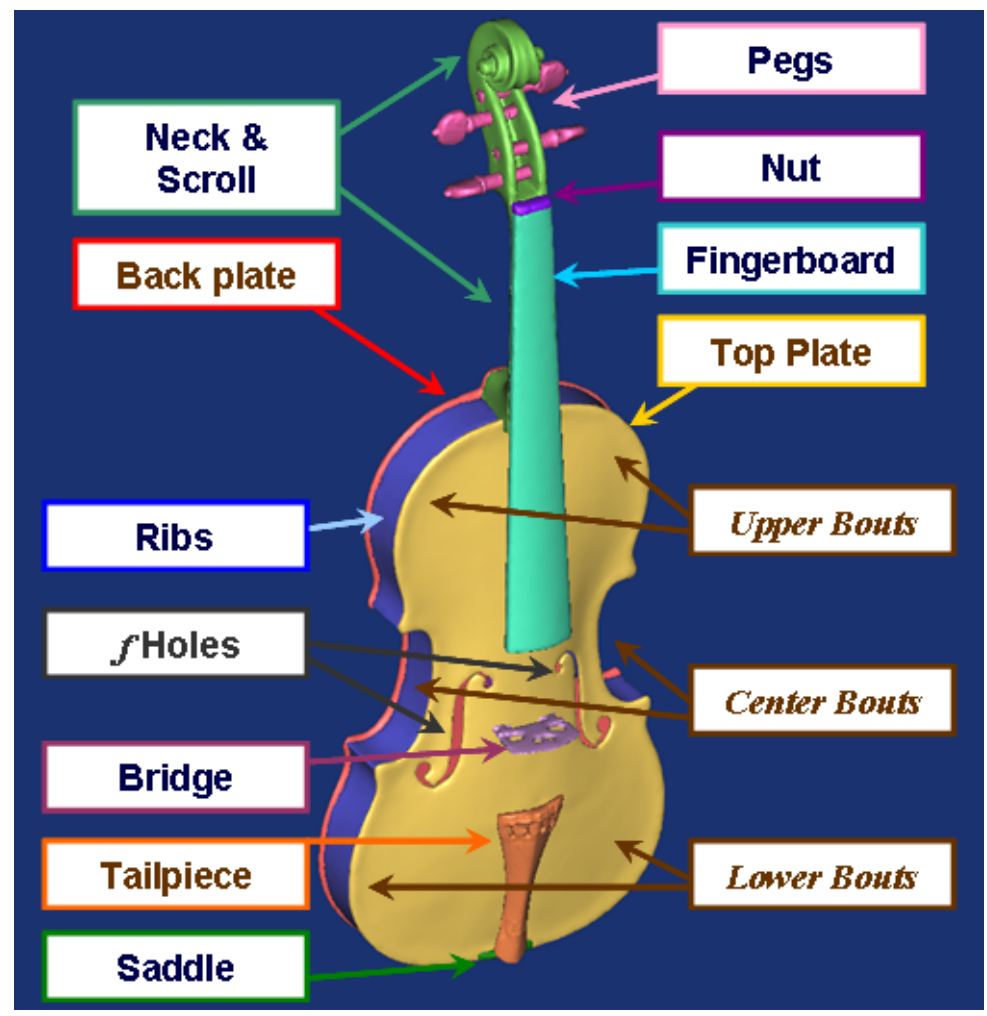

Figure A.1: Exterior parts of the violin

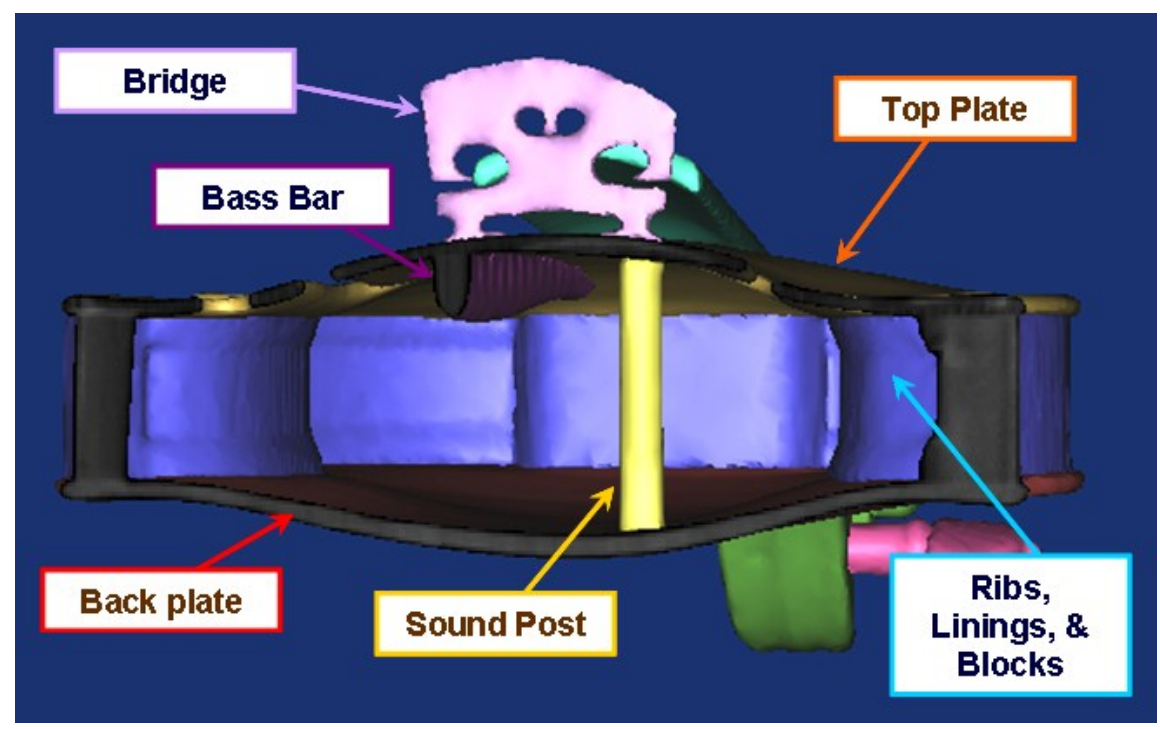

Figure A.2: Interior parts of the violin 


\section{A.4 References}

[A.1] Lord, Harold W., Gately, William S., Evensen, Harold A., "Noise Control for Engineers" Original Edition: Robert E. Krieger Pub. Co., Inc., Malabar, FL (1980). Reprint Edition: McGraw Hill Book Co., New York, NY (1987)

[A.2] Weinreich, Gabriel, "Glossary of Terms", included in the Strad3D DVD Articles, www.strad3d.org

[A.3] Rao, Singiresu S., "Mechanical Vibrations" 3rd edition, Addison-Wesley Pub. Co., Inc., Reading, MA, (1995)

[A.4] Olson, Harry F., "Music, Physics and Engineering" 2nd edition, Dover Publications, Inc., New York, NY (1967). Originally published as "Musical Engineering” McGraw Hill Book Co., Inc., New York, NY (1952)

[A.5] "A Next Generation Solution for Functional Performance Engineering LMS Virtual.Lab Acoustic Training”, LMS International (2008)

[A.6] Fletcher, Neville H., Rossing, Thomas D., "The Physics of Musical Instruments" 2nd edition, Springer Science+Business Media, LLC. New York, NY (1998)

[A.7] Allemang, R.J., "The Modal Assurance Criterion (MAC): Twenty Years of Use and Abuse", Proceedings of the International Modal Analysis Conference, Los Angleles, CA, February 2002

[A.8] U. S. Department of Agriculture, "The Encyclopedia of Wood", Skyhorse Pub., Inc., New York, NY (2007). Originally published by U.S.D.A. Washington, D.C. (1999)

[A.9] Desmet, W., et al, "Numerical Acoustics - Theoretical Manual", LMS International (1998)

[A.10] Thompson, William T., Dahleh, Marie D., "Theory of Vibration with Application" 5th edition, Prentice Hall, Inc. Upper Saddle River, NJ (1998)

[A.11] Heron-Allen, Edward, "Violin Making: A Historical and Practical Guide" Dover Publications, Inc., Mineola, NY (2005). Originally published as "Violin Making, as it was and is" 2nd edition, Ward, Lock, and Co., London, UK (1885) 


\section{Appendix B Population of Study}

Violins are almost always hand-crafted, therefore every violin is unique. Even in manufacturing there are generally a range of variations that ultimately come out of an assembly line. The focus of this study has been the 1715 Titian Stradivari, with supporting studies on one of three factory violins. In the early stages of the project several other violins were also CT scanned, and this data is available for future studies. It should be noted that CT scanning of old, Italian instruments has become something of a trend, and more data is becoming available [B.1], [B.2], \& [B.3].

The population of this study included CT scans several violins listed in Table B.1. Figure B. 1 is a comparison of sample CT slices taken through the middle of each of the violins in the study. The following is a brief history of each.

The first three are factory violins by Florea, Student Recital II, handmade in China (2008), purchased by Michigan Technological University for testing purposes. Although these arrived from the distributer with unique ID numbers in pencil on the lower treble corner of the fingerboard, for the purposes of keeping track of the violins and associated data, these were assigned names that were easier to remember: (A) X1344 "Anna", (B) X1682 "Beverly", and (C) X1686 "Clara". The following notes were made about each within a few days of their arrival in Houghton, MI: Anna had a hairline crack near the edge of treble side f-hole. After removing Beverly's chin rest, it was observed that the lower bass side rib was starting to separate from the top plate. There were some fairly deep scratches on the top plate of Clara directly under the tail piece corresponding to the $\mathrm{G}$ and $\mathrm{E}$ string tuning screws.

The next is a copy of a violin by Johann Baptiste Schweitzer, made in Germany (circa late $19^{\text {th }}$ to early $20^{\text {th }}$ cen.). Schweitzer himself worked in Pestini near Budapest, Hungary, but for some reason he was frequently copied by German luthiers in the late $19^{\text {th }}$ and early $20^{\text {th }}$ centuries [B.4].

Next is an instrument by Francois Costa, a modern French Luthier who works in Germany, and specializes in making violins in both the French and German styles, (2007). This particular instrument was made for Andrew Fein, a dealer located in St. Paul, MN.

The next violin is simply labeled "H. Clotelle". As it turns out "Clotelle" was a brand name used by the Laberte-Humbert workshops at Mirecourt from 1891 to about 1920 [B.5]. Some brands are real names of luthiers, sold by their families or used legally by their successors, while others have a link to the catalogue of the factory where they come 
from. Whether or not there was ever a real Clotelle is unknown, but a 1912 catalogue from Laberte-Humbert does list four different models under the Clotelle brand [B.5].

An unknown German instrument follows, most likely from the same time period as the Schweitzer copy and the Clotelle. This instrument was scanned in a state of disrepair, the strings were missing, and the sound post was lying on its side. It was included with the intention of it being used to compare the deflection of the violin body under the static load of the strings and sound post.

The next violin is called "The Dancla", model \#126 (1/2) by William Lewis and Son, a dealership in Chicago who sold instruments made in various workshops in Germany. This is a half-sized violin, which may be used for comparing violins of different sizes. It was also scanned in a state of disrepair, with the neck broken in addition to the strings and sound post being missing.

Next is an imitation Stainer. Apparently these were prevalent from about 1890 until WWII. The fact that it is an imitation is apparent from two details. First, the name "Stainer" is stamped on the back plate, (which Stainer never did). Second, the label actually contains a typo, which is printed "Jacobus Stainer in Abfam, prope Oenipotum 17." According to the Smithsonian Institute's online encyclopedia, Stainer's typical label read "Jacobus Stainer in Absam, prope Oenipontum fecit 16_.." "Absam" refers to Absam, Austria where Stainer lived [B.6]. Apparently, the "s" on the original handwritten label must have looked like scripted "f" to whoever made the imitation.

Finally there are the old, Italian instruments:

The 1735 Plowden by Giuseppe Guarneri "del Gesù" was named for its first recorded owner, C.H. Chichele Plowden, a collector and amateur violinist from London [B.7]. It is considered to be representative of Guarneri's middle period, most likely using a variation of del Gesù's "Grand Amati” model [B.8]. Guarneri $(1698$ - 1744) is considered to be the second greatest luthier in history. The Plowden del Gesù is currently owned by Dr. Mark Ptashne [B.7].

The 1715 Titian is considered to be one of the most important instruments from Stradivari's golden period [B.9]. The name was given to this instrument by a French dealer, Albert Caressa, because the orange-red color of the varnish reminded him of the work by the $16^{\text {th }}$ century Venetian painter, Tiziano Vecelli, more commonly known as "Titian" [B.7]. It is believed that Stradivari created geometric archtypes for his violin patterns, and that the Titian was probably constructed from Stradivari's P form [B.8]. The Titian Stradivari is currently owned by international soloist, Cho-Liang Lin, in 
whose hands "it has revealed itself to be a formidable concert instrument of unusual power and scope" [B.9].

The 1734 Willemotte is a fine example of Stradivari's late period. The violins of this period are considered quite different from those of Stradivari's golden period. For

example the arching is notably higher in instrument from the mid 1720s through the remainder of his late period violins [B.10]. The instrument was named for a collector, connoisseur, and amateur violinist, Charles of Antwerp, who purchased the instrument in 1886, and is currently owned by Dr. Mark Ptashne [B.7].

Table B.1: Population of study

\begin{tabular}{|c|c|c|c|c|c|c|c|}
\hline Letter & Name/Owner & Luthier & Dealer/Country/City & Model & Year & Size & Back \\
\hline $\mathbf{A}$ & Anna/MTU & Factory & Florea & $\begin{array}{c}\text { Student } \\
\text { Recital II }\end{array}$ & 2008 & $4 / 4$ & quarter \\
\hline B & Beverly/MTU & Factory & Florea & $\begin{array}{c}\text { Student } \\
\text { Recital II }\end{array}$ & 2008 & $4 / 4$ & quarter \\
\hline $\mathbf{C}$ & Clara/MTU & Factory & Florea & $\begin{array}{c}\text { Student } \\
\text { Recital II }\end{array}$ & 2008 & $4 / 4$ & quarter \\
\hline D & D. Pyrkosz & Copy & Germany & $\begin{array}{l}\text { Joh. Bapt. } \\
\text { Schweitzer }\end{array}$ & $\begin{array}{l}\text { late } 19 \text { th to } \\
\text { early } 20 \text { th }\end{array}$ & $4 / 4$ & slab \\
\hline $\mathbf{E}$ & L. Blough & $\begin{array}{l}\text { Francois } \\
\text { Costa }\end{array}$ & Andrew Fein & & 2007 & $4 / 4$ & slab \\
\hline $\mathbf{F}$ & D. Blough & H. Clotelle & $\begin{array}{l}\text { Laberte-Humbert } \\
\text { /Mirecourt }\end{array}$ & & c. $1890-1920$ & $4 / 4$ & slab \\
\hline G & M. Olsson & & Germany & & $\begin{array}{l}\text { late } 19 \text { th to } \\
\text { early } 20 \text { th }\end{array}$ & $4 / 4$ & quarter \\
\hline H & M.Olsson & & $\begin{array}{c}\text { William Lewis } \\
\text { \& Son }\end{array}$ & $\begin{array}{l}\text { \#126 the } \\
\text { Dancla }\end{array}$ & & $1 / 2$ & slab \\
\hline $\mathbf{J}$ & M. Olsson & $\begin{array}{c}\text { probable } \\
\text { copy }\end{array}$ & & $\begin{array}{l}\text { Jacob } \\
\text { Stainer }\end{array}$ & $\begin{array}{l}\text { late } 19 \text { th to } \\
\text { early } 20 \text { th }\end{array}$ & $4 / 4$ & quarter \\
\hline & Plowden/ & Giuseppe & & & & & \\
\hline $\mathbf{P}$ & $\begin{array}{l}\text { Dr. Mark } \\
\text { Ptashne }\end{array}$ & $\begin{array}{l}\text { Guarneri } \\
\text { del Gesù }\end{array}$ & Cremona & & 1735 & $4 / 4$ & slab \\
\hline $\mathbf{T}$ & $\begin{array}{c}\text { Titian/ } \\
\text { Cho-Liang Lin }\end{array}$ & $\begin{array}{l}\text { Antonio } \\
\text { Stradivari }\end{array}$ & Cremona & & 1715 & $4 / 4$ & quarter \\
\hline W & $\begin{array}{c}\text { Willemotte/ } \\
\text { Dr. Mark } \\
\text { Ptashne }\end{array}$ & $\begin{array}{c}\text { Antonio } \\
\text { Stradivari }\end{array}$ & Cremona & & 1734 & $4 / 4$ & slab \\
\hline
\end{tabular}




\section{Factory Violin A \\ Florea Student Recital II}

(2008)

. . .

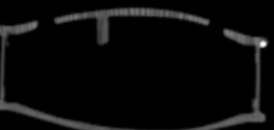

Factory Violin B

Florea Student Recital II

(2008)

-

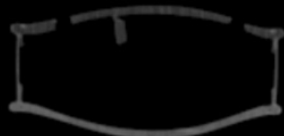

Factory Violin C

Florea Student Recital II

(2008)

. .
Copy of J.B. Schweitzer

made in Germany

(late 19th-early 20th)

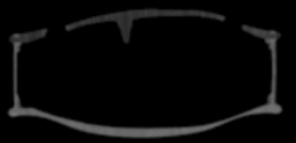

Francois Costa Andrew Fein Instruments (2007)

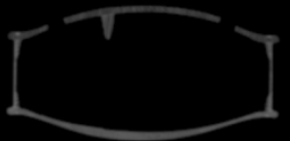

H. Clotelle

Laberte-Humbert/Mirecourt (c. 1890-1920).
Unknown

made in Germany

(late 19th-early 20th)

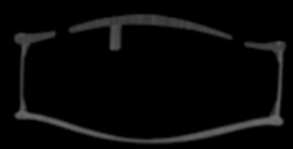

The Dancla

model \#126 1/2

William Lewis \& Son

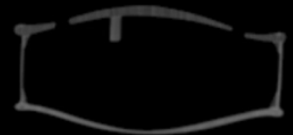

Copy of Jacob Stainer

Germany

(late 19th-early 20th)
Willemotte

Antonio Stradivari

(1734)

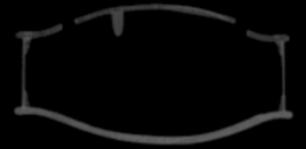

Plowden

Guarneri del Gesu

(1735)

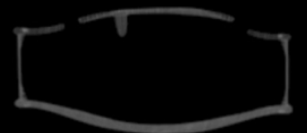

Titian

Antonio Stradivari

(1715)
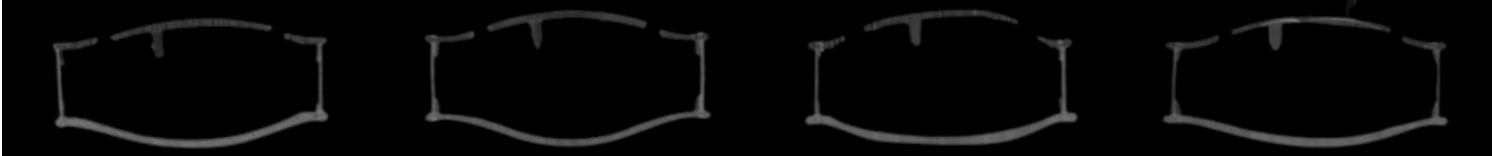

Figure B.1: Comparison of slices taken across middle section of each violin

One interesting observation was the difference in the craftsmanship. The old Italians, not surprisingly, had extremely tight tolerances, and it was difficult to determine where one piece of wood ended and another began. The factory violins on the other hand were much easier to separate since the pieces were not as tightly fit together, and there were even some observable air gaps between parts, as shown in Figure B.2. The neck of the factory violin is not quite connected to the upper end block. Also, there are portions of the ribs of the factory violin that have separated from the corner blocks. 


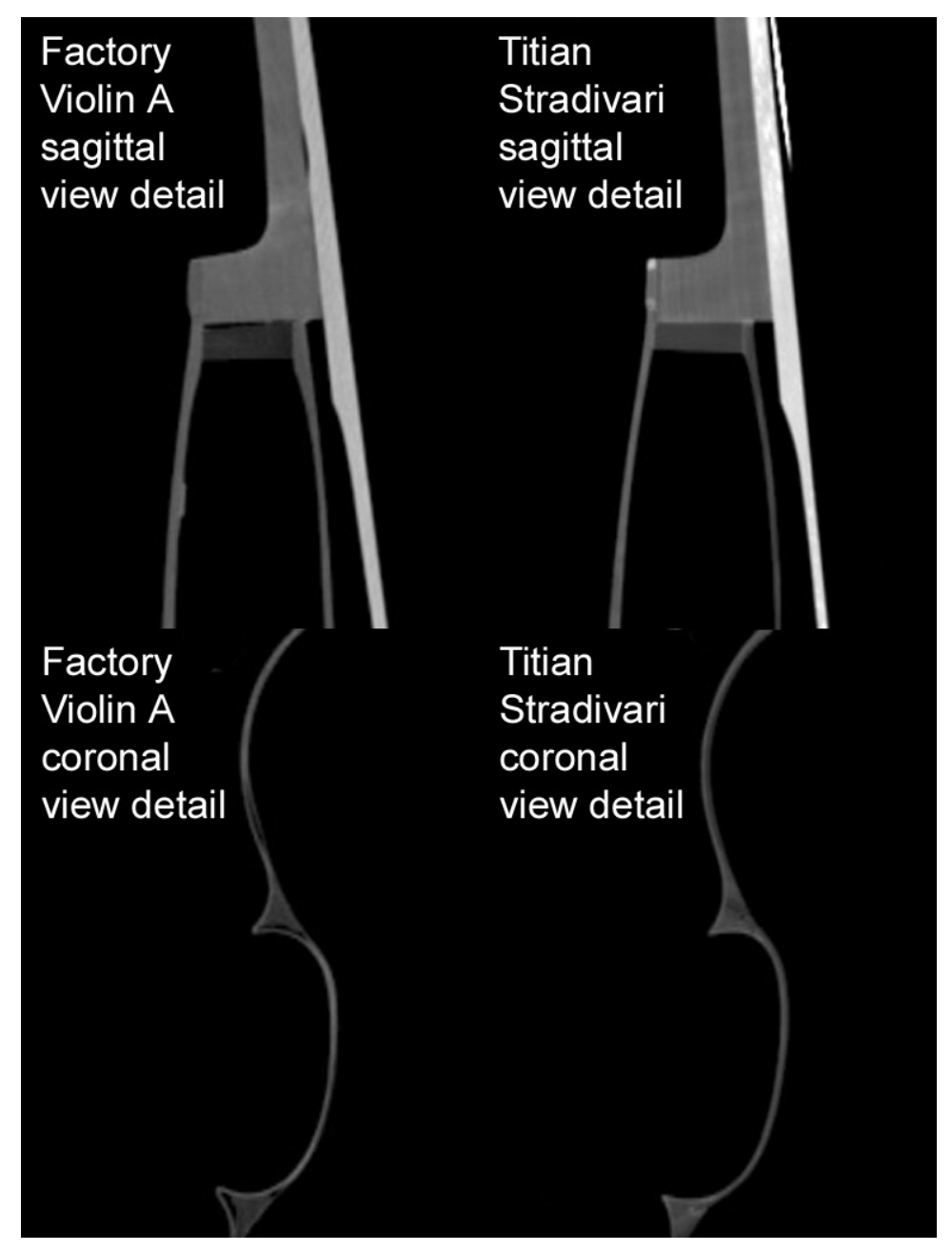

Figure B.2: Detail sagittal and coronal views of Factory violin A and the Titian Stradivari

\section{B.1 References}

[B.1] Sirr, Steven A., and Waddle, John R., "Use of CT in Detection of Internal Damage and Repair and Determination of Authenticity in High-Quality Bowed Stringed Instruments", RadioGraphics 1999; 19: 639-646.

Presented as a scientific exhibit at the 1997 RSNA scientific assembly.

[B.2] Stoel, B.C., Borman, T.M. (2008) "A Comparison of Wood Density between Classical Cremonese and Modern Violins" PLoS ONE 3(7): e2554. doi:10.1371/journal.pone.0002554

[B.3] Borman, T.M., Stoel, B.C., "CT and modal analysis of the 'Vieuxtemps' Guarneri 'del Gesù”, The Strad magazine, January 2011, pp. 68-71, www.thestrad.com.

[B.4] http://www.theviolinman.co.uk/messages/390.html, Message posts from (February 2004 - October 2006, accessed May 2009) 
[B.5] http://www.luthiers-mirecourt.com /laberte_humbert_1912.htm, (accessed July 2013)

[B.6] Smithsonian Institute, "Instrument Makers of the Stainer Family," http://www.si/resource/faq/nmah/violstai.htm, (accessed May 2009)

[B.7] Ruening, Christopher, "Provenance", included in the Strad3D DVD Articles, www.strad3d.org

[B.8] Denis, François, "Two Violin Forms: Commentary and Analyses", Translation by Pablo Alfaro and Claudia Fritz, included in the Strad3D DVD Articles, www.strad3d.org

[B.9] Zygmuntowicz, S., "The Titian Stradivari", The Strad magazine, February 2009, pp.30-34, www.thestrad.com

[B.10] "Willemotte Stradivari", excerpt from Strad3D DVD under the "About the Violins" section, www.strad3d.org 


\section{Appendix C Supporting Studies}

The following are a few supporting studies that were completed but never published. Although it was impossible to include everything that was done in support of this project, these were selected as they provided some additional insights. They are included here for readers interested in some of the finer details of the project.

\section{C.1 Wood Sample Study}

The simplest structures for testing and correlating the material properties of the different wood species are individual sample pieces of the wood commonly used in making violins. Such samples have been generously donated to the project from master luthier Joseph Curtain, a MacArthur fellow. These samples are shown in Figure C.1. The four samples on the left are from European spruce from the mountains in northern Italy, the most likely location where Stradivari obtained his wood. The two remaining samples are of Bosnian maple, also known to have been used by Stradivari. Each of these samples were cut or split "on the quarter" as they would be for making a violin with a two-piece back. Each sample was sanded smooth and CT scanned. The purpose of sanding the wood samples first is simply to improve the accuracy and stability of the FE models that were to be made from the CT scans. A complete experimental modal analysis was completed for each of these samples. The results of the first spruce sample (S1) and the first maple sample (M1) were then correlated with the FE model generated from the CT data. Experimental, Analytical and Correlation results for these to samples are presented in this section. The samples were weighed on a triple beam balance. These results are given in Table C.1. 


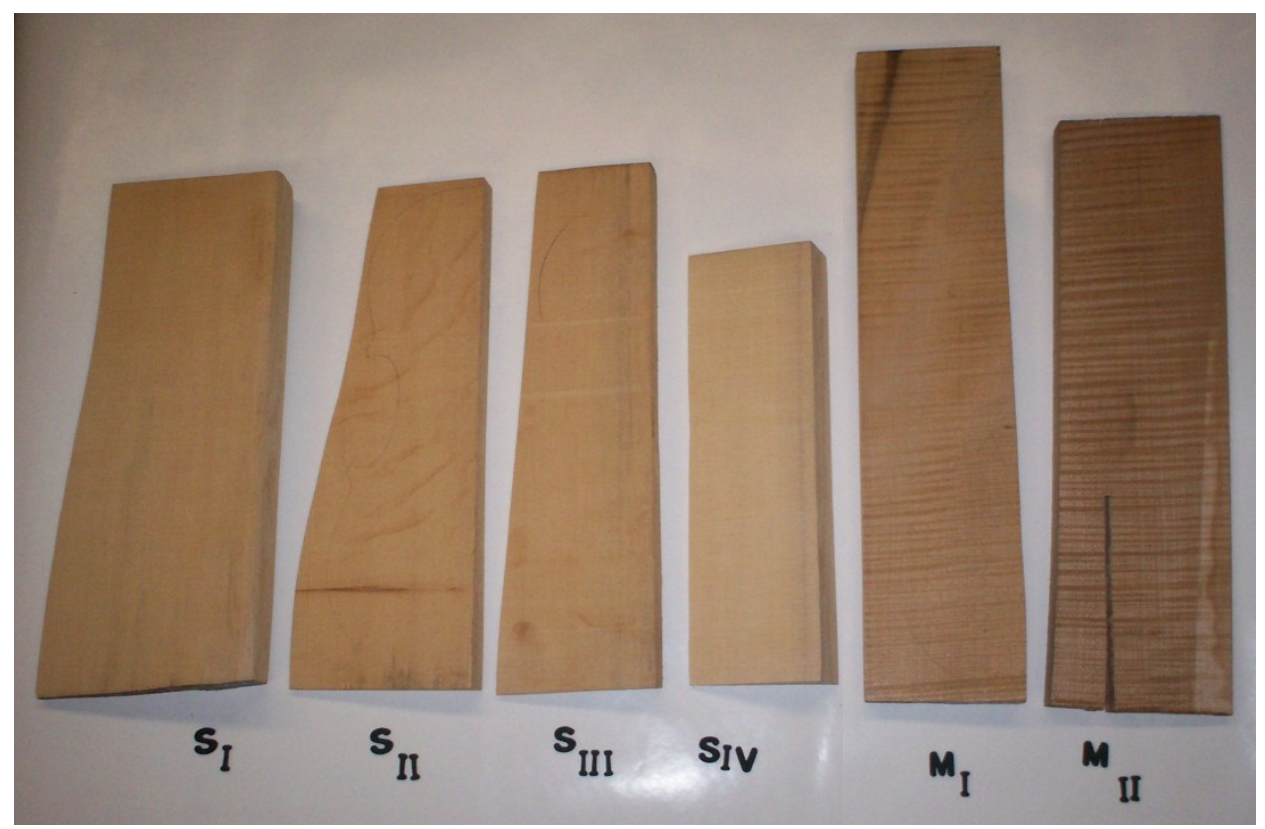

Figure C.1: Wood samples to be used in finding material properties of wood species, $\left(S_{I-I V}\right.$ : European spruce, $M_{I-I I}$ : Bosnian maple)

Table C.1: Measured mass of all wood samples

\begin{tabular}{|ccc|}
\hline Sample & Weight (lb) & Mass (grams) \\
\hline \hline Spruce 1 & 1.3750 & 623.7 \\
Spruce 2 & 0.5020 & 227.7 \\
Spruce 3 & 0.4786 & 217.1 \\
Spruce 4 & 0.5801 & 263.1 \\
\hline Maple 1 & 1.7285 & 784.0 \\
Maple 2 & 0.9668 & 438.5 \\
\hline
\end{tabular}

\section{C.1.1 Experimental Modal Data}

The experimental setup for the wood sample modal analyses was as follows. "Free-free" boundary conditions were obtained using rubber bands. Figure C. 2 shows the experimental setup for the first spruce sample. A similar set of measurement locations was used for each sample. A 5-by-9 grid was drawn on the main surface of each for the first 45 measurements locations to capture out-of-plane motion. An additional row of 9 points was selected along one edge to measure motion in the transverse direction. Two driving point accelerometers were mounted on opposite corners of the sample, one in the out-of-plane direction (point 1:+Y) and one in the transverse direction (point 54:+X). The modal impact hammer was roved over all 54 measurement locations and FRFs were 
measured and used for calculating the experimental modes. The Digital Signal Processing (DSP) parameters for the test are summarized in Table C.2.

Table C.2: DSP parameters for wood sample measurements

\begin{tabular}{|l|l|}
\hline Averaging & $5 \mathrm{Averages}$, Linear \\
\hline Bandwidth & $4096 \mathrm{~Hz}$ \\
\hline Frequency Resolution & $1.0 \mathrm{~Hz}$ \\
\hline Reference Window & Force-Exponential, 100\% \\
\hline Response Window & Exponential, 100\% \\
\hline
\end{tabular}

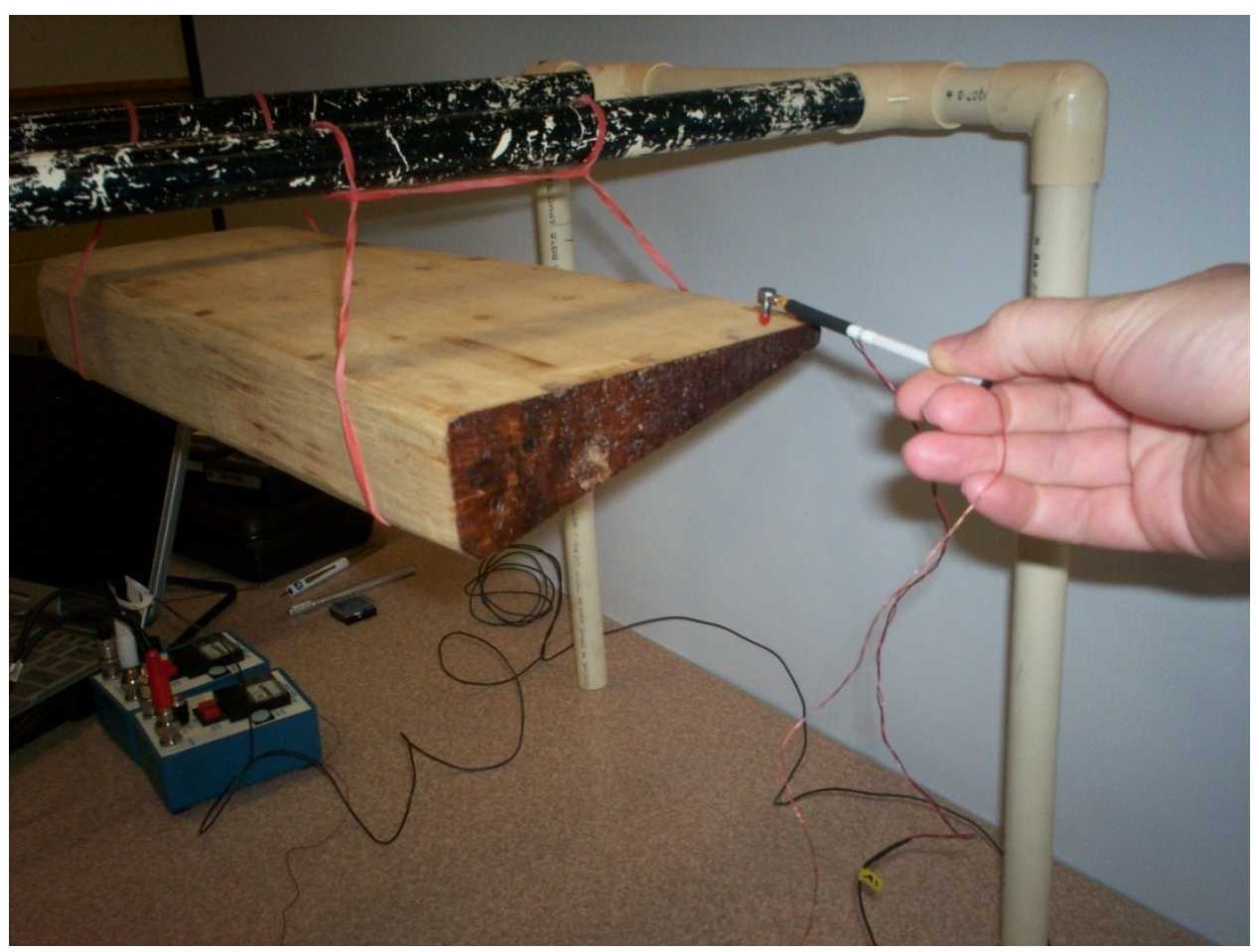

Figure C.2: Spruce sample 1 test setup

The results of the driving point FRF measurements of the first spruce sample are shown in Figure C.3, while the corresponding coherence measurements are shown in Figure C.4. Similarly, the FRF and coherence measurements for the first maple sample are shown in Figure C.5 and Figure C.6 respectively. The out-of-plane FRF measurements show little variance, and their corresponding coherence function are above 0.95 throughout the frequency range of the measured modes $(\sim 250 \mathrm{~Hz}$ to $\sim 3500 \mathrm{~Hz})$. The transverse measurements indicate a greater amount of variance in this range except where there is a large amount of transverse motion. For example, the only place where there is significant participation in the transverse direction for the spruce sample is in the $2900-3100 \mathrm{~Hz}$ 
range. The transverse coherence in the range is well above 0.95 . Similarly the maple sample only shows large transverse motion in around $1450-1650 \mathrm{~Hz}$ range, and again in the $3100-3300 \mathrm{~Hz}$ range. These regions also show coherence in the transverse direction to be over 0.95 .

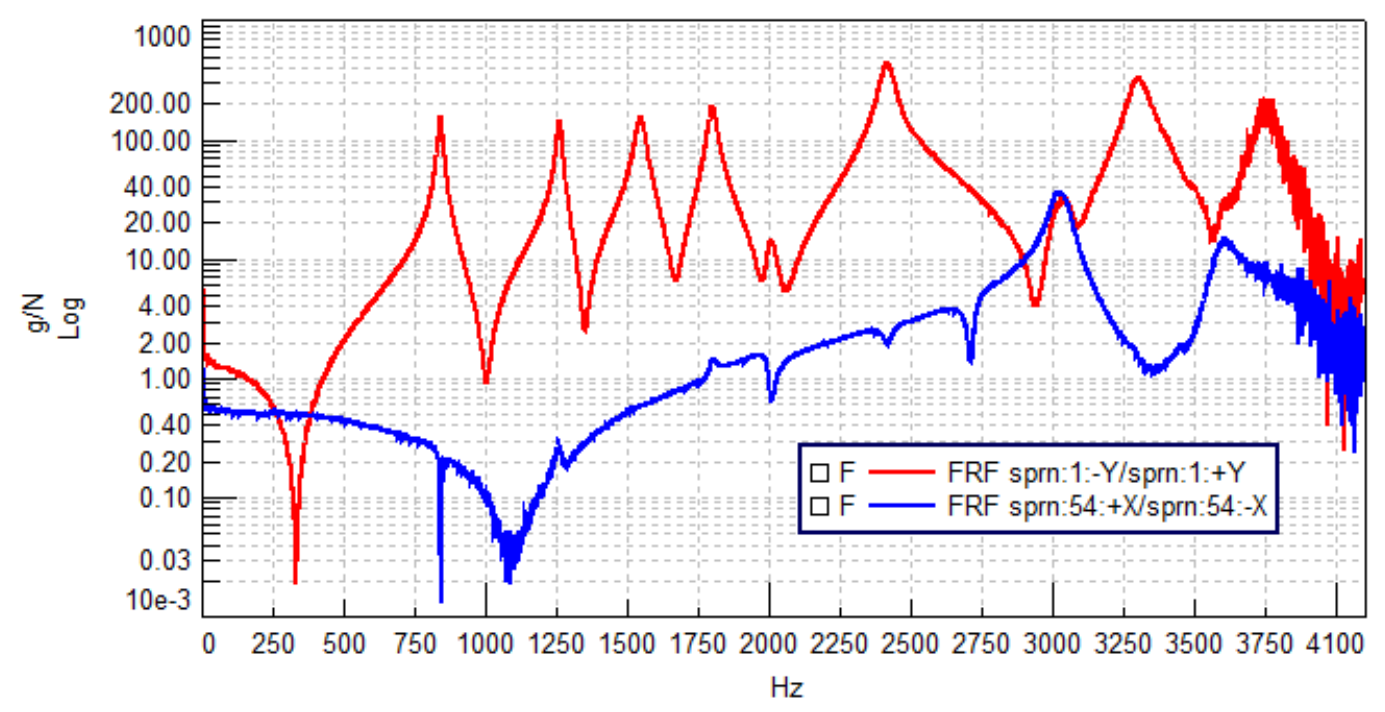

Figure C.3: Driving point FRF measurements for spruce sample 1

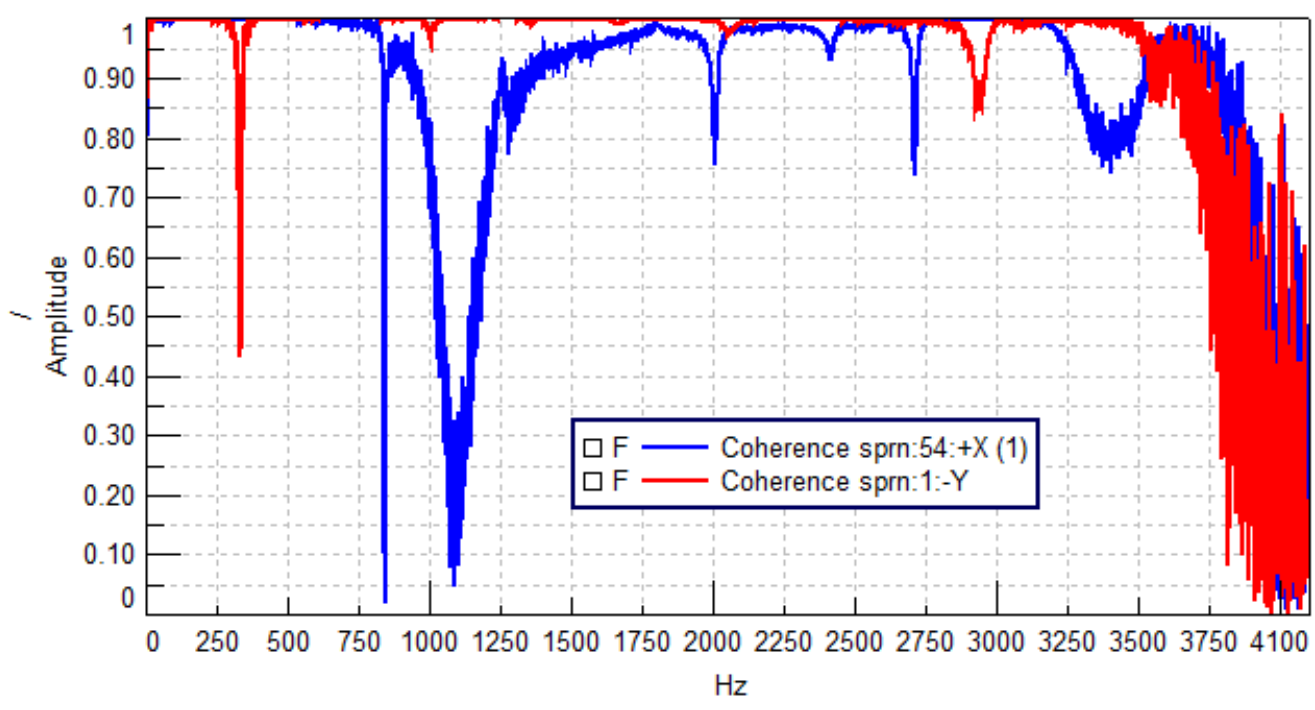

Figure C.4: Driving point coherence measurements for spruce sample 1 


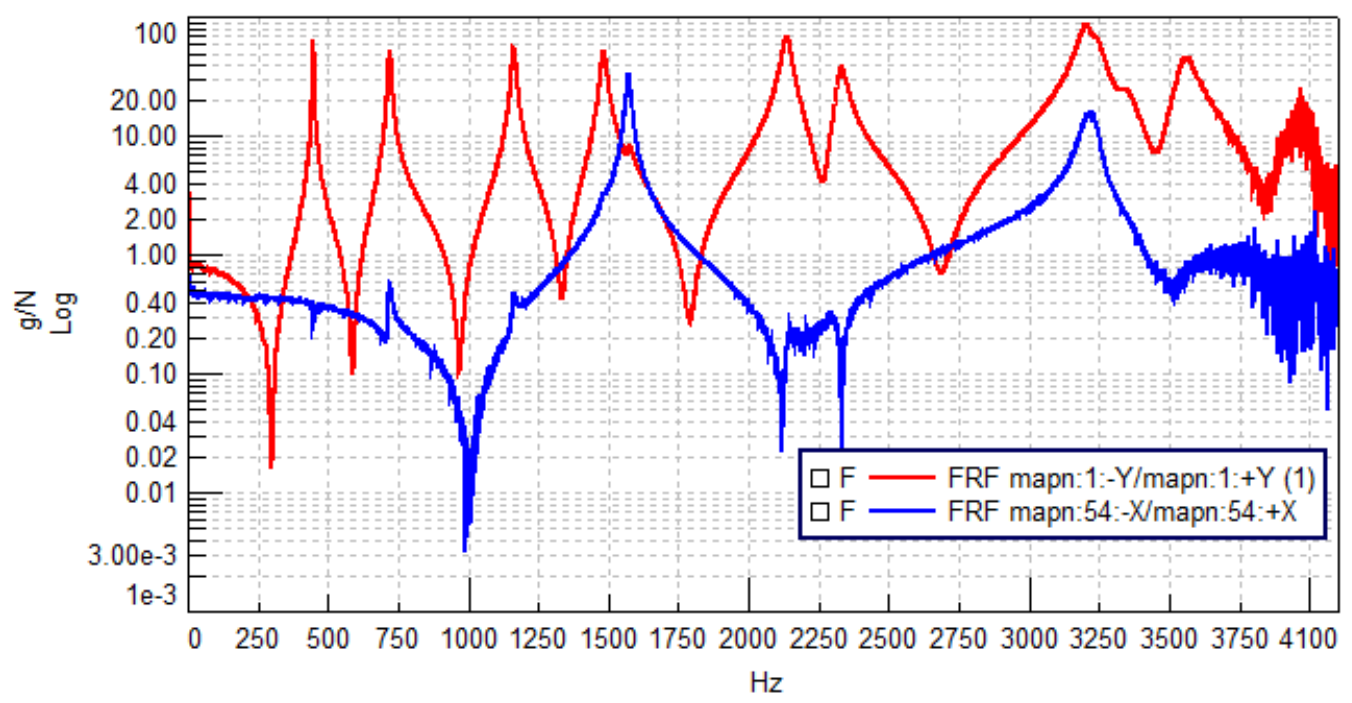

Figure C.5: Driving point FRF measurements for maple sample 1

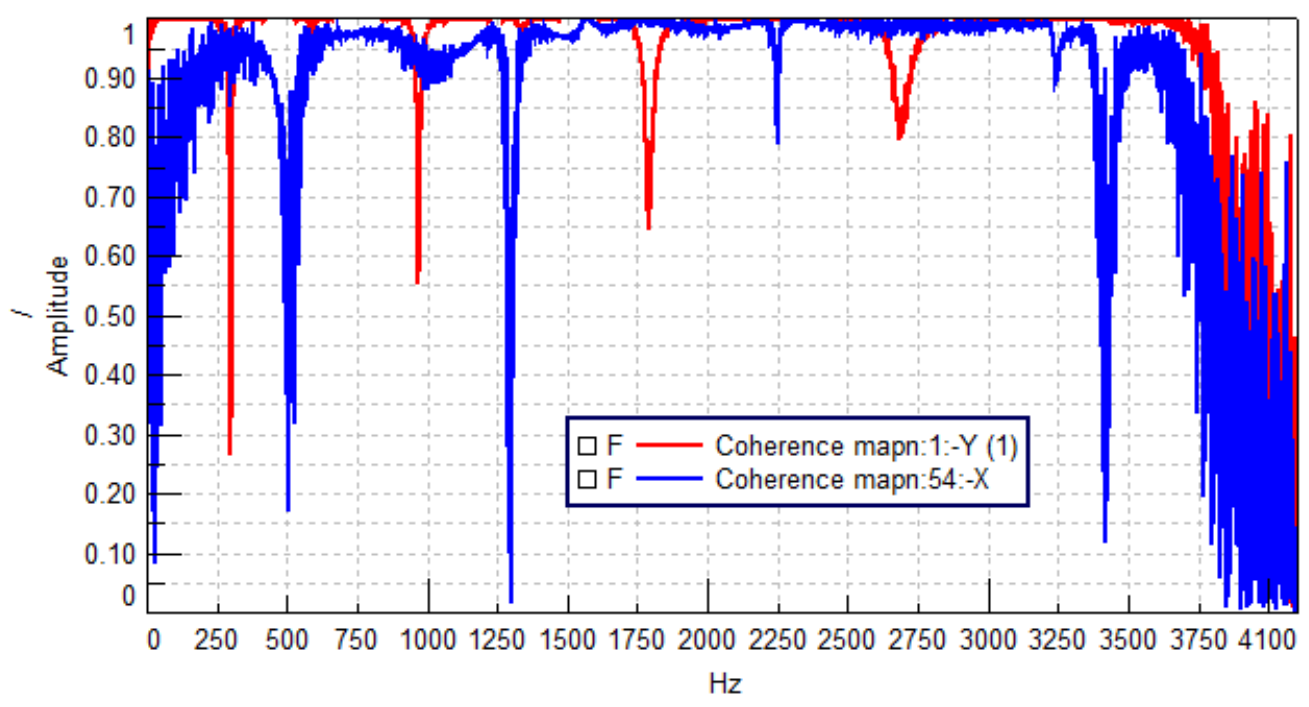

Figure C.6: Driving point coherence measurements for maple sample 1

Curve fitting of the modal data was performed using LMS Test.Lab PolyMAX. The resulting modes for spruce 1 are given in Table C.3, while those of maple 1 are given in Table C.4. 
Table C.3: Curve-fitting results from the Spruce 1 EMA

\begin{tabular}{|c|c|c|l|}
\hline $\begin{array}{c}\text { Mode } \\
\#\end{array}$ & $\begin{array}{c}\text { Frequency } \\
(\mathbf{H z})\end{array}$ & $\begin{array}{c}\text { Damping } \\
\mathbf{( \% )}\end{array}$ & Description \\
\hline 1 & 841.3 & 0.90 & $1^{\text {st }}$ torsion about Z \\
\hline 2 & 1260.9 & 0.70 & First bending YZ \\
\hline 3 & 1547.7 & 1.06 & $1^{\text {st }}$ bending XY \\
\hline 4 & 1799.8 & 0.83 & $2^{\text {nd }}$ torsion about Z \\
\hline 5 & 2003.2 & 1.02 & $2^{\text {nd }}$ torsion about X \\
\hline 6 & 2412.8 & 0.90 & $3^{\text {rd }}$ torsion about Z \\
\hline 7 & 2500.1 & 1.07 & $2^{\text {nd }}$ bending XY \\
\hline 8 & 2717.7 & 0.94 & $3 \times 3$ \\
\hline 9 & 3017.0 & 0.83 & $1^{\text {st }}$ bending XZ \\
\hline 10 & 3027.9 & 0.72 & complex \\
\hline
\end{tabular}

Table C.4: Curve-fitting results from the Maple 1 EMA

\begin{tabular}{|c|c|c|l|}
\hline $\begin{array}{c}\text { Mode } \\
\#\end{array}$ & $\begin{array}{c}\text { Frequency } \\
(\mathbf{H z})\end{array}$ & $\begin{array}{c}\text { Damping } \\
\mathbf{( \% )}\end{array}$ & Description \\
\hline 1 & 447.2 & 0.47 & $1^{\text {st }}$ bending YZ \\
\hline 2 & 719.6 & 0.70 & $1^{\text {st }}$ torsion about Z \\
\hline 3 & 1162.0 & 0.62 & $2^{\text {nd }}$ bending YZ \\
\hline 4 & 1482.3 & 0.69 & $2^{\text {nd }}$ torsion about Z \\
\hline 5 & 1572.3 & 0.54 & $1^{\text {st }}$ bending XZ \\
\hline 6 & 2135.6 & 0.76 & $3^{\text {rd }}$ bending YZ \\
\hline 7 & 2329.1 & 0.74 & $3^{\text {rd }}$ torsion about Z \\
\hline 8 & 3206.6 & 0.81 & $4^{\text {th }}$ torsion about Z \\
\hline 9 & 3233.3 & 0.70 & $2^{\text {nd }}$ bending XZ \\
\hline 10 & 3304.2 & 0.93 & $1^{\text {st }}$ bending XY \\
\hline
\end{tabular}

\section{C.1.2 Finite Element Analysis}

The initial finite element model of the spruce and maple samples were created based on CT scan data of each sample. The MIMICS by Materialise Software was used to convert the CT data into surface mesh envelopes that were imported into LMS Virtual.Lab. Each model was filled in with $2^{\text {nd }}$ order tetrahedral elements. The resulting mesh compositions for the first spruce and maple samples are given in Table C.5. Figure C.7 shows a screen capture of the wood sample FE meshes. 
Table C.5: Wood sample FE mesh compositions

\begin{tabular}{|lcc|}
\hline Sample & Nodes & Elements \\
\hline Spruce 1 & 74803 & 48926 \\
Maple 1 & 60107 & 37421 \\
\hline
\end{tabular}

(a)

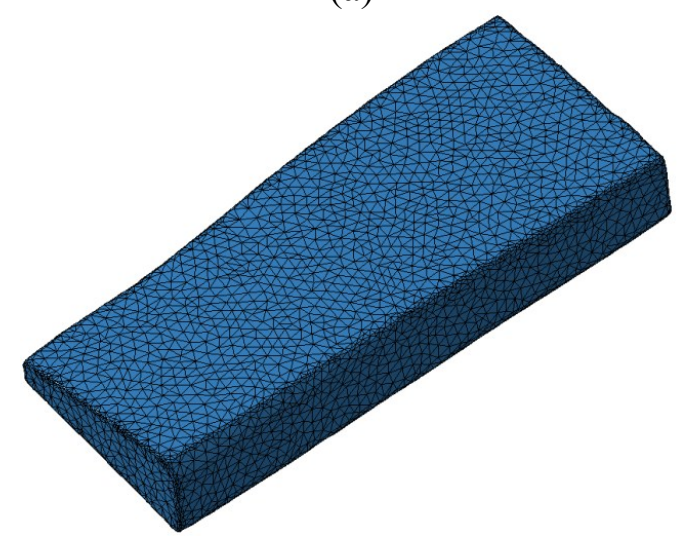

(b)

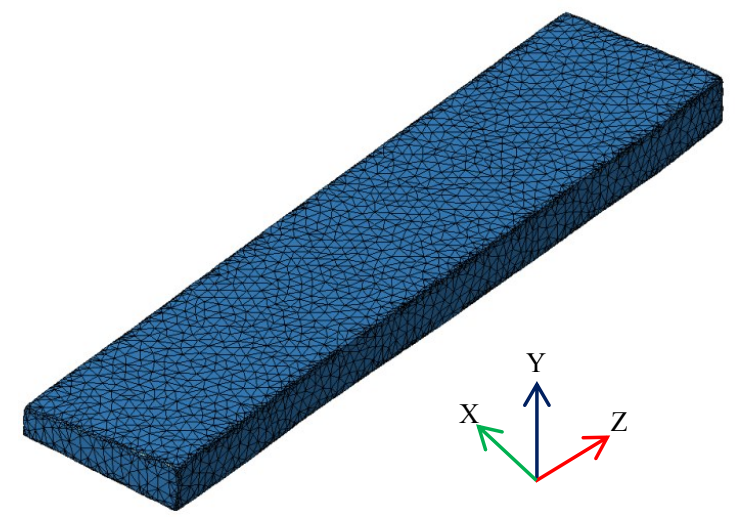

Figure C.7: Wood sample FE meshes, (a) spruce 1, (b) maple 1

The material properties for each model were initialized using book values [C.1]. These elastic ratios were converted to a MAT9 Nastran card using a custom Matlab script (see Appendix D). The actual material property values used for the FE analysis of the wood samples are listed in Table C.6.

The mass of the spruce $1 \mathrm{FE}$ model was found to be 615.5 grams, which is $\sim 1.3 \%$ lower than the measured mass of sample S1. Similarly, the mass of the maple 1 FE model was found to be 736.1 grams, which is $\sim 6.1 \%$ lower than the measured mass of sample M1.

Table C.6: Summary of material properties for wood sample FE models

\begin{tabular}{|c|c|c|c|c|c|c|c|c|c|c|}
\hline Sample & $\begin{array}{c}\text { G11 } \\
(\mathrm{MPa})\end{array}$ & $\begin{array}{c}\text { G12 } \\
(\mathrm{MPa})\end{array}$ & $\begin{array}{c}\text { G13 } \\
(\mathrm{MPa})\end{array}$ & $\begin{array}{c}\text { G22 } \\
(\mathrm{MPa})\end{array}$ & $\begin{array}{c}\text { G23 } \\
(\mathrm{MPa})\end{array}$ & $\begin{array}{c}\text { G33 } \\
(\mathrm{MPa})\end{array}$ & $\begin{array}{c}\text { G44 } \\
(\mathrm{MPa})\end{array}$ & $\begin{array}{c}\text { G55 } \\
(\mathrm{MPa})\end{array}$ & $\begin{array}{c}\text { G66 } \\
(\mathrm{MPa})\end{array}$ & $\begin{array}{c}\mathrm{RHO} \\
\left(\mathrm{kg} / \mathrm{m}^{3}\right)\end{array}$ \\
\hline \hline S1 & 1500 & 390 & 814 & 686 & 481 & 10400 & 196 & 2350 & 2430 & 350 \\
\hline M1 & 1570 & 628 & 964 & 765 & 630 & 8500 & 514 & 982 & 1730 & 570 \\
\hline
\end{tabular}

Results of the FE model normal modes analysis of spruce 1 are summarized in Table C.7. Examples of the first six FE mode shapes of spruce 1 are shown in Figure C.8. 
Table C.7: Summary of spruce 1 FE normal modes analysis

\begin{tabular}{|c|c|l|}
\hline Mode \# & Frequency $(\mathbf{H z})$ & Description \\
\hline 1 & 1457.4 & $1^{\text {st }}$ bending YZ \\
\hline 2 & 1565.5 & $1^{\text {st }}$ Torsion about Z \\
\hline 3 & 2452.5 & $1^{\text {st }}$ bending XY \\
\hline 4 & 2839.4 & $2^{\text {nd }}$ torsion about Z \\
\hline 5 & 3333.4 & $2^{\text {nd }}$ bending YZ \\
\hline 6 & 3559.9 & $2^{\text {nd }}$ torsion about X \\
\hline 7 & 3843.9 & $1^{\text {st }}$ bending XZ \\
\hline 8 & 4644.8 & Complex \\
\hline 9 & 4771.7 & $3^{\text {rd }}$ torsion about X \\
\hline 10 & 4981.1 & $3 \times 3$ \\
\hline
\end{tabular}

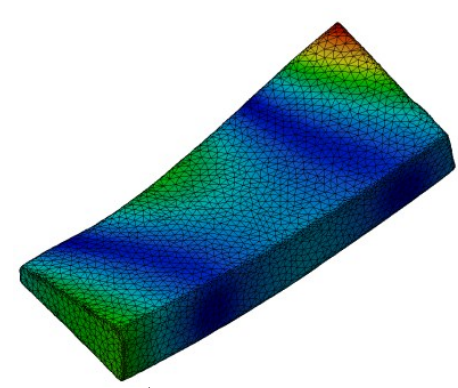

$1^{\text {st }}$ bending YZ

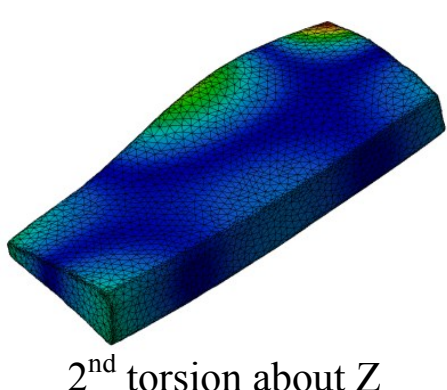

$2^{\text {nd }}$ torsion about $\mathrm{Z}$

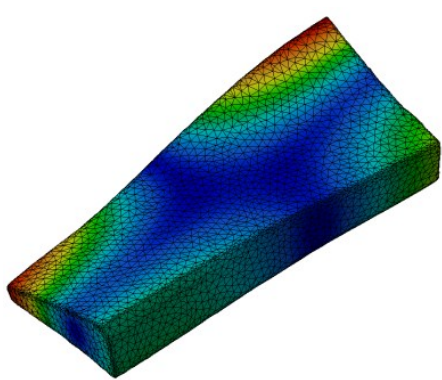

$1^{\text {st }}$ Torsion about $\mathrm{Z}$

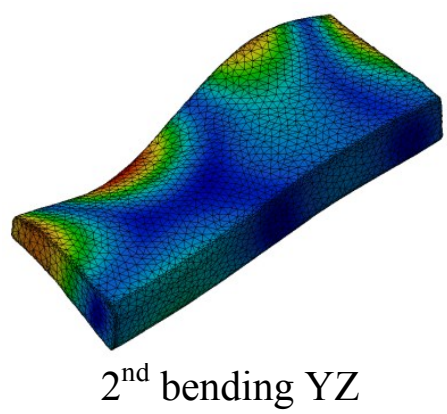

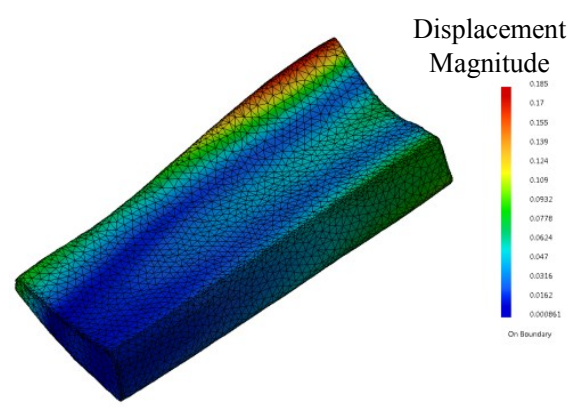

$1^{\text {st }}$ bending XY

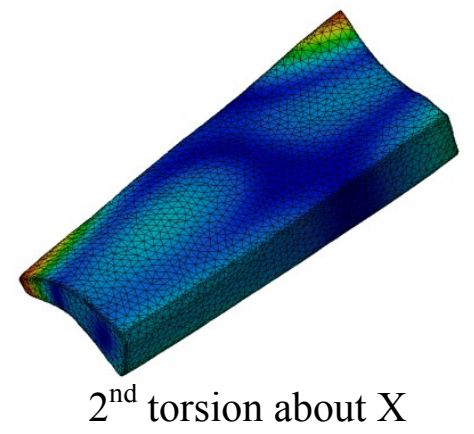

Figure C.8: Mode shapes of the spruce 1 FE model

Results of the FE model normal modes analysis of maple 1 are summarized in Table C.8. Examples of the first six FE mode shapes of maple 1 are shown in Figure C.9. 
Table C.8: Summary of maple 1 FE normal modes analysis

\begin{tabular}{|c|c|l|}
\hline Mode \# & Frequency $(\mathbf{H z})$ & Description \\
\hline 1 & 521.3 & $1^{\text {st }}$ bending YZ \\
\hline 2 & 956.7 & $1^{\text {st }}$ torsion about Z \\
\hline 3 & 1371.3 & $2^{\text {nd }}$ bending YZ \\
\hline 4 & 1842.3 & $1^{\text {st }}$ bending XZ \\
\hline 5 & 1923.6 & $2^{\text {nd }}$ torsion about Z \\
\hline 6 & 2526.0 & $3^{\text {rd }}$ bending YZ \\
\hline 7 & 2968.3 & $3^{\text {rd }}$ torsion about Z \\
\hline 8 & 3323.3 & $1^{\text {st }}$ bending XY \\
\hline 9 & 3629.2 & $2^{\text {nd }}$ torsion about X \\
\hline 10 & 3923.8 & $4^{\text {th }}$ bending YZ \\
\hline
\end{tabular}

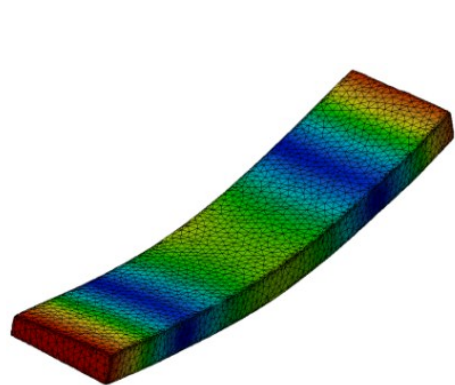

$1^{\text {st }}$ bending $\mathrm{YZ}$

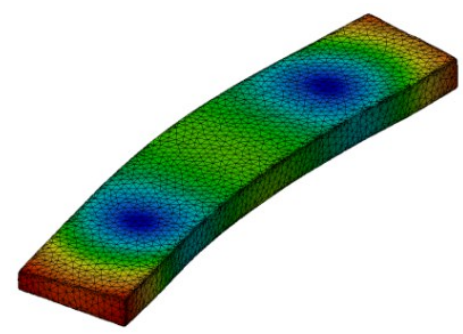

$1^{\text {st }}$ bending XZ
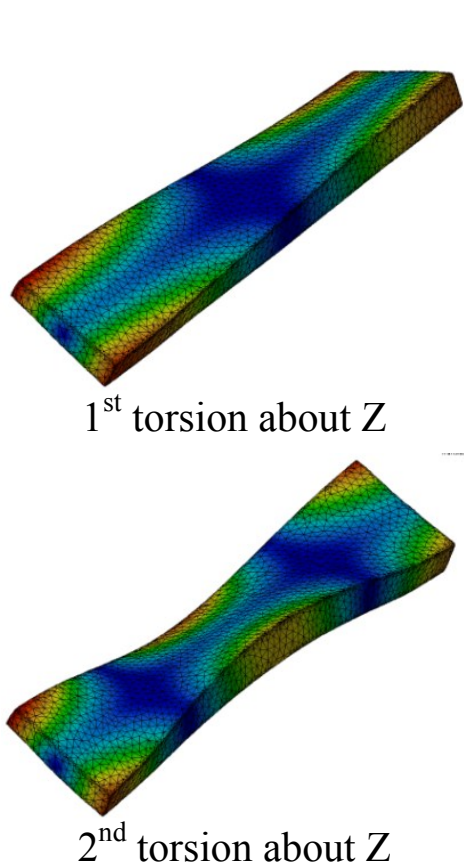
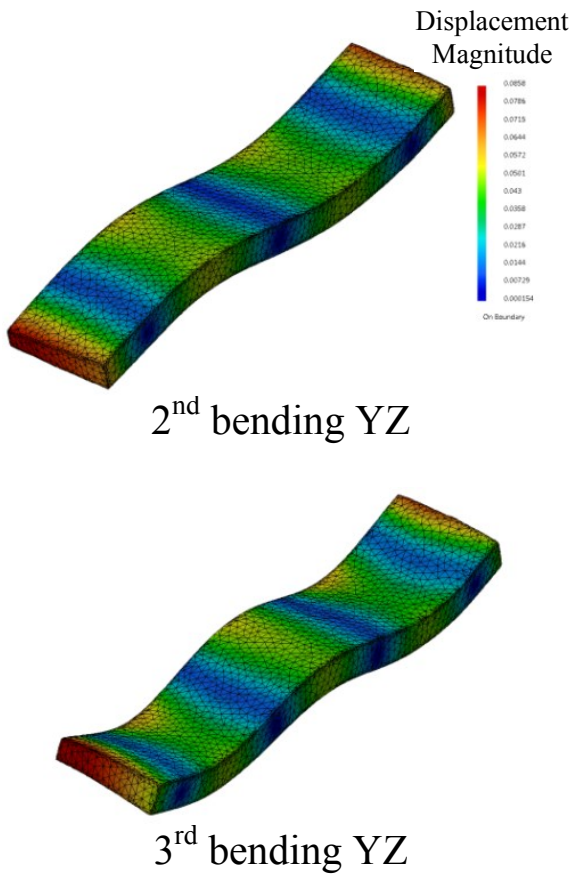

Figure C.9: Mode shapes of the maple $1 \mathrm{FE}$ model

\section{C.1.3 Correlation Results}

The results of the wood sample FE models were correlated with those from the test data using the Modal Assurance Criterion (MAC). A plot of the MAC matrix for the spruce 1 results is shown in Figure C.10. The vertical axis lists the FE modes, while the horizontal axis shows those of the test. The color scale indicates the value of the MAC between each mode pair $($ red $=1$, blue $=0)$. The subsequent mode pairs are given in Table C.9. 


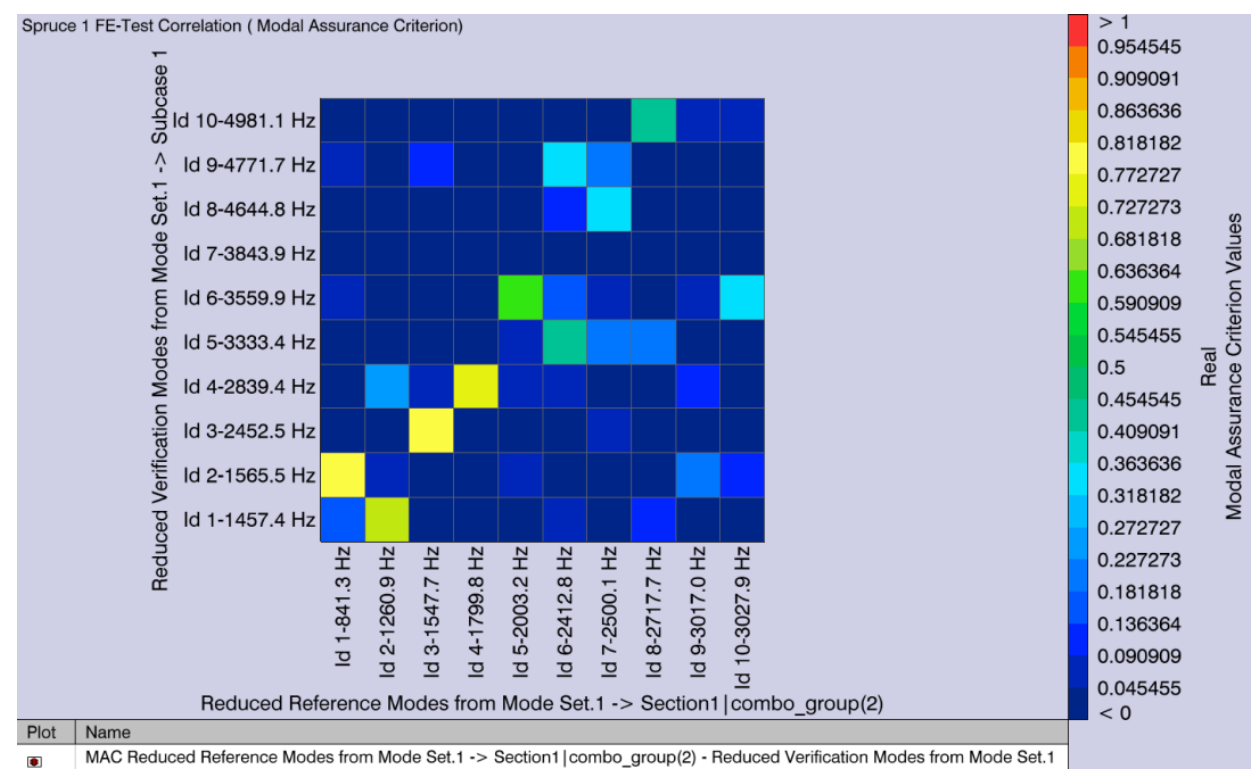

Figure C.10: MAC matrix for the Spruce 1 FE-Test correlation

Table C.9: Mode pairs of the spruce 1 FE-Test correlation

\begin{tabular}{|c|c|c|c|c|c|c|}
\hline $\begin{array}{c}\text { Test } \\
\text { Id1 }\end{array}$ & $\begin{array}{c}\text { Test Freq1 } \\
(\mathrm{Hz})\end{array}$ & $\begin{array}{c}\text { FE } \\
\mathrm{Id} 2\end{array}$ & $\begin{array}{c}\text { FE Freq2 } \\
(\mathrm{Hz})\end{array}$ & $\begin{array}{c}\text { MAC } \\
\text { Value }\end{array}$ & $\begin{array}{c}\text { Freq2-Freq1 } \\
(\mathrm{Hz})\end{array}$ & $\begin{array}{c}\text { Freq2-Freq1 } \\
(\% \text { of Freq1 })\end{array}$ \\
\hline 1 & 841.3 & 2 & 1565.5 & 0.812 & 724.18 & 86.1 \\
\hline 2 & 1260.9 & 1 & 1457.4 & 0.703 & 196.47 & 15.6 \\
\hline 3 & 1547.7 & 3 & 2452.5 & 0.804 & 904.73 & 58.5 \\
\hline 4 & 1799.8 & 4 & 2839.4 & 0.754 & 1039.67 & 57.8 \\
\hline 5 & 2003.2 & 6 & 3559.9 & 0.611 & 1556.72 & 77.7 \\
\hline 6 & 2412.8 & 5 & 3333.4 & 0.432 & 920.55 & 38.2 \\
\hline 6 & 2412.8 & 9 & 4771.7 & 0.323 & 2358.87 & 97.8 \\
\hline 7 & 2500.1 & 8 & 4644.8 & 0.362 & 2144.72 & 85.8 \\
\hline 8 & 2717.7 & 10 & 4981.1 & 0.449 & 2263.41 & 83.3 \\
\hline 10 & 3027.9 & 6 & 3559.9 & 0.356 & 531.97 & 17.6 \\
\hline
\end{tabular}

Overall the spruce $1 \mathrm{FE}$ model does not correlate well with the test data. Only the first six modes have a MAC value greater than 0.6. There is also mode switching occurring for the first two modes, as well as between the fifth and sixth modes. The average percent frequency difference for the first six modes was $\sim 55 \%$. The mode shape prediction appears to break down for the higher order modes. Reasons for the higher order modes to not correlate as well may include potential non-homogeneity in the wood sample that needs to be accounted for in this sample. It may also be possible to improve the correlation by upgrading the solid tetrahedral elements to hexahedral. This would 
require remeshing the FE model, and creating mesh maps on all surfaces. Finally, updating is needed on the material properties to bring the FE mode frequencies into alignment with those of the test.

A plot of the MAC matrix for the maple 1 results is shown in Figure C.11. Again, the vertical axis lists the FE modes, while the horizontal axis shows those of the test. The color scale indicates the value of the MAC between each mode pair (red $=1$, blue $=0$ ). The subsequent mode pairs are given in Table C.10

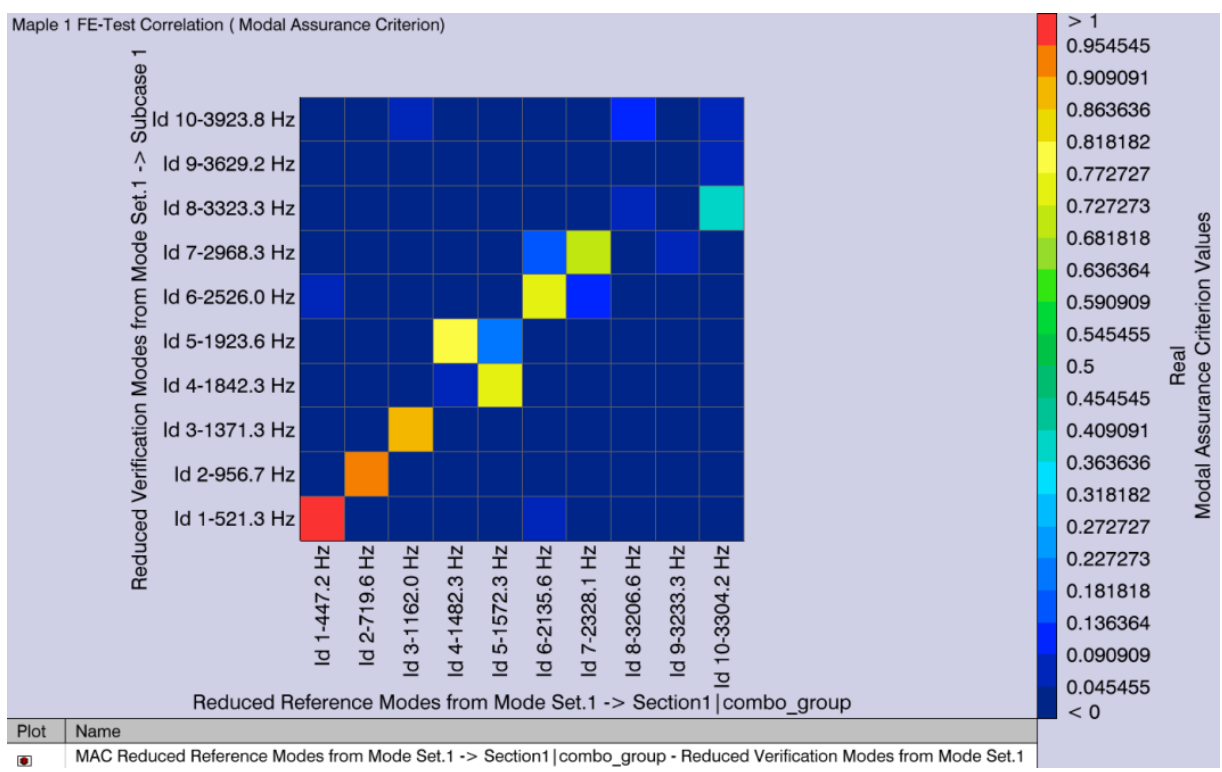

Figure C.11: MAC matrix for the maple 1 FE-Test correlation

Table C.10: Mode pairs of the maple 1 FE-Test correlation

\begin{tabular}{|c|c|c|c|c|c|c|}
\hline $\begin{array}{c}\text { Test } \\
\text { Id1 }\end{array}$ & $\begin{array}{c}\text { Test Freq1 } \\
(\mathrm{Hz})\end{array}$ & $\begin{array}{c}\text { FE } \\
\mathrm{Id} 2\end{array}$ & $\begin{array}{c}\text { FE Freq2 } \\
(\mathrm{Hz})\end{array}$ & $\begin{array}{c}\text { MAC } \\
\text { Value }\end{array}$ & $\begin{array}{c}\text { Freq2-Freq1 } \\
(\mathrm{Hz})\end{array}$ & $\begin{array}{c}\text { Freq2-Freq1 } \\
(\% \text { of Freq1 })\end{array}$ \\
\hline 1 & 447.2 & 1 & 521.3 & 0.966 & 74.13 & 16.6 \\
\hline 2 & 719.6 & 2 & 956.7 & 0.951 & 237.14 & 33 \\
\hline 3 & 1162 & 3 & 1371.3 & 0.908 & 209.36 & 18 \\
\hline 4 & 1482.3 & 5 & 1923.6 & 0.789 & 441.27 & 29.8 \\
\hline 5 & 1572.3 & 4 & 1842.3 & 0.767 & 270.02 & 17.2 \\
\hline 6 & 2135.6 & 6 & 2526 & 0.733 & 390.42 & 18.3 \\
\hline 7 & 2328.1 & 7 & 2968.3 & 0.715 & 640.24 & 27.5 \\
\hline 10 & 3304.2 & 8 & 3323.3 & 0.364 & 19.08 & 0.6 \\
\hline
\end{tabular}

The maple 1 FE results do correlate well with the test data for the first seven modes. The MAC value for all of these modes was greater than 0.7 . There does appear to be mode 
switching occurring between the fourth and fifth modes. The average percent frequency difference for the first 7 modes was $\sim 22 \%$. Again the correlation results for higher order modes are less favorable. There may be some non-homogeneity in the sample. This model would also benefit from upgrading to hexahedral elements. An adjustment to the density value would help the mass of the maple $1 \mathrm{FE}$ model match the measured mass of the sample. Finally, updating is needed in the elastic moduli to improve the frequency prediction.

\section{C.2 Cantilever Beam Study}

\section{C.2.1 Methods}

An additional set of cantilever beam tests were performed to determine the significance of glue properties. For this test a sheet of $1 / 4 "(6.35 \mathrm{~mm})$ plywood with an oak veneer was cut into three equal beams 23 " $(584.2 \mathrm{~mm})$ in length and 2.0 " $(50.8 \mathrm{~mm})$ in width. These are shown in Figure C.12.

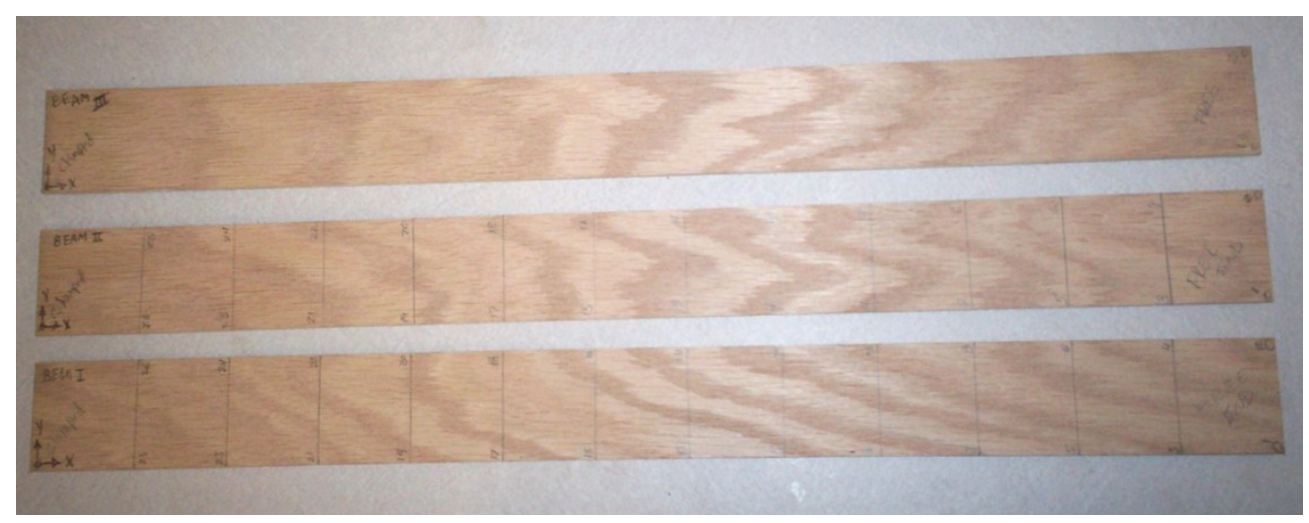

Figure C.12: Three beams cut from plywood sheet

One end of each beam was clamped to the lab bench to form a cantilever. Driving point measurements were made on the free end of all three beams, as shown in Figure C.13. A complete roving impact modal test was performed on two of the beams. This was done to confirm that the beams were dynamically similar. Two reference accelerometers were attached to the end corners of the beam using wax; one mounted vertically (beam:1:+Z), and one mounted laterally (beam:2:+Y). The hammer was impacted over 26 locations along both edges of the beam in both the vertical and lateral directions. FRFs between the force inputs and accelerometer responses were measured and used for calculating the experimental modes. The Digital Signal Processing (DSP) parameters for the test are summarized in Table C.11. 
Table C.11: DSP parameters for the plywood beams

\begin{tabular}{|l|l|}
\hline Averaging & $5 \mathrm{Averages}$, Linear \\
\hline Bandwidth & $2048 \mathrm{~Hz}$ \\
\hline Frequency Resolution & $1.0 \mathrm{~Hz}$ \\
\hline Reference Window & Force-Exponential, 100\% \\
\hline Response Window & Exponential, 100\% \\
\hline
\end{tabular}

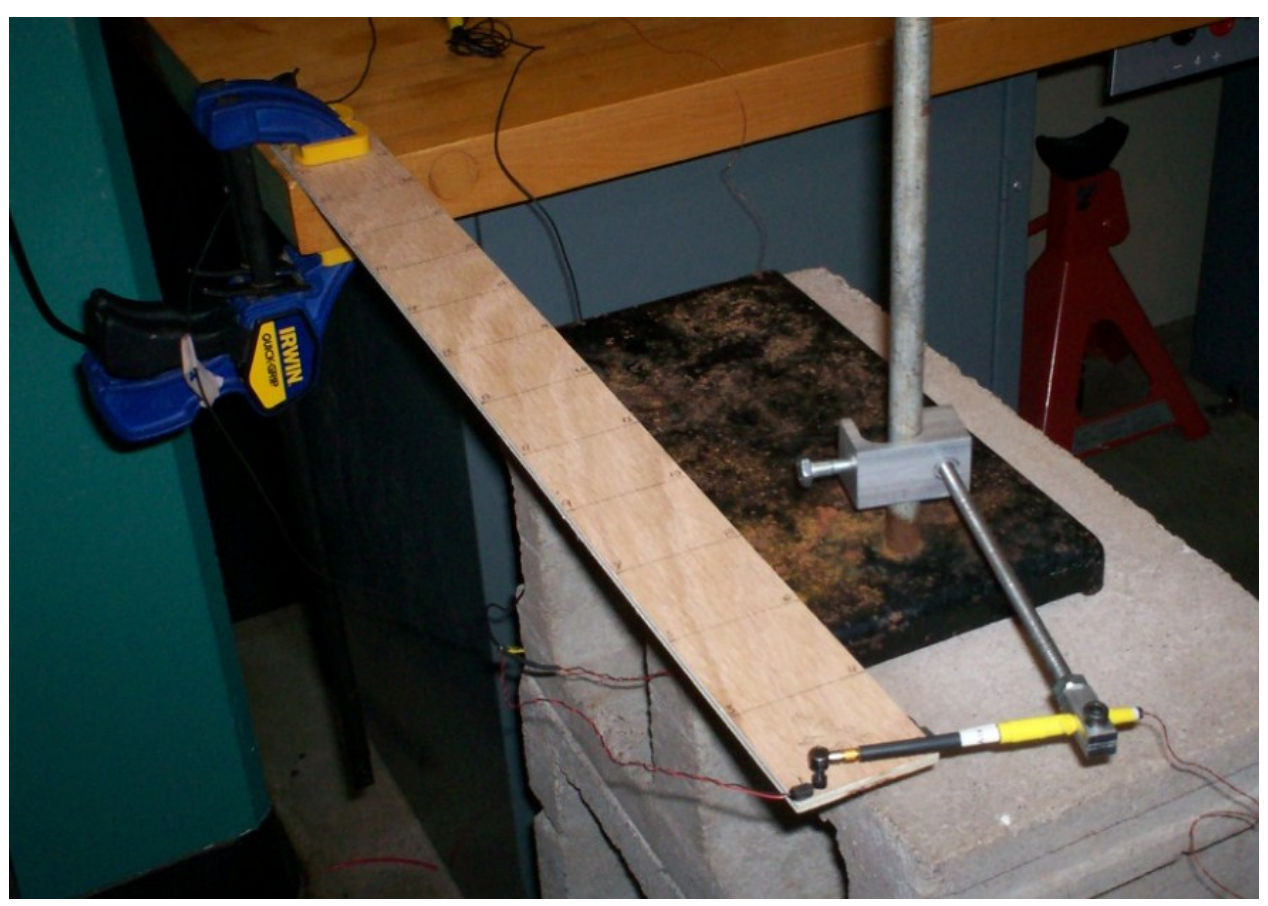

Figure C.13: Test setup of cantilever beam

Two beams were then selected and glued together. Liquid hide glue was brushed onto the surface of one beam and the second was placed directly on top. This was to maximize the area of the glue (rather than a simple lap joint) and hopefully increase the amount of influence the glue has on the final modes. A series of spring clamps were used to hold the beams together while the glue set. This process is shown in Figure C.14 and Figure C.15. 


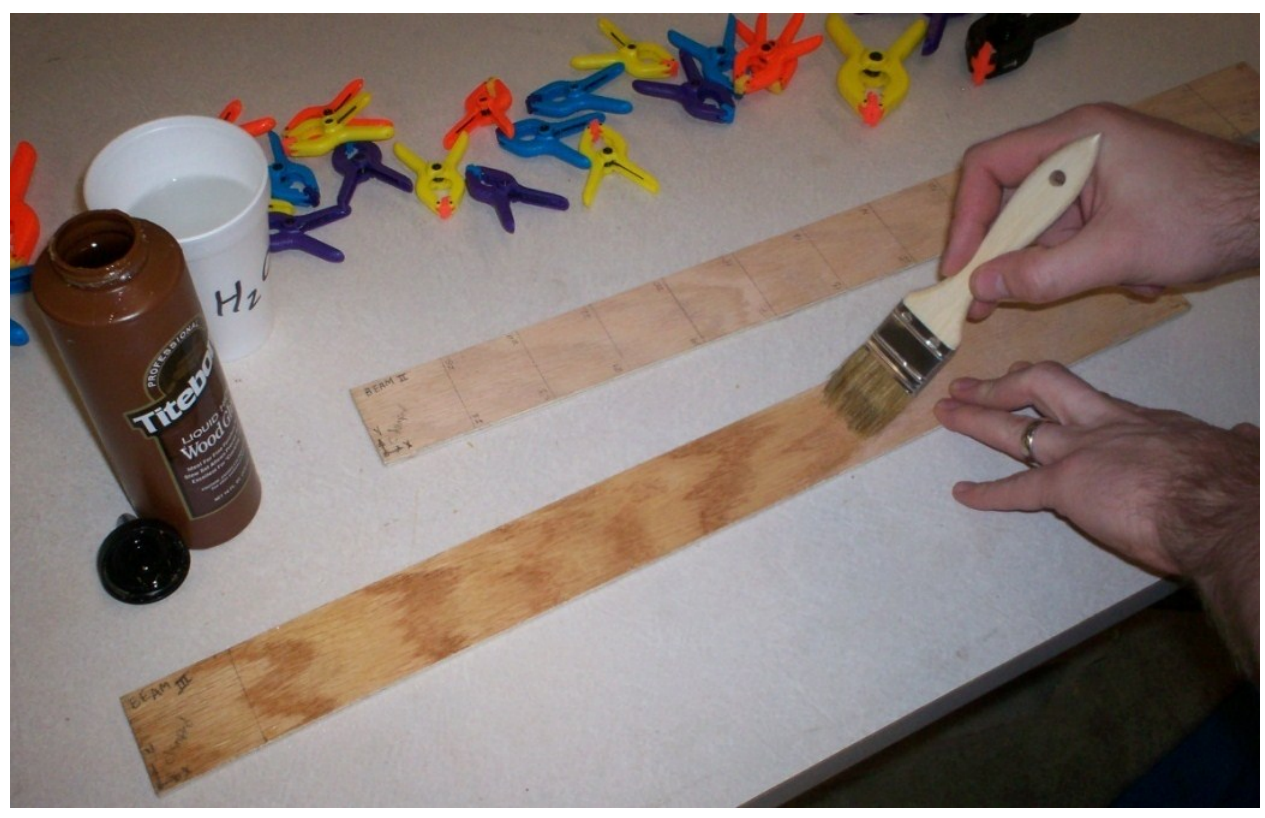

Figure C.14: Applying liquid hide glue to the surface of one beam

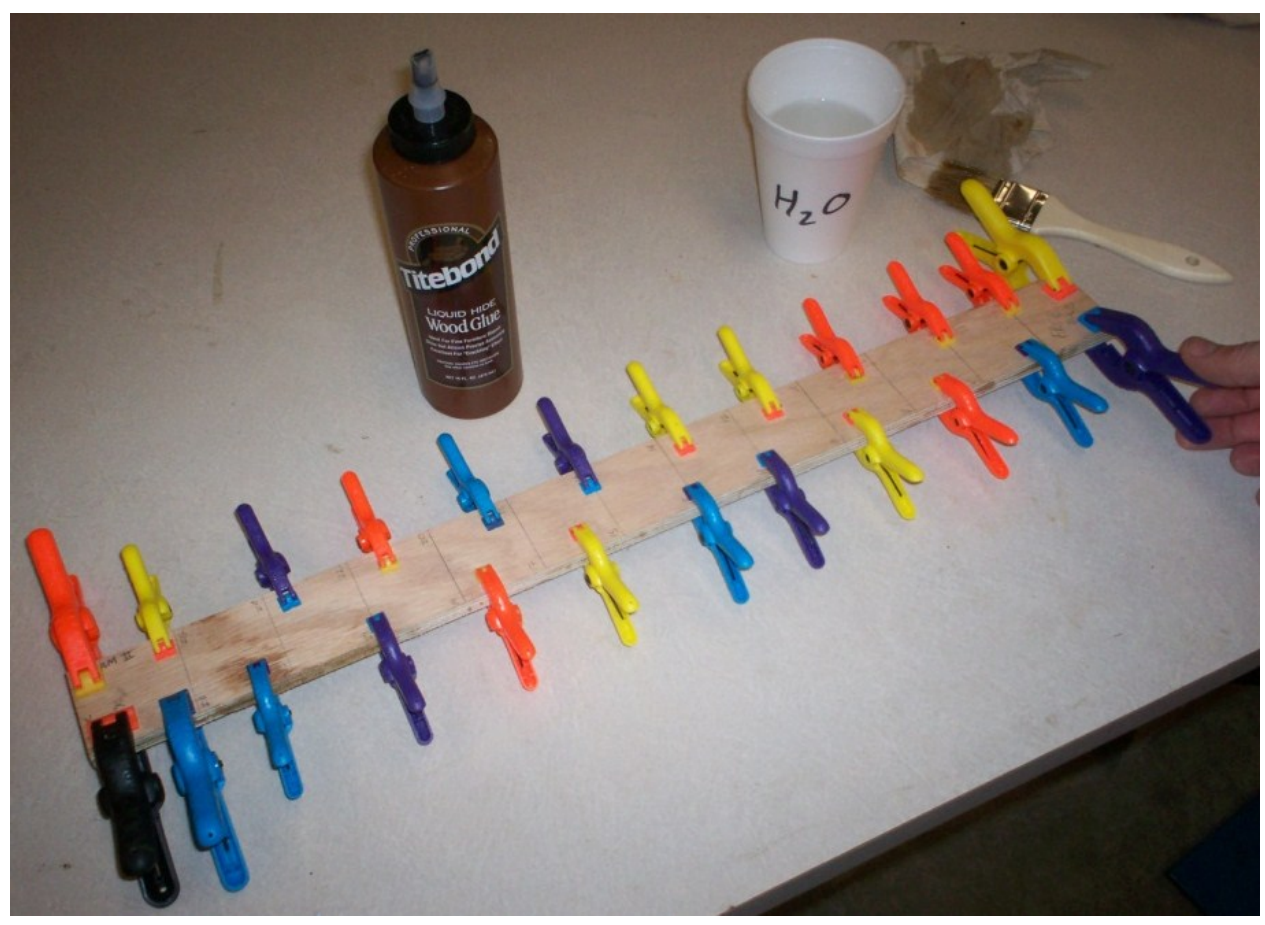

Figure C.15: Spring clamps on laminated beam

After the glue dried, the clamps were removed and the laminated beam was clamped to the lab bench as before, and another modal test was completed. It is noted that most violin makers use granular hide glue. The purpose of this testing was to determine if the 
glue properties had a significant effect on the modes of the glued beam, and if so the properties of glue would be determined from the updating procedure. Those would then be used as initial values for the two violin models and would be left as variable parameters to be updated again within each respective set of modal data. This would in theory show any significant differences between the liquid hide glue, the glue used by the violin factory, and the glue in the Titian Stardivari (not necessarily the same as what Stradivari himself originally used since the instrument has undergone repairs over the years).

\section{C.2.2 Single Beam Results}

The driving point FRFs for the single beam test are shown in Figure C.16. The red curve indicates the driving point for the vertical direction, while the blue indicates that of the lateral direction. Many more modes are observed in the vertical direction than in the lateral direction within the measured bandwidth.

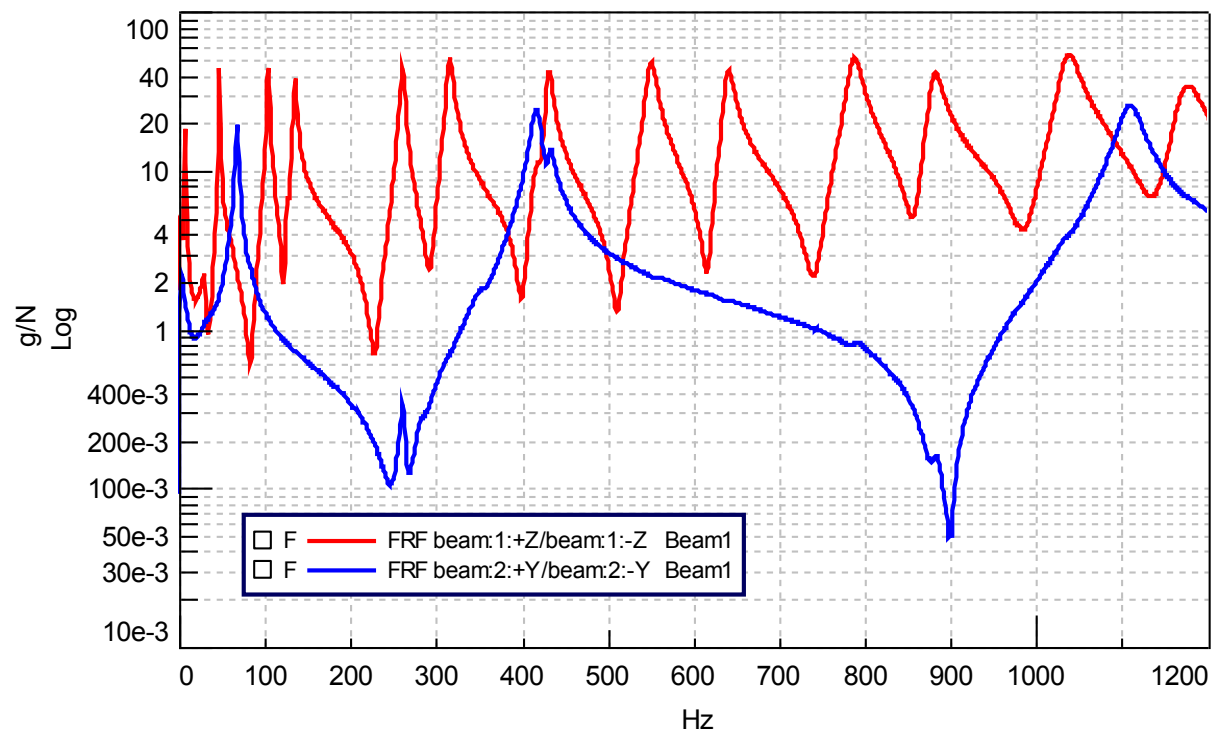

Figure C.16: Driving point FRFs for single beam

The experimental modal data for the single beam were curve fit using LMS Test.Lab PolyMAX. The resulting modes are given in Table 3. 
Table C.12: Curve-fitting results from the single beam EMA

\begin{tabular}{|c|c|c|l|}
\hline Mode \# & Frequency (Hz) & Damping (\%) & Description \\
\hline 1 & 7.402 & 2.66 & $1^{\text {st }}$ vertical bending \\
\hline 2 & 46.80 & 1.01 & $2^{\text {nd }}$ vertical bending \\
\hline 3 & 67.79 & 2.58 & $1^{\text {st }}$ lateral bending \\
\hline 4 & 104.4 & 1.30 & $1^{\text {st }}$ torsion \\
\hline 5 & 135.3 & 1.26 & $3^{\text {rd }}$ vertical bending \\
\hline 6 & 261.3 & 0.82 & $4^{\text {th }}$ vertical bending \\
\hline 7 & 316.8 & 1.15 & $2^{\text {nd }}$ torsion \\
\hline 8 & 418.8 & 1.29 & $2^{\text {nd }}$ lateral bending \\
\hline 9 & 433.0 & 0.68 & $5^{\text {th }}$ vertical bending \\
\hline 10 & 553.0 & 1.11 & $3^{\text {rd }}$ torsion \\
\hline
\end{tabular}

The finite element model of the single plywood beam was created based on the measured dimensions. The mesh contained 3765 nodes and $11702^{\text {nd }}$ order quadrangular elements. Figure C. 17 shows the FE mesh for the cantilever plywood beam. The tan elements are rigidly constrained (indicated by the red ground symbol).

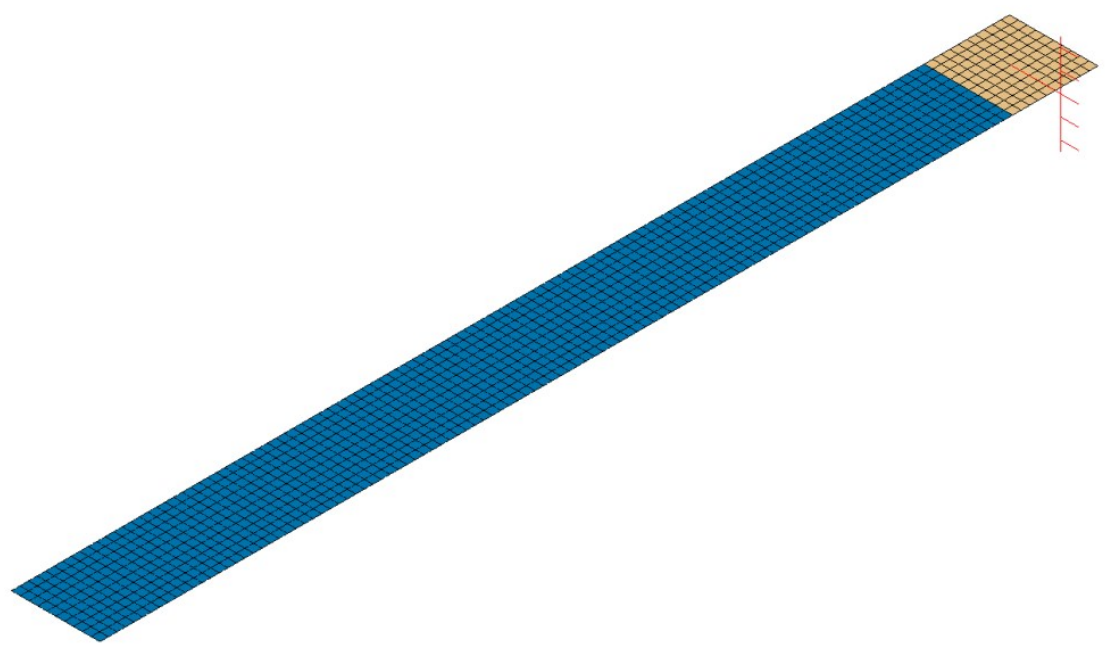

Figure C.17: FE mesh of the cantilever plywood beam

The orthotropic material properties used for the plywood beam FE model are listed in Table C.13. E1 is the Young's Modulus in the longitudinal direction, while E2 is in the lateral direction. NU12 is the Poisson's ratio in the plane of the beam. G12 is the shear modulus in the plane of the beam, while $\mathrm{G} 1 \mathrm{Z}$ and $\mathrm{G} 2 \mathrm{Z}$ are in the two vertical planes, longitudinal and lateral respectively. RHO indicates the density. 
Table C.13: Orthotropic materials for the plywood beam.

\begin{tabular}{|c|c|c|c|c|c|c|}
\hline $\begin{array}{c}\mathrm{E} 1 \\
(\mathrm{MPa})\end{array}$ & $\begin{array}{c}\mathrm{E} 2 \\
(\mathrm{MPa})\end{array}$ & NU12 & $\begin{array}{c}\text { G12 } \\
(\mathrm{MPa})\end{array}$ & $\begin{array}{c}\mathrm{G} 1 \mathrm{M} \\
(\mathrm{MPa})\end{array}$ & $\begin{array}{c}\mathrm{G} 2 \mathrm{C} \\
(\mathrm{MPa})\end{array}$ & $\begin{array}{c}\text { RHO } \\
\left(\mathrm{kg} / \mathrm{m}^{3}\right)\end{array}$ \\
\hline 3760 & 949 & 0.31 & 768 & 390 & 166 & 540 \\
\hline
\end{tabular}

Results of the initial FE model normal modes analysis are summarized in Table C.14. Examples of the first six FE mode shapes are shown in Figure C.18.

Table C.14: Summary of single plywood beam normal modes analysis

\begin{tabular}{|l|l|l|}
\hline Mode \# & Frequency (Hz) & Description \\
\hline 1 & 15.3 & $1^{\text {st }}$ vertical bending \\
\hline 2 & 76.4 & $1^{\text {st }}$ ateral bending \\
\hline 3 & 95.1 & $2^{\text {nd }}$ vertical bending \\
\hline 4 & 204.5 & $1^{\text {st }}$ torsion \\
\hline 5 & 264.3 & $3^{\text {rd }}$ vertical bending \\
\hline 6 & 447.9 & $2^{\text {nd }}$ lateral bending \\
\hline 7 & 512.4 & $4^{\text {th }}$ vertical bending \\
\hline 8 & 617.0 & $2^{\text {nd }}$ torsion \\
\hline 9 & 835.4 & $5^{\text {th }}$ vertical bending \\
\hline 10 & 1039.6 & $3^{\text {rd }}$ torsion \\
\hline
\end{tabular}




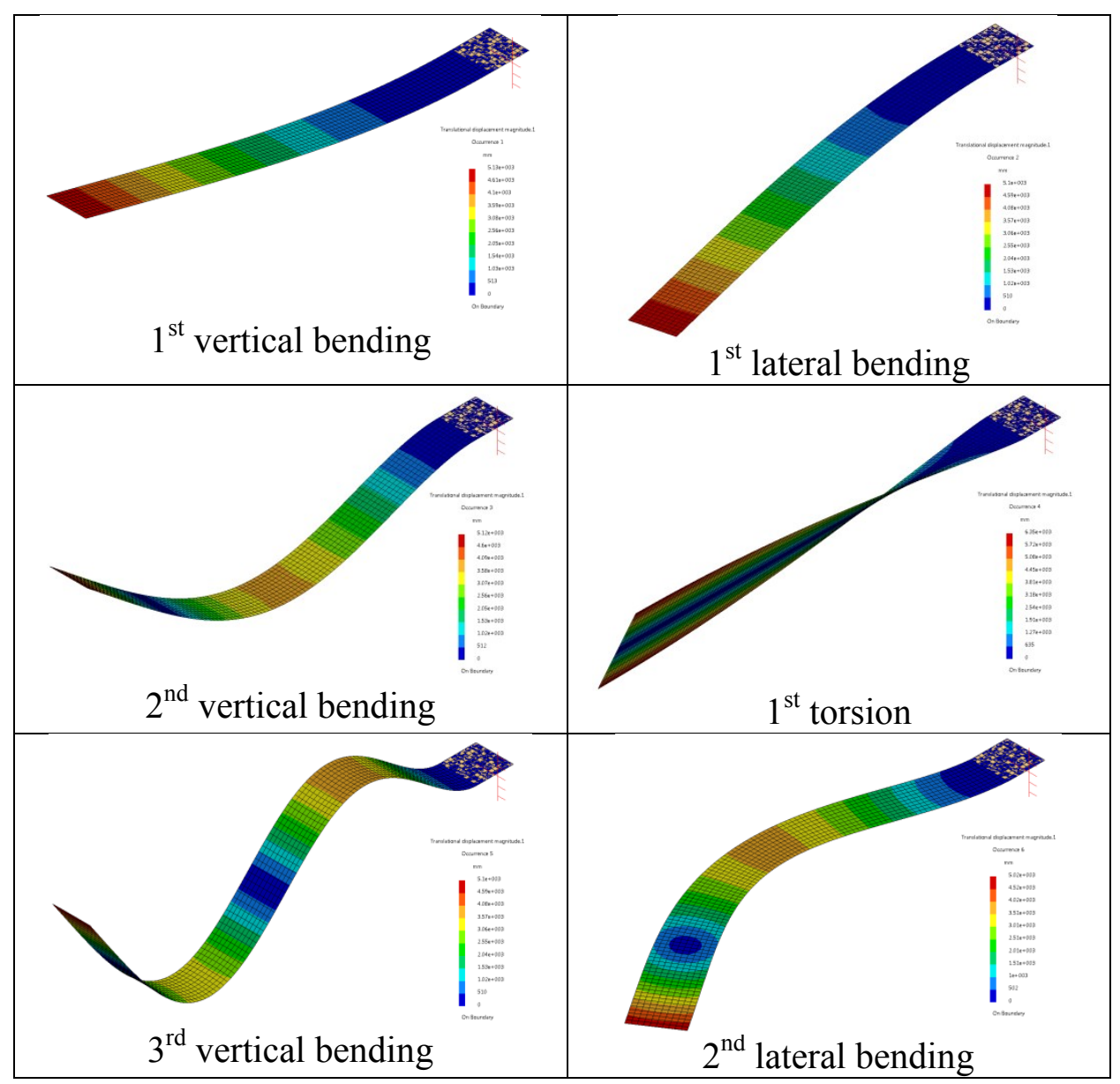

Figure C.18: Mode shapes of the single plywood beam

The results of the single plywood beam FE model were correlated with those from test data using the Modal Assurance Criterion (MAC). Optimization was used to update the material properties to improve the correlation. A plot of the final MAC matrix is shown in Figure C.19. The subsequent mode pairs are given in Figure C.19. 


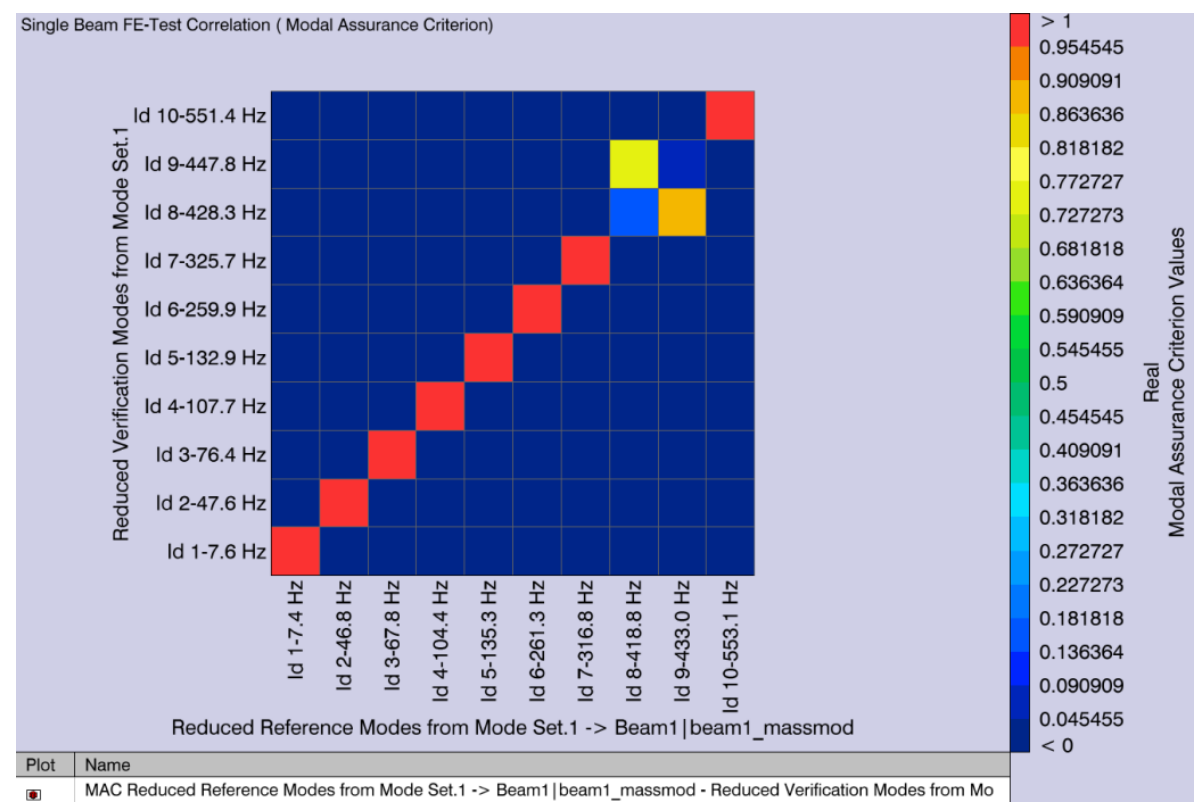

Figure C.19: MAC results for single beam after updating

Table C.15: Mode pairs of the single beam FE-test correlation after updating

\begin{tabular}{|c|c|c|c|c|c|c|}
\hline $\begin{array}{c}\text { Test } \\
\text { Id1 }\end{array}$ & $\begin{array}{c}\text { Test Freq1 } \\
(\mathrm{Hz})\end{array}$ & $\begin{array}{c}\text { FE } \\
\mathrm{Id} 2\end{array}$ & $\begin{array}{c}\text { FE Freq2 } \\
(\mathrm{Hz})\end{array}$ & $\begin{array}{c}\text { MAC } \\
\text { Value }\end{array}$ & $\begin{array}{c}\text { Freq2-Freq1 } \\
(\mathrm{Hz})\end{array}$ & $\begin{array}{c}\text { Freq2-Freq1 } \\
(\% \text { of Freq1 })\end{array}$ \\
\hline 1 & 7.4 & 1 & 7.6 & 0.977 & 0.2 & 2.7 \\
\hline 2 & 46.8 & 2 & 47.6 & 0.981 & 0.76 & 1.6 \\
\hline 3 & 67.8 & 3 & 76.4 & 0.974 & 8.59 & 12.7 \\
\hline 4 & 104.4 & 4 & 107.7 & 0.985 & 3.27 & 3.1 \\
\hline 5 & 135.3 & 5 & 132.9 & 0.982 & -2.38 & -1.8 \\
\hline 6 & 261.3 & 6 & 259.9 & 0.968 & -1.43 & -0.5 \\
\hline 7 & 316.8 & 7 & 325.7 & 0.977 & 8.88 & 2.8 \\
\hline 8 & 418.8 & 9 & 447.8 & 0.755 & 28.96 & 6.9 \\
\hline 9 & 433 & 8 & 428.3 & 0.879 & -4.72 & -1.1 \\
\hline 10 & 553.1 & 10 & 551.4 & 0.956 & -1.64 & -0.3 \\
\hline
\end{tabular}

The FE model of the single plywood cantilever beam correlates well with the test data after updating. The MAC values of all mode pairs are above 0.7 . In fact the first 7 mode pairs have MAC values above 0.9 . The average absolute percent frequency difference is $\sim 3.4 \%$. Modes 3 and 8 have largest percent frequency difference, both exceeding $5 \%$. These are both in-plane bending modes, which may be off due to boundary condition of the physical structure not being fully clamped in horizontal direction. Modes 8 and 9 are also switched, which is also due to boundary condition. The experimental data also show 
that there is a small amount of coupling between modes 8 and 9, which was not observed in the FE model. This reduced the MAC value for these two mode pairs.

\section{C.2.3 Glued Beam Results}

The driving point FRFs for the glued beam test are shown in Figure C.20. It is observed that all modes have shifted in frequency. The peaks also show increased damping due to the layer of glue.

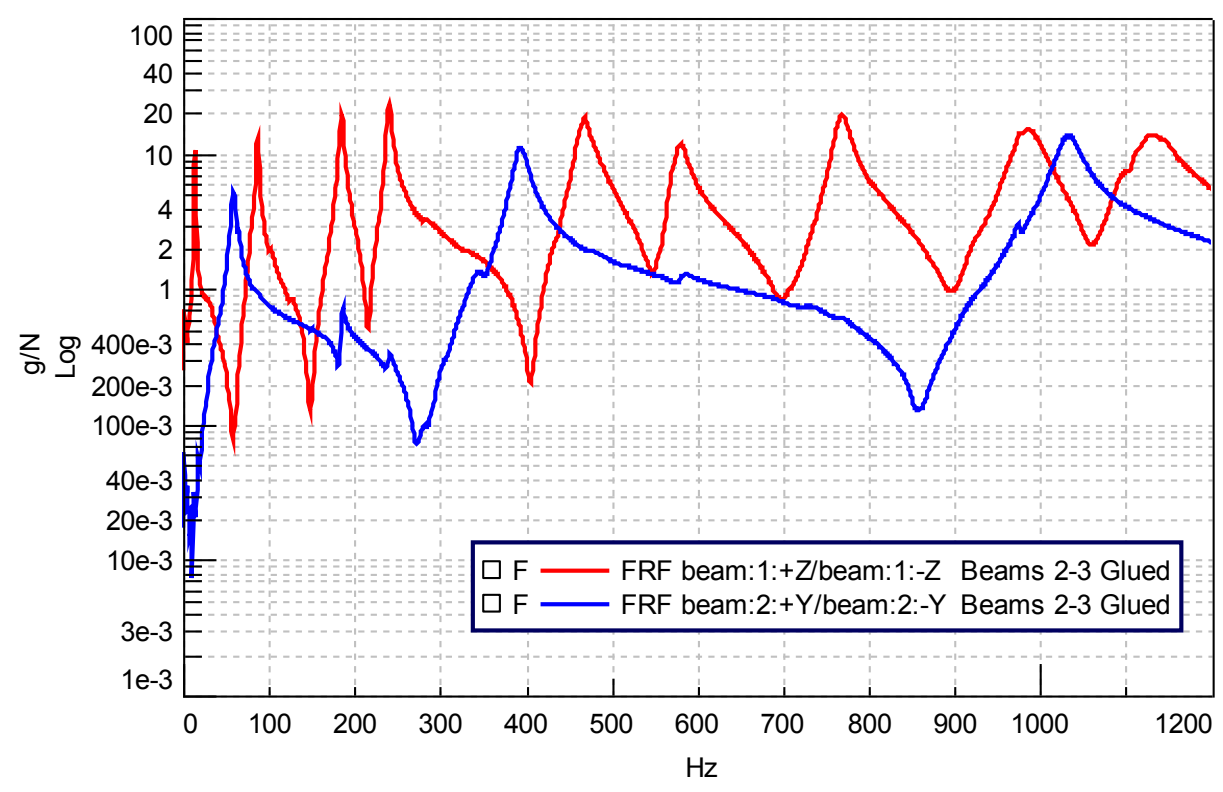

Figure C.20: Driving point FRFs for laminated beam

The experimental modal data for the laminated beam were also curve fit using LMS Test.Lab PolyMAX. The resulting modes are given in Table C.16.

Table C.16: Curve fit results for the laminated beam EMA

\begin{tabular}{|c|c|c|l|}
\hline Mode \# & Frequency (Hz) & Damping (\%) & Description \\
\hline 1 & 13.56 & 1.06 & $1^{\text {st }}$ vertical bending \\
\hline 2 & 58.42 & 4.88 & $1^{\text {st }}$ lateral bending \\
\hline 3 & 81.64 & 4.46 & $2^{\text {nd }}$ vertical bending \\
\hline 4 & 185.2 & 1.28 & $1^{\text {st }}$ torsion \\
\hline 5 & 240.3 & 1.09 & $3^{\text {rd }}$ vertical bending \\
\hline 6 & 394.8 & 1.78 & $2^{\text {nd }}$ lateral bending \\
\hline 7 & 467.6 & 1.69 & $4^{\text {th }}$ vertical bending \\
\hline 8 & 572.8 & 1.86 & $2^{\text {nd }}$ torsion \\
\hline
\end{tabular}


The finite element model of the glued beam was based on the model of the single beam. It was found that the best way to model the laminated structure was to define a composite property comprised of two $5.08 \mathrm{~mm}$ thick layers of the plywood properties from the single beam analysis with a layer of liquid hide glue in between. The initial properties used for the liquid hide glue are given in Table C.17.

Table C.17: Initial properties used for the glue layer

\begin{tabular}{|l|l|}
\hline Layer thickness & $0.04 \mathrm{~mm}$ \\
\hline Young's Modulus & $3.0 \mathrm{GPa}$ \\
\hline Poisson's Ratio & 0.30 \\
\hline Density & $395.9 \mathrm{~kg} / \mathrm{m}^{3}$ \\
\hline
\end{tabular}

The initial FE results of the laminated beam were correlated with the test data using MAC. The model was updated using optimization to adjust only the properties of the glue layer to improve the correlation. The final MAC matrix is shown in Figure C.21, while the corresponding mode pairs are shown in Table C.18.

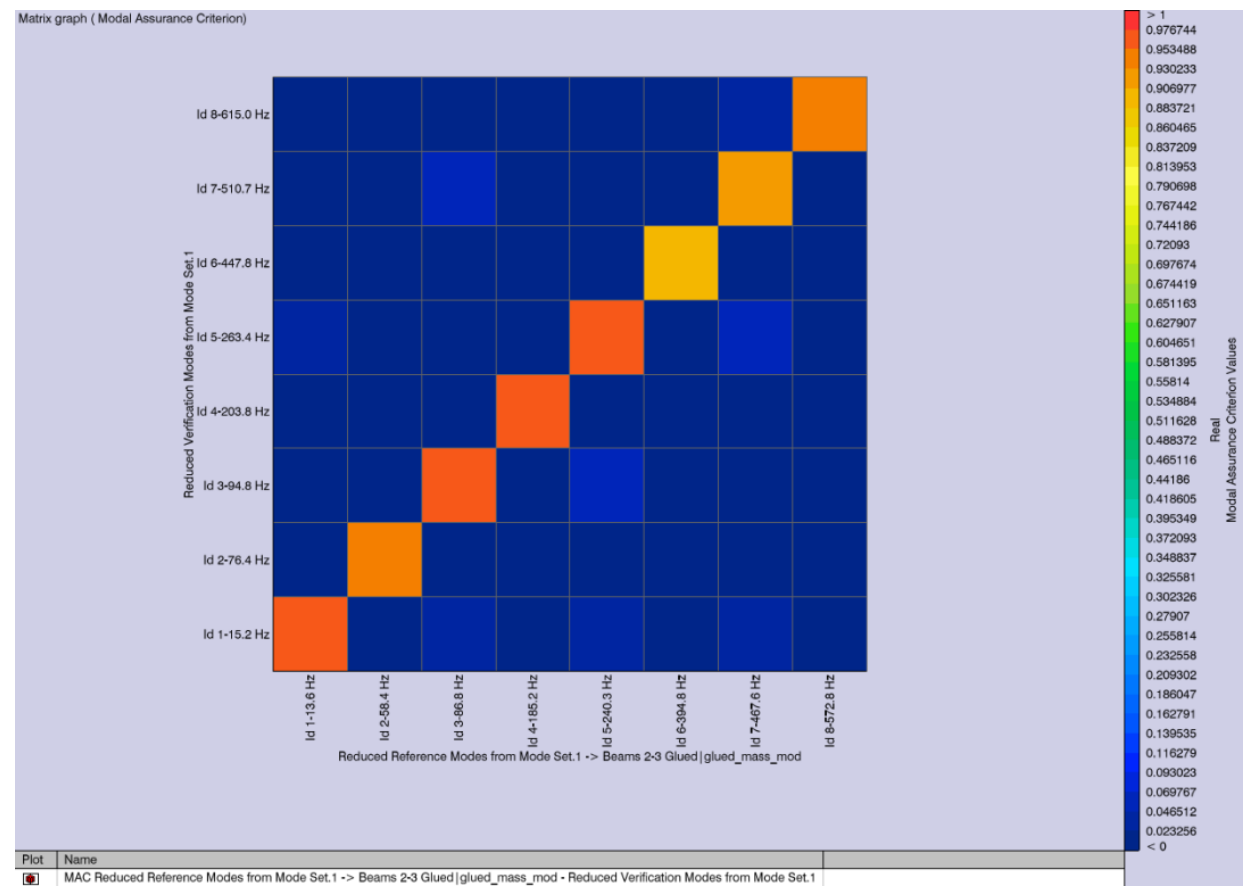

Figure C.21: MAC results for laminated beam after updating 
Table C.18: Mode pairs of the laminated beam correlation

\begin{tabular}{|l|l|l|l|l|l|l|}
\hline $\begin{array}{c}\text { Test } \\
\text { Id1 }\end{array}$ & $\begin{array}{c}\text { Test } \\
\text { Freq1 } \\
(\mathrm{Hz})\end{array}$ & FE Id2 & $\begin{array}{c}\text { FE } \\
\text { Freq2 } \\
(\mathrm{Hz})\end{array}$ & $\begin{array}{c}\text { MAC } \\
\text { Value }\end{array}$ & $\begin{array}{c}\text { Freq2- } \\
\text { Freq1 } \\
(\mathrm{Hz})\end{array}$ & $\begin{array}{c}\text { Freq2- } \\
\text { Freq1 } \\
(\% \text { of } \\
\text { Freq1 })\end{array}$ \\
\hline 1 & 13.6 & 1 & 15.2 & 0.966 & 1.64 & 12.1 \\
\hline 2 & 58.4 & 2 & 76.4 & 0.949 & 17.95 & 30.7 \\
\hline 3 & 86.8 & 3 & 94.8 & 0.973 & 8.04 & 9.3 \\
\hline 4 & 185.2 & 4 & 203.8 & 0.974 & 18.62 & 10.1 \\
\hline 5 & 240.3 & 5 & 263.4 & 0.963 & 23.11 & 9.6 \\
\hline 6 & 394.8 & 6 & 447.8 & 0.897 & 52.92 & 13.4 \\
\hline 7 & 467.6 & 7 & 510.7 & 0.921 & 43.14 & 9.2 \\
\hline 8 & 572.8 & 8 & 615 & 0.935 & 42.2 & 7.4 \\
\hline
\end{tabular}

The mode shapes FE model of the laminated beam also correlate well with those of the experiment. All mode pairs had a MAC value above 0.8 . The frequency results were less correlated, even after running the optimization for several iterations. The average percent frequency difference was $\sim 12.7 \%$. The largest percent frequency differences occur for in-plane bending modes 2 and 6 . This is again due to the boundary conditions in the lateral direction.

Table C.19: Final properties used for the glue layer

\begin{tabular}{|l|l|}
\hline Layer thickness & $0.01 \mathrm{~mm}$ \\
\hline Young's Modulus & $2.163 \mathrm{GPa}$ \\
\hline Poisson's Ratio & 0.3067 \\
\hline Density & $402.4 \mathrm{~kg} / \mathrm{m}^{3}$ \\
\hline
\end{tabular}

The final properties used for the liquid hide glue are given in Table C.19. Sensitivity analysis revealed that the mode pairs were mostly sensitive to the layer thickness, and relatively insensitive to the other material properties.

\section{C.3 Ribs Structure Study}

This study focused on the rib structure of the factory violin (B). An effort was made to measure the modes of the individual rib sections with the linings attached. The CT scans of the factory violin were used to create a finite element model of the rib structure alone. The FE results were correlated with the test data. 


\section{C.3.1 Experimental Modal Analysis}

For the rib structure (ribs, blocks, and linings) a clamping setup was used to "fix" the blocks of the structure in space. This was done to minimize the participation of the support frame so that it would not need to be included in the model. Each rib section would then have primarily local modes and a separate reference accelerometer would be needed for each rib section. Figure C.22 shows the rib structure test setup.

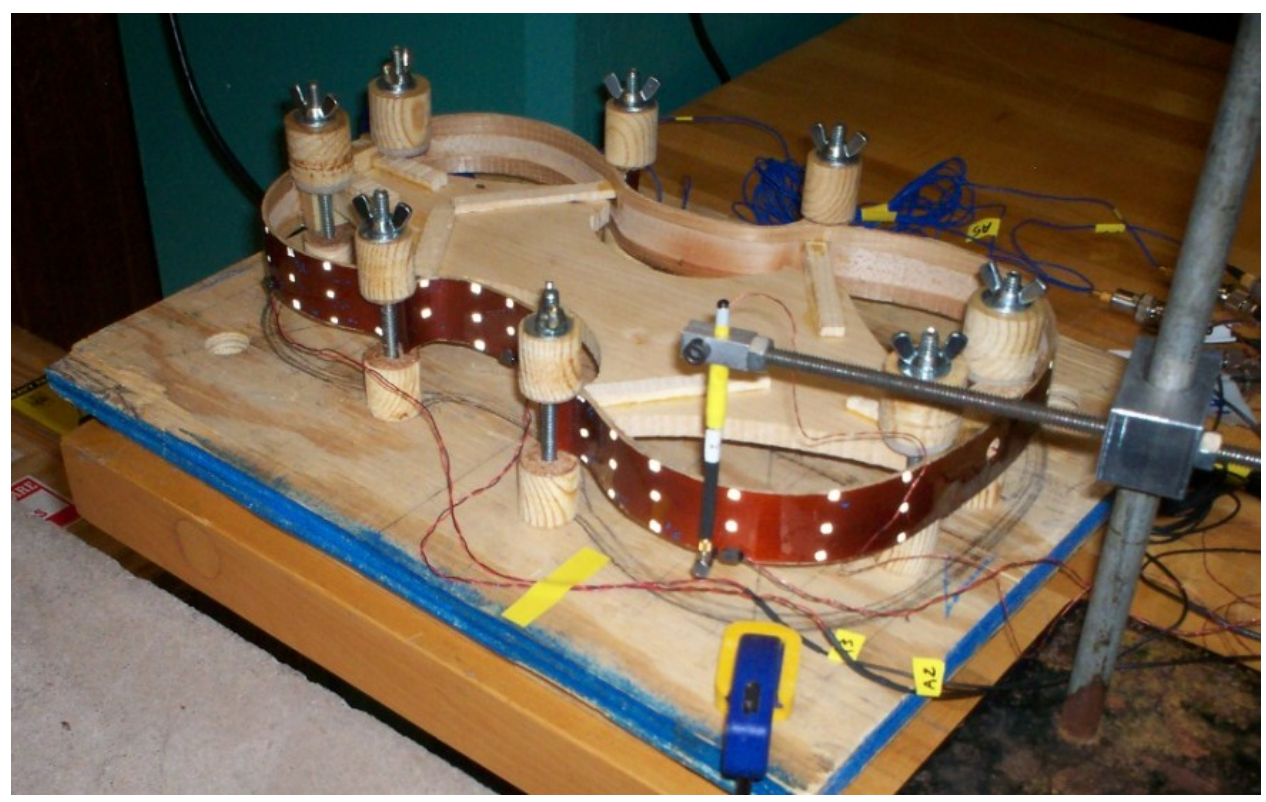

Figure C.22: Test setup for rib structure

The points were numbered from the lower bass-side and up from 1-54, then from the lower treble-side and up from 55-108. Driving points were ribs: 9 on the lower bass rib, 27 on the middle bass rib, 45 on the upper bass rib, 61 on the lower treble rib, 79 on the middle treble rib, and 97 on the upper treble rib. The impact hammer was roved over all 108 points. The DSP settings for the data acquisition are given in Table C.20.

Table C.20: DSP parameters for rib structure measurements

\begin{tabular}{|l|l|}
\hline Averaging & $5 \mathrm{Averages}$, Linear \\
\hline Bandwidth & $4096 \mathrm{~Hz}$ \\
\hline Frequency Resolution & $1.0 \mathrm{~Hz}$ \\
\hline Reference Window & Force Exponential, 100\% \\
\hline Response Window & Exponential, 100\% \\
\hline
\end{tabular}

The driving point FRFs are shown in Figure C.23. As each rib passes through individual modes, the frequency response of that rib dominates. Although similarly sized ribs (such 
as either side of the lower bouts) do have similar modes, there is enough asymmetry in the structure that these modes are well separated.

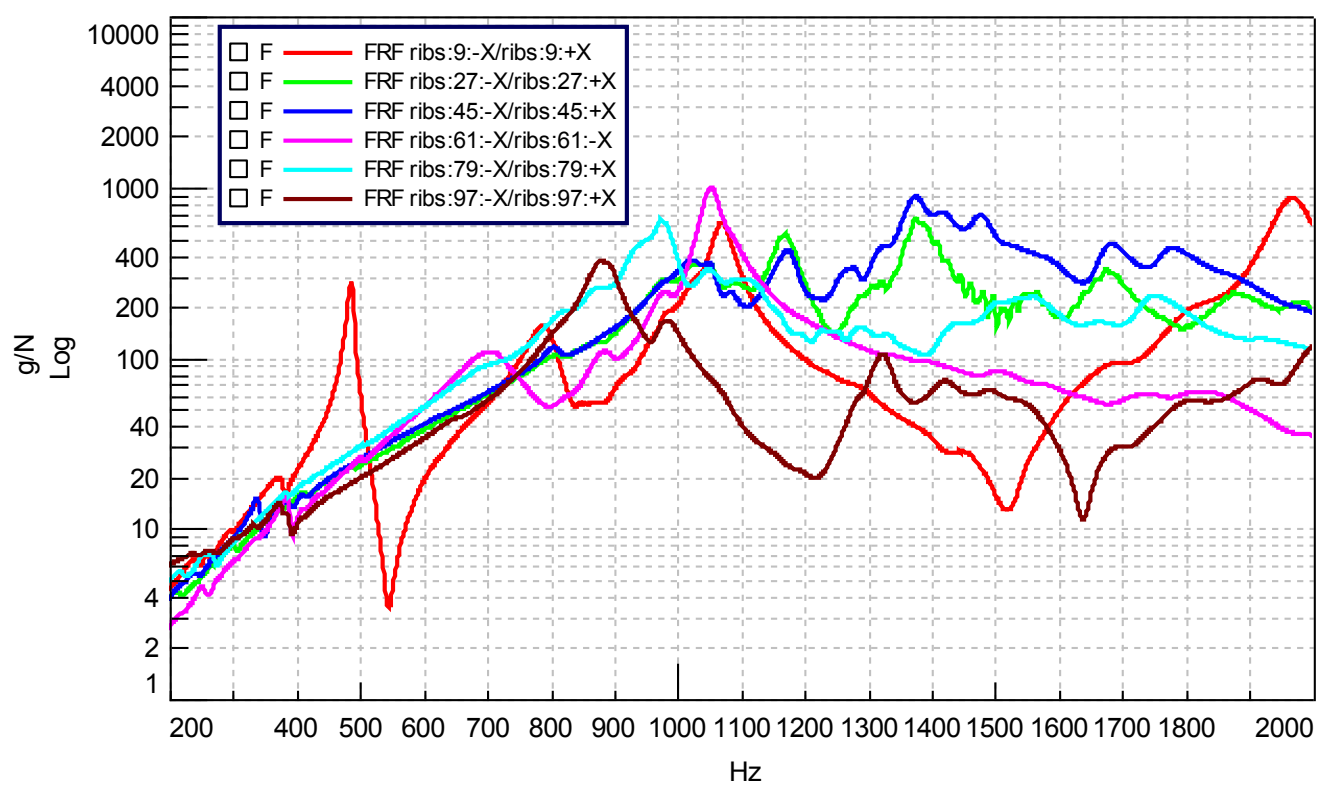

Figure C.23: Driving point FRFs of factory violin rib structure

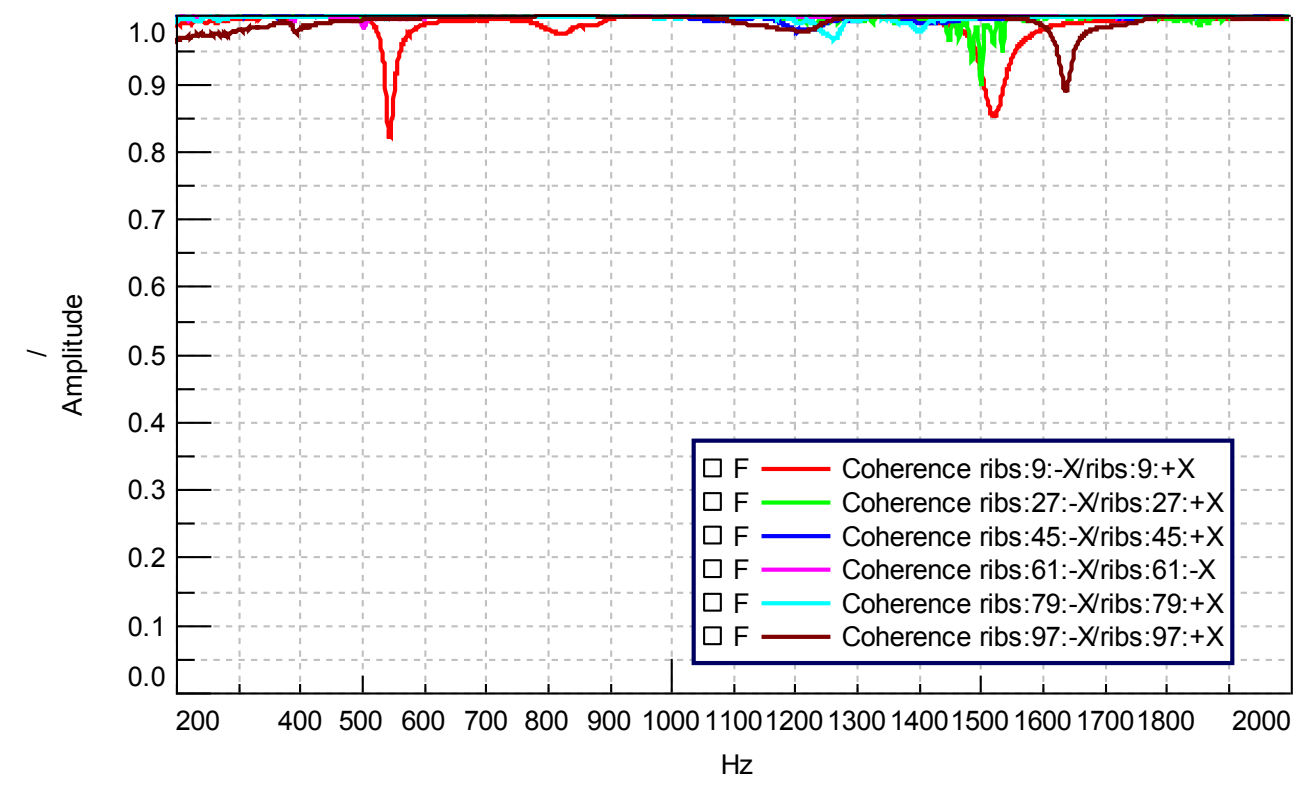

Figure C.24: Driving point coherence of factory violin rib structure

The driving point coherence functions are shown in Figure C.24. Minimal variance is observed in the frequency range of interest $(200 \mathrm{~Hz}-2000 \mathrm{~Hz})$. 
Curve fitting was performed with LMS Test.Lab PolyMAX. The resulting modes for rib structure curve fit are given in Table C.21.

Table C.21: Curve-fitting results from the rib structure EMA

\begin{tabular}{|c|c|c|l|}
\hline Mode \# & Frequency (Hz) & Damping (\%) & Description \\
\hline \hline 1 & 486.5 & 0.74 & Lower bass bow $-1^{\text {st }}$ order \\
\hline 2 & 503.5 & 1.01 & Lower treble bow $-1^{\text {st }}$ order \\
\hline 3 & 707.6 & 5.70 & Lower treble twist $-1^{\text {st }}$ order \\
\hline 4 & 801.4 & 1.38 & Upper bass bow $-1^{\text {st }}$ order \\
\hline 5 & 822.7 & 1.8 & Lower bass twist $-1^{\text {st }}$ order \\
\hline 6 & 879.3 & 3.12 & Upper treble bow $-1^{\text {st }}$ order \\
\hline 7 & 1053.6 & 1.37 & Lower treble bow $-2^{\text {nd }}$ order \\
\hline 8 & 1067.9 & 1.68 & Lower bass bow $-2^{\text {nd }}$ order \\
\hline 9 & 1105.5 & 2.42 & \\
\hline 10 & 1169.7 & 2.01 & \\
\hline
\end{tabular}

\section{C.3.2 Finite Element Model}

The finite element model of the factory violin rib structure was created based on the CT scan data of the instrument. A set of sample points was selected along the edges of the ribs to create 3D spline curves to define the surfaces of the ribs in the model. A similar process was used to create the CAD geometry of the blocks. The rib surfaces were meshed with triangular shell elements, while the blocks were meshed with solid tetrahedral elements. Rigid Bar Elements (RBEs) were used to connect the blocks to the ribs. The full mesh composition is provided in Table C.22.

Table C.22: Mesh composition of factory violin rib structure

\begin{tabular}{|l|l|}
\hline Nodes & 29936 \\
\hline TRIA6 & 2825 \\
\hline TETRA10 & 13891 \\
\hline RBE2 & 746 \\
\hline
\end{tabular}

To account for the linings a group was defined for the top and bottom two rows of shell elements of the rib surface. These were given a composite property definition consisting of $1 \mathrm{~mm}$ thick of maple rib, $0.04 \mathrm{~mm}$ thick hide glue, and $0.9 \mathrm{~mm}$ thick lining strip. The remaining shell elements were defined with a single property of $1 \mathrm{~mm}$ thick maple rib. Additional groups were created to define the grain orientation of the wood using different material axis systems. These were set for horizontal (global X), vertical (global Z), and 
two groups for $45^{\circ}$ in either direction (global $\mathrm{X} \pm 45^{\circ}$ ). Figure C.25 shows the locations of these groups.

(a)

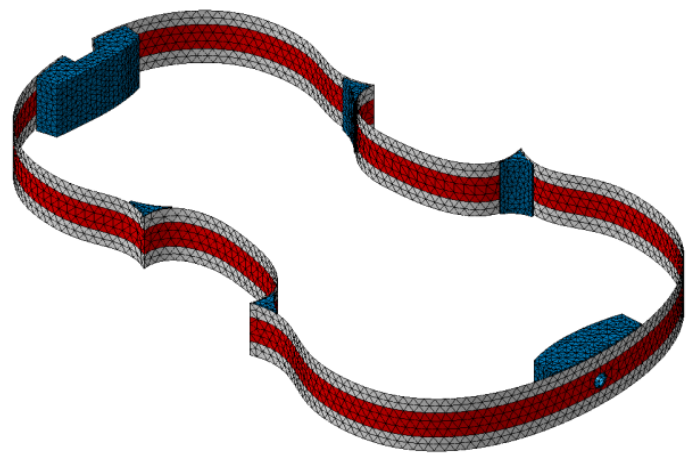

(b)

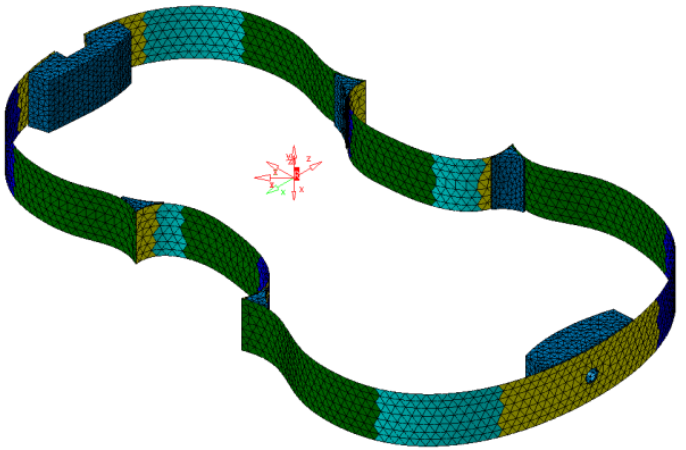

Figure C.25: Groups for defining shell properties, (a) ribs and lining groups, (b) material orientation groups

Results of the factory violin rib structure FE model normal modes analysis are summarized in Table C.23. Examples of the first six FE mode shapes are shown in Figure C.26 through Figure C.31.

Table C.23: Summary of factory violin rib structure normal modes analysis

\begin{tabular}{|c|c|l|}
\hline Mode \# & Frequency (Hz) & Description \\
\hline 1 & 484.9 & Lower bass-side bow -1 st order \\
\hline 2 & 499.0 & Lower treble-side bow -1 st order \\
\hline 3 & 728.9 & Lower treble-side twist -1 st order \\
\hline 4 & 738.0 & Upper bass-side twist -1 st order \\
\hline 5 & 741.6 & Lower treble-side twist -1 st order \\
\hline 6 & 793.2 & Upper bass-side bow -1 st order \\
\hline 7 & 824.4 & Upper treble bow -1 st order \\
\hline 8 & 1035.1 & Upper treble twist -1 st order \\
\hline \hline 9 & 1053.6 & Lower bass bow $-2^{\text {nd }}$ order \\
\hline 10 & 1085.7 & Lower treble bow $-2^{\text {nd }}$ order \\
\hline
\end{tabular}




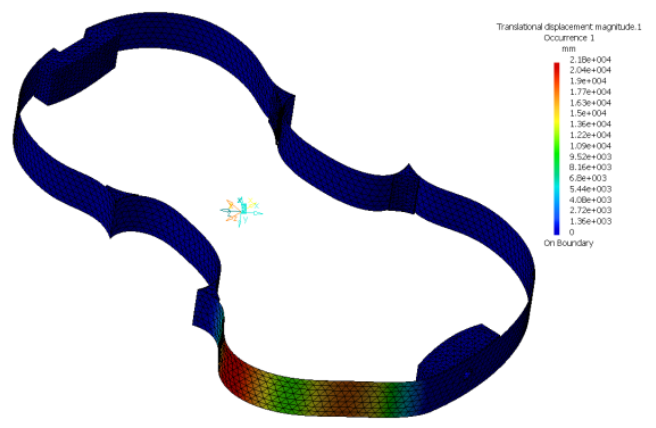

Figure C.26: Mode 1 of the factory violin rib structure (lower bass-side bow)

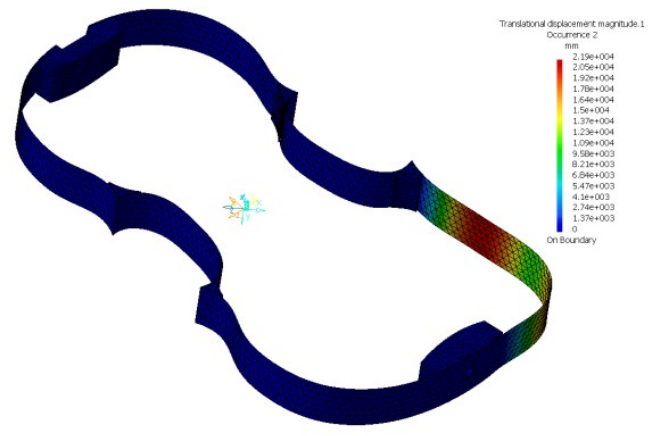

Figure C.27: Mode 2 of the factory violin rib structure (lower treble-side bow)

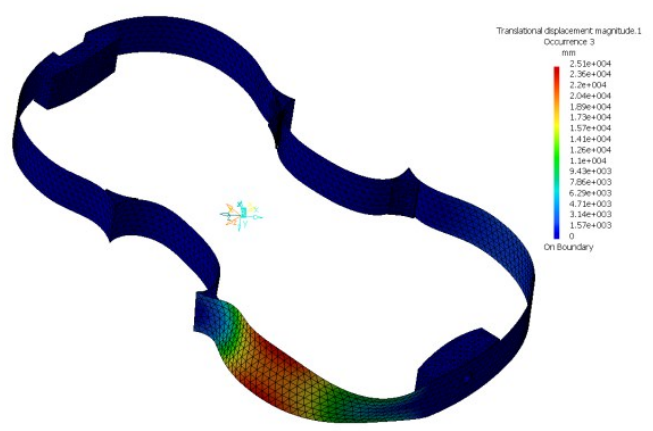

Figure C.28: Mode 3 of the factory violin rib structure (lower treble-side twist)

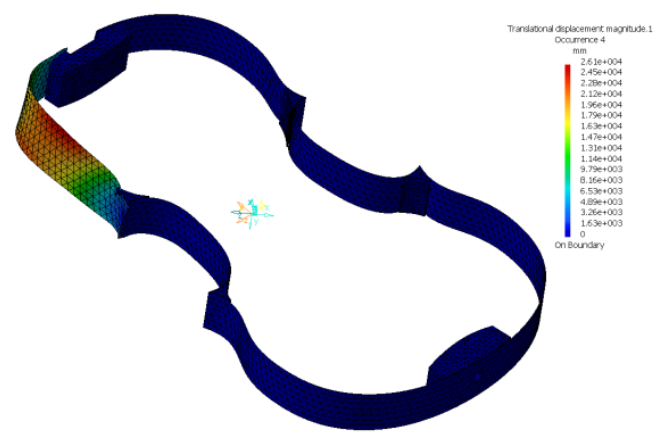

Figure C.29: Mode 4 of the factory violin rib structure (upper bass-side twist)

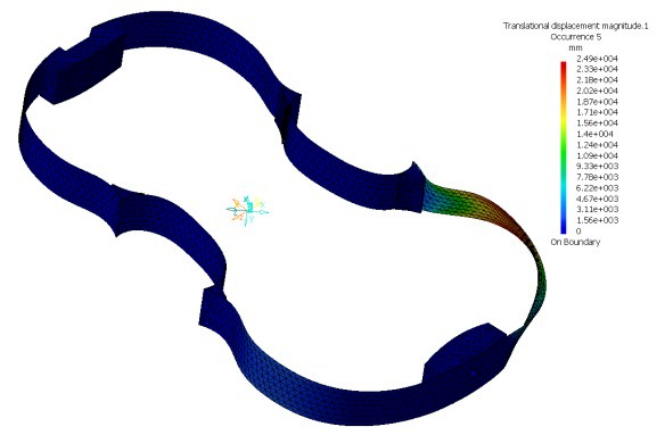

Figure C.30: Mode 5 of the factory violin rib structure (lower treble-side twist)

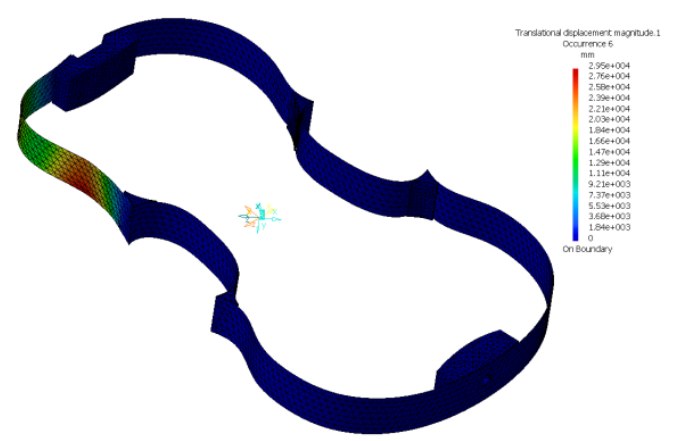

Figure C.31: Mode 6 of the factory violin rib structure (upper bass-side bow)

Michigan Technological University 


\section{C.3.3 FE-Test Correlation Results}

The results of the factory violin rib structure FE model were correlated with those from test data using the Modal Assurance Criterion (MAC). A plot of the MAC matrix is shown in Figure C.32. The subsequent mode pairs are given in Table C.24.

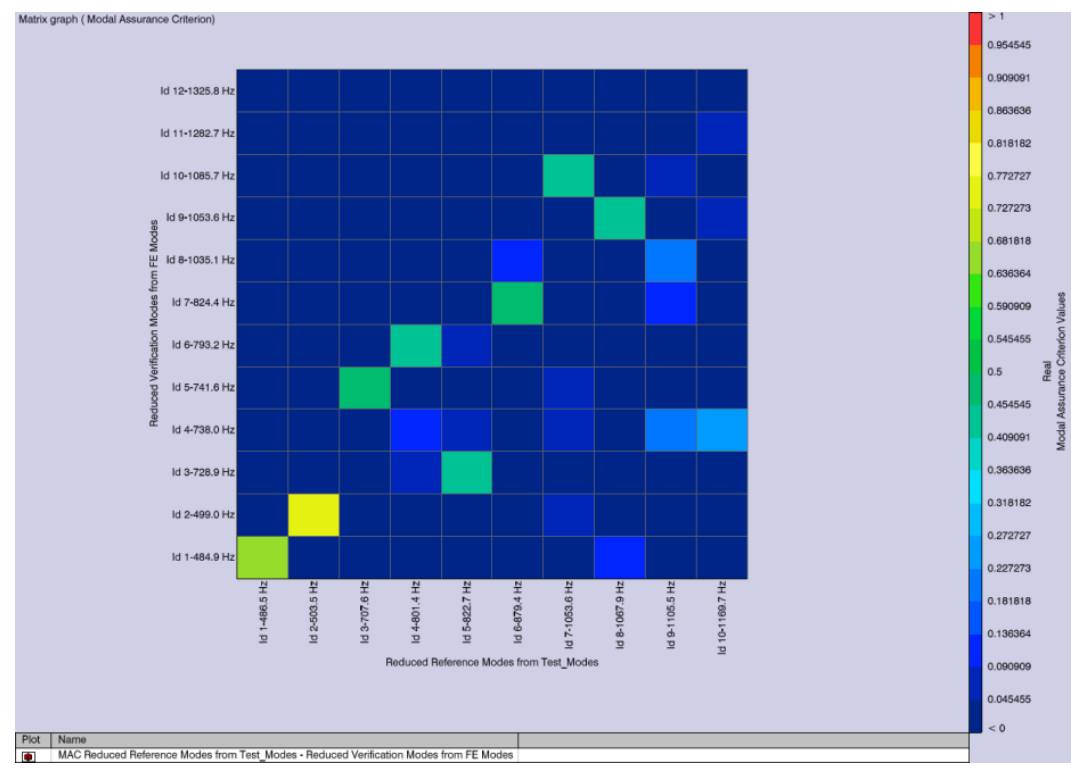

Figure C.32: MAC results for rib structure

Table C.24: Mode pairs of the rib structure correlation

\begin{tabular}{|c|c|c|c|c|c|c|}
\hline $\begin{array}{c}\text { Test } \\
\text { Id1 }\end{array}$ & $\begin{array}{c}\text { Test Freq1 } \\
(\mathrm{Hz})\end{array}$ & $\begin{array}{c}\text { FE } \\
\text { Id2 }\end{array}$ & $\begin{array}{c}\text { FE Freq2 } \\
(\mathrm{Hz})\end{array}$ & $\begin{array}{c}\text { MAC } \\
\text { Value }\end{array}$ & $\begin{array}{c}\text { Freq2-Freq1 } \\
(\mathrm{Hz})\end{array}$ & $\begin{array}{c}\text { Freq2-Freq1 } \\
(\% \text { of Freq1 })\end{array}$ \\
\hline 1 & 486.5 & 1 & 484.9 & 0.732 & -1.6 & -0.3 \\
\hline 2 & 503.5 & 2 & 499 & 0.881 & -4.51 & -0.9 \\
\hline 3 & 707.6 & 5 & 741.6 & 0.502 & 33.98 & 4.8 \\
\hline 4 & 801.4 & 6 & 793.2 & 0.475 & -8.19 & -1 \\
\hline 5 & 822.7 & 3 & 728.9 & 0.459 & -93.84 & -11.4 \\
\hline 6 & 879.4 & 7 & 824.4 & 0.546 & -54.98 & -6.3 \\
\hline 7 & 1053.6 & 10 & 1085.7 & 0.443 & 32.09 & 3 \\
\hline 8 & 1067.9 & 9 & 1053.6 & 0.47 & -14.27 & -1.3 \\
\hline 9 & 1105.5 & 8 & 1035.1 & 0.216 & -70.35 & -6.4 \\
\hline 10 & 1169.7 & 4 & 738 & 0.286 & -431.69 & -36.9 \\
\hline
\end{tabular}

It should be noted that the MAC values for the rib structure model are artificially low. Each MAC value is based on the entire mode shape vector and thus includes all degrees of freedom (DOF), even though there is only one rib section that is active in each mode. The other rib sections are relatively inactive, but do have a small amount of motion. This 
small amount of motion is sensitive to the actual boundary condition on the blocks between each section as this affects how much vibration energy from the mode of one rib section to is transmitted to another. This means that the softer constraints that existed in

the test setup allowed more transmission of energy between the rib sections than the rigid constraints of the model. The MAC computation then weights the difference between all DOFs equally, and is quite sensitive to the fact that the majority of the DOFs are on relatively inactive rib sections for any particular mode.

Despite this fact the correlation results could be better. The average MAC value for the first eight mode pairs is only $\sim 0.56$. Mode switching is prevalent throughout; however, with the exception of the 5-3 and the 6-7 mode pairs, the frequency differences of the first 8 mode pairs are all within 5\%. These results may be improved several ways. First the solid tetrahedral elements could be upgraded to hexahedral. Similarly, the shell elements could be upgraded from triangular to quadrangular. Also, it is possible that the RBEs are having a stiffening effect on the structure since they are connecting noncoincidence nodes. It would be better to equivalence the nodes between the ribs and the blocks, thereby eliminating the RBEs altogether. Finally, the constraints on the blocks could be "softened" to provide a more realistic boundary condition.

\section{C.4 Reassembled Factory Violin Measurements}

After the corpus testing was completed (see Chapter 4) the neck and scroll (still with the fingerboard attached) was reattached. An additional clamp block was made to fit over the shoulder of the neck and clamped to the corners of the corpus, as shown in Figure C.33. 


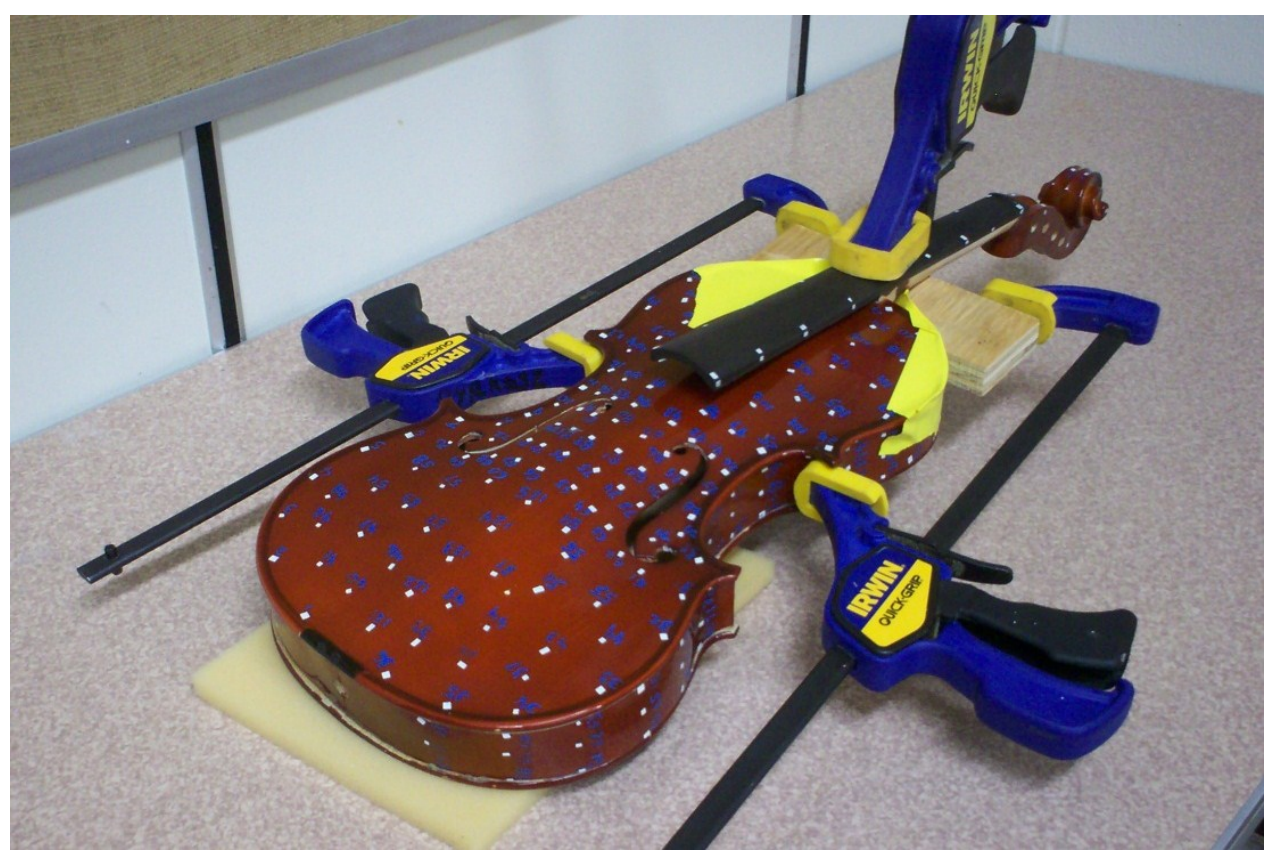

Figure C.33: Gluing the neck and scroll to the corpus

Once the neck was set, the violin was restrung and tuned. The instrument was allowed to "settle", meaning that the structure requires time to reach static equilibrium as the strain introduced by the strings is distributed to the various joints and wood fibers. As this occurs, the strings relax slightly and the violin goes flat. The violin was therefore retuned once a day for three days before it was close enough the static equilibrium that it would reliably remain in tune during the final tests. The driving point and bridge feet measurements from the original full violin model tests (see Chapter 3) were repeated to determine how much the factory violin changed from being rebuilt. As noted previously, the factory violin had a number of joints that were poorly glued. Attempts were made in the reassembly process to fit the pieces more tightly together, as such, it was expected that some of the structural modes would increase in frequency due to these joints being effectively stiffer. 


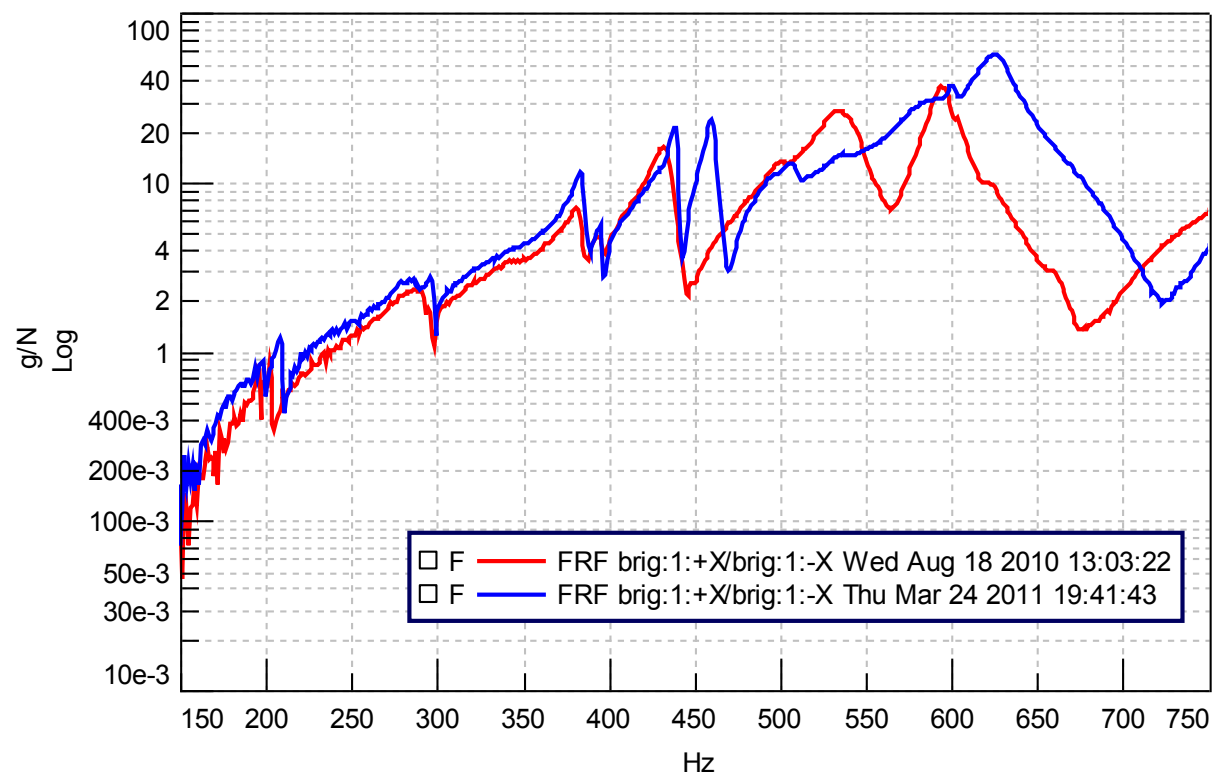

Figure C.34: Comparison of driving point FRF before and after factory violin was rebuilt

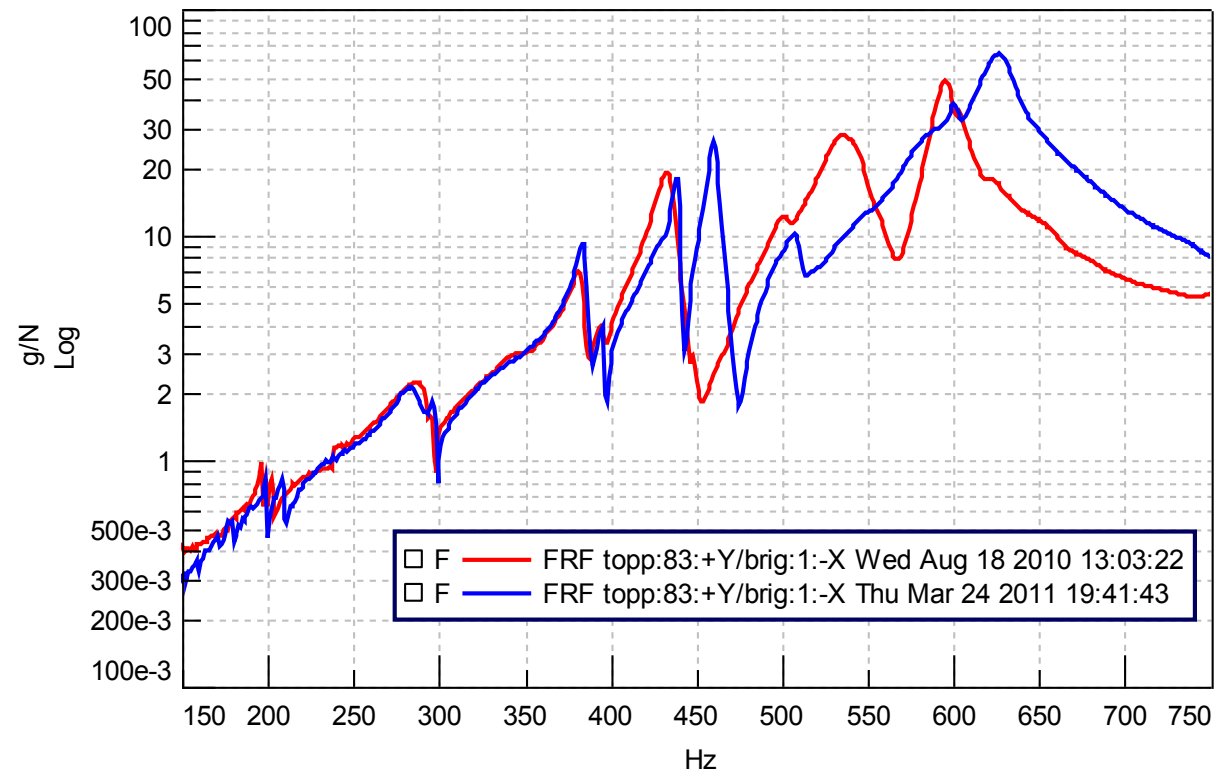

Figure C.35: Comparison of bass-side bridge foot FRF before and after factory violin was rebuilt 


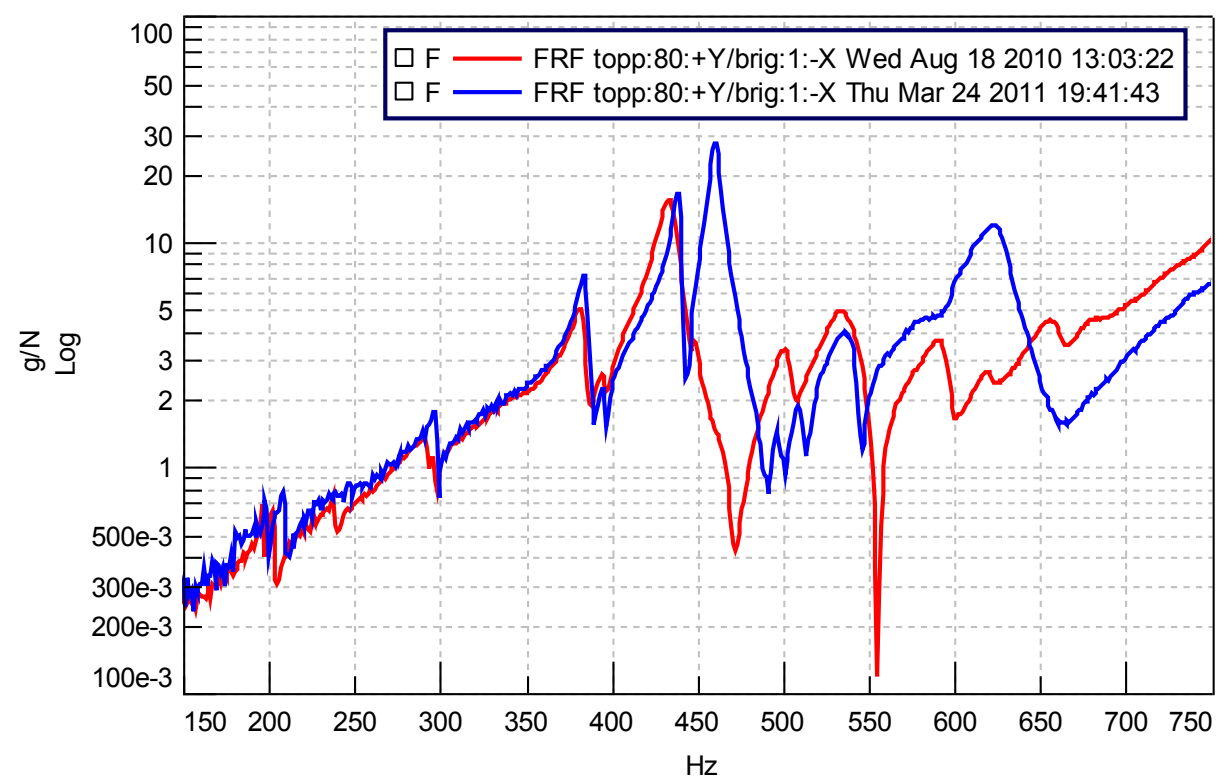

Figure C.36: Comparison of treble-side bridge foot FRF before and after factory violin was rebuilt

It is quite apparent from these results that the reassembly of the factory violin had a major impact on the dynamic behavior of the instrument. Although the frequencies below 440 $\mathrm{Hz}$ appear to have similar responses, the results are dramatically different beyond this frequency. Most notable is a peak $\sim 460 \mathrm{~Hz}$ that exists in the newly assembled instrument, which did not appear in the data from before disassembly. Aside from having stronger joints, it is also possible that the placement of the sound post may have affected the results. Other studies have shown that if a sound post is too tight or too loose, then it can have an impact on the bending modes [C.2]. Since a full modal was not performed on the new assembly it is impossible to identify which mode is associated with this peak.

\section{C.4.1 References}

[C.1] U. S. Department of Agriculture, "The Encyclopedia of Wood," Skyhorse Pub., Inc., New York, NY (2007). Originally published by U.S.D.A. Washington, D.C. (1999)

[C.2] Meinl, H., "On the frequency curves of violins," Akust. Z. 2, 22-33 (1937), Translated by Fan, C., (2007) 


\section{Appendix D Matlab Scripts}

Several scripts were created in Matlab for accomplishing various tasks. The more relevant ones have been selected for this appendix for purposes of reproducibility. The majority of these are taken up by the code for mapping plate thickness data on the shell meshes of the plate; none of the commercial software seems to have a way of doing this. These went through various iterations before being optimized. The first script "Titian FE Plate Thickness" was the final version for applying thicknesses to plates that were part of a fully assembled FE model of the Titian Stradivari violin. It calls several sub functions: Vbdfread, Vbdfedit3, VShellExtract, and VThickElement2. These have also been included in this appendix. The final script, VWoodMat, is a compact function that was created to take book values of the elastic moduli for various woods, and convert them into Nastran formatted orthotropic material cards. All scripts are thoroughly commented for clarification. Comment lines all begin with the percentage symbol, "\%".

\section{D.1 Titian FE Plate Thickness}

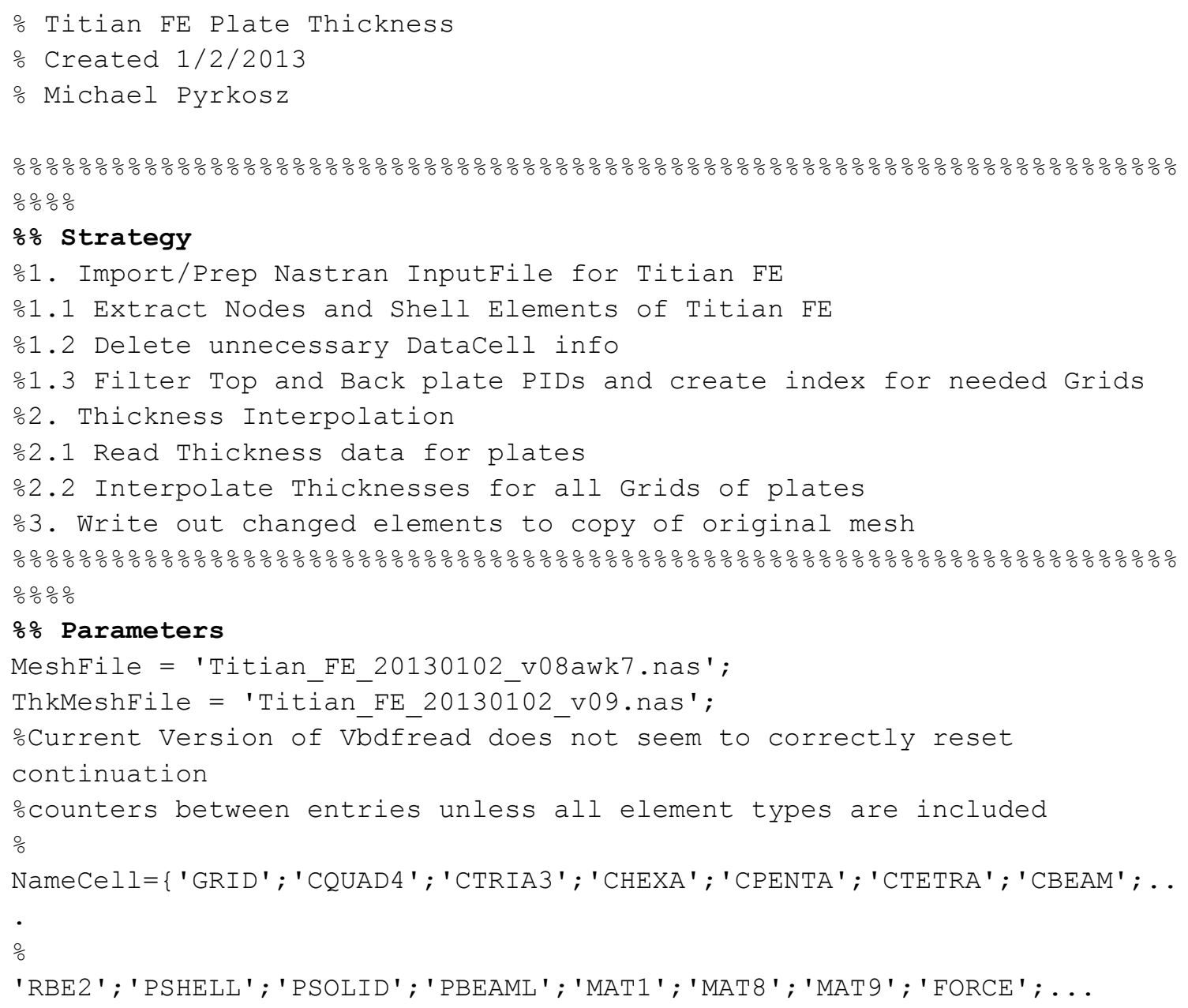




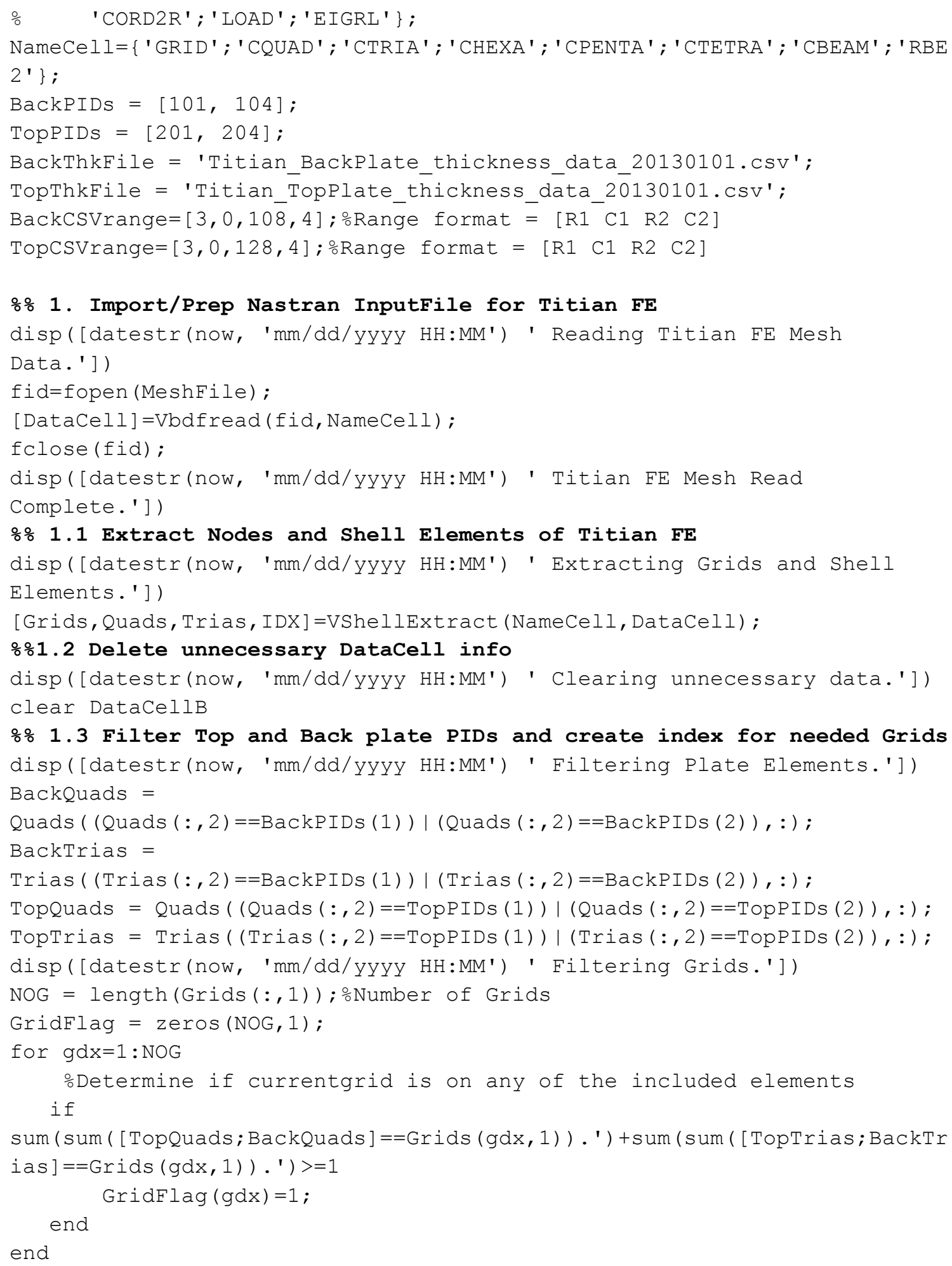




\section{D.2 Vbdfread}

function [DataCell]=Vbdfread (fid, NameCell)

음aaell]=Vbdfread (fid, NameCell)

oThis function is designed to read in NASTRAN formatted text from a Bulk

oData File (bdf) entry

\%fid = File identification from fopen

NameCell = Cell array of Bulk data entry names to be read,

\% (eg. \{'GRID'; 'CTETRA'; 'PSOLID'; 'MAT1'; etc...\})

oDataCell = Data values for the remaining field entries (fields 2 thru

9)

oNotes: long format is used when name ends in '*', since sometimes entries

ocan be mixed, it is not necessary to include the asterisk '*' in the oNameCell unless you only want to read the entries that are in large format.

oAlso, continuations such as '+' or '*' are read only, but not stored. oThus data only contains field entries for columns 2 thru 9

\section{응 Revision History}

oCreated 4/10/2010 as part of PhD Research at MTU in order to manipulate

oNastran meshs and materials in Matlab

oRev 1.1: 4/21/2011 - Added support for '+' and '*' continuation marks $\frac{\circ}{2} \operatorname{Rev} 1.2$ : 5/18/2011 - Added check if Nastran skipped symbol 'e' to denote

oexponent; if missing Matlab read as NaN instead of number

응 Count number of each Entry Type and Pre-Allocate Memory

응응응응응응응응응응응응응응응응

fseek (fid, $0,-1$ ); 을eset to beginning of file

EntryCtr=zeros (numel (NameCell), 1,'int32'); $\circ$ Counts \# of instances of each ...

oentry type; limited to 2147483647.

ContinuCtr=zeros (numel (NameCell), 1); $\circ$ Counts \# of '+' continuations for all

Ctr=0; ㄷounts \# of continuations within single entry

EntryType=1; ㅇkeep track of current entry

$\mathrm{EOF}=0$; 응 of file flag

disp ([datestr(now, 'mm/dd/yyyy HH:MM') ...

' Scanning file, and counting number of each data type...'])

while EOF =1을 End-of-File indicator not encountered 


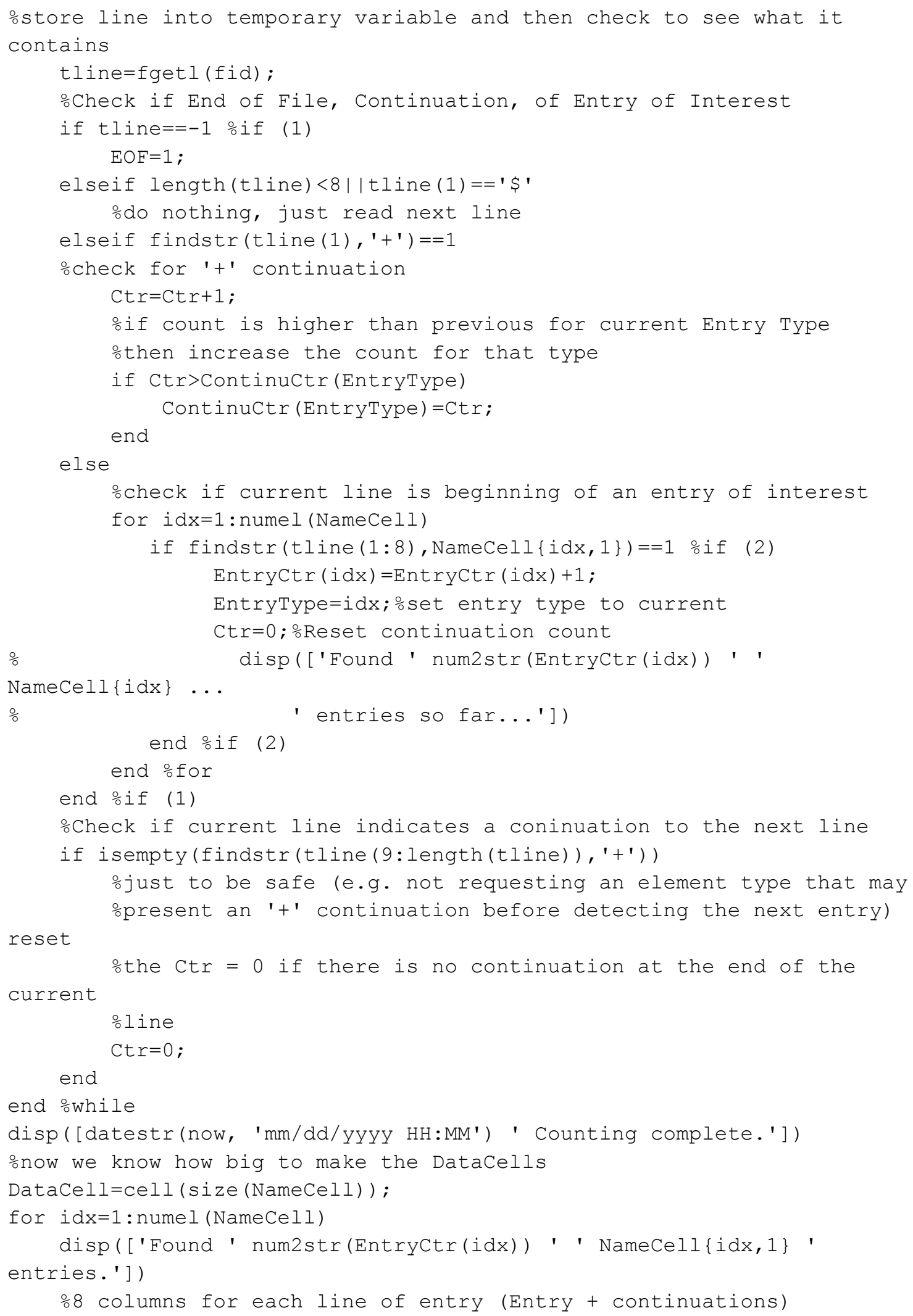




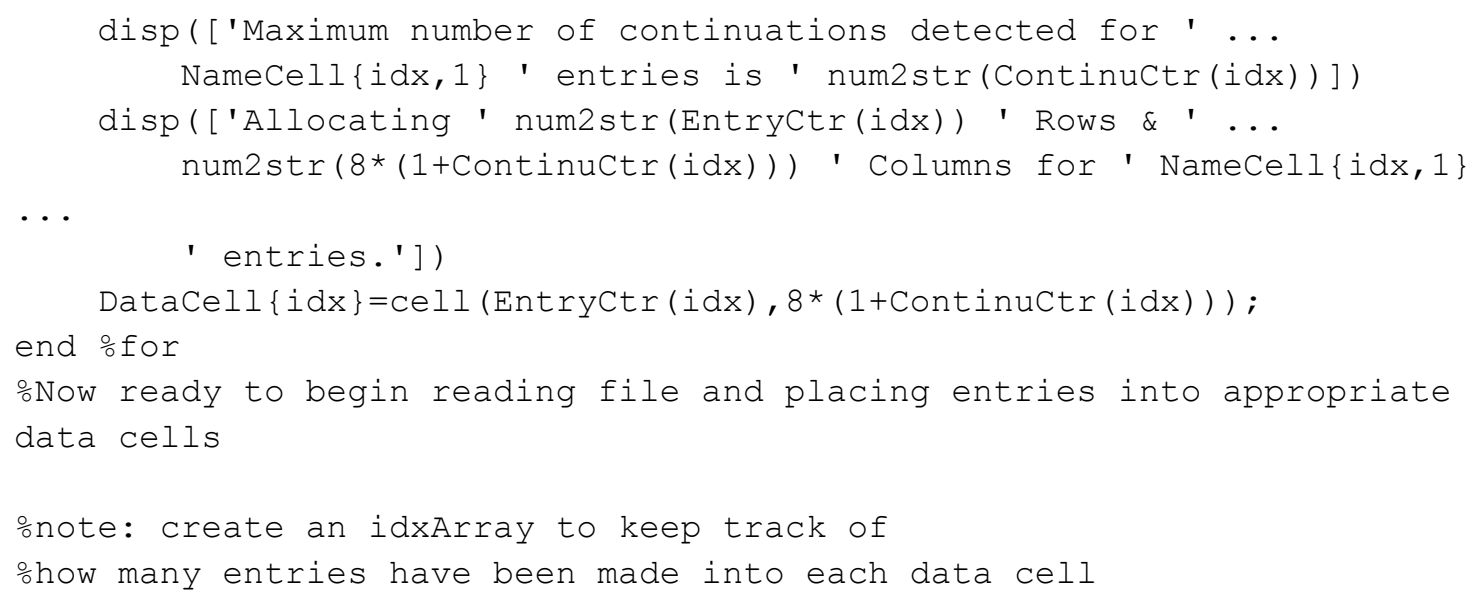




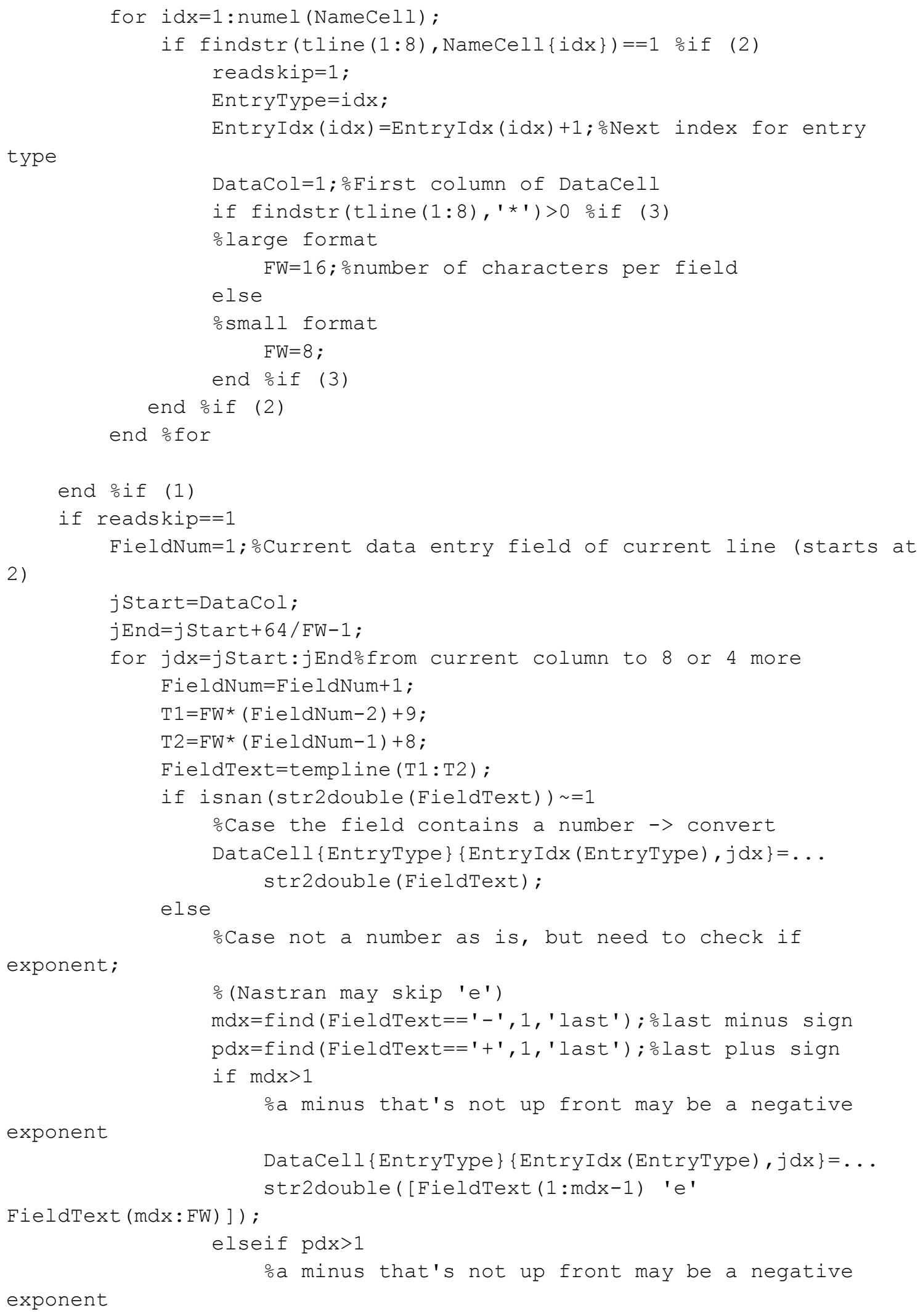




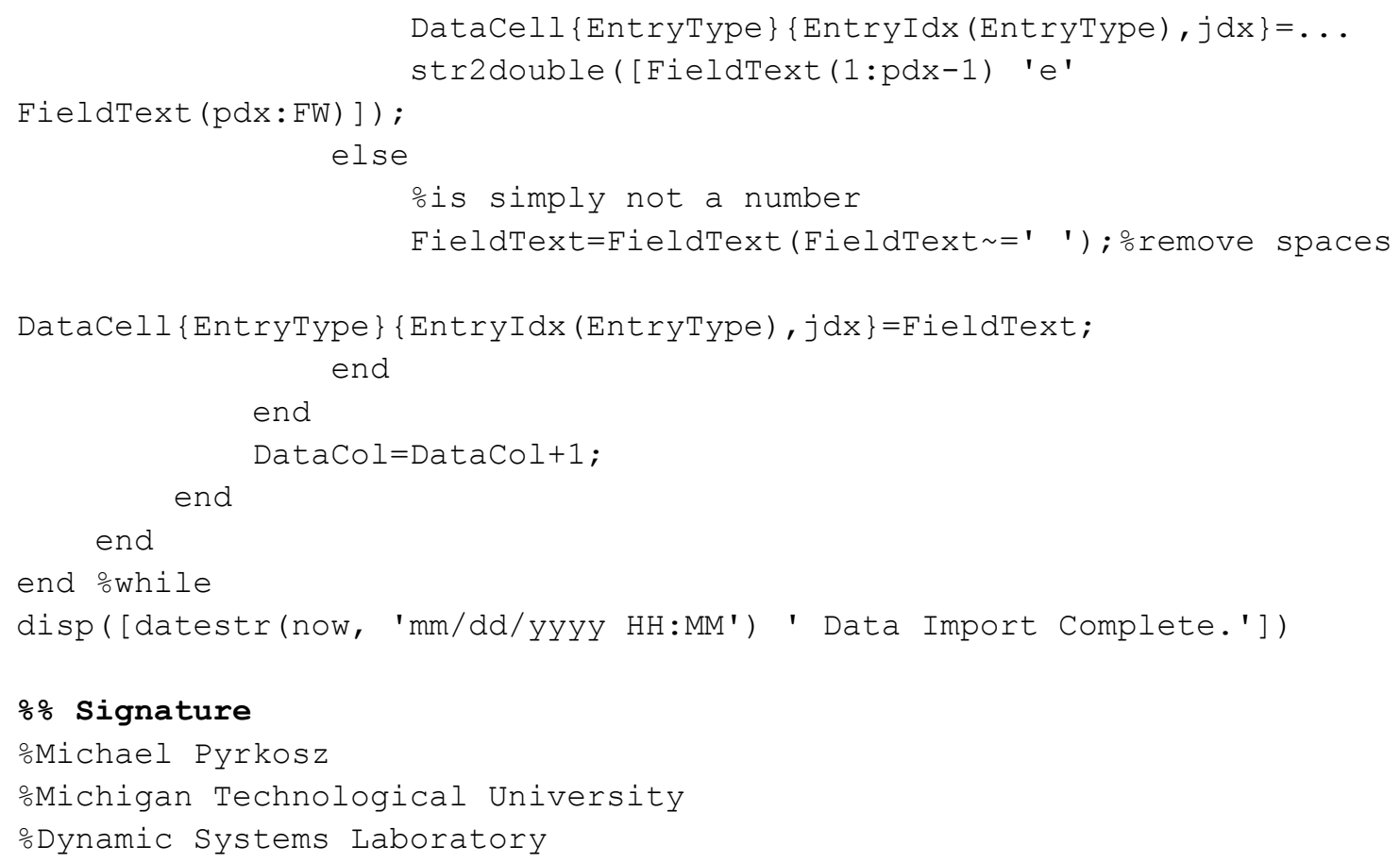

\section{D.3 Vbdfedit3}

function [ErrIDs] = Vbdfedit3 (fid, EntryStr, IDs, FieldNums, data)

으림ㅇ = Vbdfedit3 (fid, Entrystr, IDs, FieldNums, data)

oThis function is designed to edit specific entry cards of an existing oNASTRAN Bulk Data File (*.bdf)

INPUTS

ofid = File identification from fopen 
NOTES

oFor multiple line continuations '+' the line must already exist in some

oform (i.e. Nastran may skip unused lines; if new data is to be entered on othe entry a new line must be inserted into the file). Unfortunately the

oLow-level file I/O of Matlab cannot insert a line without overwriting the

onext one. One option would be to completely re-write the file, inserting

olines where needed. Another option is to use some other scripting language

osuch as AWK to add the lines.

ㅇ. 9 .

oawk '\$3==PID \{print $\$ 0, " \ldots+" ;$ print "+.."; next\}1' inputfile > outputfile

owill add a '+' to any entry with field3=3, and insert a new line after it.

oThe above AWK command requires some modification to create unique ocontinuation numbers, i.e. $\{\mathrm{N}++$ print $\$ 0$ "...+",Niprint

$"+", N, " \ldots$; next

oor something similar. Care should be taken not to repeat any numbers oalready present in the file.

oAlso, note that the new lines must contain at least as many characters as

othe needed fields (ie. replace the "..." above with the appropriate number

of spaces to align the fields).

$\frac{\circ}{\circ}$

oSupport of long format '*' is currently not included.

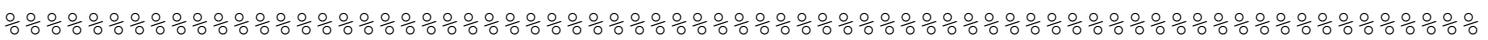
$\circ \% \frac{\circ}{0} \%$

\section{응 Revision History}

Rev 1: Created 7/1/2011 as part of LMS project: USUS20090800607 oCustom Software Development for Resonance Inspection for USCAR oR2007b: 09/20/2011 - removed 's and replaced with temp variables x1 $x 2 \times 3$

\%for backward compatability with Matlab 2007b

\%Rev 3: 01/02/2013 - updated to support continuations '+'

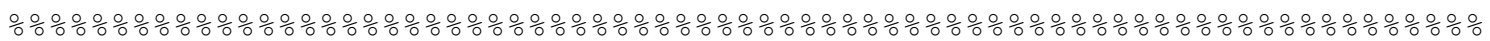
응응응응

\section{응 BDF Edit}

osizes

$[N, M]=$ size (data);

\%flag entries found 


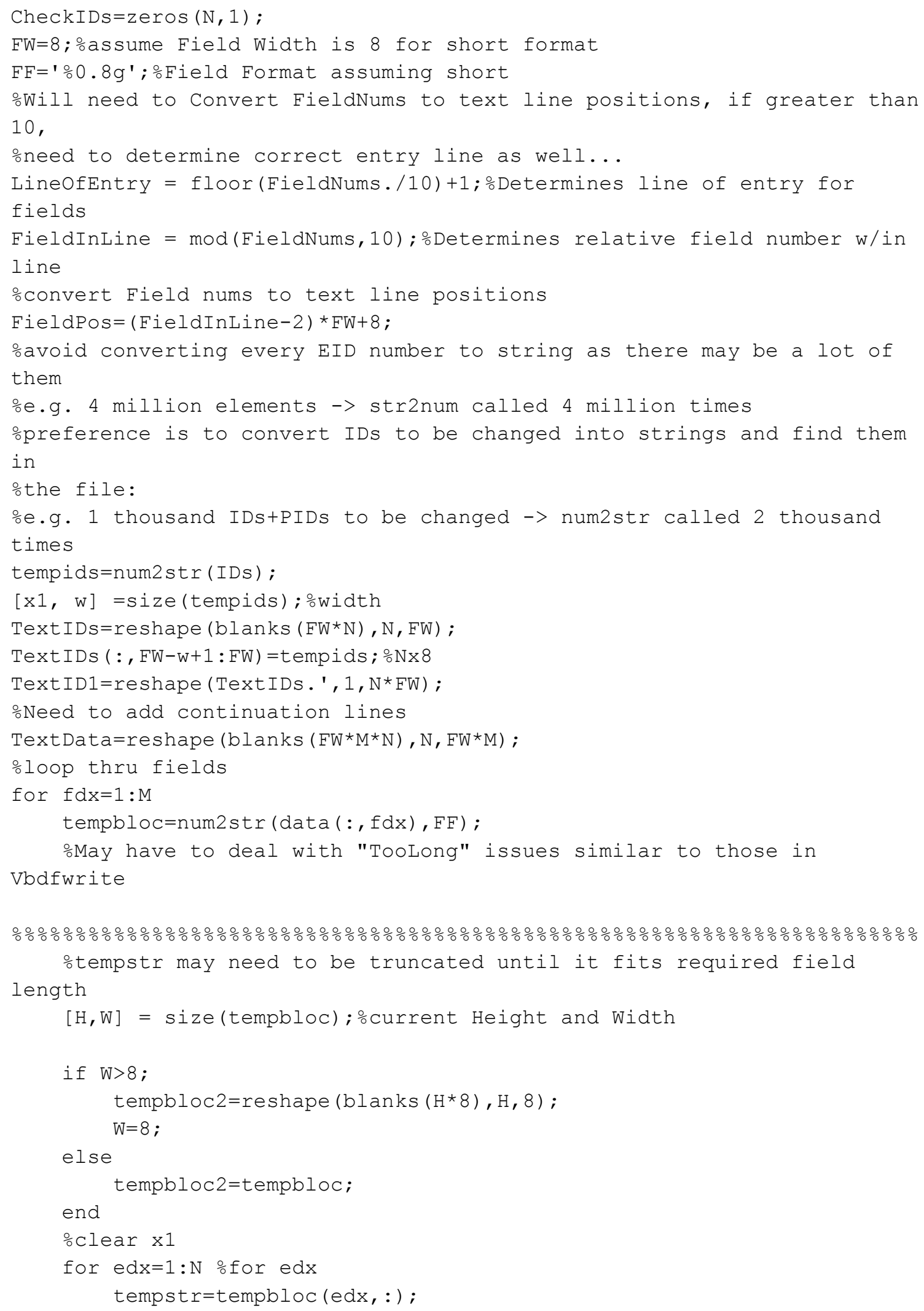




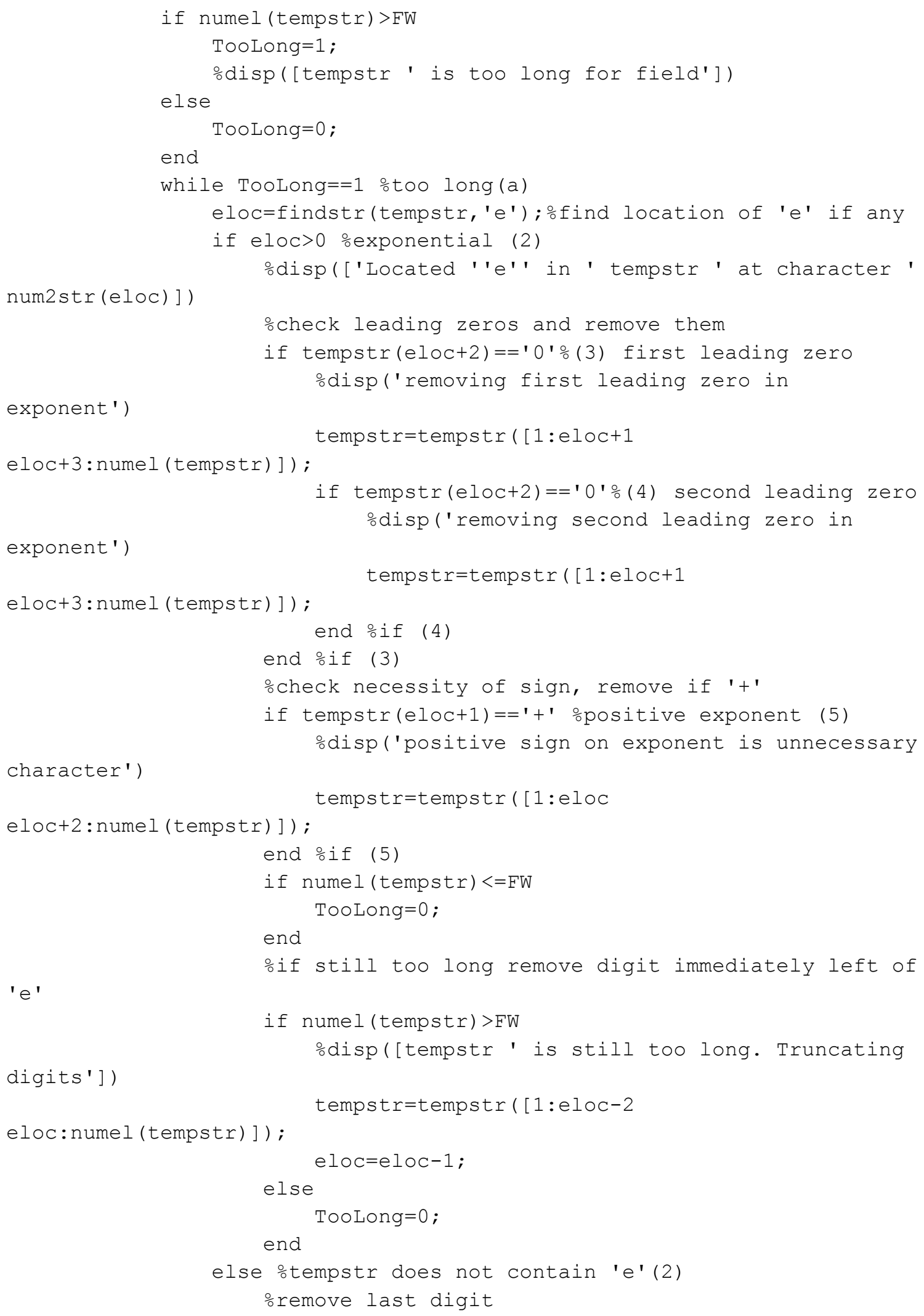




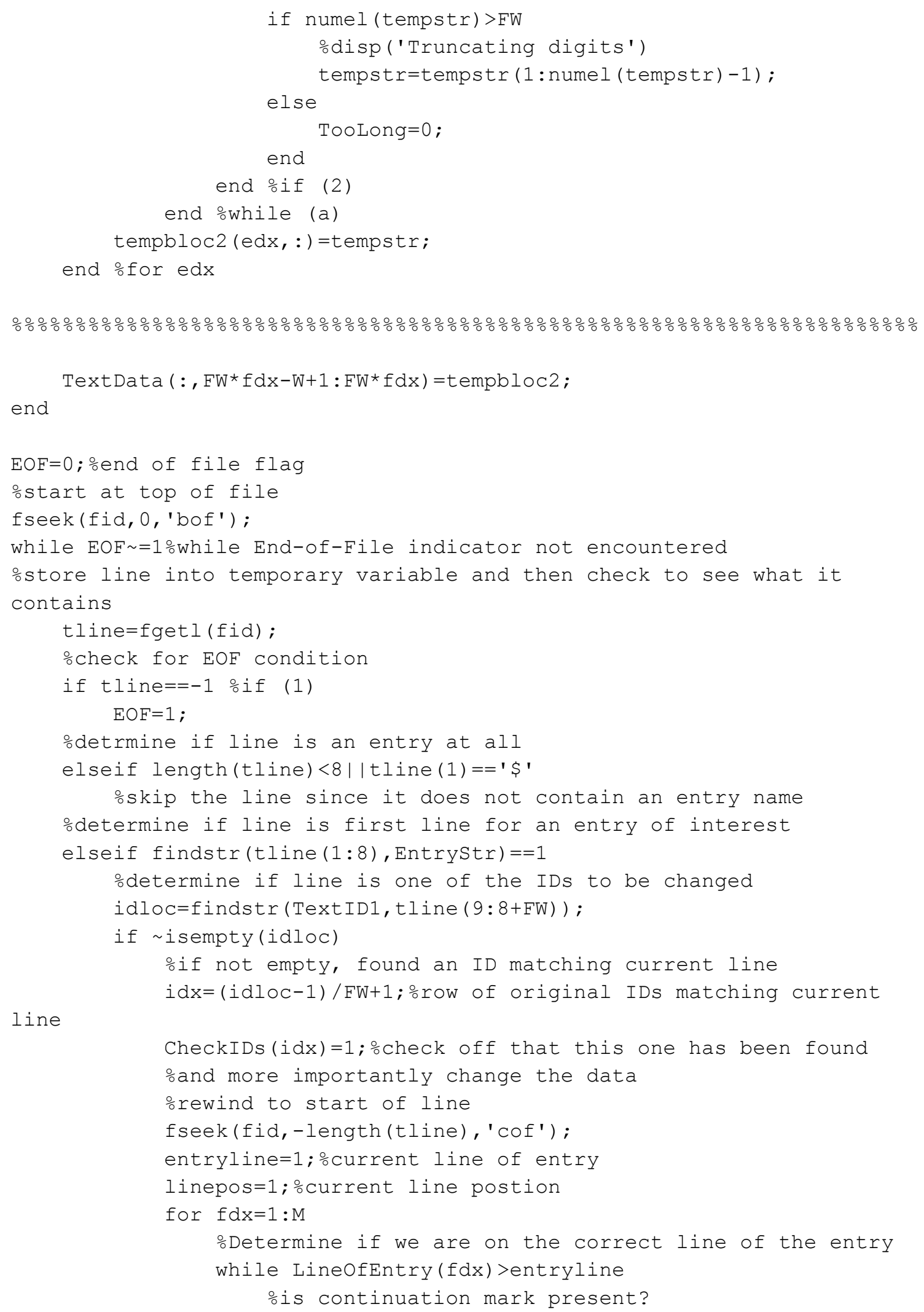




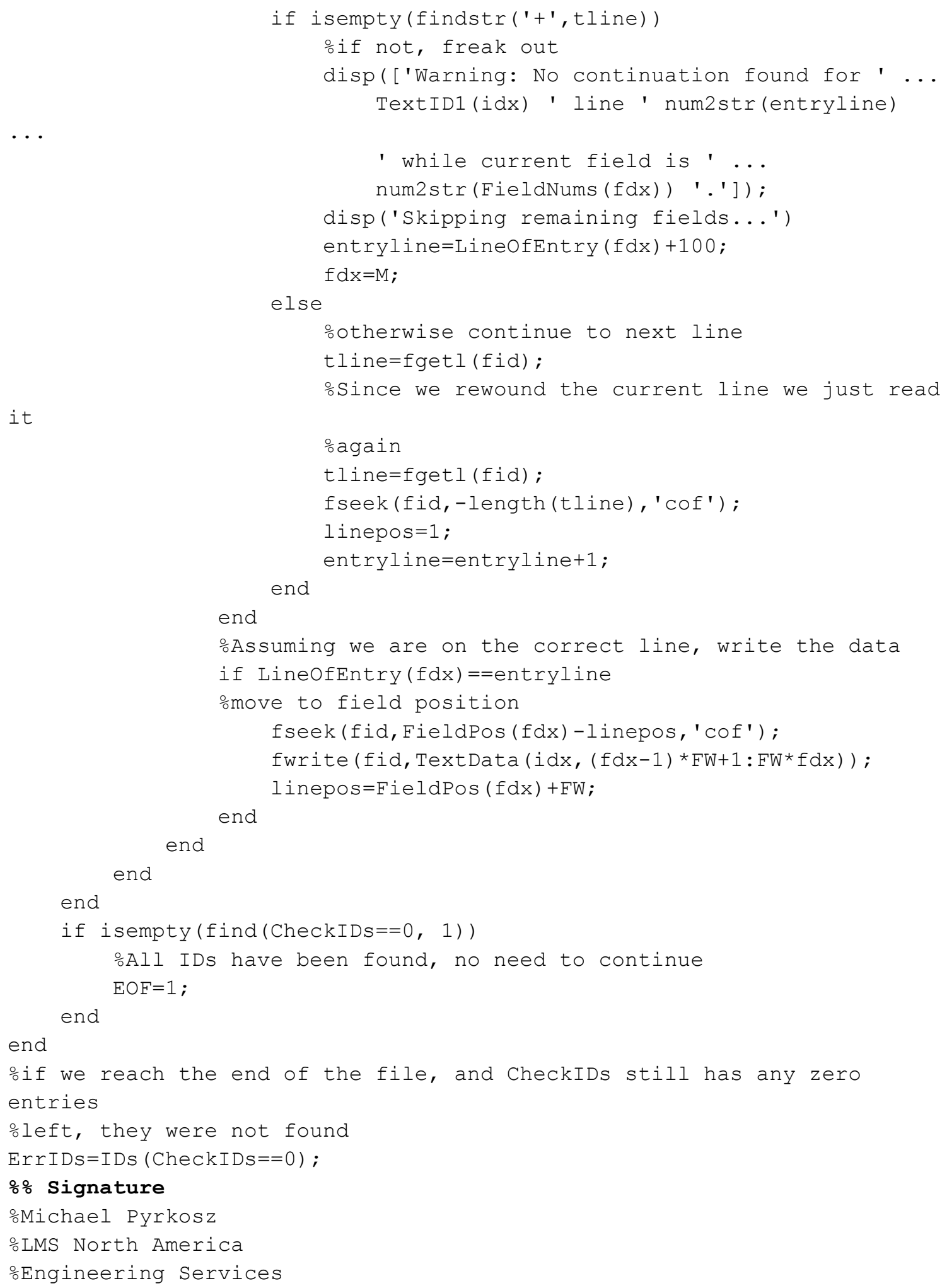




\section{D.4 VShellExtract}

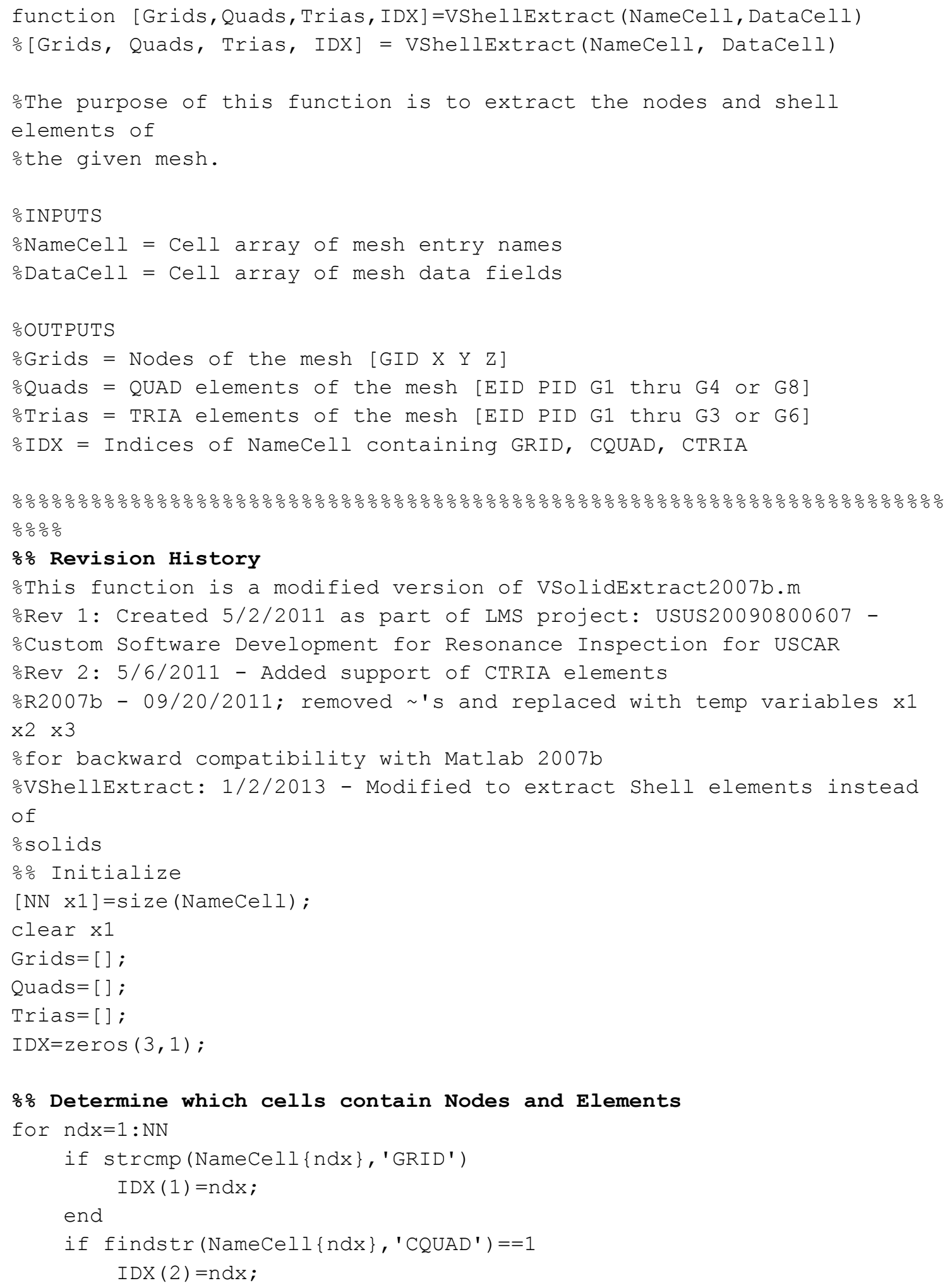




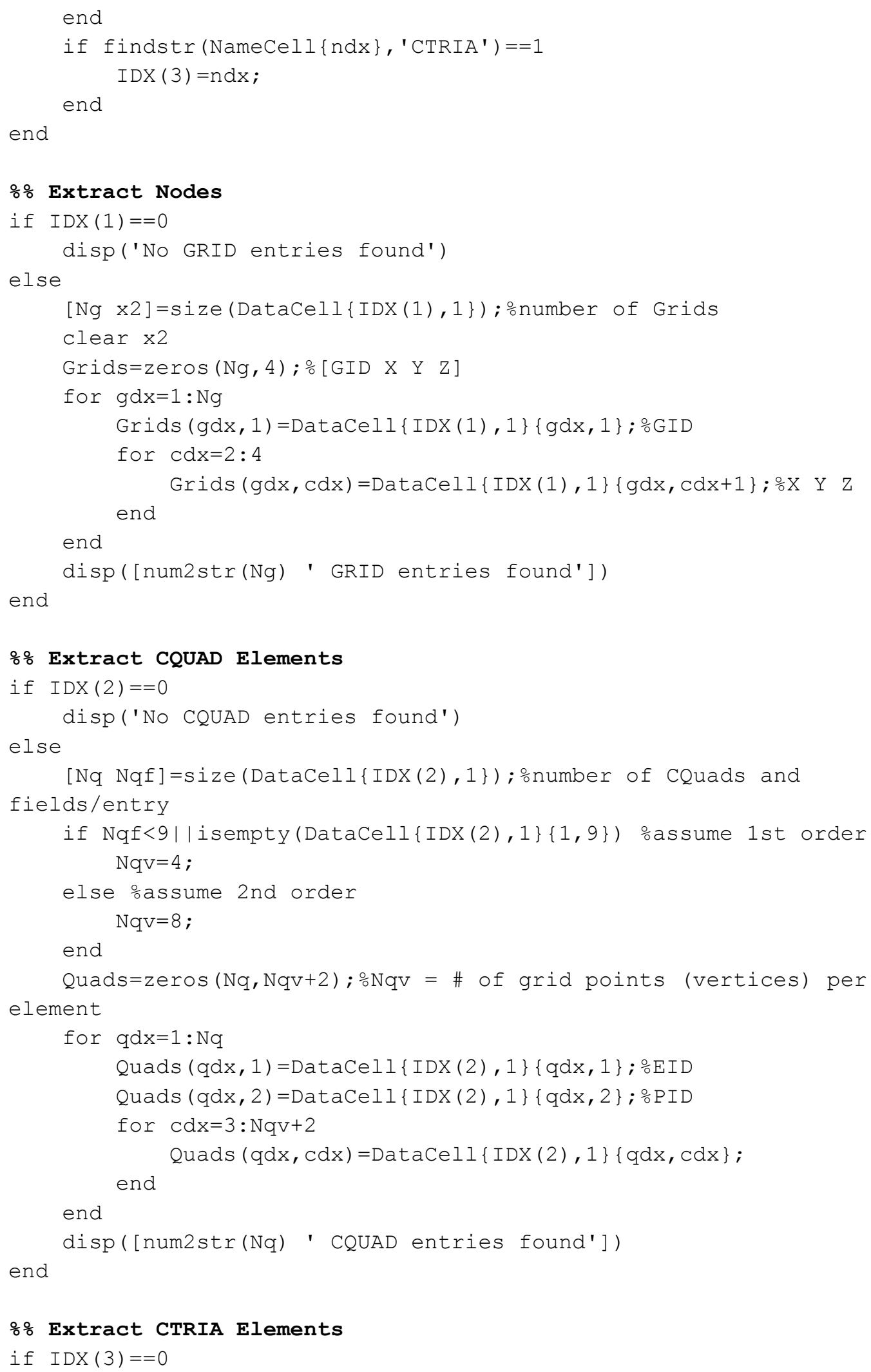

Michael Pyrkosz 


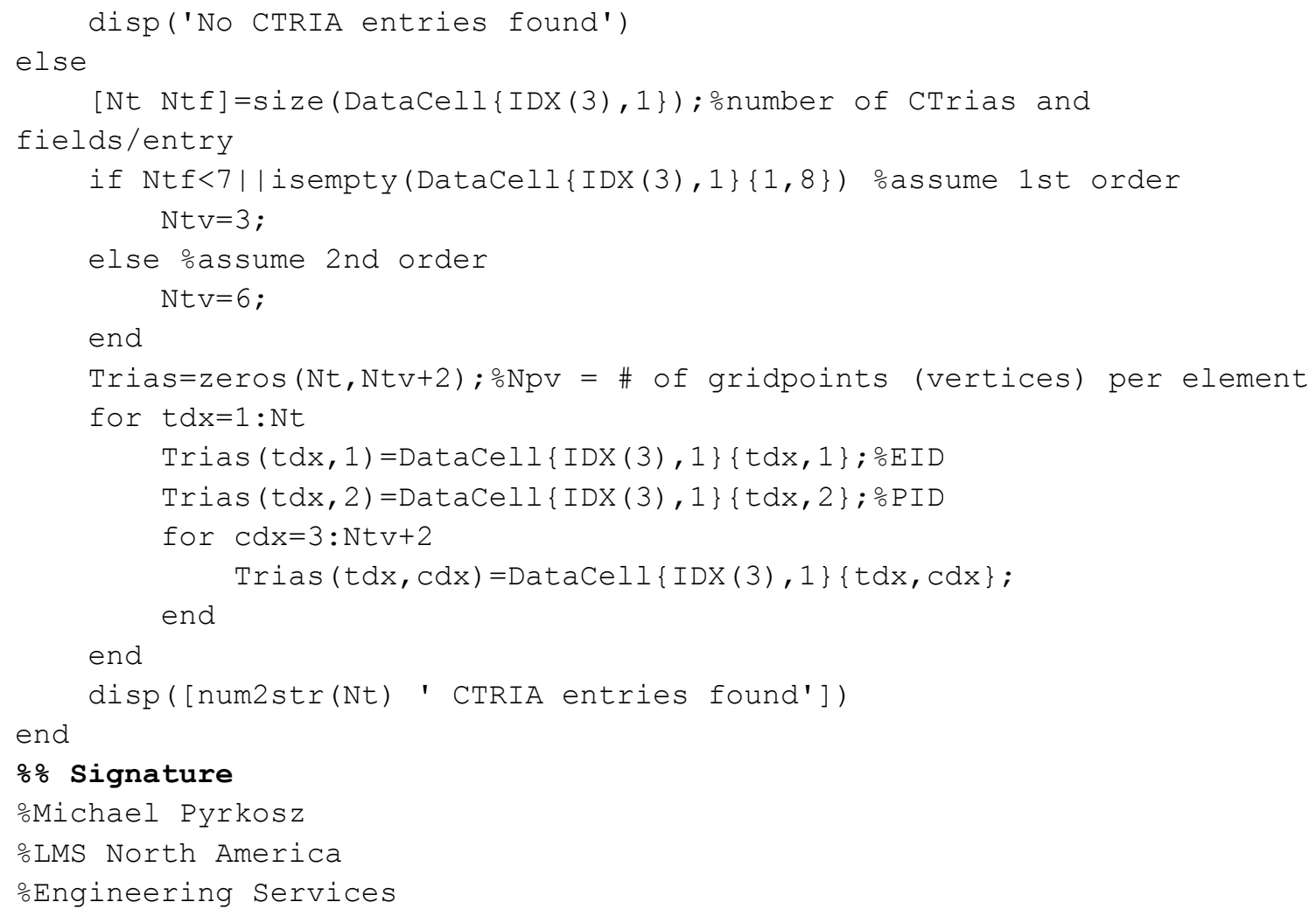

\section{D.5 VThickElement2}

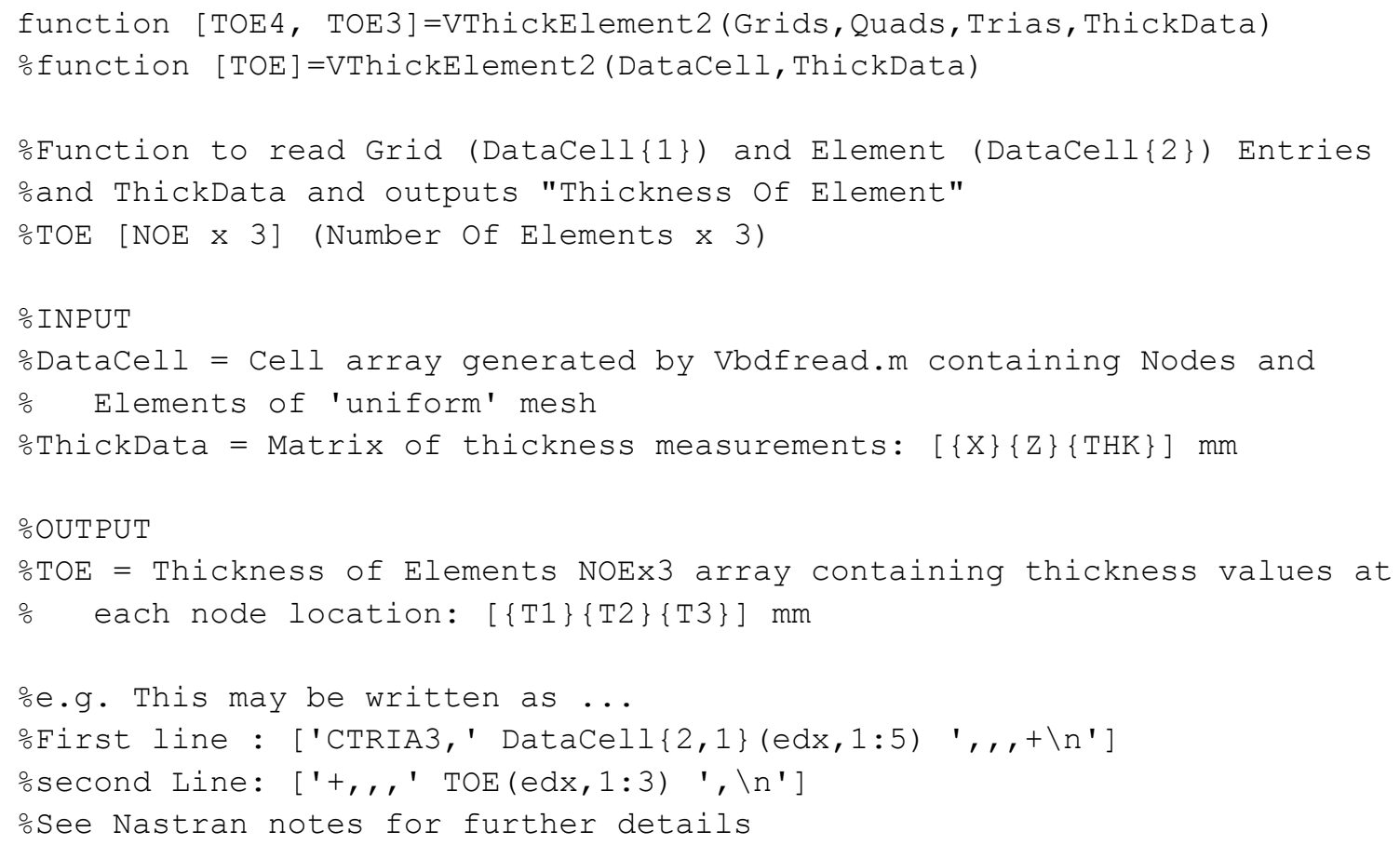




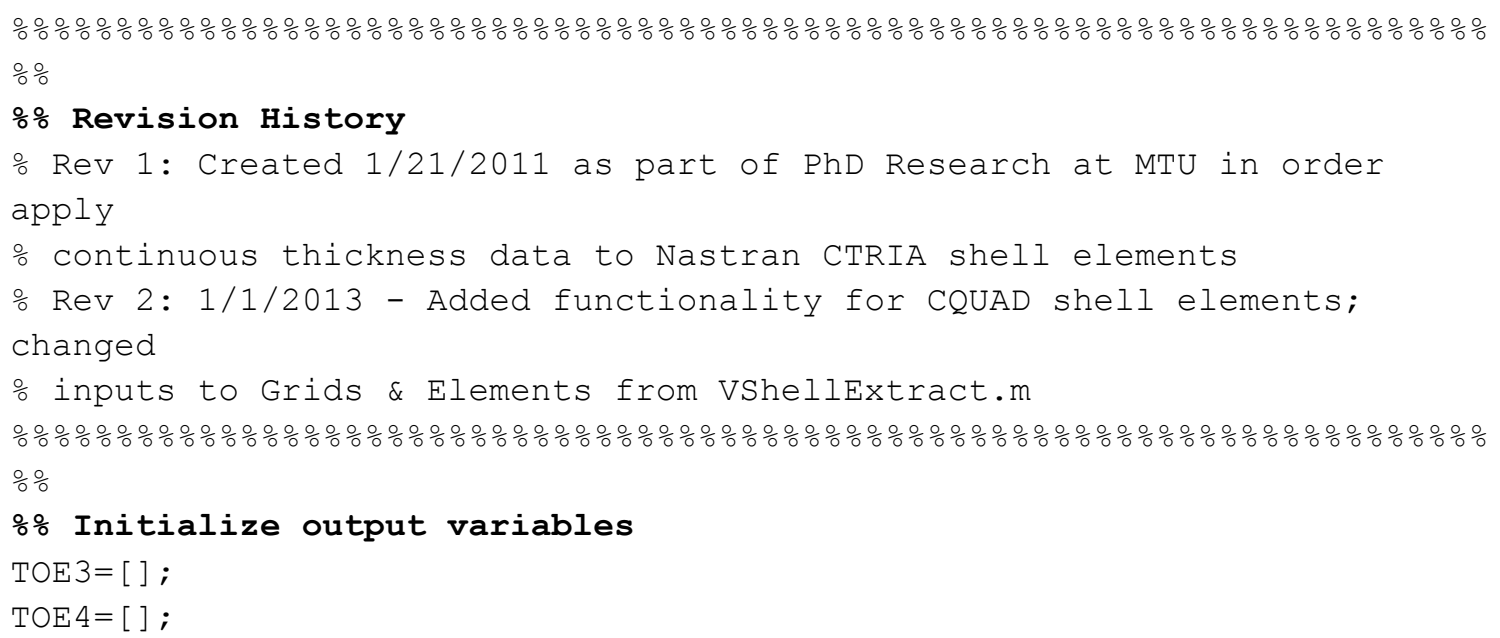

Michael Pyrkosz 


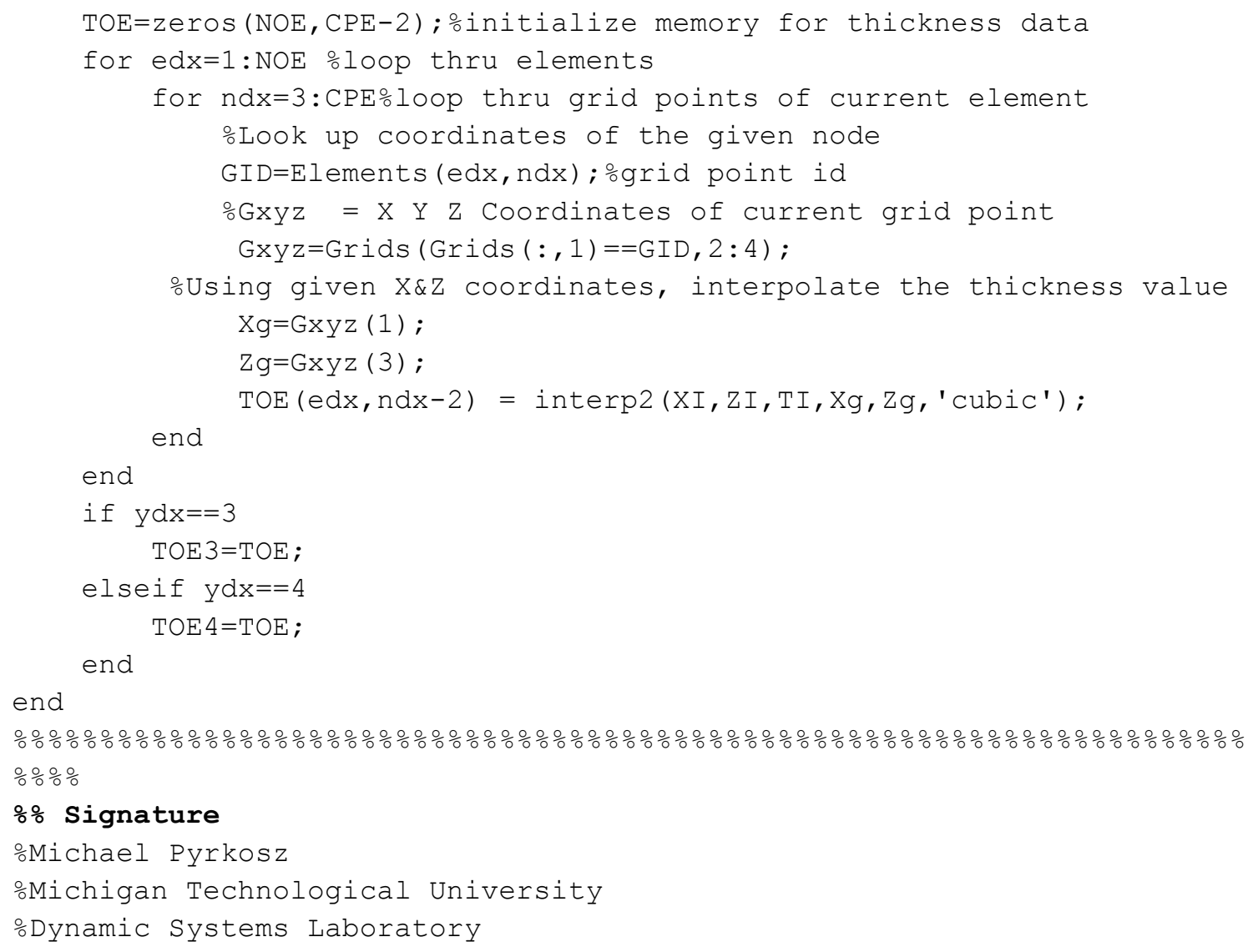

\section{D.6 VWoodMat}

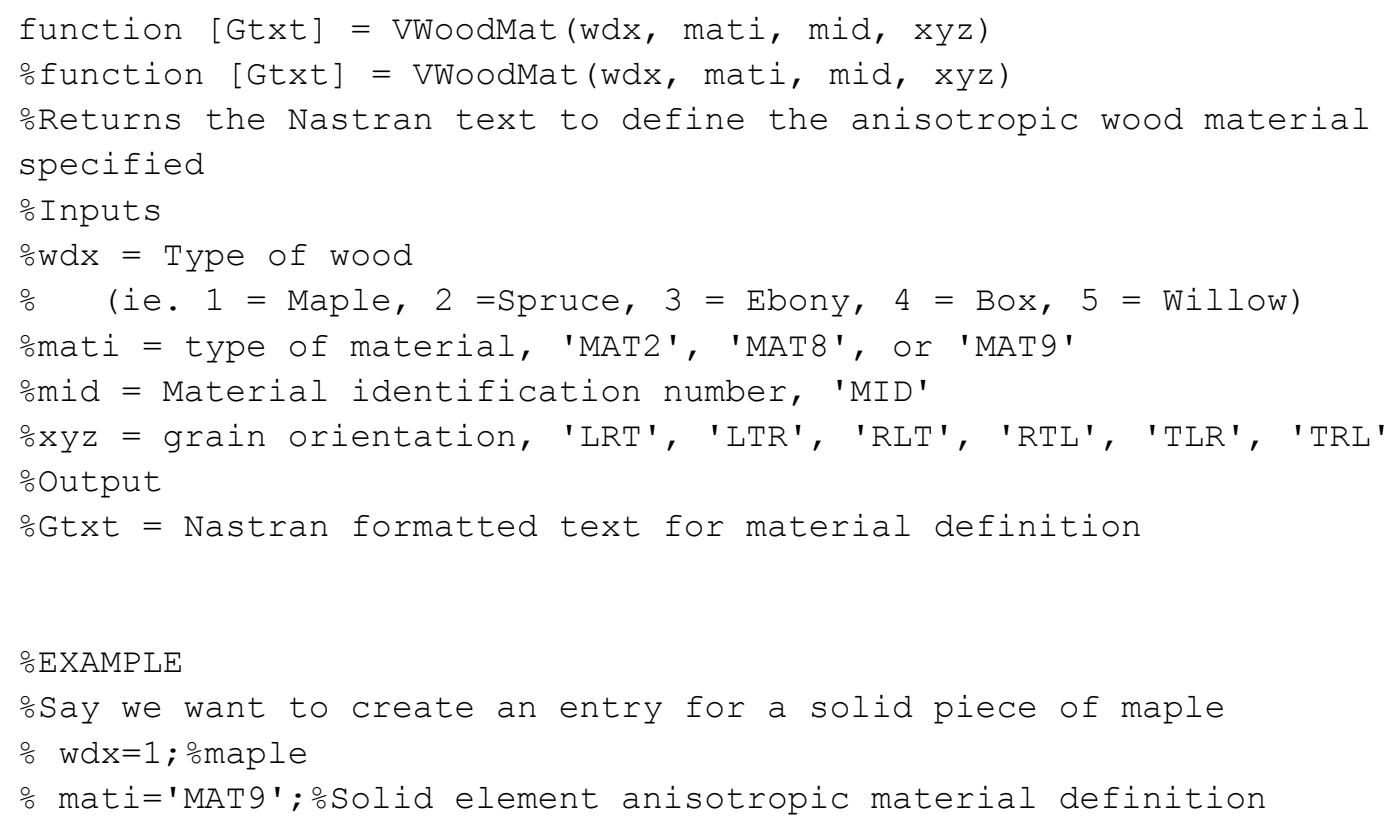




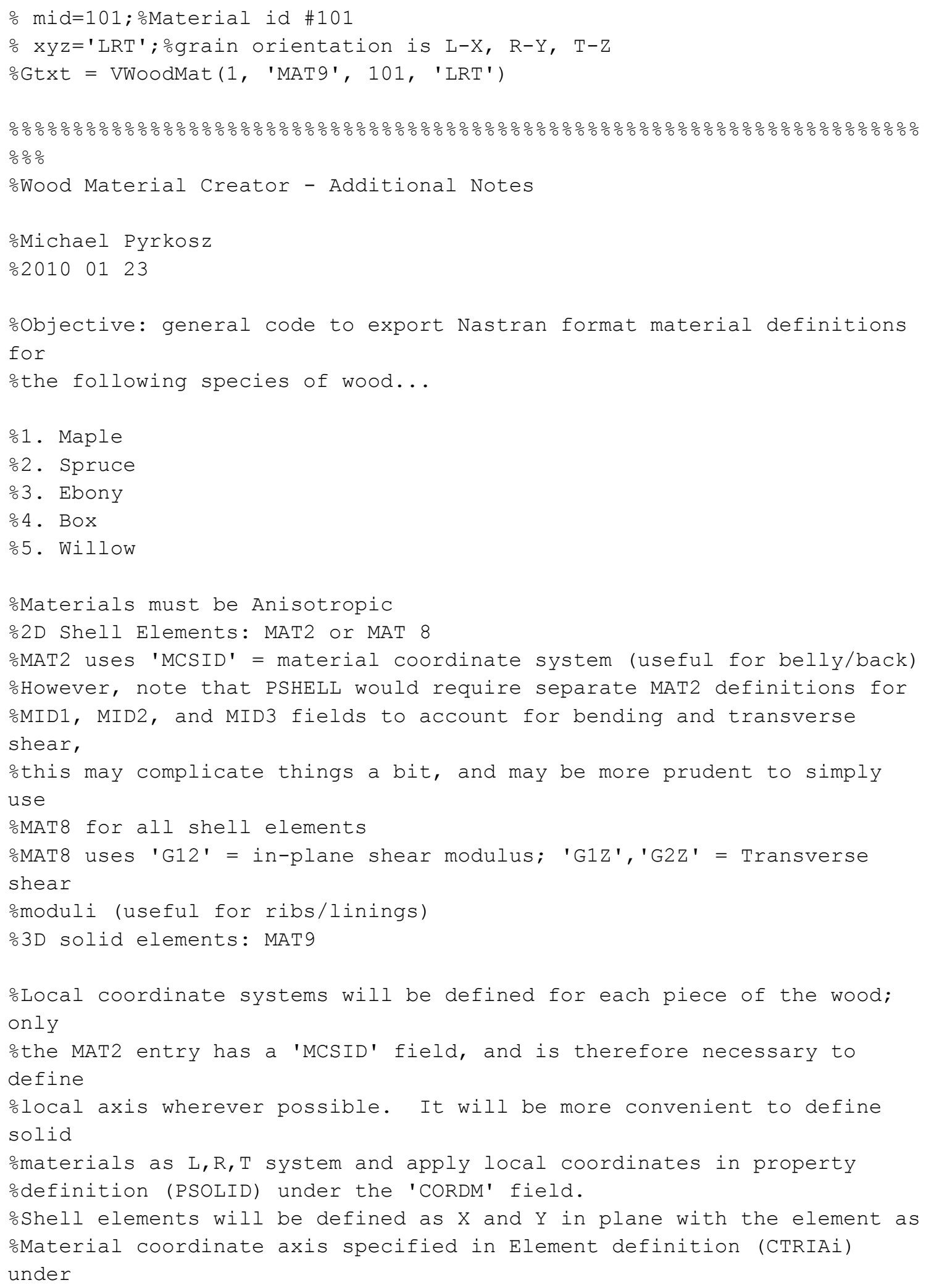




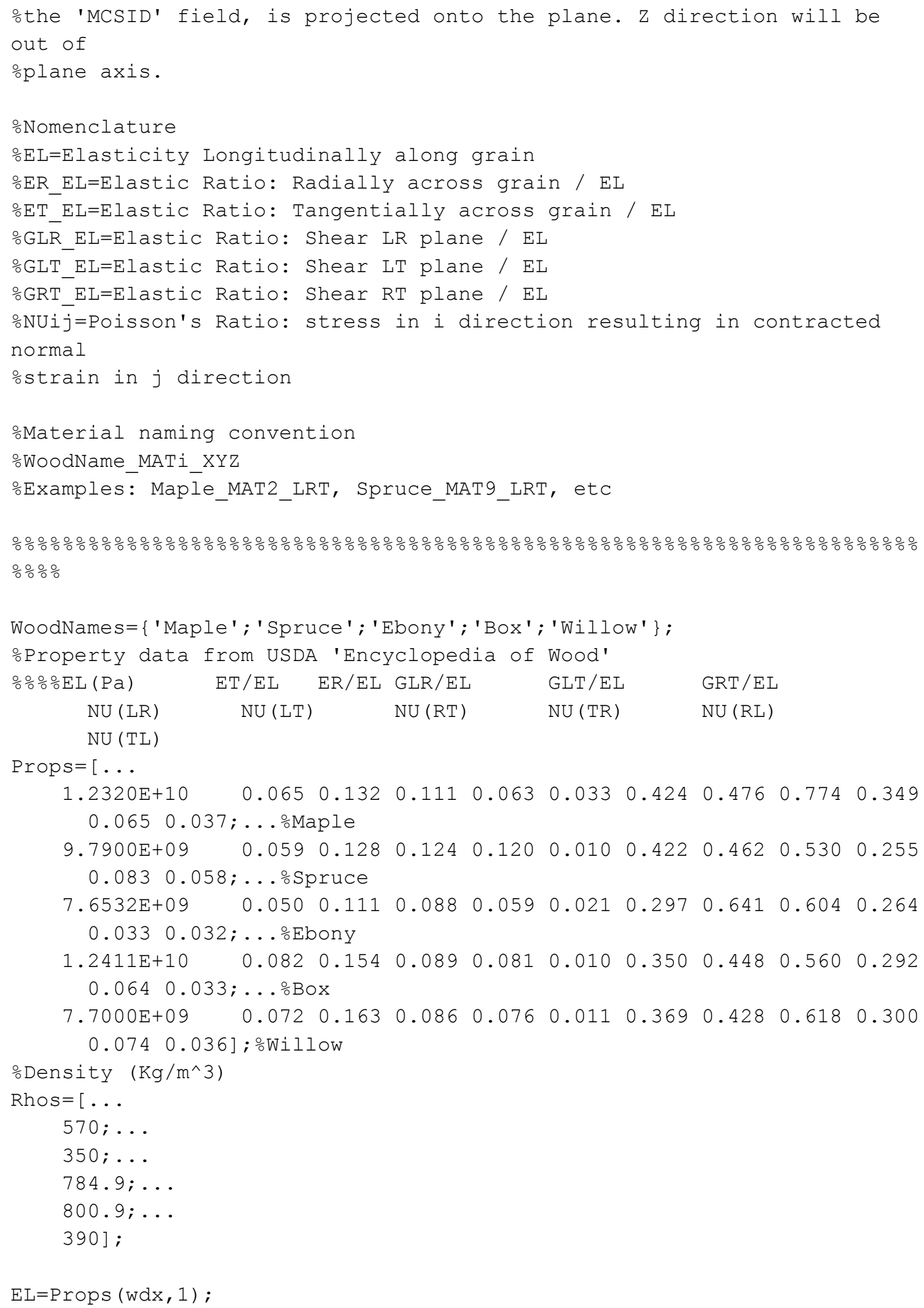




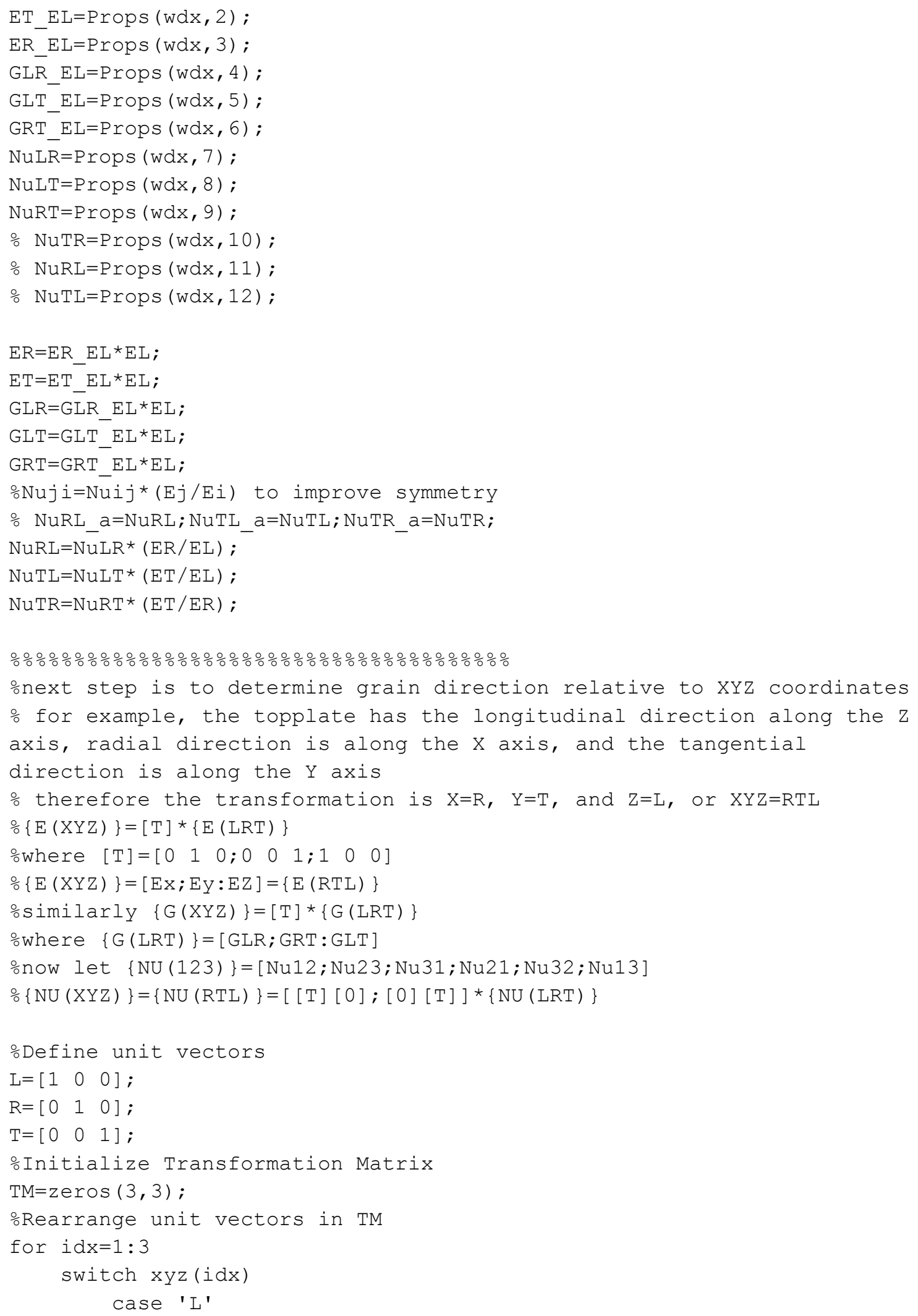




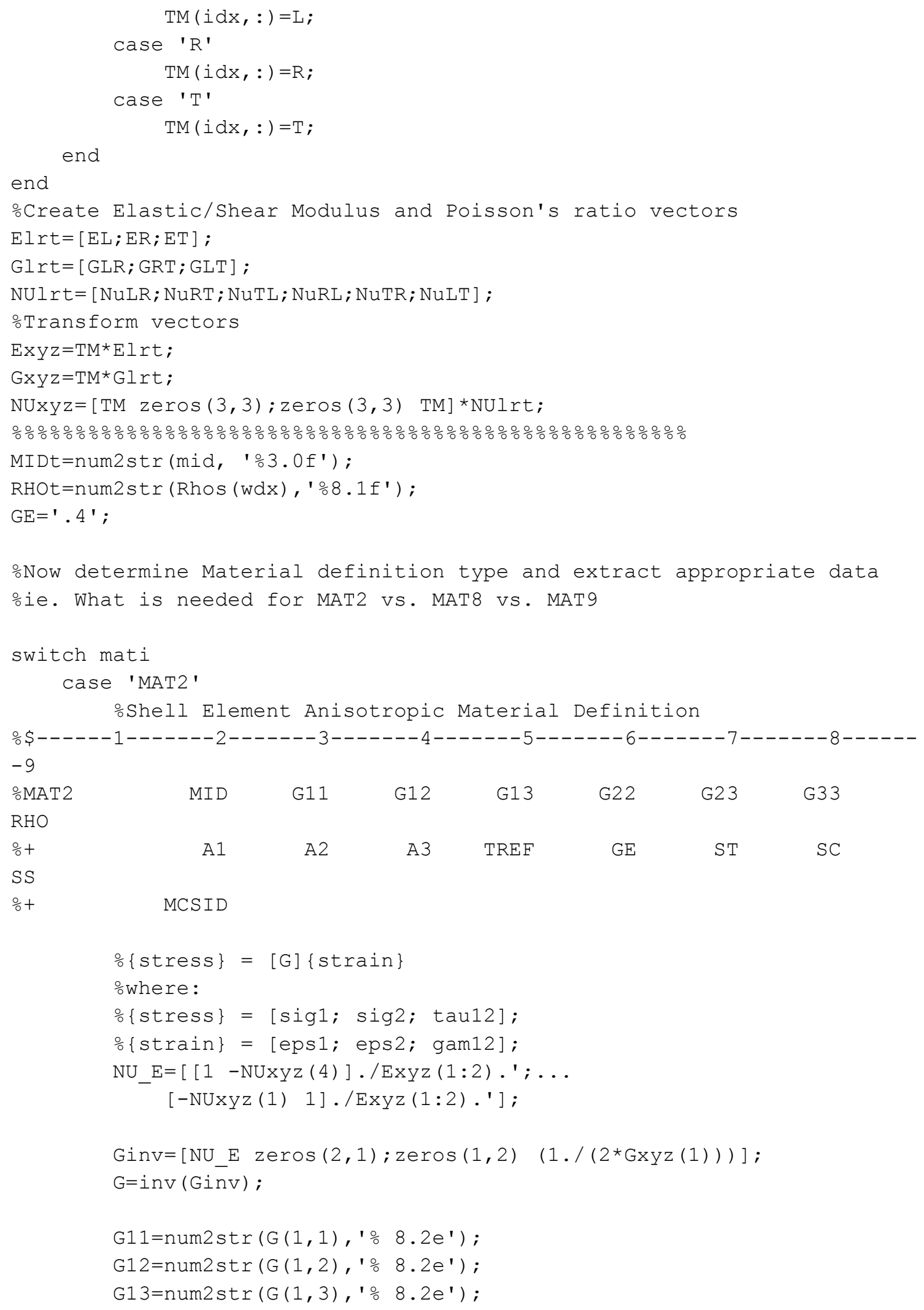




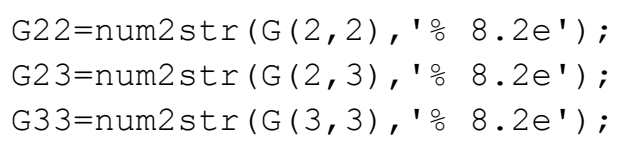




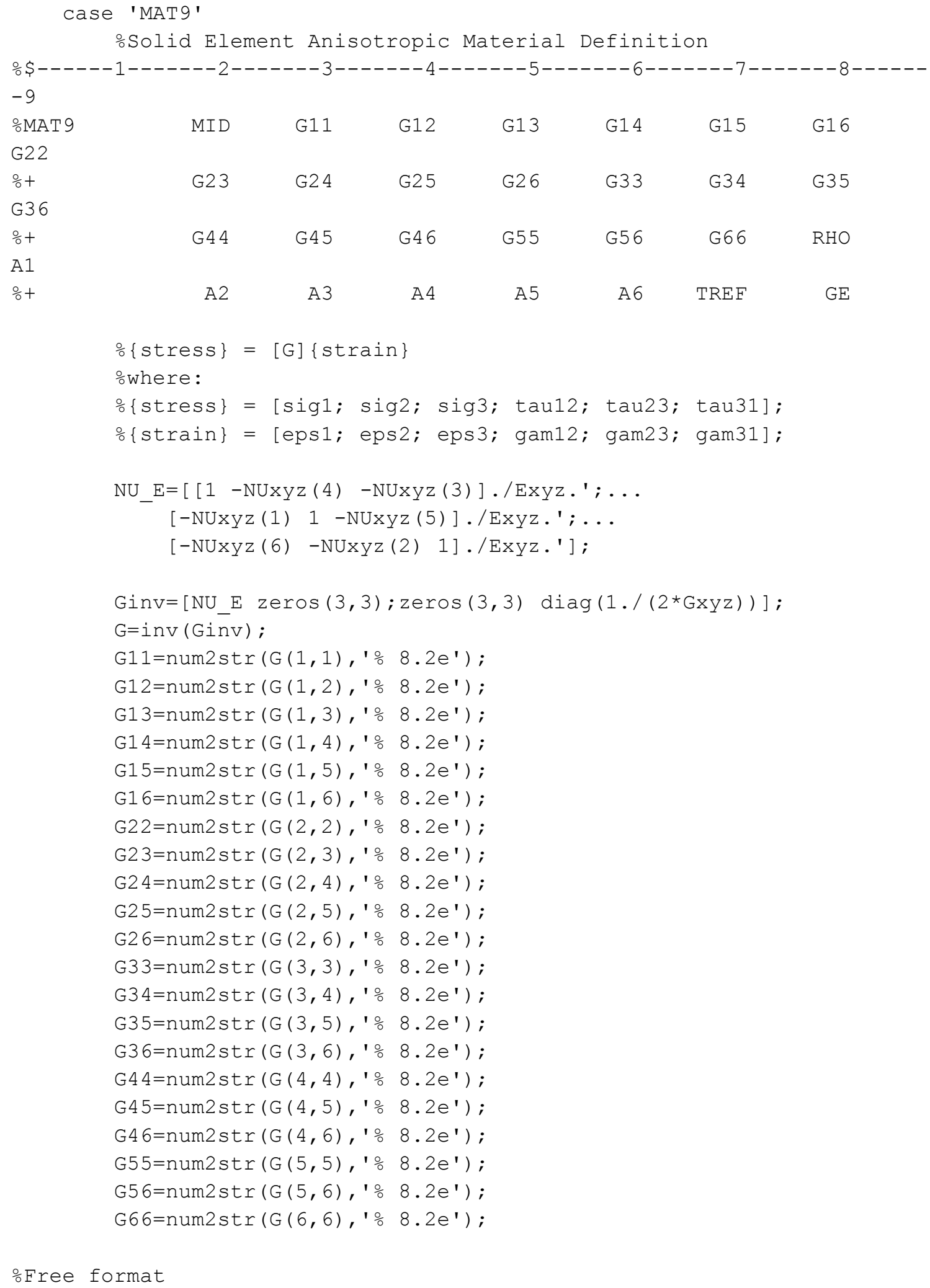




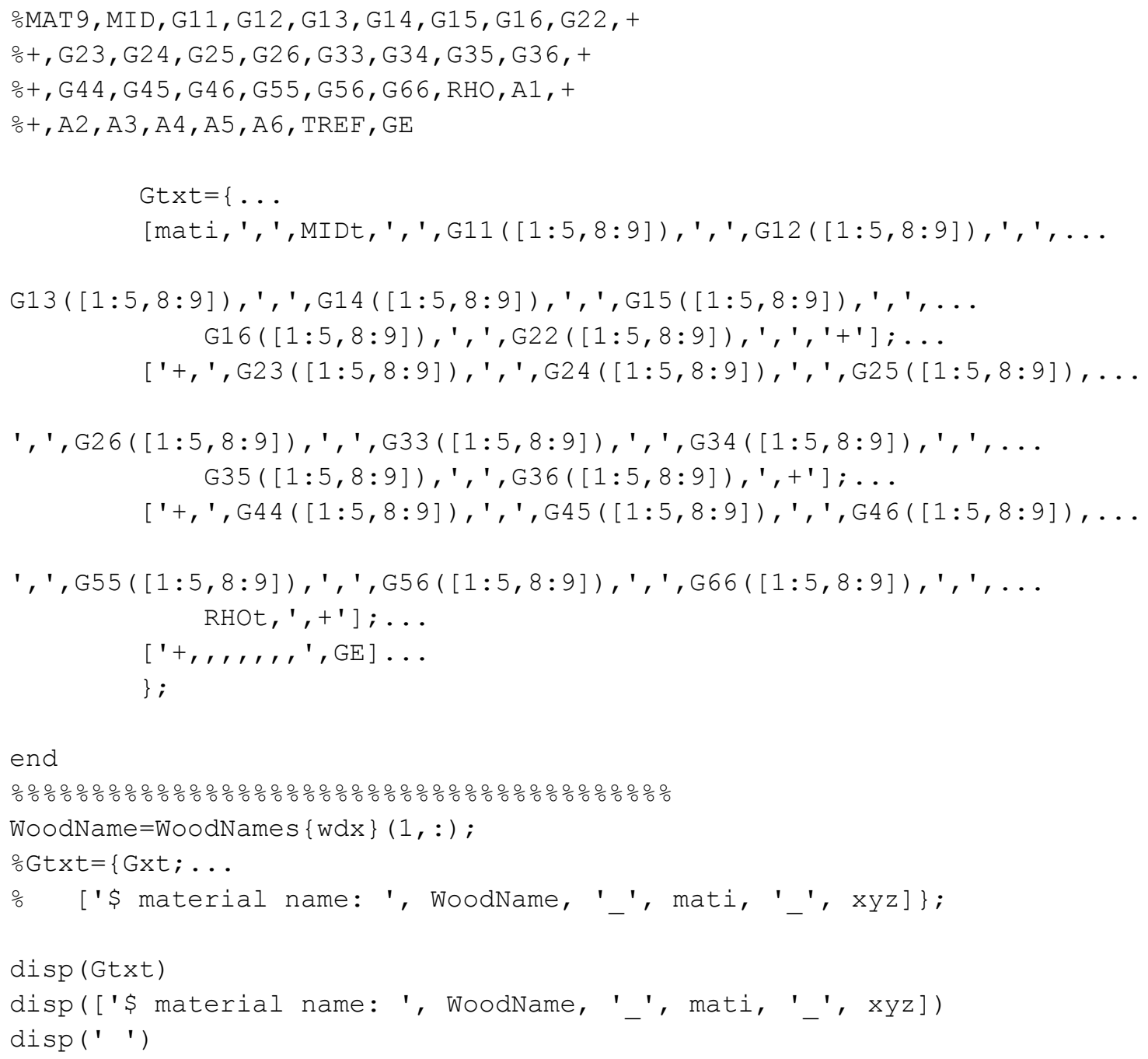




\section{Appendix E Test Support Drawings}

This appendix contains all technical drawings for various test items. This includes the Violin Experimental Modal Analysis (VEMA) stand, the hammer support arm (for hammer with flexible handle), the swing hammer adapter (for hammer with rigid handle), and the micro-hammer tip. 


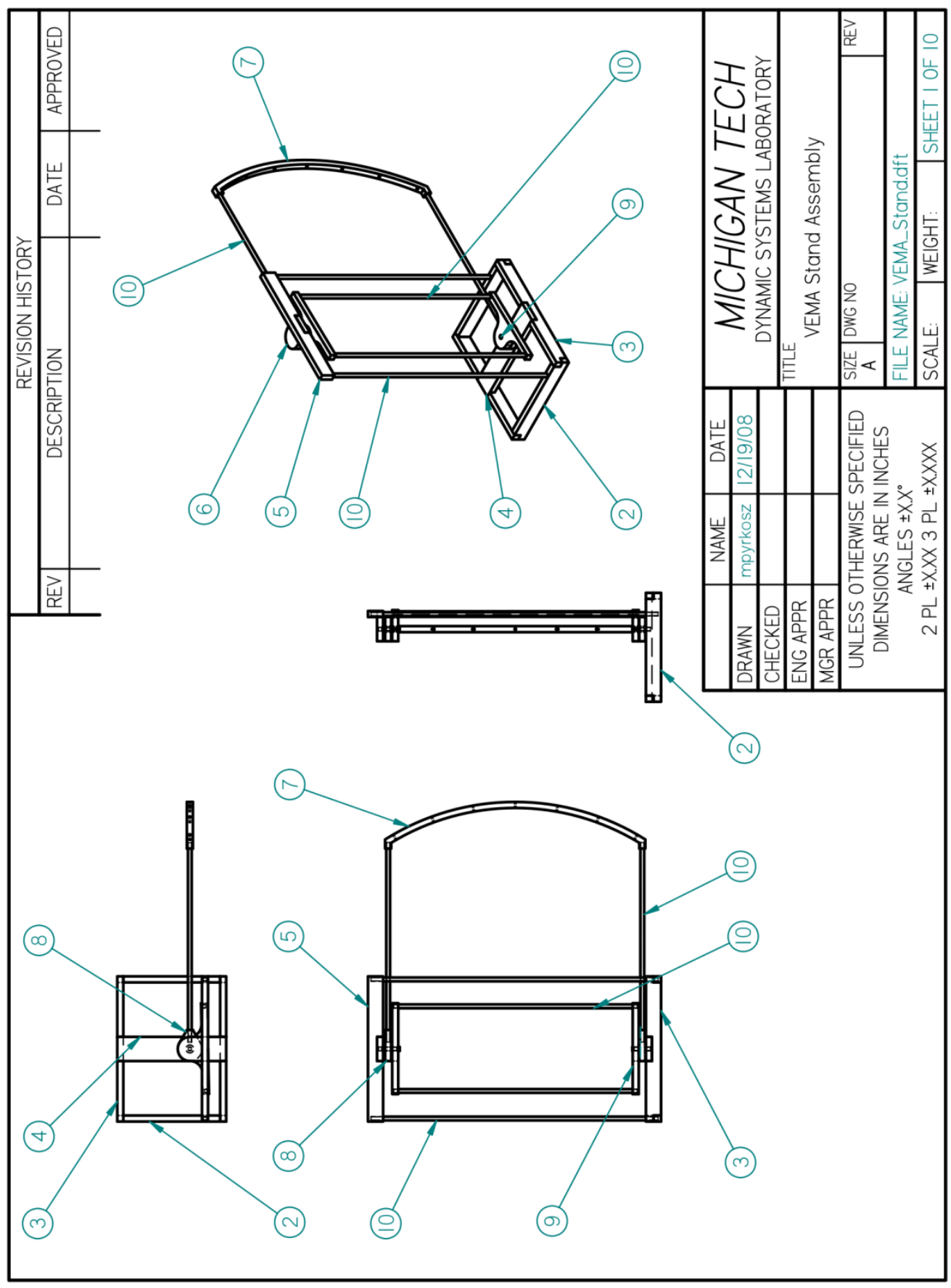

Michael Pyrkosz 


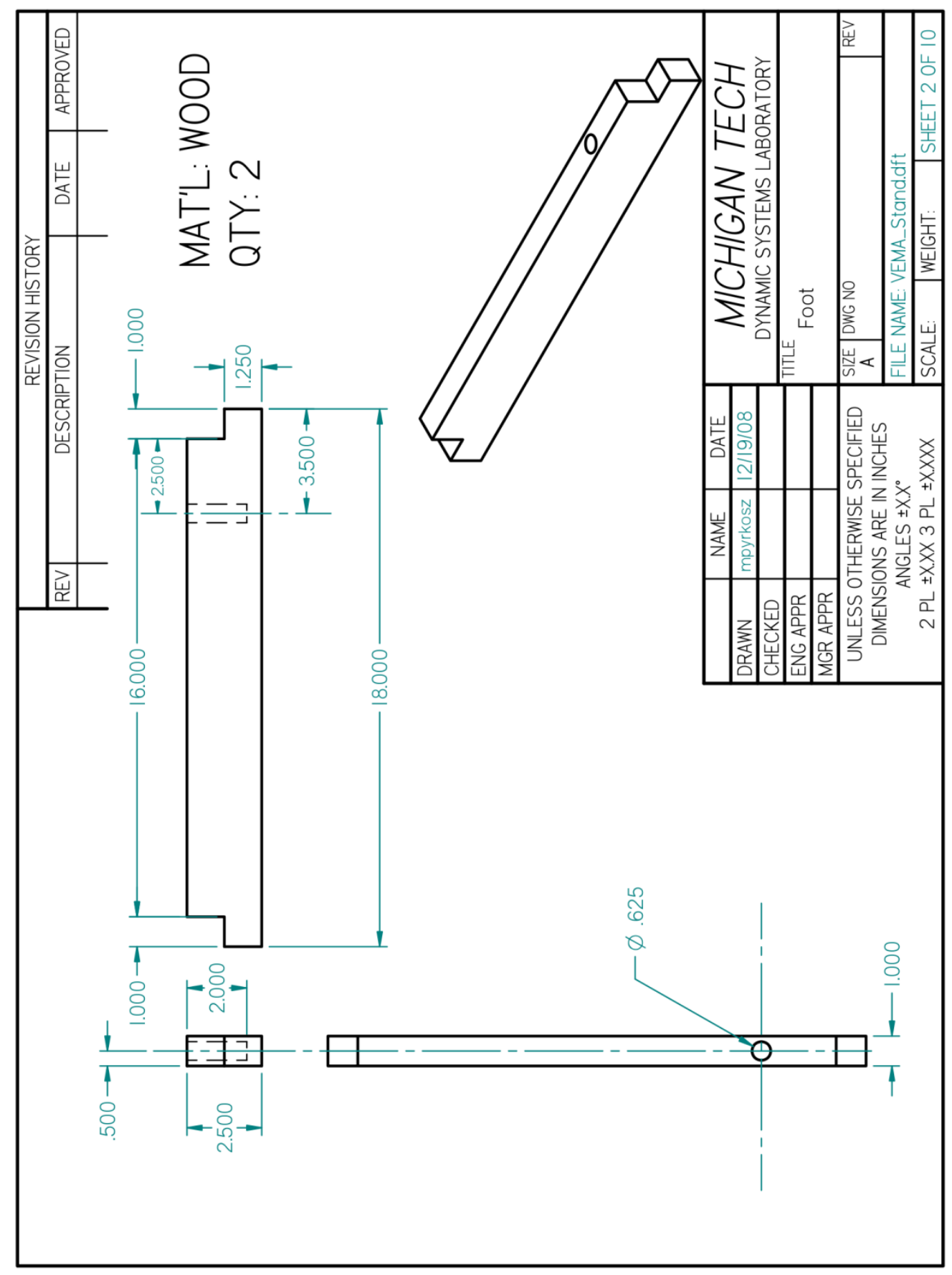

Michael Pyrkosz 


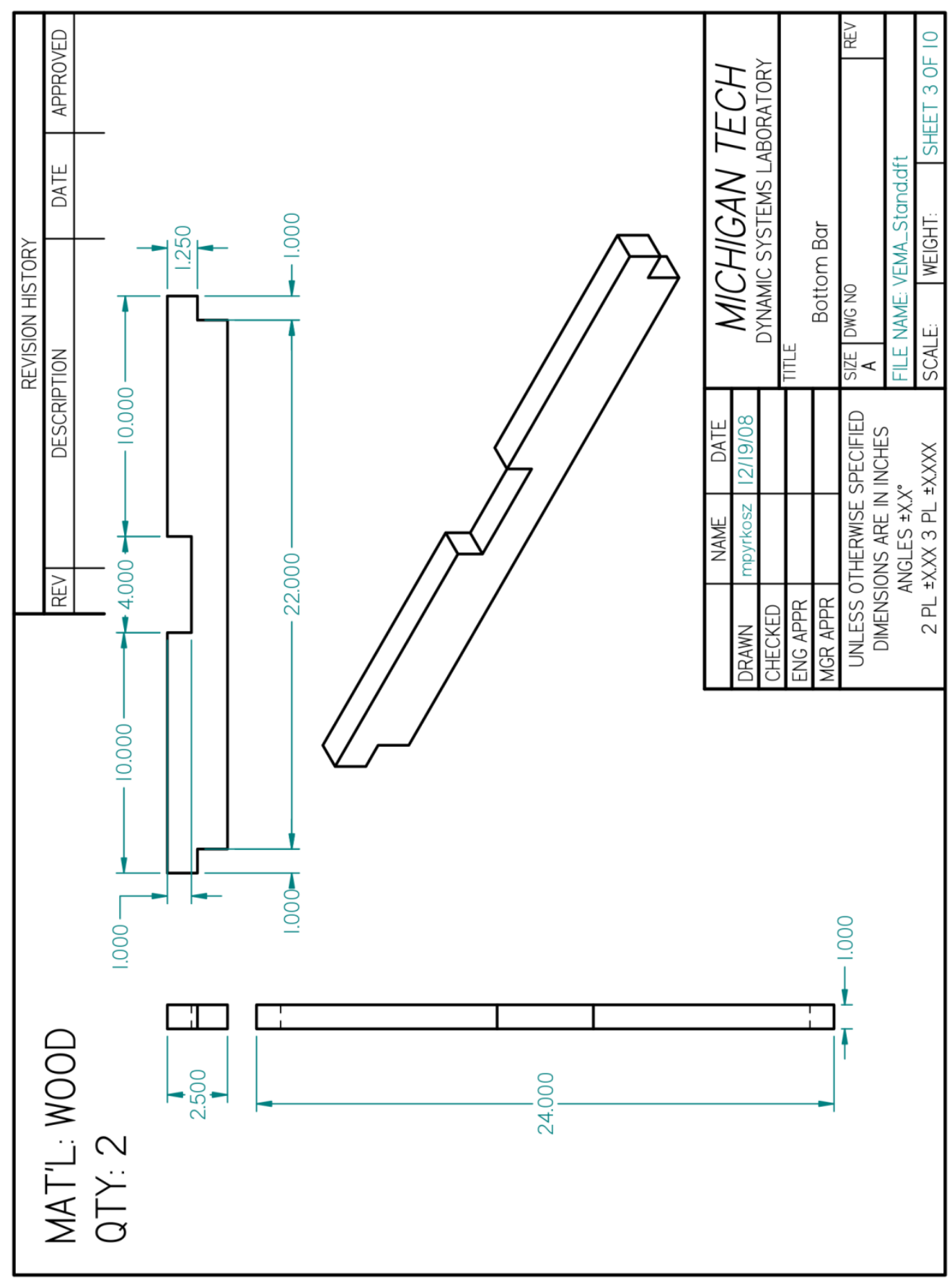




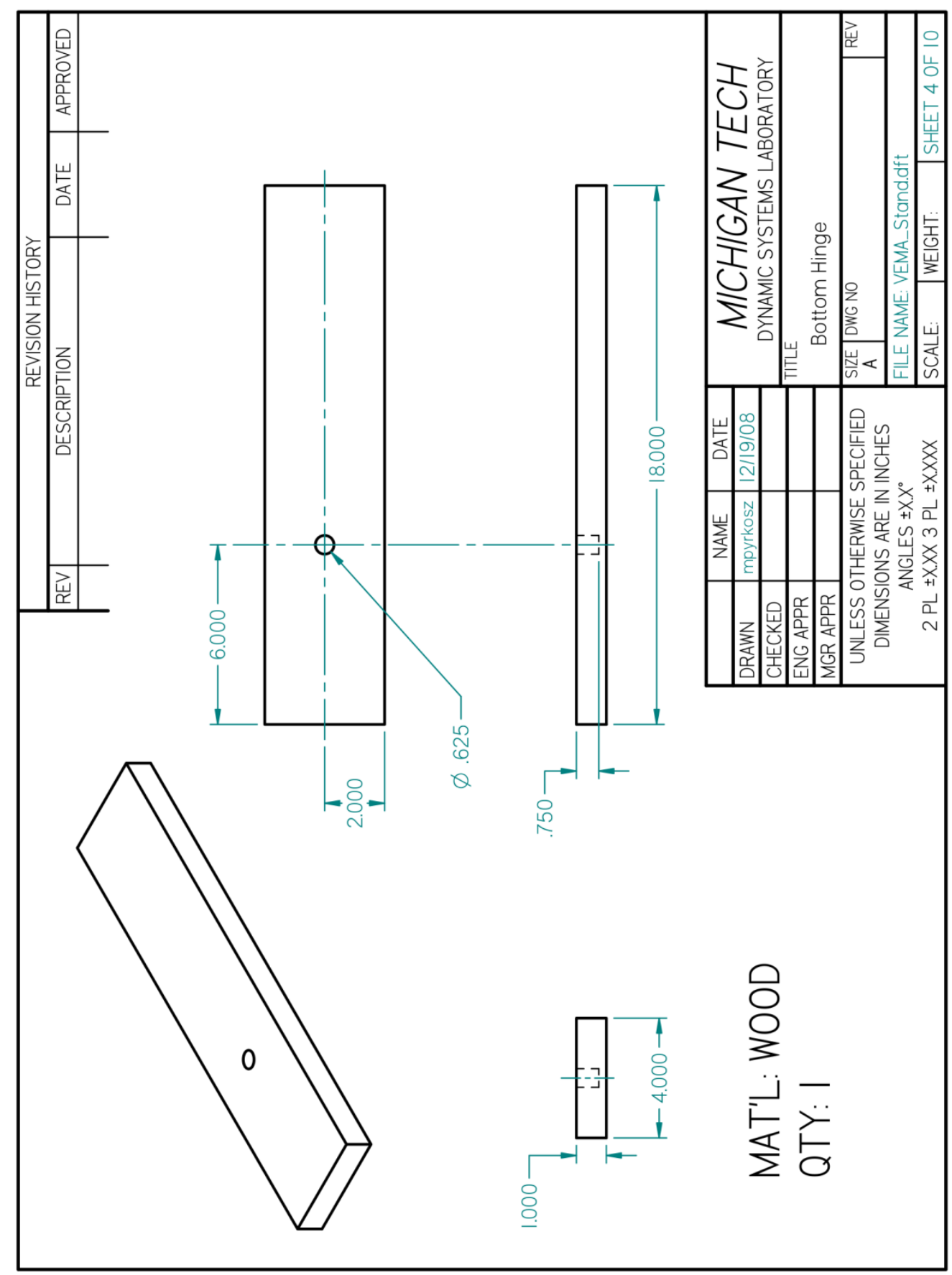

Michael Pyrkosz 


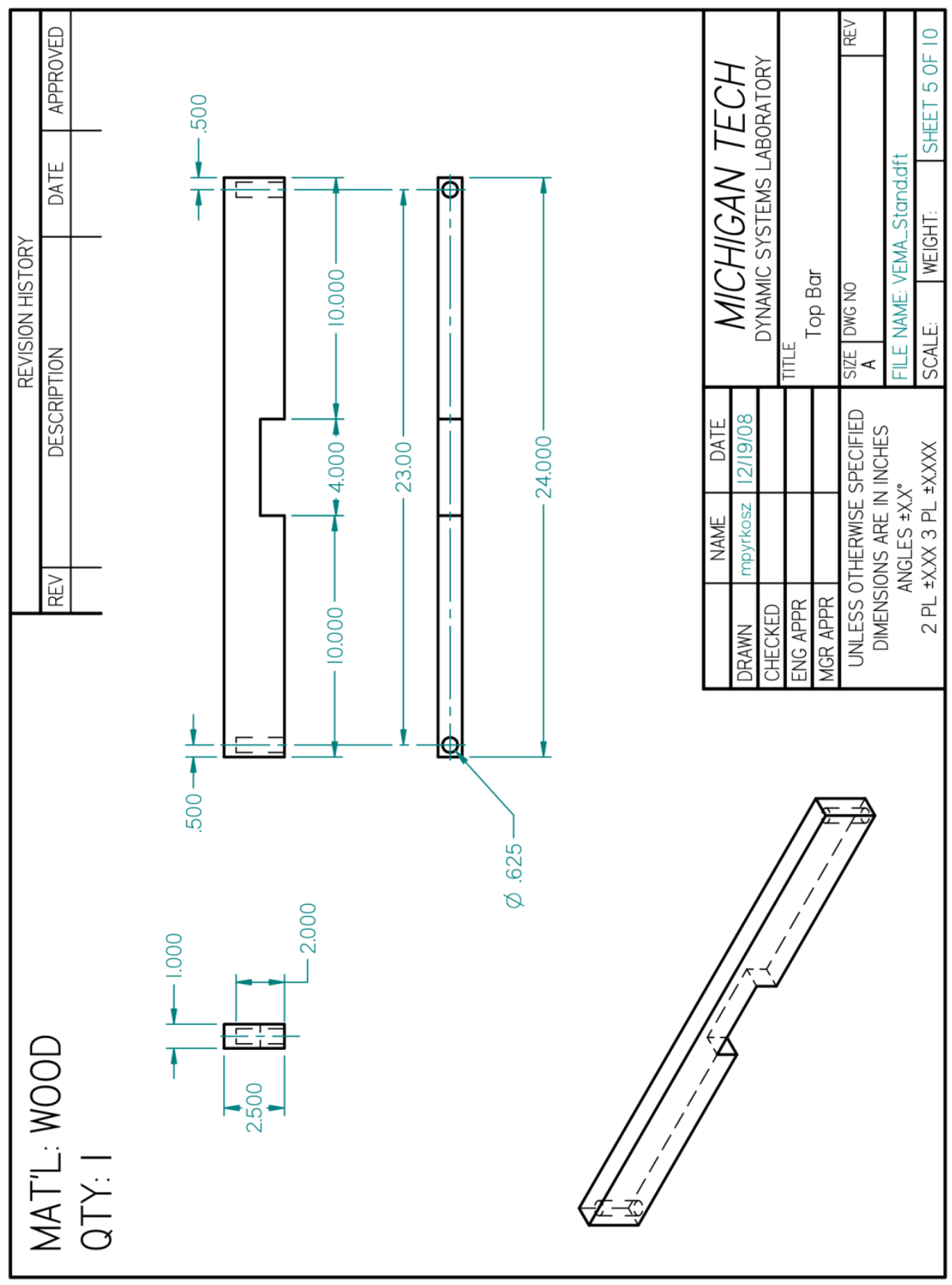




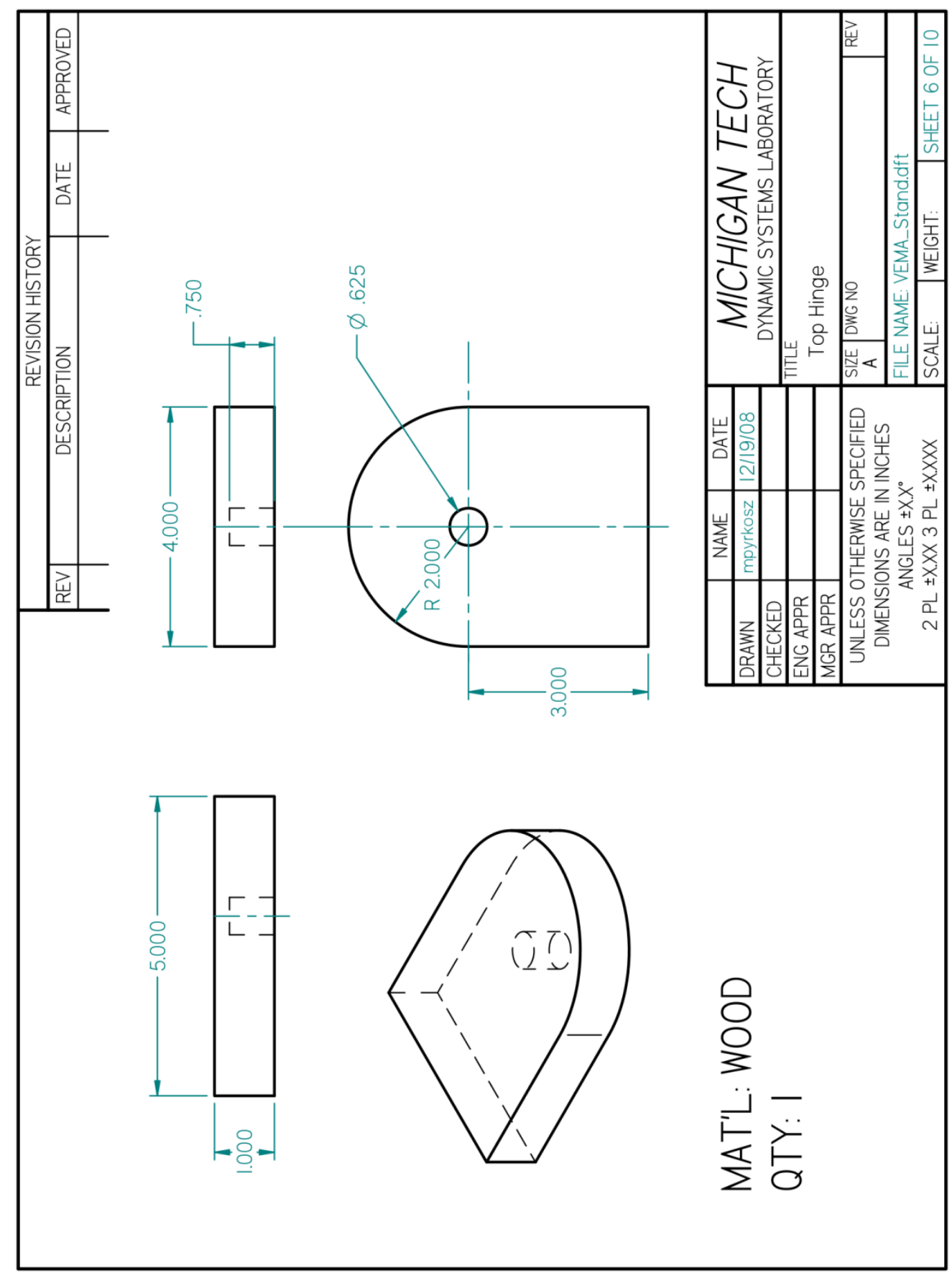

Michael Pyrkosz 


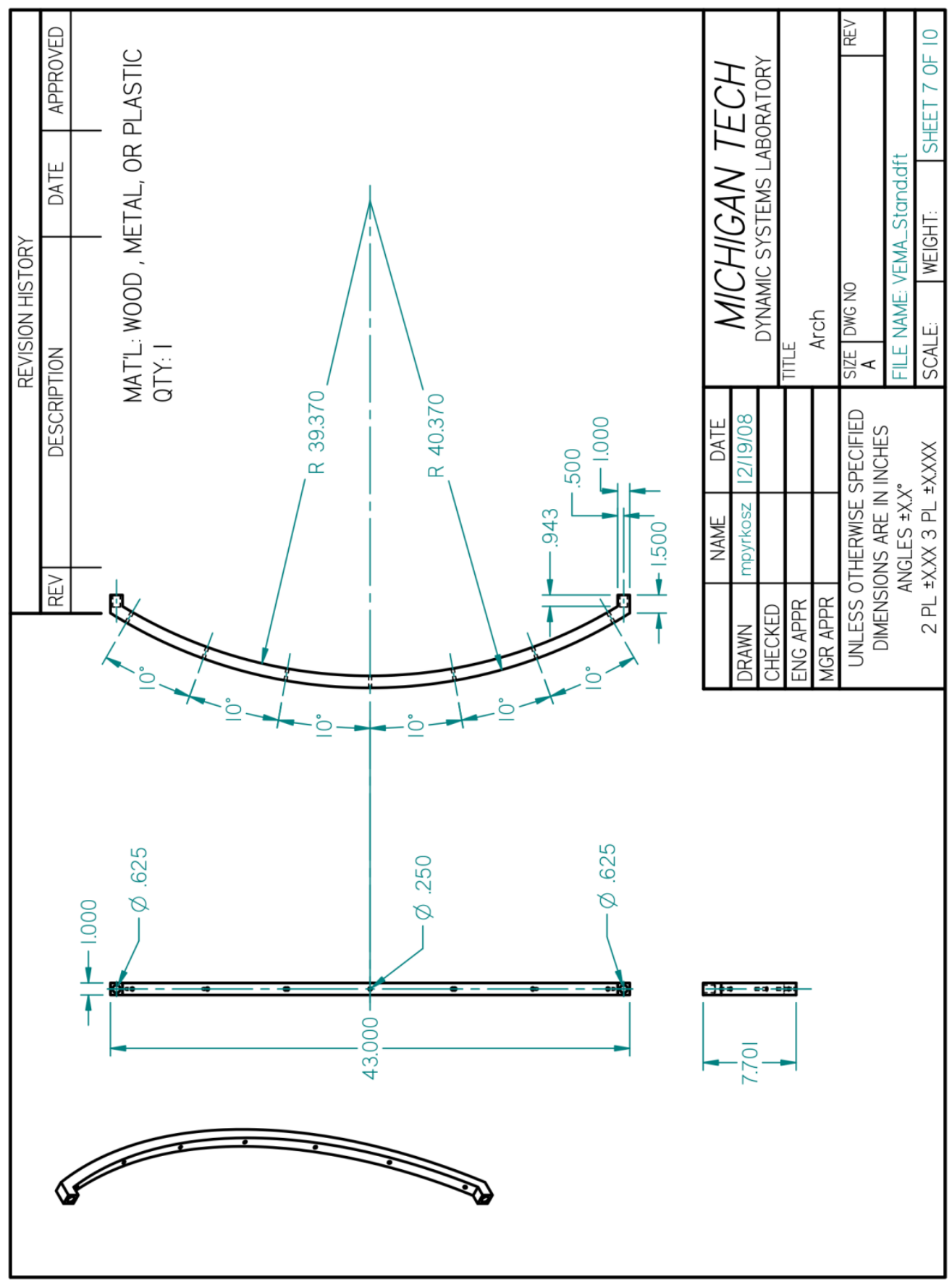




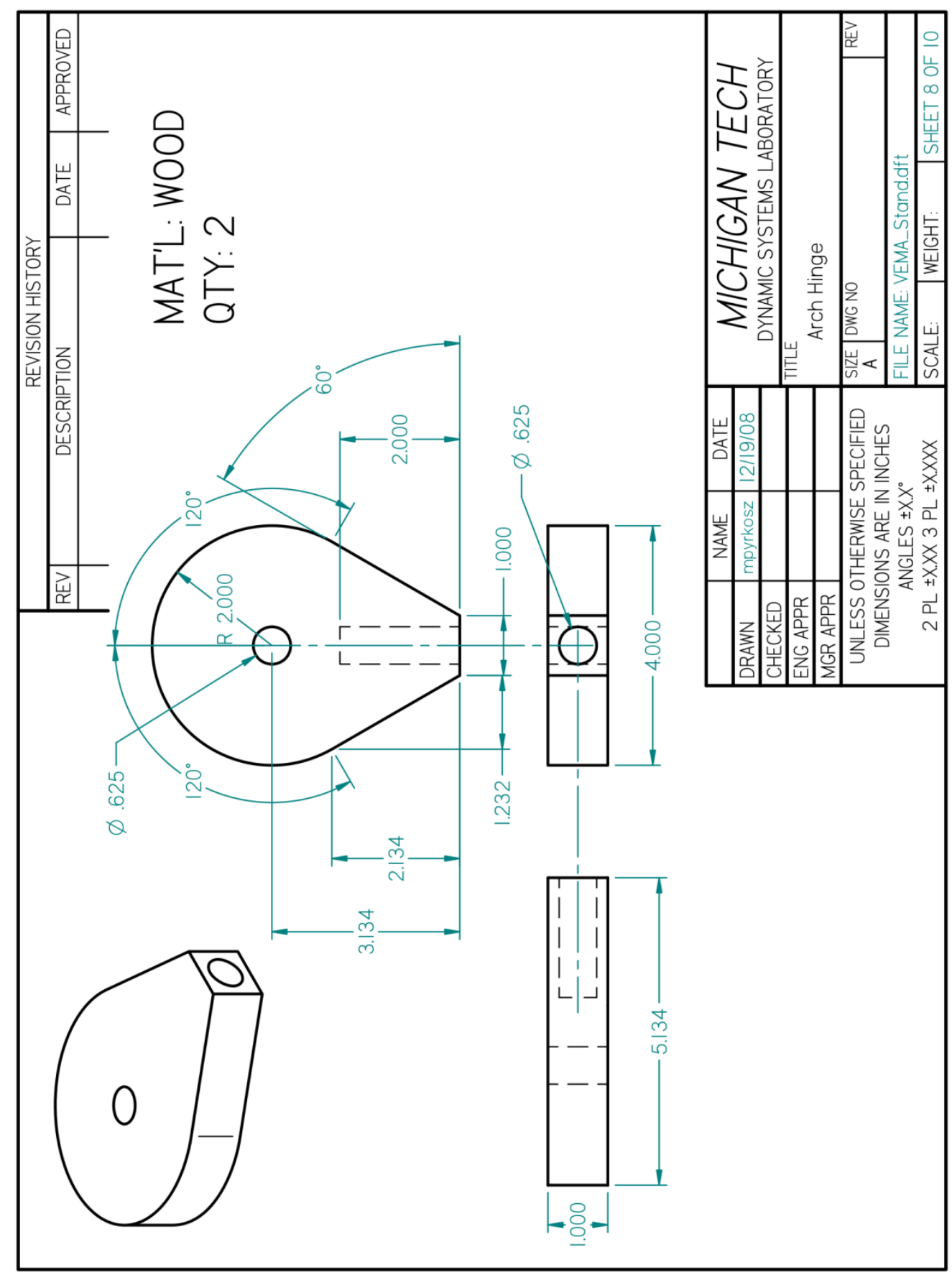

Michael Pyrkosz 


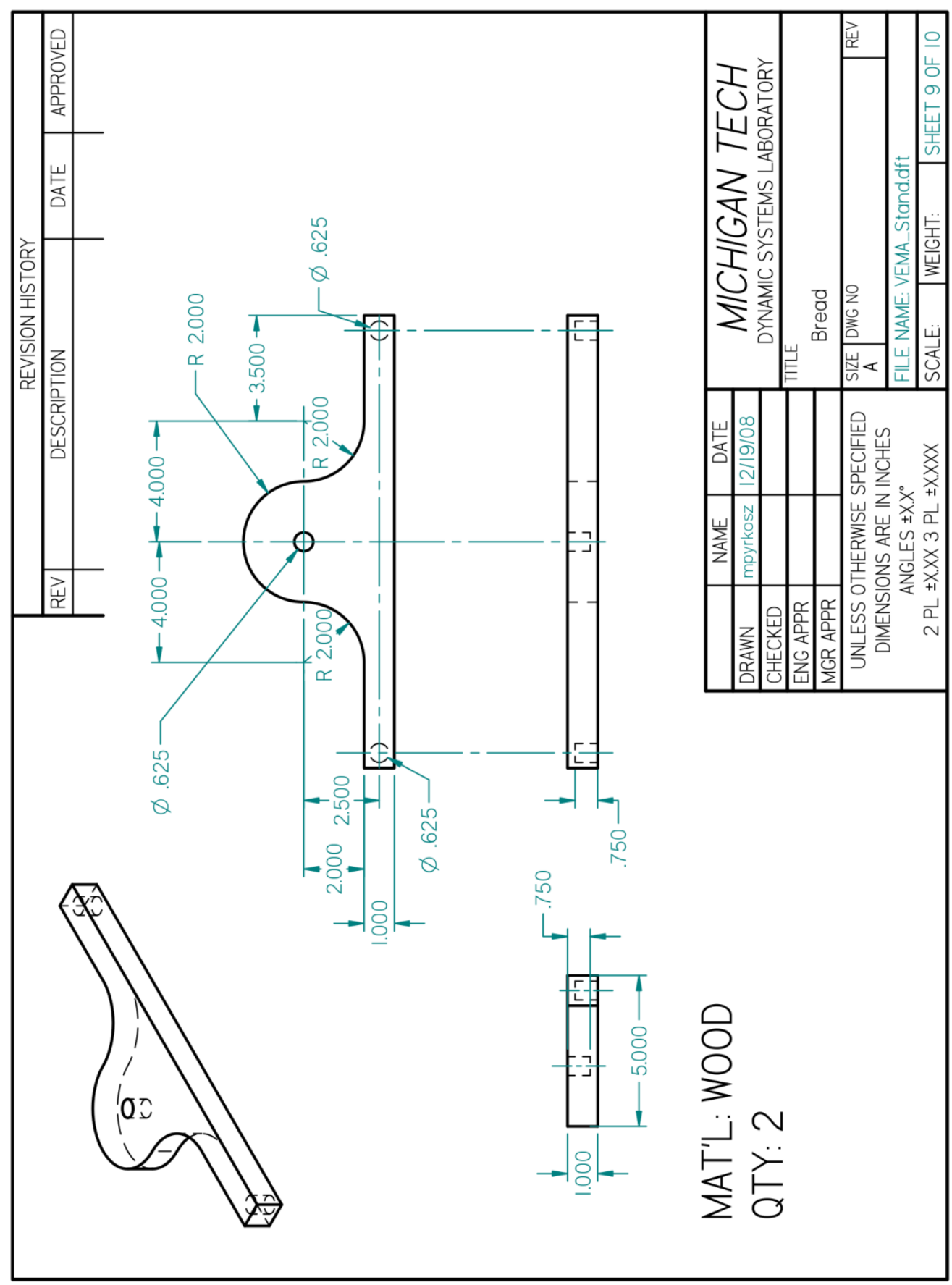




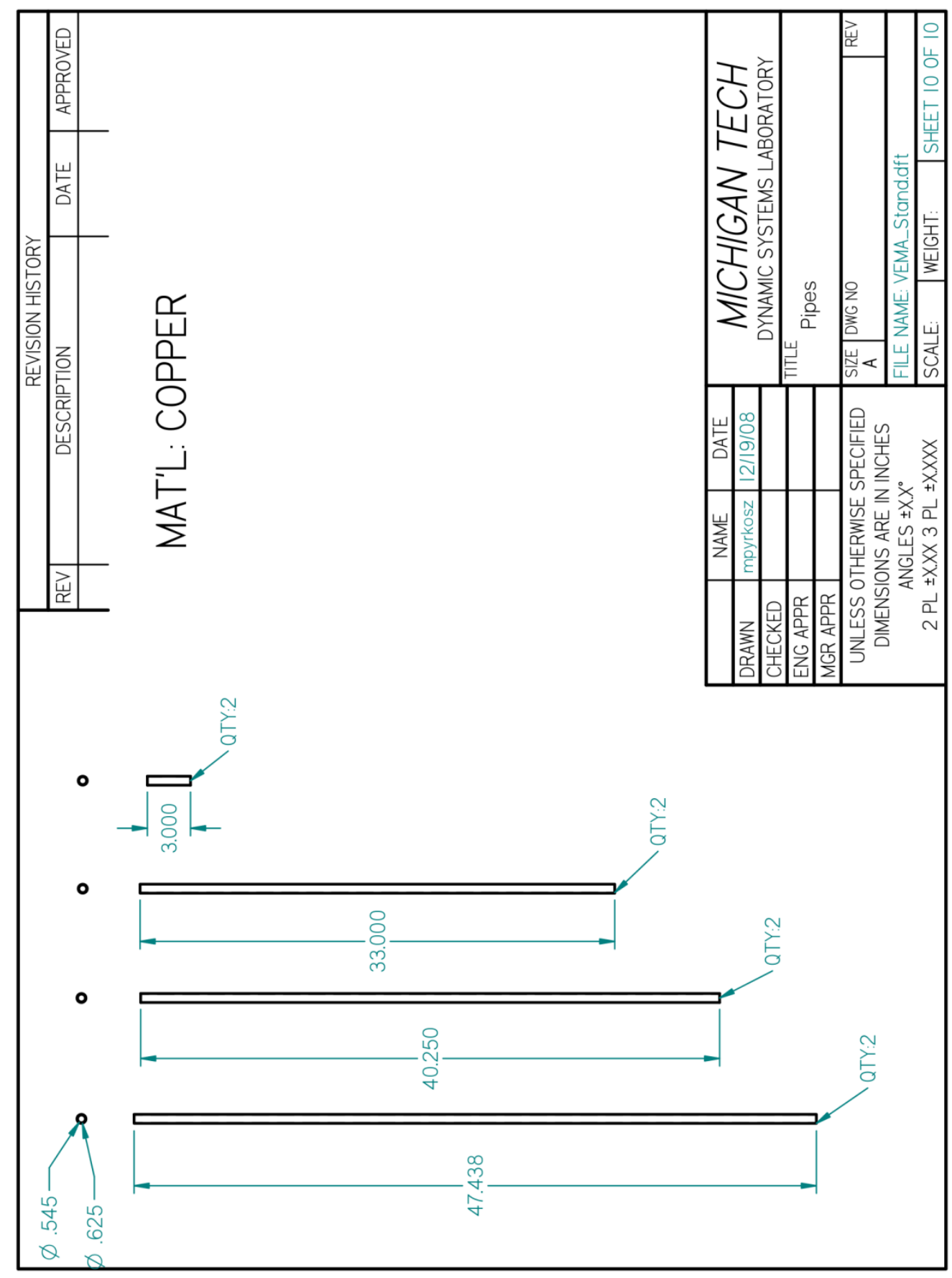

Michael Pyrkosz 


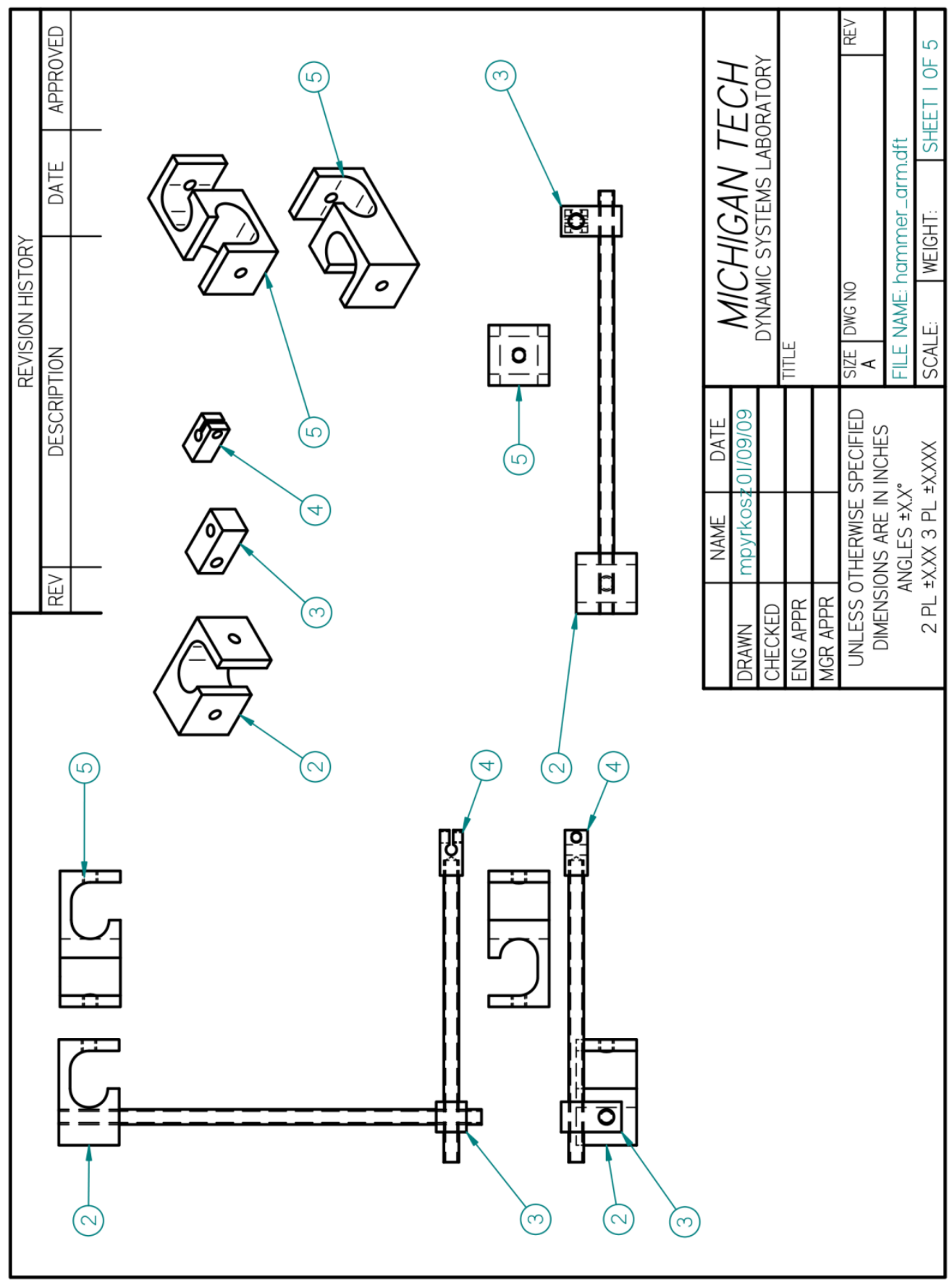




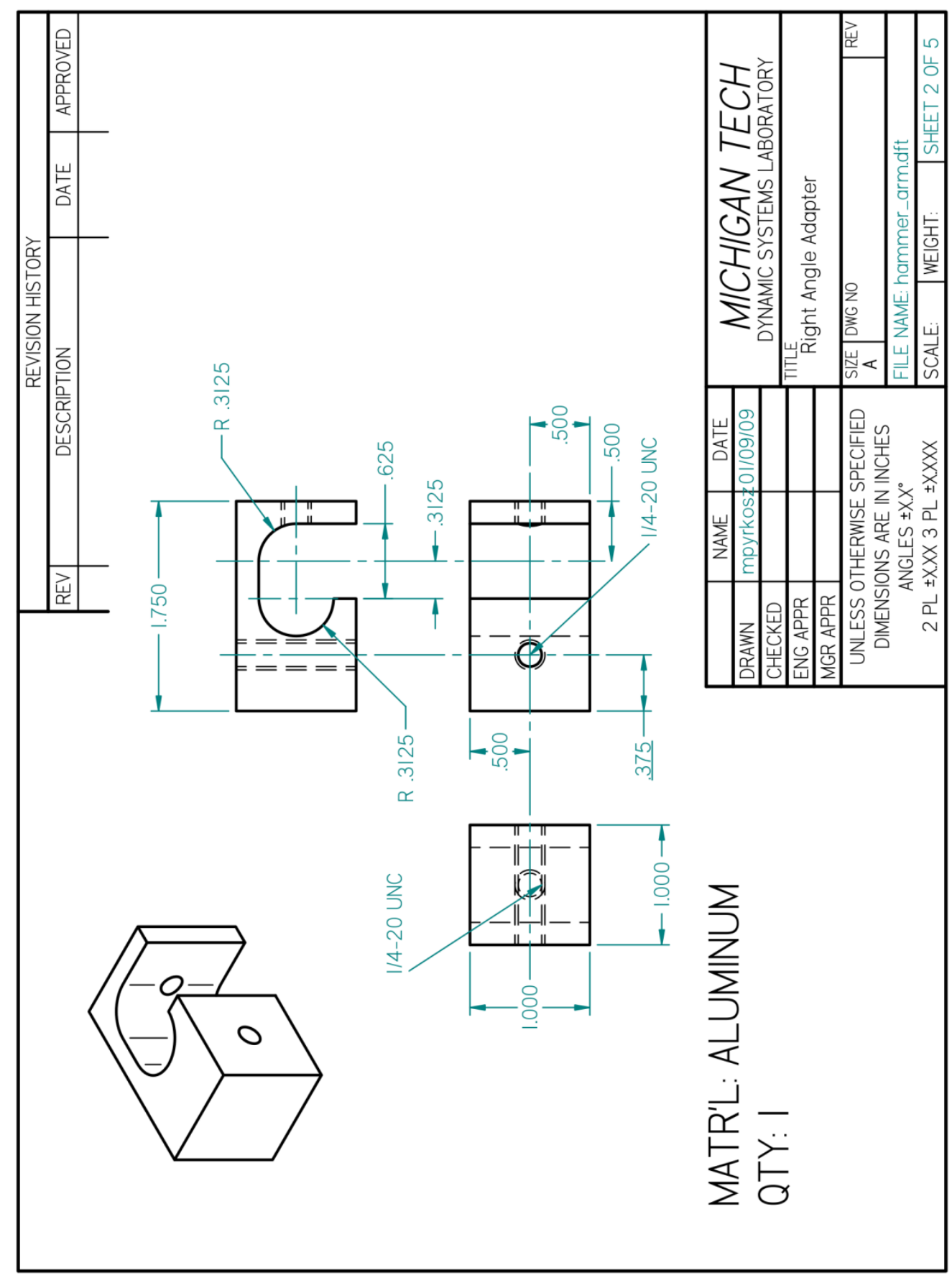

Michael Pyrkosz 


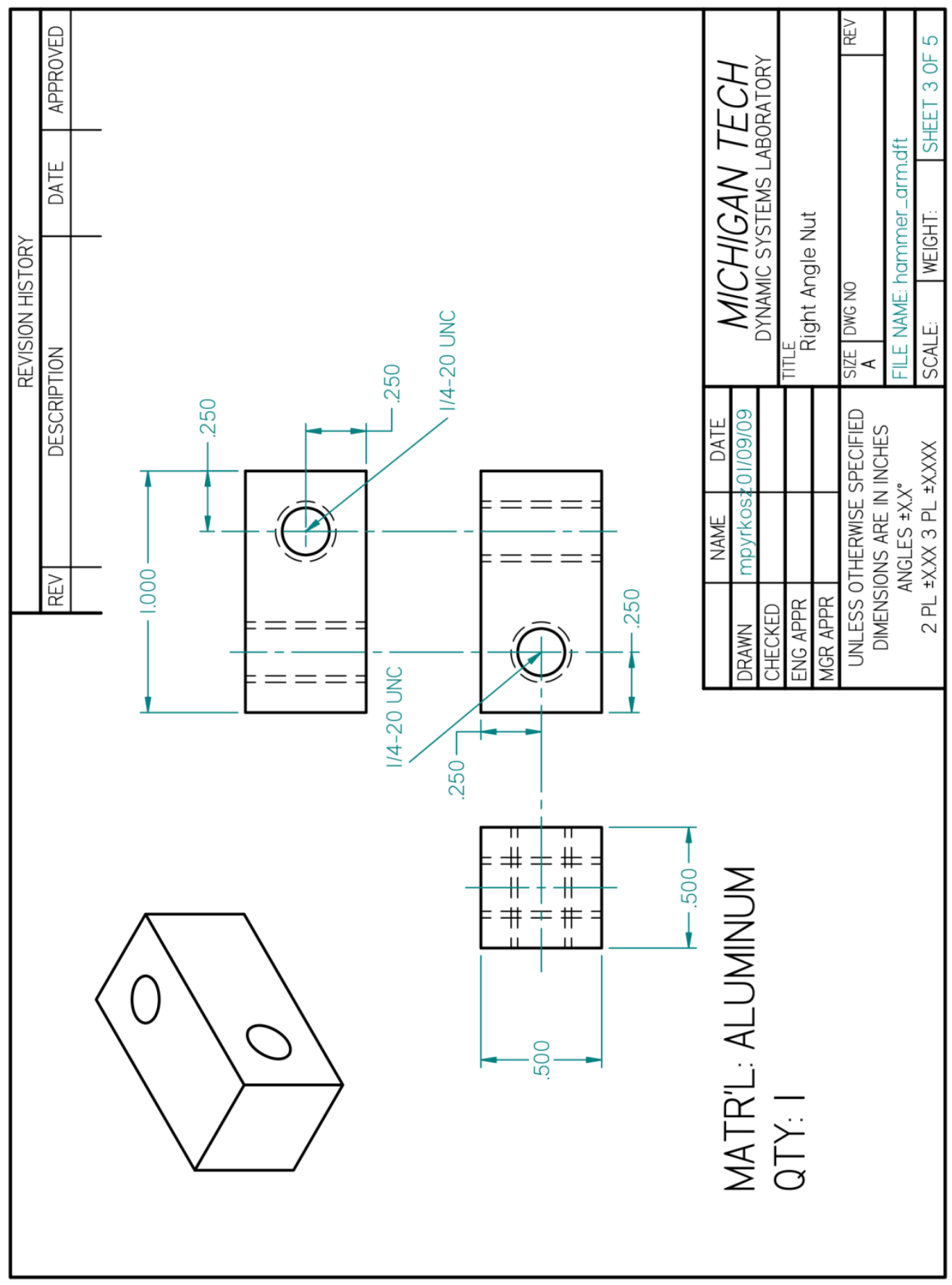




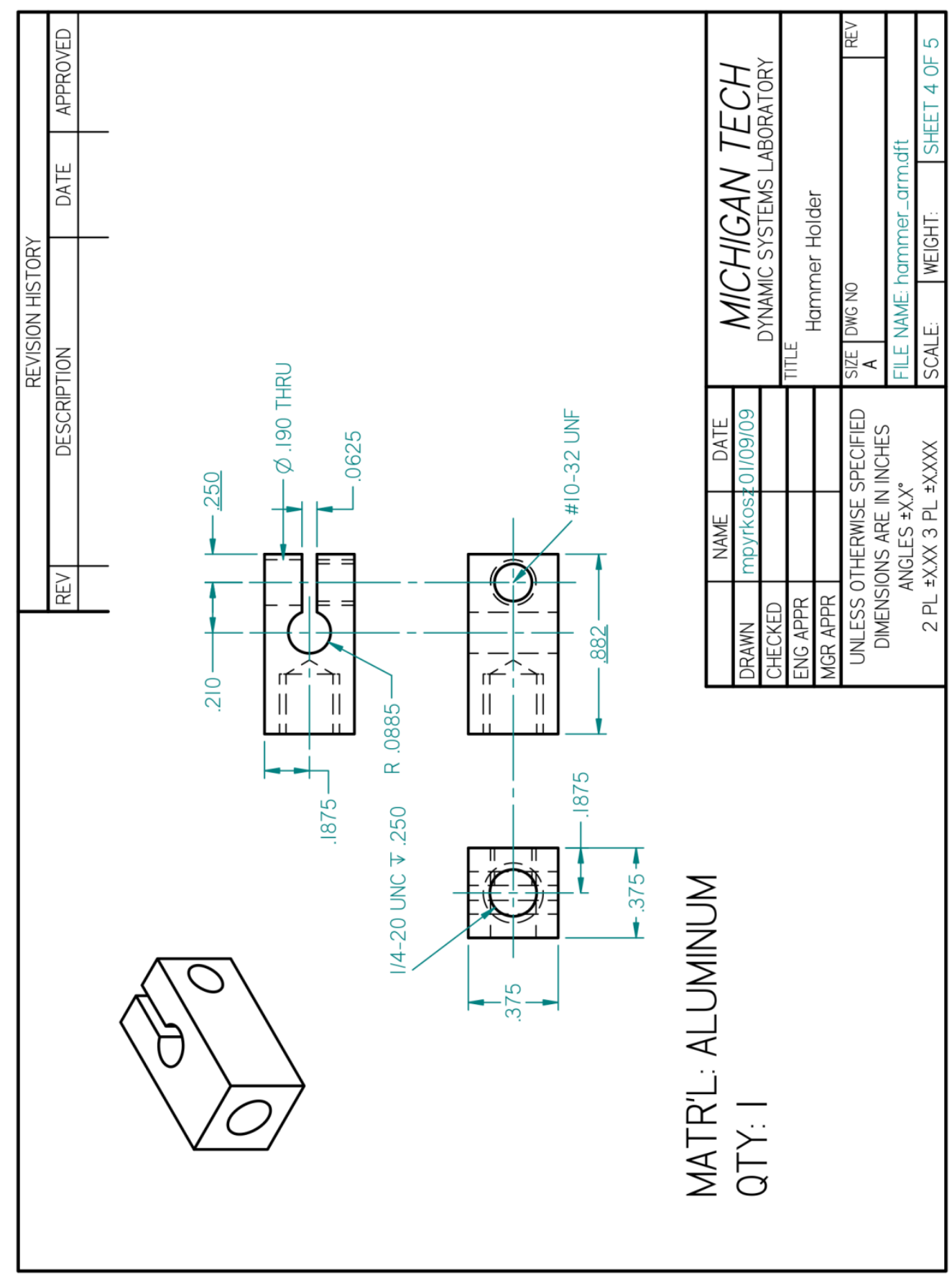

Michael Pyrkosz 


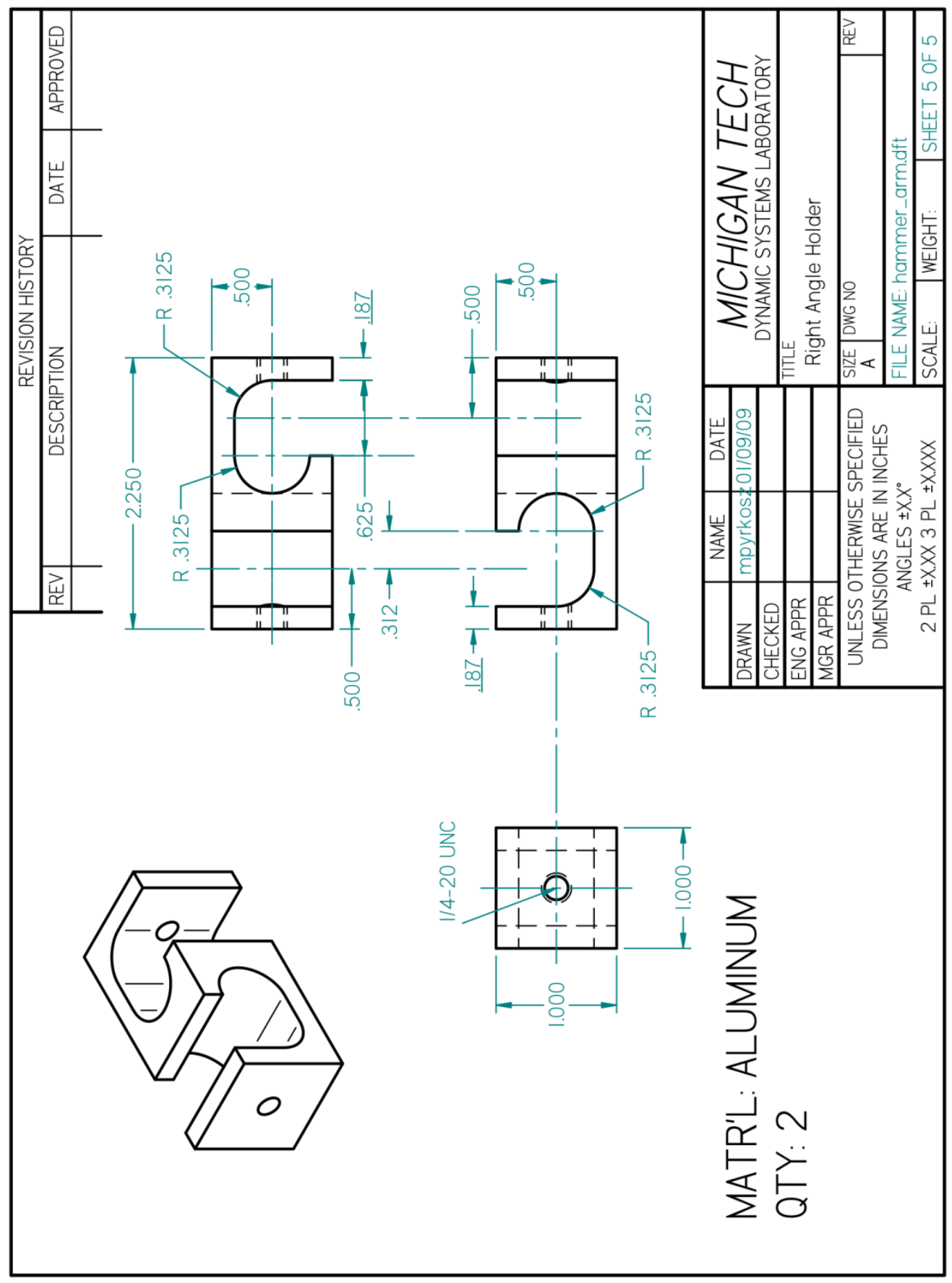




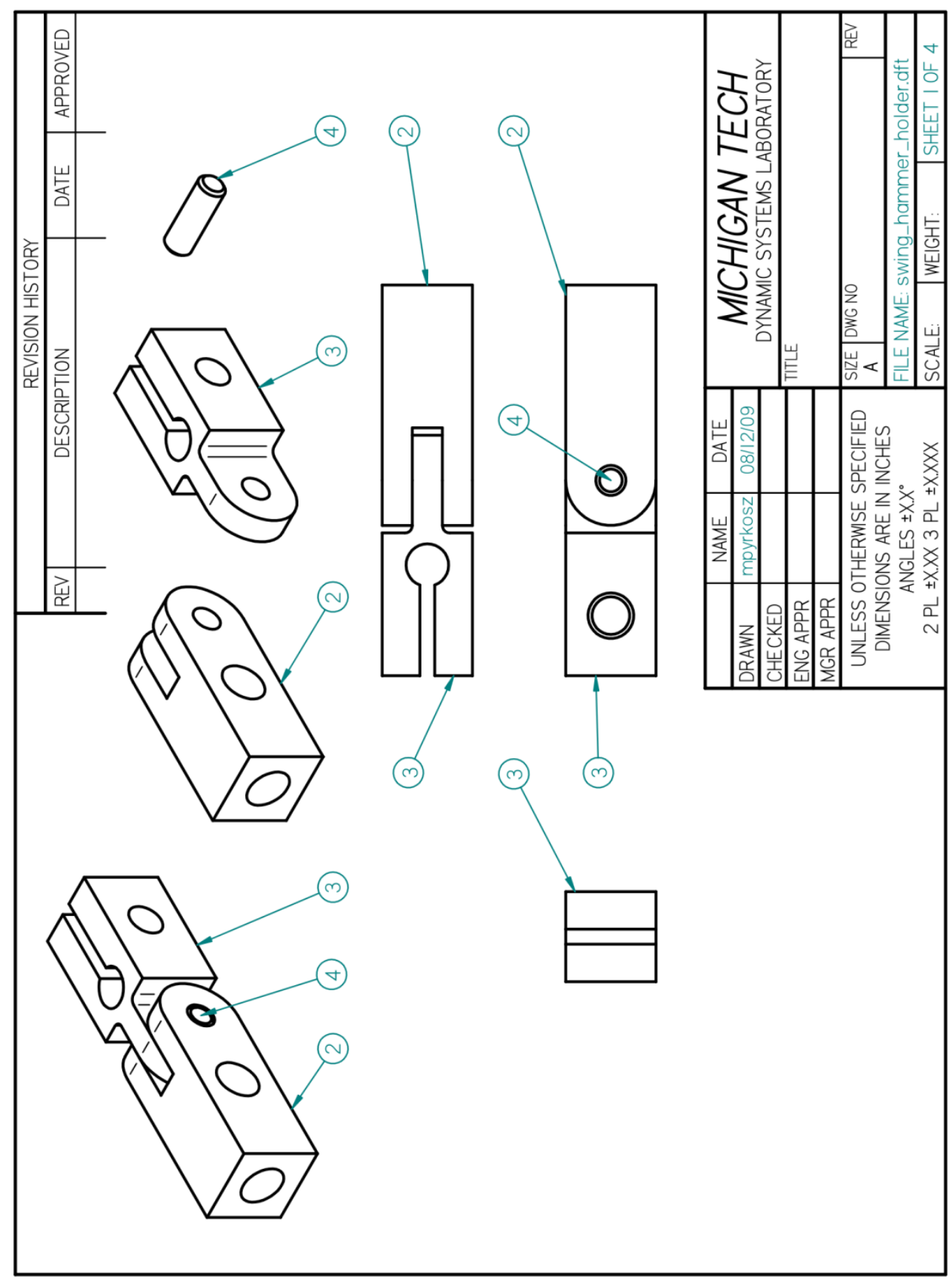

Michael Pyrkosz 


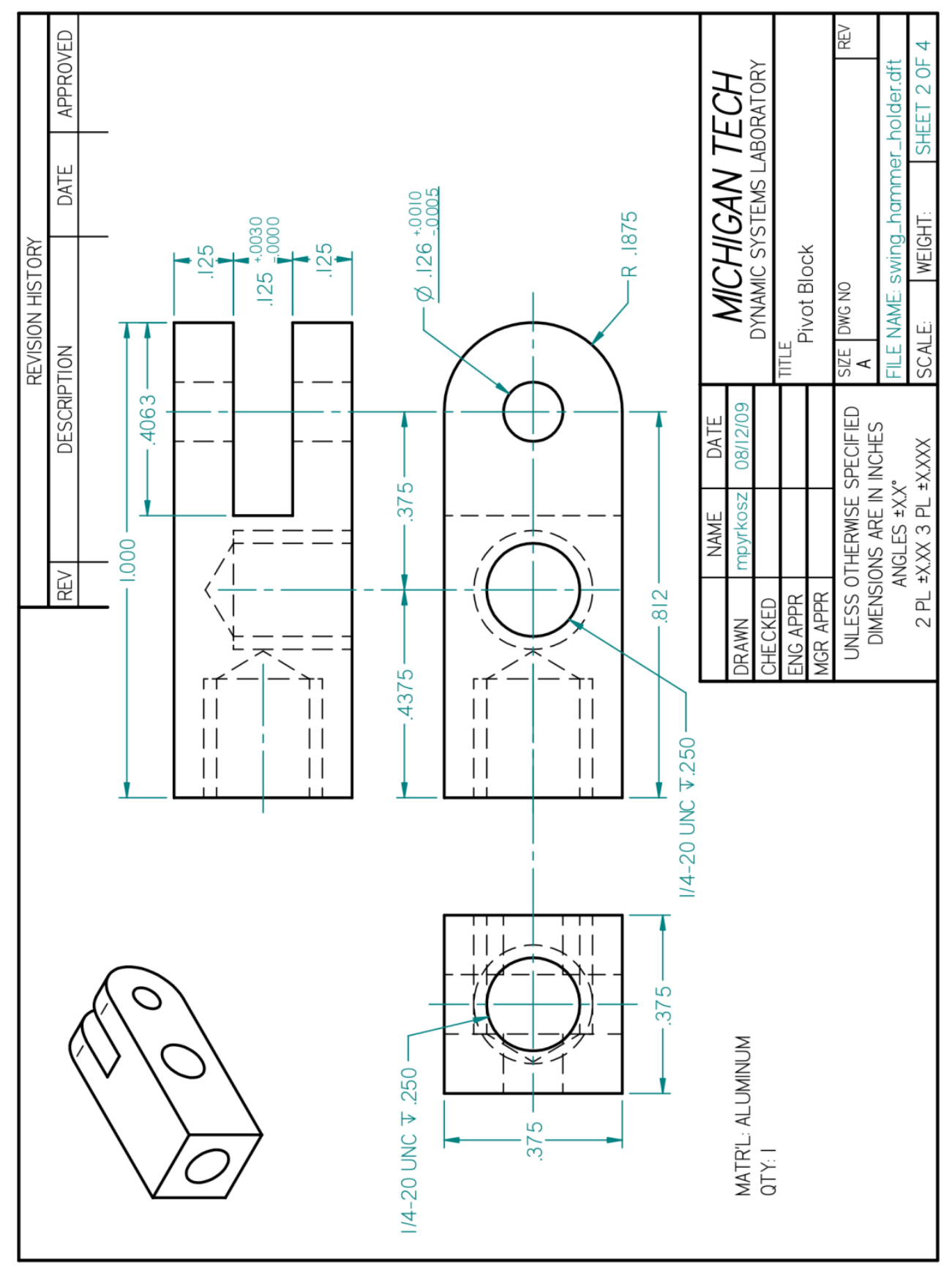




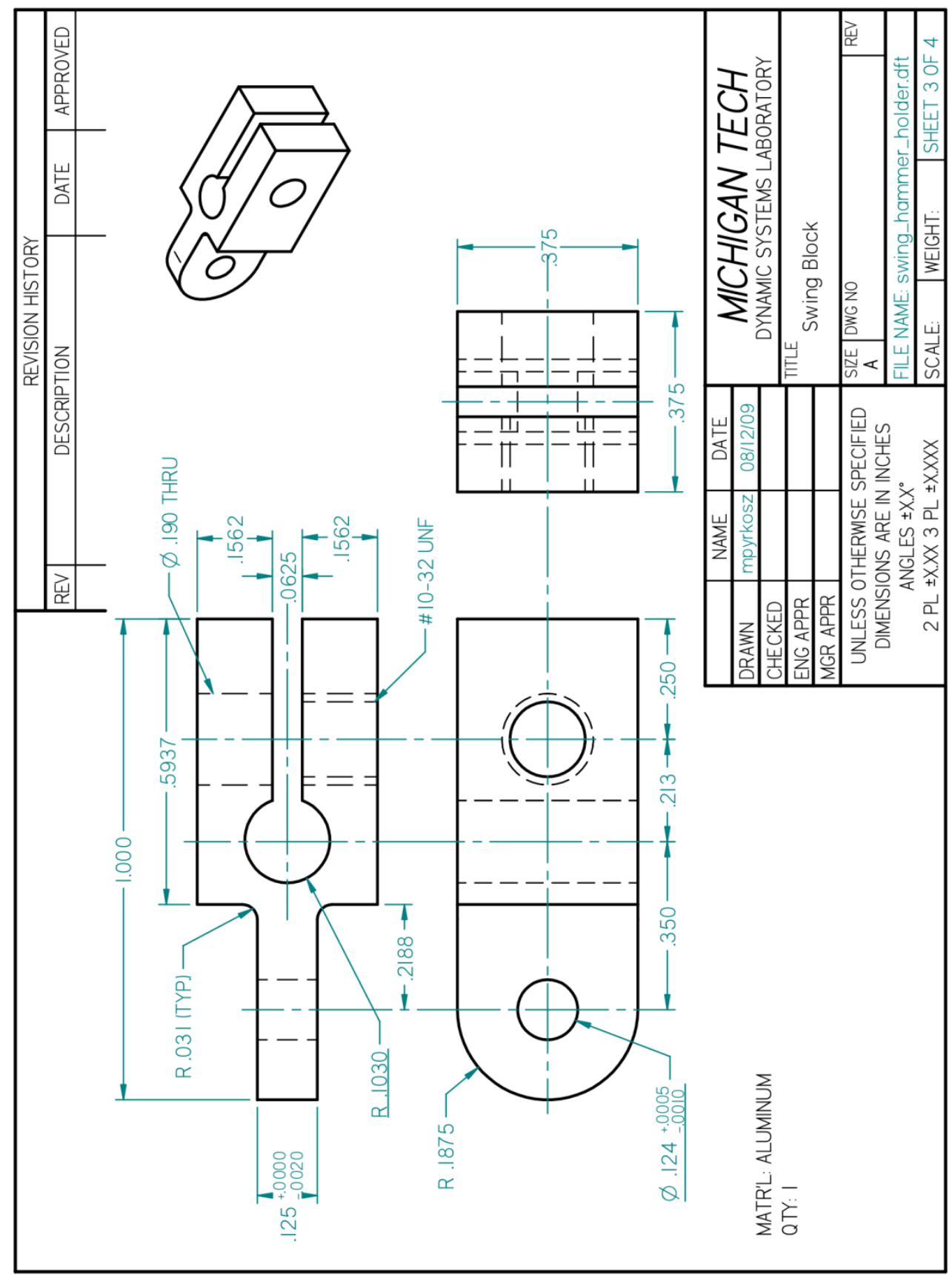

Michael Pyrkosz 


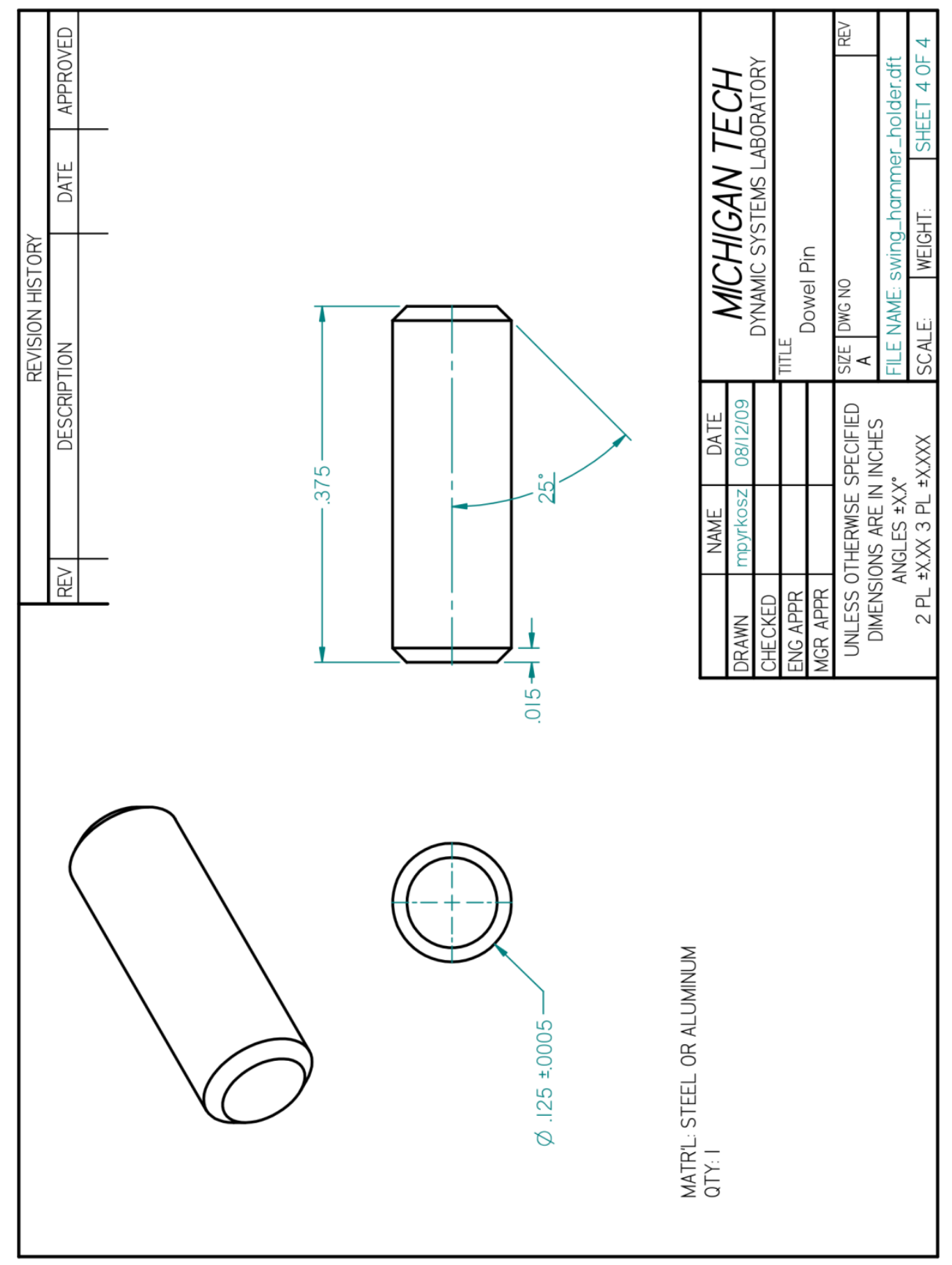




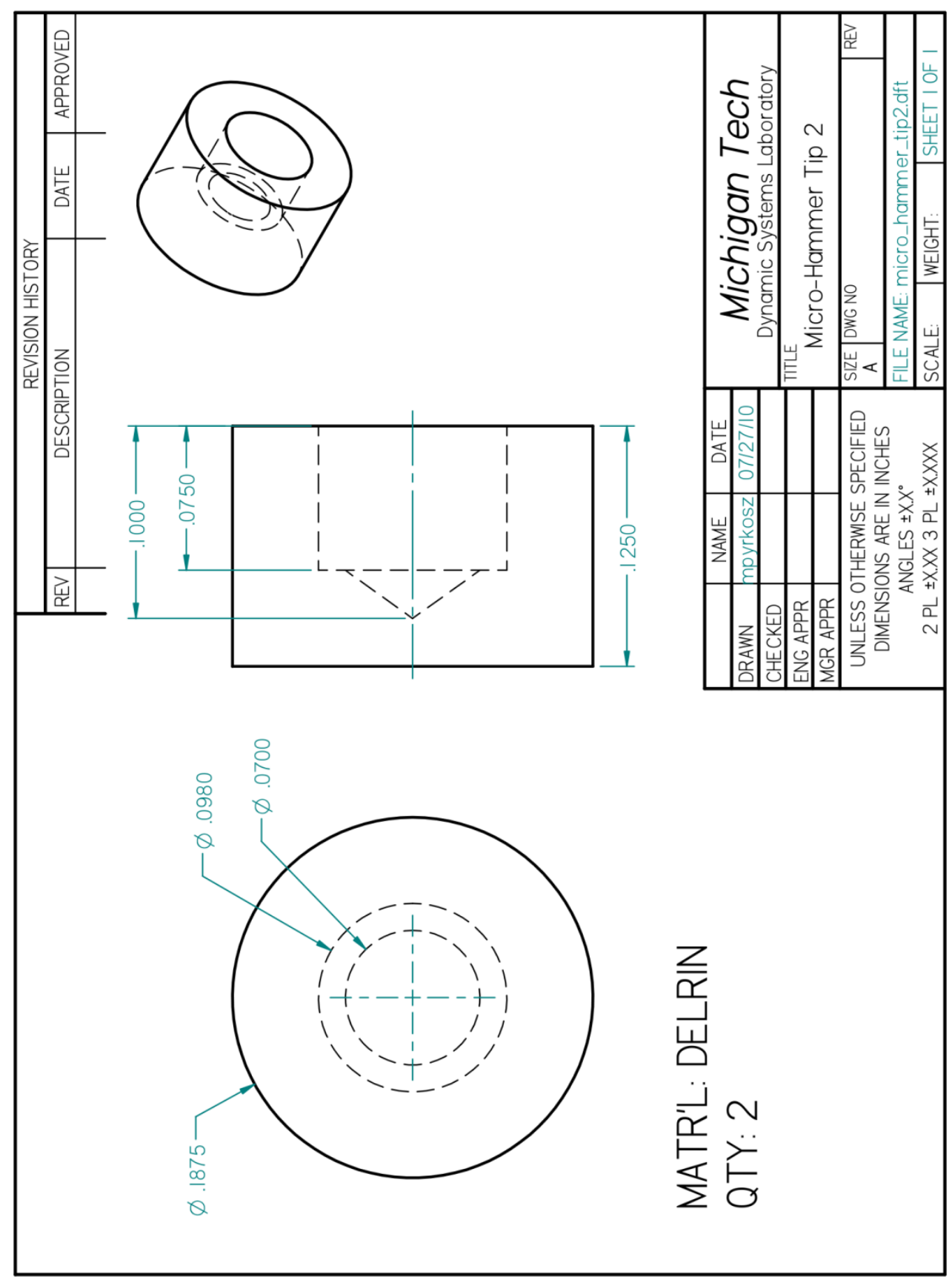

Michael Pyrkosz 
fine 